\title{
ANÁLISIS Y OPTIMIZACIÓN DE LA RESISTENCIA DE INTERFERENCIA EN MULTICASCOS
}

\section{Tesis Doctoral}

\section{DAVID FERNÁNDEZ GUTIÉRREZ}

Ingeniero Naval
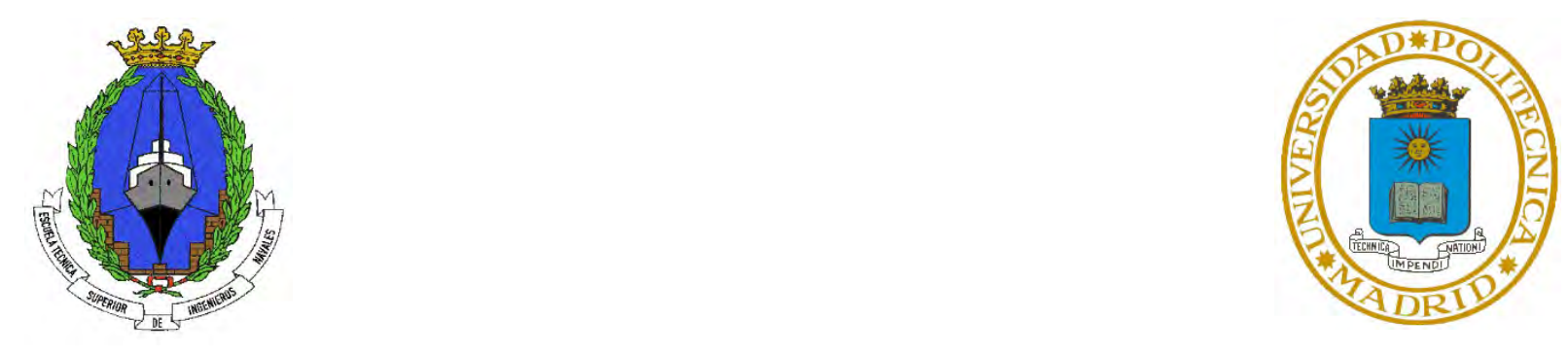

Director: ANTONIO SOUTO IGLESIAS 
Depto. de Arquitectura, Construcción, Sistemas Oceánicos y Navales Escuela Técnica Superior de Ingenieros Navales Universidad Politécnica de Madrid

\section{Análisis y optimización de la resistencia de interferencia en multicascos}

(Tesis Doctoral)

por

David Fernández Gutiérrez

Ingeniero Naval (Universidad Politécnica de Madrid, España, 2008)

MS en Ingeniería Mecánica (University of California at Berkeley, USA, 2014)

Director: Antonio Souto Iglesias

Doctor Ingeniero Naval (Universidad Politécnica de Madrid, España)

Madrid, 11 de Enero de 2016 


\section{POLITÉCNICA}

Tribunal nombrado por el Sr. Rector Magfco. de la Universidad Politécnica de Madrid, el día de de $20 \ldots$.

Presidente:

Vocal:

Vocal:

Vocal:

Secretario:

Suplente:

Suplente:

Realizado el acto de defensa y lectura de la Tesis el día de. de $20 \ldots$ en la E.T.S.I. /Facultad.

Calificación

EL PRESIDENTE

LOS VOCALES 


\section{RESUMEN}

Esta tesis presenta un estudio de la resistencia de interferencia en multicascos, término que engloba las variaciones en la resistencia al avance debidas a la interacción entre cascos. Su característica más notable es que puede ser tanto positiva como negativa, contribuyendo favorablemente en este último caso a la reducción de la resistencia total. Su análisis permanece como un área activa dentro de la comunidad hidrodinámica, si bien se ha detectado una significativa falta de información experimental sobre el flujo entre cascos.

En primer lugar se incluye una caracterización de los fenómenos de interferencia, evaluando su impacto en las diferentes componentes de la resistencia al avance. Al igual que la resistencia total, su predicción requiere el uso de técnicas experimentales o numéricas al ser inviable su solución analítica. Ambos procedimientos han sido considerados en esta tesis.

El análisis experimental de la resistencia de interferencia supone el núcleo central del trabajo realizado. Se han ensayado dos geometrías diferentes, un catamarán de servicio comercial PESBO junto con un catamarán compuesto por dos Serie 60 (S60) en paralelo. Los ensayos se han llevado a cabo en dos canales de experiencias, CEHINAV y CEHIPAR, midiéndose resistencia al avance, asiento y trimados dinámicos, y cortes de olas en la zona entre cascos. Mención especial a estos últimos, dado que a pesar de ser en la zona central donde ocurren los principales efectos de interferencia, no se encontró información previa publicada en la literatura. Su medición requirió la fabricación de un sistema de soporte específico para las probetas de medición. Por otro lado, aunque el Serie 60 es una geometría bien conocida en la comunidad hidrodinámica, a conocimiento del autor su comportamiento como multicasco no había sido aún caracterizado experimentalmente.

Los bastidores de unión se fabricaron de forma que permitieran variar la separación entre cascos. Además, los multicascos son en general embarcaciones de alta velocidad mostrando asientos y trimados dinámicos significativos. Para cuantificar su efecto en la resistencia total y de interferencia se construyó un sistema de fijación del modelo al carro que los restringiera, 
posibilitando comparar los resultados en esta condición con los del modelo libre. Como resultado, se han obtenido resultados experimentales de las dos geometrías estudiadas con múltiples separaciones, incluyendo un solo casco, y con modelo fijo y libre.

Una de las principales utilidades de los resultados experimentales es servir como referencia para validar modelos de predicción. Como primera aplicación, los datos experimentales obtenidos con el catamarán S60 se han contrastado con las predicciones numéricas de Yeung et al. (2004). El análisis numérico de la resistencia de interferencia se completa con la realización de simulaciones numéricas utilizando dos códigos substancialmente diferentes: Tdynlin y CD-Adapco Star-CCM+. Los cortes de olas obtenidos en las simulaciones se comparan con los valores experimentales, mostrándose además los campos de presiones y velocidades calculados sobre el casco.

La principal aportación de esta tesis es una extensa caracterización experimental de los fenómenos de interferencia, con la intención de servir de referencia para futuros estudios con multicascos. El análisis de los resultados permite comprender mejor el impacto que la interacción entre cascos, así como las variaciones dinámicas de las condiciones de navegación, tienen en las diferentes componentes de la resistencia. Por otro lado, permite apreciar la dependencia de la resistencia de interferencia con la velocidad y separación entre cascos, de cara a su utilización como parámetro de diseño. 


\section{ABSTRACT}

This thesis presents a study of the interference resistance in multihulls, which comprises all the variations in the ship resistance due to the interaction between hulls. Its most remarkable characteristic is that it can be positive or negative, contributing in the second case to the reduction of the total ship resistance. Its study remains an active area in the hydrodynamic community, although there is a significant lack of published experimental data regarding the flow between hulls.

First of all, the interference phenomena are characterized, evaluating the impact in the different components of the ship resistance. Similar to the total resistance, their prediction requires experimental or numerical techniques, as no analytic solutions are known. Both procedures have been considered in this thesis.

The experimental analysis of the interference resistance is the core of the work done. Two different geometries have been tested, a commercial catamaran PESBO and a catamaran composed by two parallel Series 60 (S60). The tests have been carried out in two towing tanks, CEHINAV and CEHIPAR, measuring ship resistance, dynamic sinkage and trim, and wave cuts in-between the hulls. Special mention to the wave cuts, as no previous published information was found regarding this zone, even if it is where the main interference effects happen. Their measurement required building a support tripod for the probes, specifically designed for this experimental campaign. In addition, although the Series 60 is a well known hull for experimental and computational analyses, to the author's knowledge its behavior as a multihull had not yet been experimentally described.

The connection frames between the hulls were built so they allowed adjusting the hull spacing. Furthermore, multihulls are usually high-speed vessels, acquiring significant dynamic sinkage and trim. To quantify the effect of these dynamic variations of the interference resistance, a clamping system was developed to fixed the model and compare the results with the free model condition. Thus, experimental results have been obtained for various hull 
separations with the two geometries analyzed, including a single hull, and with free and fixed model.

One of the main applications of experimental results is to serve as a reference to validate prediction models. As a first step, the obtained experimental data with the catamaran S60 have been compared with the numerical predictions of Yeung et al. (2004). The numerical analysis of the interference resistance is completed with the inclusion of numerical simulations using two codes substantially different: Tdynlin and CD-Adapco Star-CCM+. The wave cuts obtained from the simulations are compared with the experimental ones, showing also the pressure and velocity fields over the hulls.

The main contribution of this thesis is an extensive experimental characterization of the interference phenomena, aiming to serve as reference for future studies about multihulls. The analysis of the results provides insights into the impact that the interaction between hulls, as well as the dynamic variations of the sailing conditions, have in the different components of the ship resistance. Moreover, it allows us to observe the dependence of the interference resistance on the velocity and hull spacing, useful towards its use as a design parameter. 


\section{ÍNDICE}

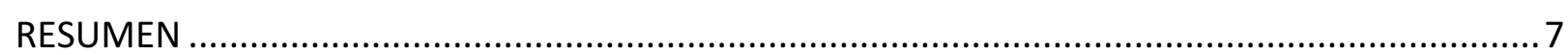

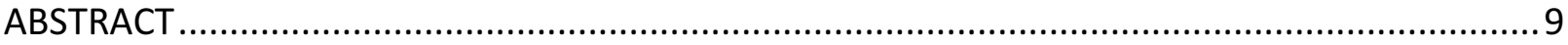

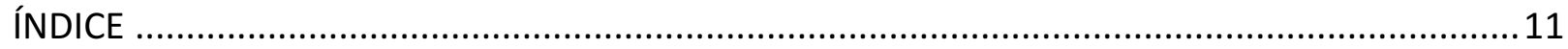

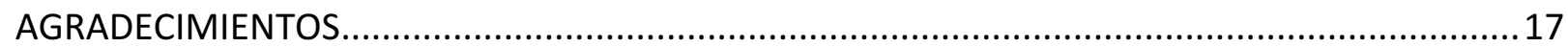

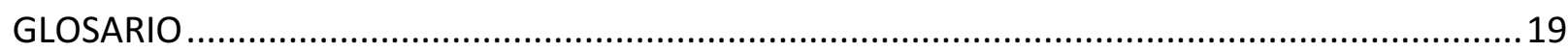

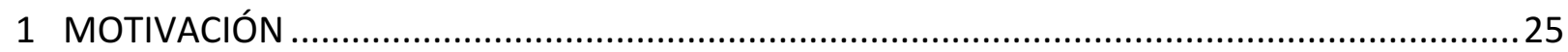

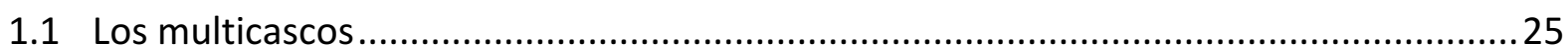

1.2 Diferencias frente a un monocasco ...................................................................... 27

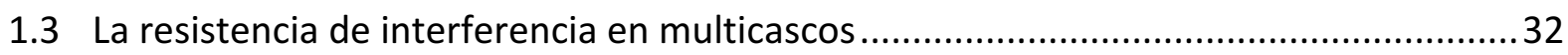

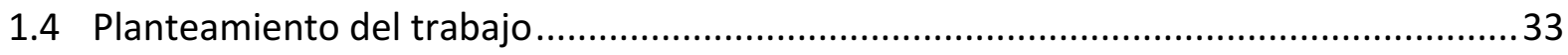

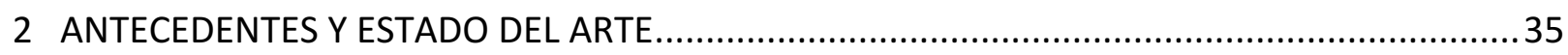

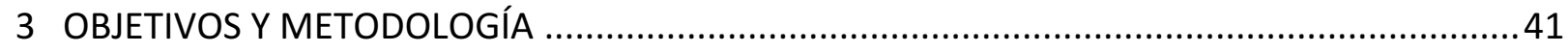

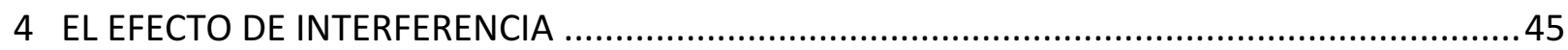

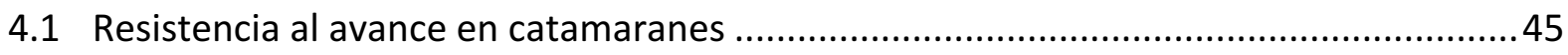

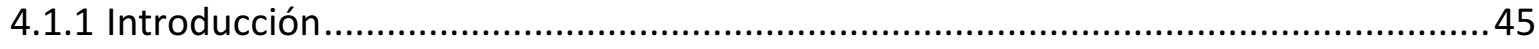

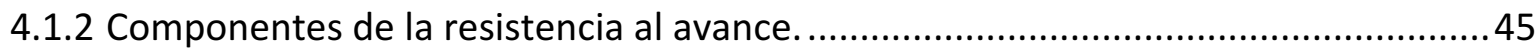

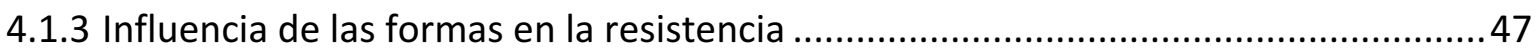

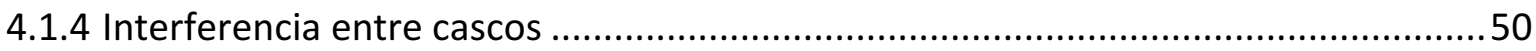

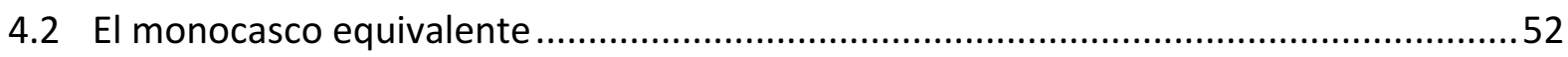

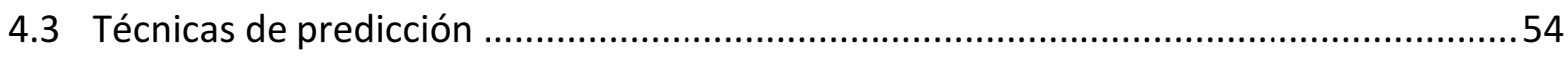




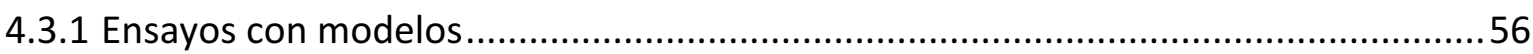

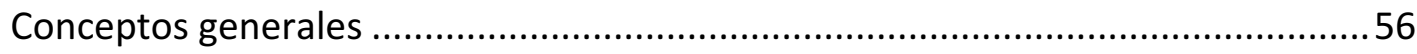

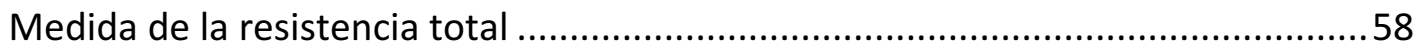

Medida de las componentes de resistencia .................................................62

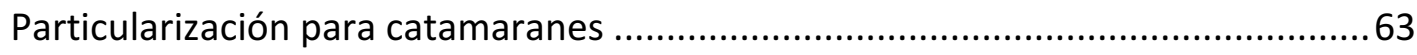

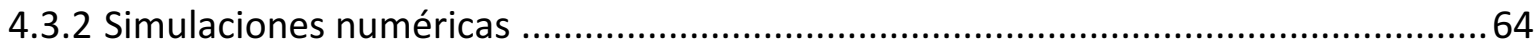

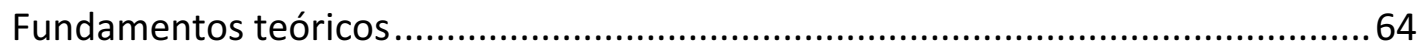

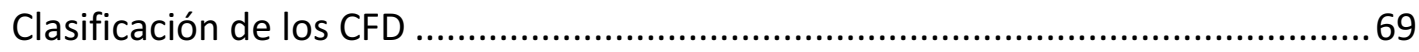

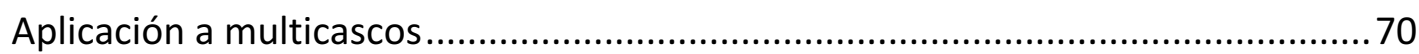

5 HERRAMIENTAS EMPLEADAS Y CONFIGURACIONES ESTUDIADAS ..................................... 73

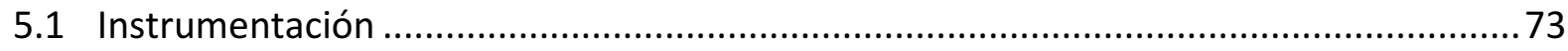

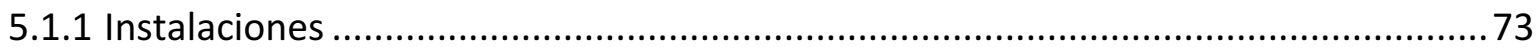

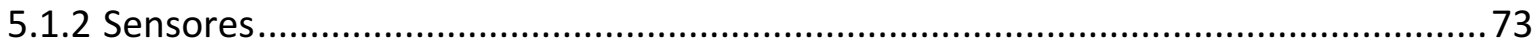

5.1.3 Ajuste de la separación entre cascos ................................................................... 76

5.1.4 Desarrollo del sistema de trimado fijo del CEHINAV ................................................77

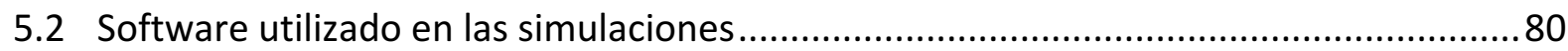

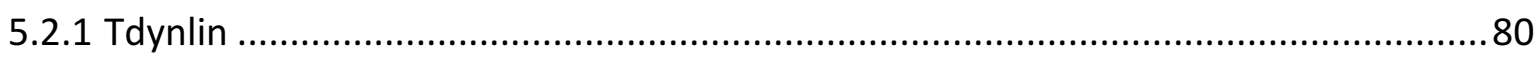

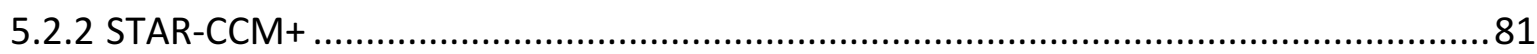

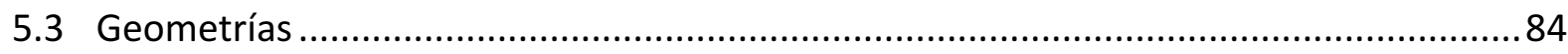

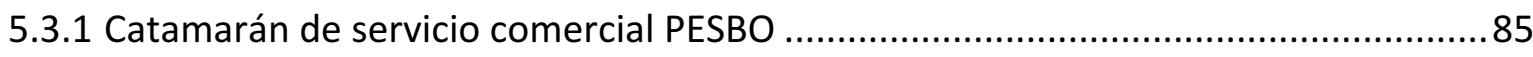

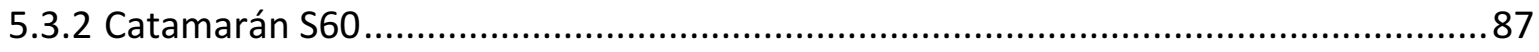

6 RESULTADOS EXPERIMENTALES .............................................................................. 91

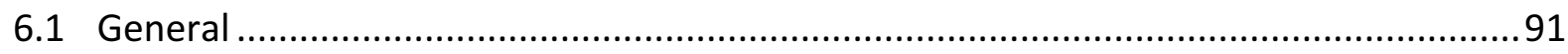

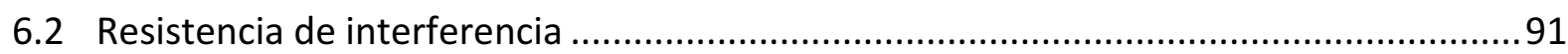




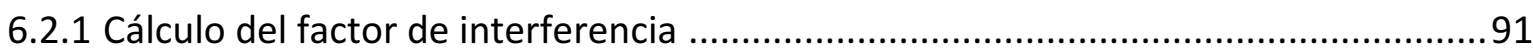

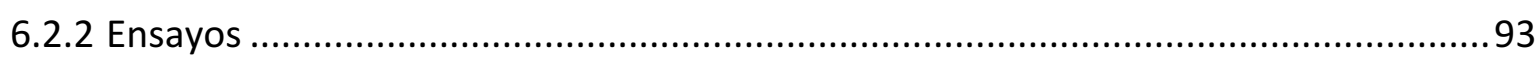

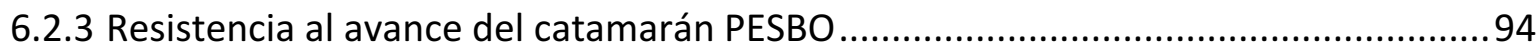

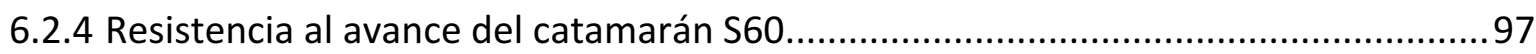

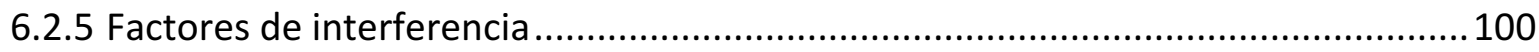

6.2.6 Comparativa con los resultados numéricos de Yeung et al. (2004) .......................103

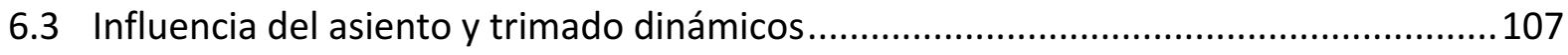

6.4 Estudio experimental del tren de olas en la zona entre cascos................................112

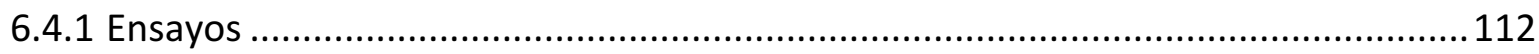

6.4.2 Impacto del trimado en los cortes de olas........................................................ 114

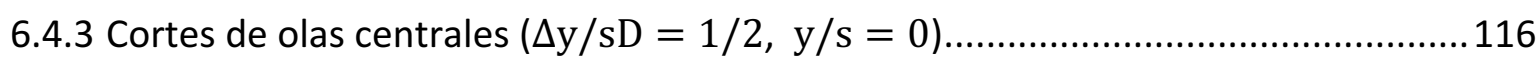

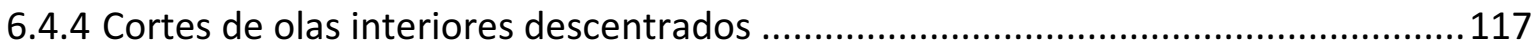

6.5 Análisis global de los resultados experimentales ..................................................120

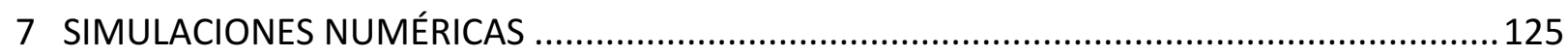

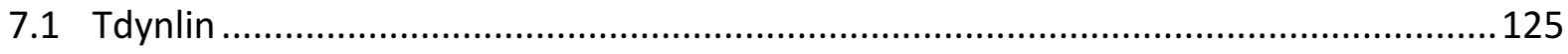

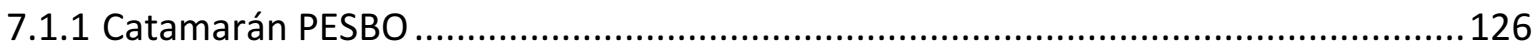

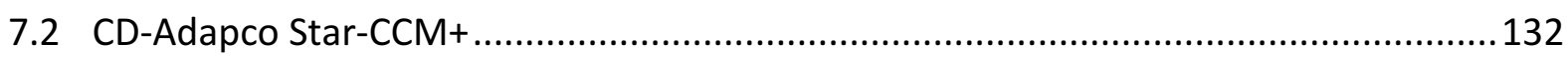

7.2.1 Configuración de los parámetros de cálculo..................................................... 132

Dominio de cálculo .................................................................................... 133

Parámetros de generación de la malla ........................................................... 135

Modelos físicos, condiciones iniciales y de contorno ........................................ 135

Visualización de los resultados ..................................................................... 138

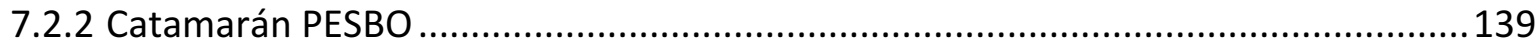

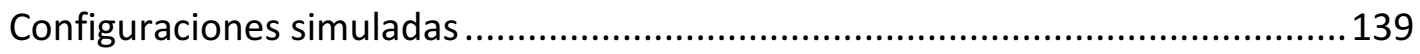

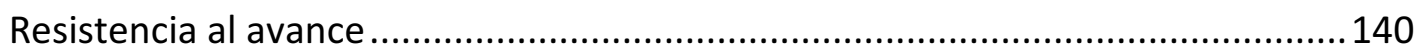

ANÁLISIS Y OPTIMIZACIÓN DE LA RESISTENCIA DE INTERFERENCIA EN MULTICASCOS 


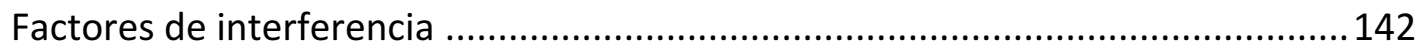

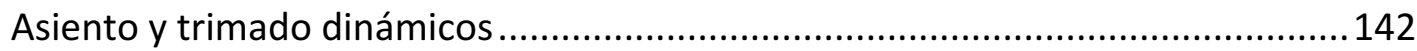

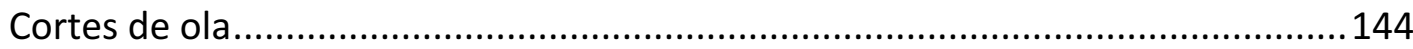

Campo de presiones y elevaciones.......................................................... 145

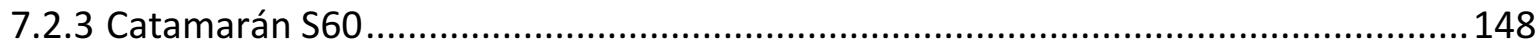

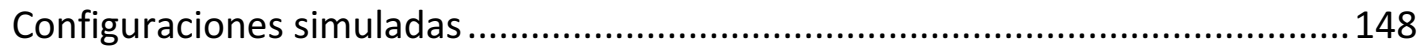

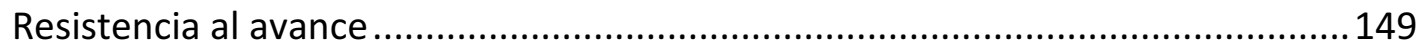

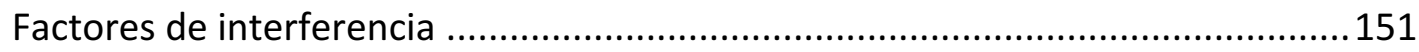

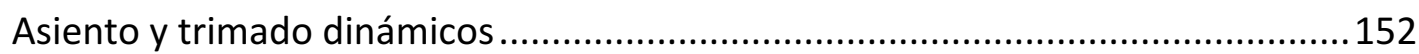

Campo de presiones y elevaciones.............................................................. 154

7.3 Análisis global de las simulaciones numéricas....................................................... 158

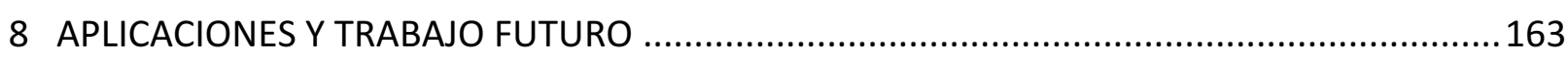

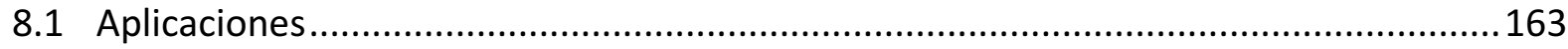

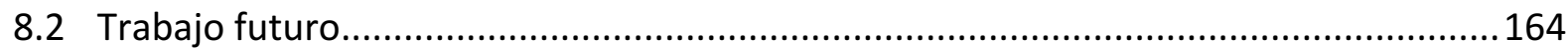

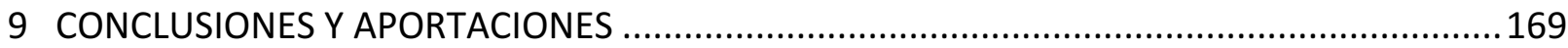

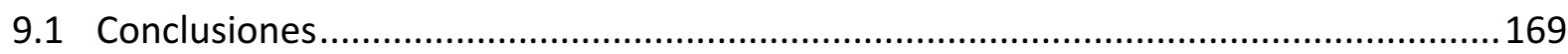

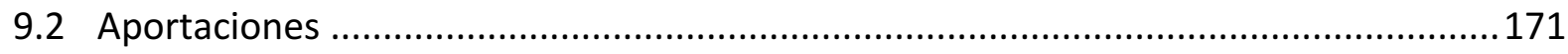

PUBLICACIONES REALIZADAS DURANTE ESTA TESIS ....................................................... 173

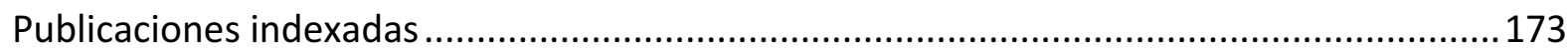

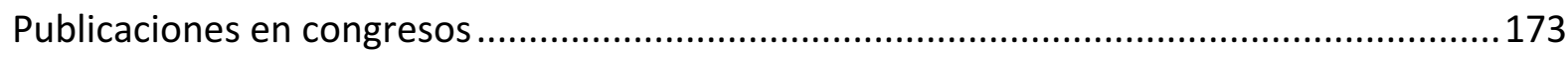

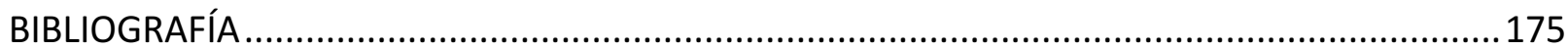

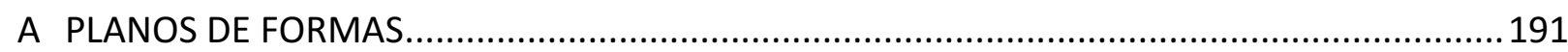

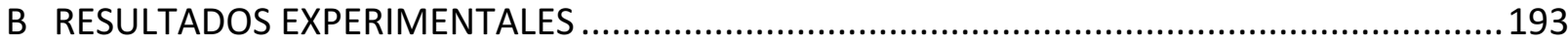

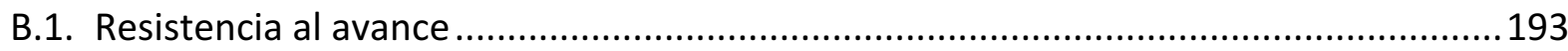




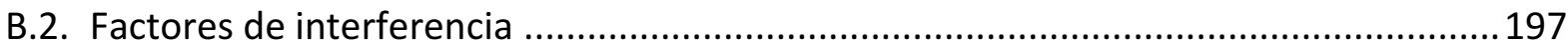

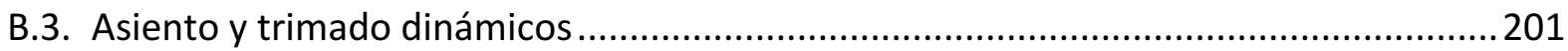

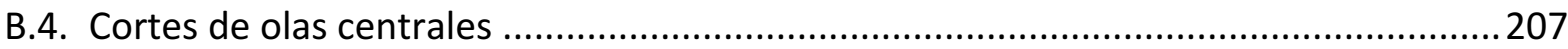

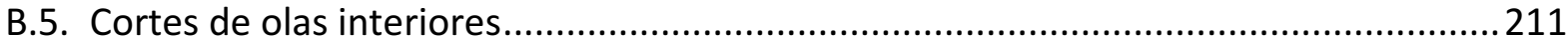

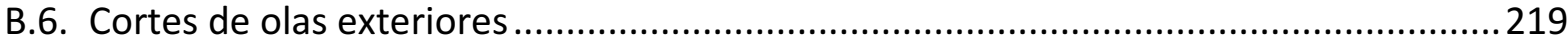

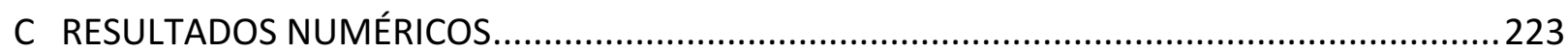

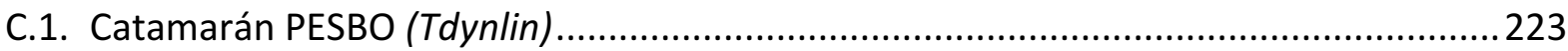

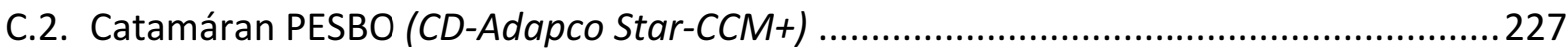

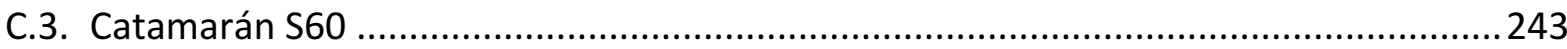




\section{AGRADECIMIENTOS}

Mi más profundo agradecimiento a mi director, Antonio Souto Iglesias, por haberme empujado a seguir adelante y confiado en mí durante todos estos años. A ti va dedicada esta tesis con todo el merecimiento. Gracias también a todos los miembros del tribunal, donde quiero añadir también a Luis Pérez Rojas, por los innumerables consejos y sugerencias.

Junto con ellos, muchas son las personas que me vienen a mi mente, y cuya ayuda ha sido indispensable para poder conseguir terminar este trabajo. Muchas gracias a Carlos López Pavón, Roque Velasco, Elkin y el personal del CEHINAV y CEHIPAR por el fantástico trabajo realizado durante los ensayos experimentales. De igual manera, llevar a cabo todas las simulaciones numéricas habría sido mucho más complicado sin la inestimable ayuda de Patricia Izaguirre. No puedo dejar de agradecer a la gente de Berkeley, y en particular a Rachael, A.J. y Lu, por escucharme siempre y ayudarme cada vez que lo he necesitado.

Sin duda, todo lo anterior no hubiera sido posible sin el apoyo y soporte de mi familia, quien siempre ha estado a mi lado. A mis padres, mis hermanas y mi novia, gracias por inspirarme día a día y acompañarme en todas estas aventuras a las que me lanzo. Por último, y no por ello menos importante, la lista de amigos, profesores y compañeros de trabajo con los que he compartido tantas experiencias inolvidables estos años es innumerable. Cada vez vamos haciendo el mapa más pequeño, ipero estoy seguro que seguiremos viéndonos en el camino!

A todos vosotros, gracias. 


\section{GLOSARIO}

\section{ACRÓNIMOS}

BEM Método de elementos de contorno (Boundary Element Method)

CFD Dinámica de fluidos computacional (Computational Fluid Dynamics)

CEHINAV Grupo de Investigación del Canal de Ensayos Hidrodinámicos de la ETSIN (UPM)

CEHIPAR Canal de Ensayos Hidrodinámicos de El Pardo

DNS Resolución numérica directa de las ecuaciones de Navier-Stokes (Direct Navier-Stokes Simulations

ETSIN Escuela Técnica Superior de Ingenieros Navales

ITTC Conferencia Internacional de Canales de Ensayos (International Towing Tank Conference)

LES Large Eddy Simulations

MSI Índice de mareo (Motion Sickness Index)

RANS Reynolds Averaged Navier-Stokes

RAO Operador de respuesta en amplitud (Response Amplitude Operator)

RI Resistencia de interferencia

S60 Geometría base de la Serie $60 \operatorname{con} C_{B}=0.60$

UPM Universidad Politécnica de Madrid

WC $\quad$ Corte de olas

\section{VARIABLES (Caracteres latinos)}

$\begin{array}{ll}a & \text { Aceleración } \\ B & \text { Manga } \\ B M & \text { Radio metacéntrico } \\ C_{A A} & \text { Coeficiente de resistencia aerodinámica } \\ C_{A P} & \text { Coeficiente de resistencia por apéndices } \\ C_{B} & \text { Coeficiente de bloque }\end{array}$


$C_{F} \quad$ Coeficiente de fricción

$\Delta C_{F} \quad$ Incremento del coeficiente de fricción debido a la rugosidad del casco

$C_{F G} \quad$ Coeficiente de resistencia for formas

$C_{R} \quad$ Coeficiente de resistencia residual

$C_{T} \quad$ Coeficiente de resistencia total

$C_{W} \quad$ Coeficiente de resistencia por formación de olas

$C R \quad$ Coeficiente de rozamiento

$e \quad$ Energía interna

F $\quad$ Fuerza

$\vec{f} \quad$ Fuerzas volumétricas

Fr Número de Froude $\left(L_{P P}\right.$ se toma como longitud de referencia, salvo que esta sea especificada con un subíndice)

$\mathrm{Fr}_{h} \quad$ Número de Froude en base a la profundidad

FS Superficie libre

GM Brazo metacéntrico

$g \quad$ Aceleración de la gravedad

$h \quad$ Profundidad

$\overline{\bar{I}} \quad$ Tensor identidad

$I_{W} \quad$ Inercia o segundo momento de área de la flotación

$I_{\eta} \quad$ Integral del cuadrado de elevaciones del corte de olas

$I_{\eta, F S} \quad$ Integral del cuadrado de elevaciones de la superficie libre

$I F \circ I F_{W} \quad$ Factor de interferencia en base a la resistencia por formación de olas

$I F_{p} \quad$ Factor de interferencia en base al campo de presiones

$I F_{R} \quad$ Factor de interferencia en base a la resistencia total

$I F_{S \& T} \quad$ Factor de interferencia debida al asiento y trimado dinámico

$k \quad$ Factor de forma, también

KB Altura del centro de carena

$K G \quad$ Altura del centro de gravedad 
$L \quad$ Eslora (si no se especifica el tipo con un subíndice se asume $L_{P P}$ )

$L_{P P} \quad$ Eslora entre perpendiculares

m Masa

$N \quad$ Número de paneles en sentido longitudinal

$Q \quad$ Fuente volumétrica de calor (radiación por ejemplo)

$p \quad$ Presión

$\vec{q} \quad$ Vector de conducción de calor

$R \quad$ Resistencia al avance ( $R_{T}$ si no se especifica el tipo con un subíndice)

$\vec{r} \quad$ Vector posición, $\vec{r}=(x, y, z)$

$R_{A} \quad$ Resistencia aerodinámica

$R_{F} \quad$ Resistencia de fricción

$R_{I} \quad$ Resistencia de Interferencia

$R_{P} \quad$ Resistencia de presión

$R_{R} \quad$ Resistencia residual

$R_{T} \quad$ Resistencia total

$\delta R_{T} \quad$ Diferencia entre la resistencia total con modelo libre y fijo, adimensionalizada con la $R_{T}$ del modelo libre

$R_{V} \quad$ Resistencia viscosa

$R_{W} \quad$ Resistencia por formación de olas

$R_{W B} \quad$ Resistencia por formación de olas rompientes

$R_{W P} \quad$ Resistencia por deformación de la superficie libre

$R_{W T} \quad$ Resistencia de estela

Re Número de Reynolds ( $L_{P P}$ se toma como longitud de referencia, salvo que esta sea especificada con un subíndice)

$S \quad$ Superficie mojada

$\mathcal{S} \quad$ Superficie delimitadora del volumen de control del dominio fluido

$s \quad$ Separación entre crujías de cada casco del catamarán

$S_{D} \quad$ Separación entre los costados interiores de cada casco del catamarán 
$T$

Calado

Temperatura (utilizado en las ecuaciones de Navier-Stokes)

$t$

$u$

V

$v$

$\vec{v}$

$v$

$V_{c r}$

w

WS

$x$

$y$

$\Delta y$

Z

$\Delta z$

Tiempo

Velocidad longitudinal

Velocidad (magnitud)

Volumen de control del dominio fluido

Vector velocidad, representando el campo de velocidades. $\vec{v}=(u, v, w)$

Velocidad transversal

Velocidad crítica

Velocidad vertical

Superficie mojada

Posición longitudinal medida desde la perpendicular de proa en sentido proa-popa

$y \quad$ Posición lateral del corte de olas

y Distancia entre la posición transversal del corte de olas y el costado del casco del catamarán más próximo

Posición vertical

Desplazamiento vertical

\section{VARIABLES (Caracteres griegos)}

$\beta$

$\Delta$

$\eta \quad$ Elevación de la superficie libre

$\lambda \quad$ Segundo coeficiente de viscosidad

$\lambda_{m} \quad$ Factor de escala del modelo

$\lambda_{w} \quad$ Longitud de ola

$\mu$

$v$ fricción

Desplazamiento Viscosidad cinemática
Factor de interferencia combinado del factor de forma y resistencia de

Viscosidad dinámica, también denominado primer coeficiente de viscosidad 


$\begin{array}{ll}\rho & \text { Densidad } \\ \sigma & \text { Factor de interferencia de la resistencia friccional } \\ \overline{\bar{\sigma}} & \text { Tensor de esfuerzos de Cauchy } \\ \tau & \text { Factor de interferencia de la resistencia por formación de olas } \\ \phi & \text { Factor de interferencia del factor de forma } \\ \Omega & \text { Factor de interferencia de la resistencia residual }\end{array}$

\section{SUBÍNDICES}

D Cada uno de los cascos de un multicasco (demihull)

$\mathrm{MH} \quad$ Multicasco (multihull) 


\section{MOTIVACIÓN}

En las últimas décadas se ha podido apreciar un crecimiento en la demanda de embarcaciones de alta velocidad, tanto para el transporte comercial como para usos militares ${ }^{1}$. En este sector, los multicascos y en particular los catamaranes juegan un papel destacado al ofrecer una menor resistencia al avance. Una de las particularidades de este tipo de embarcaciones es la interacción existente entre los cascos, denominada interferencia.

En esta tesis se analizará experimental y numéricamente la influencia que este efecto tiene en la resistencia al avance, con el fin de optimizar el comportamiento hidrodinámico de dichas embarcaciones.

\subsection{Los multicascos}

Un multicasco como su propio nombre indica es una embarcación con más de un casco, también denominado flotador, pudiéndose clasificar en:

- Proas. Disponen de dos cascos diferentes, actuando uno como flotador principal y el otro como estabilizador.

- Catamaranes. Presentan dos cascos simétricos desde crujía.

- Trimaranes. Presentan un casco central principal y luego dos cascos simétricos, uno en cada costado, que actúan como estabilizadores.

- Embarcaciones con múltiples flotadores. Llevan el concepto de multicasco a su máximo exponente, contando con más de tres cascos. Son mucho menos frecuentes, siendo la configuración más utilizada los pentamaranes, o embarcaciones de 5 cascos.

Su utilización tiene su origen en las embarcaciones utilizadas en la Polinesia para desplazarse entre islas vecinas (tipo proas). Estos se dieron cuenta que si añadían a sus canoas unos flotadores externos situados a una cierta distancia se mejoraba la estabilidad de la

\footnotetext{
${ }^{1}$ https://en.wikipedia.org/wiki/List of multihulls
} 
embarcación de una forma más sencilla que mediante lastres para bajar la posición del centro de gravedad.

Debido a su origen, los términos con los que se denominan cada tipo de flotador provienen de las lenguas malayo-polinesias ${ }^{2}$ :

- Vaka. Este término representa al flotador principal, que en castellano se puede traducir como canoa.

- Ama. Este término representa los flotadores que tienen función de estabilizadores

- Aka. Este término representa la estructura que une los distintos flotadores (amas).

De esta forma, semánticamente un multicasco tipo proa se compone de un "vaka" y un "ama”, unidos por un "aka", un catamarán se compone de dos "vakas" sujetos por el "aka", y un trimarán tiene un "vaka" y dos "amas", uno en cada costado", unidos por el "aka".

A lo largo de la historia se han empleado de forma puntual en multitud de aplicaciones, destacando la náutica deportiva así como el transporte de pasajeros.

Los multicascos son bastante populares para la competición, especialmente en Europa y Australia. Hay muchos tipos de multicascos en diferentes categorías. Entre las categorías más relevantes cabe destacar el Hobie Cat 16, Formula 18, A-cats, la clase Tornado (LaverónSimavilla et al. (2008)) y recientemente las clases AC45, AC62 y AC72 de Copa América (ACRM (2014a), ACRM (2014b) y ACRM (2013) respectivamente).

En la náutica de recreo destaca su uso en el Caribe y en el Pacífico sur. Aparecen menos a menudo en EE.UU. aunque cada vez son más frecuentes.

Ya dentro de los multicascos de pasaje con propulsión mecánica hay ferries de más de $100 \mathrm{~m}$ en funcionamiento ${ }^{3}$, pudiendo alcanzar velocidades por encima de los 40 nudos. Un tipo

\footnotetext{
${ }^{2}$ https://en.wikipedia.org/wiki/Multihull y https://en.wikipedia.org/wiki/Catamaran
} 
particular de estos con una geometría destacada son los catamaranes tipo "wavepiercing" (Fig. 1).

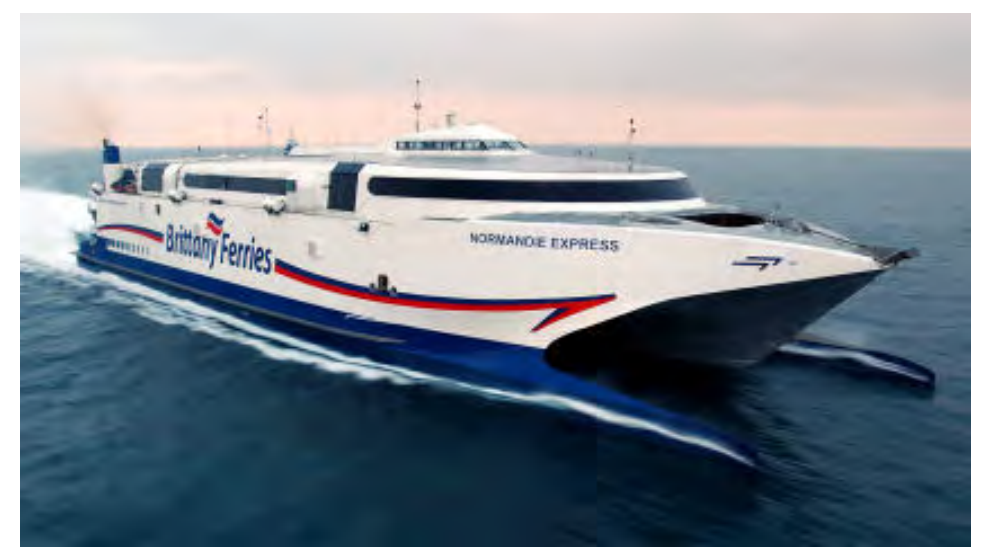

Fig. 1. Imagen del Fast Ferry tipo wavepiercing "HSC Normandie Express"

\subsection{Diferencias frente a un monocasco}

Un multicasco, como su nombre indica presenta varios cascos a lo largo de la manga del buque. Como consecuencia, la relación $B / L_{P P}$ es claramente mayor que en un monocasco.

Este hecho motiva la principal diferencia entre ambos tipos de geometrías, que es el aumento significativo de la estabilidad transversal, o lo que es lo mismo, el brazo metacéntrico. De acuerdo con la formulación general de estabilidad de buques, el parámetro característico de dicha magnitud es el GM (Aláez (1980)):

$$
G M=K B+B M-K G
$$

${ }^{3}$ El fast ferry "HSC Benchijigua Express" es el multicasco comercial de mayor tamaño construido hasta la fecha. Botado en 2004, es un trimarán con una eslora total de $126.65 \mathrm{~m}$ y una manga de $30.4 \mathrm{~m}$, pudiendo alcanzar una velocidad máxima de 42 nudos.

http://en.wikipedia.org/wiki/HSC Benchijigua Express 
donde $K B$ y $K G$ representan la altura del centro de carena y gravedad respectivamente y $B M$ es el radio metacéntrico. La configuración de un multicasco afecta principalmente a este último, el cual se calcula según:

$$
B M=\frac{I_{W}}{\text { Vol. Desplazado }}
$$

donde $I_{W}$ es la inercia o segundo momento de área de la flotación. $I_{W}$ depende cuadráticamente de la distancia al eje de simetría, significativamente mayor en el caso de catamaranes. Este hecho conlleva un aumento importante del BM. La Fig. 2 muestra cómo con el mismo valor neto del área en la flotación, el valor de $I_{W}$ es mucho mayor en el caso del catamarán. De hecho en la práctica lo que sucede es que se afinan más aún los cascos para reducir la formación de olas, manteniendo inercias significativas con superficies en la flotación menores que las del monocasco.
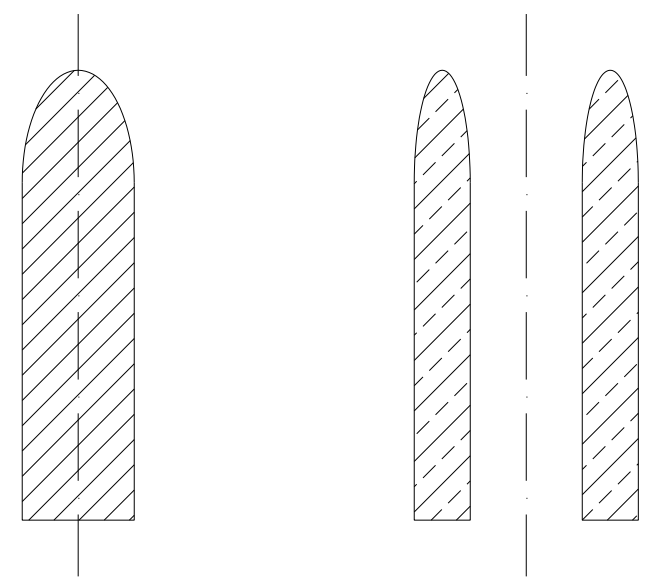

Fig. 2. Diferencia en el aspecto de la superficie de flotación para un monocasco y un multicasco para el mismo valor de área.

Por el contrario, al subdividir el volumen desplazado en varios cascos el calado de la embarcación es menor, por lo que el valor del $K B$ disminuirá. Esta reducción no obstante es significativamente inferior al aumento del $B M$. Este hecho permite trabajar con valores del $K G$ mayores. 
Una mejor estabilidad permite reducir las necesidades de lastrado, aumenta la seguridad del buque ante hundimientos y además en el caso de veleros incrementa el empuje máximo del viento que el buque es capaz de absorber ${ }^{4}$. Esto explica que estas embarcaciones sean más rápidas que sus monocascos equivalentes en condiciones de navegación semejantes. Además los movimientos son menores lo que mejora la confortabilidad a bordo, hecho importante en los buques de pasaje.

Otro aspecto que debe ser comparado es la operatividad del buque. La reducción de calado es positiva, dado que reduce las restricciones que pueda tener la embarcación de cara al posible paso por canales o atraque en puertos. Por el contrario el aumento de la manga, si bien aumenta la superficie en cubierta lo que es beneficioso de cara a la capacidad de almacenamiento, puede ocasionar problemas a la hora de atravesar canales. Es importante en este apartado tener en cuenta el tamaño del buque. Si es un buque de gran eslora $\left(L_{P P}>\right.$ $100 \mathrm{~m}$ ), una configuración tipo catamarán puede dar lugar a mangas apreciables que realmente supongan problemas.

En lo que respecta a la maniobrabilidad, en general al aumentar la manga es menor la de un multicasco respecto a un monocasco.

También se debe evaluar la respuesta de ambos tipos de configuraciones ante diferentes condiciones de navegación. Dado que las formas en un multicasco son más finas, su comportamiento en olas es mejor al reducirse los impactos sobre los cascos. Esta ventaja se torna en desventaja ante grandes oleajes que puedan dar lugar a impactos sobre la superestructura de unión entre los cascos. La amplificación dinámica de las cargas inducidas por dichos impactos pueden dañar gravemente la estructura.

${ }^{4}$ La acción del viento sobre el velamen se puede asemejar al de una fuerza aplicada en el centro vélico, que se traduce en un momento escorante importante. A mayor estabilidad transversal mayor momento escorante que se puede contrarrestar y por ende mayor fuerza admisible absorbida sobre el centro vélico, lo que representa un mayor empuje efectivo del viento. 
La configuración de multicascos es recomendable para velocidades de diseño elevadas, ya que se reduce la resistencia al avance. Cada uno de los cascos es más esbelto, reduciendo la resistencia por formación de olas que a altas velocidades es la componente predominante. No obstante, para velocidades bajas es más adecuada una configuración de monocasco, al ser los efectos de fricción más relevantes. Como la superficie mojada total es inferior en estos últimos la resistencia al avance es menor.

La manga de cada uno de los cascos es más pequeña, pudiendo surgir dificultades a la hora de situar los elementos propulsores. En un monocasco la maquinaria propulsora puede estar centralizada en una cámara de máquinas. Sin embargo en un multicasco, en cada uno de los cascos que estén dotados de elementos propulsores deberán tener su propia cámara de máquinas. Además, el disponer de menor manga será una dificultad añadida a la hora de emplazar todos los equipos.

Por otro lado la necesidad de duplicar sistemas lleva asociada una mayor seguridad ante accidentes o averías. Existen buques donde se debe asegurar su operatividad a pesar de que se produzcan daños, tanto estructurales como en la propulsión. Al emplear una configuración tipo catamarán se consigue cumplir con gran parte de estos requisitos de forma intrínseca.

Por último se encuentran los aspectos constructivos. En este apartado la configuración en monocasco es claramente mejor. La experiencia acumulada en la construcción naval a lo largo de la historia, tanto desde el punto de vista constructivo como operacional es muy grande. Además el volumen en un multicasco está peor aprovechado, ya que lo que realmente se está efectuando es una subdivisión de espacios y servicios. Esto implica un aumento de las dificultades tanto de fabricación del forro como del armamento de los servicios interiores. Como consecuencia la dificultad y el coste de construcción de un monocasco por lo general es menor que el de un multicasco equivalente. La Tabla 1 resume las conclusiones obtenidas al comparar la configuración de un monocasco frente a un multicasco. 
Tabla 1. Valores representativos del buque proyectado.

\begin{tabular}{|l|c|c|}
\hline PARÁMETRO & MONOCASCO & MULTICASCO \\
\hline Estabilidad & & MEJOR \\
\hline Restricciones de calado & & MEJOR \\
\hline Restricciones de manga & MEJOR $^{5}$ & \\
\hline Necesidades de lastrado & & MEJOR \\
\hline Superficie en cubierta & & MEJOR \\
\hline Maniobrabilidad & MEJOR & MEJOR \\
\hline Comportamiento en olas & & MEJOR \\
\hline Resistencia al avance a velocidades altas & & \\
\hline Resistencia al avance a velocidades bajas & MEJOR & \\
\hline Sistema propulsor & MEJOR & MEJOR \\
\hline Seguridad ante accidentes y averías & & MEJOR \\
\hline Experiencia constructiva & & \\
\hline Dificultad y coste de construcción & & \\
\hline
\end{tabular}

Como se puede deducir de la tabla anterior, no se puede afirmar categóricamente que ninguna de las dos configuraciones es mejor o peor, sino que dependerá de las necesidades de cada diseño en particular. Por norma general atendiendo a la rentabilidad de la inversión se utilizan monocascos.

${ }^{5}$ Especialmente para buques de esloras grandes $(L>100 \mathrm{~m})$.

${ }^{6}$ Siempre y cuando no se alcancen alturas de ola que impacten sobre la estructura de unión de los cascos, hecho que puede dar lugar a pantocazos con graves consecuencias estructurales. 


\subsection{La resistencia de interferencia en multicascos}

Centrándonos en la resistencia al avance, un multicasco, a diferencia de un buque convencional, presenta varios cuerpos flotantes unidos por la superestructura. La interacción entre los cascos introduce una nueva componente adicional, que se denomina Resistencia de Interferencia $\left(R_{I} \circ \mathrm{RI}\right)$.

La mayor particularidad que tiene esta componente es que puede presentar valores tanto positivos como negativos. Es decir, gracias a los efectos de interferencia se puede conseguir una reducción de la resistencia total frente a lo que se esperaría a partir de los datos del monocasco equivalente, a semejanza del comportamiento de un bulbo de proa. De forma teórica, Chen \& Sharma (1997) muestran cómo la resistencia por formación de olas puede ser nula en multicascos moviéndose en canales poco profundos a alta velocidad. No obstante, dicha reducción se produce de forma puntual en rangos específicos de velocidades dependientes de la separación entre cascos.

En cualquier caso, la interferencia entre cascos es un fenómeno que debe ser tenido en cuenta a la hora de acometer el diseño de un multicasco. Su correcta estimación puede permitir realizar pequeñas modificaciones que permitan reducir la resistencia al avance, con el consiguiente ahorro en la explotación del buque. Por el contrario, no tenerla en cuenta puede dar lugar a definir una configuración innecesariamente ineficiente desde el punto de vista hidrodinámico. El objetivo será conseguir un diseño que cumpliendo con las limitaciones del proyecto pueda beneficiarse lo máximo posible de los efectos de interacción en las condiciones de navegación previstas.

El análisis de este efecto desde diferentes puntos de vista es el núcleo de esta tesis, y a ello estarán dedicados los siguientes capítulos. 


\subsection{Planteamiento del trabajo}

Aunque existen estudios previos en la bibliografía que tratan este fenómeno, la mayoría de ellos se centran en los valores de resistencia sin estudiar los fenómenos físicos involucrados, y más concretamente en el tren de olas generado. Como consecuencia existe una cierta falta de información experimental, especialmente del flujo entre cascos.

Adicionalmente, uno de los parámetros que tiene una influencia relevante en el efecto de interferencia es la variación del asiento y trimado dinámicos entre el catamarán y el monocasco equivalente. Sin embargo, la información experimental de este hecho es también limitada. Para cuantificarlo se ha realizado un amplio estudio experimental, ensayando la misma configuración con modelo fijo y libre.

El presente trabajo pretende solventar dicha falta de información aportando a la comunidad científica una extensa base experimental y numérica de los efectos de interferencia con diferentes configuraciones. Para ello se cubrirán los siguientes puntos:

- Análisis de la bibliografía existente (capítulo 2).

- Definición de los objetivos a alcanzar y la metodología seguida (Capítulo 3).

- Descripción del efecto de interferencia, las causas que lo producen y los efectos que provoca (capítulo 4). Mención especial al debate sobre el concepto de monocasco equivalente, dado lo ambiguo de su definición (sección 4.2).

- Descripción de la instrumentación y software empleado así como de las geometrías estudiadas (capítulo 5).

- Análisis experimental y numérico de la resistencia de interferencia:

- Obtención y análisis de resultados experimentales con diferentes geometrías tipo catamarán (capítulo 6). Se incluyen resultados de resistencia, asiento y trimado dinámicos, y cortes de olas. La sección 6.5 realiza un análisis global de los diferentes resultados obtenidos

- Realización de simulaciones numéricas con las geometrías ensayadas con herramientas CFD (capítulo 7). 
- Aplicaciones prácticas de considerar la resistencia de interferencia y trabajo futuro (capítulo 8).

- Conclusiones y aportaciones (capítulo 9). 


\section{ANTECEDENTES Y ESTADO DEL ARTE}

Se pueden encontrar en la bibliografía diferentes estudios cuyo objetivo es conseguir embarcaciones más eficientes recurriendo a la utilización de multicascos (Martínez \& Castells (2005)). Migali et al. (2001), Fang et al. (2009) o Muscat-Fenech \& Rosa (2014) evalúan el comportamiento de configuraciones tipo trimarán, Tarafder et al. (2010) tetramarán ${ }^{7}$, o Wu \& Moan (2006) pentamarán. No obstante, la mayoría de los estudios se centran en la configuración tipo catamarán, bien convencional o SWATH ${ }^{8}$ (Pérez Arribas (2000)).

Dentro de las diferentes áreas de estudio, existen publicaciones que analizan los problemas estructurales particulares de este tipo de embarcaciones. Heggelund \& Moan (2002) analizan de forma global la resistencia longitudinal y a torsión de un catamarán de 60 m evaluando las tensiones y deformaciones en su estructura. Ge et al. (2005) estudian el efecto de los impactos de slamming. Chen \& Guedes-Soares (2008) calculan la resistencia última de cascos fabricados con materiales compuestos, y Librescu et al. (2007) analizan la respuesta de estructuras tipo sándwich ante impactos. Otras publicaciones se centran en aspectos constructivos, con la utilización de nuevos materiales o mejores sistemas de propulsión, como el artículo de Moraes et al. (2007).

Centrándonos en el comportamiento hidrodinámico, múltiples publicaciones analizan diferentes vertientes, destacando el comportamiento en la mar y la resistencia al avance. Una de las principales aplicaciones de este tipo de embarcaciones es el transporte de pasajeros. Minimizar las aceleraciones es esencial para maximizar el confort. En esta línea se encuentran los artículos publicados por Inoue \& Kamruzzaman (2008) o Centeno et al. (2001) donde se analiza la influencia de la separación de cascos en los movimientos de un catamarán. Davis \& Holloway (2003) evalúan la operatividad de 14 geometrías diferentes calculando su Índice de Mareo (MSI). Por otro lado, los estudios de resistencia al avance buscan en general minimizar

\footnotetext{
${ }^{7}$ Multicasco con cuatro cascos, menos frecuente en la industria.

${ }^{8}$ De sus siglas en inglés Small Waterplane Area Twin Hull (catamaranes de poca área en la flotación)
} 
ésta optimizando los diferentes parámetros de los que depende. Varias publicaciones evalúan la relevancia de las diferentes componentes de la resistencia en multicascos, en los que ya se incluye la resistencia de interferencia (Turner \& Taplin (1968), Aláez (1991), Insel \& Molland (1991)).

Gran parte de los estudios existentes consideran altas velocidades $(F r>0.5)$, condición típica en este tipo de embarcaciones (Molland et al. (1996), Migali et al. (2001), Moraes et al. (2004), Sahoo et al. (2004)). La navegación en aguas poco profundas es otro de los aspectos analizados, relevante dado el pequeño calado de los multicascos (Insel \& Doctors (1995), Jiankang et al. (2001), Tarafder \& Suzuki (2008a), Aubault \& Yeung (2012)). Algunos estudios combinan ambas condiciones evaluando el comportamiento en aguas poco profundas a velocidades supercríticas ${ }^{9}$ (Chen et al. (2003b), Chen et al. (2003a), Molland et al. (2004)) e incluso añaden el efecto de navegar en canales estrechos (Chen \& Sharma (1997)). El hecho de navegar a alta velocidad provoca variaciones dinámicas de las condiciones de navegación que es necesario tener en cuenta. Gourlay (2008) analiza esta variación, importante para evitar posibles colisiones con el fondo al navegar en aguas poco profundas.

El oleaje generado por los buques puede llegar a afectar negativamente al entorno (tanto al medio marino como a la costa), mayor aún en el caso de las embarcaciones rápidas. Se dan situaciones donde incluso se establecen límites máximos de velocidad en determinadas zonas por este motivo. La estimación por tanto de la propagación hacia la costa del oleaje generado por estos buques puede ser importante en determinados casos. Esta se puede efectuar por métodos numéricos, pero exige una potencia de cálculo muy grande. Por ello existen artículos que tratan precisamente de proponer métodos alternativos que reduzcan estas exigencias, como el de Day \& Doctors (2001) o Janson et al. (2003).

\footnotetext{
${ }^{9}$ Velocidad superior a la velocidad crítica, $V_{c r}$, definida como

$$
F r_{h}=1 \rightarrow V_{c r}=\sqrt{g h}
$$

donde $g$ es la aceleración de la gravedad y $h$ es la profundidad. Este es un punto importante en el que surgen diferencias significativas en las condiciones de navegación.
} 
Existen en la literatura muy pocos estudios realizados con embarcaciones a escala real, consistiendo la mayoría de ellos en modelos numéricos y/o ensayos en canal. Destaca el trabajo de Davis et al (2005) donde se analiza el comportamiento en la mar un catamarán tipo wavepiercing de $86 \mathrm{~m}$, obteniéndose de forma experimental los operadores de respuesta en amplitud (RAO) de los movimientos del buque en los seis grados de libertad. En esta línea, Macfarlane (2009) presenta una metodología de ensayos utilizada con un catamarán de recreo, o Lee et al. (2007) presenta el proyecto real de un pequeño catamarán. Se partió de una geometría y se fue optimizando mediante ensayos numéricos y en un canal de experiencias hasta conseguir las formas óptimas tras haber probado con varias geometrías alternativas.

Desde un punto de vista teórico, la mayoría de los estudios usan la teoría lineal para predecir la resistencia por formación de olas (Insel et al. (1994), Yeung et al. (2004), Yeung (2005)). Bajo la aproximación de cuerpos esbeltos, adecuada para los flotadores en multicascos, el flujo sobre el casco puede ser reproducido por una distribución de fuentes/sumideros sobre el plano de crujía cuya intensidad es proporcional a la pendiente horizontal de la superficie media del casco. La integral de Michell (Michell (1898)) puede usarse entonces para obtener la resistencia, como se muestra en los trabajos de Tuck (1987) y Tuck et al. (2002). Doctors (2006) extiende esta teoría a cascos con popas de espejo, Pérez Arribas (2000) la aplica en catamaranes tipo SWATH, y Lunde (1951), Yeung (1978) y Liu \& Yue (1996) consideran su utilización en condiciones no estacionarias. Por otro lado, Miller et al. (2006), Broglia et al. (2011), Zaghi et al (2011) o He et al. (2015) resuelven directamente las ecuaciones de NavierStokes, siendo capaces de estimar aceptablemente los valores de resistencia y predecir los efectos de interferencia a altos números de Froude. Dado que el flujo es diferente en cada banda de cada uno de los cascos, cada casco se comporta como un perfil sustentador cobrando importancia la predicción de las cargas laterales. Lin (1974) y Peng (2001) superponen una distribución de fuentes/sumideros y dobletes para predecir tanto la resistencia por formación de olas como las fuerzas laterales. Posteriormente Wan \& Yeung (2012) presentan un completo modelo numérico para la predicción del flujo cruzado entre cascos. 
A nivel de diseño, los efectos de interferencia que constituyen el eje central de esta tesis dependen de la geometría, orientación y separación de los cascos. Existen estudios que analizan el efecto de la utilización de cascos asimétricos, como los de Zaraphonitis et al. (2001) o Chen et al. (2003b). Kumar et al. (2005) evalúa la ventaja de introducir bulbos en el plano central. La introducción de una separación longitudinal entre los cascos aumenta la asimetría del modelo, potenciando los efectos de interferencia (Söding (1997), Yeung \& Wan (2008), Yu (2015)). Bruzzone et al. (1999) consideran la influencia de la geometría de cada casco, comparando formas simétricas con otras asimétricas que tienen el mismo desplazamiento en diferentes condiciones de navegación. En esta línea, McDonald et al. (2011) comparan configuraciones tipo $\operatorname{TriSWACH}^{10}$, trimarán convencional y monocasco. Pérez Arribas (2000) propone un método de generación de formas para catamaranes tipo SWATH buscando minimizar la resistencia al avance. Con respecto a la separación, se pueden encontrar diversos estudios numéricos que analizan su impacto en la resistencia de interferencia (Insel et al. (1994), Tarafder \& Suzuki (2007)). Sarles et al. (2011) analiza la resistencia de interferencia con diferentes cascos de sección semielíptica variando también su separación.

La principal aplicabilidad de los efectos de interferencia es la cancelación de los trenes de olas de cada casco, lo que en definitiva reduce la resistencia por formación de olas (Chen \& Sharma (1997), Chen et al. (2003a), Tuck (2005)). Chen \& Huang (2004) presentan un método de optimización donde se va modificando sistemáticamente una geometría inicial en base al tren de olas generado para minimizar la resistencia de las formas finales. La mayoría de los estudios asumen modelo fijo y teoría lineal, lo que reduce las exigencias computacionales y permite por tanto ser implementados en un entorno de optimización en las fases iniciales del proyecto (Tuck \& Lazauskas (1998), Moraes et al. (2007), Yeung \& Wan (2008)).

En general, la mayoría de los trabajos se centran únicamente en los valores de resistencia al avance (Bruzzone et al. (1999), Sahoo et al. (2004), Zaghi et al (2011), Pavkov \& Morabito

\footnotetext{
${ }^{10}$ Trimarán con casco central de pequeña área en la flotación, cuyas siglas provienen de su nombre en inglés: Trimaran Small Waterplane Area Center Hull
} 
(2014), o Couser et al. (1998) que incluyen también la medición de fuerzas laterales). Molland et al. (1996), Lugni et al. (2004) o Migali et al. (2001) presentan varias campañas experimentales, si bien en general hay una cierta carencia de resultados experimentales en cuanto al tren de olas, especialmente en la zona entre cascos. Este hecho motivó al CEHINAV ${ }^{11}$ a iniciar una campaña experimental y numérica para estudiar el flujo en la zona central en catamaranes. Souto-Iglesias et al. (2005) y Souto-Iglesias et al. (2007) presentan los datos obtenidos en la primera fase de ensayos con un catamarán de pesca comercial. Los ensayos iniciales permitían el movimiento libre en el plano vertical, dificultando la identificación de los efectos de interferencia al encontrarse diferencias significativas en los trimados dinámicos. Posteriormente, este estudio fue ampliado con nuevos ensayos con trimado fijo, junto con un nuevo modelo consistente en dos Serie 60 en paralelo. El análisis completo del trabajo realizado durante estos años da lugar a esta tesis, cuyos principales resultados son presentados por Souto-Iglesias et al. (2012).

${ }^{11}$ Grupo de investigación del Canal de Ensayos Hidrodinámicos de la Escuela Técnica Superior de Ingenieros Navales (ETSIN) de la Universidad Politécnica de Madrid (UPM). 


\section{OBJETIVOS Y METODOLOGÍA}

El principal objetivo de esta tesis es indagar en el impacto que la separación de los cascos y geometría tienen en la resistencia de interferencia en aguas profundas, con especial énfasis en el análisis del flujo entre cascos. Dicho estudio ha sido abordado desde dos puntos de vista, experimental y numérico, con el fin adicional de servir como referencia para validar futuros modelos de predicción, así como evaluar las ventajas/dificultades de los ya existentes.

La resistencia de interferencia es un fenómeno complejo, dado que en esencia engloba las variaciones de todas las componentes de la resistencia al avance de un multicasco frente a un monocasco. La secuencia seguida procede de forma recursiva tal y como muestra la Fig. 3.

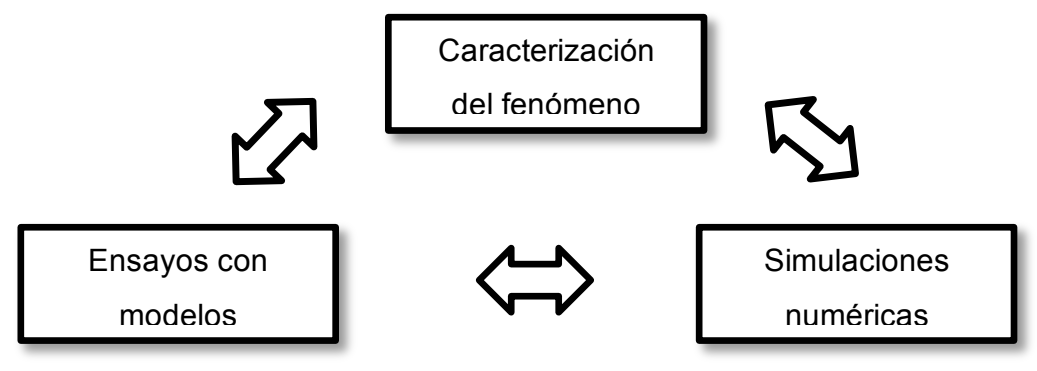

Fig. 3. Esquema de la metodología seguida

El primer paso es caracterizar el fenómeno, profundizando en la resistencia de interferencia en sí, y discernir las diferentes variables de las depende. Idealmente, el objetivo debería ser encontrar una solución analítica que permita calcular exactamente su valor. Desafortunadamente dicha solución aún no se conoce, lo que nos lleva a buscar simplificaciones que permitan aproximar los valores de resistencia como son precisamente los modelos numéricos. Por otro lado, los ensayos experimentales permiten cuantificar sistemáticamente la influencia de cada variable y evaluar la precisión de los modelos numéricos. Toda la información recogida permite volver de nuevo al primer paso y caracterizar mejor la resistencia de interferencia, comenzando de nuevo el proceso. 
El origen de este proyecto surge tras un estudio de resistencia al avance de un catamarán de servicio comercial llevado a cabo en el CEHINAV. En éste se observaron variaciones significativas en la resistencia al variar la separación entre cascos, lo que llevo a analizar los efectos de interferencia. En primer lugar se realizaron simulaciones numéricas con el código de paneles Tdynlin ${ }^{12}$, siendo necesario modificar este para poder considerar multicascos (Souto-Iglesias et al. (2005)). Experimentalmente, se procedió a realizar una nueva campaña midiendo la resistencia al avance y realizando cortes de olas tanto centrales como exteriores con diversas configuraciones, incluyendo un solo casco (Souto-Iglesias et al. (2007)).

Una de las principales fuentes de incertidumbre observadas fue el efecto del asiento y trimado dinámicos, diferentes en el multicasco y en el monocasco. Este parámetro fue medido, pero su influencia debía ser analizada con más detalle. De hecho, la mayoría de las simulaciones numéricas asumen modelo fijo, mientras que los ensayos se hacen con trimado libre. Por ello, la segunda fase se centró en cuantificar este hecho utilizando el monocasco base de la Serie 60 con $C_{B}=0.60$ (S60) (Kim \& Jenkins (1981), Fernández-Gutiérrez (2010)). En primer lugar se fabricó un sistema que permitiera realizar ensayos con asiento y trimado fijo. De esta forma se puede comparar la respuesta hidrodinámica de las distintas configuraciones para la misma condición de navegación, y validar así mejor los resultados de Tdynlin. En paralelo, utilizando el código comercial $\mathrm{CD}$-Adapco Star- $\mathrm{CCM}+{ }^{13}$ se realizó el mismo análisis numéricamente al poder asumir modelo fijo o libre, utilizándose los datos experimentales para evaluar su precisión a la hora de calcular el asiento y trimado dinámicos.

En una tercera fase, además del catamarán comercial de la primera fase se incluyó una nueva configuración con dos S60 en paralelo, ensayándose también con trimado libre (Souto-Iglesias et al. (2012)). Dado el mayor rango de velocidades como catamarán, la geometría original

\footnotetext{
12 Tdynlin es un código potencial de paneles del CEHINAV, inicialmente desarrollado por el Prof. SoutoIglesias (Souto-Iglesias (2001)). Uno de los objetivos del CEHINAV es su expansión para considerar diferentes tipos de buques, por lo que este proyecto sirvió para desarrollar y validar una nueva opción: multicascos.

${ }^{13}$ http://www.cd-adapco.com/products/star-ccm
} 
embarcaba agua lo que obligó a extender la obra muerta verticalmente para evitarlo. En los ensayos se midieron resistencia, asiento y trimado. Por otro lado, a pesar de ser un modelo bien conocido en la comunidad hidrodinámica (Todd (1963), Kim \& Jenkins (1981), Toda et al. (1992), Longo et al. (1993), Nakatake \& Takeshi (1994), Tarafder \& Suzuki (2008b)), su comportamiento como catamarán no había sido aún analizado experimentalmente. El objetivo fue por tanto caracterizar un estándar de validación para modelos numéricos de resistencia al avance en multicascos. En esta línea, los resultados obtenidos por Yeung et al. (2004), que usan también un catamarán S60 como caso de estudio, se contrastaron con los datos experimentales recogidos.

En conclusión, la Tabla 2 resume los objetivos concretos y la metodología seguida para cumplirlos. 
Tabla 2. Objetivos y metodología

\begin{tabular}{|c|c|c|c|}
\hline OBJETIVO & \multicolumn{3}{|c|}{ METODOLOGÍA } \\
\hline $\begin{array}{l}\text { Estudio experimental de la } \\
\text { resistencia de interferencia }\end{array}$ & $\begin{array}{l}\text { Realización de ensa } \\
\text { Canales De Ensayo: } \\
\text { - } \text { CEHINAV } \\
\text { - } \quad \text { CEHIPAR }^{14} \\
\end{array}$ & $\begin{array}{l}\text { s con modelos a escala } \\
\text { Geometrías: } \\
\text { - } \quad \text { Catamarán de } \\
\text { servicio comercial } \\
\text { - Catamarán } 560\end{array}$ & $\begin{array}{ll}\text { Variables Medidas: } \\
\text { - } & \text { Resistencia al avance } \\
\text { - } & \text { Cortes de olas } \\
& \text { centrales y laterales } \\
\text { - } & \text { Asiento } \\
\text { - } & \text { Trimado }\end{array}$ \\
\hline $\begin{array}{l}\text { Estudio numérico de la } \\
\text { resistencia de interferencia }\end{array}$ & \multicolumn{3}{|c|}{$\begin{array}{l}\text { Realización de simulaciones con diferentes herramientas numéricas: } \\
\text { - Tdynlin } \\
\text { Código de paneles } \\
\quad \text { Implementación de la opción de cálculo con catamaranes } \\
\text { O Simulaciones con asiento y trimado fijo } \\
\text { CD-Adapco Star-CCM+ } \\
\text { Código comercial, que permite incorporar efectos viscosos y modelos de } \\
\text { turbulencia } \\
\text { O Simulaciones con asiento y trimado fijo o libre }\end{array}$} \\
\hline Análisis del flujo entre cascos & $\begin{array}{l}\text { - Desarrollo de } ~ \\
\text { - Comparativa d } \\
\text { (catamarán y } \mathrm{n}\end{array}$ & $\begin{array}{l}\text { istema para medir cort } \\
\text { s cortes de ola centrale } \\
\text { ocasco equivalente) }\end{array}$ & $\begin{array}{l}\text { de ola entre cascos } \\
\text { catamarán) y laterales }\end{array}$ \\
\hline $\begin{array}{l}\text { Análisis de la influencia del } \\
\text { asiento y trimado en la } \\
\text { resistencia de interferencia }\end{array}$ & $\begin{array}{l}\text { Comparativa en los } \\
\text { trimado: } \\
\text { - } \quad \text { Desarrollo de } ~ \\
\text { de libertad al e } \\
\text { - Simulaciones } n \\
\text { la misma malla }\end{array}$ & $\begin{array}{l}\text { ores de resistencia con } \\
\text { istema de fijación para } \\
\text { yar modelos en canal d } \\
\text { éricas en ambas condic } \\
\text { cial }\end{array}$ & $\begin{array}{l}\text { in restringir el asiento y } \\
\text { der restringir dichos grados } \\
\text { ensayos } \\
\text { es con Star-CCM+ utilizando }\end{array}$ \\
\hline $\begin{array}{l}\text { Establecimiento de estándar de } \\
\text { validación para modelos } \\
\text { numéricos de resistencia al } \\
\text { avance en multicascos }\end{array}$ & 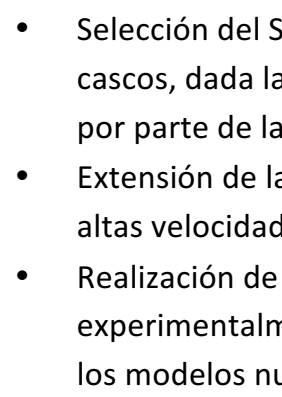 & $\begin{array}{l}\text { como geometría de ref } \\
\text { mplia experiencia con es } \\
\text { munidad hidrodinámica } \\
\text { bra muerta para evitar e } \\
\text { sayos con diferentes ser } \\
\text { te esta geometría y ser } \\
\text { éricos }\end{array}$ & $\begin{array}{l}\text { encia para las formas de los } \\
\text { modelo como monocasco } \\
\text { toarcar agua al ensayar a } \\
\text { raciones para caracterizar } \\
\text { de referencia para validar }\end{array}$ \\
\hline $\begin{array}{l}\text { Validación de los modelos } \\
\text { numéricos }\end{array}$ & $\begin{array}{l}\text { - } \text { Cálculo con mi } \\
\text { - } \text { Precisión de St } \\
\text { como la resiste } \\
\text { - } \text { Resistencia de } \\
\text { (2004) basado }\end{array}$ & $\begin{array}{l}\text { cascos en Tdynlin } \\
\text {-CCM+ para predecir el a } \\
\text { ia y elevaciones de la su } \\
\text { terferencia del modelo p } \\
\text { la aproximación de casc }\end{array}$ & $\begin{array}{l}\text { ento y trimado dinámicos, así } \\
\text { erficie libre. } \\
\text { opuesto por Yeung et al. } \\
\text { s esbeltos. }\end{array}$ \\
\hline
\end{tabular}

${ }^{14}$ Canal de Ensayos Hidrodinámicos de El Pardo, Madrid, España 


\section{EL EFECTO DE INTERFERENCIA}

\subsection{Resistencia al avance en catamaranes}

\subsubsection{Introducción}

La inercia se define como la propiedad de los cuerpos de no modificar su estado de reposo o movimiento si no es por la acción de una fuerza.

Consecuentemente, cuando un buque comienza a navegar debe atravesar una fase transitoria en la que vence su propia inercia hasta alcanzar las condiciones deseadas. Por otro lado, al navegar altera el medio en el que se encuentra. Para ello deberá emplear energía dado que el entorno se opondrá a dichos cambios, entre los que cabe destacar:

- Desplazamiento de las partículas de fluido alrededor del buque (de aire y agua) debido a los efectos de fricción viscosa

- Efectos de presión (fuerzas normales sobre el casco y superestructura, deformación de la superficie libre)

- Turbulencia, tanto del aire como del agua

- Spray

- Olas rompientes

El conjunto de todos ellos da lugar a una componente básicamente constante que deberá vencer de forma continua, y que es lo que se denomina resistencia al avance. Optimizar esta tiene como objetivo minimizar el grado en que el buque afecta al entorno y conseguir así avanzar con la mínima resistencia.

\subsubsection{Componentes de la resistencia al avance.}

La subdivisión de fenómenos físicos provocados por un buque en su avance conduce de forma natural a una subdivisión de la resistencia total $\left(R_{T}\right)$ en diferentes componentes (SNAME (1941)), tal y como se resume en la Fig. 4. Si bien todas ellas están interrelacionadas de forma muy compleja de modelizar, en la práctica normalmente se consideran independientes para 
facilitar su análisis. Su predicción es una de las áreas más importantes en construcción naval, como se presenta en la sección 4.3.

William Froude (Froude (1868), Froude (1874)) fue el primero que subdividió la resistencia total en una componente debida a la fricción, $R_{F}$, englobando el resto de factores en lo que denominó resistencia residual, $R_{R}$.

$$
R_{T}=R_{F}+R_{R}
$$

Posteriormente, Hughes (1954) identificó la resistencia por formación de olas, $R_{W}$, consecuencia directa de la componente normal de las fuerzas sobre el casco. Debido a la diferencia en cuanto a viscosidad dinámica y densidad entre el aire y agua ${ }^{15}$, la componente de resistencia aerodinámica, $R_{A}$, tradicionalmente se desprecia frente a la resistencia hidrodinámica. No obstante la resistencia varía con el cuadrado de la velocidad de encuentro, alcanzando valores un orden de magnitud superiores en el caso del viento. Por ello, en determinadas condiciones de navegación ambas componentes pueden llegar a ser comparables.

El efecto de la viscosidad es difícil de modelizar. El enfoque clásico sigue la aproximación propuesta por Prandtl (1904) de considerar los efectos viscosos únicamente en una delgada zona en contacto con la superficie del casco, denominada capa límite. Esta simplificación permite obtener resultados muy próximos a la realidad, pudiendo combinar los efectos de fricción y presión normal para determinar la resistencia viscosa, $R_{V}$. La dificultad reside en delimitar dicha zona, cuya solución analítica sólo se conoce en contadas ocasiones (Schlichting et al. (2000)).

${ }^{15}$ Propiedades de aire y agua a $20^{\circ} \mathrm{C}$ y $1 \mathrm{~atm}$

\begin{tabular}{lcccc} 
& \multicolumn{3}{c}{ Aire } & Agua \\
Densidad, & $\rho$ & 1.2 & 1000 & $\mathrm{~kg} / \mathrm{m}^{3}$ \\
Viscosidad dinámica, & $\mu$ & $0.18 \cdot 10^{-4}$ & $10 \cdot 10^{-4}$ & $\mathrm{~kg} /(\mathrm{m} \cdot \mathrm{s})$ \\
Viscosidad cinemática, & $v$ & $0.15 \cdot 10^{-4}$ & $0.01 \cdot 10^{-4}$ & $\mathrm{~m} / \mathrm{s}$
\end{tabular}




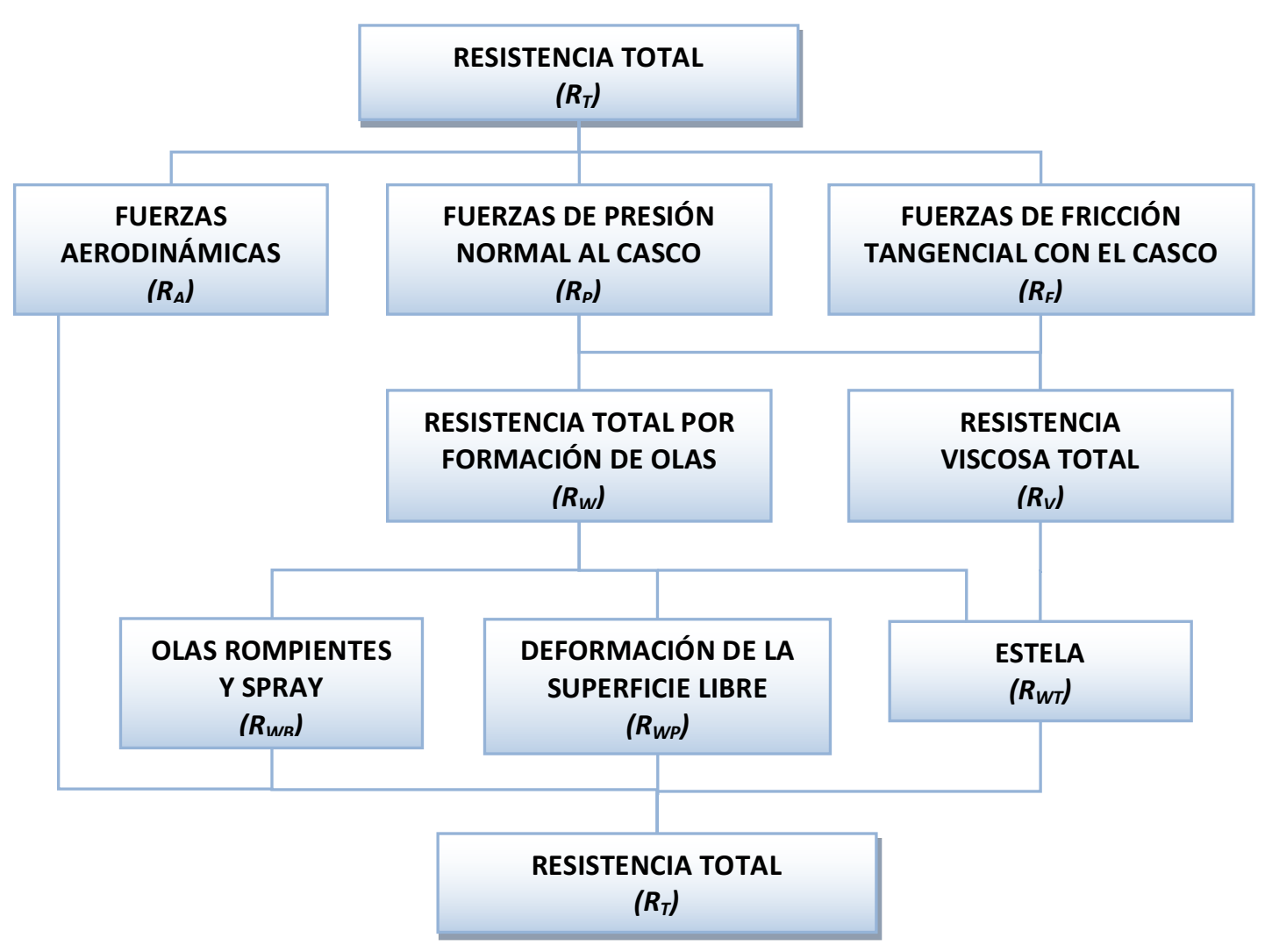

Fig. 4: Subdivisión de la resistencia total en componentes.

La resistencia por formación de olas se puede subdividir a su vez en tres componentes, de forma similar a Insel (1990). Por un lado se encuentra la deformación pura de la superficie libre, $R_{W P}$, en donde se engloban los fenómenos de difracción, refracción y radiación. La disipación de energía en forma de olas rompientes y spray, $R_{W B}$, también supone una parte importante de la resistencia al avance. Por último está la perturbación del dominio fluido en la zona sumergida, donde al incluir los efectos viscosos se obtiene el campo final de velocidades o estela. La energía necesaria para generar dicha perturbación es lo que se denomina resistencia de estela, $R_{W T}$.

\subsubsection{Influencia de las formas en la resistencia}

El casco de un buque es un cuerpo esbelto y diseñado para evitar la separación del flujo al igual que un perfil aerodinámico. Presenta por ello una superficie alisada con el menor 
número posible de discontinuidades ${ }^{16}$. Si bien existen series de formas tipo y recomendaciones generales, no hay un claro óptimo sino que dependerá de las características concretas de cada embarcación.

En general, aumentar la eslora entre perpendiculares de un buque, $L_{P P}$, para un determinado desplazamiento es beneficioso para reducir la resistencia al avance. No obstante, una mayor eslora aumenta significativamente el coste del buque y no siempre es posible debido a restricciones de navegación y/o atraque. Aumentar el calado, $T$, también es beneficioso y conlleva un menor coste añadido, aunque hay que tener en cuenta las restricciones de los puertos, canales, astilleros, etc. El aumento de manga, $B$, por el contrario tiende a aumenta la resistencia al avance, si bien es esencial para garantizar una estabilidad adecuada. Normalmente los buques presentan relaciones $L_{P P} / B$ entre 4 y 7 , y $B / T$ entre 2 y 3 . A priori cuanto más esbelto sea el buque menor oleaje generará, si bien la superficie mojada es mayor a igualdad de desplazamiento. Por ello las embarcaciones rápidas tienden a maximizar la relación $L_{P P} / B$, mientras que embarcaciones lentas donde predomina la resistencia de fricción muestran valores mucho más bajos.

Caracterizadas las dimensiones delimitadoras del casco, el siguiente paso es definir sus formas concretas donde los requisitos operacionales del buque juegan un papel fundamental.

- El coeficiente de bloque, $C_{B}$, en buques de peso muerto alcanza valores por encima de 0.8 , mientras que en los buques de volumen oscila normalmente entre 0.6 y 0.7 .

- La curva de áreas es uno de los diagramas más importantes a la hora de optimizar el casco. En ella se resume la distribución del volumen desplazado, la posición longitudinal del centro de carena, así como la extensión de los cuerpos de proa, central y popa. Junto con estos, la pendiente y curvatura en las zonas de entrada y salida son parámetros a tener en cuenta de cara a la resistencia, tal y como resume Baquero (2002).

\footnotetext{
${ }^{16}$ En el caso de incluirse, como codillos o quillas de balance, se diseñan siguiendo líneas de corriente para perturbar mínimamente el flujo en condiciones normales de navegación.
} 
- Transversalmente, las formas se pueden categorizar en $U$ o V (Fig. 5). De forma natural el buque presenta formas tipo $U$, con el fondo y costados planos unidos mediante un pantoque curvo. Las formas en $\mathrm{V}$ por el contrario facilitan el desplazamiento lateral del agua al navegar en olas, evitando así los pantocazos en el fondo que pueden dañar la estructura. Ambos tipos pueden combinarse en una misma sección, cambiando de uno a otro en diferentes alturas.

- Una de los elementos estructurales más estudiados para mejorar el comportamiento hidrodinámico son los bulbos de proa y/o popa. Tanto en embarcaciones de alta como de baja velocidad han mostrado su eficiencia a la hora de reducir la resistencia al avance. Semejante al casco, se pueden clasificar atendiendo a sus secciones transversales en tipo gota, diamante u óvalo (Fig. 6). En general cuanto más sumergido más eficiente, si bien muestran un peor comportamiento con mala mar.

- El tipo y número de propulsores es crucial para las formas de popa. En el caso de propulsión convencional estas deben acomodar la salida del eje y la hélice mientras que si se emplean PODs pueden ser más tendidas introduciendo un quillote central para garantizar la estabilidad de rumbo.

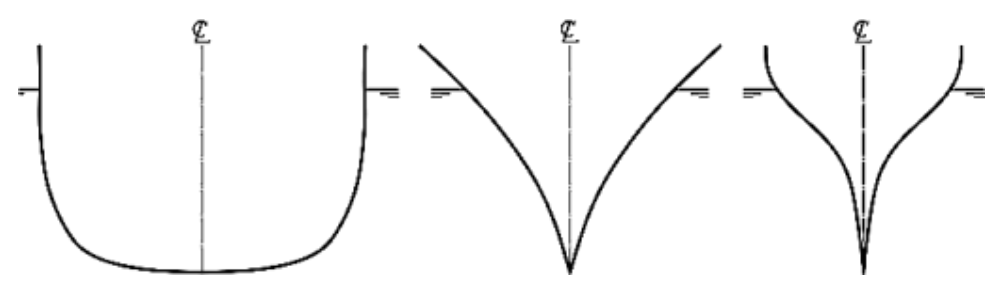

Fig. 5: De izquierda a derecha, formas en $U, V$ y combinación de ambas
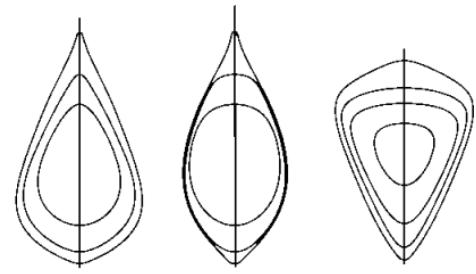

Fig. 6: De izquierda a derecha, bulbo tipo gota, óvalo y diamante (Alvariño et al. (1991))

En función del tipo de buque existen en la literatura multitud de regresiones que pueden ser utilizadas para dimensionar el buque en las fases iniciales de proyecto. SNAME (1941), Alvariño et al. (1991) o Baquero (2002) realizan un detallado resumen de dichas formulaciones, cuyo análisis queda fuera del alcance de este trabajo. 
Particularizando en multicascos, cada uno de los cascos es mucho más esbelto que el de un monocasco a igualdad de eslora, como se vio en el apartado 1.2 (Fig. 2). Son por ello configuraciones adecuadas para embarcaciones rápidas al minimizar la resistencia por formación de olas, a pesar aumentar significativamente la superficie mojada y por ello la resistencia de fricción (Bruzzone et al. (1999)). De cara su dimensionamiento, se puede acudir a diferentes series sistemáticas de catamaranes existentes en la literatura, como la serie NPL (Bailey (1976)) extendida a catamaranes en la Universidad de Southampton (Molland et al. (1996)).

\subsubsection{Interferencia entre cascos}

El análisis hecho de las componentes de la resistencia total en el apartado 4.1.2 se realizó de una forma general, sin especificar el tipo de buque. ¿Qué sucede en el caso de multicascos? Un primer enfoque puede ser suponer la independencia del comportamiento hidrodinámico de cada casco por separado. No obstante, esta hipótesis es bastante imprecisa dado que existe una clara interferencia entre ambos, que se manifiesta en la diferencia apreciable a simple vista entre el flujo entre cascos y el flujo exterior.

Dicha interacción es bastante compleja de analizar. Insel (1990) e Insel \& Molland (1991) presentan un estudio en profundidad de los efectos de interferencia en cada una de las componentes, entre los que se distingue:

- Variación en el flujo sobre los cascos.

- La distribución de presiones y velocidades en cada costado respecto al plano de crujía de cada casco es diferente.

- La zona entre cascos actúa como una tobera resultando en velocidades más altas, lo que conlleva un aumento de la resistencia de fricción y modifica el factor de forma.

- Un flujo cruzado puede ocurrir bajo la quilla, lo que deriva en una componente de arrastre que normalmente se desprecia en los monocascos. 
- Dado que los campos de velocidades al unirse los flujos a popa de los cascos son diferentes, se incrementa la aparición de vórtices y spray con el consiguiente aumento de la resistencia.

- La estructura de la capa límite varía debido a la variación de la velocidad, modificando la resistencia viscosa.

- La superficie mojada efectiva es diferente al incidir sobre cada casco las olas generadas por el resto, afectando a la resistencia de fricción.

- Variación en el tren de olas

- Debido a la variación de la distribución de presiones, el oleaje generado es diferente. Esto conlleva obviamente variaciones en la resistencia por formación de olas.

- En determinadas condiciones, los trenes de olas de cada casco se cancelan entre sí reduciendo la elevación total, y por ende la resistencia por formación de olas.

- Los trenes de olas divergentes reflejados en los cascos adyacentes aumentan la complejidad del campo de elevaciones final, aumentando el número de componentes a tener en cuenta.

- En los planos de simetría entre cascos, el efecto del oleaje siempre se superpone, duplicando la altura del oleaje. Esto puede dar lugar a olas rompientes y spray a altas velocidades.

- La diferencia entre las velocidades en ambos costados del casco modifica el oleaje generado en la zona de popa.

El estudio individual de cada uno de estos fenómenos es importante a la hora de identificar posibles modificaciones favorables en las formas de cada casco. No obstante requiere predecir con exactitud el campo de velocidades y elevaciones, lo que computacionalmente es costoso y propenso a errores significativos. Por ello, de forma práctica se suele mantener la subdivisión previa de la resistencia total y añadir un nuevo término denominado resistencia de interferencia, $R_{I}$, que engloba todos efectos derivados de la interacción entre cascos. 


$$
R_{I}=R_{M H}-\sum_{i} R_{D_{i}}
$$

Donde $R_{M H}$ es la resistencia del multicasco ${ }^{17}$ y $R_{D_{i}}$ es la resistencia individual de cada casco ${ }^{18}$. Por otra parte, para poder cuantificar la relevancia de los efectos de interferencia se emplea la variable adimensional denominada factor de interferencia, $I F_{R}$. Como alternativa se puede utilizar el factor de interferencia del oleaje, $I F_{W}$, el cual se basa únicamente en la resistencia por formación de olas. Idealmente, el valor del factor de interferencia se debe mantener lo más bajo posible, y negativo si se puede.

$$
\begin{gathered}
I F_{R}=\frac{R_{M H}-\sum_{i} R_{D_{i}}}{\sum_{i} R_{D_{i}}} \\
I F_{W}=\frac{R_{W, M H}-\sum_{i} R_{W, D_{i}}}{\sum_{i} R_{W, D_{i}}}
\end{gathered}
$$

Tanto experimental como numéricamente es muy difícil sino imposible reproducir los fenómenos viscosos con exactitud. Por ello aunque el $I F_{W}$ no incluye todos los fenómenos de interferencia, presenta varias ventajas a nivel práctico. En primer lugar, evita incorporar los problemas derivados de los fenómenos de escala asociados con la componente viscosa, como se explica en el apartado 4.3.1. Por otro lado puede emplearse directamente con modelos numéricos potenciales que asumen flujo no viscoso, mucho más sencillos como se presenta en el apartado 4.3.2. En ambos casos, valores negativos representan situaciones de interferencia favorable, mientras que valores positivos conllevan una interferencia desfavorable.

\subsection{El monocasco equivalente}

En las definiciones de la $R_{I}$ e $I F$ se puede apreciar cómo se toma como referencia la suma de las resistencias de cada casco por separado, o lo que es igual, la condición de interferencia nula. Esta es la práctica habitual en la comunidad hidrodinámica y la seguida en este trabajo.

\footnotetext{
${ }^{17}$ El subíndice ${ }_{\mathrm{MH}}$ procede de su nombre en inglés, MultiHull

${ }^{18}$ El subíndice D procede de su nombre en inglés, Demihull
} 
Siguiendo esta definición es posible obtener valores negativos de $R_{I}$, lo que lleva a concluir que la interacción entre cascos contribuye a reducir la resistencia.

No obstante, es importante detenerse en el concepto de monocasco equivalente para poder comparar acertadamente los multicascos con otros tipos de embarcaciones. Simplemente eliminar la interferencia entre cascos no es realista, ya que no está asociada a ninguna configuración geométrica factible. Es necesario por tanto definir una serie de criterios a satisfacer independientes del tipo de configuración para poder identificar el monocasco equivalente. A tal fin podemos destacar:

- Formas

- Eslora

- Desplazamiento

- Velocidad

- Superficie en cubierta

- Volumen de carga

- Estabilidad transversal

En última instancia, los criterios a utilizar para la elección entre un monocasco o multicasco dependerán de las características operativas de cada embarcación. Habitualmente se suele fijar la igualdad de eslora, desplazamiento y velocidad, como ya se utilizó en el apartado 1.2. Este criterio deja libertad para la optimización de las formas de cada configuración por separado, lo que sin duda introduce una fuente de error significativa dado el gran abanico de posibilidades. En cualquier caso, este enfoque resulta muy útil en las fases iniciales del proyecto, donde únicamente se requiere una estimación aproximada del comportamiento de cada configuración a la hora de escoger la más idónea.

Científicamente el $I F$ es más adecuado al fijar las formas y permitir cuantificar los efectos de interferencia para poder comparar diferentes multicascos. Al incluir el monocasco equivalente es preciso especificar el criterio seguido para definirlo y tener en cuenta el enfoque cualitativo de dicha comparativa. Este estudio supone una continuación lógica a este trabajo, y permitirá 
entender mejor las virtudes de los multicascos. En una futura fase se realizará una búsqueda de monocascos potencialmente equivalentes y se optimizarán sus formas para satisfacer los diferentes criterios mencionados anteriormente, pudiendo así ser comparados con mayor precisión.

\subsection{Técnicas de predicción}

La resistencia al avance es causa de uno de los principales gastos de operación de un buque (consumo de combustible), y por ello su minimización es uno de los aspectos más importantes en su diseño. Un posible forma de conseguirlo es reducir el coeficiente de fricción del casco, como analizan Izaguirre Alza et al. (2010). No obstante, la propia geometría del casco es el factor más importante de cara a la resistencia al avance. Disponer de técnicas precisas de predicción que permitan optimizar las formas del buque antes de ser construido es esencial para poder conseguir un diseño eficiente. Es más importante aún si se tienen en cuenta las grandes dimensiones de los buques y la elevada dificultad de realizar modificaciones a posteriori (Fig. 7). Todo ello explica la enorme importancia de esta área, la cual persiste hoy en día como una de las más activas en ingeniería naval.

Para conocer a priori la resistencia al avance sería necesario resolver las ecuaciones de NavierStokes. Dado que no se conoce su solución analítica exacta ${ }^{19}$ es necesario realizar simplificaciones que permitan obtener soluciones aproximadas. Inicialmente se emplearon ensayos con modelos, estimando teóricamente parte de las componentes de resistencia para compensar los fenómenos de escala (sec. 4.3.1). En los últimos años, el enorme aumento de las capacidades de cálculo de los ordenadores ha impulsado el uso de modelos numéricos para la optimización de formas (sec. 4.3.2). Ambos enfoques se apoyan mutuamente, como

${ }^{19}$ Si bien existen soluciones exactas de las ecuaciones de Navier-Stokes, sólo son válidas para casos muy particulares, y en ningún caso sobre un dominio finito y tensor de deformaciones no nulo $\left((\operatorname{def} \vec{v})_{i j}=\frac{\partial v_{i}}{\partial x_{j}}+\frac{\partial v_{j}}{\partial x_{i}} \neq \overline{\bar{O}}\right.$, en notación indicial donde $\vec{v}$ representa el campo de velocidades). La demostración de la existencia y unicidad de una solución general es uno de los siete problemas del milenio según el Clay Mathematics Institute. http://www.claymath.org/millennium-problems 
indica Pérez Rojas (2009), proporcionando los mejores resultados al emplearse conjuntamente.

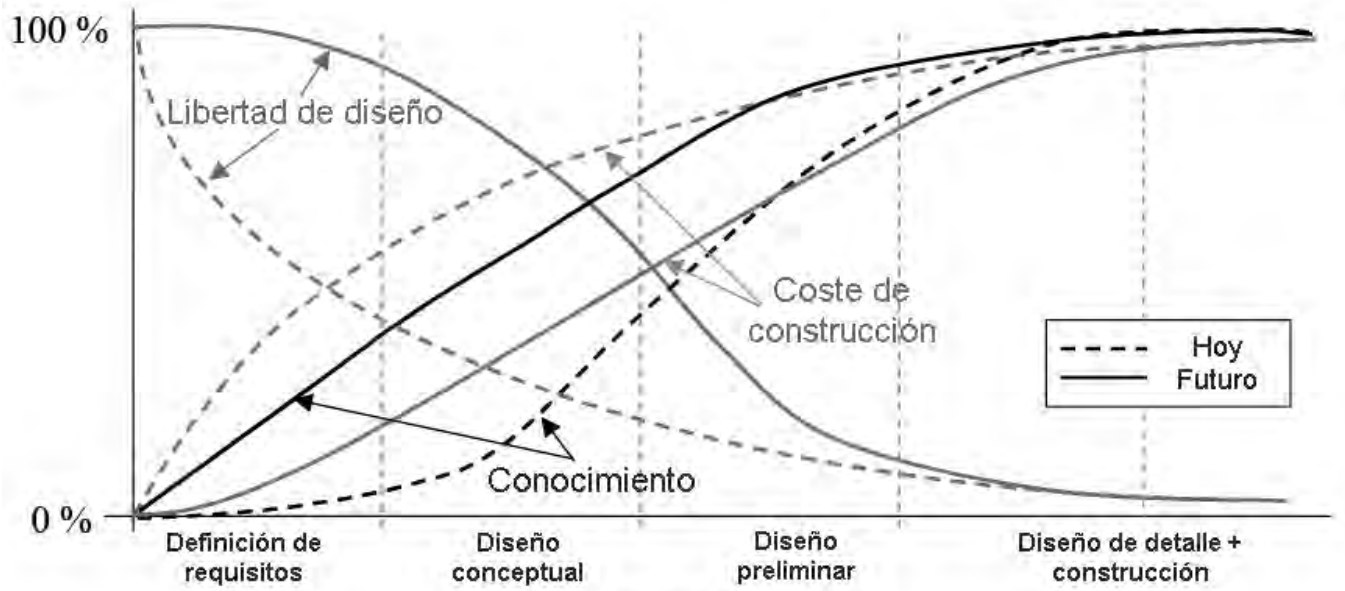

Secuencia de Diseño

Fig. 7: Variación de los costes de construcción, libertad para realizar cambios, y conocimiento basado en las técnicas de predicción a lo largo de la secuencia de diseño (adaptada de Rigterink (2014))

Una de las divergencias habituales entre los resultados de los ensayos y las simulaciones numéricas es la inclusión del trimado y asiento dinámicos. En los ensayos se suele dejar el modelo lo más libre posible, restringiendo únicamente el movimiento lateral. Las simulaciones numéricas por el contrario tienden a fijar la posición del buque para poder definir explícitamente las condiciones de contorno y discretizar el dominio fluido. Esto simplifica enormemente los cálculos, si bien introduce una discrepancia con respecto a la realidad como presentan Kim \& Jenkins (1981) o Subramani et al. (2000). En general no supone un problema, dado que normalmente los resultados numéricos se emplean cualitativamente como herramientas comparativas entre diferentes geometrías. No obstante, es importante resaltar que la tecnología actual permite tanto realizar ensayos con modelo fijo como simulaciones con modelo libre, tal y como se efectuó en las fases iniciales de este trabajo (Fernández-Gutiérrez (2010)). Su aplicación en el estudio de multicascos es directa, como se detalla en la sección 6.3. El análisis con modelo fijo y libre del multicasco y de los cascos por separado permite cuantificar la relevancia del asiento y trimado dinámicos tanto en la resistencia total como en la componente de interferencia. 
Los siguientes apartados profundizan en las diferentes técnicas existentes para predecir la resistencia al avance y sus diferentes componentes.

\subsubsection{Ensayos con modelos}

\section{Conceptos generales}

La utilización de modelos a escala para predecir la resistencia se basa en el establecimiento de unas leyes de similitud que permitan extrapolar las mediciones a escala real. Para poder establecer una correlación exacta es necesario conseguir una similitud dinámica ${ }^{20}$, la cual implica similitud geométrica ${ }^{21}$ y cinemática ${ }^{22}$.

Normalmente los ensayos de resistencia al avance en canales de experiencias hidrodinámicas se realizan bajo las siguientes suposiciones:

- Los efectos de tensión superficial y compresibilidad del agua se consideran despreciables dadas las grandes dimensiones y baja velocidad del buque.

- La estructura del buque es infinitamente rígida lo que evita tener que considerar las vibraciones del casco o los fenómenos de interacción fluido-estructura, y por ende reproducir a escala la respuesta estructural del casco

- Los ensayos se centran en la obra viva, no incluyendo la componente aerodinámica

Quedan por lo tanto como componentes dominantes los efectos gravitacionales (generación de oleaje) y viscosos, consistente con la subdivisión presentada anteriormente en la Fig. 4 (sec. 4.1.2). Un análisis dimensional muestra cómo el parámetro que define la relevancia de los efectos gravitacionales es el número de Froude, Fr, mientras que en el caso de los efectos viscosos es el número de Reynolds, $R e$.

${ }^{20}$ Los coeficientes adimensionales de todas las fuerzas actuantes son iguales a escala modelo y escala real. No hay que olvidar que se incluyen tanto las fuerzas excitadoras como las reacciones y deformaciones de la estructura.

${ }^{21}$ El modelo simplemente es una reproducción a escala de la estructura real.

${ }^{22}$ El flujo a escala modelo es una reproducción a escala del flujo a escala real, donde hay que incluir tanto la escala geométrica (líneas de corriente) como la escala temporal (velocidades). 


$$
\begin{aligned}
& \sum F=F_{\text {grav }}+F_{\text {vis }}=m a=F_{\text {inercia }} \\
& \left.\left.\begin{array}{rl}
F_{\text {inercia }} & \propto \frac{\rho V^{2}}{L} \\
F_{\text {grav }} & \propto \rho g \\
F_{\text {visc }} & \propto \frac{\mu V}{L^{2}}
\end{array}\right\} \begin{array}{l}
\frac{F_{\text {inercia }}}{F_{\text {grav }}} \propto \frac{V^{2}}{g L} \propto \frac{V}{\sqrt{g L}} \\
\frac{F_{\text {inercia }}}{F_{\text {visc }}} \propto \frac{\rho V L}{\mu}=\frac{V L}{v}
\end{array}\right\} \\
& F r=\frac{V}{\sqrt{g L}} \\
& R e=\frac{\rho V L}{\mu}
\end{aligned}
$$

donde $m$ representa la masa, $a$ aceleración, $F$ fuerza, $\rho$ densidad, $V$ velocidad, $L$ longitud característica, $g$ aceleración de gravedad, $\mu$ viscosidad dinámica y $v$ viscosidad cinemática. Desafortunadamente, a escala modelo es prácticamente imposible igualar ambos. Asumiendo que el campo gravitatorio es el mismo, con un factor de escala geométrica $\lambda_{m}$, la única posibilidad es variar el fluido circundante de forma que las viscosidades cinemáticas tengan un ratio $\lambda_{m}^{3 / 2}$.

$$
\begin{aligned}
& \lambda_{m}=\frac{L_{m}}{L} \\
& F r=F r_{m} \Rightarrow \frac{V}{\sqrt{g L}}=\frac{V_{m}}{\sqrt{g L_{m}}} \Rightarrow \frac{V_{m}}{V}=\sqrt{\frac{L_{m}}{L}}=\sqrt{\lambda_{m}} \\
& R e=R e_{m} \Rightarrow \frac{\rho V L}{\mu}=\frac{\rho_{m} V_{m} L_{m}}{\mu_{m}} \Rightarrow \frac{V_{m}}{V}=\frac{\rho / \mu}{\rho_{m} / \mu_{m}} \frac{L}{L_{m}}=\frac{v_{m}}{v} \frac{1}{\lambda_{m}} \\
& \left.\begin{array}{l}
F r=F r_{m} \\
R e=R e_{m}
\end{array}\right\} \Rightarrow \frac{v_{m}}{v}=\lambda_{m}^{3 / 2}
\end{aligned}
$$

En el caso de estructuras grandes, como es el caso de los buques esto es inviable ${ }^{23}$. Los buques navegan en regímenes de $R e \approx 10^{8}$, demasiado altos para poder ser reproducidos en

${ }^{23}$ La viscosidad cinemática del agua es $v \approx 10^{-6} \mathrm{~m}^{2} / \mathrm{s}$, por lo que para un factor de escala normal de 1:10 la viscosidad cinemática requerida sería $v_{m} \approx 3 \cdot 10^{-8}$, reduciéndose cuanto menor sea la escala. Alcanzar valores tan bajos sólo es posible usando substancias especiales, como algunos 
túneles de viento o canales de ensayos. Por ello los ensayos se realizan por lo general igualando el $F r$ y se añade una corrección para tener en cuenta las diferencias en la componente viscosa. Esto es lo que se denomina similitud dinámica parcial. Se distinguen dos tipos de procedimientos para ello:

- Medida de la resistencia total del modelo y estimación de la resistencia de fricción mediante fórmulas empíricas.

- Medidas directas de las diferentes componentes.

\section{Medida de la resistencia total}

Los métodos basados en la medida de la resistencia total y la estimación de la resistencia de fricción son muy prácticos de cara a la extrapolación de la resistencia a escala real. Esto se debe a que medir el valor total de la resistencia es relativamente sencillo. Basta conectar el modelo al carro de remolque mediante una célula de carga y restringir los movimientos laterales sin afectar al resto. A cambio se pierde información relativa a la relevancia de los diferentes fenómenos físicos que tienen lugar.

Este tipo de métodos fue empleado por primera vez por William Froude (Froude (1868), Froude (1874)). En primer lugar subdividió la resistencia al avance en dos componentes: resistencia de fricción y resistencia residual $\left(R_{F}\right.$ y $R_{R}$ respectivamente). La resistencia de fricción según W. Froude es equivalente al de una placa plana con la misma superficie mojada y puede ser obtenida de acuerdo a la siguiente expresión:

$$
R_{F}=f S V^{n}
$$

donde $S$ es la superficie mojada, $V$ velocidad de avance, y $f$ y $n$ son constantes función de la eslora y naturaleza de la superficie. Por otro lado, la resistencia residual engloba el resto de factores y puede ser deducida a partir de la diferencia entre la resistencia total medida y la resistencia viscosa calculada a partir de la expresión anterior. Además, Froude supuso que las

gases a muy baja temperatura. Esto queda completamente fuera del alcance de un canal de ensayos. 
resistencias residuales del modelo y del buque están relacionadas por la relación de desplazamientos:

$$
\left(\frac{R_{R}}{\Delta}\right)_{M O D E L O}=\left(\frac{R_{R}}{\Delta}\right)_{B U Q U E}
$$

No obstante, esta igualdad se cumplía si tanto el buque como el modelo avanzaban a igualdad de lo que posteriormente se denominó número de Froude, $\operatorname{Fr}$ (ec. 4.6). Como ya se demostró, dicha conclusión proviene de realizar un análisis dimensional de cada variable. Este hecho pone de manifiesto una vez más la utilidad de emplear coeficientes adimensionales en vez de magnitudes absolutas. Permite evitar posibles confusiones derivadas de la utilización de sistemas de unidades distintos, y además comparativamente es mucho más indicativo al no estar distorsionado por las dimensiones reales de cada geometría. De esta forma se define el coeficiente de resistencia total al avance, $C_{T}$, como

$$
C_{T}=\frac{R_{T}}{\frac{1}{2} \rho S V^{2}}
$$

Utilizando coeficientes adimensionales y siguiendo el método propuesto por W. Froude, el coeficiente de resistencia total del buque se obtiene según la siguiente expresión:

$$
C_{T}(\text { Buque })=C_{F}(\text { Buque })+\left[C_{T}\left(\text { Modelo }-C_{F}(\text { Modelo })\right]\right.
$$

donde los valores de los coeficientes de fricción, $C_{F}$, se estiman mediante una línea de fricción pactada por los canales de experiencias, y el valor del coeficiente de resistencia total del modelo se obtiene mediante ensayos de remolque. A la hora de adimensionalizar la resistencia de fricción se puede emplear la ecuación (4.13) y utilizar la misma magnitud de referencia que en la ecuación (4.15). Siguiendo esta hipótesis se realizaron una serie de experiencias con placas planas dando lugar a mejoras en la formulación a emplear para obtener la resistencia de fricción, como las descritas por Schoenherr (1932), Hughes (1954) o Hughes (1966). 
La ITTC estudió este aspecto y en 1957 propuso una nueva fórmula de la curva de fricción, conocida como ITTC-57 (ITTC (1957)), y que se sigue empleando en la actualidad.

$$
C_{F, I T T C-57}=\frac{0.075}{[\log (R e)-2]^{2}}
$$

Las definiciones propuestas del coeficiente de fricción equiparan la superficie mojada a la de una placa plana, sin tener en cuenta la influencia de las formas a la hora de variar la velocidad del flujo. Tratando de incluir este hecho, Hughes (1954) propuso un método en el que se subdivide el coeficiente de resistencia total en tres componentes:

- Coeficiente de resistencia de fricción de una placa plana, $C_{F}$

- Coeficiente de resistencia de fricción debida a las formas del buque, $C_{F G}$

- Coeficiente de resistencia por formación de olas, $C_{W}$

Realmente la resistencia de fricción debida a las formas se puede considerar como un incremento de la resistencia de fricción de una placa plana. Dicho incremento se introduce matemáticamente como un factor de forma $(1+k)$. De esta forma:

$$
C_{T}=(1+k) C_{F}+C_{W}
$$

Para la estimación del factor de forma Hughes propuso la realización de ensayos a muy baja velocidad $(\mathrm{Fr}<0.1)$, donde el valor de $C_{W}$ es despreciable. El problema reside en que a tan baja velocidad la transición de flujo laminar a turbulento se produce mucho más aguas abajo. Esto modifica la estructura de la capa límite respecto a las condiciones normales de navegación, y por ende la resistencia de fricción. Una alternativa mucho más realista es utilizar el método propuesto por Prohaska (1966). Se fundamenta en que la resistencia residual a baja velocidad $(0.1<F r<0.2)$ es función del $F r$ :

$$
C_{R}=a F r^{n}
$$


Donde $a$ y $n$ son constantes de ajuste, y según las recomendaciones de la ITTC toman valores de 1 y 4 respectivamente. Tras realizar una serie de ensayos a diferentes velocidades dentro de este rango de $F r$ y representar $C_{T} / C_{F}$ versus $a F^{4} / C_{F}$, la línea recta obtenida tras realizar un ajuste de mínimos cuadrados intersectará el eje de ordenadas en el valor del factor de forma $(1+k)$. Las recomendaciones de la ITTC señalan no obstante que debe tenerse cuidado con los buques con geometrías especiales, tales como con bulbos de proa muy cerca de la superficie o grandes popas de espejo.

$$
\begin{gathered}
C_{T}=(1+k) C_{F}+F r^{4} \\
(1+k)=\frac{F r^{4}}{C_{F}}-\frac{C_{T}}{C_{F}}
\end{gathered}
$$

Este planteamiento fue introducido en las recomendaciones de la ITTC en 1978, en lo que se denominó Método de Predicción de la Resistencia al Avance ITTC-78 para buques de una sola hélice (ITTC (1978)). En ella se subdividía la resistencia en cuatro componentes:

- Resistencia friccional, $C_{F}$, obtenida según la línea ITTC-57 (ITTC (1957)).

- Resistencia residual, $C_{R}$

- Incremento por rugosidad del casco, $\Delta C_{F}$. Para un modelo se puede suponer 0

- Resistencia del aire, $C_{A A}$. Para un modelo $\sin$ superestructuras se puede suponer 0

$$
C_{T}=(1+k) C_{F}+C_{R}+\Delta C_{F}+C_{A A}
$$

Finalmente, en la actualidad tras la celebración de la $26^{\text {th }}$ ITTC Conference, las recomendaciones operativas para la estimación de la resistencia al avance se recogen en la referencia ITTC (2011). A modo de resumen decir que se subdivide la resistencia al avance en cinco componentes. Se mantienen los cuatro términos anteriores, introduciéndose como quinto elemento el valor de la resistencia de los apéndices, $C_{A P}$. Este se mide experimentalmente ensayando en el canal el modelo con y sin apéndices y restando los valores de las resistencias totales obtenidas:

$$
C_{T}=(1+k) C_{F}+C_{R}+\Delta C_{F}+C_{A P}+C_{A A}
$$




\section{Medida de las componentes de resistencia}

La segunda opción a la hora de determinar la resistencia al avance consiste en la medida directa de las componentes de la resistencia. En este apartado hay dos tipos de métodos:

- Medición de las fuerzas de fricción y las fuerzas de presión sobre la superficie del casco.

- Medición de la energía cedida al entorno en forma de formación de olas por los efectos de presión más la cedida en forma de estela por los efectos de fricción. De acuerdo a la conservación de la cantidad de movimiento, dicha energía es igual a la necesaria para desplazar el buque a la velocidad deseada.

Medir las fuerzas de fricción y presión requiere distribuir células de carga por todo el casco. Como resultado se obtiene una representación discreta de las fuerzas actuantes sobre el buque, cuya integración proporciona directamente las componentes de la resistencia al avance. La dificultad reside en conseguir capturar correctamente los máximos y mínimos de presión y fricción. La medición del campo de elevaciones y velocidades es relativamente más sencilla, aunque requiere un tratamiento posterior más complejo. Mediante el uso de sensores puntuales de elevación ${ }^{24}$ se pueden obtener cortes de olas que sirven para reconstruir la deformación de la superficie libre. Dumez \& Cordier (1997) presentan un análisis en detalle de las técnicas más comunes dentro de este tipo: cortes longitudinales (LCM), descomposición discreta (DDM) o identificación de una distribución de singularidades (SDIM). La medición de la estela requiere utilizar sensores de velocidad sumergidos, como tubos de Pitot. Para poder obtener el campo completo de velocidades es necesario realizar múltiples mediciones, lo que eleva considerablemente el tiempo necesario para completar los ensayos.

Se puede apreciar cómo ambos tipos exigen una instrumentación mucho más sofisticada que la medida de la resistencia total, siendo además mucho más propensos a errores. Por ello su

\footnotetext{
${ }^{24}$ Entre los sensores más comunes destacan las probetas capacitivas, probetas resistivas o sensores ultrasónicos.
} 
utilización es mucho menos frecuente. Cabe destacar la toma de cortes de ola como procedimiento adicional a la medición de la resistencia total. Si bien por sí mismos proporcionan una información limitada, son muy útiles para validar los modelos numéricos.

\section{Particularización para catamaranes}

A la hora de medir la resistencia de un multicasco se puede emplear directamente cualquiera de los métodos anteriores. Los métodos basados en medir la resistencia total resultan más adecuados si sólo se desea medir la resistencia de interferencia. Simplemente es necesario realizar ensayos con el multicasco y cada uno los cascos por separado. Estos no obstante no aportan luz sobre los fenómenos físicos involucrados que se presentaron en la sec. 4.1.4, siendo más conveniente para ello la medición por separado de cada componente.

Insel (1990) presenta un análisis detallado de las modificaciones a introducir en la formulación basada en la medición de la resistencia total para incluir los efectos de interferencia en el que propone:

- Modificación a la formulación de ITTC-57

$$
C_{T, C A T}=C_{F, C A T}+C_{R, C A T}=\sigma C_{F}+\Omega C_{R}
$$

- Modificación a la formulación de ITTC-78

$$
\begin{aligned}
C_{T} & =\left(1+k_{C A T}\right) C_{F, C A T}+C_{W, C A T} \\
& =(1+\phi k) \sigma C_{F}+\tau C_{W} \\
& =(1+\beta k) C_{F}+\tau C_{W}
\end{aligned}
$$

donde $\sigma$ es el factor de interferencia de la resistencia friccional, $\Omega$ el factor de interferencia de la resistencia residual, $\phi$ el factor de interferencia del factor de forma, $\tau$ el factor de interferencia de la resistencia por formación de olas y $\beta$ el factor de interferencia combinado del factor de forma y resistencia de fricción.

Una de las principales causas de reducción de la resistencia de interferencia son los fenómenos de cancelación del oleaje. La realización de cortes de olas, especialmente en la zona entre cascos, permite cuantificar este fenómeno. La medición del asiento y trimado 
dinámicos es necesaria para evaluar las diferentes condiciones de navegación. Además, la toma de fotografías y vídeos del flujo sobre el casco permite estimar las variaciones en la superficie mojada.

\subsubsection{Simulaciones numéricas}

\section{Fundamentos teóricos}

A pesar de la valiosa información que se obtiene de los ensayos con modelos, no hay que olvidar que la predicción teórica de la resistencia al avance sigue siendo el procedimiento ideal. En esta línea, muchos son los esfuerzos realizados para conseguir soluciones aproximadas a las ecuaciones de Navier-Stokes aplicadas al flujo alrededor de un buque (Pérez Rojas (1994)). Debido a la complicada geometría del casco y la existencia de una superficie libre, de forma práctica es posible únicamente obtener soluciones discretas a las ecuaciones de gobierno. No obstante, para poder alcanzar una resolución admisible es necesario utilizar un gran número de elementos, lo que inevitablemente conduce a la utilización de herramientas computacionales o CFD (Computational Fluid Dynamics).

A pesar de ser necesario emplear técnicas numéricas, es recomendable siempre extender la formulación analítica lo más lejos posible para minimizar las desviaciones con la solución exacta. Volviendo de nuevo al origen, las variables que caracterizan el dominio fluido son la densidad $\rho$, presión $p$, velocidad $\vec{v}=(u, v, w)$, y temperatura $T$. Cada una de ellas es función de la posición ${ }^{25} \vec{r}=(x, y, z)$, y tiempo $t$, y están relacionadas a través de las ecuaciones de Navier-Stokes. Estas se basan en la conservación de masa (4.26), cantidad de movimiento (4.27) y energía (4.28).

$$
\frac{d}{d t} \int_{\mathcal{V}(t)} \rho d \mathcal{V}=0
$$

${ }^{25}$ En este trabajo se emplea un sistema de coordenadas cartesiano para expandir los diferentes operadores, si bien la formulación presentada es válida para cualquier sistema de coordenadas. 


$$
\begin{aligned}
& \frac{d}{d t} \int_{\mathcal{V}(t)} \rho \vec{v} d \mathcal{V}=\underbrace{\int_{\mathcal{V}(t)} \rho \vec{f} d \mathcal{V}}_{\text {fuerzas volumétricas }}+\underbrace{\int_{\mathcal{S}(t)} \overline{\bar{\sigma}} d \overrightarrow{\mathcal{S}}}_{\text {fuerzas superficiales }} \\
& \frac{d}{d t} \int_{\mathcal{V}(t)} \rho\left(e+\frac{1}{2}|\vec{v}|^{2}\right) d \mathcal{V} \\
& =\underbrace{\int_{\mathcal{V}(t)} \rho \vec{f} \cdot \vec{v} d \mathcal{V}}_{\text {tasa de trabajo volumétrico }} \\
& +\underbrace{\int_{\mathcal{S}(t)}(\overline{\bar{\sigma}} \vec{v}) \cdot d \overrightarrow{\mathcal{S}}}_{\text {tasa }} \\
& +\underbrace{\int_{\mathcal{S}(t)}-\vec{q} \cdot d \overrightarrow{\mathcal{S}}}_{\text {tasa de conducción de calor }} \\
& +\underbrace{\int_{\mathcal{V}(t)} \rho Q d \mathcal{V}}_{\text {tasa de generación de calor }}
\end{aligned}
$$

donde $\mathcal{V}$ representa el dominio fluido, $\mathcal{S}$ el límite de dicho dominio, $\overline{\bar{\sigma}}$ el tensor de esfuerzos de Cauchy ${ }^{26}, \vec{f}$ las fuerzas volumétricas ${ }^{27}$, $e$ la energía interna, $\vec{q}$ el vector de conducción de calor, y $Q$ es una fuente volumétrica de calor (radiación por ejemplo). Dado que las expresiones anteriores deben satisfacerse para cualquier dominio fluido, asumiendo que este no presenta discontinuidades pueden ser manipuladas para obtener su formulación diferencial, más común en la práctica (Kundu et al. (2012), Savaş (2014)):

$$
\frac{\partial \rho}{\partial t}+\operatorname{div}(\rho \vec{v})=0
$$

\footnotetext{
${ }^{26} \mathrm{El}$ agua se puede considerar como un fluido newtoniano, por lo que $\overline{\bar{\sigma}}$ se puede expandir de acuerdo a la ecuación (4.32)

${ }^{27}$ En el caso del flujo alrededor del casco con superficie libre, la única fuerza volumétrica actuante es la gravitatoria $\vec{f}=(0,0,-g)$, donde $g$ es la aceleración de la gravedad. No obstante la formulación general considera otras posibles componentes como por ejemplo las fuerzas electromagnéticas.
} 


$$
\begin{aligned}
& \rho \frac{\partial \vec{v}}{\partial t}+\rho(\operatorname{grad} \vec{v}) \vec{v}=\operatorname{div} \overline{\bar{\sigma}}+\rho \vec{f} \\
& \rho \frac{\partial e}{\partial t}+\rho(\operatorname{grad} e) \cdot \vec{v}=\overline{\bar{\sigma}} \cdot \operatorname{grad} \vec{v}+\operatorname{div}(-\vec{q})+\rho Q \\
& \overline{\bar{\sigma}}=-p \overline{\bar{I}}+\lambda \operatorname{div} \vec{v} \overline{\bar{I}}+\mu \operatorname{def} \vec{v} \\
& \operatorname{div}()=\frac{\partial}{\partial x}+\frac{\partial}{\partial y}+\frac{\partial}{\partial z} \\
& \operatorname{grad}(\quad)=\left(\frac{\partial}{\partial x}, \frac{\partial}{\partial y}, \frac{\partial}{\partial z}\right) \\
& \operatorname{def} \vec{v}=(\operatorname{grad} \vec{v})+(\operatorname{grad} \vec{v})^{*} \\
& =\left[\begin{array}{ccc}
2 \frac{\partial u}{\partial x} & \frac{\partial u}{\partial y}+\frac{\partial v}{\partial x} & \frac{\partial u}{\partial z}+\frac{\partial w}{\partial x} \\
\frac{\partial v}{\partial x}+\frac{\partial u}{\partial y} & 2 \frac{\partial v}{\partial y} & \frac{\partial v}{\partial z}+\frac{\partial w}{\partial y} \\
\frac{\partial w}{\partial x}+\frac{\partial u}{\partial z} & \frac{\partial w}{\partial y}+\frac{\partial v}{\partial z} & 2 \frac{\partial w}{\partial z}
\end{array}\right] .
\end{aligned}
$$

donde $\mu$ es el primer coeficiente de viscosidad, $\lambda$ el segundo coeficiente de viscosidad, $(\quad)^{*}$ el traspuesto del tensor, e $\overline{\bar{I}}$ el tensor identidad.

Expandir el tensor de esfuerzos permite diferenciar entre los esfuerzos normales o de presión y los esfuerzos cortantes. No obstante, hay seis variables de estado y sólo cinco ecuaciones por lo que es necesaria una nueva expresión, linealmente independiente de las anteriores, para que el problema esté correctamente planteado. Esta es precisamente la ecuación de estado que vincula las variables termodinámicas del fluido (4.39), que junto con las anteriores completa las ecuaciones de gobierno en mecánica de fluidos:

$$
\frac{\partial \rho}{\partial t}+\operatorname{div}(\rho \vec{v})=0
$$




$$
\begin{aligned}
\rho \frac{\partial \vec{v}}{\partial t}+\rho(\operatorname{grad} & \vec{v}) \vec{v} \\
& =-\operatorname{grad} p+\operatorname{grad}(\lambda \operatorname{div} \vec{v})+\operatorname{div}(\mu \operatorname{def} \vec{v}) \\
& +\rho \vec{f} \\
\rho \frac{\partial e}{\partial t}+\rho(\operatorname{grad} e) \cdot \vec{v} & \\
& =\underbrace{\operatorname{div}(-\vec{q})-p \operatorname{div} \vec{v}}_{\text {disipación de energía por fricción }} \\
& +\lambda=f(p, T) .
\end{aligned}
$$

Para completar la formulación del problema es necesario definir las condiciones iniciales y de contorno. Las primeras dependerán de las condiciones de navegación a simular, mientras que las segundas en el caso del flujo alrededor del casco de un buque son:

- Sobre el casco:

- Condición de impenetrabilidad, por lo que la velocidad normal al casco es igual que la velocidad del casco

- En el caso de incluir los fenómenos viscosos es necesario añadir la condición de no deslizamiento, por lo que la velocidad tangencial es igual a la velocidad del casco

- En la superficie libre:

- Condición cinemática, según la cual las partículas en la superficie libre permanecen en la superficie libre

- Condición dinámica, según la cual la presión sobre la superficie libre es igual a la presión atmosférica

- En el fondo, si se consideran aguas poco profundas y se incluye el fondo como límite del dominio fluido:

- Condición de impenetrabilidad

- Condición de no deslizamiento si se incluyen los efectos viscosos

- En el cierre del dominio fluido lejos del buque: 
- Condición de radiación, según la cual las perturbaciones introducidas por el buque desaparecen en el infinito

Como se mencionó anteriormente, no se conoce una solución exacta a este sistema de ecuaciones en derivadas parciales no lineales. Entramos por tanto en el campo de las posibles aproximaciones que permitan obtener resultados suficientemente representativos.

Antes de abordar numéricamente las expresiones anteriores, bajo ciertas condiciones particulares es posible simplificar estas cancelando parte de sus términos:

- Si el problema es estacionario todos los términos de derivadas temporales desaparecen.

- Si el fluido es incompresible la densidad es constante (González Gutiérrez \& Bermejo (2005)).

- Si el flujo es potencial todos los términos viscosos desaparecen. Esto cobra especial relevancia al aplicar la teoría de capa límite propuesta por Prandtl (1904) presentada anteriormente, donde el flujo exterior que representa una de las condiciones de contorno es precisamente flujo potencial.

- Si el flujo es barotrópico $(\rho=f(p))$, la ecuación de energía está desacoplarla y no es necesario incluirla para obtener el campo de velocidades.

Por otro lado, las mediciones experimentales muestran habitualmente oscilaciones aleatorias de las variables de estado, en lo que se conoce como flujo turbulento. No sólo son funciones del espacio y tiempo, sino que deben ser tratadas como valores estocásticos. Este hecho aumenta aún más la complejidad matemática del problema, siendo su tratamiento una de las principales áreas de investigación en mecánica de fluidos. Las diferentes alternativas propuestas se pueden clasificar en las siguientes categorías:

- Resolución numérica directa de las ecuaciones de Navier-Stokes, conocido por sus siglas en inglés DNS (Direct Numerical Simulations). A priori es el más exacto, si bien la potencia computacional requerida es enorme, no siendo factible su aplicación naval a día de hoy. 
- Utilización de modelos de turbulencia que reduzcan los requisitos de cálculo, como por ejemplo

- RANS (Reynolds Averaged Navier-Stokes)

- LES (Large Eddy Simulations)

- Suposición de flujo laminar, válido para valores de $R e$ bajos

\section{Clasificación de los CFD}

El espectro de herramientas CFD existentes es muy grande, abarcando desde sencillos códigos encaminados a resolver problemas específicos hasta complejos entornos de simulación. Atendiendo a su funcionalidad se pueden clasificar en:

- Códigos privados orientados a problemas específicos. Representan el primer paso al abordar un nuevo problema, siendo típicos en el mundo académico y en centros de I+D como parte de sus paquetes de herramientas.

- Códigos open source, semejantes a los anteriores pero encaminados a beneficiarse de la colaboración entre instituciones.

- Entornos de desarrollo open source. Estos van un paso más allá al estar diseñados para su aplicación en múltiples problemas, minimizando la duplicación de código. OpenFoam ${ }^{28}$ destaca en este tipo, siendo cada vez más utilizado en la industria y academia.

- Paquetes comerciales de simulación, mucho más complejos y versátiles. Integran múltiples modelos numéricos, con una interfaz de usuario que facilita el pre y postproceso. Normalmente permiten el desarrollo de macros para poder extender las opciones de cálculo de acuerdo a las necesidades del usuario. CD-Adapco Star CCM+ ${ }^{29}$ o Ansys $\mathrm{CFD}^{30}$ se encuentran entre los más utilizados.

Particularizando en las aplicaciones navales, normalmente se asume el agua como un fluido incompresible y sin variaciones térmicas. Centrándose en la conservación de masa y cantidad

\footnotetext{
${ }^{28}$ http://www.openfoam.org/index.php

${ }^{29} \mathrm{http}: / /$ www.cd-adapco.com/products/star-ccm\%C2\%AE

${ }^{30} \mathrm{http}: / / \mathrm{www}$.ansys.com/Products/Simulation+Technology/Fluid+Dynamics
} 
de movimiento, se pueden considerar combinaciones de las siguientes simplificaciones y condiciones de contorno:

- Potenciales o viscosos

- Con o sin superficie libre

- Estacionarios o transitorios

- Con o sin superficies sustentadoras

Por otro lado, con respecto a la discretización del dominio fluido y sus límites se pueden subdividir en:

- Códigos 3D volumétricos con mallas tridimensionales que discretizan el dominio fluido completo. Permiten incorporar los modelos de turbulencia y efectos viscosos.

- Códigos 3D de paneles que asumen flujo potencial que únicamente discretizan las superficies delimitadoras del dominio fluido. Se basan en el método de elementos de contorno o BEM (Boundary Element Method) para obtener el campo de velocidades y presiones.

- Códigos 2D, útiles para problemas particulares como sloshing o con cuerpos de revolución.

\section{Aplicación a multicascos}

En general, la mayoría de los CFD orientados a calcular el flujo alrededor de un monocasco pueden ser empleados con multicascos, como por ejemplo Sahoo et al. (2004) realiza con Shipflow $^{31}$. No obstante, existen ciertas particularidades que hacen de los multicascos un caso merecedor del desarrollo de herramientas específicas para mejorar la precisión de los resultados o reducir los requerimientos computacionales.

A la hora de definir el dominio de cálculo, es beneficioso asignar una nueva zona para el flujo entre cascos que permita aplicar operadores específicos para capturar los fenómenos de interferencia. Por ejemplo numéricamente es recomendable aproximar las derivadas en la

\footnotetext{
${ }^{31}$ http://www.flowtech.se/
} 
dirección física de propagación del flujo, que en el caso de la cara interior de los cascos es hacia el plano de crujía.

Pero sin duda la característica más relevante de los multicascos desde un punto de vista hidrodinámico es la gran esbeltez de sus cascos. Como se anticipó en el estado del arte, este hecho los convierte es ideales para la aplicación de la teoría lineal como muestra Tuck (1987). En este apartado cabe destacar los códigos propuestos por Insel (1990), Peng (2001) o Yeung et al. (2004).

Dichos algoritmos buscan minimizar los requisitos computacionales con el fin de analizar múltiples condiciones y optimizar el diseño final. Sin embargo, las predicciones de la resistencia son relativamente limitadas al asumir flujo potencial. En el otro extremo se encuentran los códigos que resuelven directamente las ecuaciones de Navier-Stokes incorporando modelos de turbulencia y viscosidad. La inclusión de multicascos en estos permite alcanzar un mayor grado de precisión a cambio de demandar una potencia de cálculo significativamente mayor. Ejemplo de ello son los trabajos de Miller et al. (2006) y He et al. (2015) con el CFDShip-lowa ${ }^{32}$, o de Broglia et al. (2011) y Zaghi et al (2011) con el CFD del CNR-Insean ${ }^{33}$.

\footnotetext{
${ }^{32}$ http://www.iihr.uiowa.edu/shiphydro/cfd-code/

${ }^{33}$ Canal de Ensayos Hidrodinámicos de Italia http://www.insean.cnr.it/en
} 


\section{HERRAMIENTAS EMPLEADAS Y CONFIGURACIONES ESTUDIADAS}

\subsection{Instrumentación}

\subsubsection{Instalaciones}

Las campañas experimentales de este estudio se han llevado a cabo en los canales de aguas tranquilas del CEHINAV y CEHIPAR. La Tabla 3 resume las principales características de ambos.

Tabla 3. Características de los canales de ensayos utilizados

\begin{tabular}{|l|r|r|}
\hline & \multicolumn{1}{|c|}{ CEHINAV } & \multicolumn{1}{c|}{ CEHIPAR } \\
\hline Longitud & $100.0 \mathrm{~m}$ & $320.0 \mathrm{~m}$ \\
Anchura & $3.8 \mathrm{~m}$ & $12.5 \mathrm{~m}$ \\
Profundidad & $2.2 \mathrm{~m}$ & $6.5 \mathrm{~m}$ \\
Velocidad máxima del carro: & $3.5 \mathrm{~m} / \mathrm{s}$ & $10 \mathrm{~m} / \mathrm{s}$ \\
\hline
\end{tabular}

\subsubsection{Sensores}

El procedimiento experimental seguido en ambas instalaciones ha sido semejante, remolcando los modelos a diferentes velocidades y midiéndose la resistencia total al avance, el asiento y trimado. En el caso del CEHINAV se midieron además cortes de olas centrales y laterales. La Tabla 4 resume los sensores empleados para medir cada uno de ellos, para lo cual se siguieron los siguientes procedimientos:

- La velocidad del carro es controlada por encoders que miden las revoluciones del motor eléctrico en el caso del CEHINAV, o las de ruedas del carro en el CEHIPAR. EI CEHINAV cuenta con un segundo encoder con rodamiento específico con el fin de cuantificar mejor la incertidumbre en las medidas y detectar posibles desviaciones, como se discute en detalle más adelante. 
- La resistencia al avance se mide mediante células de carga instaladas en los carros de remolque (Fig. 8). En el caso de los ensayos con modelo fijo en el CEHIPAR se empleó un dinamómetro de seis componentes para poder restringir completamente los movimientos del modelo en relación al carro (Fig. 9).

- La medida del asiento y trimado se realiza de forma diferente en el CEHINAV y CEHIPAR. En el primero se emplean láseres de posición para determinar los desplazamientos del modelo relativos al carro en sentido longitudinal y vertical en proa y popa (Fig. 10). Por el contrario, el CEHIPAR utiliza encoders en las articulaciones de los brazos de sujeción para medir la rotación de estos y determinar así su posición exacta y por ende el desplazamiento del modelo (Fig. 11).

- La toma de cortes de ola en el CEHINAV se efectúa mediante sensores puntuales de elevación de la superficie libre tipo probeta (Fig. 12). Al sumergirse parcialmente, la conductividad de los electrodos varía. Para posicionar el modelo respecto a las probetas se emplea un láser adicional orientado transversalmente al canal. Al avanzar el carro llega un momento en que este incide en el haz de laser modificando su señal. Conocidas la distancia entre el láser, la probeta y el modelo se pueden posicionar correctamente la situación de este último respecto al corte de olas medido (Fig. 13). Se emplearon tres probetas para los cortes laterales junto con una probeta central. Las primeras están sujetas a un soporte dispuesto transversalmente al canal y sujeto a uno de los costados de éste. La probeta central por otro lado se posiciona mediante una estructura tipo trípode apoyada en el fondo (Fig. 14), diseñada ex profeso para esta campaña experimental. De esta forma se pueden tomar medidas entre los cascos sin interferir con ellos a su paso. 


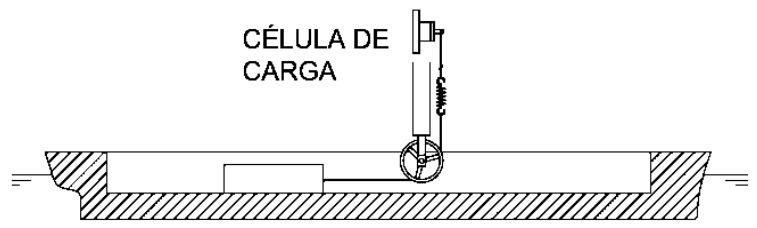

Fig. 8. Esquema de instalación de la célula de carga para el caso de movimiento vertical libre

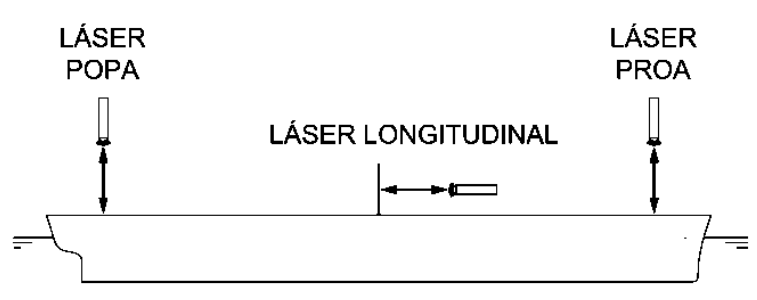

Fig. 10. Esquema de instalación los láseres para la medida del asiento y trimado en el CEHINAV

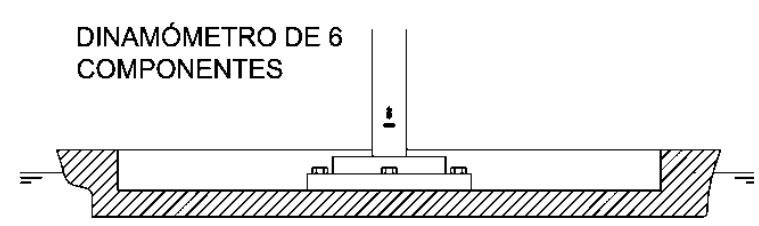

Fig. 9. Esquema de instalación del dinamómetro de 6 componentes para el caso de modelo fijo

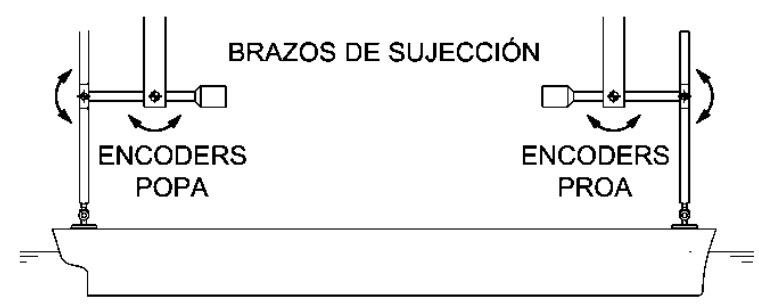

Fig. 11. Esquema de instalación de los encoders para la medida del asiento y trimado en el CEHIPAR

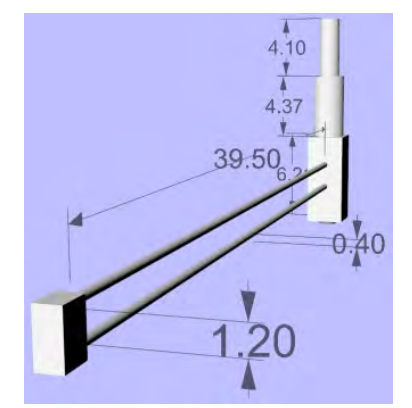

Fig. 12 Probeta para medir elevaciones de Ia superficie libre del CEHINAV

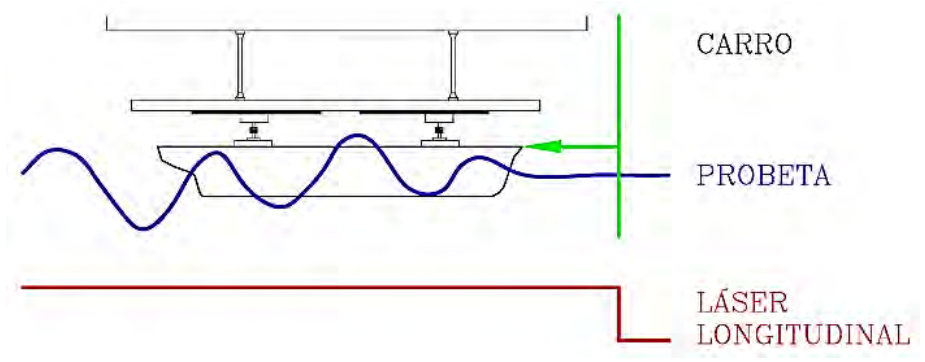

Fig. 13. Posición relativa del carro respecto a la medida de la probeta

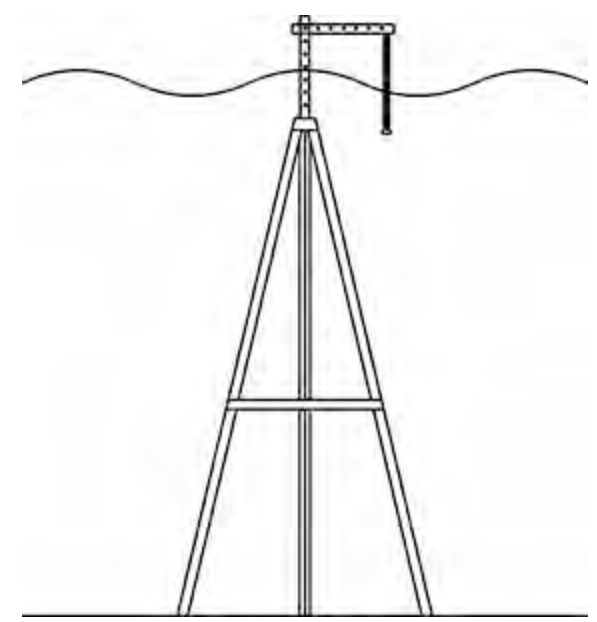

Fig. 14. Dispositivo para la medida de cortes de olas centrales 
Tabla 4. Características técnicas de los sensores utilizados en el CEHINAV ${ }^{34}$

\begin{tabular}{|l|l|}
\hline \multirow{3}{*}{ Velocidad } & $\begin{array}{l}\text { Encoder principal modelo Dinapar } 625 \\
\text { Frecuencia máxima: } 100 \mathrm{kHz} \\
\text { Pulsos por vuelta: } 2000 \text { (precisión: } 0.055 \mathrm{~mm} \text { ) }\end{array}$ \\
\cline { 2 - 3 } & $\begin{array}{l}\text { Encoder secundario modelo Heidenhain ROD-426 } \\
\text { Frecuencia máxima: } 100 \mathrm{kHz} \\
\text { Pulsos por vuelta: } 5000 \text { (precisión: } 0.100 \mathrm{~mm} \text { ) }\end{array}$ \\
\hline Resistencia & $\begin{array}{l}\text { Célula de carga Kempf \& Remmers Gmbh R57-IX } \\
\text { Precisión: } \pm 0.01 \mathrm{~g}\end{array}$ \\
\hline $\begin{array}{l}\text { Asiento y } \\
\text { trimado }\end{array}$ & $\begin{array}{l}\text { Láseres para medir la el desplazamiento horizontal y vertical en proa y popa } \\
\text { Modelo DLS-2000 } \\
\text { Precisión: } \pm 0.75 \mathrm{~mm} \\
\text { Rango: } 300 \mathrm{~mm} \\
\text { Resolución: } 0.076 \mathrm{~mm}\end{array}$ \\
\hline $\begin{array}{l}\text { Cortes de } \\
\text { ola }\end{array}$ & $\begin{array}{l}\text { Probetas de tipo resistivo fabricadas en el CEDEX } \\
\text { Diámetro de los electrodos: } 4 \text { mm }\end{array}$ \\
\hline
\end{tabular}

\subsubsection{Ajuste de la separación entre cascos}

Este trabajo gira alrededor del análisis de la relación entre la separación entre cascos y la resistencia de interferencia, con el fin de optimizar la resistencia al avance. Experimentalmente por tanto es esencial contar con una estructura de conexión entre cascos que permita ajustar su separación de forma sistemática. Su fabricación fue una de las primeras tareas llevadas a cabo, consistiendo en dos bastidores de madera ubicados en los extremos de proa y popa (Fig. 15). Únicamente se consideraron configuraciones tipo catamarán en base a las limitaciones de manga del CEHINAV. En las bases de los bastidores se perforaron guías para atornillar los cascos, cuyo espaciado se calibró para proporcionar tres posibles separaciones. Además, los propios bastidores sirven para unir el modelo al carro al permitir ubicar los puntos de conexión en el plano de crujía como en un monocasco.

\footnotetext{
${ }^{34}$ Las especificaciones técnicas de los sensores del CEHIPAR no fueron obtenidas por razones de confidencialidad

${ }^{35}$ Centro de Estudios y Experimentación de Obras Públicas http://www.cedex.es
} 

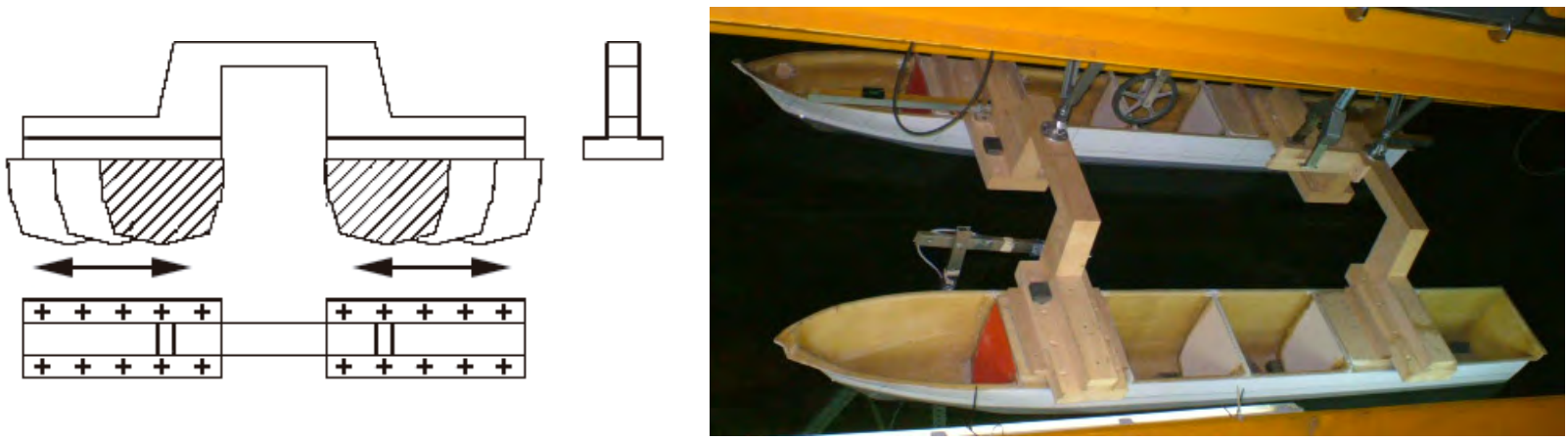

Fig. 15. Sistema de ajuste de la separación entre cascos

Como resultado se obtuvo un sistema robusto dada la gran sección transversal de los bastidores, si bien poco flexible al permitir únicamente tres separaciones posibles. Como mejora se construyó un bastidor de segunda generación consistente en perfiles de aluminio más ligero (Fig. 16). Al igual que en el caso anterior, el bastidor sirve de interconexión entre el carro y los cascos, los cuales van atornillados al bastidor. No obstante, el bastidor cuenta con número mucho mayor de puntos de sujeción ampliando significativamente el rango de separaciones. En el futuro será recomendable revisar este sistema para poder incluir posibles separaciones longitudinales y poder así evaluar la utilización de configuraciones asimétricas, en la línea presentada por Yu (2015).

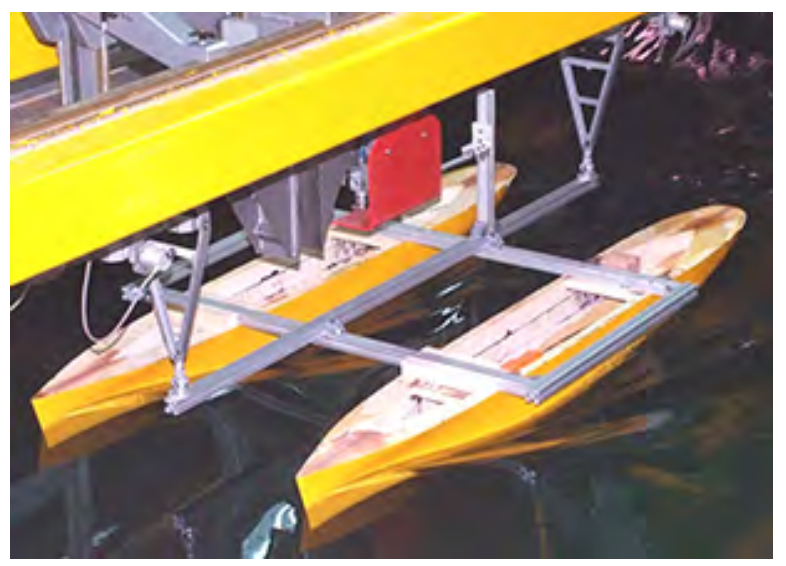

Fig. 16. Bastidor de unión de los cascos de segunda generación

\subsubsection{Desarrollo del sistema de trimado fijo del CEHINAV}

Tras los primeros ensayos en el CEHINAV se observó la utilidad de poder restringir los movimientos verticales del modelo para cuantificar los efectos del asiento y trimado 
dinámicos. Al no disponer de un dinamómetro de seis componentes, se optó por fabricar una estructura de soporte más sencilla pero suficientemente robusta (Fernández-Gutiérrez (2010)).

El sistema diseñado se basa en una estructura rígida fija al carro y unida al modelo mediante un sistema de guías especiales con holguras mínimas para restringir al máximo el movimiento vertical. A lo largo de dichas guías se deslizan unos carritos fijados al modelo y dotados de unos rodamientos de muy bajo rozamiento que minimizan la resistencia añadida por este sistema.
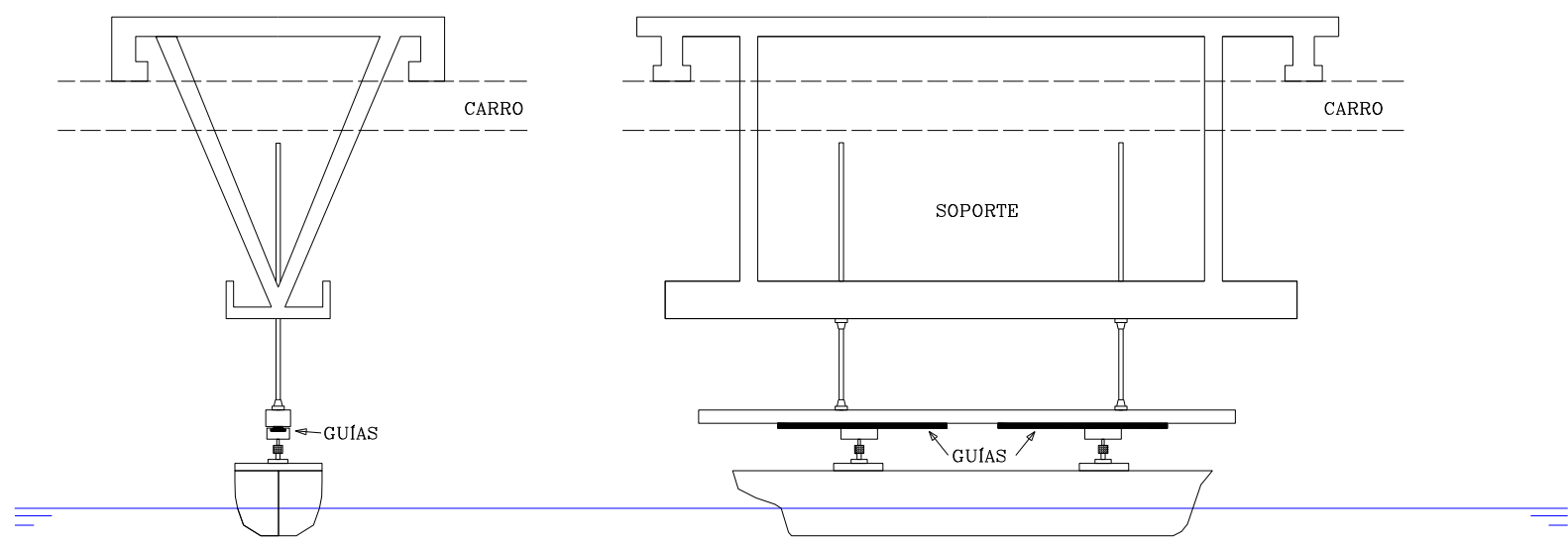

Fig. 17. Sistema de trimado fijo fabricado en el CEHINAV

Éste sistema permite seguir utilizando la misma célula de carga que en los ensayos con el modelo libre, si bien hay que añadir el rozamiento de las guías. Esto requiere calibrar el sistema previo a los ensayos aplicando fuerza longitudinal de forma gradual mediante una polea y pesas. Debe tenerse en cuenta que el coeficiente de rozamiento depende de la fuerza vertical actuante sobre las guías, la cual difiere en cada ensayo ${ }^{36}$ y que se simulará también mediante pesas como muestra la Fig. 18.

${ }^{36}$ A pesar de encontrarse en equilibrio hidrostático, la distribución de presiones sobre el casco varía al desplazarse el modelo. Esto induce una componente vertical dinámica, que es precisamente la que el sistema de fijación debe restringir. Ver la sección 6.3 para una explicación más detallada. 


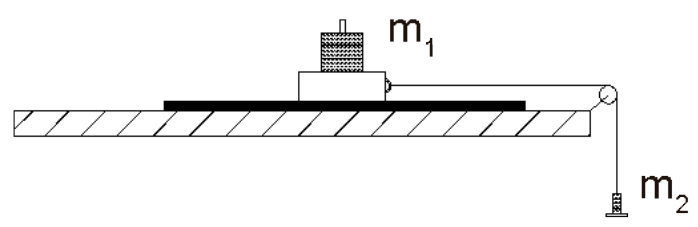

Fig. 18. Esquema del procedimiento de medición del rozamiento del sistema de trimado fijo del CEHINAV

Al incrementar progresivamente la fuerza longitudinal se alcanza un punto en el que los rodamientos comienzan a desplazarse. Este punto permite determinar el coeficiente de

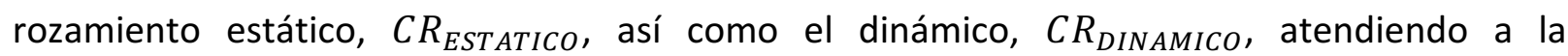
aceleración del carrito una vez empieza a desplazarse.

$$
\begin{gathered}
\sum F=m a \\
P-F_{R}=m a \Rightarrow P-N C R=m a \\
m_{2} g-m_{1} g C R=m_{1} \frac{2 s}{t^{2}} \\
C R_{E S T A ́ T I C O}=\frac{m_{2}}{m_{1}} \\
C R_{\text {DINÁMICO }}=\frac{m_{2} g-m_{1} \frac{2 s}{t^{2}}}{m_{1} g}
\end{gathered}
$$

Tras calibrarse para un rango de fuerzas verticales sobre las guías entre 0 y $6.6 \mathrm{~kg}$ se apreció como los valores de los coeficientes de rozamiento eran prácticamente constantes, tomándose como:

- $\quad$ CR $R_{\text {ESTÁTICO }}=0.0114$

- $C R_{\text {DINÁMICO }}=0.0015$

Este sistema fue validado posteriormente con un Serie 60 tal y como describe FernándezGutiérrez (2010). Los datos de los ensayos muestran como estos coeficientes se traducen en fuerzas de fricción del orden de $0.5 \%$ de la resistencia total, valor inferior a la propia precisión de las medidas. Y lo que es más importante, consiguen reducir los movimientos verticales en un $96 \%$ demostrando su aplicabilidad para el fin al que fueron diseñados. 


\subsection{Software utilizado en las simulaciones}

\subsubsection{Tdynlin}

Tdynlin $^{37}$ es un código de paneles con superficie libre desarrollado en el grupo de investigación del CEHINAV y empleado para el diseño y optimización de carenas (Berenguer et al. (2002), Pérez Arribas et al. (2004)). Su origen se remonta al trabajo de Souto-Iglesias (2001), basándose en el método de Dawson (1977) y posterior generalización de Bruzzone (1994). Tiene como objetivo calcular el flujo potencial alrededor del casco, el tren de olas generado y la resistencia al avance empleando singularidades de Rankine (fuentes). En un primer paso se calcula el flujo alrededor del casco sin considerar la superficie libre, o para ser más exactos asumiendo que esta no se deforma. Para asegurarse que las elevaciones son nulas se añade una copia reflejada del casco que convierte a la superficie libre en un plano de simetría, creando lo que se denomina modelo doble. El siguiente paso es determinar la deformación real de la superficie libre. Para ello se utilizan los valores de presión y velocidad en el plano de simetría calculados en el paso previo para linearizar la condición de contorno dinámica sobre la superficie libre. La condición de radiación se impone mediante el uso de operadores numéricos up-wind. El código está escrito en C, pudiéndose compilar tanto para Linux como para Windows.

El programa funciona como un módulo dentro del entorno de simulación $\mathrm{GiD}^{38}$, el cual se utiliza para el pre y post-proceso. Tdynlin ofrece una alternativa sencilla frente a Tdyn ${ }^{39}$, el cual también hace uso del entorno GiD si bien ofrece un completo paquete de simulación basado el cálculo finitesimal (FIC-FEM) ${ }^{40}$. Permite así incorporar fenómenos viscosos junto con el asiento y trimado dinámicos, como presenta en detalle García Espinosa et al. (2002).

\footnotetext{
${ }^{37}$ http://canal.etsin.upm.es/proyectos/cfd/cfd.html

${ }^{38}$ http://www.gidhome.com/

${ }^{39}$ http://www.compassis.com/compass/en/Productos/Tdyn+CFD\%2BHT

${ }^{40}$ De sus siglas en inglés Finite Increment Calculus -Finite Element Methods (Cálculo de Incrementos Finitos - Método de Elementos Finitos)
} 
GiD permite importar la geometría del casco en formato IGES para generar la malla superficial, la cual debe incluir también la extensión de la superficie libre a calcular (Fig. 19). Una vez asignadas las diferentes zonas y generada la malla, Tdynlin se encarga de resolver el campo de velocidades, presiones y elevaciones, empleándose GiD de nuevo para su visualización (Fig. 20). Adicionalmente, GiD permite la extracción de cortes de ola a determinadas separaciones laterales, muy útil para poder validar los resultados con datos experimentales.

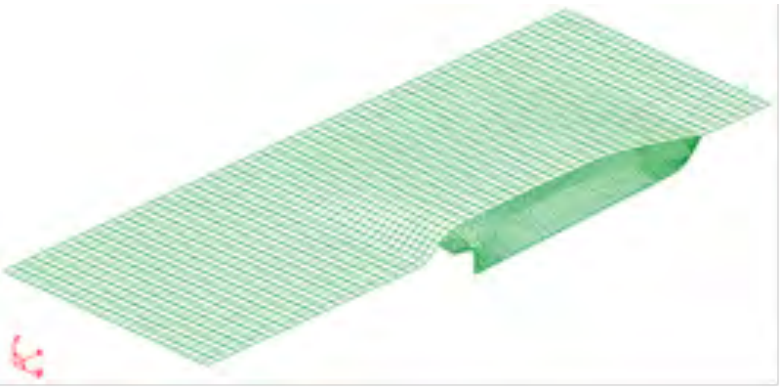

Fig. 19. Mallado generado en GiD para su uso con Tdynlin

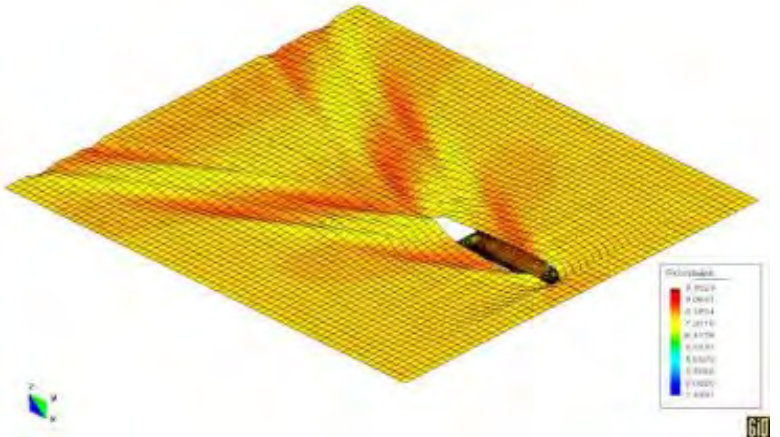

Fig. 20. Tren de olas calculado por el ETSIN-CFD para un buque pesquero

La implementación y validación del módulo de catamaranes en Tdynlin es precisamente uno de los objetivos iniciales de este trabajo, por lo que su utilización se remonta a las primeras fases. Ello requirió reprogramar las condiciones de la superficie libre identificando específicamente la zona entre cascos para poder utilizar un operador diferencial específico. Dado que los fenómenos de oleaje son mayormente potenciales, Tdynlin permite evaluar rápidamente las posibles cancelaciones de los trenes de olas de cada casco, la cual es una de las principales causas de interferencia.

\subsubsection{STAR-CCM+}

CD-Adapco Star-CCM+ es uno de los principales paquetes CFD comerciales ${ }^{41}$ (Izaguirre Alza (2012), Perić (2013), Atreyapurapu et al. (2014)). Está basado en el método de volúmenes finitos y proporciona un entorno de simulación completo, desde la definición geométrica de la

\footnotetext{
${ }^{41}$ http://www.cd-adapco.com/products/star-ccm\%C2\%AE
} 
región de cálculo hasta el post-proceso de los resultados obtenidos. El programa permite múltiples opciones de simulación y está orientado a trabajar con geometrías 3D y mallas volumétricas, si bien estas pueden simplificarse a mallas bidimensionales en casos especiales.

La interfaz está diseñada para conducir al usuario intuitivamente a lo largo del proceso. A medida que se van seleccionando los diferentes modelos a usar, se activan las opciones disponibles para cada uno de ellos siguiendo un esquema piramidal. En primer lugar sólo están disponibles las opciones de edición geométrica necesarias para establecer el dominio de cálculo. El programa parte de geometrías tridimensionales, las cuales pueden ser importadas en diferentes formatos (IGES, STEP, etc.) de modeladores 3D como Rhinoceros, Catia o AutoCAD. Una vez definida esta se activan las opciones de mallado y modelos físicos (Tabla 5 y Tabla 6). Una vez configurados todos ellos se ejecuta la simulación, ofreciendo múltiples opciones de visualización de los resultados. Cabe destacar la posibilidad de integrar algoritmos de cálculo programados en Java, lo que amplifica las posibilidades del programa y permite adaptarlo a las necesidades específicas del usuario. 
Tabla 5. Opciones principales de los modelos de mallado en Star-CCM+

\begin{tabular}{|c|c|}
\hline OPCIONES PRINCIPALES & 2ㅇ NIVEL \\
\hline $\begin{array}{l}\text { - Mallado Superficial: } \\
\text { ○ Surface Remesher. } \\
\text { Reajusta la calidad de los elementos del mallado } \\
\text { ○ Surface Wrapper. } \\
\text { En el caso que la región definida no esté cerrada } \\
\text { se encarga de remallar para cerrar las aberturas } \\
\text { existentes } \\
\text { - Mallado Volumétrico } \\
\circ \text { Advancing Layer Mesher } \\
\circ \text { Polyhedral mesher } \\
\circ \text { Tetrahedral mesher } \\
\circ \text { Thin mesher } \\
\circ \text { Trimmer. ajusta las celdas generadas a las } \\
\text { superficies geométricas }\end{array}$ & $\begin{array}{l}\text { - Prism Layer. } \\
\text { Permite aumentar la densidad de mallado en la } \\
\text { superficie para tener en cuenta los efectos de capa } \\
\text { límite } \\
\text { - Extruder }\end{array}$ \\
\hline
\end{tabular}

Tabla 6. Opciones principales de los modelos físicos en Star-CCM+

\begin{tabular}{|c|c|c|c|}
\hline 1er NIVEL & 2ㅇ NIVEL & 3er NIVEL & OPCIONALES \\
\hline $\begin{array}{l}\text { - Op. espaciales: } \\
\text { ○ Axisimétrico } \\
\text { ○ Tridimensional } \\
\text { ○ Bidimensional } \\
\text { - Op. Temporales: } \\
\circ \text { Inestable explícito } \\
\circ \text { Balance armónico } \\
\circ \text { Inestable implícito } \\
\circ \text { Inestable PISO } \\
\circ \text { Estacionario } \\
\text { Op. de materiales: } \\
\circ \text { Gas } \\
\circ \text { Líquido } \\
\circ \text { Sólido } \\
\circ \text { Mezcla multifase } \\
\circ \text { Gas } \\
\text { (multicomponente) } \\
\circ \text { Líquido } \\
\text { (multicomponente) } \\
\circ \text { Sólido } \\
\text { (multicomponente) }\end{array}$ & $\begin{array}{l}\text { - Op. de movimiento: } \\
\circ 6 \text { Grados de } \\
\text { Libertad } \\
\circ \text { Morpher } \\
\circ \text { Marco de ref. móvil } \\
\circ \text { Movimiento de } \\
\text { cuerpo rígido } \\
\circ \text { Estacionario } \\
\text { - Régimen de } \\
\text { viscosidad: } \\
\circ \text { Viscoso } \\
\circ \text { Laminar } \\
\circ \text { Turbulento } \\
\text { - Op. de multifase: } \\
\circ \text { Flujo multifásico } \\
\text { segregado } \\
\circ \text { Volumen de fluido } \\
\text { (VOF) }\end{array}$ & $\begin{array}{l}\text { - 6-DOF } \\
\text { ○ Mov. Embebido } \\
\text { ○ Morphing } \\
\text { ○ Movimiento } \\
\text { - Modelos Turbulencia } \\
\text { ○ K-Epsilon } \\
\circ \text { K-Omega } \\
\text { - SST (Menter) } \\
\text { - Standard } \\
\text { (Wilcox) } \\
\circ \text { Tensiones Reynolds } \\
\circ \text { Spalart-Allmaras } \\
\circ \text { Y+ }\end{array}$ & $\begin{array}{l}\frac{\text { Cambio de fase }}{\text { - Cavitación }} \\
\text { Transición } \\
\text { - Gamma Re-Theta } \\
\text { - Supresión turbulencia } \\
\text { Modelos físicos } \\
\text { opcionales: } \\
\text { - Fluido isotérmico } \\
\text { segregado } \\
\text { - Radiación } \\
\text { - Tensión superficial } \\
\text { - Gravedad } \\
\text { - Ajuste calidad celdas } \\
\text { - Temperatura } \\
\text { - multifásica segregada } \\
\text { - Dscalar pasivo }\end{array}$ \\
\hline
\end{tabular}




\subsection{Geometrías}

De cara a la realización de los ensayos se han utilizado dos modelos diferentes de catamaranes tanto en el CEHINAV como en el CEHIPAR:

- Catamarán de servicio comercial PESBO

- Catamarán S60

La geometría de ambos cascos es idéntica en cada configuración, siendo necesario analizar únicamente uno de ellos para evaluar los efectos de interferencia. Por ello, de aquí en adelante denominaremos monocasco al flotador independiente ${ }^{42}$. No hay confundir este con el concepto de monocasco equivalente discutido en el apartado 4.2. La Tabla 7 recoge sus dimensiones principales.

Tabla 7. Dimensiones principales a escala modelo de las geometrías estudiadas

\begin{tabular}{|l|r|r|r|r|}
\hline & & \multicolumn{1}{|c|}{ PESBO } & \multicolumn{1}{c|}{ S60 } & \\
\hline Eslora entre perpendiculares ${ }^{43}$ & $L_{P P}$ & 2208 & 2500 & $\mathrm{~mm}$ \\
\hline Manga de cada casco & $B_{D}$ & 241 & 333 & $\mathrm{~mm}$ \\
\hline Calado de diseño & $T$ & 120 & 133 & $\mathrm{~mm}$ \\
\hline Volumen desplazado de cada casco & $\nabla_{D}$ & 0.0440 & 0.0663 & $\mathrm{~m}^{3}$ \\
\hline Superficie mojada de cada casco & $W S_{D}$ & 0.885 & 1.062 & $\mathrm{~m}^{2}$ \\
\hline Coeficiente de bloque & $C_{B}$ & 0.653 & 0.600 & \\
\hline Eslora/manga de cada casco & $L_{P P} / B_{D}$ & 9.28 & 7.51 & \\
\hline Manga/calado de cada casco & $B_{D} / T$ & 1.98 & 2.50 & \\
\hline
\end{tabular}

${ }^{42}$ Se identificará con el subíndice $\mathrm{D}$ procedente de su nombre en inglés, demihull

43 Toda la información presentada en este trabajo corresponde a escala modelo. De cara a su extrapolación al buque real, el factor de escala del catamarán PESBO es $\lambda_{m}=1: 12.5$. En el caso del S60, la eslora de referencia de la serie sistemática es $400 \mathrm{ft}$ (121.92 m, Todd (1963)), lo que implica $\lambda_{m} \approx 1: 49$ 
A su vez, con cada geometría se han considerado diferentes separaciones entre cascos, caracterizándose cada una de ellas mediante la separación entre crujías, $s$, o la separación entre los costados interiores, $s_{D}$ (Fig. 21). Ambas variables pueden ser adimensionalizadas con la eslora $L_{P P}$ o manga del monocasco, $B_{D}$.

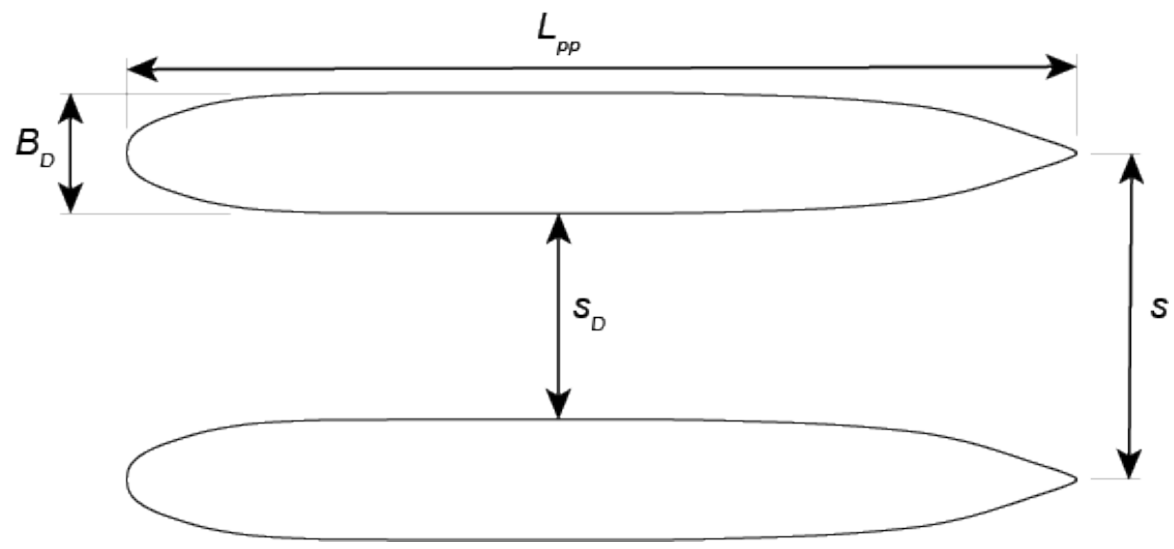

Fig. 21. Variables empleadas para caracterizar la separación entre cascos

\subsubsection{Catamarán de servicio comercial PESBO}

Este modelo corresponde a un catamarán ya construido y destinado al transporte de carga para dar servicio a unas granjas marinas. Durante la primera campaña de ensayos en 2004 se apreciaron efectos de interferencia considerándose merecedores de un estudio en detalle, lo que constituye el germen de este trabajo. Cada uno de los cascos es simétrico con respecto a su plano de crujía (Fig. 22), con una tolerancia de fabricación de $\pm 1 \mathrm{~mm}$. El apéndice $A$ contiene el plano de formas en detalle, pudiéndose descargar su definición 3D en formato IGES en http://canal.etsin.upm.es/ftp/2012/S60/.

Utilizando esta geometría se ensayaron múltiples velocidades en el rango de $F r$ de 0.1 a 0.550 en diferentes fases de la realización de este trabajo. Respecto a la separación entre cascos, la Tabla 8 muestra las tres separaciones seleccionadas usando el bastidor original de unión descrito anteriormente (sec. 5.1). 

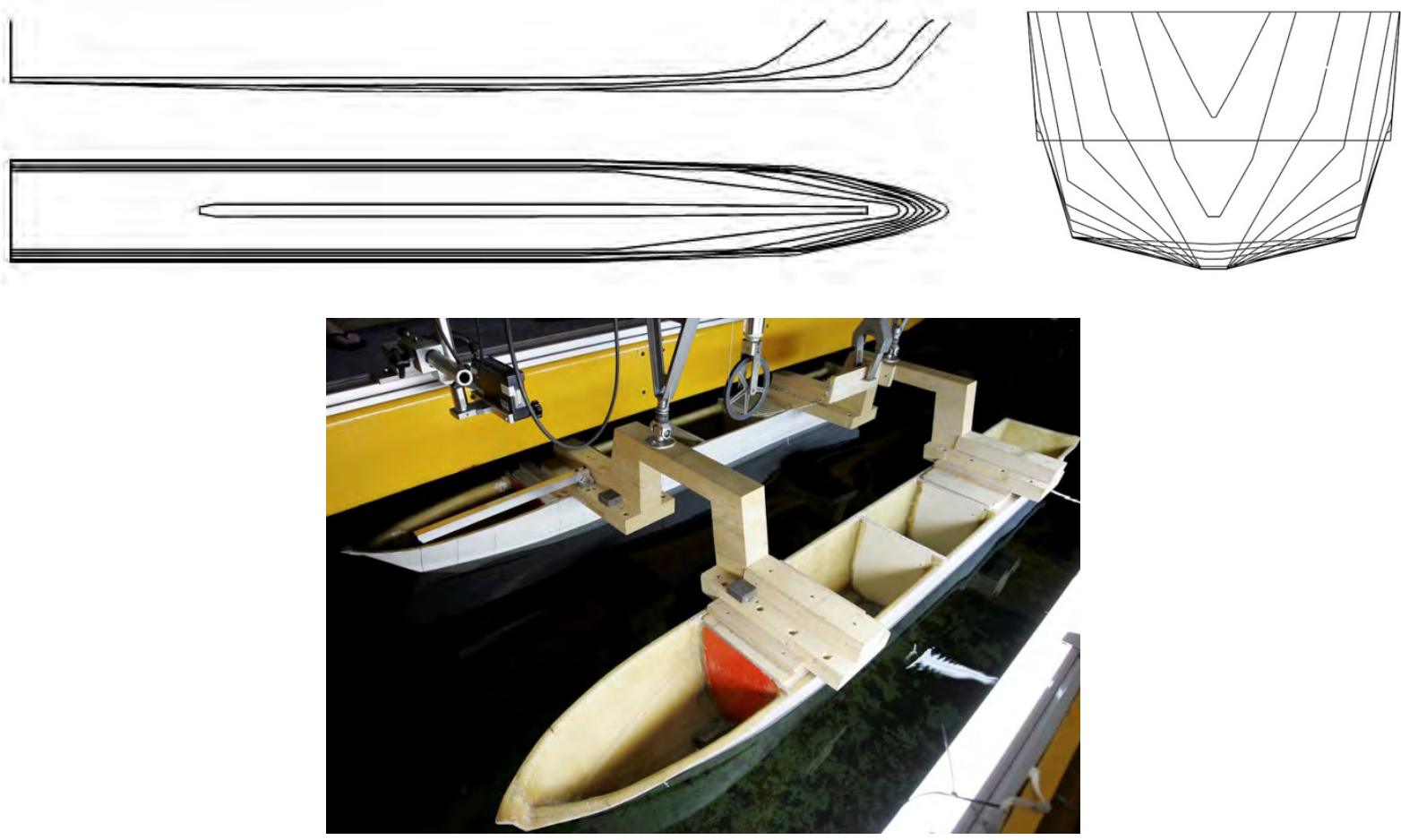

Fig. 22. Formas de los cascos del catamarán de servicio PESBO

Tabla 8. Separaciones ensayadas con el catamarán de servicio PESBO

\begin{tabular}{|l|c|c|c|c|}
\hline Configuración & $\begin{array}{c}\text { Espacio entre cascos, } \\
\boldsymbol{s}_{\boldsymbol{D}}(m)\end{array}$ & $\begin{array}{c}\text { Separación, } \\
\boldsymbol{S}(m)\end{array}$ & $\begin{array}{c}\boldsymbol{s} / \boldsymbol{L} \\
(-)\end{array}$ & $\begin{array}{c}\boldsymbol{s} / \boldsymbol{B}_{\boldsymbol{D}} \\
(-)\end{array}$ \\
\hline Sep. 1 & 0.220 & 0.458 & 0.207 & 1.926 \\
\hline Sep. 2 & 0.420 & 0.658 & 0.298 & 2.768 \\
\hline Sep. 3 & 0.620 & 0.858 & 0.388 & 3.609 \\
\hline
\end{tabular}




\subsubsection{Catamarán 560}

Como complemento a los ensayos anteriores se decidió construir y ensayar un catamarán compuesto por dos Serie 60 (Todd (1963)) en paralelo. Sus dimensiones principales son relativamente similares a las del catamarán de servicio PESBO como se puede apreciar en la Tabla 7, si bien las formas geométricas de cada casco son significativamente diferentes:

- El S60 no tiene cuerpo cilíndrico frente al el largo cuerpo cilíndrico mostrado por el PESBO

- El S60 muestra una popa de crucero convencional en contraste con la popa de espejo en el caso del PESBO

- El S60 no presenta codillos, mientras que el PESBO muestra dos claros codillos
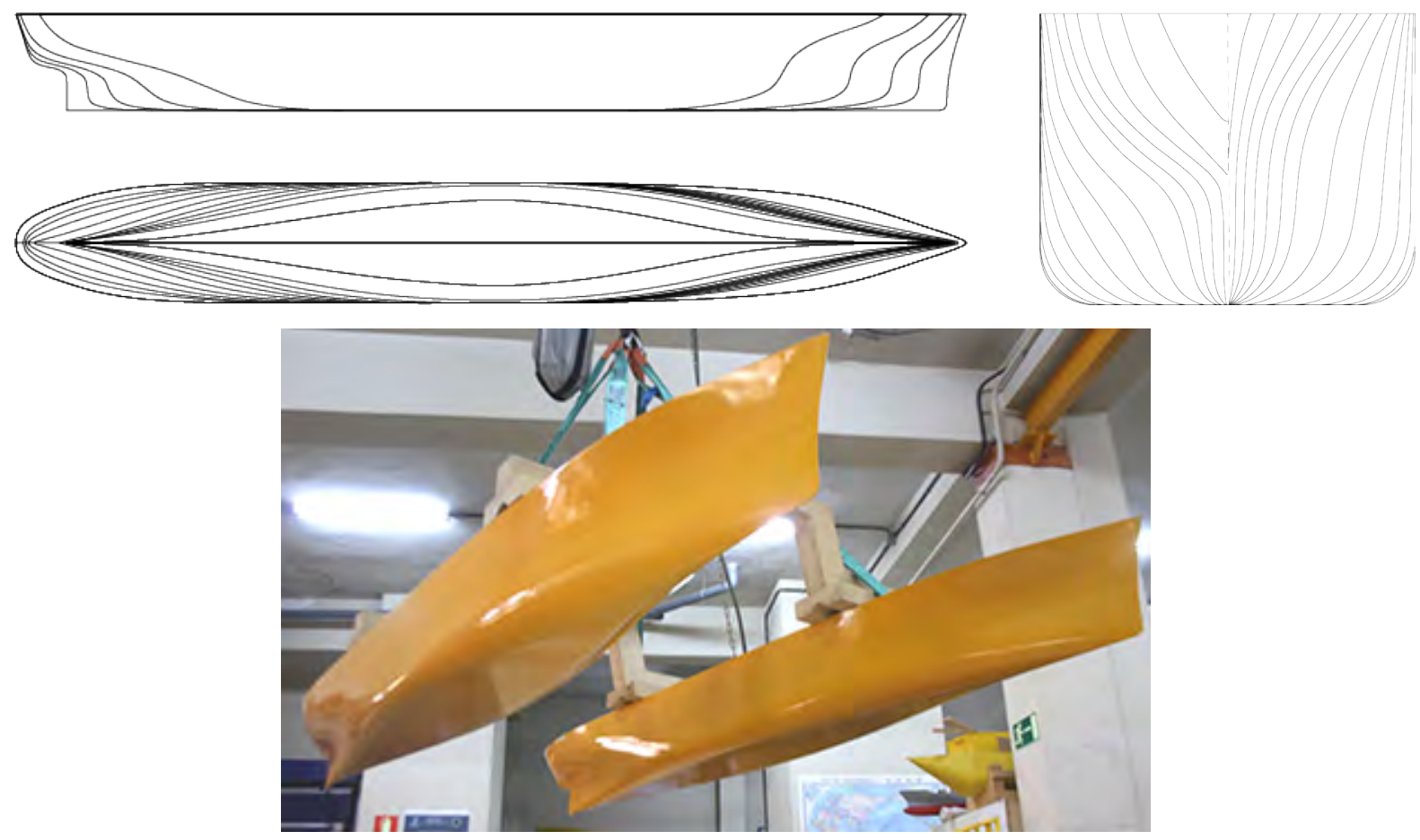

Fig. 23. Modelo del catamarán $\mathrm{S} 60$ antes de ser puesto en el agua

En primer lugar, la comparativa entre las respuesta de ambas configuraciones permite evaluar de forma cualitativa el impacto de la geometría en la resistencia de interferencia. En cualquier caso, la principal ventaja del catamarán S60 es la posibilidad de aprovechar la gran cantidad de información disponible de su comportamiento como monocasco (Todd (1963), Kim \& Jenkins (1981), Toda et al. (1992), Longo et al. (1993), Nakatake \& Takeshi (1994), Tarafder \& 
Suzuki (2008b)). Representa por tanto una configuración ideal como estándar de análisis para multicascos. La extensa campaña experimental realizada tiene como fin proporcionar una base sólida de validación para modelos numéricos, como el de Yeung et al. (2004) quienes emplean también un catamarán $\mathrm{S} 60$ como caso de estudio.

Previamente a la fabricación del modelo, su geometría fue redefinida a partir del modelo 3D en IGES utilizado como referencia en el CFD Workshop en Tokio (1994). Ello fue motivado por la falta de una adecuada continuidad entre la multitud de superficies que conformaban el casco (Fig. 24). Más aún, el oleaje generado al cubrirse un rango de $F r$ entre 0.15 y 0.55 , por encima del habitual en un Serie 60, requería extender verticalmente la superficie del casco para evitar el embarque de agua. Tras el proceso de modelización, la geometría actualizada coincide prácticamente con la geometría original como muestra la Fig. 25. Al igual que con el PESBO, ésta se ofrece a la comunidad hidrodinámica como estándar digital para futuros estudios, pudiéndose descargar en http://canal.etsin.upm.es/ftp/2012/S60/ .

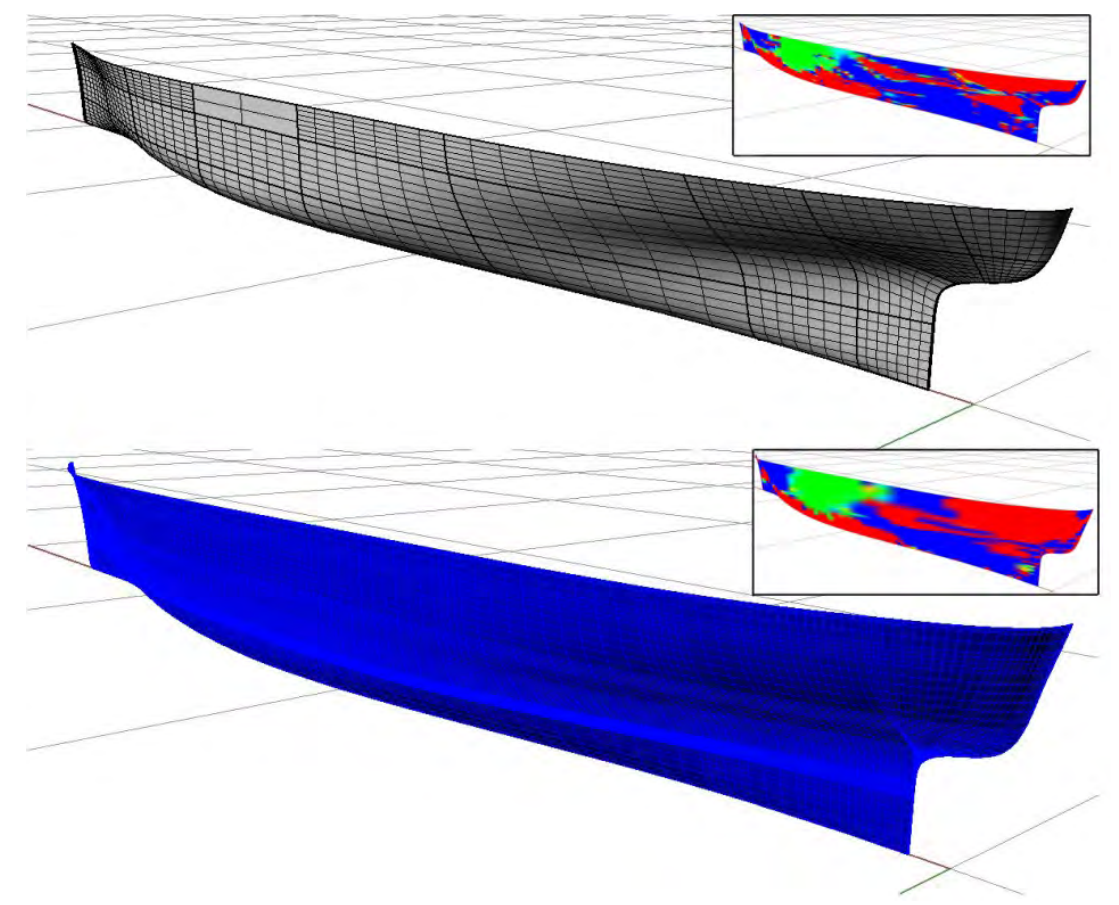

Fig. 24. Aspecto original (superior) y redefinido (inferior) del casco del Serie 60. Los detalles en la esquina superior derecha de cada modelo muestran la curvatura de la superficie. 


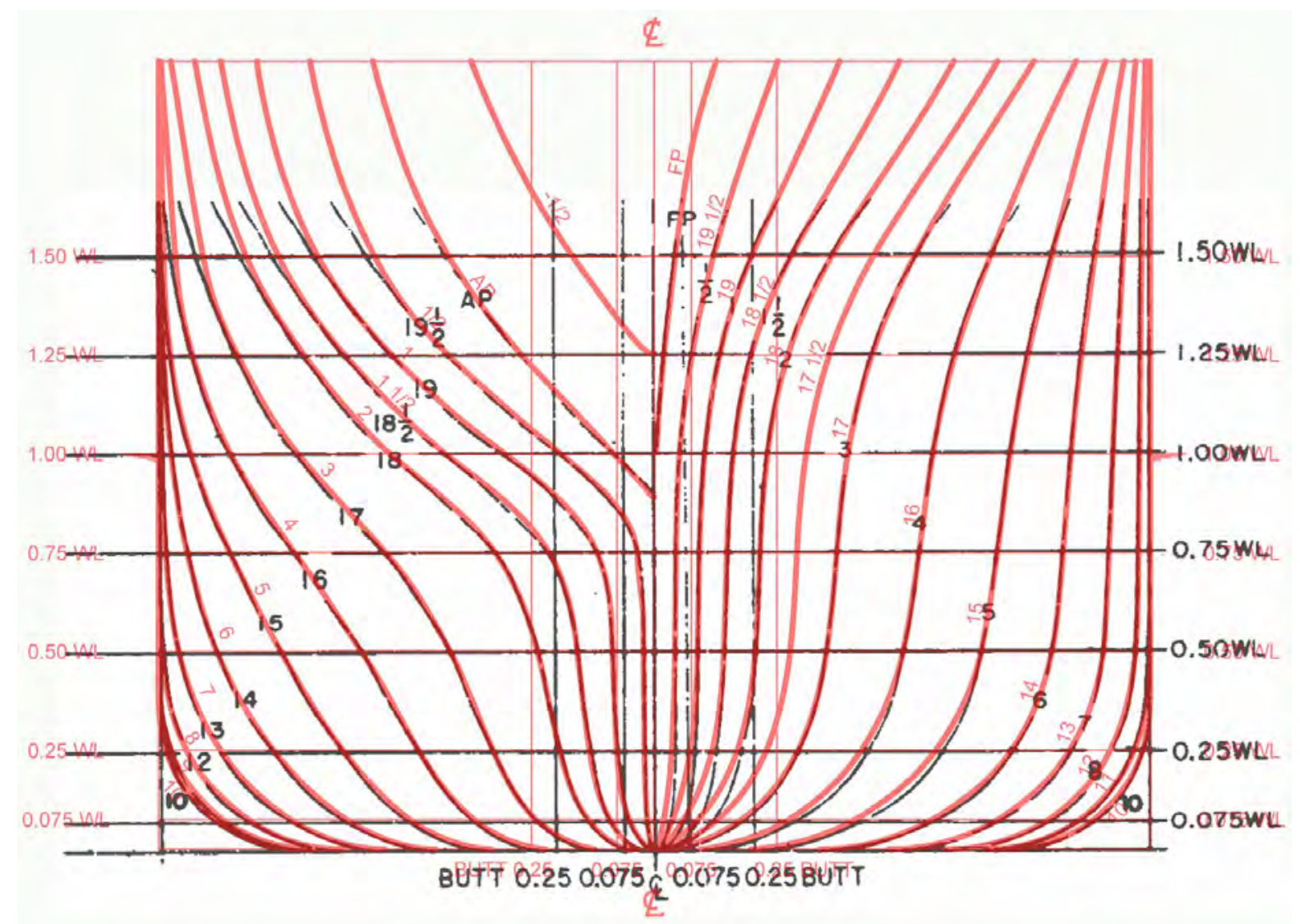

Fig. 25. Comparativa de entre la caja de cuadernas original (negro, Todd (1963)) y la redefinida en el presente estudio (rojo) 
Respecto a las separaciones estudiadas, utilizando el segundo bastidor de unión se analizaron cuatro posibles configuraciones como muestra la Tabla 9. Estos valores fueron escogidos de acuerdo a los resultados mostrados por Yeung et al. (2004), donde $s / L=0.226$ corresponde al caso de máxima interferencia favorable, mientras que $s / L=0.388$ muestra la mayor interferencia desfavorable. Las otras dos separaciones se añaden para refinar la matriz de datos incluyendo un valor intermedio y otro extremo, escogido para resultar en cuatro separaciones equiespaciadas.

Tabla 9. Separaciones ensayadas con el catamarán 560

\begin{tabular}{|l|c|c|c|c|}
\hline Configuración & $\begin{array}{c}\text { Espacio entre cascos, } \\
\boldsymbol{s}_{\boldsymbol{D}}(m)\end{array}$ & $\begin{array}{c}\text { Separación, } \\
\boldsymbol{s}(m)\end{array}$ & $\begin{array}{c}\boldsymbol{s} / \boldsymbol{L} \\
(-)\end{array}$ & $\begin{array}{c}\boldsymbol{s} / \boldsymbol{B}_{\boldsymbol{D}} \\
(-)\end{array}$ \\
\hline Sep. 1 & 0.232 & 0.565 & 0.226 & 1.697 \\
\hline Sep. 2 & 0.435 & 0.768 & 0.307 & 2.306 \\
\hline Sep. 3 & 0.638 & 0.971 & 0.388 & 2.916 \\
\hline Sep. 4 & 0.841 & 1.174 & 0.470 & 3.525 \\
\hline
\end{tabular}




\section{RESULTADOS EXPERIMENTALES}

\subsection{General}

La campaña experimental realizada constituye el núcleo central de este trabajo. Las siguientes secciones recogen los resultados obtenidos en los diferentes ensayos realizados, cuyo análisis se subdivide en cuatro categorías principales:

- Resistencia al avance, $R_{T}$ (sec. 6.2.2 - 6.2.4), en base a la cual se obtiene los factores de interferencia (sec. 6.2.5). Se incluye en este apartado una comparativa con las predicciones teóricas de Yeung et al. (2004) (sec. 6.2.6)

- Asiento y trimado dinámicos, con el fin de cuantificar en qué medida las variaciones dinámicas de las condiciones de navegación afectan a los fenómenos de interferencia (sec. 6.3)

- Cortes de ola, incluyendo cortes centrales y laterales (sec. 6.4). Se incluye una comparativa entre los cortes de olas exteriores del catamarán y monocasco para seleccionar el más adecuado de cara a visualizar los efectos de interferencia (sec. 6.4.2).

- Análisis global de los resultados experimentales anteriores (sec. 6.5)

\subsection{Resistencia de interferencia}

\subsubsection{Cálculo del factor de interferencia}

Como se presentó en el apartado 4.1.4, el parámetro empleado para cuantificar los efectos de interferencia es el factor de interferencia, bien en función de la resistencia total, $I F_{R}$, o la resistencia por formación de olas, $I F_{W}$.

A efectos prácticos la utilización del $I F_{R}$ o el $I F_{W}$ no supone una gran diferencia. En este trabajo se emplea la curva de fricción de ITTC-57, por lo que la resistencia viscosa del 
multicasco es igual a la suma de las componentes de cada casco por separado ${ }^{44}$. Se cancelan así mutuamente en el numerador no variando en absoluto su valor o signo. Por tanto ambos factores muestran por igual las condiciones de interferencia favorable o desfavorable así como su intensidad relativa al resto, siendo únicamente diferente su magnitud absoluta. Respecto al factor de forma, este es fundamental en la extrapolación a escala real. Sin embargo, su impacto en los factores de interferencia es pequeño por las mismas razones, al afectar únicamente a la componente viscosa.

Como se verá más adelante al analizar los valores medidos, la principal causa encontrada de las interferencias favorables reside en la interacciones del oleaje. Con el fin de minimizar el impacto de los efectos viscosos, en este trabajo se empleará únicamente el $I F_{W}$, el cual se denominará $I F$ de aquí en adelante. Además, la igualdad de los cascos permite estimar la resistencia total excluyendo los fenómenos de interferencia como el doble de la resistencia del monocasco.

$$
\sum_{i} R_{D_{i}}=2 R_{D}
$$

En base a las suposiciones anteriores, el cálculo del factor de interferencia se realiza de acuerdo al siguiente proceso:

- Determinación de la resistencia de interferencia a partir de los valores de resistencia total del multicasco y del monocasco:

$$
R_{I}=R_{M H}-\sum_{i} R_{D_{i}}=R_{M H}-2 R_{D}
$$

- Deducción de la componente de fricción de acuerdo a la descomposición de Hughes (1954) mostrada en la ec. (4.18), utilizando la curva de fricción ITTC-57 (ec. (4.17)) y el método de Prohaska (1966) para estimar el factor de forma (Tabla 10). Dado que cada

${ }^{44}$ La estimación de la resistencia viscosa de acuerdo a la curva de ITTC-57 es función únicamente de la velocidad y superficie mojada en la situación de equilibrio, la cual está libre de cualquier efecto de interferencia. 
casco en las geometrías estudiadas es idéntico, se asume que el factor de forma es el mismo para el catamarán que para cada casco.

$$
\begin{gathered}
R_{W}=R_{T}-(1+k) R_{F} \\
C_{F, I T T C-57}=\frac{0.075}{[\log (R e)-2]^{2}}
\end{gathered}
$$

Tabla 10. Factores de forma, $k$, de las geometrías estudiadas

\begin{tabular}{|l|l|}
\hline Catamarán de servicio PESBO & 0.2400 \\
\hline Catamarán $560^{45}$ & 0.0673 \\
\hline
\end{tabular}

- Determinación del factor de interferencia de interferencia en la resistencia por formación de olas, definido en la ecuación (4.4)

$$
I F=I F_{W}=\frac{R_{W, M H}-\sum_{i} R_{W, D_{i}}}{\sum_{i} R_{W, D_{i}}}=\frac{R_{W, M H}-2 R_{W, D}}{2 R_{W, D}}
$$

\subsubsection{Ensayos}

El análisis de la resistencia de interferencia es el eje central del presente trabajo, por lo que su cuantificación es uno de los apartados más importantes. Con diferencia supone el mayor esfuerzo experimental llevado a cabo, cubriendo un amplio espectro de configuraciones y velocidades resumido en la Tabla 11. Los vídeos de los experimentos se pueden descargar como material adicional en http://canal.etsin.upm.es/ftp/2012/S60/. Los siguientes apartados presentan en detalle los resultados obtenidos en relación a la resistencia de interferencia, la cual requiere previamente determinar la resistencia al avance.

45 Obtenido a partir de la formulación de Min \& Kang (2010), quienes analizaron a fondo la dependencia del factor de forma y el $R e$ 
Tabla 11. Ensayos de resistencia al avance

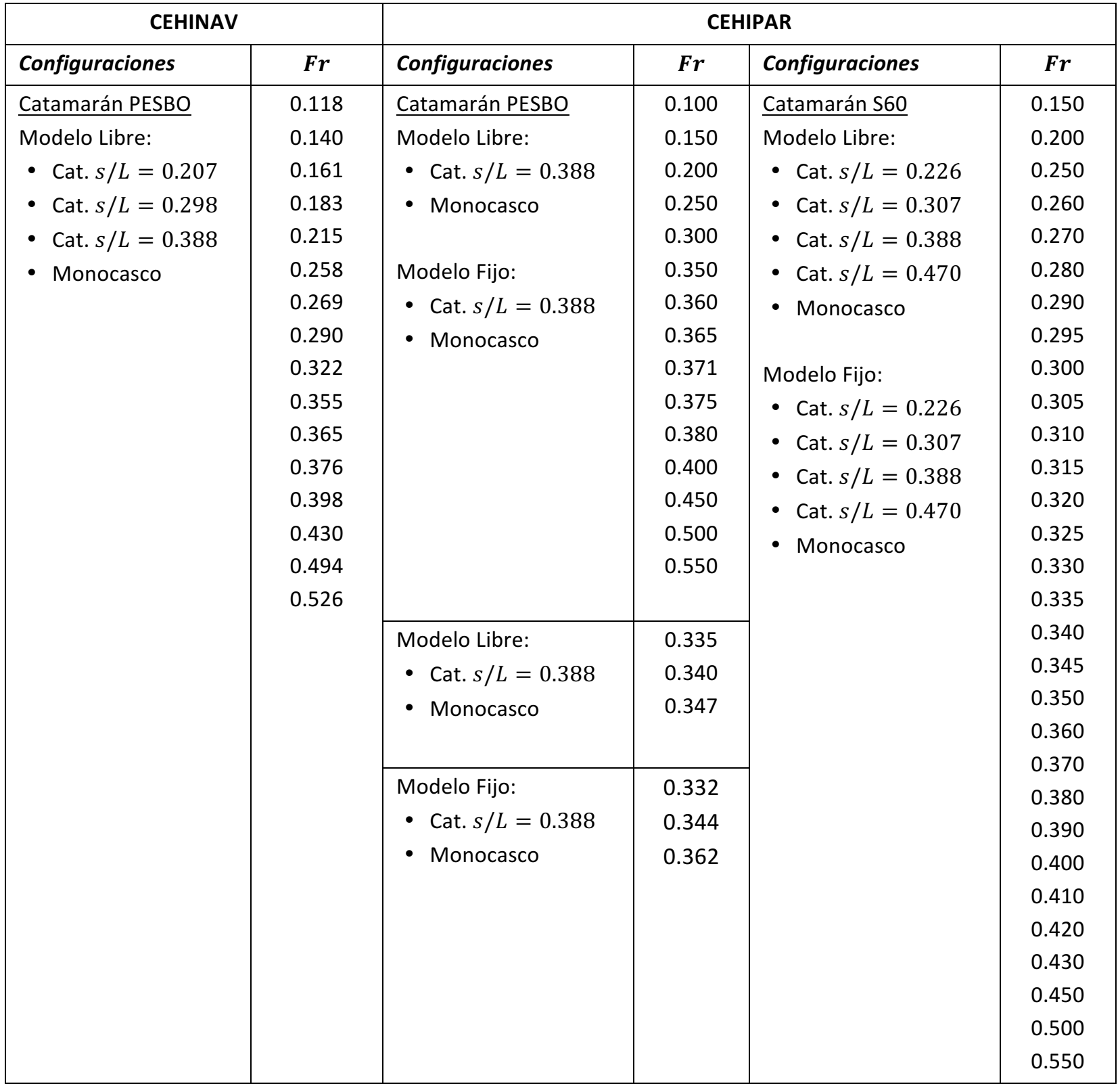

\subsubsection{Resistencia al avance del catamarán PESBO}

La Fig. 26 y Fig. 27 recogen las diferentes curvas de $R_{T}$ medidas, incluyendo el doble de la resistencia del monocasco como referencia para identificar las zonas de interferencia favorable. Los ensayos iniciales con el catamarán PESBO se realizaron en el CEHINAV únicamente con modelo libre, analizando las tres separaciones posibles con el bastidor inicial (Tabla 8). Posteriormente los ensayos en el CEHIPAR confirmaron los valores medidos en el CEHINAV, añadiendo las condiciones de modelo fijo. En esta segunda fase con condición de 
trimado fijo únicamente se analizó la separación mayor $(s / L=0.388)$ al ser la que muestra mayores efectos de interferencia.

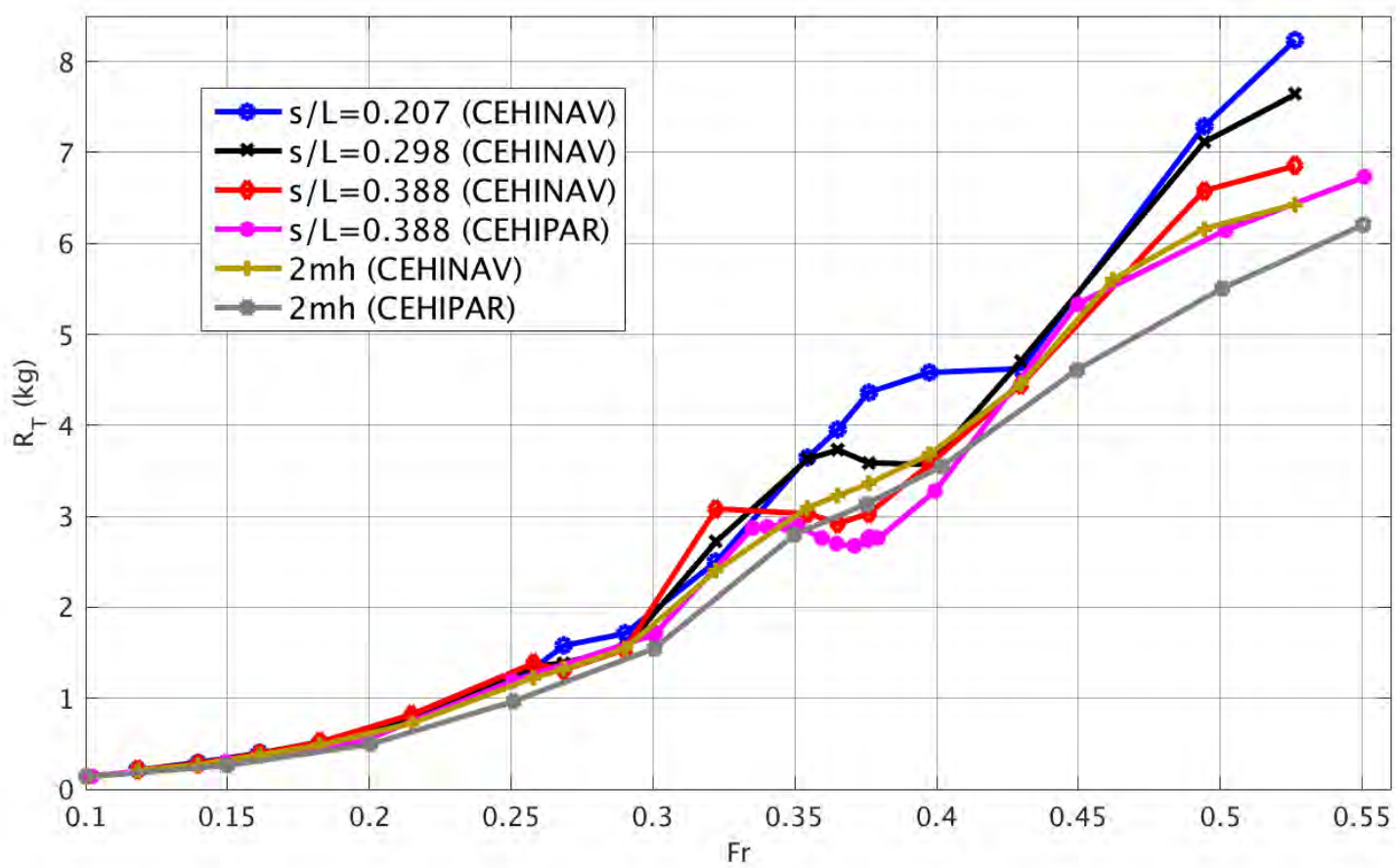

Fig. 26. Curvas de $R_{T}$ en función del Fr del cat. PESBO, modelo libre

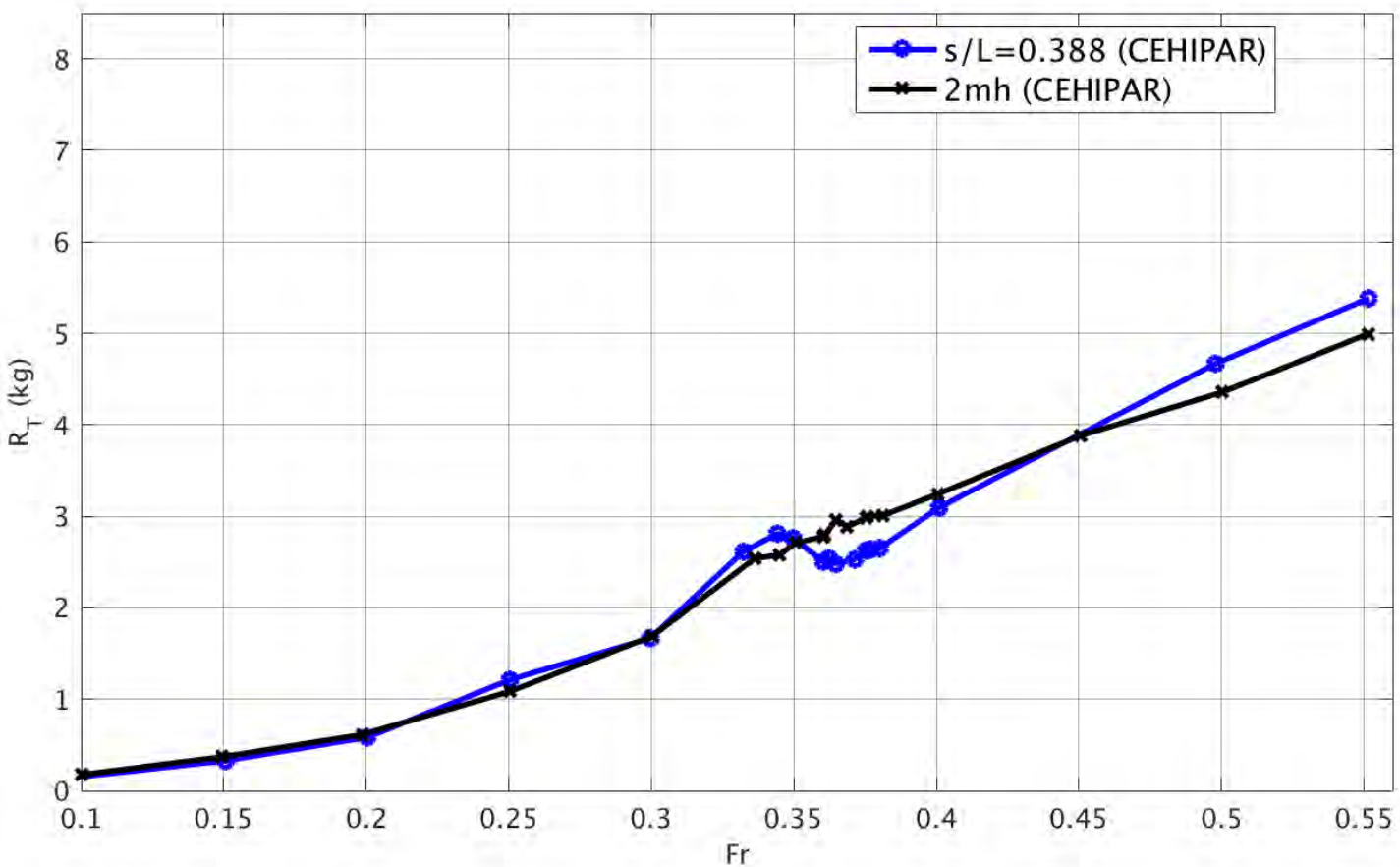

Fig. 27. Curvas de $R_{T}$ en función del Fr del cat. PESBO, modelo fijo ( $s / L=0.388$ ) 
La Fig. 28 amplia la zona de la joroba para visualizar mejor las diferencias significativas entre las condiciones de modelo fijo y libre, como se analiza en detalle en la Sec. 6.3. Estas incluyen también los valores calculados de $R_{W}$ empleados para determinar los factores de interferencia. Los marcadores indican los valores medidos, interpolándose con splines de acuerdo con la técnica desarrollada por Souto-Iglesias et al. (2012) para mostrar una curva continua. Se puede apreciar como las curvas de $R_{T}$ y $R_{W}$ muestran tendencias similares, lógico ya que la resistencia de fricción se calcula analíticamente por lo que crece de forma suave y continua.

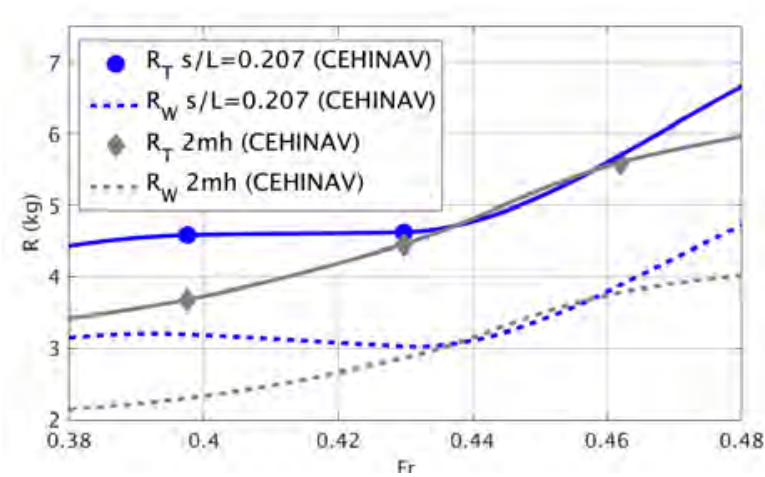

(a) $S / L=0.207$. Modelo libre

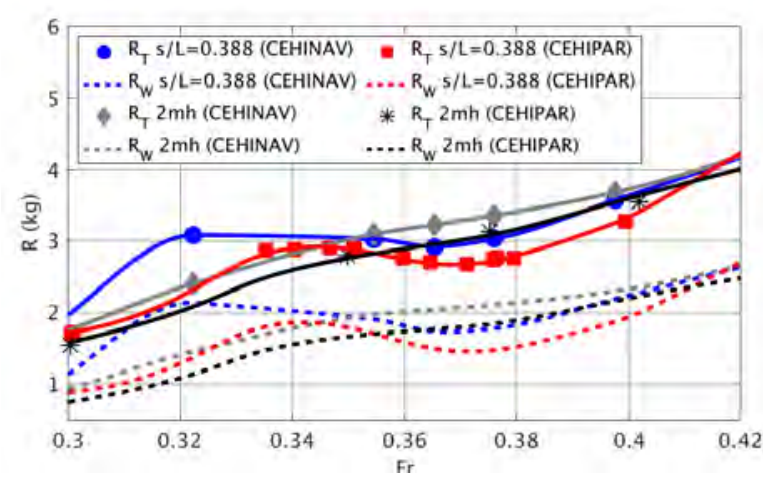

(c) $s / L=0.388$. Modelo libre

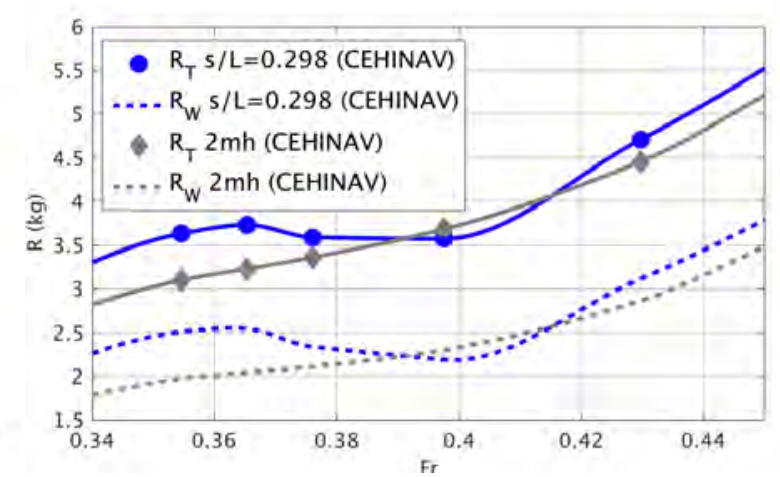

(b) $s / L=0.298$. Modelo libre

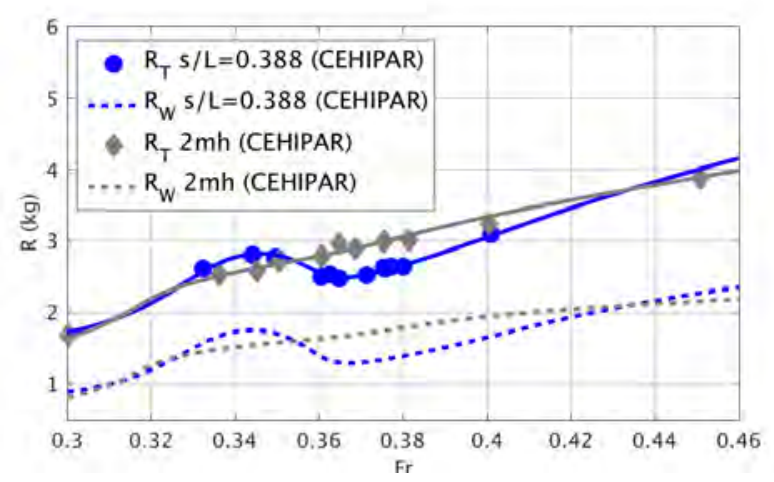

(d) $s / L=0.388$. Modelo fijo

Fig. 28. Detalle de las curvas de $R_{T}$ y $R_{W}$ del cat. PESBO

A bajas velocidades, la distancia entre cascos no influye en absoluto en la resistencia, comenzando a mostrar pequeñas diferencias con $F r$ entre 0.25 y 0.30 . La región de $F r$ entre 0.30 y 0.45 muestra las mayores diferencias entre el catamarán y el monocasco. A pesar que 
los últimos muestran una curva de resistencia monótona creciente, el catamarán muestra una zona donde la resistencia es constante e incluso decrece. Esta "joroba" es típica de este tipo de embarcaciones, como muestran Turner \& Taplin (1968) o Zaraphonitis et al. (2001). La localización exacta de esta zona depende de la separación entre cascos, comenzando antes cuanto mayor sea la separación. Por último se puede apreciar una disminución de la pendiente de las curvas de resistencia en el extremo superior de valores de $F r$. Esto puede indicar una segunda joroba, consistente con los resultados de Molland et al. (1996), aunque al no poder alcanzar velocidades más altas fue imposible comprobarlo.

\subsubsection{Resistencia al avance del catamarán S60}

El rango de $F r$ cubierto en este caso es mayor que con el catamarán PESBO, dado que a priori no estaba claro dónde ocurrirían las mayores interferencias. Las Fig. 29-Fig. 31 muestran las curvas de resistencia resultantes en todas las condiciones analizadas, al igual que con el PESBO anteriormente. En el caso de la separación menor $(s / L=0.226)$ con el modelo libre no se pudo alcanzar el rango superior de velocidades debido al embarque de agua en popa procedente del oleaje generado por el modelo.

Tanto los modelos libres como fijos muestran como patrón general una reducción de la resistencia a medida que se aumenta la separación, tendiendo hacia la condición de no interferencia. Como ocurre con el PESBO, se puede apreciar una ligera joroba entre $\mathrm{Fr}=0.30$ y $F r=0.40$ típica en catamaranes. La separación $s / L=0.307$ con modelo libre alcanza una situación de planeo como muestra la estabilización de la curva de resistencia para $F r=0.55$. En el caso de la separación $s / L=0.388$ las diferencias en resistencia entre el monocasco y el catamarán disminuyen para altos Fr. Esta tendencia es todavía más clara en el caso de la separación mayor $(s / L=0.470)$, como se discute más adelante en la sec. 6.3. 


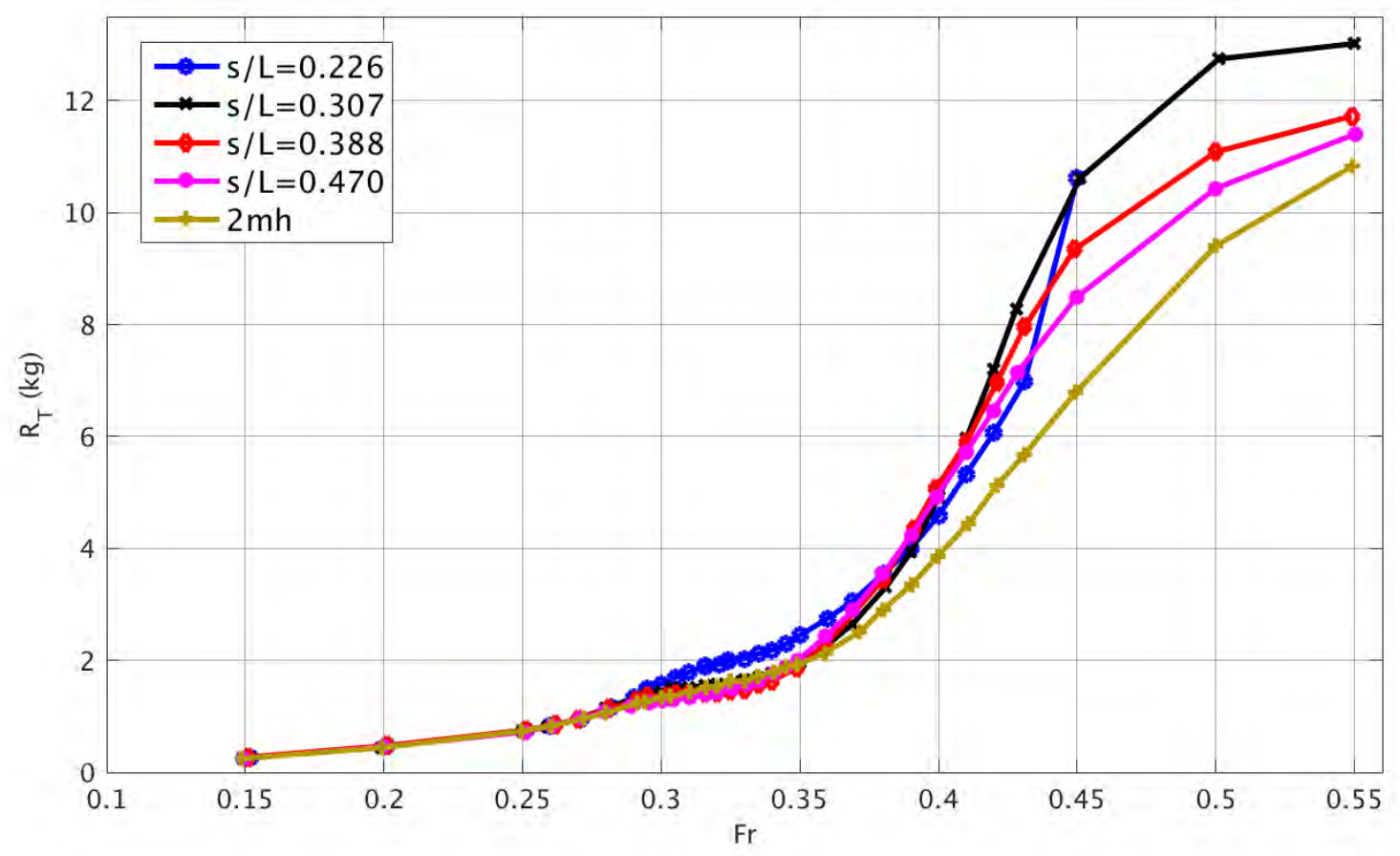

Fig. 29. Curvas de $R_{T}$ en función del Fr del cat. S60, modelo libre

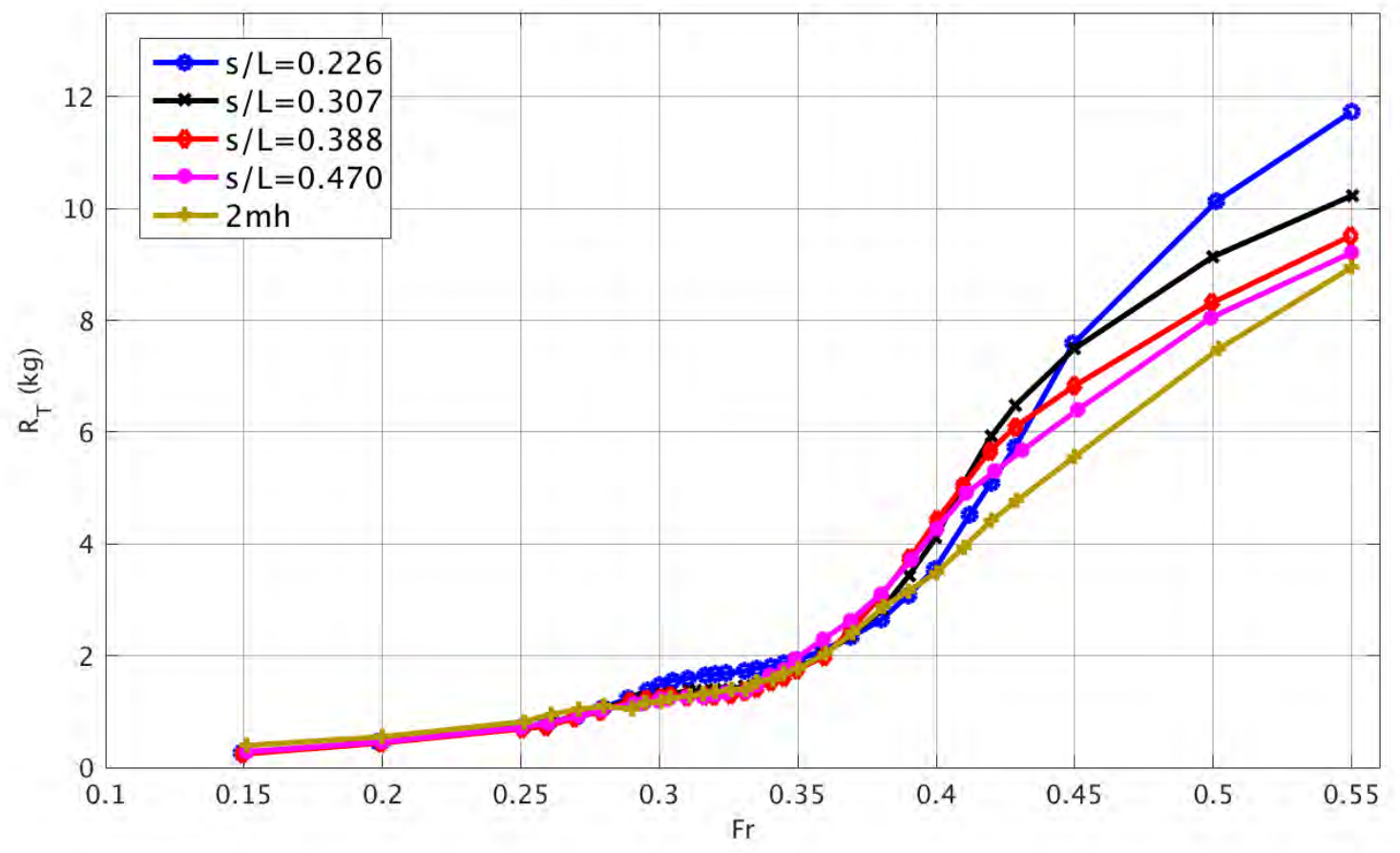

Fig. 30. Curvas de $R_{T}$ en función del Fr del cat. S60, modelo fijo 


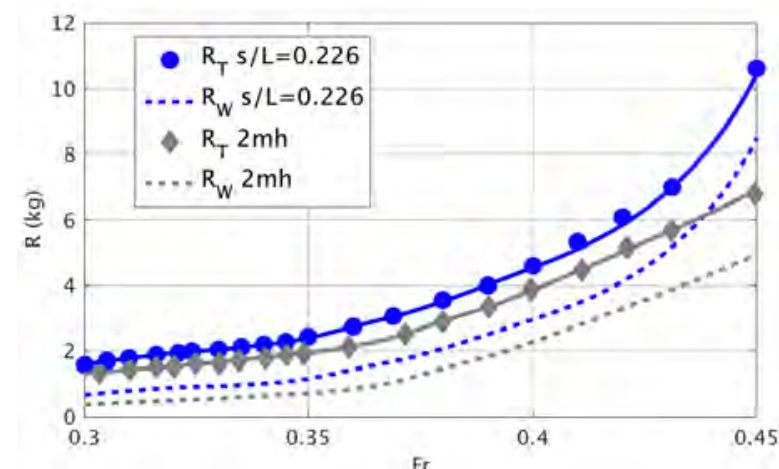

(a) $s / L=0.226$. Modelo libre

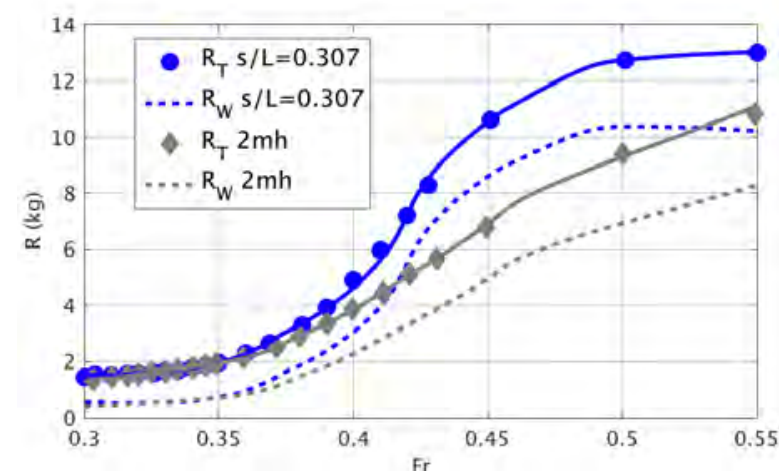

(c) $s / L=0.307$. Modelo libre

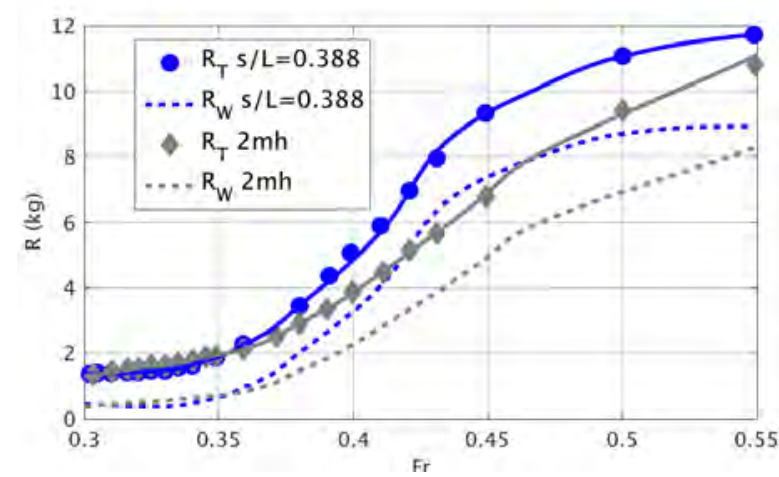

(e) $s / L=0.388$. Modelo libre

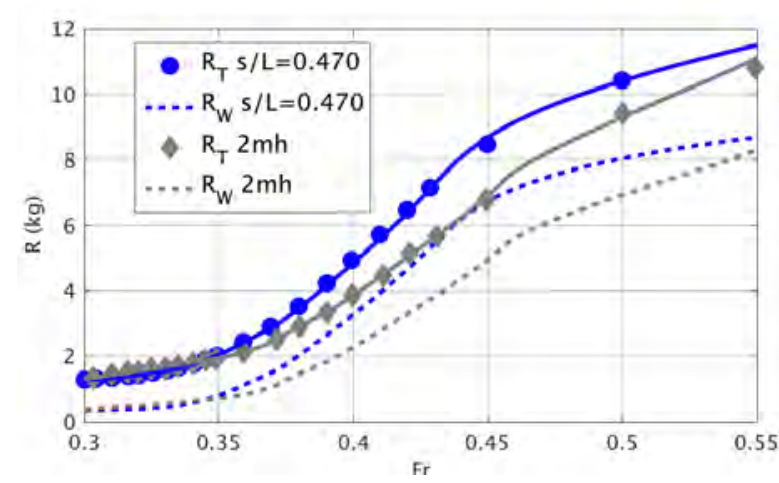

(g) $s / L=0.470$. Modelo libre

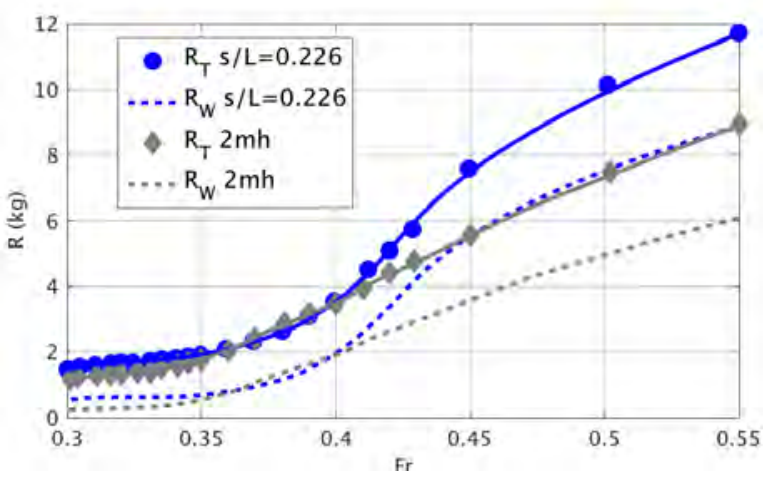

(b) $s / L=0.226$. Modelo fijo

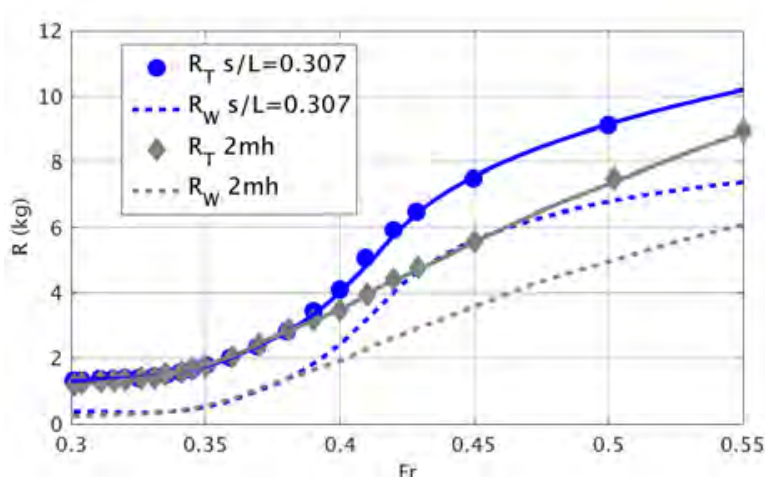

(d) $s / L=0.307$. Modelo fijo

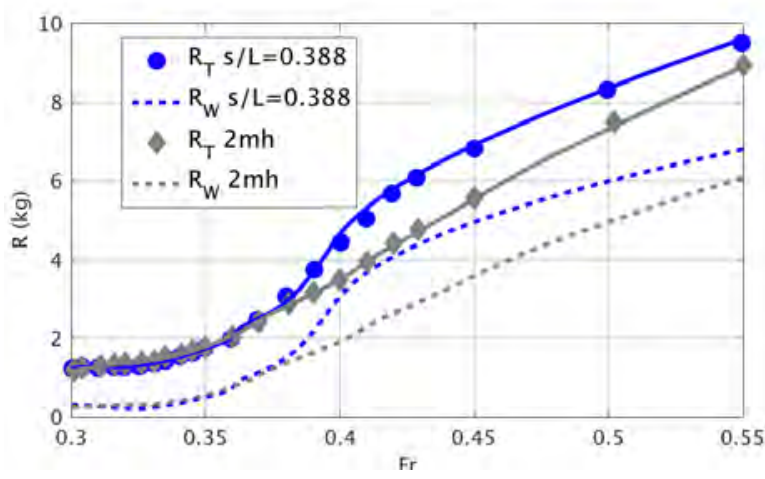

(f) $s / L=0.388$. Modelo fijo

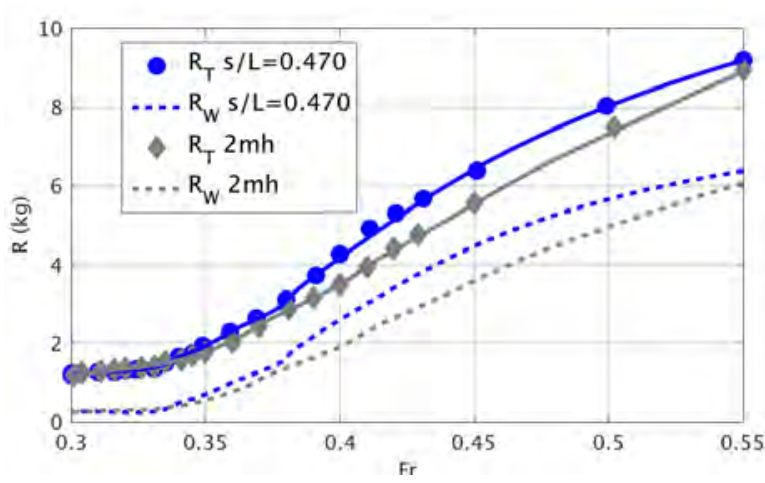

(h) $s / L=0.470$. Modelo fijo

Fig. 31. Detalle de las curvas de $R_{T}$ y $R_{W}$ del cat. $S 60$ 


\subsubsection{Factores de interferencia}

Los resultados anteriores muestran zonas donde la interferencia es favorable reduciendo la resistencia total frente al valor esperado a partir. El IF permite apreciar mejor la relevancia de dichos fenómenos de interferencia, calculándose siguiendo el procedimiento presentado en la sec. 6.2.1. Las Fig. 32-Fig. 35 muestran gráficamente los resultados obtenidos, recogiéndose en la Tabla 12 los rangos específicos de $F r$ donde la interferencia es favorable, así como los valores máximos y mínimos de este. En el caso de las mediciones de resistencia en el CEHIPAR, con $\mathrm{Fr}<0.27$ no tienen precisión suficiente para mostrar diferencias entre el monocasco y catamarán. Esto provoca oscilaciones irreales en los valores del $I F$, por lo que se no se han tenido en cuenta.

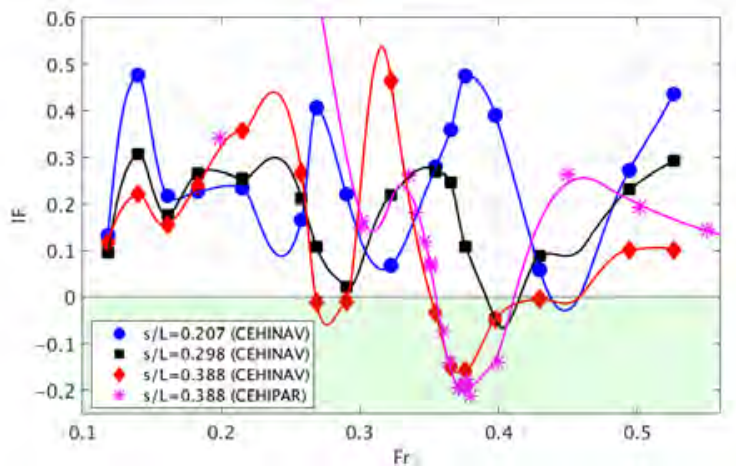

Fig. 32. IF del cat. PESBO con modelo libre

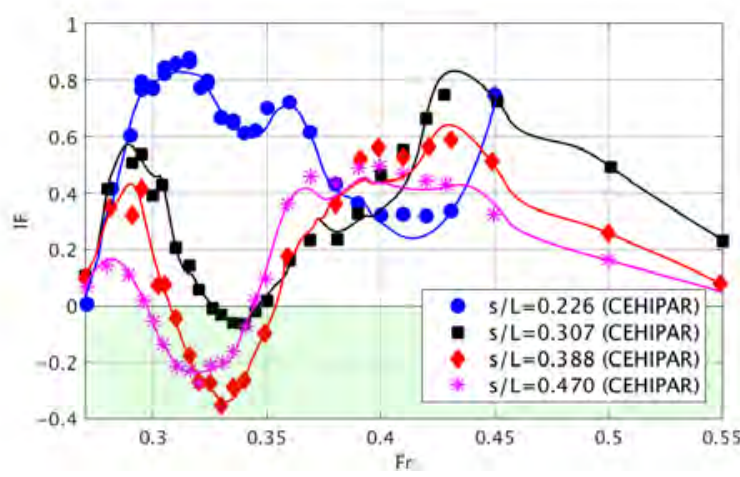

Fig. 34. IF del cat. 560 con modelo libre

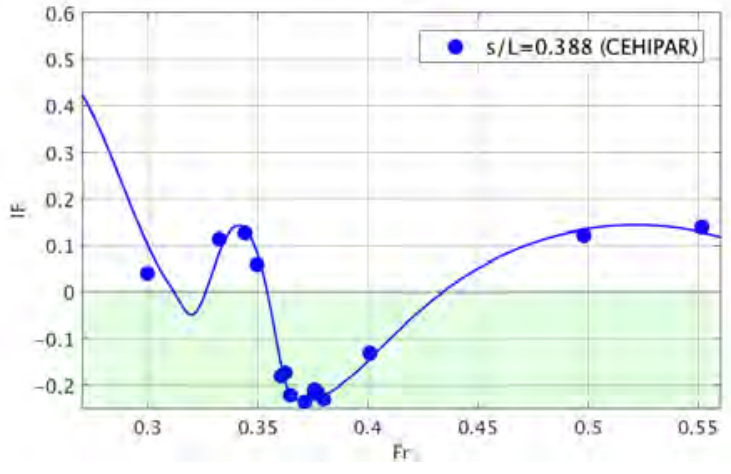

Fig. 33. IF del cat. PESBO con modelo fijo

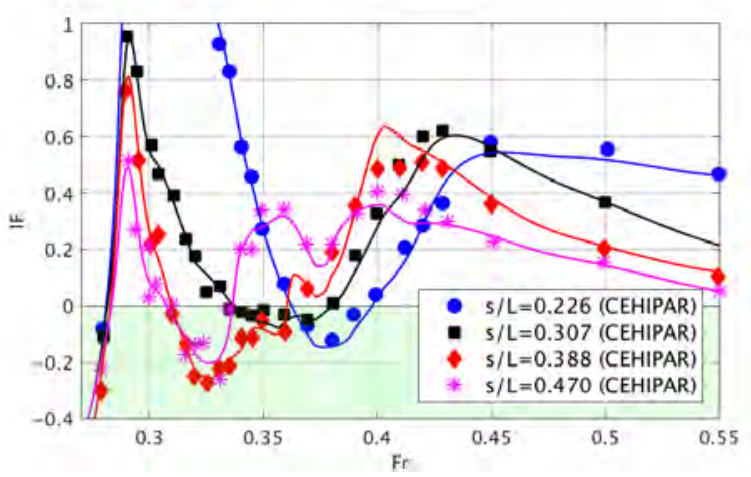

Fig. 35. IF del cat. $S 60$ con modelo fijo 
Tabla 12. Rangos de Fr significativos en las curvas de $\boldsymbol{R}_{T}$ y extremos del IF

\begin{tabular}{|c|c|c|c|c|c|c|}
\hline \multirow[t]{2}{*}{ Configuración } & \multicolumn{2}{|c|}{ Joroba de la $R_{T}$} & \multicolumn{2}{|c|}{ Interferencia favorable } & \multirow[t]{2}{*}{ Max IF } & \multirow[t]{2}{*}{ Min IF } \\
\hline & Fr inicial & Fr final & Fr inicial & Fr final & & \\
\hline \multicolumn{7}{|l|}{ Catamarán PESBO } \\
\hline$s / L=0.207$, modelo libre & 0.38 & 0.43 & - & - & 0.476 & 0.057 \\
\hline$s / L=0.298$, modelo libre & 0.36 & 0.40 & 0.39 & 0.41 & 0.292 & -0.046 \\
\hline$s / L=0.388$, modelo libre & 0.33 & 0.37 & 0.27 & 0.29 & 0.474 & -0.156 \\
\hline & & & 0.35 & 0.43 & & \\
\hline$s / L=0.388$, modelo fijo & 0.34 & 0.37 & 0.36 & 0.43 & 0.140 & -0.237 \\
\hline \multicolumn{7}{|l|}{ Catamarán S60 } \\
\hline$s / L=0.226$, modelo libre & 0.27 & 0.34 & - & - & 0.878 & 0.000 \\
\hline$s / L=0.226$, modelo fijo & 0.27 & 0.35 & 0.36 & 0.40 & 1.404 & -0.122 \\
\hline$s / L=0.307$, modelo libre & 0.28 & 0.33 & 0.33 & 0.35 & 0.749 & -0.065 \\
\hline$s / L=0.307$, modelo fijo & 0.27 & 0.33 & 0.34 & 0.38 & 0.955 & -0.051 \\
\hline$s / L=0.388$, modelo libre & 0.27 & 0.33 & 0.31 & 0.35 & 0.588 & -0.352 \\
\hline$s / L=0.388$, modelo fijo & 0.28 & 0.32 & 0.31 & 0.36 & 0.761 & -0.275 \\
\hline$s / L=0.470$, modelo libre & 0.27 & 0.33 & 0.30 & 0.34 & 0.493 & -0.273 \\
\hline$s / L=0.470$, modelo fijo & 0.27 & 0.33 & 0.31 & 0.33 & 0.516 & -0.262 \\
\hline
\end{tabular}

En el caso del PESBO, las tres separaciones ensayadas muestran una tendencia oscilatoria de amplitud creciente a medida que aumenta el Fr. En los extremos inferiores y superiores las tres curvas son similares $(F r<0.25$ y $F r>0.42)$, con valores muy bajos, uniformes y positivos en el rango inferior y apuntando a una nueva oscilación en el rango superior. Sin embargo, la zona intermedia muestra notables diferencias en función de la separación, tanto en la amplitud como en la frecuencia de las oscilaciones. Es de destacar cómo la separación menor $(s / L=0.207)$ está prácticamente en oposición de fase con la mayor $(s / L=0.388)$, mientras que la intermedia $(s / L=0.298)$ muestra un compromiso entre ambas. La amplitud de las oscilaciones responde al mismo patrón, con valores máximos para la separación mayor y menor. En relación a las posibles interferencias favorables (IF negativos), a priori cuanto 
mayor es la separación mayor es su extensión e intensidad como muestra la Tabla 12, alcanzándose los mínimos con menores $\mathrm{Fr}$.

Las curvas del S60 muestran un comportamiento semejante, si bien con menores oscilaciones. Al igual que con el PESBO, el modelo libre con la separación menor $(s / L=0.226)$ no muestra ninguna zona de interferencia favorable. Además, en el rango superior de $F r$ los efectos de interferencia tienden a disminuir en todas las separaciones y el $I F$ converge hacia un valor nulo, consistente con los resultados de Zaghi et al (2011). En la zona intermedia las curvas vuelven de nuevo a depender de la separación. Las interferencias más favorables se obtienen con $s / L=0.388$, al igual que con el catamarán PESBO en el cual no se pudo considerar la separación mayor utilizada con el S60 $(s / L=0.470)$. Esta última separación muestra a su vez una reducción de la intensidad en los efectos de interferencia. Tanto en el catamarán S60 como en el PESBO existen diferencias apreciables entre el modelo libre y el modelo fijo. Esto pone de manifiesto la relevancia de las variaciones dinámicas en la condición de navegación, como se analiza en detalle en la sec. 6.3.

Los resultados anteriores permiten extraer las siguientes conclusiones:

- El comportamiento oscilatorio proviene de las jorobas en la curva de resistencia del catamarán, las cuales se entrecruzan con la curva monótona creciente del monocasco. Dichas jorobas ocurren a menores $F r$ a medida que la separación es mayor, explicando como el $I F$ muestra la misma tendencia.

- El aumento de las oscilaciones con el $F r$ pone de manifiesto el mayor impacto de los efectos de interferencia debidos al oleaje.

- La interferencia favorable proviene mayoritariamente de la cancelación de los trenes de olas, lo que explica el mejor comportamiento con la separación mayor. Este hecho se analizará más en detalle en la sec. 6.3. En cualquier caso no hay que olvidar que esta tendencia se debe revertir al seguir aumentando la separación como muestran los ensayos con el catamarán S60. Asintóticamente el IF tiende a cero para separaciones infinitas debido a la difusión y radiación de la energía del oleaje, como muestran la respuesta más atenuada con $s / L=0.470$. 
- Las olas rompientes y spray, así como la aceleración del flujo al reducir la separación conllevan siempre una interferencia desfavorable, explicando cómo la separación menor nunca alcanza valores del IF negativos a pesar de mostrar amplitudes semejantes a la separación mayor.

- La geometría de los cascos y la separación están intrínsecamente ligadas a la hora de determinar los fenómenos de interferencia, al no existir una correlación clara de la intensidad de las oscilaciones con la separación. El análisis del campo de elevaciones usando técnicas numéricas aporta luz en este caso, como se discute en el capítulo 7.

- La precisión en la predicción de los valores de interferencia es crítico para la toma de decisiones. Las variaciones drásticas del $I F$ con el $F r$ pueden propiciar justo el efecto contrario al esperado a la hora de escoger una separación si no se tiene la información correcta.

- Las variaciones dinámicas de la condición de navegación inducen variaciones significativas en los valores de interferencia. Deben por tanto ser tenidas en cuenta directa o indirectamente.

\subsubsection{Comparativa con los resultados numéricos de Yeung et al. (2004)}

Uno de los objetivos de este trabajo es proporcionar una base experimental que sirva para validar modelos de predicción de los efectos de interferencia. Como primera aplicación se pueden comparar los resultados obtenidos con los presentados por Yeung et al. (2004), quienes precisamente calculan el IF de un catamarán S60 en un amplio rango de separaciones y velocidades. El modelo numérico propuesto se basa en la teoría lineal, asumiendo flujo potencial, modelo fijo y buque esbelto.

La Fig. 36 permite comparar los valores experimentales con las predicciones numéricas, donde se pueden apreciar diferencias significativas entre ambos. En general, los valores numéricos subestiman el $I F$, con máximos de 0.3 frente al 0.7 experimental. Aunque de acuerdo con Yeung et al. (2004) las interferencias más favorables ocurren con $s / L=0.226$ y $F r=0.33$, esto no sucede en los valores experimentales. Con $F r=0.33$ precisamente ocurren los efectos más desfavorables, desplazándose el mínimo de $I F$ a alrededor de $F r=0.38$. Para la 
separación $s / L=0.307$ se observa como los picos del $I F$ se obtienen experimentalmente a $F r=0.43$ en vez del $F r=0.38$ previsto numéricamente. Los valores mínimos del $I F$ muestran un desfase similar. Este patrón se repite en el resto de separaciones, en las que los picos y valles del $I F$ en los experimentos se retrasan frente a los obtenidos numéricamente. A velocidades altas, los valores calculados por Yeung et al. (2004) convergen con los resultados experimentales, especialmente con los del modelo fijo.

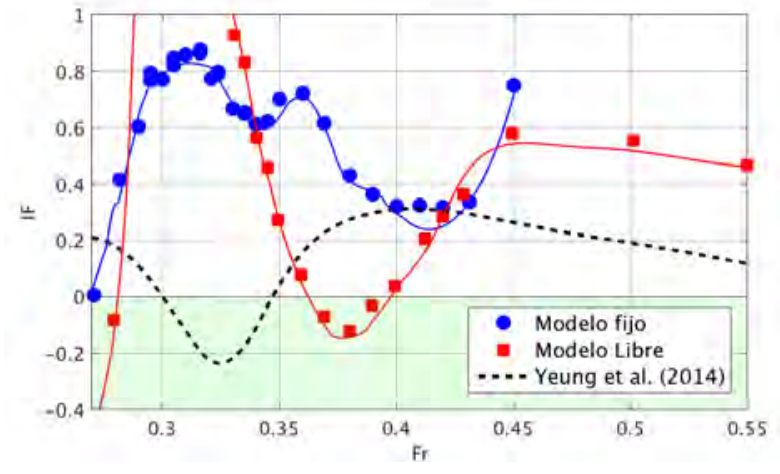

(a) $s / L=0.226$

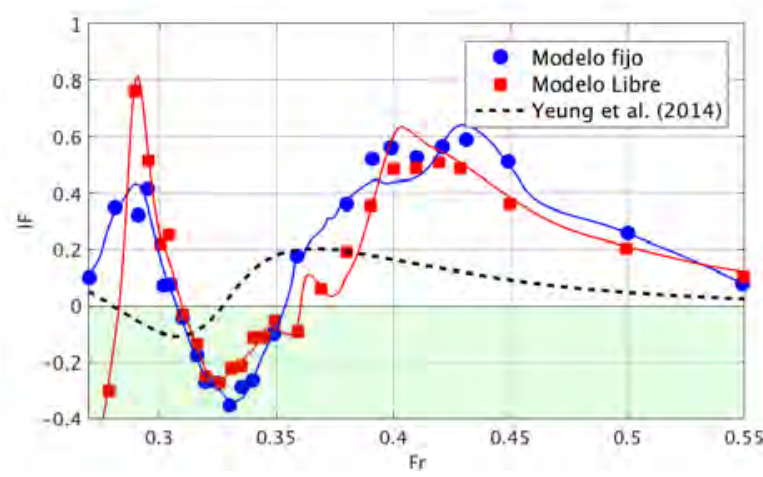

(c) $s / L=0.388$

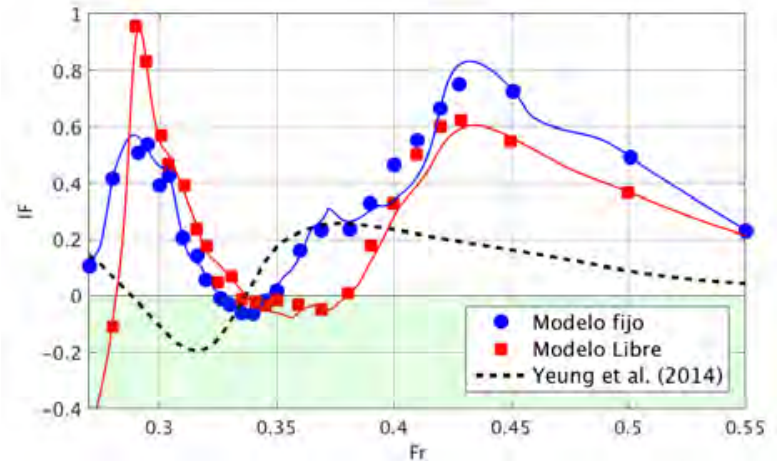

(b) $s / L=0.307$

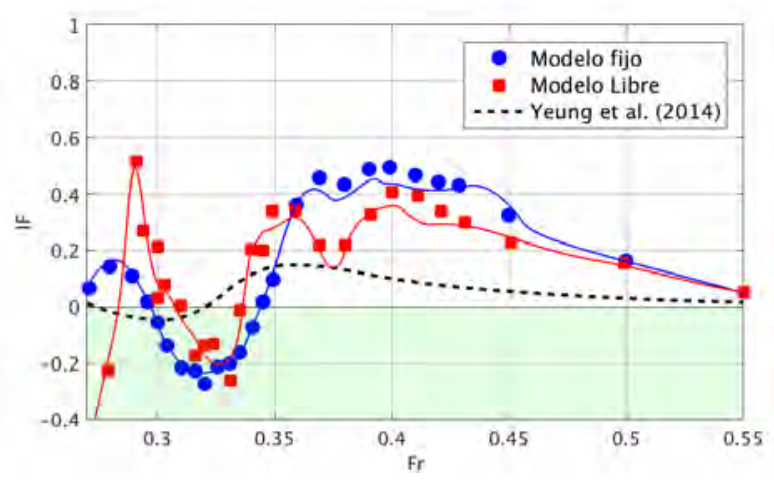

(d) $s / L=0.470$

Fig. 36. IF medidos del cat. S60 para las diferentes separaciones vs. valores calculados por Yeung et al. (2004)

Utilizando un diagrama de contorno bidimensional se puede apreciar mejor la interrelación de la velocidad y la separación con el $I F$, como se muestra en la Fig. 37. Esta recoge una reproducción de la figura original presentada por Yeung et al. (2004) en mi rango de estudio, así como las generadas a partir de los resultados experimentales para el modelo libre y fijo respectivamente utilizando una interpolación spline 2D. La escala de color utilizada para los 
resultados de Yeung et al. (2004) es diferente, debido a que su rango de $I F$ es significativamente más bajo que los experimentales.

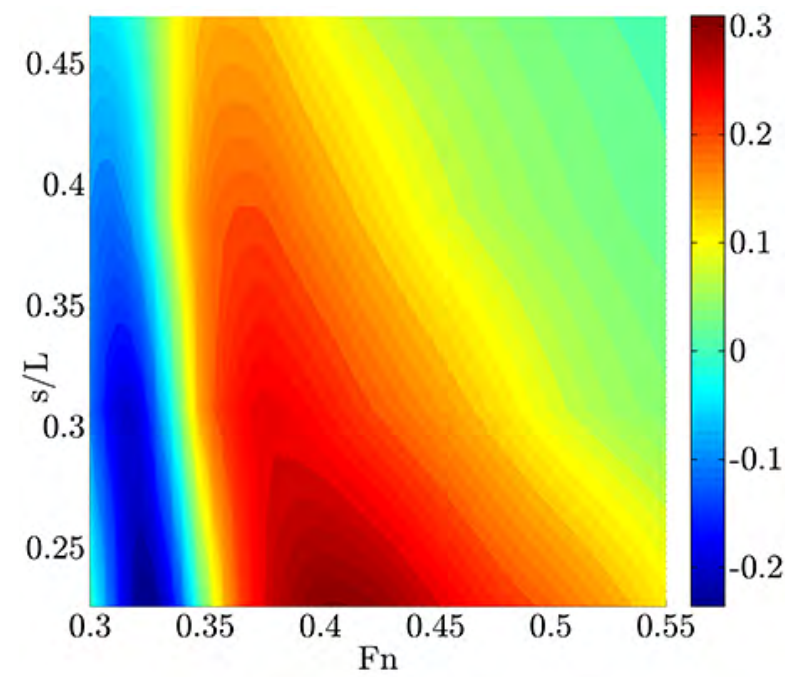

(a) Yeung et al. (2004)
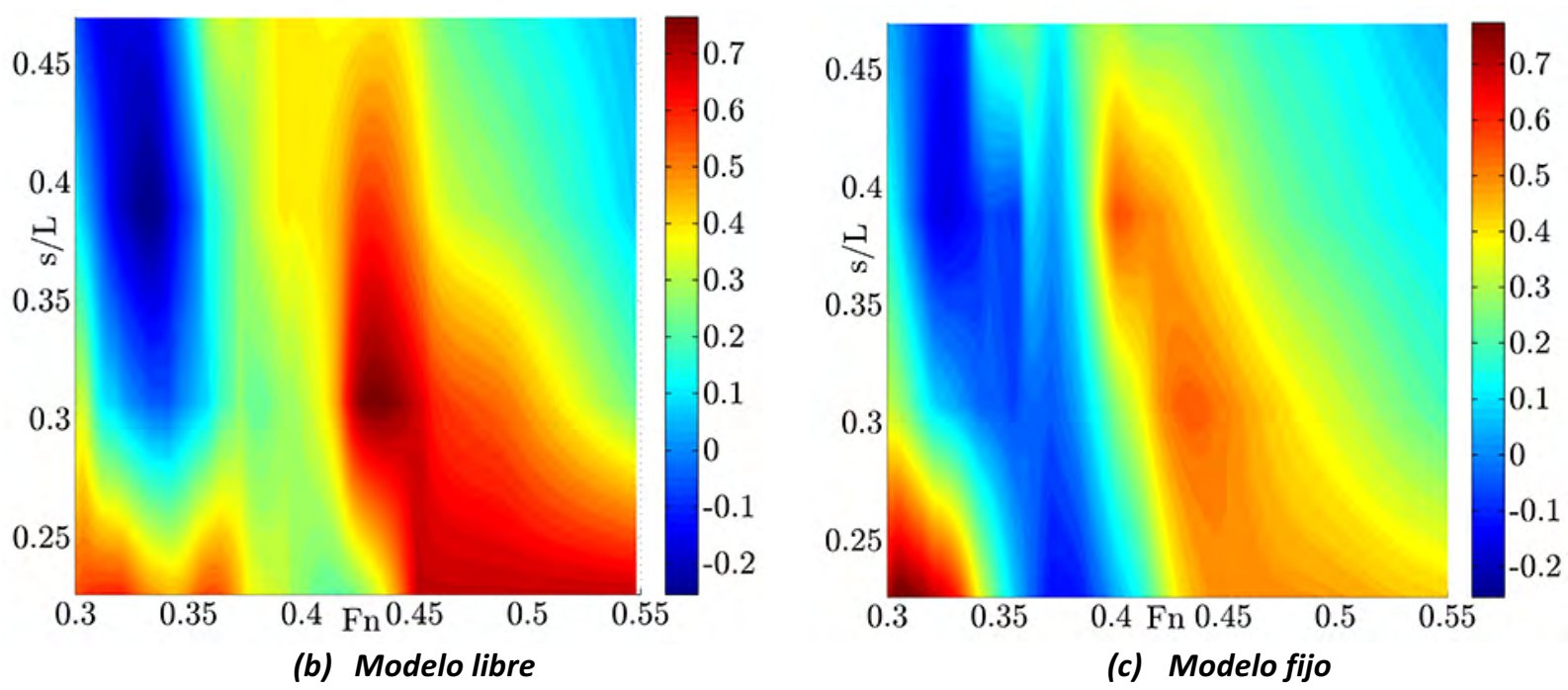

Fig. 37. Diagramas de contorno del IF como función del Fr y $s / L$

Las tendencias observadas en las curvas individuales (Fig. 36) son ahora más claras. Globalmente existen similitudes en los patrones de interferencia, si bien también se aprecian algunas diferencias. Comparando los datos experimentales del modelo libre (la cual es la configuración a esperar a escala real) con los de Yeung et al. (2004) muestra que la situación de máxima interferencia favorable sucede con $F r=0.33$ y con un $I F \operatorname{similar}(\approx-0.2)$, pero con una separación entre cascos superior (0.4 en vez de 0.266). Este $F r$ es similar al encontrado por Zaghi et al (2011) con un modelo más esbelto. Por otro lado, las interferencias 
desfavorables son mayores en los datos experimentales, con máximos del orden de 0.7 en vez del 0.3 calculado teóricamente. Es significativo que este máximo no ocurre para la separación más pequeña, como es el caso de Zaghi et al (2011). Además, los experimentos muestran una transición más suave que el modelo teórico entre las regiones de interferencia favorable y desfavorable. 


\subsection{Influencia del asiento y trimado dinámicos}

El buque al navegar altera el campo de velocidades a su alrededor respecto a la situación en reposo, y por ende la distribución de presiones sobre el casco. Como el empuje es la integral de presiones sobre el casco, su valor y su punto de aplicación serán diferentes. Consecuentemente, el equilibrio de fuerzas entre el peso y el empuje es diferente al desplazarse que en reposo. Este fenómeno es lo que se denomina asiento y trimado dinámico. A mayor velocidad de avance mayor es la importancia de dichas variaciones dinámicas, como muestra González Álvarez-Campana (1998).

El objetivo en este trabajo es cuantificar su impacto en la resistencia al avance para poder estimar el error cometido en las simulaciones que no lo tienen en cuenta. Los gráficos mostrados en el apartado anterior muestran cómo dicho error no es despreciable, apreciándose claras diferencias en las curvas de resistencia (Fig. 26-Fig. 31) y consecuentemente IF (Fig. 32-Fig. 37). La Fig. 38 resume las desviaciones encontradas, $\delta R_{T}$, adimensionalizadas con la resistencia del modelo libre (ec. (6.5))

$$
\delta R_{T}=\frac{R_{T, \text { libre }}-R_{T, f i j o}}{R_{T, \text { libre }}}
$$

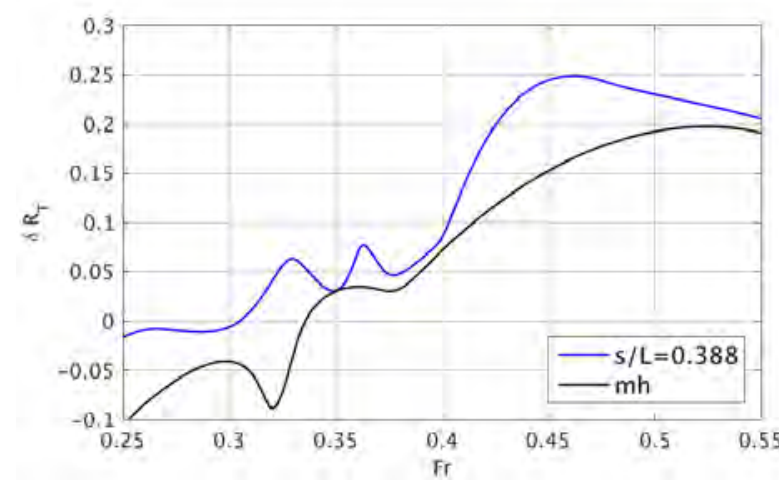

(a) Catamarán PESBO

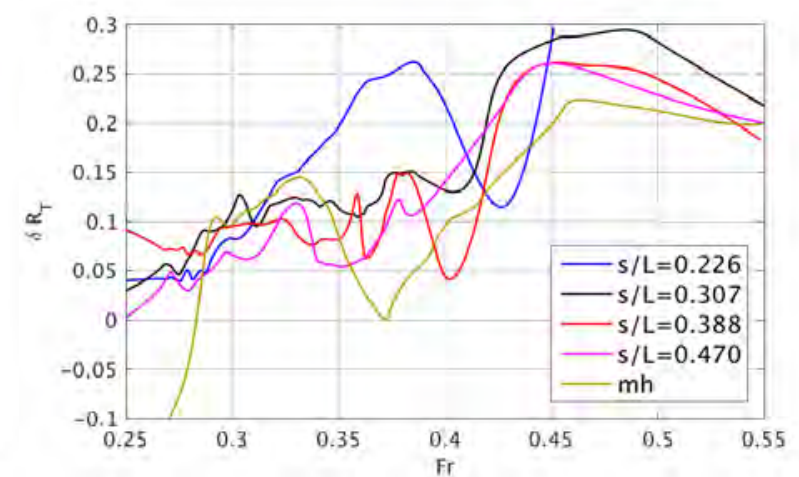

(b) Catamarán 560

Fig. 38. Curvas de $\delta R_{T}$ de las configuraciones ensayadas

En general, se puede apreciar cómo la resistencia del modelo libre es mayor que el modelo fijo en todos los casos $\left(\delta R_{T}>0\right)$, pudiéndose observar mejor a altas velocidades. Este resultado 
es consistente con las conclusiones extraídas por Kim \& Jenkins (1981) y Fernández-Gutiérrez (2010) para un monocasco Serie 60 o Moraes et al. (2004) para un Wigley. Las diferencias encontradas son mayores en el catamarán que en el monocasco, aumentando con la separación entre cascos. Su relevancia reside en el hecho de que el modelo libre es precisamente el que reproduce el comportamiento real.

Centrándonos en los factores de interferencia, las curvas de $I F$ del PESBO muestran una tendencia similar, con ciertas diferencias en el rango $0.30 \leq F r \leq 0.34$. En el caso del S60, la respuesta del modelo libre presenta mayores diferencias con el modelo fijo, mostrando el primero generalmente un comportamiento más desfavorable (Fig. 36). Las desviaciones más significativas en el $I F$ entre el modelo libre y fijo se aprecian con la separación menor $(s / L=$ 0.226). En el caso de la separación $s / L=0.307$, estas se concentran en el rango $0.35 \leq F r \leq$ 0.40. Las diferencias con las separaciones mayores son menos acusadas. Los diagramas de contorno mostrados en la Fig. 37 permiten observar la interrelación entre la velocidad y separación a la hora de evaluar el efecto del asiento y trimado dinámicos. Éstos muestran como la transición entre las zonas de interferencia favorable y desfavorable es más rápida con el modelo fijo. Además, las separaciones menores con modelo fijo muestran zonas de interferencia favorable leve, contrariamente al modelo libre donde siempre es desfavorable. Las regiones de interferencia favorable son semejantes en el modelo libre y fijo, aunque como se indicó anteriormente son más intensos en el caso del modelo libre. En resumen, el modelo libre tiende a amplificar los fenómenos de interferencia, tanto favorables como desfavorables.

La comparativa de la diferencia entre monocasco y multicasco con y sin trimado fijo permite evaluar la componente de interferencia debida a la variación en las condiciones dinámicas de navegación, $I F_{S \& T}$. La Fig. 39 muestra los resultados obtenidos, donde el $I F_{S \& T}$ se define de acuerdo a la ecuación (6.6) utilizando la resistencia por formación de olas como referencia al igual que el $I F$, así como el hecho de que los dos cascos son idénticos 


$$
\begin{gathered}
I F_{S \& T}=\frac{\left(R_{W, M H \text { libre }}-2 R_{W, D \text { libre }}\right)-\left(R_{W, M H \text { fijo }}-2 R_{W, D \text { fijo }}\right)}{2 R_{W, D \text { libre }}} \\
=I F_{\text {libre }}-I F_{\text {fijo }} \frac{R_{W, D \text { fijo }}}{R_{W, D \text { libre }}}
\end{gathered}
$$

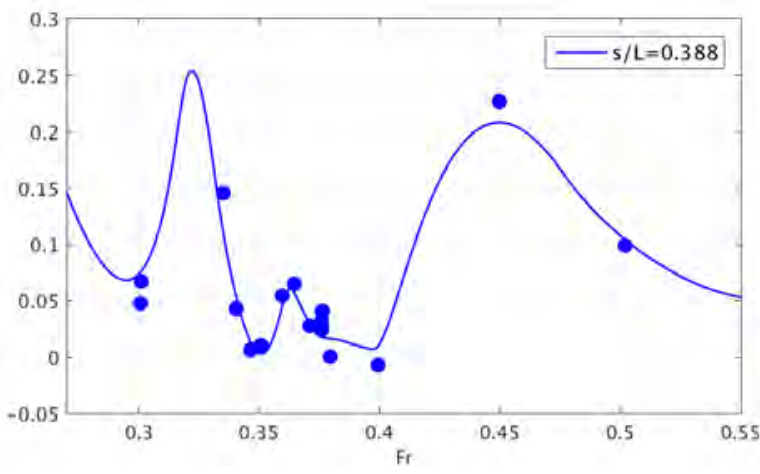

(a) Catamarán PESBO

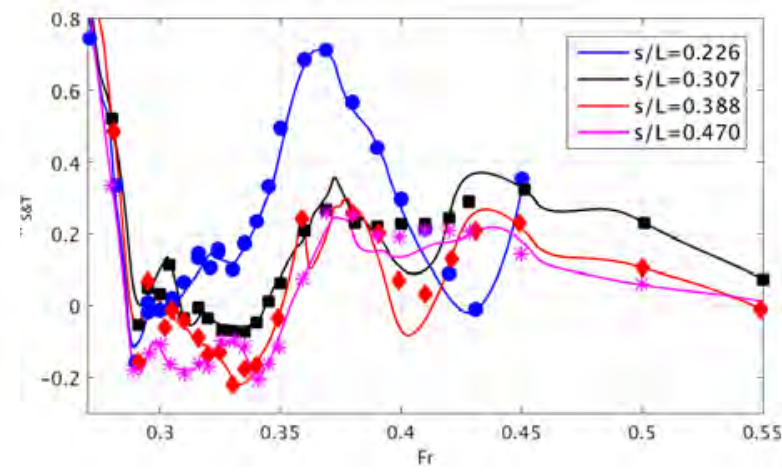

(b) Catamarán 560

Fig. 39. IF $F_{S \& T}$ de las configuraciones ensayadas

Se puede apreciar cómo las curvas del $I F_{S \& T}$ muestran oscilaciones del mismo orden que el $I F$. Dado que los $I F$ con modelo libre y fijo tienen órdenes de magnitud semejante, se concluye por tanto que al fijar el modelo se introduce un cierto desfase en los fenómenos de interferencia. Este hecho pone de manifiesto una vez más la sensibilidad del $I F$ con el $F r$ debido a su carácter oscilatorio. A pesar de predecir la amplitud correctamente, pequeñas desviaciones del $\mathrm{Fr}$ al que ocurren pueden derivar en una interferencia entre cascos totalmente diferente. De acuerdo a las curvas de $I F_{S \& T}$ del S60, dicho desfase depende débilmente de la separación al mostrar las diferentes separaciones ensayadas un comportamiento similar. La separación menor es la que se aleja en mayor medida del patrón general. Esto indica cómo la fijación del modelo tiene un impacto mayor en los mecanismos de interferencia que surgen al aproximar los cascos (olas rompientes, spray, aceleración del flujo, etc.).

Para poder correlacionar las diferencias encontradas con el asiento y trimado dinámicos, estos se midieron experimentalmente en los ensayos de resistencia. La Fig. 40 y Fig. 41 muestran los 
resultados obtenidos para cada geometría, adimensionalizados con la longitud típica ${ }^{46} V^{2} / g$ como se muestran en las ecuaciones (6.7) y (6.8) (Kim \& Jenkins (1981)).

$$
\begin{gathered}
\text { Trimado }=-\left(\Delta z_{\text {proa }}-\Delta z_{\text {popa }}\right) \frac{2 g}{V^{2}} \\
\text { Asiento }=-\left(\Delta z_{\text {proa }}+\Delta z_{\text {popa }}\right) \frac{g}{V^{2}}
\end{gathered}
$$

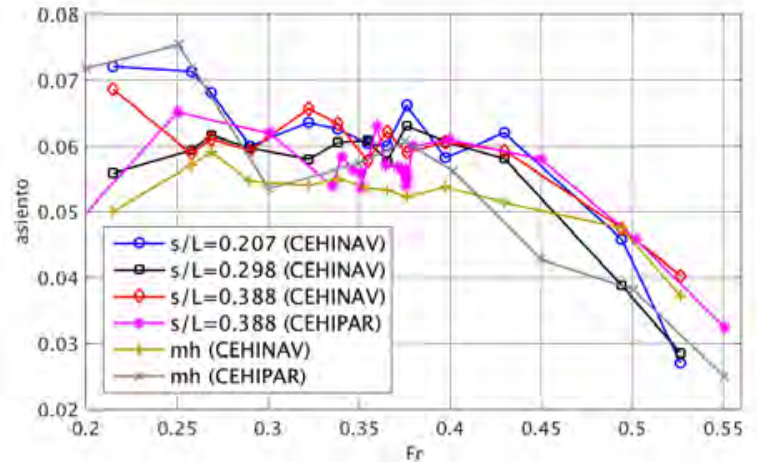

(a) Catamarán PESBO

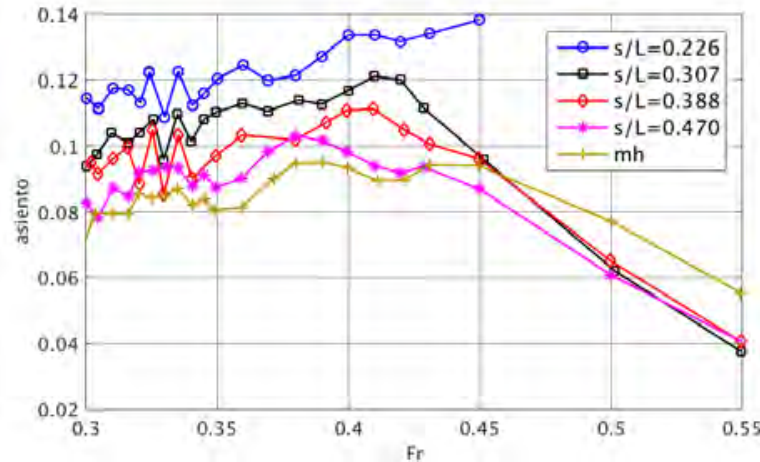

(b) Catamarán 560

Fig. 40. Asiento dinámico función del Fr

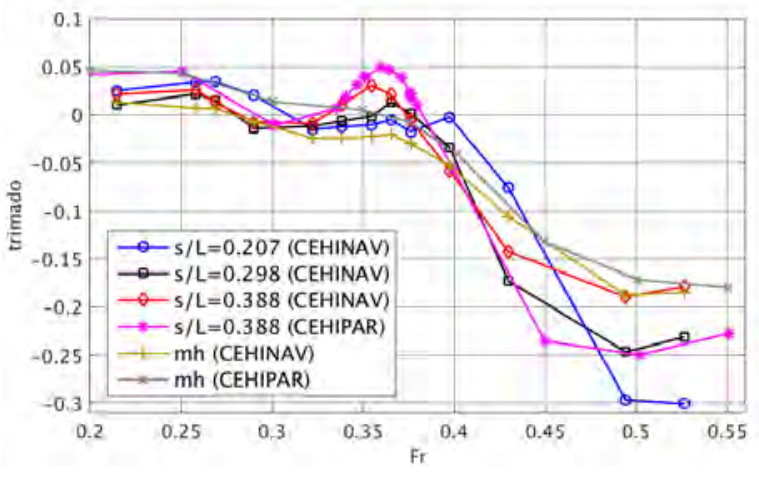

(a) Catamarán PESBO

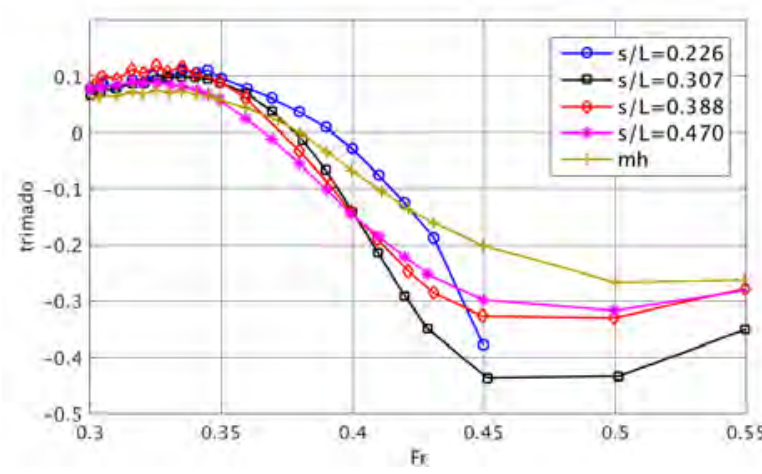

(b) Catamarán S60

Fig. 41. Trimado dinámico función del Fr

En el caso del PESBO, aunque el asiento es significativo ( $\sim 10 \%$ del calado) su comportamiento es muy similar en el monocasco y el catamarán. Respecto al trimado, sus valores absolutos

\footnotetext{
${ }^{46}$ Longitud de ola característica sobre $2 \pi$
} 
son bajos (valores adimensionales entre -0.06 y +0.06 lo que equivale $a \pm 0.3^{\circ}$ ) con pequeñas variaciones. Se puede apreciar una reducción de su valor alrededor de $F r=0.37$, lo que podría explicar la interferencia favorable encontrada a esta velocidad con el modelo libre. Atendiendo a la separación entre cascos, no se aprecia que tenga un impacto significativo en el asiento y trimado dinámicos, especialmente en la zona intermedia $(0.3<F r<0.4)$ donde los efectos de interferencia son más acusados. Esto explica el menor efecto en la resistencia de interferencia respecto al S60. Sin embargo, las diferencias crecen a medida que aumenta el $F r$, mostrando cómo las variaciones dinámicas deben ser tenidas en cuenta en multicascos de alta velocidad.

El catamarán S60 muestra valores de asiento pequeños, del mismo orden que el PESBO, lógico dado que ambas no son embarcaciones de planeo. Sin embargo, el trimado es significativamente mayor en el caso del S60. Al comparar el asiento y trimado dinámicos del monocasco y catamarán $\mathrm{S} 60$ se pueden observar diferencias significativas en el rango $0.30 \leq$ $F r \leq 0.42$. Para la separación menor $(s / L=0.226)$ el asiento del catamarán es un $50 \%$ mayor. En el caso del trimado, en todos los casos las diferencias son más patentes para valores altos del $F r$. En el rango $0.38 \leq F r \leq 0.45$ se puede observar un incremento significativo de éste que debe ser causado por las características geométricas del Serie 60 . Estos hechos sin duda motivan los IF mayores del S60 como se indicó anteriormente. No obstante, las diferencias entre el $I F$ del modelo fijo y libre muestran un comportamiento oscilatorio que difiere de las diferencias en el asiento, las cuales siguen un patrón monótono.

En resumen, no se puede afirmar por tanto que existe una correlación directa entre el $I F$ y el asiento y trimado dinámicos, si bien sí una importante interrelación. Dichos efectos dinámicos están condicionados por las formas del casco. La visualización del campo de velocidades y presiones permite identificar las zonas críticas y sugerir posibles modificaciones a las formas para ajustar la respuesta dinámica del buque. Los CFD juegan un papel muy importante en este aspecto, pudiendo explotar todo su potencial como herramientas comparativas (capítulo 7). No obstante, el análisis y optimización detallada de formas del buque atendiendo a consideraciones dinámicas queda fuera del alcance de este trabajo. 


\subsection{Estudio experimental del tren de olas en la zona entre cascos}

\subsubsection{Ensayos}

El análisis de la resistencia al avance en la sección anterior muestra cómo los efectos de cancelación del oleaje juegan un papel central en la posibilidad de alcanzar interferencias favorables (sec. 6.2.5), tal y como sugiere Chen et al. (2003b). El tren de olas generado por cada casco varía en función del $F r$, no sólo en amplitud sino en la posición de los máximos y mínimos. Pueden por tanto variar de una situación en las que las componentes de cada casco se superponen a otra en la que se cancelan entre sí, como representan la Fig. 42 y Fig. 43 . La primera corresponde a la condición de máximo oleaje resultante, mientras que la segunda representa la situación más favorable minimizando la altura del oleaje y por lo tanto el $I F$.

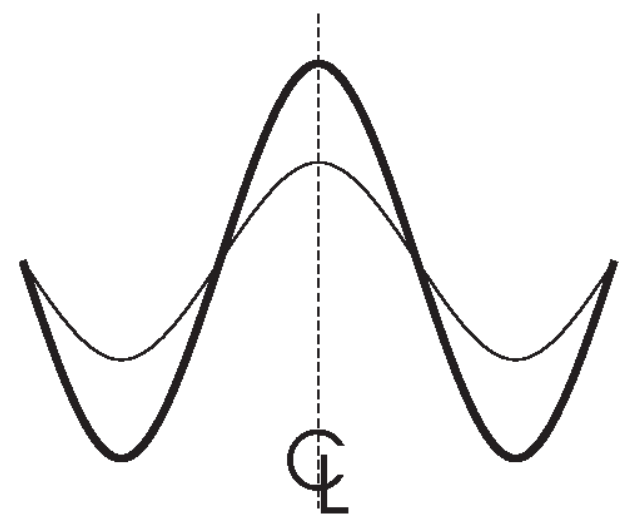

Fig. 42. Situación de interacción máxima donde los trenes de olas de cada casco se solapan, duplicando la altura del oleaje resultante

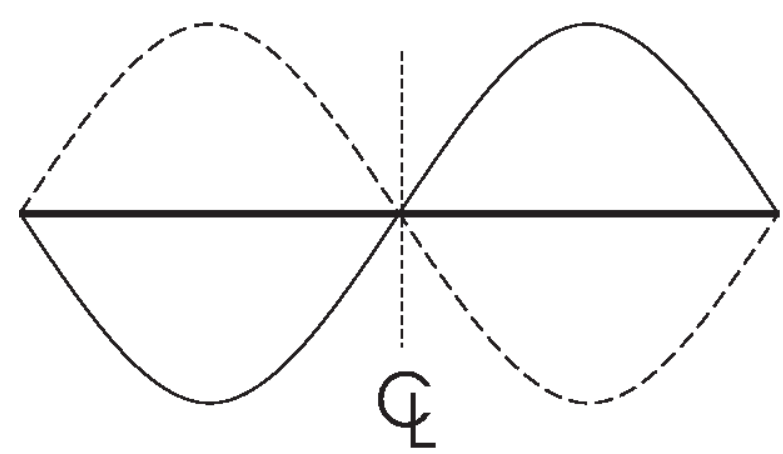

Fig. 43. Situación de interacción mínima donde los trenes de olas de cada casco se cancelan entre sí, resultando en una elevación nula

Como se presentó en el apartado 4.3.1, la medición del campo completo de elevaciones es muy costosa y propensa a errores. Una alternativa mucho más sencilla y fiable es la toma de múltiples cortes de olas longitudinales. Con el fin de comprender mejor los fenómenos de cancelación, otra parte importante de este trabajo ha consistido en la caracterización del campo de elevaciones. La zona entre cascos es la más interesante de cara a la interferencia, y sin embargo se detectó en ella una carencia de datos experimentales en la literatura. Por ello se construyó un soporte especial que permitiera tomar cortes de olas en esta zona, como se presentó en el apartado 5.1. 
La Tabla 13 resume los ensayos de cortes de olas realizados, los cuales se llevaron a cabo únicamente con el catamarán PESBO en el CEHINAV. Estos cubren tanto las diferentes separaciones en la configuración catamarán como monocasco. Los cortes de olas exteriores del catamarán y del monocasco se han medido a la misma distancia del costado del casco que los tomados en la zona entre cascos. Además del plano de crujía se han incluido tres posiciones intermedias para poder estudiar la variación de las elevaciones en sentido transversal. Respecto a las velocidades, se han escogido cuatro $\mathrm{Fr}$ representativos de las diferentes regiones observadas en las curvas de resistencia.

La Fig. 44 muestra gráficamente el significado de las diferentes variables empleadas para caracterizar los cortes de olas. La distancia entre el corte de olas al costado del casco, $\Delta y$, se adimensionaliza utilizando distancia entre cascos, $s_{D}$, medida entre costados. $\Delta y / s_{D}=1 / 2$ corresponde por tanto al corte de olas en el plano de crujía. Alternativamente, $\Delta y$ también se puede adimensionalizar con la manga del monocasco, $B_{D}$, para indicar la proximidad del corte de olas al casco independientemente de la separación. $\Delta y$ no incluye direccionalidad, por lo que puede indicar tanto cortes interiores como exteriores. Para distinguir entre ambos se utiliza la posición transversal respecto al plano de crujía, $y$, la cual se adimensionaliza con la separación $s$ medida entre crujías. Dado que el origen del sistema de coordenadas es diferente en el catamarán y en el monocasco (Fig. 44), los valores de $y / s$ difieren entre ambos aunque los cortes se tomen a la misma $\Delta y$. Por último, en los gráficos de cortes de olas presentados la posición longitudinal, $x$, y las elevaciones, $\eta$, se adimensionalizan con la eslora del modelo. El origen se toma en la perpendicular de proa, correspondiendo $x / L=1$ a la perpendicular de popa. 
Tabla 13. Ensayos de cortes de olas con el cat. PESBO

\begin{tabular}{|c|c|c|c|c|c|c|}
\hline \multirow[t]{3}{*}{ Configuración } & \multirow[t]{3}{*}{$F r$} & \multirow[t]{3}{*}{$\Delta y / s_{D}$} & \multirow[t]{3}{*}{$\Delta y / B_{D}$} & \multicolumn{3}{|c|}{$y / s$} \\
\hline & & & & \multirow{2}{*}{$\begin{array}{c}\text { cortes } \\
\text { interiores }\end{array}$} & \multicolumn{2}{|c|}{ cortes exteriores } \\
\hline & & & & & catamarán & monocasco \\
\hline \multirow{3}{*}{$\begin{array}{l}\text { Modelo Libre: } \\
\text { - Cat. } s / L=0.207 \\
\text { - Monocasco }\end{array}$} & \multirow{3}{*}{$\begin{array}{l}0.322 \\
0.375 \\
0.398 \\
0.430\end{array}$} & $1 / 2$ & 0.462 & 0.0 & 1.000 & 0.500 \\
\hline & & $1 / 3$ & 0.308 & 0.080 & 0.920 & 0.420 \\
\hline & & $1 / 4$ & 0.231 & 0.120 & 0.880 & 0.380 \\
\hline \multirow{4}{*}{$\begin{array}{l}\text { Modelo Libre: } \\
\text { - Cat. } s / L=0.298 \\
\text { - Monocasco }\end{array}$} & \multirow{4}{*}{$\begin{array}{l}0.322 \\
0.375 \\
0.398 \\
0.430\end{array}$} & $1 / 2$ & 0.882 & 0.0 & 1.000 & 0.500 \\
\hline & & $1 / 3$ & 0.588 & 0.106 & 0.894 & 0.394 \\
\hline & & $1 / 4$ & 0.441 & 0.160 & 0.840 & 0.340 \\
\hline & & $1 / 6$ & 0.294 & 0.213 & 0.787 & 0.287 \\
\hline \multirow{4}{*}{$\begin{array}{l}\text { Modelo Libre: } \\
\text { - Cat. } s / L=0.388 \\
\text { - Monocasco }\end{array}$} & \multirow{4}{*}{$\begin{array}{l}0.322 \\
0.375 \\
0.398 \\
0.430\end{array}$} & $1 / 2$ & 1.303 & 0.0 & 1.000 & 0.500 \\
\hline & & $1 / 3$ & 0.868 & 0.120 & 0.880 & 0.380 \\
\hline & & $1 / 4$ & 0.651 & 0.181 & 0.819 & 0.319 \\
\hline & & $1 / 6$ & 0.434 & 0.241 & 0.759 & 0.259 \\
\hline
\end{tabular}

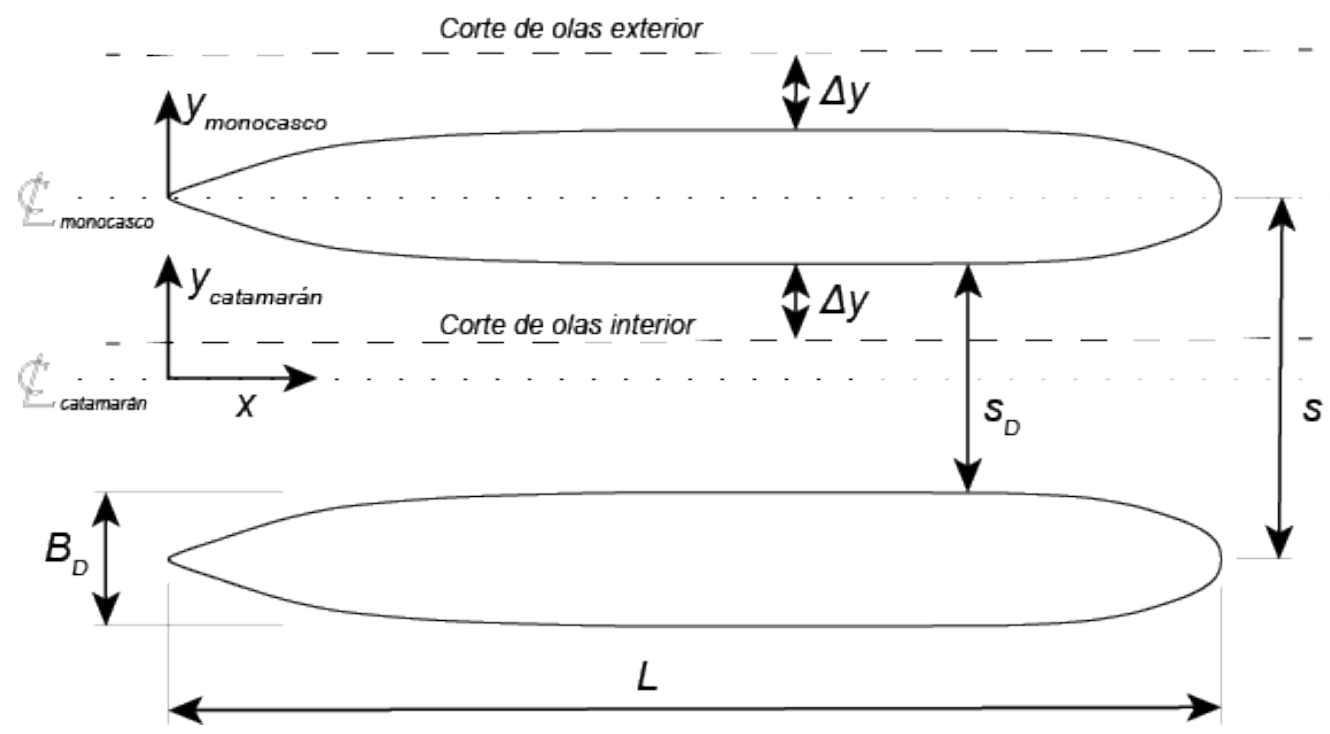

Fig. 44. Variables empleadas para identificar los cortes de ola

\subsubsection{Impacto del trimado en los cortes de olas}

Las mediciones del asiento y trimado dinámicos con modelo libre (sec. 6.3) muestran cómo las condiciones de navegación del catamarán y monocasco no son exactamente iguales. Este hecho introduce diferencias al comparar los cortes de olas de uno y otro que no provienen de 
la interacción entre los trenes de olas de cada casco, dificultando apreciar los efectos de cancelación. Con el fin de minimizar el impacto del asiento y trimado dinámicos se escogió el catamarán PESBO para la realización de este análisis, al mostrar el catamarán y monocasco valores parecidos (ver análisis correspondiente a la Fig. 40 y Fig. 41).

Como alternativa al monocasco se podrían utilizar los cortes de olas exteriores del catamarán ya que a priori los primeros no están influenciados por el segundo casco. La comparativa entre los cortes de olas exteriores con ambas configuraciones permite evaluar el posible impacto de dichas variaciones dinámicas de la condición de navegación. La separación mayor $(s / L=$ $0.388)$ y $F r=0.322$ adquiere el asiento y trimado más semejantes al monocasco, si bien los cortes de olas del catamarán y monocasco muestran claras diferencias (Fig. 45). Por el contrario, la separación menor $(s / L=0.207)$ y $F r=0.430$ muestra las mayores diferencias pero los cortes de olas son mucho más similares que en el caso anterior (Fig. 46). El apéndice B.6 contiene el conjunto total de cortes medidos. Esta falta de correlación lleva a concluir la independencia de los cortes de olas con el trimado en el caso del PESBO. Por otro lado, las diferencias significativas encontradas indican la existencia de interferencias incluso en los cortes de olas exteriores del catamarán. Se concluye por tanto que los cortes de olas del monocasco son más adecuados para el análisis de los fenómenos de cancelación del oleaje que los exteriores del catamarán.

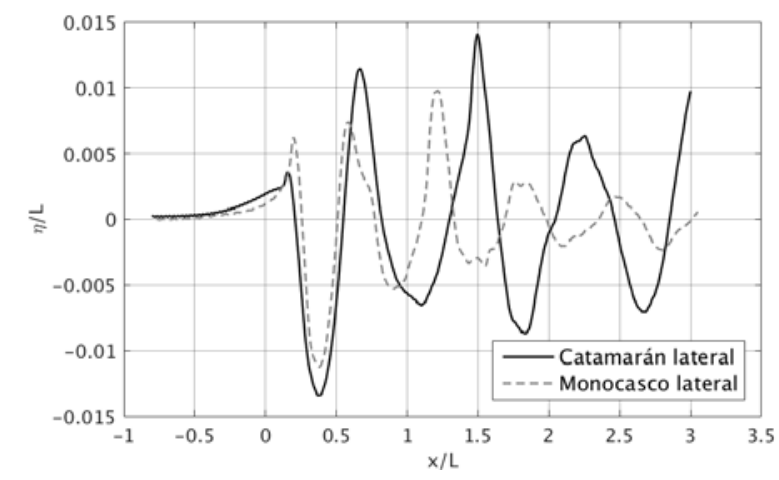

Fig. 45. Comparativa entre el corte de olas exterior del cat. PESBO con $s / L=0.388$ y el catamarán con $\mathrm{Fr}=0.332$ y a $\Delta y / s_{D}=1 / 2$

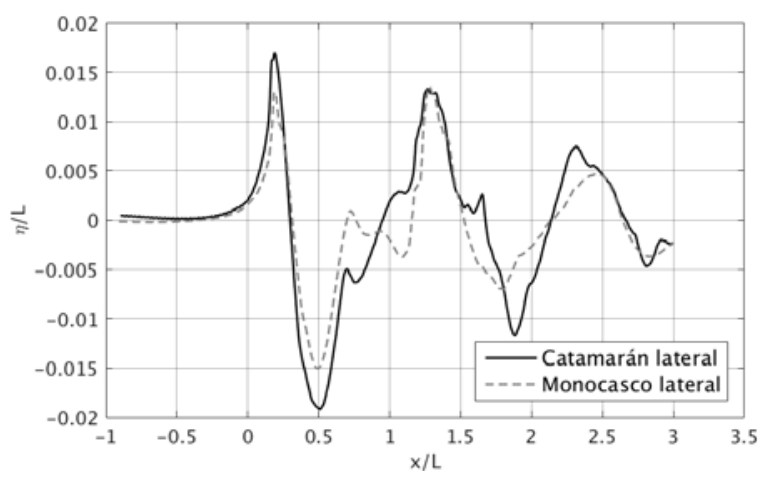

Fig. 46. Comparativa entre el corte de olas exterior del cat. PESBO con $S / L=0.207$ y el catamarán con $F r=0.430$ y a $\Delta y / s_{D}=1 / 2$ 


\subsubsection{Cortes de olas centrales $\left(\Delta y / s_{D}=1 / 2, y / s=0\right)$}

El plano de crujía representa un plano de simetría por lo que los trenes de olas procedentes de cada casco deben sumarse siempre. Es interesante comprobar esta conclusión analítica comparando los cortes de olas centrales del catamarán con los del monocasco a la misma distancia lateral. De producirse un solapamiento perfecto, las elevaciones medidas con el catamarán deberían ser iguales al doble de las medidas con el monocasco. La Fig. 47 muestra esta comparativa para varias condiciones representativas, recogiéndose el conjunto completo el anexo B.4.

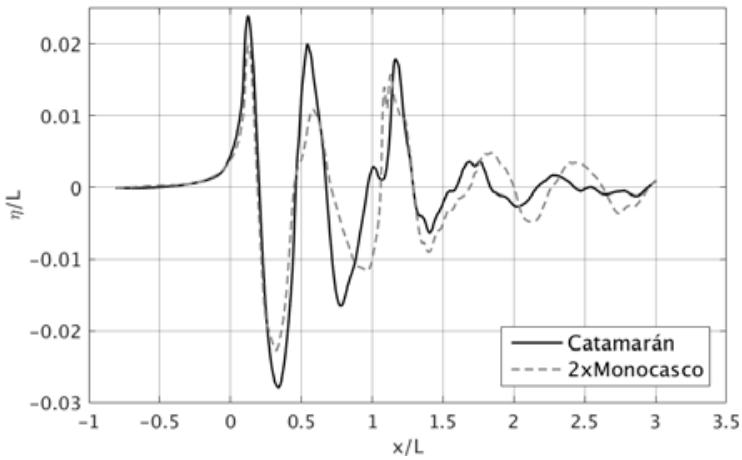

(a) $s / L=0.207 \quad F r=0.322$

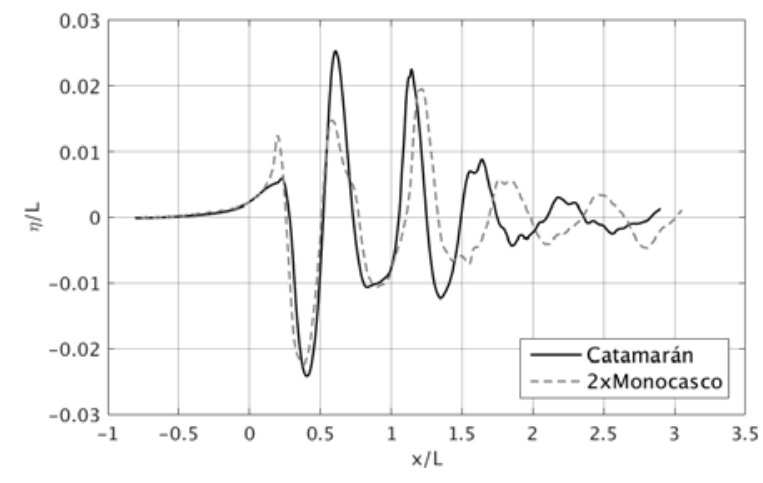

(c) $s / L=0.388 \quad F r=0.322$

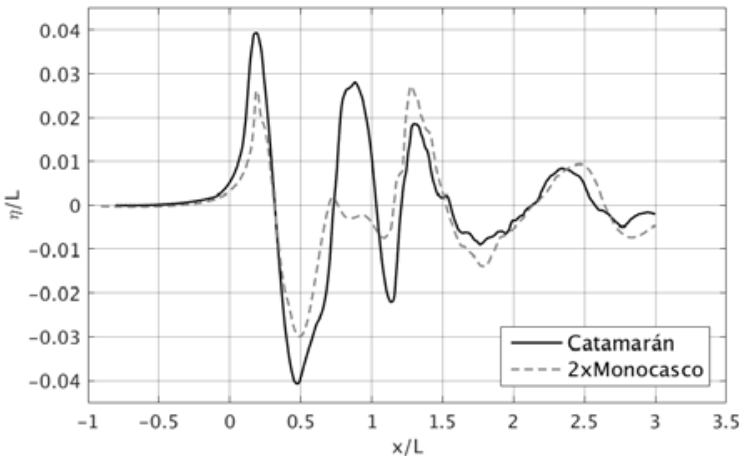

(b) $s / L=0.207 \quad F r=0.430$

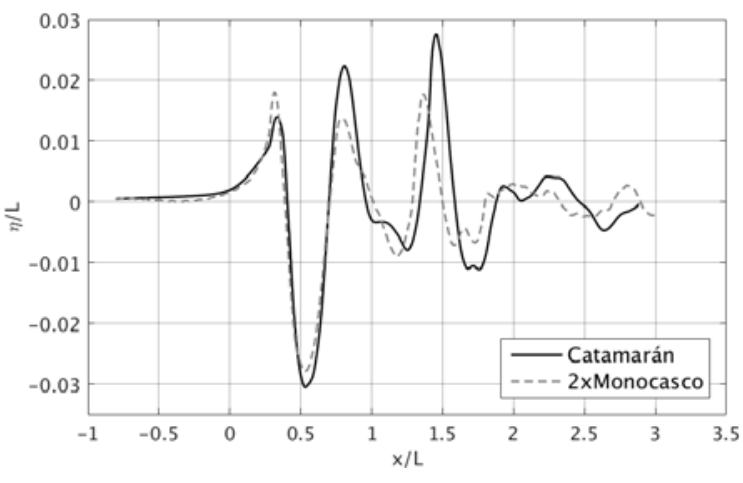

(d) $s / L=0.388 \quad F r=0.375$

Fig. 47. Cortes de olas centrales representativos del cat. PESBO vs. doble del corte de ola del monocasco la misma distancia lateral

La Fig. 47(a) con $s / L=0.207$ muestra cómo la posición longitudinal, $x / L$, del primer pico del catamarán ocurre antes que la correspondiente en la Fig. 47 (c) con $s / L=0.388$. Esto resulta 
de la menor separación en la primera a pesar de tener igual $F r$, lo que provoca que los trenes de olas de cada casco se encuentren antes.

La comparativa de la Fig. 47(a) y Fig. 47(b) muestra el incremento esperado de la longitud de olas con el $\mathrm{Fr}$ independientemente de la separación.

La observación más interesante se obtiene analizando la Fig. 47(d). Esta corresponde a la condición de máxima interferencia favorable de acuerdo a la Fig. 32 (sec. 6.2.5). Sin embargo, las elevaciones en el corte de olas central permanecen semejantes al doble del monocasco, siendo incluso las del catamarán ligeramente superiores. Este resultado corrobora cómo el plano de crujía actúa como un plano de simetría, por lo que los fenómenos de cancelación no se pueden apreciar con cortes de olas centrales. Es necesario por tanto recurrir a cortes de olas descentrados en la zona entre cascos, como se analiza en el apartado siguiente.

\subsubsection{Cortes de olas interiores descentrados}

Para poder analizar las elevaciones de la superficie libre en la zona interior se han tomado cortes de olas interiores descentrados, a $\frac{1}{6}, \frac{1}{4}$ y $\frac{1}{3}$ de la distancia entre cascos. Al igual que en el caso anterior, se han tomado también cortes de olas a la misma distancia lateral con el monocasco (Tabla 13). Sin embargo, en este caso no es interesante duplicar las elevaciones del monocasco para compararlas con las del monocasco, dado que estas no se toman en un plano de simetría. Fuera del plano de crujía, las elevaciones deben tener longitudes de ola semejantes al depender principalmente del $F r$, aunque mostrarán un desfase y diferente amplitud permitiendo su mutua cancelación.

La Fig. 48 muestra los cortes de olas interiores asociados con las zonas más representativas de interferencia favorable y desfavorable, recogiéndose el conjunto completo en el apéndice B.5. En primer lugar, se aprecia claramente en la Fig. 48(a) y Fig. 48(b) como los primeros picos del catamarán son del mismo orden que los del monocasco al no existir interacción entre los trenes de olas aún. Los dos picos siguientes del catamarán son aproximadamente el doble que 
en el monocasco, lo que significa que los dos trenes de olas están en fase. Esto concuerda con el hecho de que estas figuras corresponden a la separación menor $(s / L=0.207)$ a $F r=$ 0.375, que de acuerdo con la Fig. 32 muestra los mayores $I F$. Por otro lado, en la Fig. 48(c) y Fig. 48(d) la amplitud del primer seno en el catamarán está mucho más próxima a la del monocasco, especialmente en el corte a $\Delta y / s_{d}=1 / 4$. Este hecho es indicativo de una interacción favorable entre los trenes de olas que tiende a reducir la elevación final, consistente con los valores mínimos de $I F$ mostrados en esta condición.
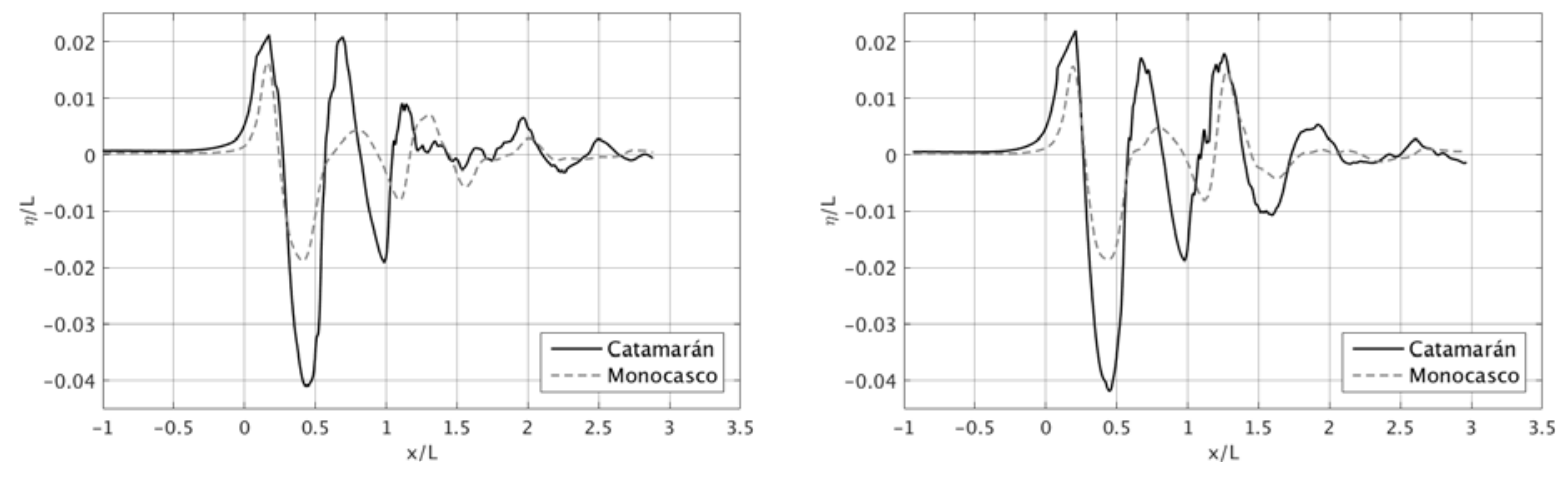

(a) $s / L=0.207 \quad F r=0.375 \quad \Delta y / s_{D}=1 / 4$

(b) $s / L=0.207 \quad F r=0.375 \quad \Delta y / s_{D}=1 / 3$
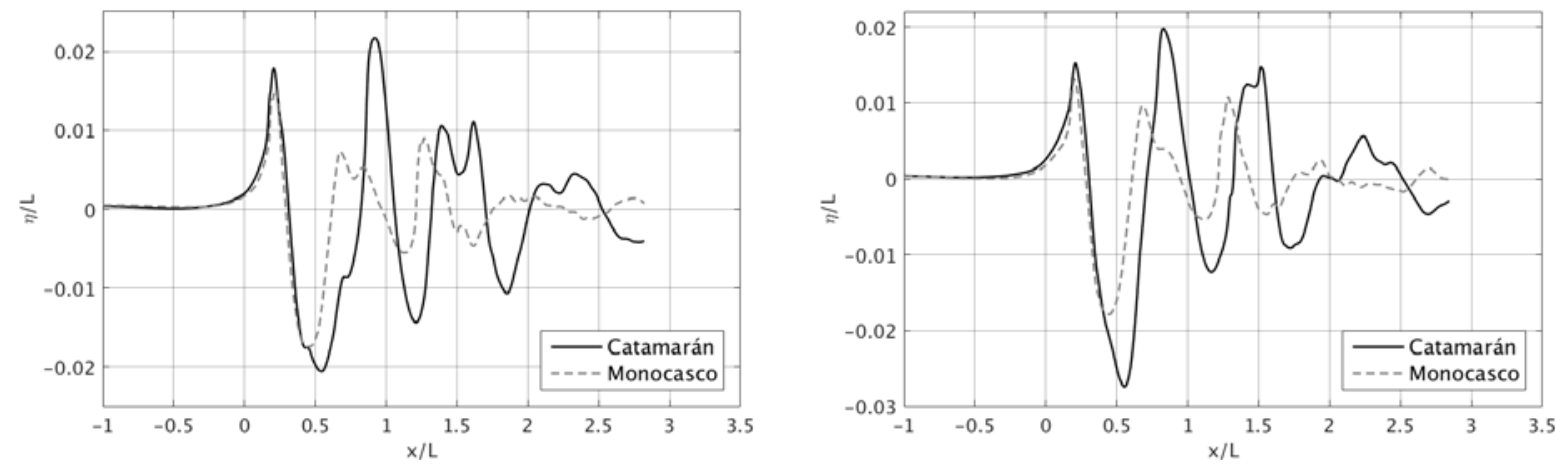

(c) $s / L=0.388 \quad F r=0.375 \quad \Delta y / s_{D}=1 / 4$

(d) $s / L=0.388 \quad F r=0.375 \quad \Delta y / s_{D}=1 / 3$

Fig. 48. Cortes de olas interiores descentrados del cat. PESBO vs. monocasco a la misma distancia lateral

Los análisis previos se basan en observaciones cualitativas de los cortes de olas. No obstante, no hay que olvidar que en última instancia dichos cortes están encaminados a evaluar las interferencias en la $R_{W}$, la cual depende de la integral del cuadrado de las elevaciones. Limitando dicha integral a la sección 2D medida en un corte de olas se obtiene un parámetro 
que permite cuantificar las diferencias entre cortes, $I_{\eta}$. Dicho parámetro se calcula de acuerdo a la ecuación (6.9), donde se utilizan los valores adimensionales de las elevaciones y posiciones longitudinales para generalizar los resultados. La Tabla 14 muestra los valores resultantes correspondientes a los cortes mostrados en Fig. 48.

$$
I_{\eta}=\frac{1}{L^{3}} \int_{\text {Wave Cut }} \eta^{2} d x
$$

Tabla 14. Integración del cuadrado de las elevaciones de los cortes de olas experimentales

\begin{tabular}{|c|c|c|c|c|}
\hline Configuración & $\frac{\Delta y}{s_{d}}$ & $I_{\eta, c a t a m a r a ́ n}$ & $I_{\eta, \text { monocasco }}$ & $\frac{I_{\eta, \text { catamarán }}}{I_{\eta, \text { monocasco }}}$ \\
\hline $\begin{array}{l}\text { PESBO, modelo libre } \\
s / L=0.207 \\
F r=0.375\end{array}$ & $\begin{array}{l}1 / 3 \\
1 / 4\end{array}$ & $\begin{array}{l}5.06 \mathrm{E}-4 \\
4.46 \mathrm{E}-4\end{array}$ & $\begin{array}{l}1.19 \mathrm{E}-4 \\
1.05 \mathrm{E}-4\end{array}$ & $\begin{array}{l}4.24 \\
4.25\end{array}$ \\
\hline $\begin{array}{l}\text { PESBO, modelo libre } \\
s / L=0.388 \\
F r=0.375\end{array}$ & $\begin{array}{l}1 / 3 \\
1 / 4 \\
1 / 6\end{array}$ & $\begin{array}{l}3.02 \mathrm{E}-4 \\
2.66 \mathrm{E}-4 \\
2.34 \mathrm{E}-4\end{array}$ & $\begin{array}{l}1.01 \mathrm{E}-4 \\
9.93 \mathrm{E}-5 \\
1.00 \mathrm{E}-4\end{array}$ & $\begin{array}{l}3.00 \\
2.68 \\
2.34\end{array}$ \\
\hline
\end{tabular}

En una situación hipotética donde el corte de olas del catamarán es exactamente el doble que el del monocasco, el ratio entre los valor de $I_{\eta}$ del catamarán y monocasco debería ser 4 . Si el ratio es superior a cuatro es un claro indicador de una interferencia desfavorable, como sucede con $s / L=0.207$ y $F r=0.375$. Por el contrario, para $s / L=0.388$ y $F r=0.375$ su valor es inferior a cuatro indicando cómo la interferencia es favorable, consistente en ambos casos con los $I F$ calculados anteriormente (Fig. 32). Queda claro cómo el $I F$ y el $I_{\eta}$ están íntimamente relacionados, mostrando una vez más cómo la interacción entre los trenes de olas juega un papel clave a la hora de obtener una interferencia favorable. 


\subsection{Análisis global de los resultados experimentales}

Las secciones previas han abordado los efectos de interferencia desde diferentes perspectivas. No obstante, en última instancia todos los efectos están interconectados al corresponder a una determinada geometría navegando a una velocidad y estado de la mar específicos. El paso final por tanto es entrelazarlas para adquirir una perspectiva global de este fenómeno.

Una de las primeras disquisiciones es definir la condición de interferencia nula que sirva de referencia para determinar la $R_{I}$. A priori, evaluando la respuesta de cada casco por separado se obtendría dicha referencia. La dificultad reside en cómo tratar las diferencias en el asiento y trimado dinámicos, lo que lleva a la decisión entre ensayos con modelo libre o fijo. Dado que el concepto de monocasco representa una condición virtual, idealmente debería tener el mismo asiento y trimado que el multicasco. En la práctica sin embargo esto es normalmente inviable, utilizándose como alternativa ensayos con modelo fijo del multicasco y monocasco. Se asegura así que la condición de navegación es la misma, si bien se pierde la influencia que el asiento y trimado dinámicos tienen en la resistencia. No obstante, se ha observado cómo para valores moderados del $F r$ las diferencias en las condiciones dinámicas de navegación son pequeñas. Más aún, el análisis de los cortes de olas sugiere cómo dichas diferencias no están correlacionadas con el $I F$. Se recomienda por tanto en este caso la utilización de ensayos con modelo libre, más próximos a la realidad. De hecho, la comparativa entre modelo libre y fijo pone de manifiesto cómo los segundos subestiman sistemáticamente la resistencia al avance, con el consiguiente riesgo a la hora de extrapolar resultados (sec. 6.3). En cualquier caso, las mediciones del asiento y trimado muestran cómo estos adquieren valores significativos para $\mathrm{Fr}>0.50$, con diferencias apreciables entre el multicasco y monocasco. Se recomienda por tanto emplear el modelo libre para el multicasco y el modelo fijo para el monocasco preajustando el asiento y trimado de acuerdo a los valores dinámicos del multicasco. Las embarcaciones estudiadas en este trabajo no están diseñadas para planear, por lo que apenas se superó el $\mathrm{Fr}=0.50$. En el futuro se espera extender el rango de velocidades utilizando nuevas geometrías de planeo y semi-planeo. 
Centrándonos en los fenómenos de interferencia, los ensayos de resistencia demuestran ser los más eficientes a la hora de mostrar su intensidad global. Esto se debe a que reproducen prácticamente todas sus componentes excepto los efectos viscosos dependientes del $R e$, diferente en el modelo y buque real. Este hecho, clave en los ensayos con modelos, se tiene en cuenta estimando la resistencia viscosa analíticamente para poder discernir la resistencia por formación de olas. De cara a cuantificar dichos fenómenos de interferencia es recomendable utilizar el $I F$. Al tratarse de un parámetro adimensional, permite comparar directamente diferentes geometrías y velocidades. Respecto a su definición, dadas las dificultades a la hora de reproducir o predecir los efectos viscosos se recomienda el uso de la $R_{W}$ para definir el $I F$, como se explicó en el apartado 6.2.2. El análisis de los valores medidos del $I F$ es sin duda el que ha aportado conclusiones más relevantes respecto a los fenómenos de interferencia, recogidas en detalle en el apartado 6.2.5. Entre ellas cabe destacar el mayor impacto de los efectos de interferencia debidos al oleaje, siendo la cancelación entre los trenes de olas la responsable principal de interferencias favorables.

A la hora de profundizar en las diferentes causas de interferencia, las curvas de $R_{I}$ o IF proporcionan una información limitada que debe ser ampliada con otros métodos experimentales y numéricos (sec. 4.3). Destacan para tal fin los cortes de olas para la $R_{W}$ y las simulaciones numéricas para visualizar el flujo sobre el casco. En lo que respecta a las variaciones de la energía disipada en olas rompientes y spray, por el momento no se conoce un método directo que permita cuantificarlas experimentalmente.

Los cortes de olas proporcionan un método relativamente sencillo para analizar el campo de elevaciones, y con multitud de aplicaciones. En primer lugar han servido para visualizar y cuantificar la importancia de los efectos de cancelación a través del $I_{\eta}$, consistentes con los $I F$, así como la imposibilidad de observar estos en el plano de crujía. Un detalle que merece la pena revisar es la falta de correlación encontrada entre las elevaciones en el costado exterior del multicasco y monocasco, sus asientos y trimados dinámicos y el $I F$. Este hecho induce a considerar la existencia de interferencias que afectan al tren de olas exterior, si bien no está claro cuál es su origen. En principio se achacan a la variación del campo de presiones sobre y 
bajo el casco, transmitiendo las perturbaciones a la zona exterior. Cabe destacar que en todo este análisis se ha supuesto un comportamiento lineal del oleaje. La inclusión en el futuro de fenómenos no lineales permitirá comprender mejor la interacción entre los trenes de olas tanto en la zona entre cascos como en la exterior.

Una de las principales aplicaciones de los resultados experimentales es su utilización para validar modelos numéricos. La sec. 6.2.6 muestra una primera comparativa de los datos medidos con las predicciones numéricas presentadas por Yeung et al. (2004) utilizando la teoría de cuerpos esbeltos. La dificultad para obtener resultados fiables reside en el comportamiento oscilatorio del $I F$, con variaciones acusadas en función del $F r$ y separación. Como consecuencia, pequeñas desviaciones en el cálculo del IF pueden llevan a estimar las zonas de interferencia favorable precisamente donde se encuentran las condiciones más desfavorables. Esta importante sensibilidad pone en duda la utilización del IF como variable de diseño en base a resultados meramente numéricos.

En lo referente a las variables de diseño, el $s / L$ es el parámetro más sencillo a ajustar para conseguir interferencias favorables. En principio, los valores de resistencia y elevaciones de la superficie libre indican como las separaciones mayores en el rango estudiado muestran un mejor comportamiento. No obstante, no hay un claro óptimo sino que se deberá buscar un equilibrio entre

- Separaciones demasiado grandes que muestren una atenuación significativa del oleaje

- Separaciones demasiado pequeñas que generen olas rompientes, spray y una excesiva aceleración del flujo, que conllevan una interferencia desfavorable

- Separaciones con oscilaciones del $I F$ relativamente moderadas, de forma que haya un riesgo contenido de caer en zonas muy desfavorables

No obstante, las formas de los cascos son las que en última instancia determinan la respuesta hidrodinámica del multicasco, y por ende los efectos de interferencia resultantes. Prueba de ello son las diferencias encontradas entre el catamarán PESBO y el S60 tanto en resistencia como en asiento y trimado dinámicos o cortes de olas. A pesar de las directrices generales 
referentes al diseño de formas vistas en el apartado 4.1.3, el uso de CFD es especialmente útil en este apartado. El capítulo 7 a continuación se centra en este aspecto, evaluando la capacidad para evaluar los efectos de interferencia mediante simulaciones numéricas. 


\section{SIMULACIONES NUMÉRICAS}

La utilización de herramientas numéricas es un complemento ideal a la toma de datos experimentales. Sus dos principales virtudes residen en la facilidad para visualizar el campo de presiones y velocidades sobre el caso y elevaciones en la superficie libre, así como para analizar múltiples geometrías. En este trabajo se han empleado para tal fin dos códigos substancialmente diferentes: Tdynlin y Star-CCM+, presentados en el apartado 5.2.

\subsection{Tdynlin}

Tras la primera campaña experimental con el catamarán PESBO se consideró útil implementar un nuevo módulo de catamaranes para el código de paneles Tdynlin desarrollado en el CEHINAV. Los datos experimentales se podrían usar para su validación, empleando a posteriori los resultados numéricos para optimizar las formas de catamaranes. A cambio de un esfuerzo de programación relativamente sencillo al tratarse de un código potencial interno, se aumentaban las capacidades numéricas del CEHINAV.

Como se explicó en el apartado 4.3.2, los códigos potenciales desprecian los efectos viscosos para simplificar el modelo numérico y reducir significativamente los requisitos computacionales. Se emplean por tanto para predecir los efectos de presión, como las elevaciones de la superficie libre, no siendo tan adecuados para estimar la $R_{T}$. Su mayor utilidad es servir como herramienta comparativa al requerir un tiempo de cálculo bajo, por lo que pueden lidiar con un gran número de geometrías.

En base a las consideraciones anteriores, las simulaciones realizadas con Tdynlin dentro de este proyecto reproducen los ensayos experimentales de cortes de olas (Tabla 13, sec. 6.4). Estos se utilizan como herramienta de validación, cuantificando la precisión de los resultados en base a las diferencias en el $I_{\eta}$. Tdynlin únicamente permite simulaciones con modelo fijo, por lo que se ha ajustado manualmente el asiento y trimado en cada simulación de acuerdo a los valores experimentales (Fig. 40 y Fig. 41). 


\subsubsection{Catamarán PESBO}

Una malla adecuada es muy importante para conseguir resultados precisos. Como se indicó en la sec. 5.2.1, Tdynlin se ejecuta como un módulo dentro del entorno de simulación $\mathrm{GiD}^{47}$. Esto permite utilizar las herramientas integradas en este último para la generación de la malla y visualización de los resultados, como describe más en detalle Souto-Iglesias (2001).

Para el casco se utilizaron inicialmente 1944 paneles. Sin embargo, con la separación menor se observaron inestabilidades numéricas en la zona entre cascos, lo que obligó ampliar el número de paneles a 3260 para mejorar la resolución (Fig. 49).
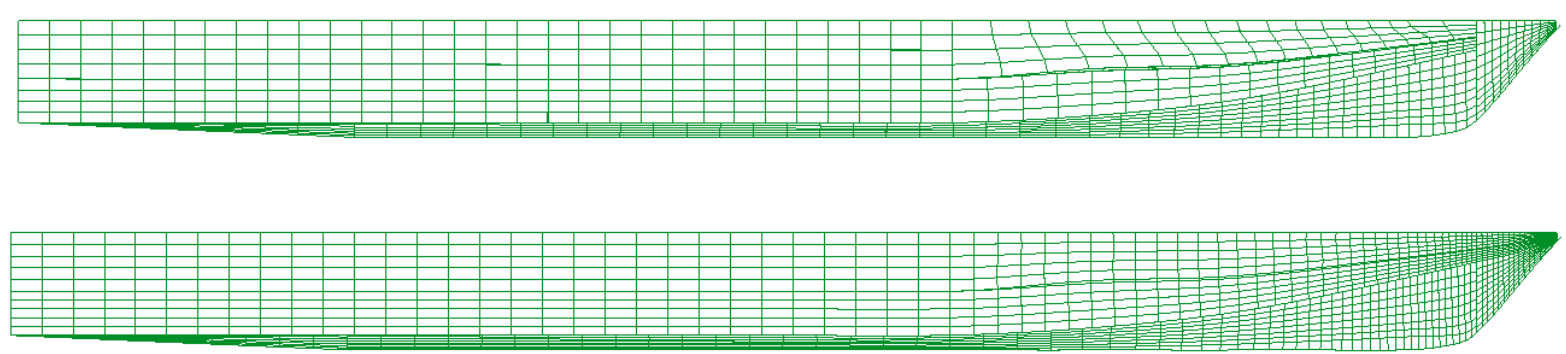

牱

Fig. 49. Comparativa entre el mallado del casco del PESBO con 1944 paneles (superior) y 3260 paneles (inferior)

En lo que respecta a la superficie libre, es importante destacar que este catamarán tiene popa de espejo (ver Fig. 22 y Fig. 49), algo muy común en este tipo de barcos al abaratar su construcción y permitir una mejor disposición general de los elementos de la cámara de máquinas. Desde el punto de vista hidrodinámico, normalmente el flujo en esta parte del buque no se ajusta bien a las hipótesis de flujo potencial. Por ello, se simplifica el problema y se asume en el modelo aquí desarrollado que las líneas de corriente que vienen del costado se encuentra agujas abajo del buque a una distancia aproximada de $L / 4$, dejando sin modelar la parte de la superficie libre entre la popa de espejo y el punto de encuentro de esas líneas de corriente, como se observa en la Fig. 50.

\footnotetext{
${ }^{47}$ http://www.gidhome.com/
} 
En la tesis doctoral de Souto-Iglesias (2001) se investigó la posibilidad de desarrollar modelos potenciales lineales para condiciones de popa de espejo seco. Podría ser interesante valorar la aplicación de esos modelos a este caso. También podría ser interesante estudiar la estructura de las olas que se forman en esa zona, tratando de optimizar el diseño de las formas en esa parte del casco con el objetivo de minimizar la generación de oleaje en la misma, como estudian Martínez Legazpi et al. (2012) y Martínez Legazpi et al. (2015).

Volviendo sobre la superficie libre del Catamarán PESBO, la extensión considerada es $3.5 L \times 1.8 L$ dejando una eslora de margen a proa del casco. Además, dado que la configuración geométrica es simétrica respecto al plano de crujía, sólo es necesario mallar la mitad del dominio. El parámetro escogido para determinar el número óptimo de paneles es la longitud de ola, $\lambda_{w}$, calculada según la ec. (7.1) Para cada $\lambda_{w}$ se requieren entre 15 y 20 paneles de acuerdo con Souto-Iglesias (2001), por lo se ha escogido un valor intermedio de 18. La ec. (7.2) proporciona el número de paneles $N$ a emplear para una longitud $l$. En sentido transversal es necesario distinguir entre la zona exterior y la interior entre cascos. En la primera se emplea la expresión (7.2) directamente, mientras que en la zona interior se llegó a utilizar el doble de paneles para mejorar la resolución. Teniendo en cuenta estas directrices, la Tabla 15 muestra los paneles empleados para cada simulación.

$$
\begin{gathered}
\lambda_{w}=2 \pi F r^{2} L \\
N=18 \frac{l}{2 \pi F r^{2} L}
\end{gathered}
$$

Una vez generada la malla (Fig. 50), Tdynlin calcula el campo de elevaciones de la superficie libre así como velocidades y presiones sobre la obra viva (Fig. 51 y Fig. 52). Cabe recordar que el principal objetivo en esta fase es la de validar los resultados numéricos. Para ello se han extraído cortes de olas centrales a partir del campo de elevaciones para compararlos con los experimentales. La Fig. 53 muestra los casos correspondientes a la mayor y menor separación con el $\mathrm{Fr}$ más alto y bajo. El conjunto completo de resultados se recoge en el apéndice C.1. 
Tabla 15. Paneles empleados en la superficie libre con el Tdynlin para el cat. PESBO

\begin{tabular}{|c|c|ccccc|}
\hline Configuración & $\boldsymbol{F r}$ & & \multicolumn{3}{c}{$\mathbf{N}$} & \\
& & Longitudinal & Transv. Interior & Transv. Exterior & Total \\
\hline$\underline{s / L=0.207}$ & 0.322 & 97 & 4 & 47 & 4947 \\
& 0.375 & 72 & 4 & 35 & 2808 \\
& 0.398 & 64 & 3 & 31 & 2176 \\
& 0.410 & 55 & 3 & 27 & 1650 \\
\hline$\underline{s / L=0.298}$ & 0.322 & 100 & 7 & 38 & 4500 \\
& 0.375 & 72 & 4 & 34 & 2736 \\
& 0.398 & 65 & 7 & 38 & 2925 \\
& 0.410 & 56 & 7 & 38 & 2520 \\
\hline$\underline{s / L=0.388}$ & 0.322 & 100 & 10 & 35 & 4500 \\
& 0.375 & 72 & 5 & 33 & 2736 \\
& 0.398 & 65 & 10 & 25 & 2275 \\
& 0.410 & 56 & 10 & 35 & 2520 \\
\hline
\end{tabular}

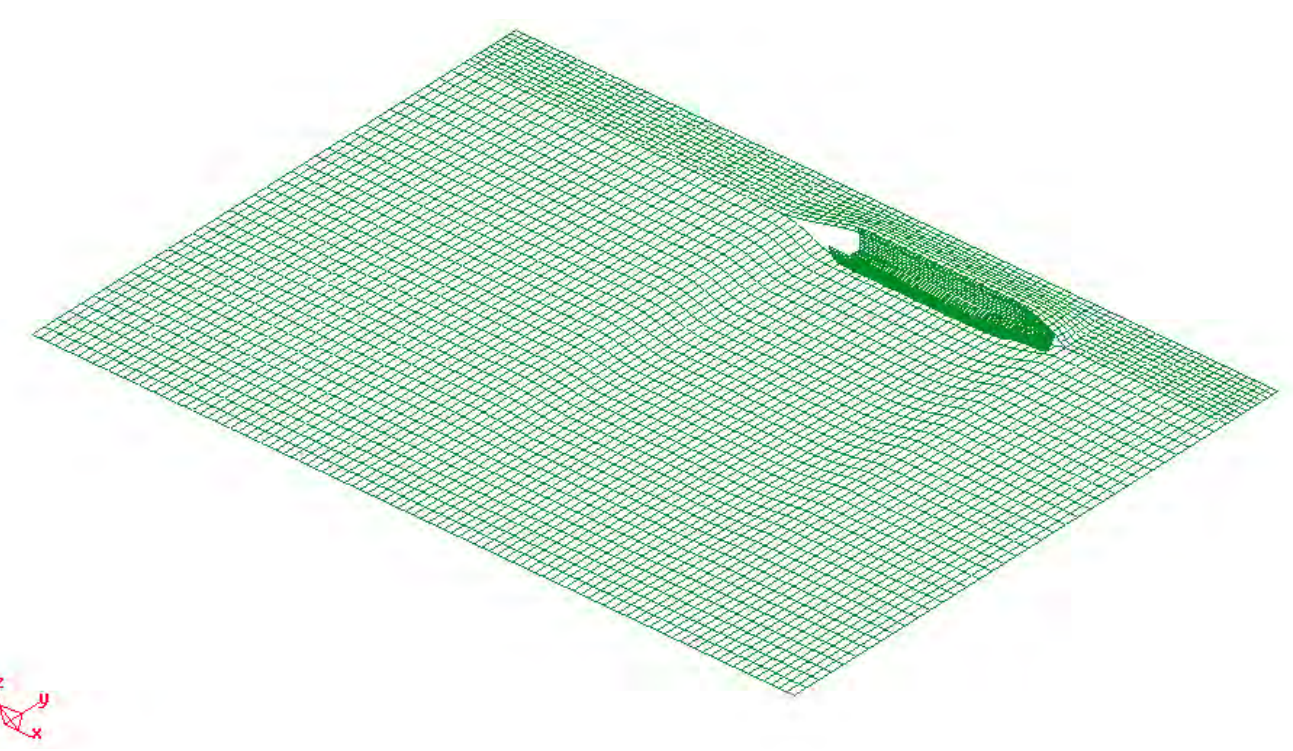

Fig. 50. Aspecto del mallado del cat. PESBO en Tdynlin para $s / L=0.388$ y $F r=0.322$ 


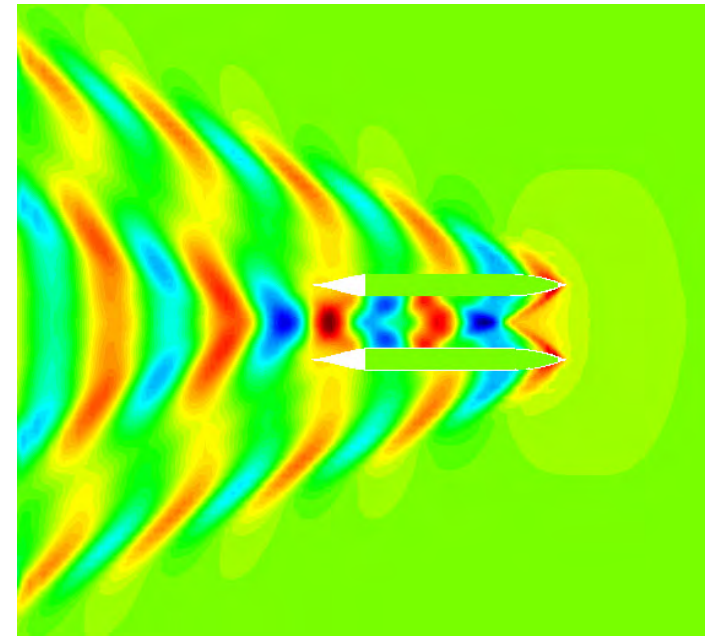

Fig. 51. Elevaciones de la superficie libre calculadas con Tdynlin. Cat. PESBO, $s / L=388$ y $F r=0.372$

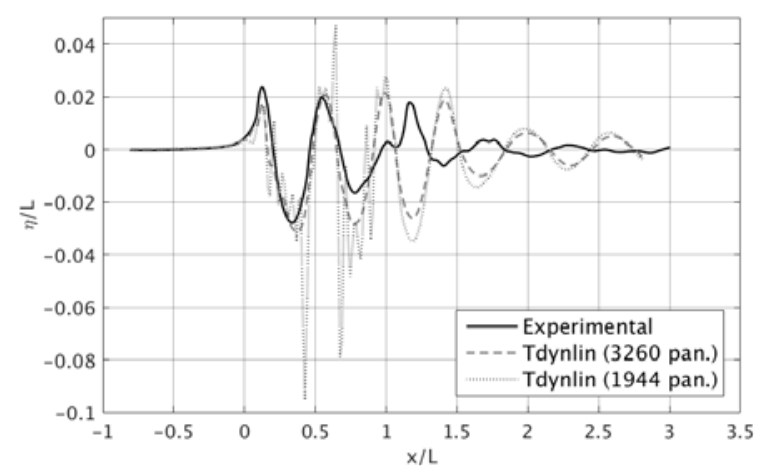

(a) $s / L=0.207 \quad F r=0.322$

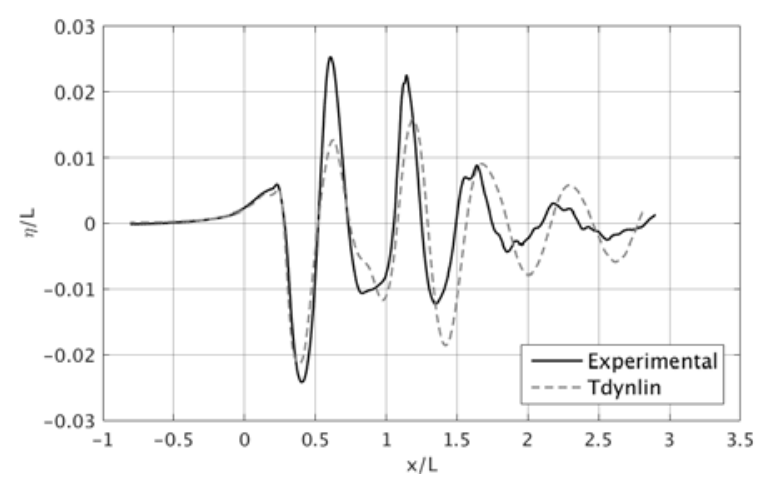

(c) $s / L=0.388 \quad F r=0.322$

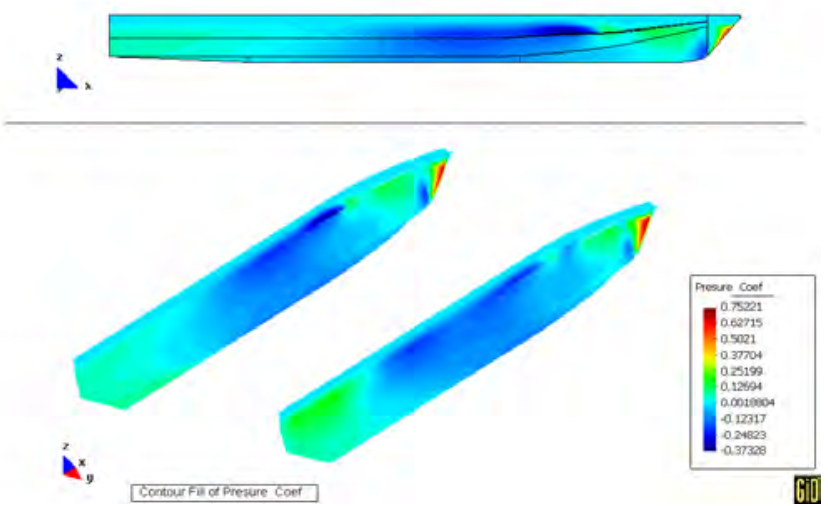

Fig. 52. Presiones sobre el casco calculadas con Tdynlin. Cat.PESBO, $\frac{s}{L}=388$ y Fr $=0.372$

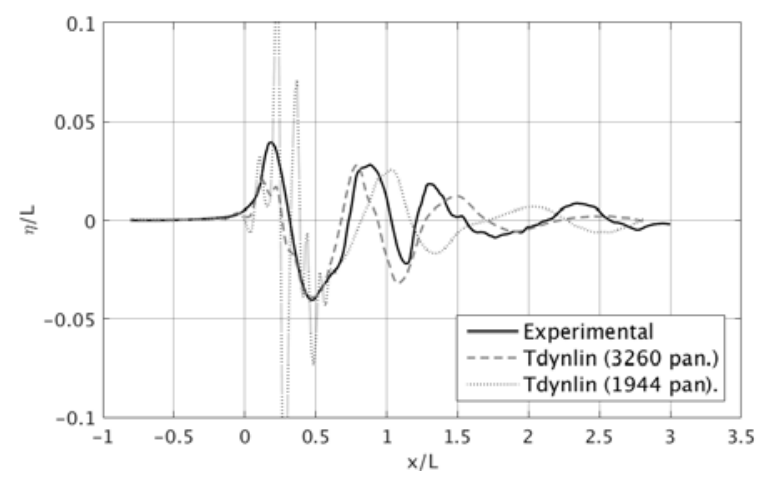

(b) $s / L=0.207 \quad F r=0.430$

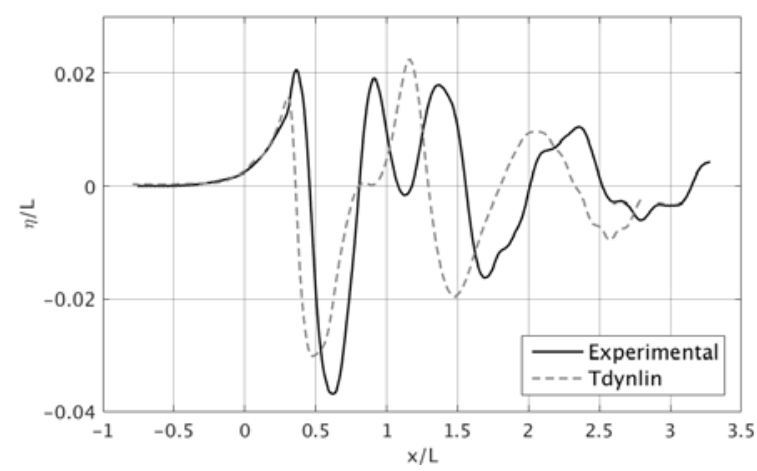

(d) $s / L=0.388 \quad F r=0.430$

Fig. 53. Comparativa de los cortes de ola centrales del cat. PESBO calculados con Tdynlin vs. experimentales 
Se aprecia como los resultados de Tdynlin muestran una dependencia con el $F r$ y especialmente la separación, esencial para servir como herramienta comparativa. Mención especial cabe para la Fig. 53(a) y Fig. 53(b) donde se incluyen los resultados con los dos mallados del casco considerados (1944 vs. 3260 paneles) con $s / L=0.207$. Los resultados con el mallado menos denso muestran claras inestabilidades numéricas. Su explicación reside en el modelo numérico empleado, donde se emplean singularidades de Rankine como se explicó en la sec. 5.2.1. Cuanto más cerca se posicionan los cascos, mayor es la interacción entre dichas singularidades provocando las oscilaciones observadas para $s / L=0.322$. Al aumentar el número de paneles, y por ende singularidades, la intensidad individual de cada una se reduce a la vez que se obtiene una discretización más fiel de la geometría original y con paneles con una relación de aspecto mejor. Como resultado se reducen los errores numéricos, eliminando las oscilaciones tal y como se comprueba con el mallado más denso.

Centrándonos en la correlación con los cortes experimentales, con $s / L=0.207$ y $F r=0.322$ el corte de olas numérico reproduce relativamente bien el periodo del corte experimental hasta $x / L=0.7$. De este punto en adelante los resultados numéricos difieren de los experimentales. Lo mismo sucede con la velocidad alta $(F r=0.430)$. En el caso de la separación mayor $(s / L=0.388)$, los resultados numéricos se aproximan mucho más a los experimentales, sin mostrar en este caso las inestabilidades de la separación menor a pesar de utilizar el mallado menos denso. Para $F r=0.322$, las elevaciones experimentales son superiores a las numéricas, aunque las pendientes son muy similares hasta $x / L=1.5$. En el caso de $F r=0.430$, las elevaciones son muy similares, aunque los valores experimentales muestran un máximo que no se reproduce en los numéricos.

Finalmente, el parámetro $I_{\eta}$ (integral del cuadrado de elevaciones) permite cuantificar las diferencias en los cortes de olas al igual que se efectuó al analizar los cortes experimentales. La Tabla 16 resume los valores obtenidos, los cuales confirman las conclusiones anteriores. Los cortes experimentales muestran el aumento esperado del $I_{\eta}$ con la velocidad, si bien los datos numéricos muestran un comportamiento más errático. Proporcionalmente los valores numéricos y experimentales convergen a medida que aumenta la velocidad, esperable al 
corresponder a longitudes de ola mayores que se capturan mejor con el modelo numérico. La separación menor es la que muestra mayores errores, síntoma de nuevo de la pérdida de precisión debido a la proximidad entre las singularidades de ambos cascos.

Tabla 16. $I_{\eta}$ de los cortes de olas centrales del cat. PESBO calculados con Tdynlin

\begin{tabular}{|c|c|c|c|c|}
\hline Configuración & $\boldsymbol{F r}$ & $\boldsymbol{I}_{\boldsymbol{\eta}, \text { Tdynlin }}$ & $\boldsymbol{I}_{\boldsymbol{\eta}, \text { experimental }}$ & $\frac{\boldsymbol{I}_{\boldsymbol{\eta}, \text { Tdynlin }}}{\boldsymbol{I}_{\boldsymbol{\eta}, \text { catamarán }}}$ \\
\hline$s / L=0.207$ & 0.322 & $5.11 \mathrm{E}-4$ & $2.60 \mathrm{E}-4$ & 1,96 \\
& 0.375 & $9.15 \mathrm{E}-4$ & $5.32 \mathrm{E}-4$ & 1,72 \\
& 0.398 & $6.01 \mathrm{E}-4$ & $7.06 \mathrm{E}-4$ & 0,85 \\
& 0.430 & $6.43 \mathrm{E}-4$ & $7.98 \mathrm{E}-4$ & 0,81 \\
\hline$s / L=0.298$ & 0.322 & $2.21 \mathrm{E}-4$ & $2.42 \mathrm{E}-4$ & 0,91 \\
& 0.375 & $4.70 \mathrm{E}-4$ & $3.70 \mathrm{E}-4$ & 1,27 \\
& 0.398 & $5.26 \mathrm{E}-4$ & $4.55 \mathrm{E}-4$ & 1,16 \\
& 0.430 & $5.02 \mathrm{E}-4$ & $5.50 \mathrm{E}-4$ & 0,91 \\
\hline$S / L=0.388$ & 0.322 & $2.06 \mathrm{E}-4$ & $2.41 \mathrm{E}-4$ & 0,85 \\
& 0.375 & $4.39 \mathrm{E}-4$ & $3.63 \mathrm{E}-4$ & 1,21 \\
& 0.398 & $3.63 \mathrm{E}-4$ & $3.56 \mathrm{E}-4$ & 1,02 \\
& 0.430 & $4.24 \mathrm{E}-4$ & $5.10 \mathrm{E}-4$ & 0,83 \\
\hline
\end{tabular}




\subsection{CD-Adapco Star-CCM+}

En una segunda fase se trató de superar las limitaciones de flujo potencial y asiento y trimado fijos del Tdynlin para mejorar la precisión de los resultados y predecir con más exactitud los efectos de interferencia. No obstante, la inclusión de flujo viscoso y modelo libre en Tdynlin exigiría reprogramarlo prácticamente desde el principio, por lo que se consideró más eficiente utilizar el software comercial CD-Adapco Star-CCM+ disponible en el CEHINAV (Izaguirre Alza (2012)).

Star-CCM+ es uno de los entornos de simulación CFD más completos y populares del mercado. No sólo incluye de por sí los modelos numéricos requeridos debidamente validados, sino que además facilita enormemente el proceso de mallado y visualización de resultados durante el post-proceso. Sus características principales se presentaron en la Sec. 5.2.2. A lo largo del proyecto se han empleado múltiples versiones, desde la v4.04 a la más reciente v10.04. Esto ha permitido aprovechar el proceso de depuración y actualización continuo por parte de CDAdapco para mejorar la eficiencia, estabilidad y precisión de las simulaciones.

Centrándonos en este trabajo en particular, la utilización de CD-Adapco Star-CCM+ tiene como objetivo permitir:

1. Evaluar la precisión de los resultados numéricos simulando las condiciones ensayadas con el catamarán PESBO y S60.

2. Analizar el campo de presiones y velocidades sobre el casco, evaluando la influencia de la geometría de los cascos en los fenómenos de interferencia.

\subsubsection{Configuración de los parámetros de cálculo}

A pesar de simularse dos geometrías diferentes, ambas presentan dimensiones relativamente semejantes. Esto ha permitido homogeneizar los parámetros de cálculo y poder comparar así los resultados obtenidos con cada geometría, minimizando las desviaciones puramente numéricas. 


\section{Dominio de cálculo}

El primer paso para poder llevar a cabo las simulaciones es definir el dominio de cálculo, denominado región en Star-CCM+, el cual debe ser un volumen cerrado. En este caso, se ha empleado un volumen prismático como muestra la Fig. 54, delimitado por cuatro grupos de superficies: CenterPlane, Inlet, Outlet, Hull. Una de las particularidades del problema a resolver es su simetría respecto al plano de crujía, lo que permite modelizar sólo una de las bandas reduciendo así las necesidades computacionales.
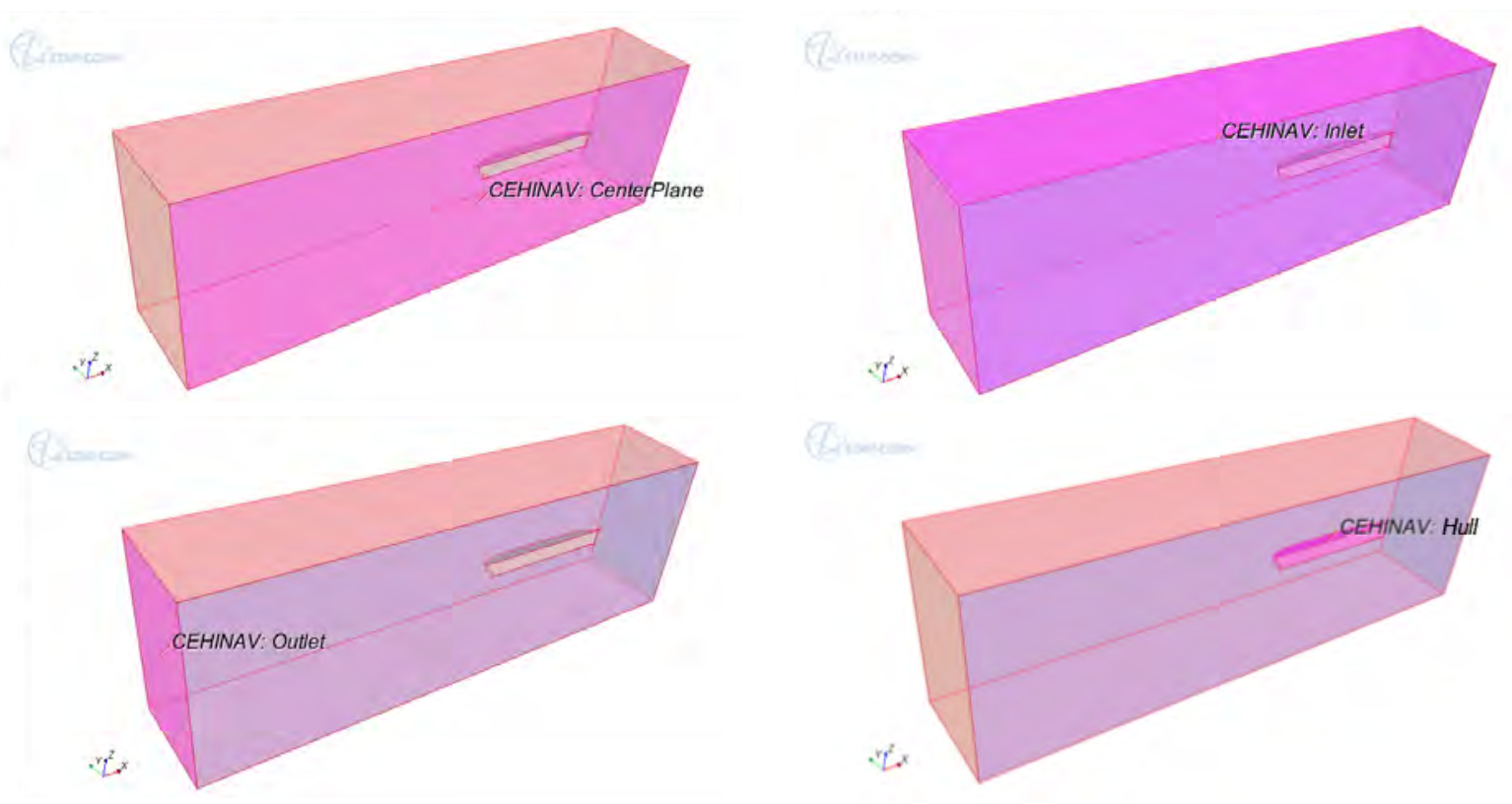

Fig. 54: Identificación de cada uno de los grupos de superficies que definen el contorno de la región de estudio

Tanto el PESBO como el $\mathrm{S} 60$ son modelos ensayados en el CEHINAV, por lo que se utilizaron las dimensiones del canal como referencia para establecer los límites de dicho dominio:

- LONGITUD: Una eslora a proa del modelo y dos esloras a popa. En total cuatro esloras. Tomando como referencia la eslora del modelo más largo (S60), 2.5 m, la longitud total del dominio de cálculo es de $10 \mathrm{~m}$.

- ANCHURA: Se ha tomado la semi-manga del canal (1.9 m).

- AltuRA: La profundidad del canal es $2.2 \mathrm{~m}$, y por encima de la flotación se ha analizado hasta $1 \mathrm{~m}$. Por lo tanto la altura total del dominio es de $3.2 \mathrm{~m}$. 
El caso del monocasco es computacionalmente menos exigente que el catamarán, al necesitar resolver el flujo únicamente sobre uno de los costados del casco. Sin embargo, es necesario intersectar el plano central de simetría con el casco y asegurarse que los bordes de unión están apropiadamente conectados. En el caso del catamarán S60, el modelo CAD presentaba pequeñas desalineaciones que imposibilitaba conectar ambas superficies con exactitud. Para solventar esta dificultad, se utilizó el modelo de mallado Wrapper (ver Tabla 5) con un tamaño de celda muy pequeño para redefinir la geometría base.

Por último, cada superficie delimitadora tiene un comportamiento diferente, el cual se especifica en Star-CCM+ seleccionando el tipo de superficie entre las opciones disponibles. La Tabla 17 identifica las superficies empleadas y su tipo.

Tabla 17. Comportamiento de las superficies delimitadoras

\begin{tabular}{|l|l|}
\hline SUPERFICIE & COMPORTAMIENTO \\
\hline $\begin{array}{l}\text { CenterPlane } \\
\text { El plano de crujía }\end{array}$ & $\begin{array}{l}\text { Symmetry plane } \\
\text { Como su propio nombre indica, es un plano de simetría }\end{array}$ \\
\hline $\begin{array}{l}\text { Inlet } \\
\text { Las superficies por donde se espera } \\
\text { la entrada de agua }\end{array}$ & $\begin{array}{l}\text { Velocity inlet } \\
\text { Superficie de entrada de fluido en el que la velocidad } \\
\text { es conocida. }\end{array}$ \\
\hline $\begin{array}{l}\text { Outlet } \\
\text { Las superficies por donde se espera } \\
\text { la salida de agua }\end{array}$ & $\begin{array}{l}\text { Pressure outlet } \\
\text { Superficie de salida de fluido sobre la que se especifica } \\
\text { una presión estática de referencia. }\end{array}$ \\
\hline $\begin{array}{l}\text { Hull } \\
\text { La superficie del casco, junto con la } \\
\text { cubierta }\end{array}$ & $\begin{array}{l}\text { Wall } \\
\text { Superficies rígidas que no dejan pasar el flujo. Sobre } \\
\text { ellas se puede definir si tener en cuenta efectos de } \\
\text { fricción viscosa o no. }\end{array}$ \\
\hline
\end{tabular}

${ }^{48}$ Las paredes del canal, aunque no dejan pasar fluido, se incluyen en el Inlet para evitar problemas de convergencia en la solución. 
Parámetros de generación de la malla

Una vez definido el dominio, el siguiente paso es discretizarlo, generando una malla volumétrica. De las opciones que permite el programa (Tabla 5) se han empleado los siguientes modelos:

- Surface Remesher

- Trimmer

- Prism Layer Mesher

La superficie libre es una región de especial interés, por lo que se refinará la malla en esta zona. Star-CCM+ permite crear volúmenes de control donde redefinir los parámetros de mallado. Como la propagación del oleaje está delimitada por el ángulo de Kelvin $\left(19.28^{\circ}\right)$, se ha orientado dicho volumen de control según este ángulo. La otra región de interés importante es el casco, y en particular los bordes de las superficies que lo componen. Por lo tanto en estas zonas se aumentará también la densidad de mallado. La Tabla 18 resume los principales parámetros de cada una de las zonas.

Tabla 18. Parámetros de mallado empleados en Star-CCM+

\begin{tabular}{|c|l|l|}
\hline GENERAL & \multicolumn{1}{|c|}{ SUSPERFICIE LIBRE } & \multicolumn{1}{c|}{ CASCO } \\
\hline - Tamaño base: $0.5 \mathrm{~m}$ & - Tamaño base: $0.5 \mathrm{~m}$ & • Tamaño base: $0.01 \mathrm{~m}$ \\
& ○ Dirección X: $0.1 \mathrm{~m}$ & - Tamaño bordes: $0.005 \mathrm{~m}$ \\
& ○ Dirección Y: $0.1 \mathrm{~m}$ & • Capa límite \\
& ○ Dirección Z: $0.002 \mathrm{~m}$ & ○ Anchura: $0.005 \mathrm{~m}$ \\
& - Volumen de control & № capas: 4 \\
& orientado 20 y altura & \\
& $\pm 0.08 \mathrm{~m}$ & \\
\hline
\end{tabular}

Modelos físicos, condiciones iniciales y de contorno

El cálculo del flujo alrededor de un buque conlleva el análisis de un cuerpo con seis grados de libertad localizado en la interfase entre dos fluidos, el cual se desplaza a un alto $R e$ y 
generando un tren de olas. Por ello, dentro de las opciones de cálculo del Star-CCM+ se han empleado las siguientes:

- Tridimensional

- No estacionario implícito

- 6 grados de libertad (6-DOF)

- Movimiento en los 6 grados de libertad

- Mezcla multifásica ${ }^{49}$

- Multifase euleriana

\section{- Volumen de fluido (VOF)}

○ Flujo segregado

- Ecuación de estado multifásica

- Turbulento

○ RANS

○ SST (Menter) K-Omega

○ All y+ Wall Treatment

- Gravedad

Si bien es cierto que el problema es estacionario, la convergencia es más rápida si se utiliza un método implícito no estacionario. El paso de tiempo se debe escoger para captar correctamente el fenómeno físico que se está resolviendo, en este caso la generación del oleaje. A su vez, puede necesitarse realizar muchas iteraciones en cada paso de tiempo cuando se esperan variaciones súbitas (típico en problemas de impacto), o bien pocas si la evolución es progresiva (problemas estacionarios, como en este trabajo). Teniendo en cuenta estas consideraciones, se escogieron:

- Paso de tiempo para el modelo no estacionario: $0.01 \mathrm{~s}$

- Condiciones de parada:

○ № máximo de iteraciones en cada paso de tiempo: 5

- Tiempo físico máximo de cálculo: $10 \mathrm{~s}$.

Por otro lado, la utilización del modelo 6-DOF requiere especificar las condiciones iniciales para su aplicación, caracterizadas mediante las siguientes variables:

- Tiempo para liberación ${ }^{50}: 0.1 \mathrm{~s}$.

\footnotetext{
${ }^{49}$ Se ha definido para las simulaciones la densidad del agua $997.561 \mathrm{~kg} / \mathrm{m}^{3}$ y la del aire $1.1841 \mathrm{~kg} / \mathrm{m}^{3}$

${ }^{50}$ Para los cálculos en los que se deja algún grado de libertad al movimiento, el tiempo para liberación indica el tiempo inicial en el que se mantiene el cuerpo fijo para evitar que las diferencias entre las condiciones iniciales y la solución final provoquen oscilaciones excesivas.
} 
- $\operatorname{Rampa}^{51}: 5.0 \mathrm{~s}$

En cuanto a los grados de libertad, el programa permite definir cuáles se dejan libres y cuáles están restringidos. En este caso dependerá de si está simulando una condición de trimado libre o no:

- Modelo fijo: Todos los grados de libertad están restringidos

- Modelo libre: Se permiten desplazamientos según el eje Z (asiento) y rotaciones alrededor del eje $\mathrm{Y}$ (trimado)

El último paso es definir la situación inicial del campo de velocidades del fluido y del reparto de agua/aire (condiciones iniciales), así como las condiciones de entrada/salida de fluido durante la realización de los cálculos (condiciones de contorno). Para su caracterización se ha definido una ola plana incidente, opción disponible en Star-CCM+ con los modelos físicos seleccionados. Esta corresponde a una condición sin oleaje, sólo con viento y corriente estacionarios, como es el caso de los ensayos de resistencia en aguas tranquilas. Para su definición es necesario especificar las siguientes variables:

- Punto de la superficie libre

- Dirección vertical

- Velocidad del aire

- Velocidad del agua

El punto y la dirección permiten al programa situar la superficie libre correctamente. El punto escogido es $[0.0,0.0,0.0] \mathrm{m}$ al estar el origen en la superficie libre. La dirección vertical es $[0.0,0.0,1.0]$, que indica desde el punto anterior hacia donde se sitúa el aire. Por otro lado, la velocidad del aire y del agua serán precisamente las velocidades a las que se desee realizar la simulación, por lo que se ajustarán en cada caso.

\footnotetext{
${ }^{51}$ Una vez liberado el modelo, la rampa indica el tiempo en el que gradualmente se van aplicando las fuerzas. El objetivo es el mismo que el del tiempo de liberación, evitar oscilaciones excesivas.
} 
La ventaja de utilizar este método radica en la facilidad con la que se definen las condiciones iniciales y de contorno. En lo que respecta a las primeras, para ajustar el reparto de agua/aire basta definir la mezcla multifásica en función de la ola. Se ubica el agua en el volumen de fluido de alta densidad de la ola, y el aire en el volumen de fluido de baja densidad. En lo que respecta al campo de velocidades es el de la ola. En el caso de las condiciones de contorno, para las superficies de entrada de fluido (velocity inlet) se define el campo de velocidades igual al de la ola, mientras que en las superficies de salida de fluido (pressure outlet) es la presión hidrostática calculada en función de la ola.

\section{Visualización de los resultados}

Star-CCM+ proporciona una serie de herramientas de post-proceso para analizar los resultados. En este trabajo se han utilizado las siguientes opciones:

- Ubicaciones geométricas:

- Iso-superficie que representa la superficie libre (Valor del VOF $=0.5$ )

- Cortes longitudinales de la superficie libre para extraer los cortes de olas

- Informes, monitores y gráficas asociadas de las siguientes variables:

- Resistencia longitudinal

- Asiento dinámico

○ Trimado dinámico

- Equilibrio de fuerzas verticales

- Equilibrio de momentos de trimado

- Gráficas

- Gráfica XY que contiene los cortes de olas calculados a partir del campo de elevaciones de la superficie libre

La función de estos elementos es doble. Una vez finalizados los cálculos, permiten analizar las variables de interés. Por otro lado, durante la simulación muestran la variación de todas estas variables en las iteraciones sucesivas, comprobando si la solución converge y físicamente tiene sentido. 


\subsubsection{Catamarán PESBO}

Los siguientes apartados recogen los principales resultados obtenidos en las simulaciones del catamarán PESBO con Star-CCM+. El conjunto completo de resultados se incluye más adelante en el apéndice C.2.

\section{Configuraciones simuladas}

Con el catamarán PESBO se han realizado un total de 32 simulaciones, como muestra la Tabla 19. Se han escogido las mismas velocidades y separaciones simuladas con Tdynlin, añadiendo el casco del monocasco, y considerando modelo fijo y libre para cada configuración.

En primer lugar, esto permite validar las elevaciones calculadas de la superficie libre con los cortes de olas experimentales, al igual que se realizó con Tdynlin. Por otro lado, la inclusión del monocasco permite calcular los factores de interferencia obtenidos numéricamente y correlacionarlos con los valores presentados anteriormente (sec. 6.2.4 y 6.2.5). Por último, estas configuraciones se escogieron como representativas de los fenómenos de interferencia favorable y desfavorable, por lo que permiten correlacionar el campo de presiones y velocidades sobre el casco con los fenómenos de interferencia.

Tabla 19. Matriz de simulaciones realizadas con el catamarán PESBO en Star-CCM+ y características de la malla empleada

\begin{tabular}{|c|c|c|c|c|c|}
\hline \multicolumn{3}{|c|}{ Configuración } & \multicolumn{2}{c|}{ Mallado } \\
\hline Condición & $F r$ & Separación & Na Nodos & No Caras & Na Celdas \\
\hline Modelo fijo & 0.322 & $S / L=0.207$ & 2210985 & 5924359 & 1981884 \\
Modelo libre & 0.375 & $S / L=0.298$ & 2215326 & 5936435 & 1984702 \\
& 0.398 & $S / L=0.388$ & 2223898 & 5964249 & 1994102 \\
& 0.410 & Monocasco & 1672761 & 4133130 & 1385337 \\
\hline
\end{tabular}

Una vez introducidos los parámetros de discretización y cálculo descritos en la sección. 7.2.1., Star-CCM+ genera la malla automáticamente. La Fig. 55 muestra el aspecto final de ésta para 
la separación menor del multicasco. La Fig. 56 a su vez detalla la discretización de la superficie libre para la separación mayor.
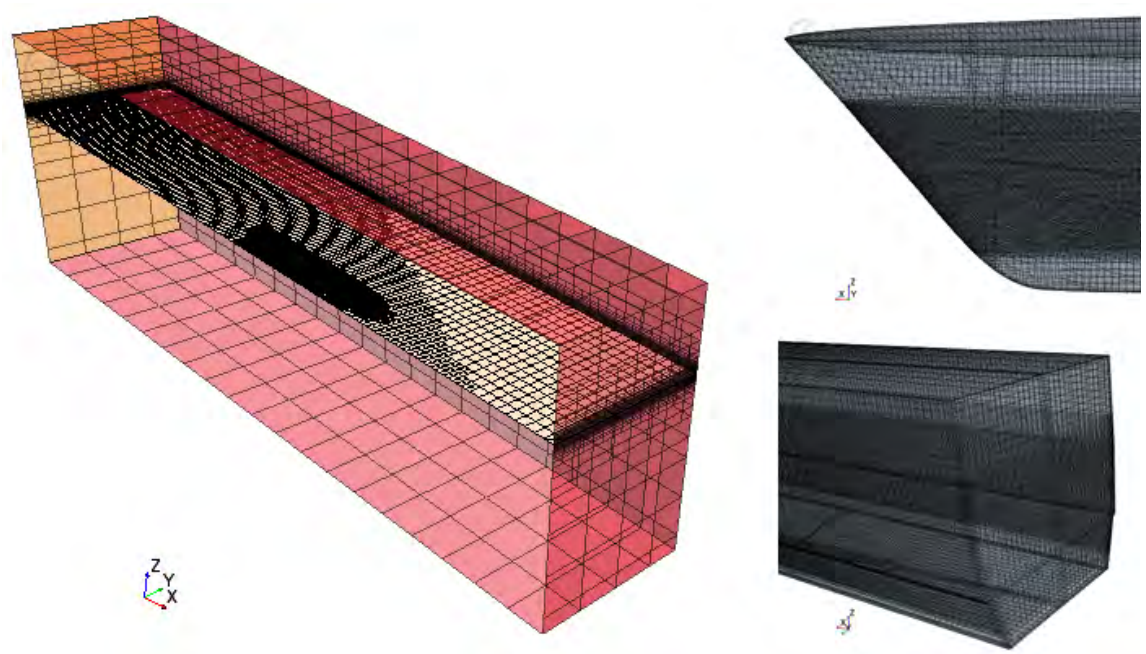

Fig. 55. Aspecto final del mallado del volumen de control del cat. PESBO en Star-CCM+ $(s / L=0.207)$ y detalle de la zona de proa (superior derecha) y popa (inferior derecha)

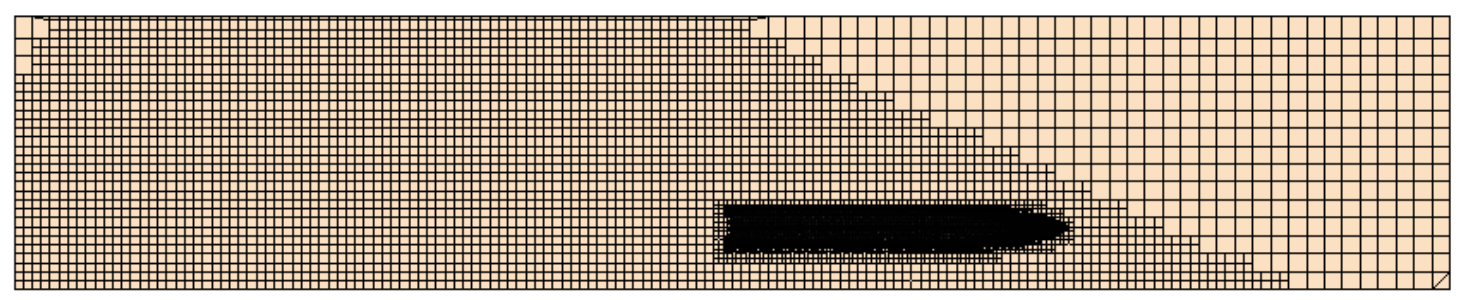

Fig. 56. Mallado de la superficie libre del cat. PESBO en Star-CCM+ $(s / L=0.388)$

\section{$\underline{\text { Resistencia al avance }}$}

Una vez completada cada simulación, se han calculado los valores de resistencia total al avance y asiento y trimado dinámicos. La Fig. 57 muestra gráficamente el conjunto de valores calculados en cuanto a resistencia. Se puede apreciar cómo el modelo libre presenta mayor resistencia que el modelo fijo, al igual que en los ensayos. No obstante, las conclusiones más interesantes se obtienen al comparar los valores calculados con los valores experimentales, como muestra la Fig. 58. Los valores calculados por Star-CCM+ muestran diferencias menores entre el catamarán y el monocasco que las curvas experimentales, lo cual dificulta apreciar la joroba mostrada en los últimos. Aún así, los valores calculados reproducen correctamente las 
tendencias medidas experimentalmente: con la separación menor, $s / L=0.207$, la resistencia del catamarán es superior al monocasco en todos los casos; al aumentar la separación, la resistencia del catamarán pasa a ser inferior al monocasco en los $F r$ intermedios; al fijar el modelo, el rango donde este fenómeno ocurre se extiende al $F r$ superior.

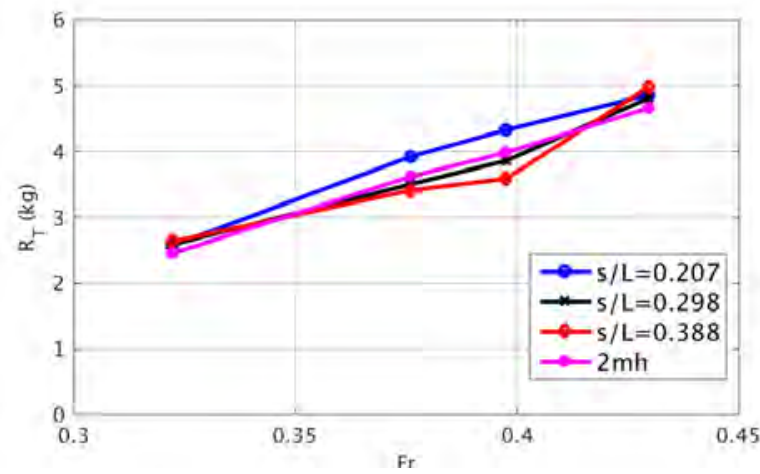

(a) Modelo libre

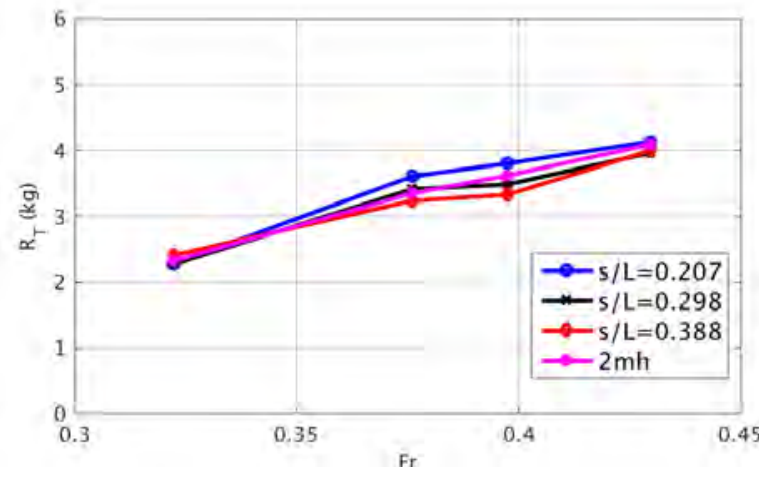

(b) Modelo fijo

Fig. 57. Curvas de $\boldsymbol{R}_{T}$ en función del Fr del cat. PESBO calculadas con Star-CCM+

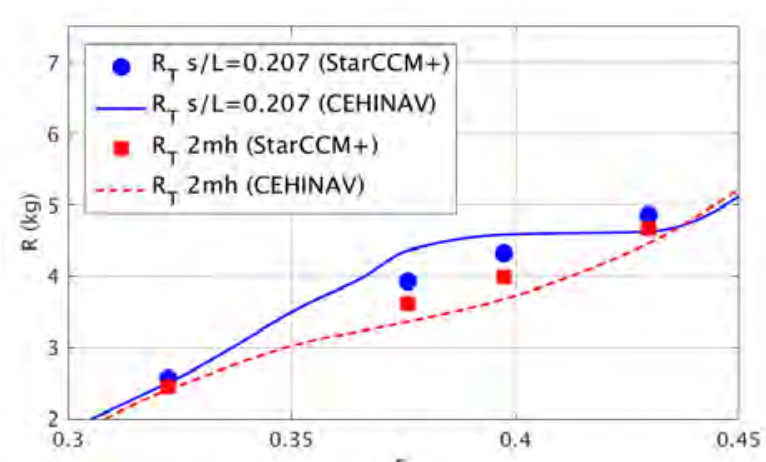

(a) $s / L=0.207$. Modelo libre

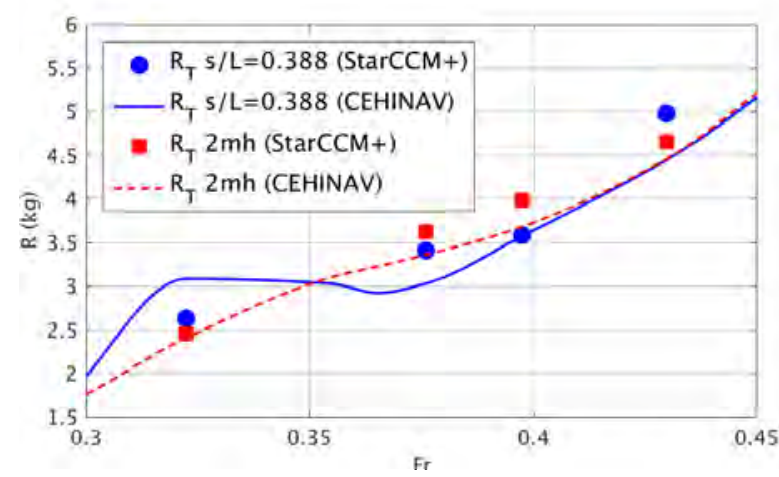

(c) $s / L=0.388$. Modelo libre

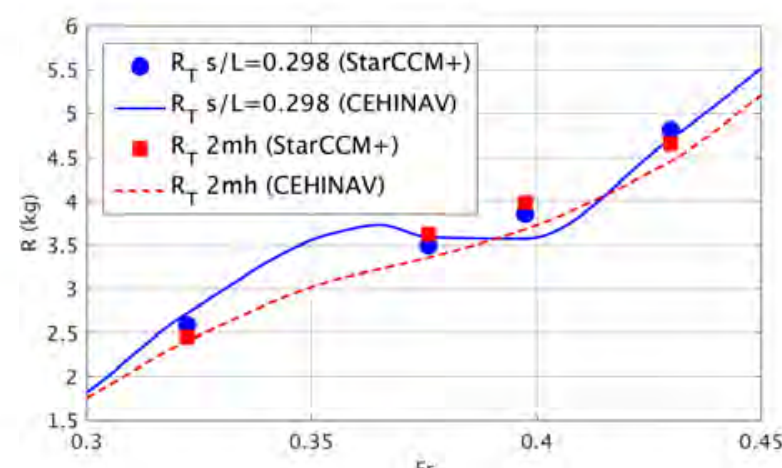

(b) $s / L=0.298$. Modelo libre

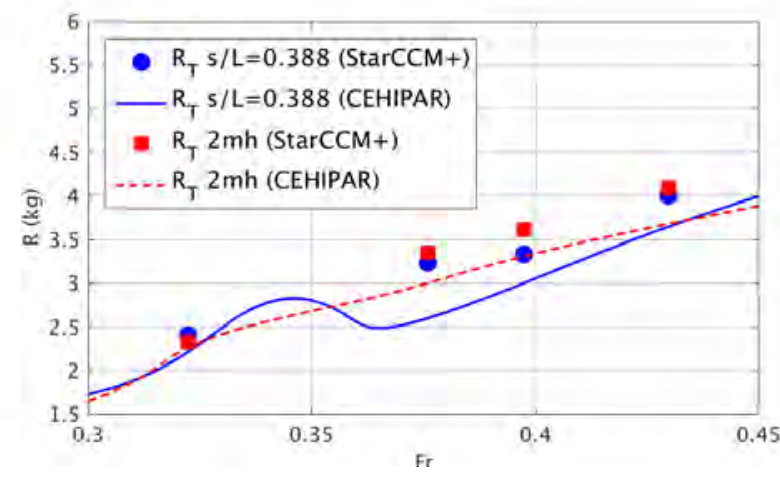

(d) $s / L=0.388$. Modelo fijo

Fig. 58. Detalle de las curvas de $\boldsymbol{R}_{T}$ del cat. PESBO calculadas con Star-CCM+ vs. experimentales 


\section{Factores de interferencia}

Al igual que en el caso de los datos experimentales, a partir de las curvas de resistencia podemos calcular los factores de interferencia, IF. La Fig. 64 muestra la comparativa con los valores medidos experimentalmente. Como se anticipó al analizar las curvas de resistencia, las simulaciones arrojan diferencias menores entre el catamarán y el monocasco, lo que se traduce en magnitudes del IF más bajas. No obstante, las tendencias en cuanto a las zonas de interferencia positiva y negativa son consistentes con los valores experimentales en prácticamente todos los casos.

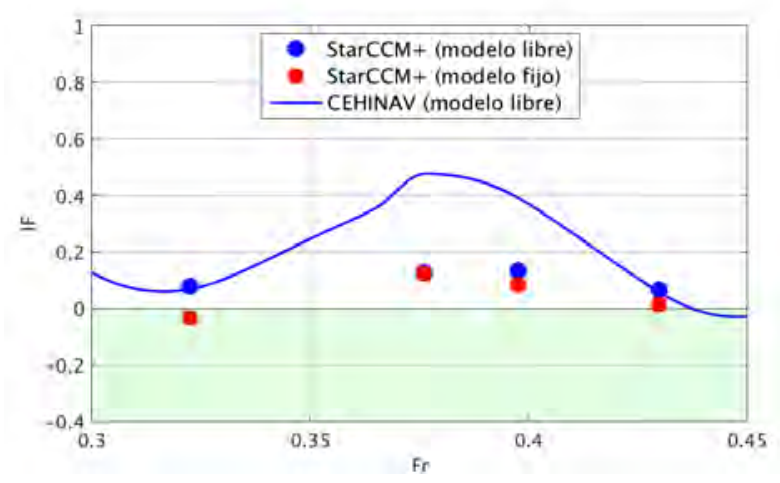

(a) $s / L=0.207$

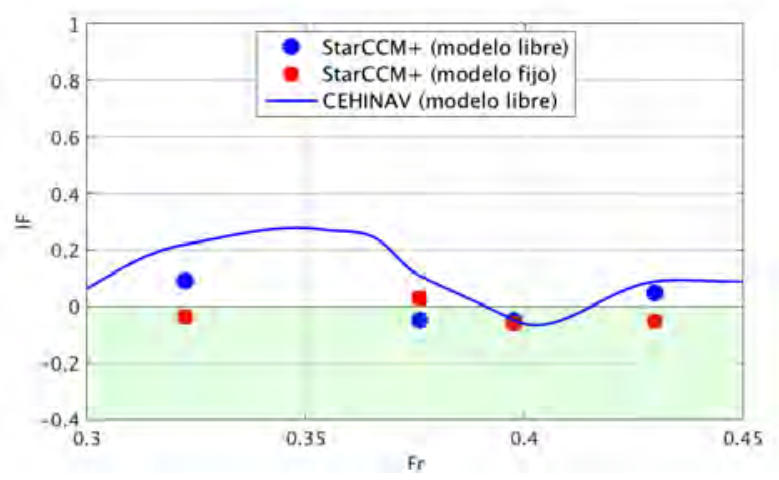

(b) $s / L=0.298$

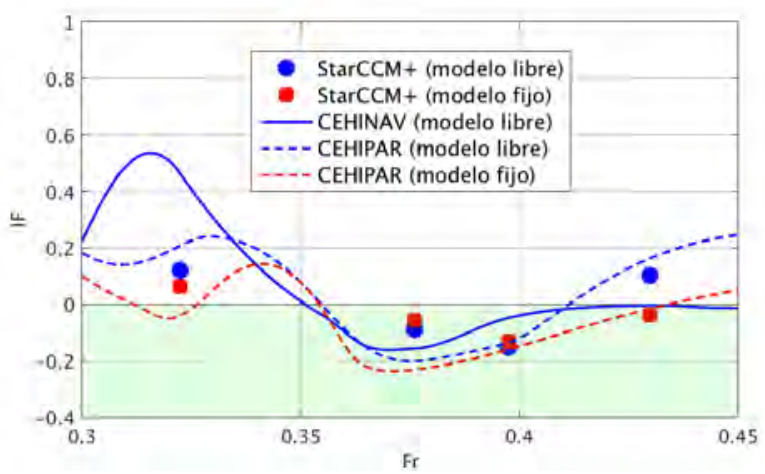

(c) $s / L=0.388$

Fig. 59. IF del cat. PESBO calculados con Star-CCM+ vs. valores experimentales

\section{Asiento y trimado dinámicos}

La Fig. 60 muestra las curvas de trimado y asiento dinámicos adimensionales (ec. (6.7) y (6.8)) calculados en las condiciones de trimado libre, comparándolas con los datos experimentales. 


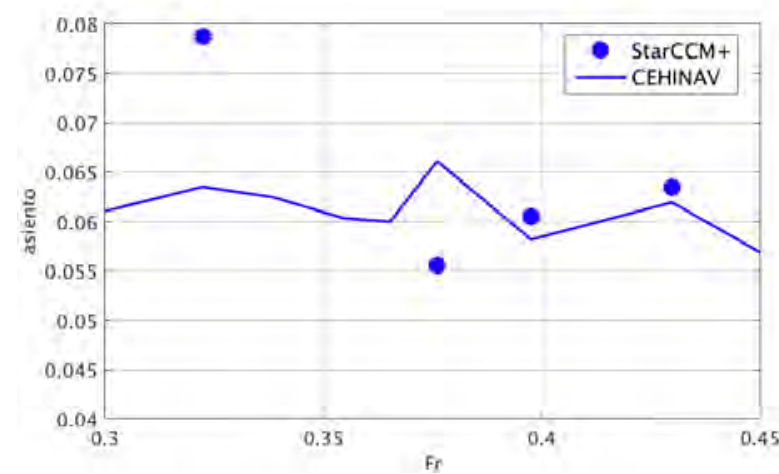

(a) Asiento dinámico $(s / L=0.207)$

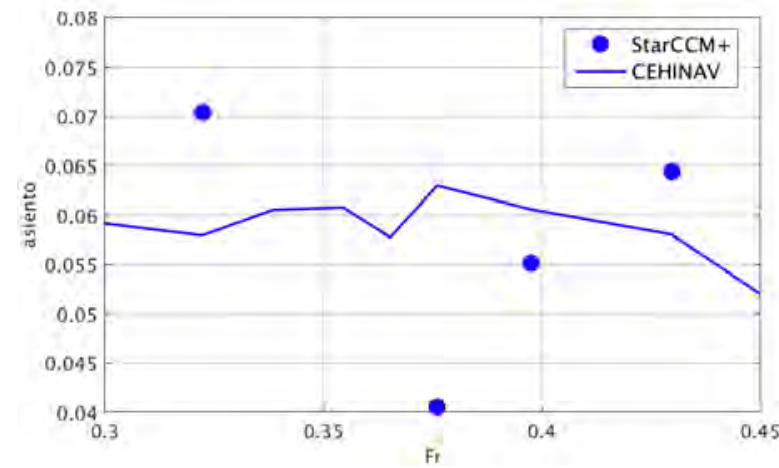

(c) Asiento dinámico $(s / L=0.298)$

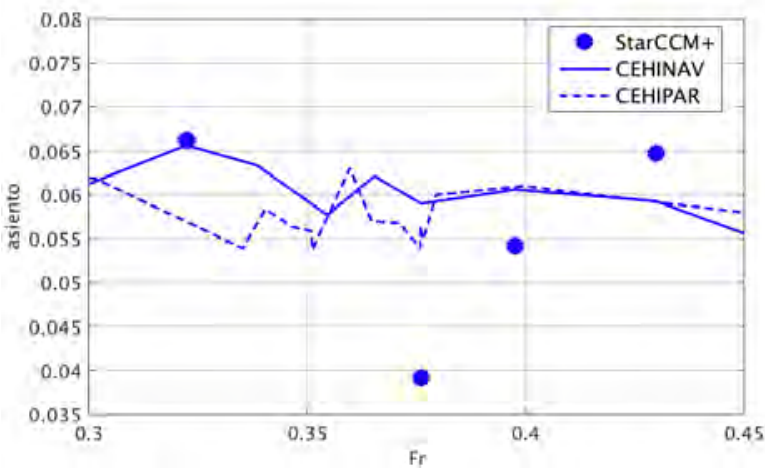

(e) Asiento dinámico $(s / L=0.388)$

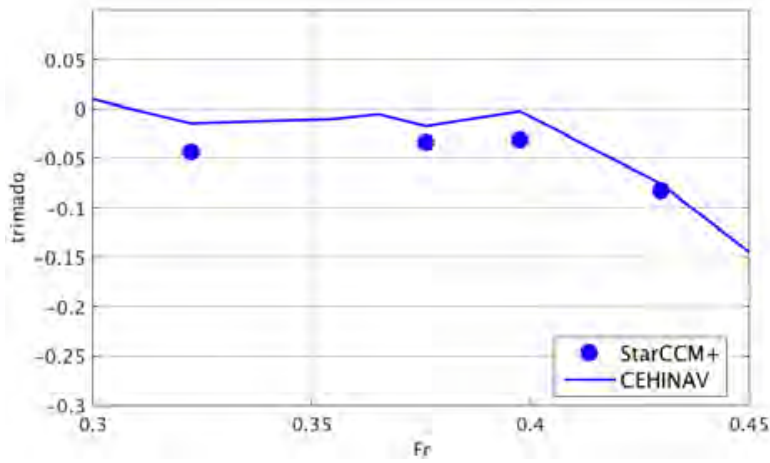

(b) Trimado dinámico $(s / L=0.207)$

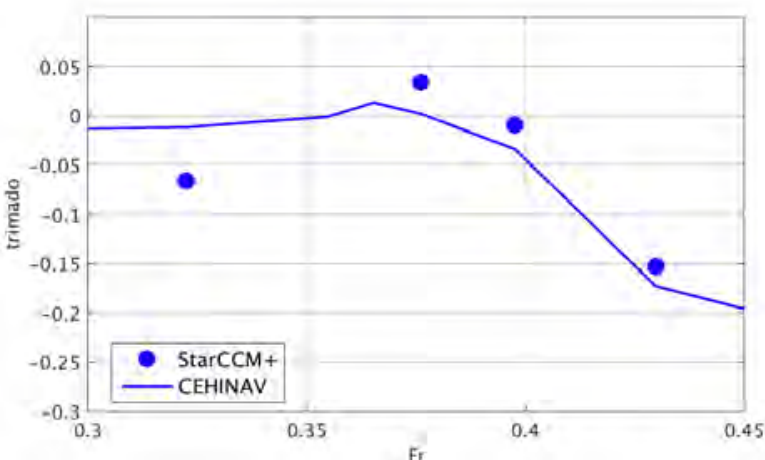

(d) Trimado dinámico $(s / L=0.298)$

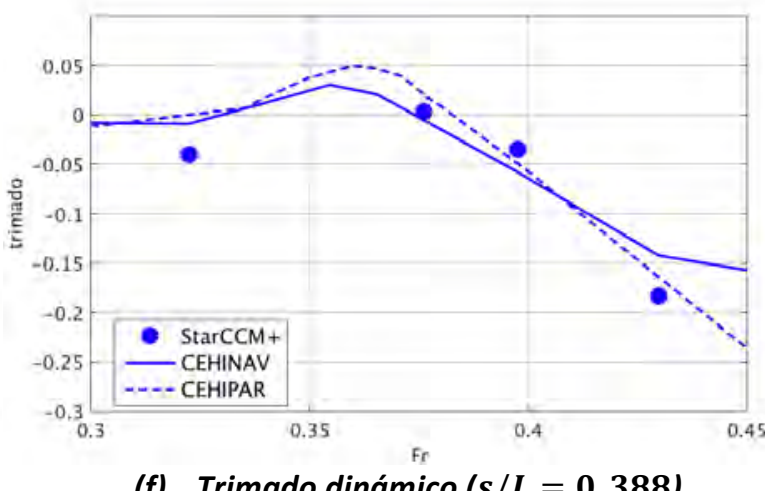

(f) Trimado dinámico $(s / L=0.388)$

Fig. 60. Asiento y trimado dinámicos del cat. PESBO calculados con Star-CCM+ vs. valores experimentales

Los valores predichos por Star-CCM+ aproximan adecuadamente los valores experimentales, especialmente en el caso del trimado donde se puede apreciar claramente la correlación entre ambos. En el caso del asiento, a pesar de encontrarse desviaciones mayores hay que tener en cuenta su rango de variaciones significativamente menor frente al del trimado. 


\section{Cortes de ola}

Al igual que con el Tdynlin (sec. 7.1.1), la Fig. 61 muestra los cortes de olas centrales calculados con Star-CCM+ para los casos correspondientes a las separaciones mayor y menor con el Fr más alto y bajo. La correlación encontrada entre los cortes de olas numéricos y experimentales es buena, extendiéndose al conjunto completo de cortes calculados con esta geometría recogido en el apéndice C.2. La precisión de los resultados es mejor en la zona de proa donde los efectos potenciales son dominantes, a semejanza de Tdynlin, reproduciéndose con Star-CCM+ mejor el comportamiento aguas abajo. En cuanto al asiento y trimado dinámicos, las diferencias encontradas al ser incluidos son pequeñas. No obstante, su consideración conlleva en prácticamente todos los casos un acercamiento a los datos experimentales. Permite además capturar mejor la amplitud de los máximos, la cual tiende a ser infra-estimada. Por último, se observan ciertas inestabilidades en la zona del espejo, síntoma de posibles olas rompientes.

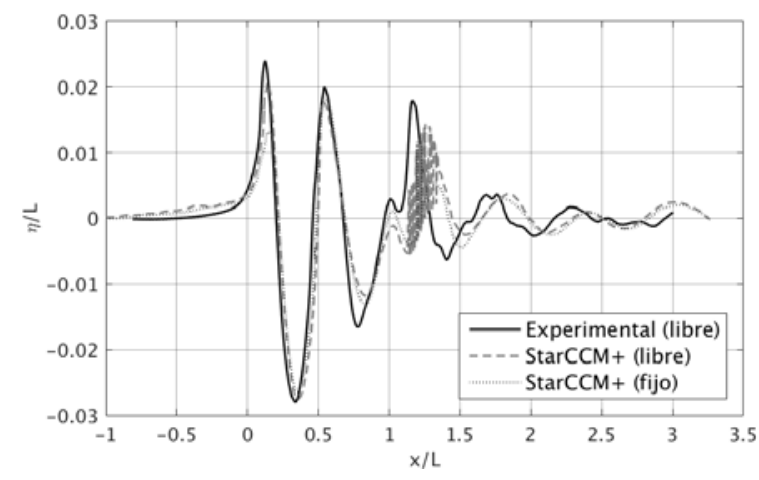

(a) $s / L=0.207 \quad F r=0.322$

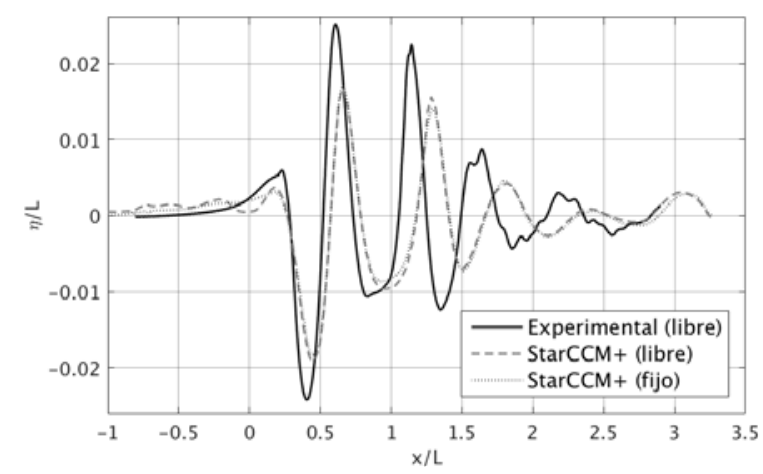

(c) $s / L=0.388 \quad F r=0.322$

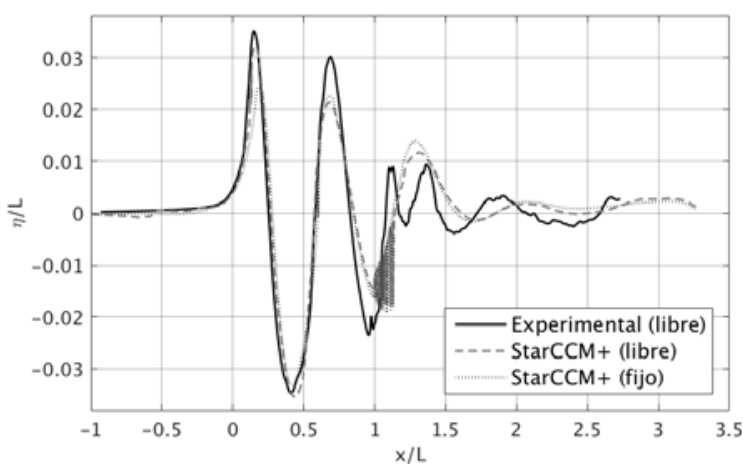

(b) $s / L=0.207 \quad F r=0.430$

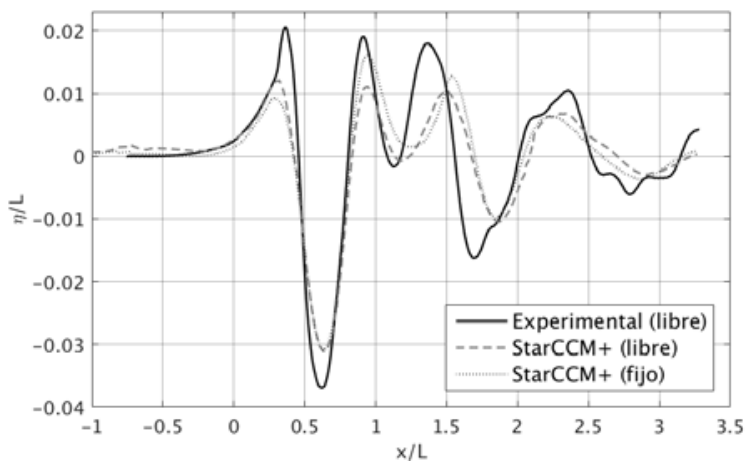

(d) $s / L=0.388 \quad F r=0.430$

Fig. 61. Comparativa de los cortes de ola centrales del cat. PESBO calculados con Star-CCM+ vs. experimentales 


\section{Campo de presiones y elevaciones}

Como se anticipó anteriormente, una de las principales utilidades de las herramientas numéricas es la visualización completa del campo presiones sobre el casco, así como de elevaciones de la superficie libre. La Fig. 62(a) y Fig. 63(a) muestran resultados correspondientes a las condiciones de interferencia más desfavorable, recogiéndose en la Fig. 62(b) y Fig. 63(b) los de interferencia más favorable.

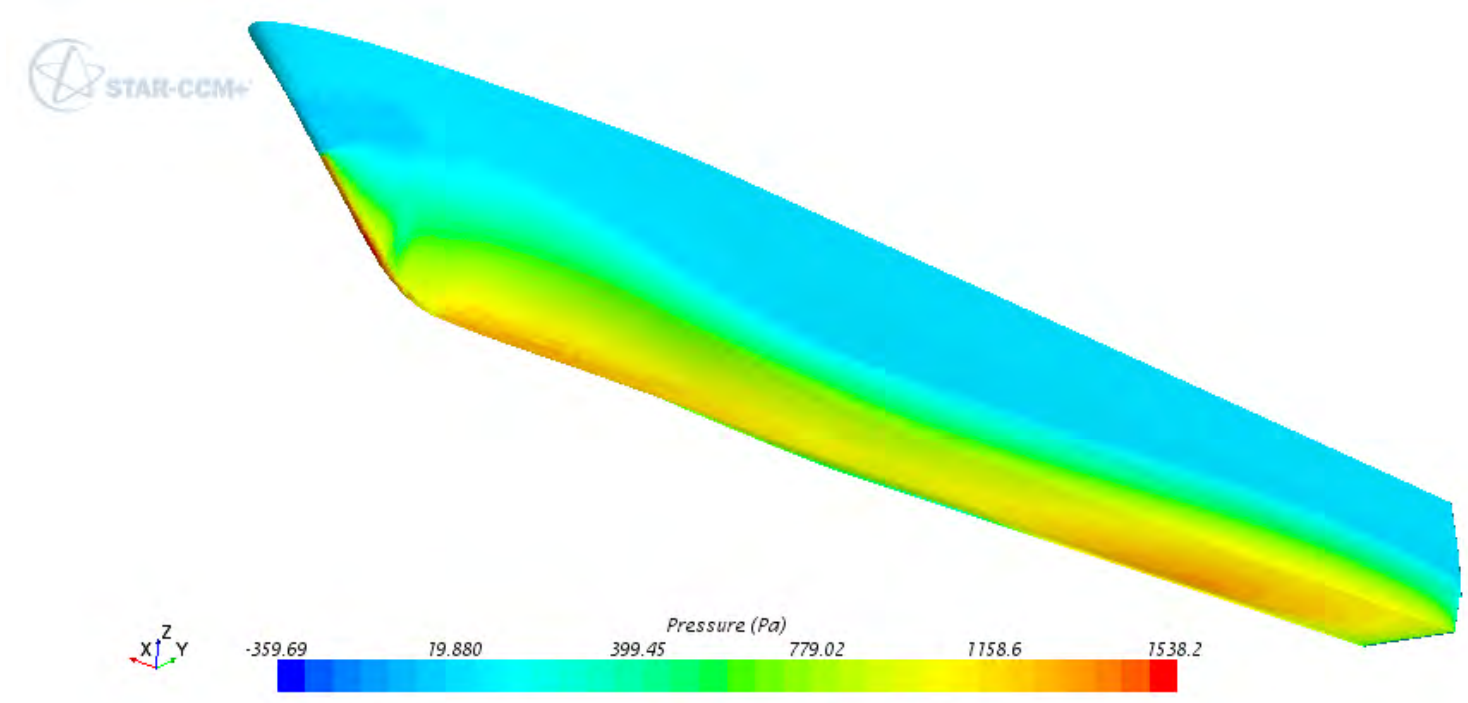

(a) $s / L=0.207, F r=0.375$, modelo libre

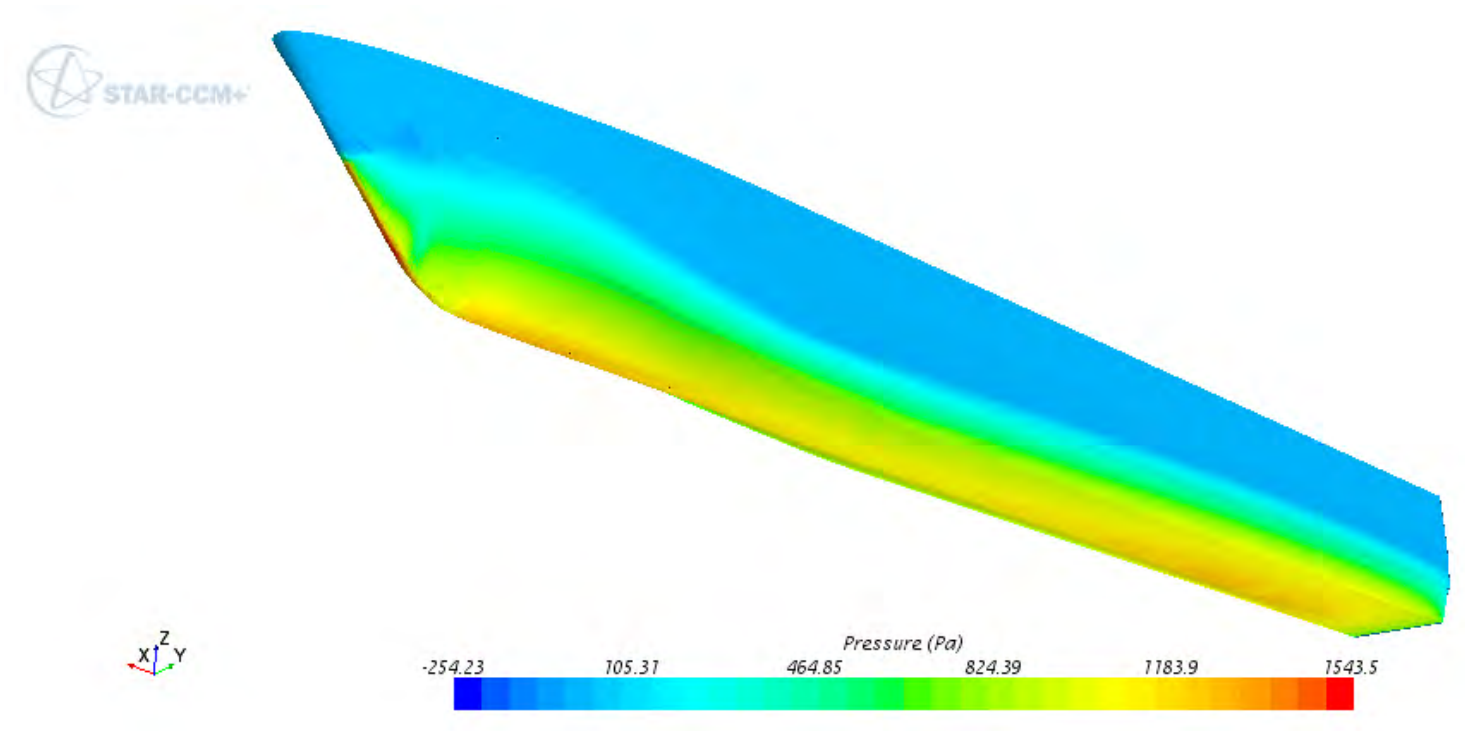

(b) $s / L=0.388, F r=0.375$, modelo libre

Fig. 62. Campos de presiones sobre el casco del cat. PESBO calculados con Star-CCM+ 

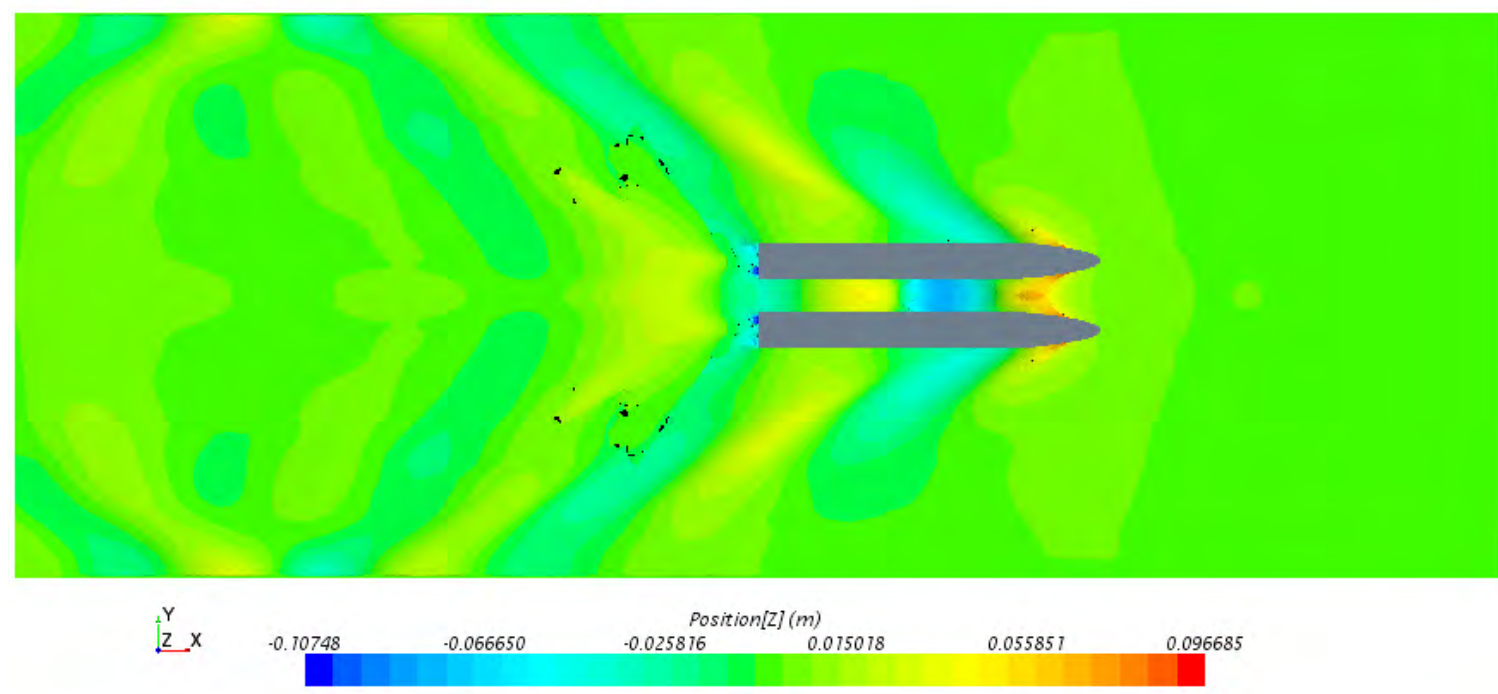

(a) $s / L=0.207, F r=0.375$, modelo libre

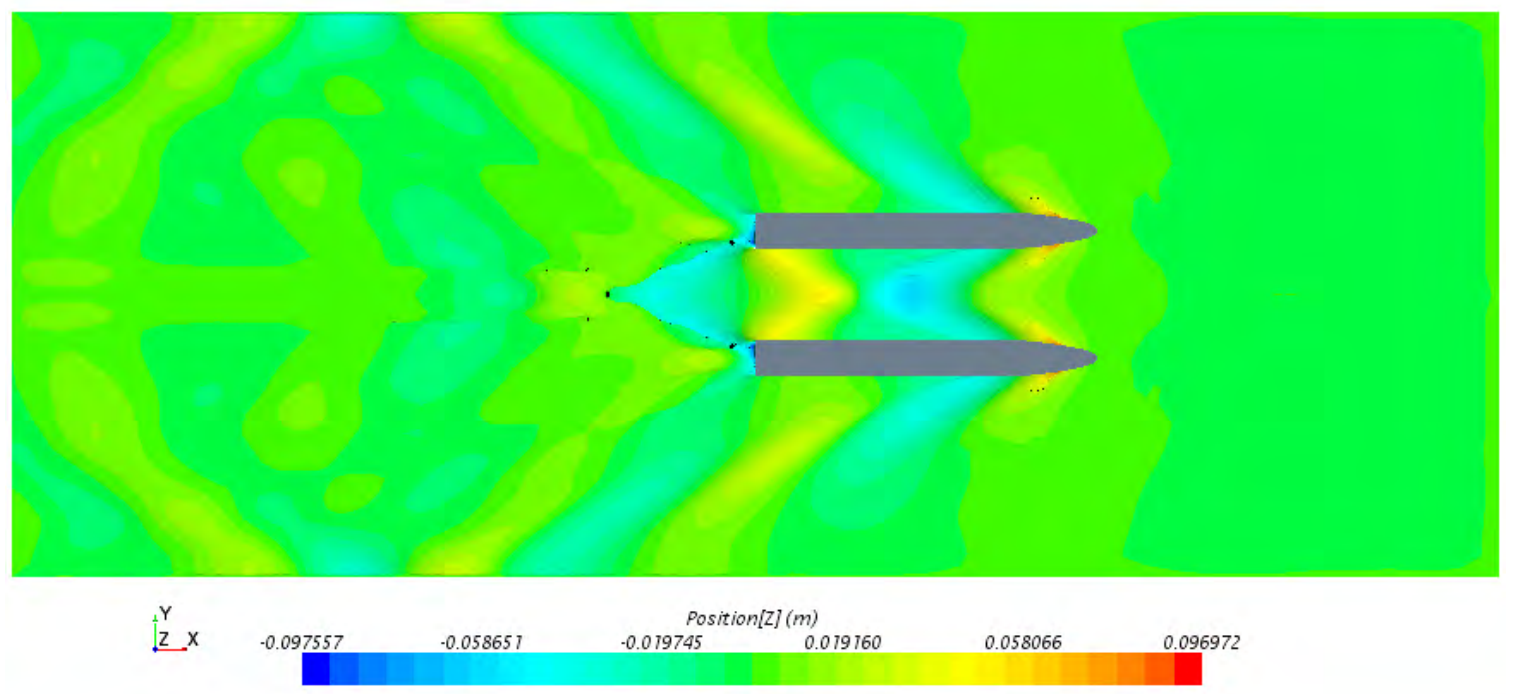

(b) $s / L=0.388, F r=0.375$, modelo libre

Fig. 63. Campo de elevaciones de la superficie libre con el cat. PESBO calculados con Star-CCM+

En primera instancia, el análisis del campo de presiones sobre el casco no arroja una gran luz sobre su relación con los fenómenos de interferencia. En ambos casos, el perfil de presiones responde al esperado en una embarcación de este tipo, siendo las diferencias entre ambas un orden de magnitud inferior excepto en zonas puntuales. 
Por el contrario, los campos de elevaciones en la Fig. 63 muestran diferencias significativas, confirmando las conclusiones extraídas del análisis experimental de cortes de olas (sec. 6.4). La separación menor, $s / L=0.207$, muestra amplitudes mayores del oleaje en la zona entre cascos, consistente con el hecho de corresponder a la condición de interferencia más desfavorable. En el caso de la separación mayor, $s / L=0.388$, la zona de interferencia se extiende a una mayor superficie si bien el campo de elevaciones resultante presenta amplitudes menores. Dado que la energía cedida al oleaje depende del cuadrado de las elevaciones, esto se traduce en una menor resistencia y por ende una interferencia favorable. 


\subsubsection{Catamarán $S 60$}

\section{Configuraciones simuladas}

En el caso del catamarán $\mathrm{S} 60$ se han simulado un total de 50 casos. Se han escogido cinco velocidades representativas, simulándose las condiciones de modelo fijo y libre para cada separación como resume la Tabla 20. Los Fr simulados se escogieron para capturar las zonas de máxima y mínima interferencia (Fig. 34 y Fig. 35), y a la vez cubrir de forma relativamente uniforme el rango de velocidades ensayadas. La Fig. 64 muestra el aspecto final de la malla generada para la separación menor, detallándose en la Fig. 65 la discretización de la superficie libre en el caso de la separación mayor.

Tabla 20. Matriz de simulaciones realizadas con el catamarán S60 en Star-CCM+ y características de la malla empleada

\begin{tabular}{|c|c|c|c|c|c|}
\hline \multicolumn{3}{|c|}{ Configuración } & \multicolumn{3}{|c|}{ Mallado } \\
\hline Condición & $\boldsymbol{F r}$ & Separación & Na Nodos & № Caras & Na Celdas \\
\hline Modelo fijo & 0.335 & $s / L=0.226$ & 2568644 & 7038364 & 2373726 \\
\hline \multirow[t]{4}{*}{ Modelo libre } & 0.350 & $s / L=0.307$ & 2565194 & 7029914 & 2370098 \\
\hline & 0.376 & $s / L=0.388$ & 2553965 & 6997958 & 2359666 \\
\hline & 0.400 & $s / L=0.470$ & 2571069 & 7046855 & 2375600 \\
\hline & 0.430 & Monocasco & 1446962 & 3905374 & 1332251 \\
\hline
\end{tabular}
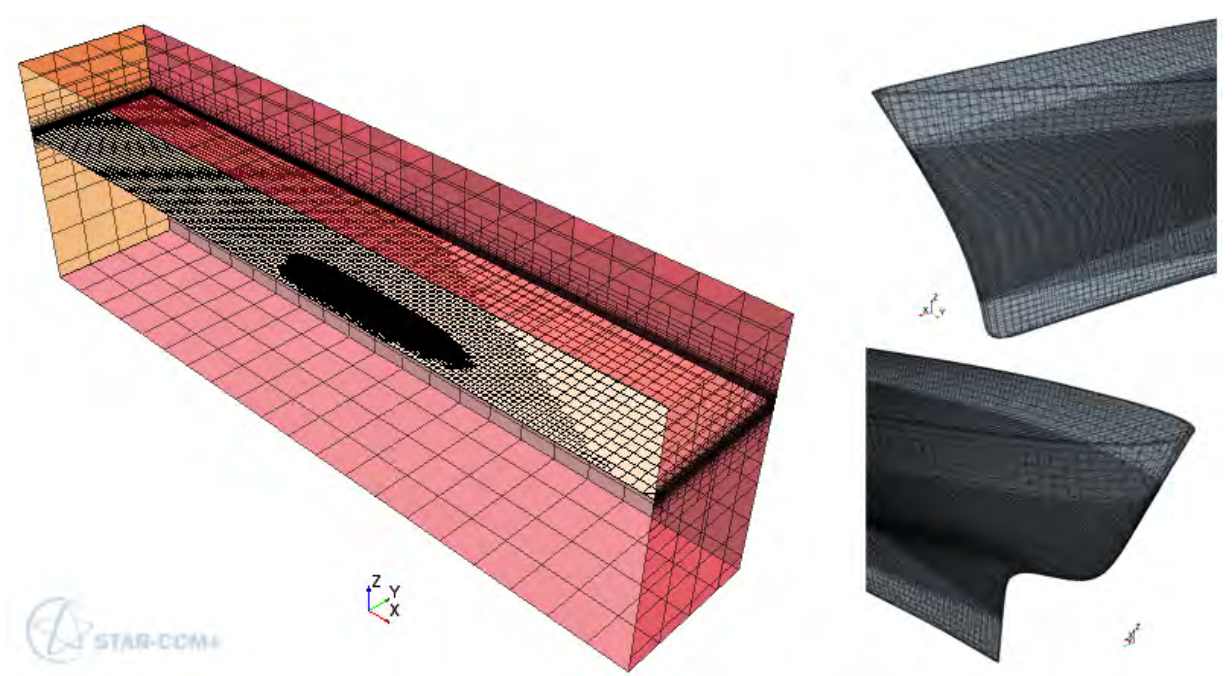

Fig. 64. Aspecto final del mallado del volumen de control del cat. $S 60$ en Star-CCM+ $(s / L=0.226)$ y detalle de la zona de proa (superior derecha) y popa (inferior derecha) 


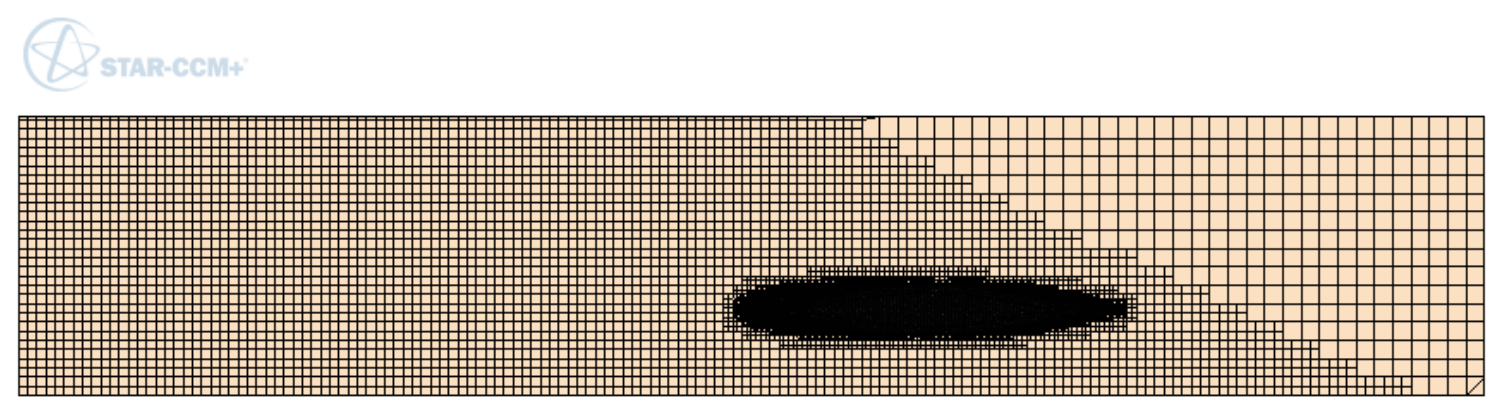

Fig. 65. Mallado de la superficie libre del cat. S60 en Star-CCM+ (s/L=0.470)

\section{$\underline{\text { Resistencia al avance }}$}

Una vez completadas las simulaciones, la Fig. 66 recoge el conjunto de valores calculados en cuanto a resistencia. Al igual que con el cat. PESBO, los valores de resistencia obtenidos con el modelo libre son mayores que al restringir el asiento y trimado dinámicos. Por otro lado, al comparar las predicciones numéricas con las curvas experimentales (Fig. 67), se aprecia cómo en este caso las desviaciones encontradas son mucho menores. La comparativa del catamarán y monocaso muestra de nuevo como Star-CCM+ es capaz de capturar correctamente la relación entre ambos, indicando en este caso más claramente las jorobas encontradas experimentalmente.

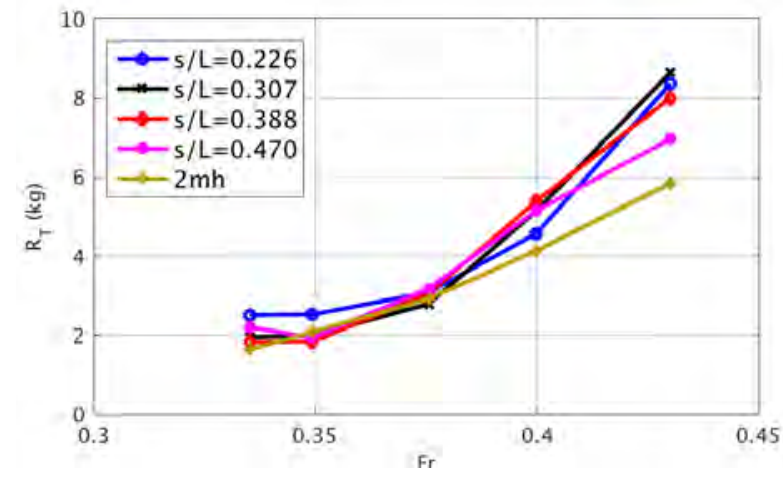

(a) Modelo libre

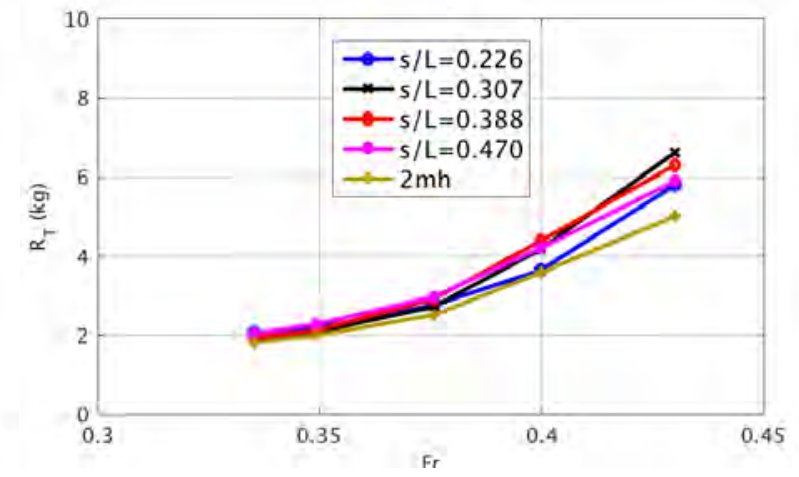

(b) Modelo fijo

Fig. 66. Curvas de $R_{T}$ en función del Fr del cat. S60, modelo libre 


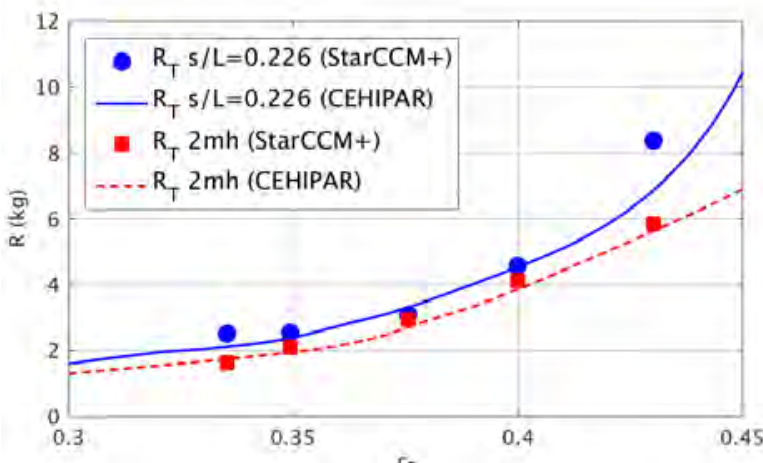

(a) $s / L=0.226$. Modelo libre

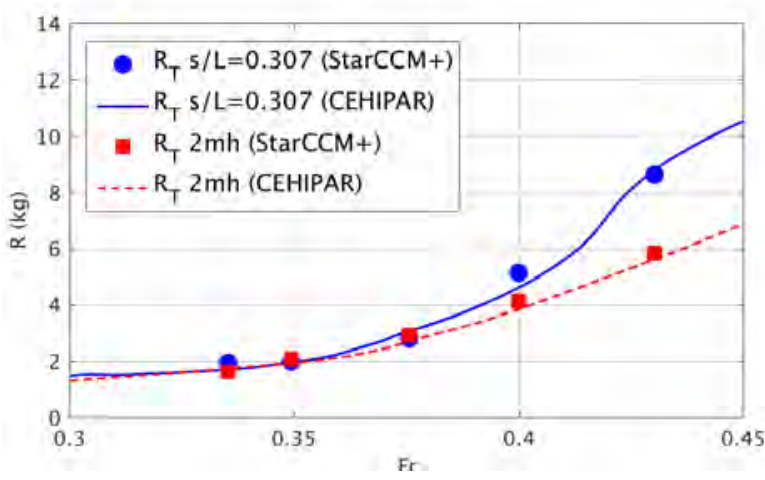

(c) $s / L=0.307$. Modelo libre

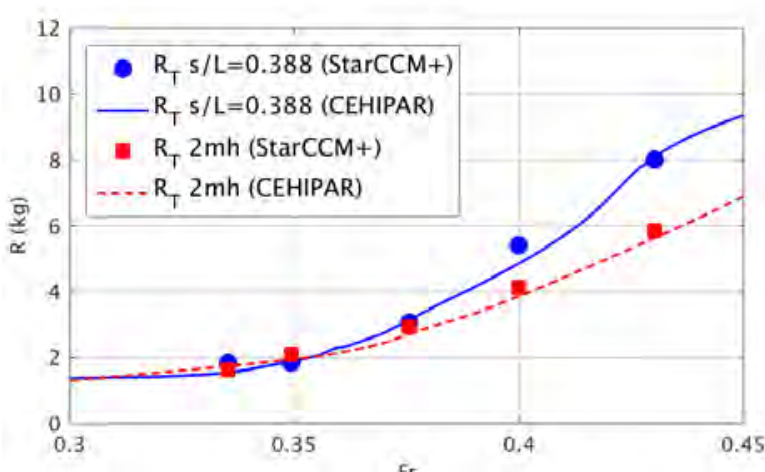

(e) $s / L=0.388$. Modelo libre

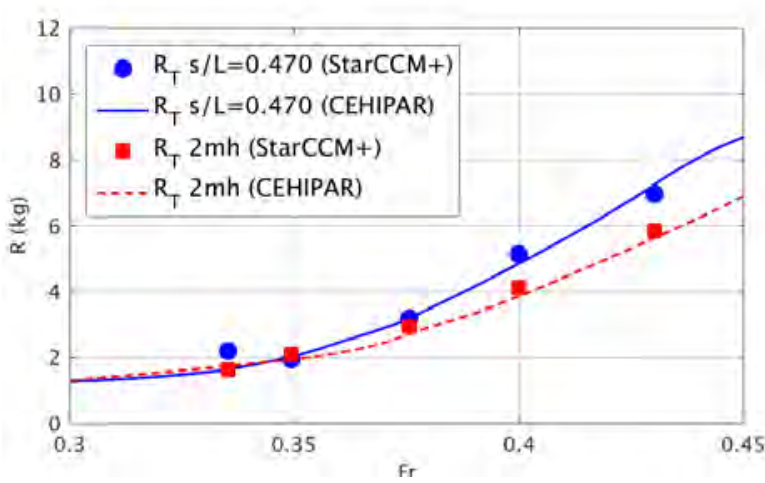

(g) $s / L=0.470$. Modelo libre

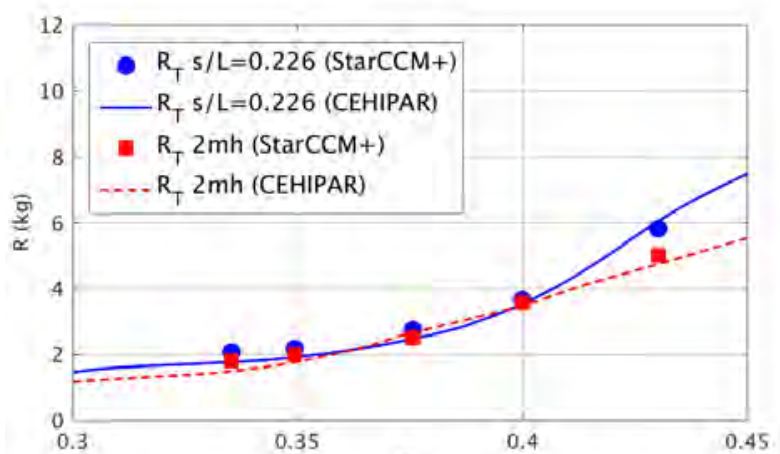

(b) $s / L=0.226$. Modelo fijo

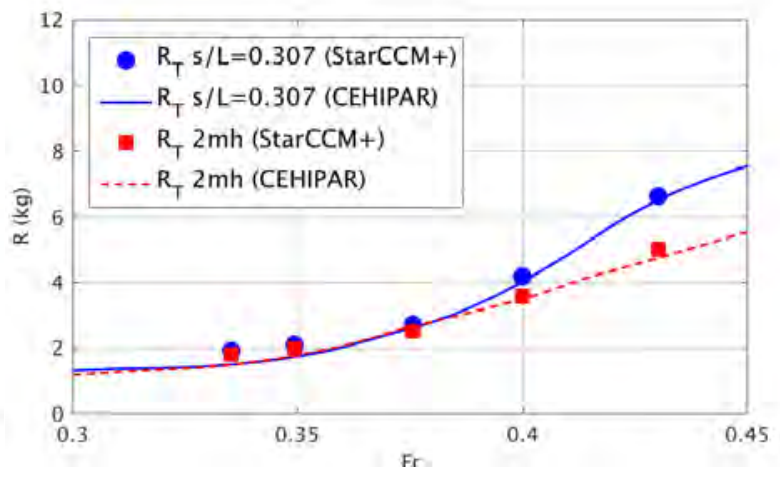

(d) $s / L=0.307$. Modelo fijo
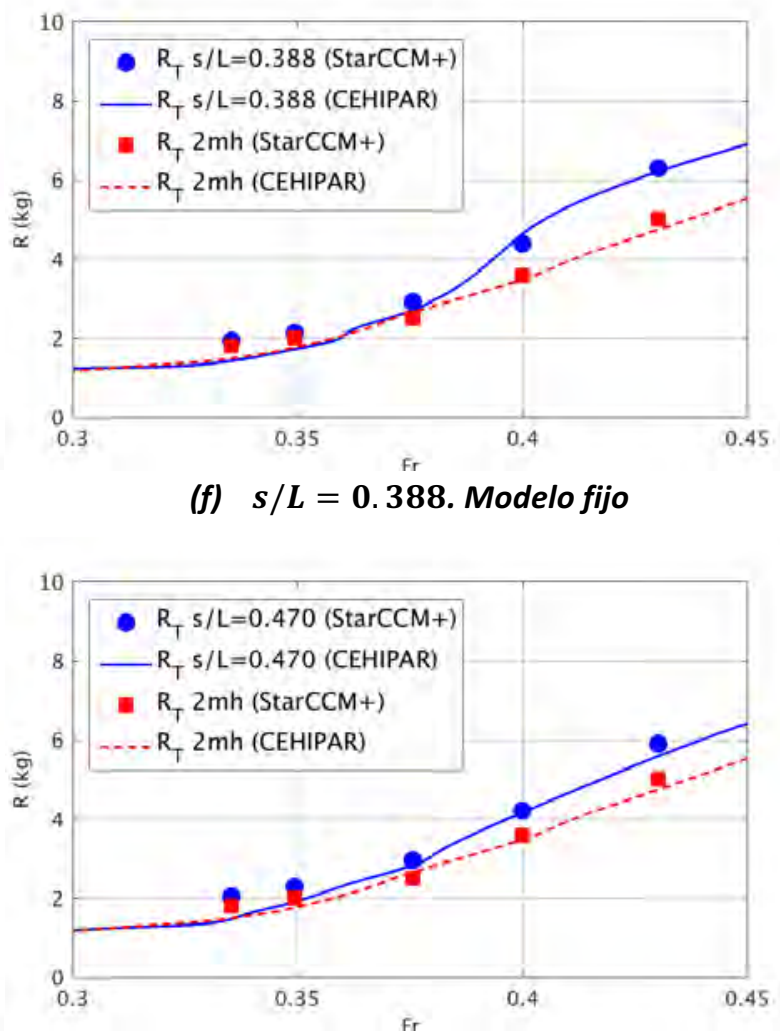

(h) $s / L=0.470$. Modelo fijo

Fig. 67. Valores calculados de $\boldsymbol{R}_{T}$ el catamarán $S 60$ con Star-CCM+ vs. mediciones 


\section{Factores de interferencia}

La Fig. 68 muestra los valores del $I F$ obtenidos a partir de los valores de resistencia calculados con Star-CCM+. Las predicciones numéricas muestran una buena concordancia con las curvas experimentales, encontrándose las mayores desviaciones con el $\mathrm{Fr}$ menor donde predominan los efectos viscosos. No obstante, al analizar la posición relativa entre los valores del $I F$ numéricos del modelo fijo y libre se observa como en general no coinciden con las curvas experimentales en la zona central. Más aún, a pesar del reducido número de puntos calculados, se puede apreciar desviaciones significativas en la ubicación de los mínimos del $I F$ (mayor interferencia favorable) calculados frente a los experimentales. Ambos hechos son síntoma de la dificultad de Star-CCM+ para predecir las zonas de interferencia favorable, al igual que se observó con el modelo simplificado de Yeung et al. (2004).

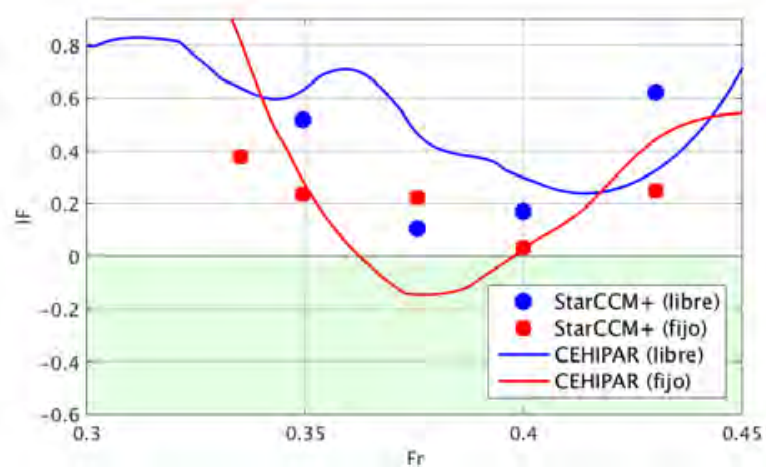

(a) $s / L=0.226$

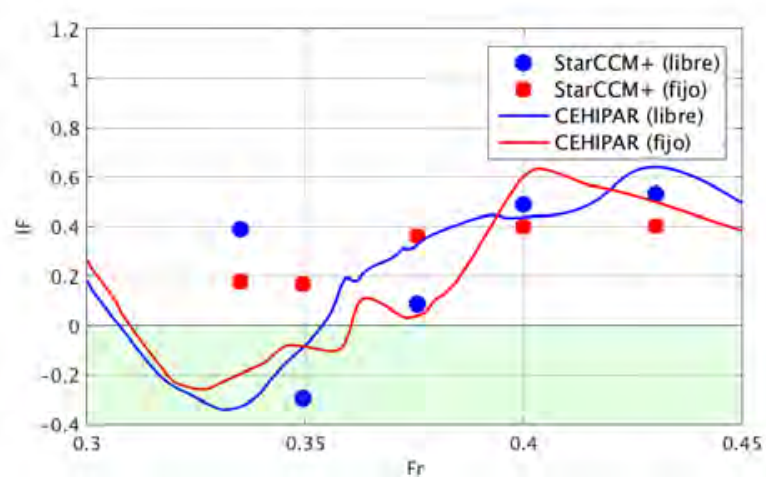

(c) $s / L=0.388$

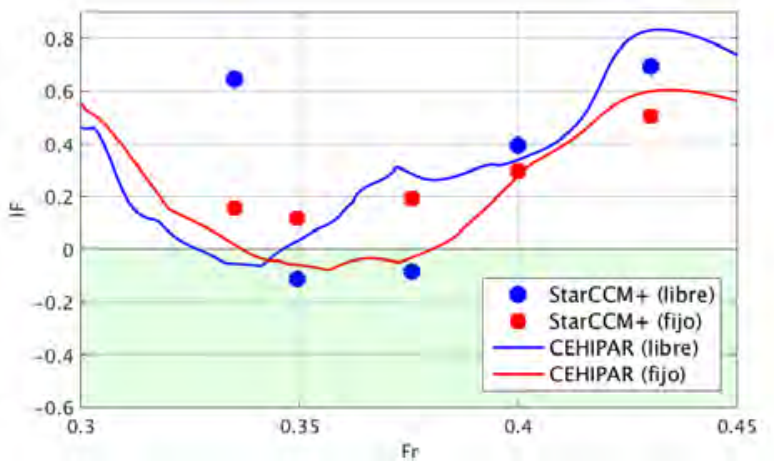

(b) $s / L=0.307$

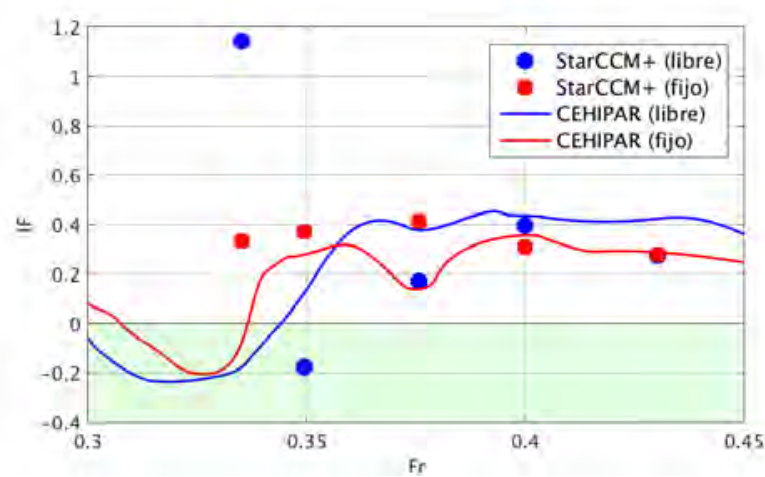

(d) $s / L=0.470$

Fig. 68. IF del cat. S60 calculados con Star-CCM+ vs. valores experimentales 


\section{Asiento y trimado dinámicos}

La Fig. 69 muestra las curvas de asiento y trimado dinámicos adimensionales (ec. (6.7) y (6.8)) calculados en las condiciones de trimado libre, comparándolas con los datos experimentales. En ambos casos los valores calculados reproducen correctamente el comportamiento experimental, mostrando desviaciones aún menores que con el cat. PESBO.

En el caso del asiento, la tendencia inicial es a aumentar a medida que aumenta el $\mathrm{Fr}$, revirtiéndose al alcanzar $\mathrm{Fr}$ altos y acercarse a la zona de planeo. Las curvas de trimado a su vez presentan valores iniciales relativamente constantes disminuyendo a continuación a medida que aumenta el Fr. A pesar de reproducir la tendencia experimental, es necesario mencionar el desfase sistemático que se aprecia en estas últimas para todas las separaciones. La buena correlación entre las simulaciones numéricas y experimentales en cuanto a resistencia inducen a atribuir esta desviación como parte del post-proceso de las diferentes variables calculadas. 


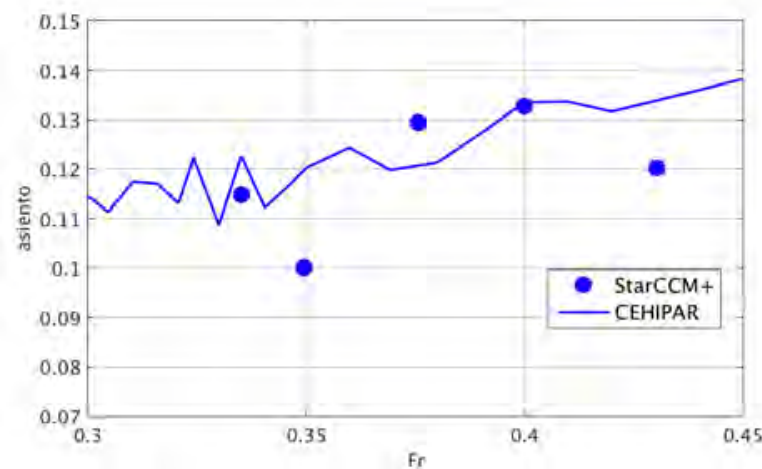

(a) Asiento dinámico $(s / L=0.226)$

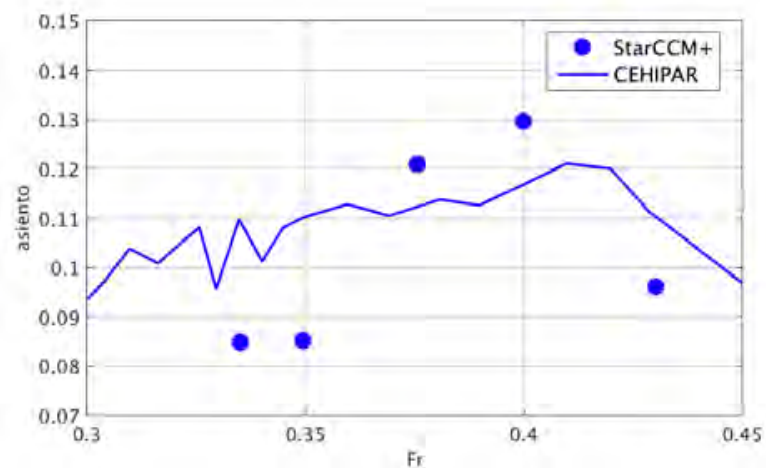

(c) Asiento dinámico $(s / L=0.307)$

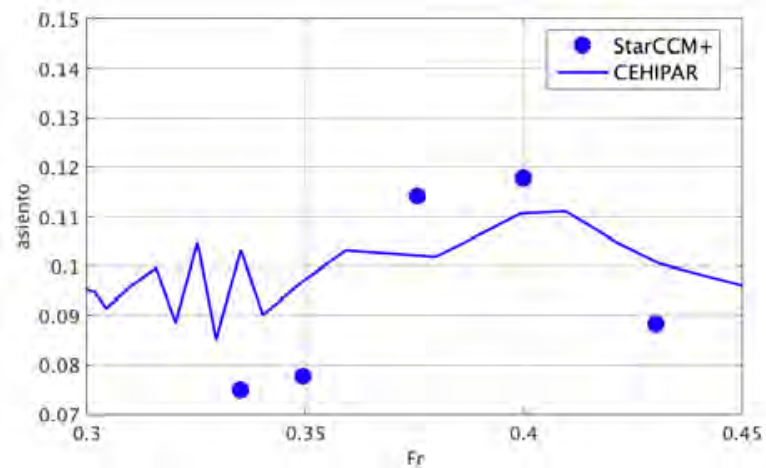

(e) Asiento dinámico $(s / L=0.388)$

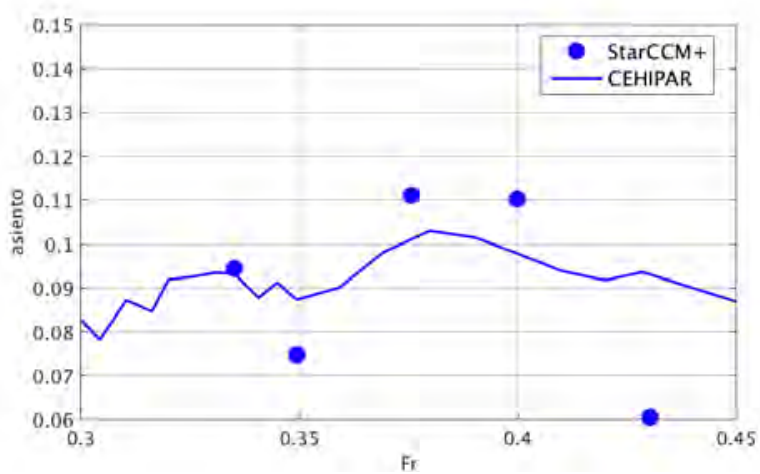

(g) Asiento dinámico $(s / L=0.470)$

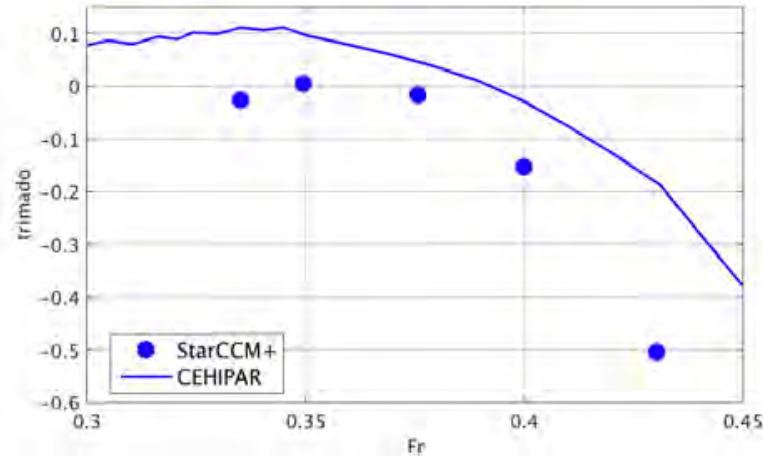

(b) Trimado dinámico $(s / L=0.226)$

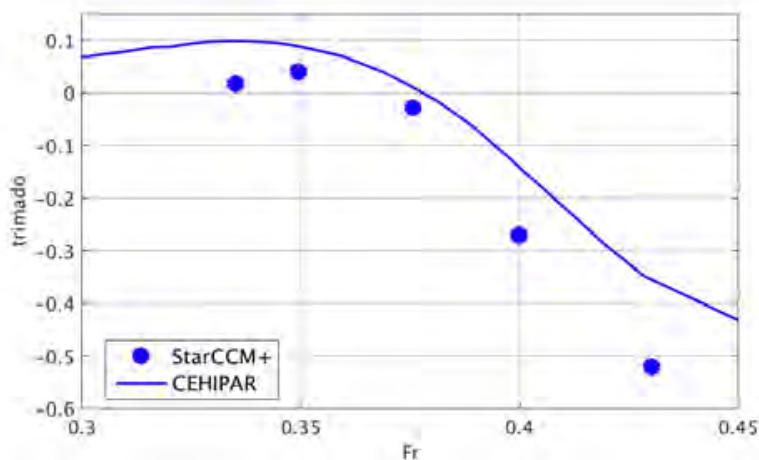

(d) Trimado dinámico $(s / L=0.307)$

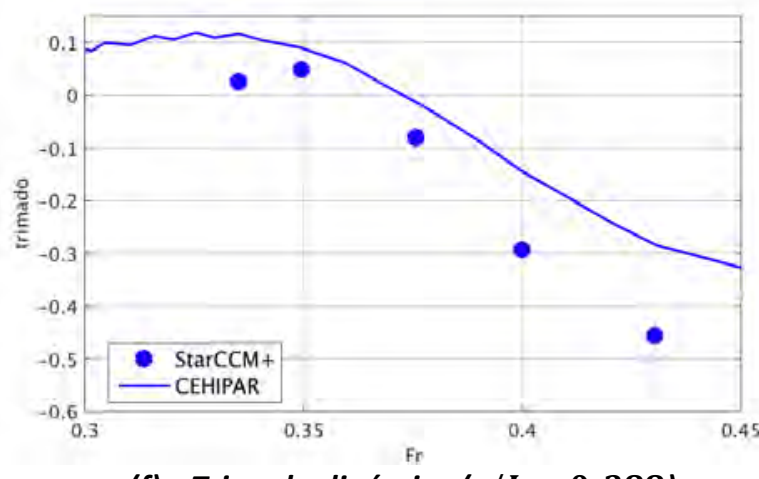

(f) Trimado dinámico $(s / L=0.388)$

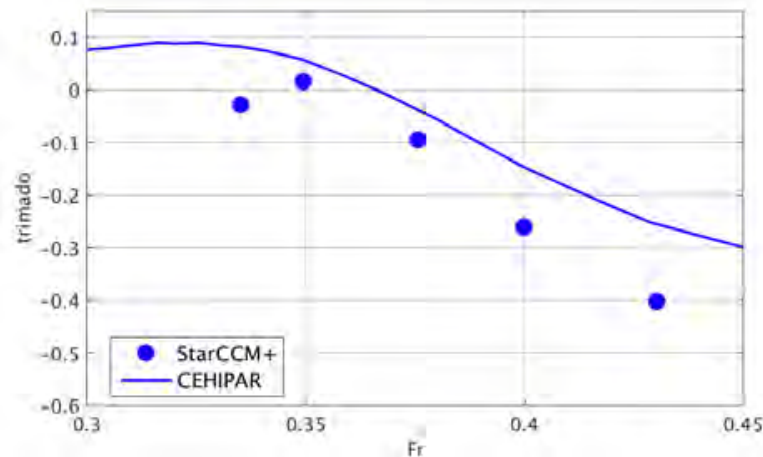

(h) Trimado dinámico $(s / L=0.470)$

Fig. 69. Asiento y trimado dinámicos del cat. $S 60$ calculados con Star-CCM+ vs. valores experimentales 


\section{Campo de presiones y elevaciones}

Una vez cotejados los resultados numéricos con los datos experimentales, el siguiente paso es analizar el campo de presiones sobre el casco junto con las elevaciones de la superficie libre. A semejanza de lo realizado con el cat. PESBO, la Fig. 70(a) y Fig. 71(a) muestran resultados correspondientes a las condiciones de interferencia más desfavorable del cat. S60, recogiéndose en la Fig. 70(b) y Fig. 71(b) los de interferencia más favorable, recogiéndose el conjunto completo de resultados en el apéndice Error! Reference source not found..

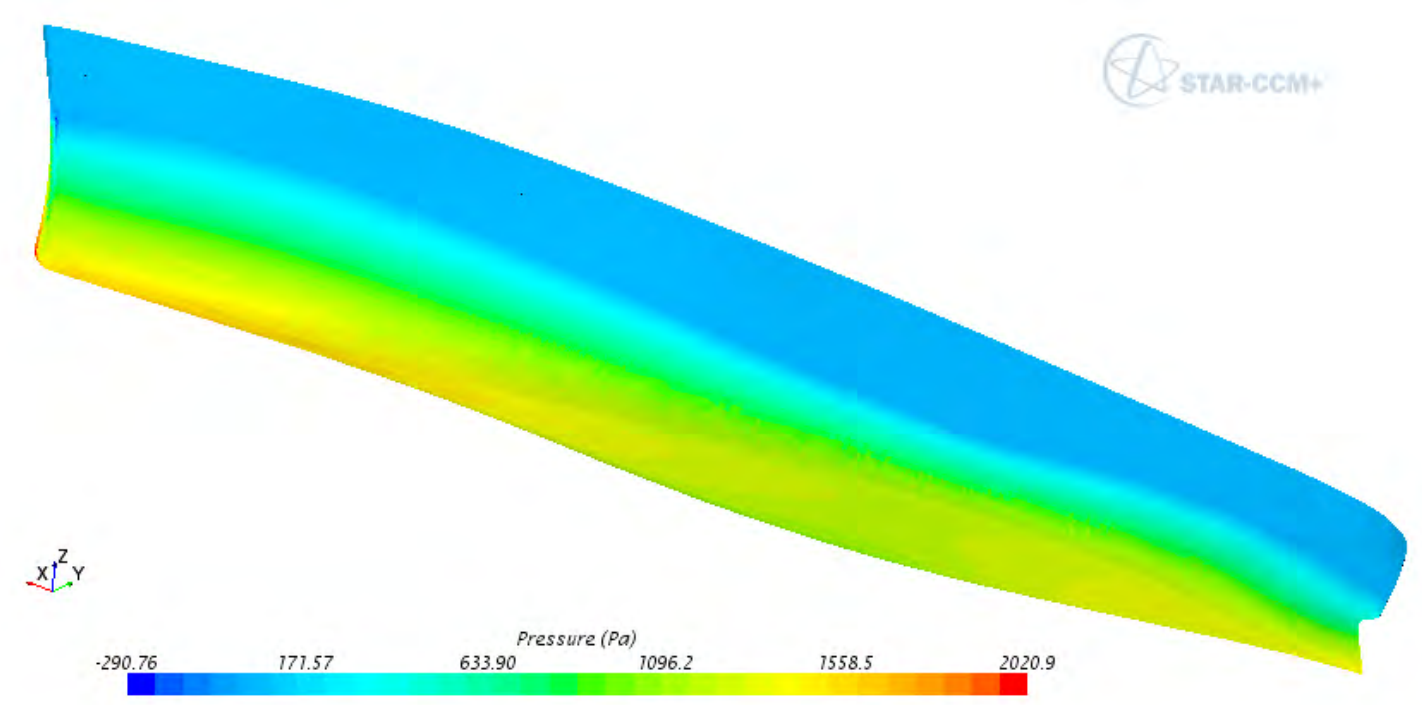

(a) $s / L=0.226, F r=0.335$, modelo libre

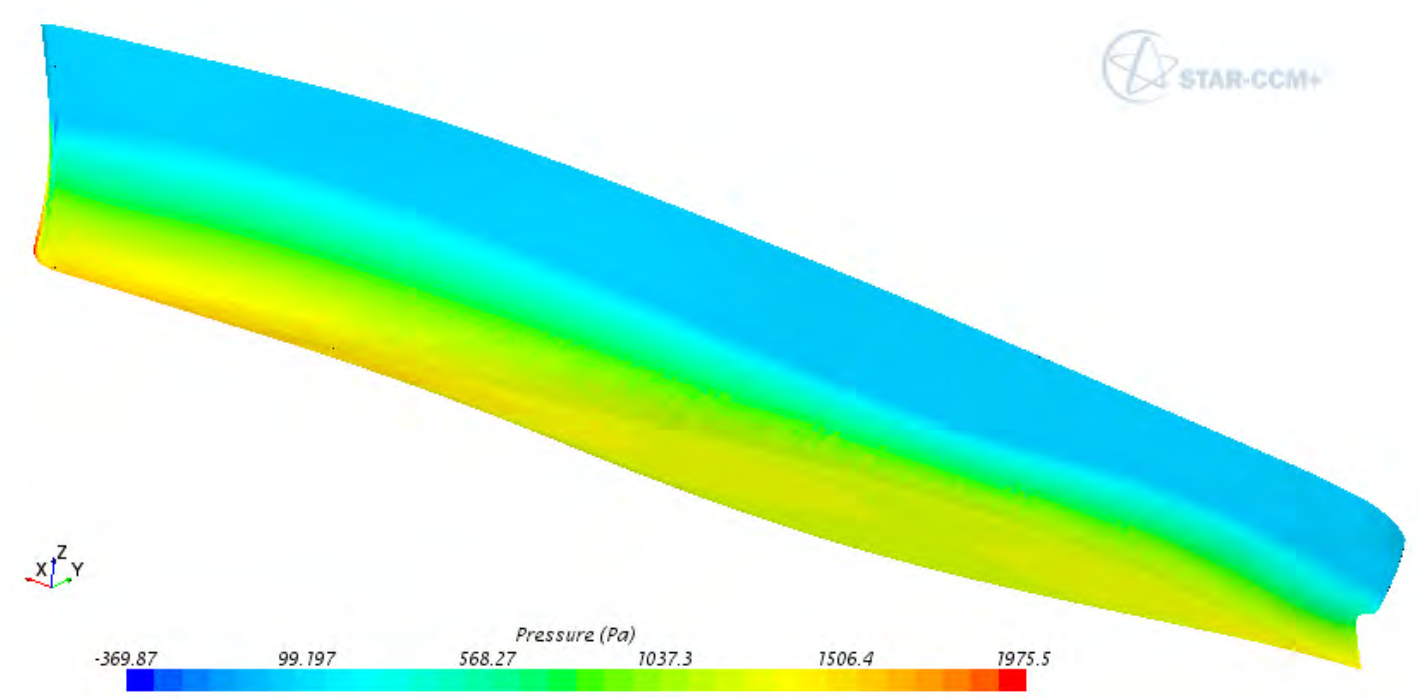

(b) $s / L=0.388, F r=0.335$, modelo libre

Fig. 70. Campos de presiones sobre el casco del cat. 560 calculados con Star-CCM+ 


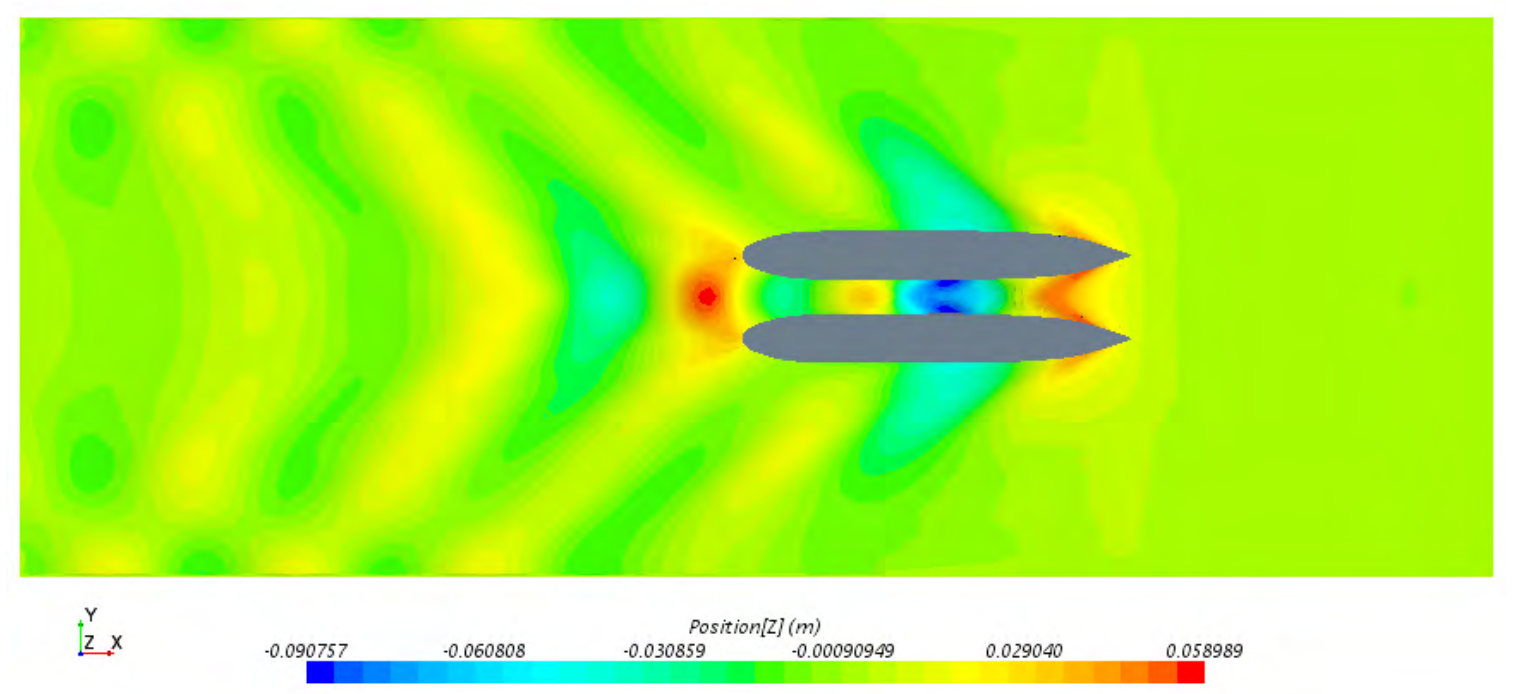

(a) $s / L=0.226, F r=0.335$, modelo libre

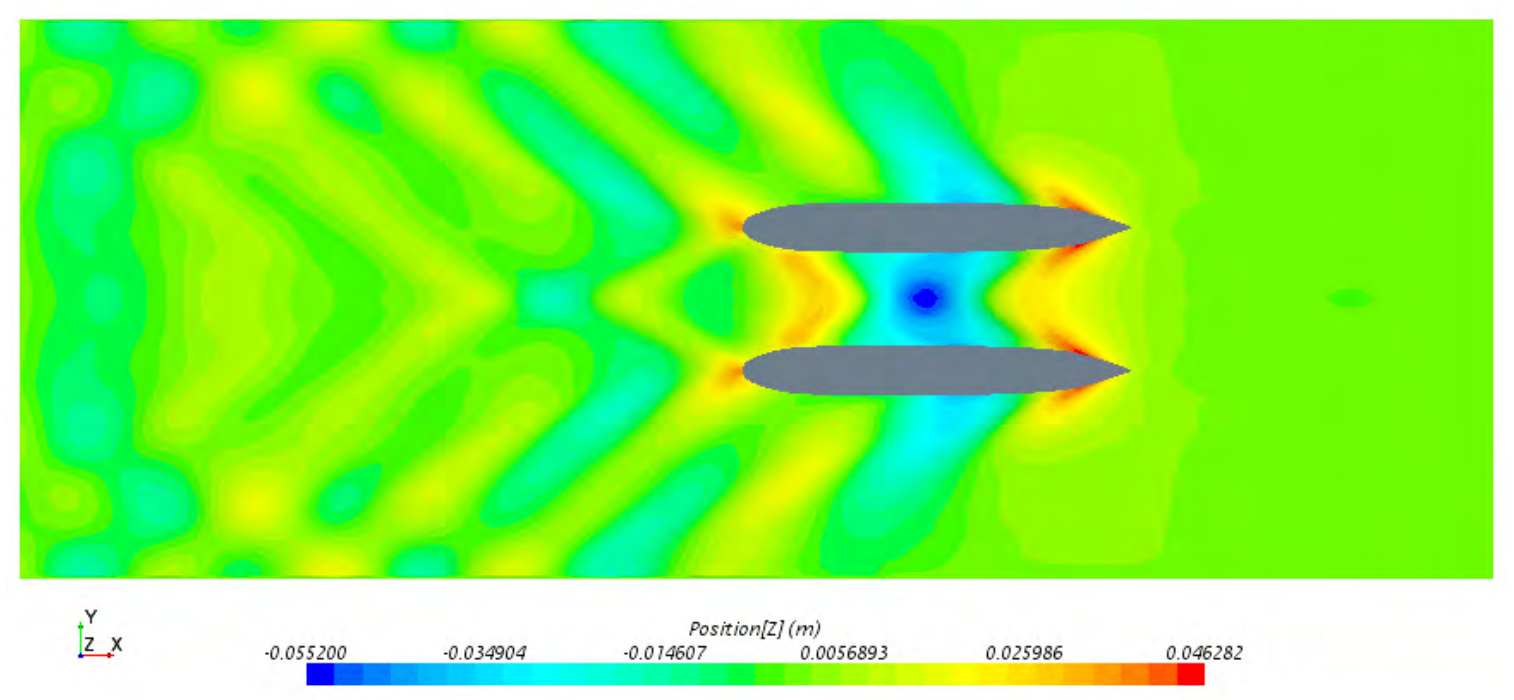

(b) $s / L=0.388, F r=0.335$, modelo libre

Fig. 71. Campo de elevaciones de la superficie libre con el cat. S60 calculados con Star-CCM+

Es interesante también analizar la respuesta a altos $F r$ donde todas las configuraciones muestran una interferencia desfavorable. La Fig. 72 muestra los resultados obtenidos para la separación $s / L=0.307$, la cual muestra la respuesta más desfavorable para $F r=0.430(F r$ simulado más alto). 


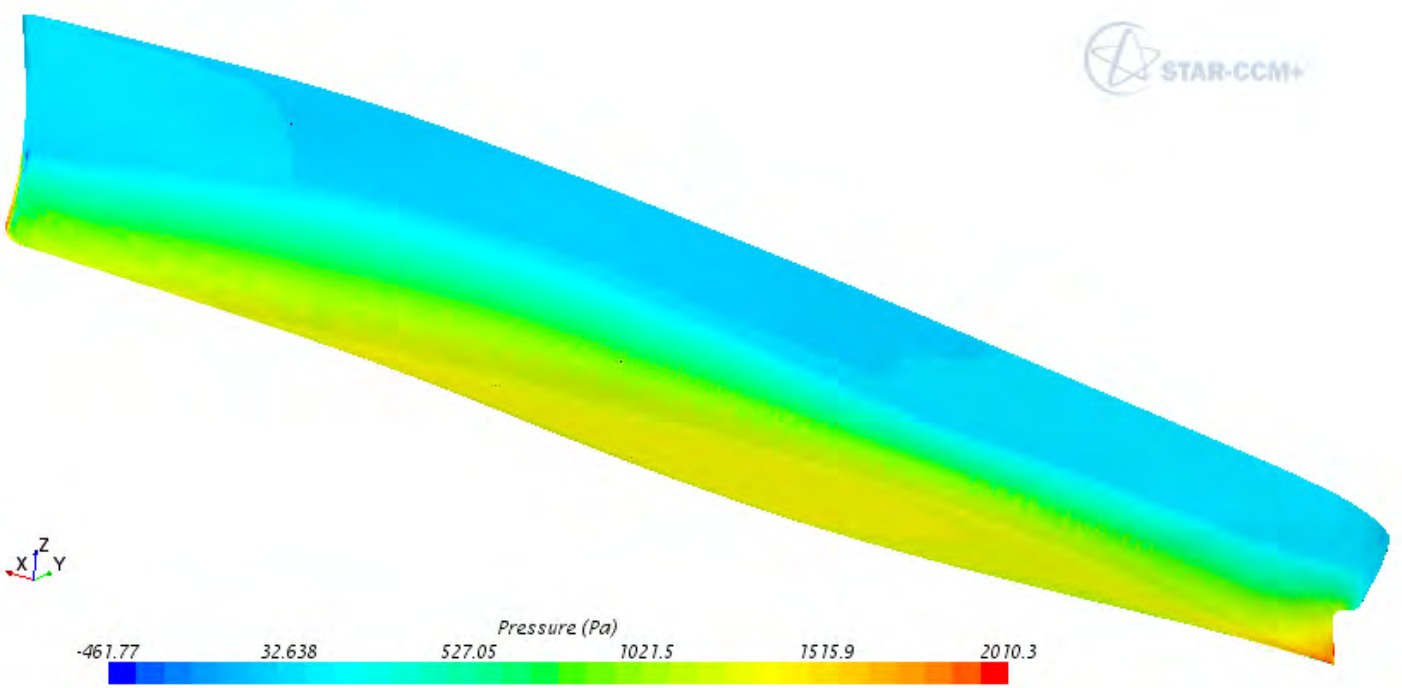

(a) Presiones sobre el casco
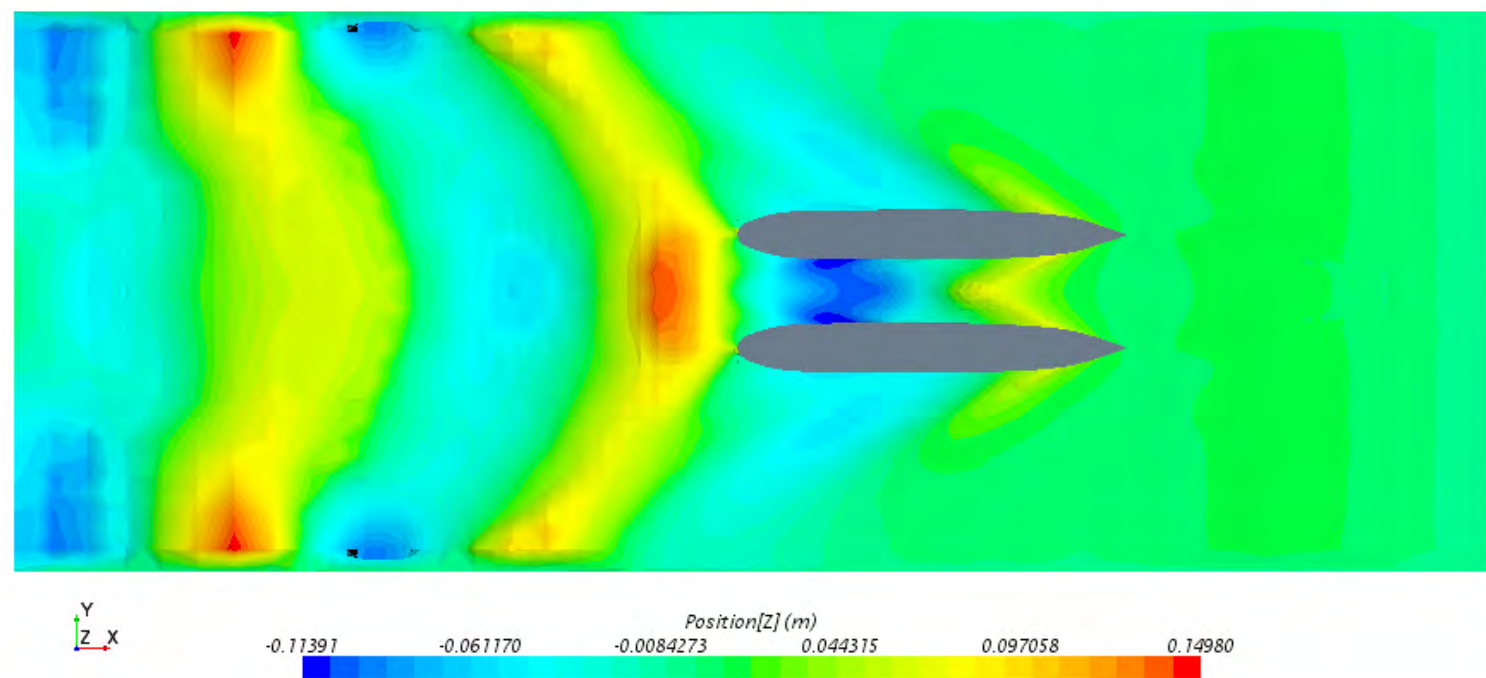

(b) Elevaciones de la superficie libre

Fig. 72. Campo de presiones y elevaciones calculados con Star-CCM+ para el catamarán S60, con modelo libre, $s / L=0.307, F r=0.430$

Al igual que se observó con el cat. PESBO, no se observan diferencias apreciables entre ambos casos en el campo de presiones sobre el casco. Respecto a las elevaciones de la superficie libre, de nuevo las amplitudes del oleaje en la Fig. 71 con $s / L=0.388$ son significativamente menores $^{52}$ que con $s / L=0.266$, a pesar de extenderse la zona de interferencia sobre una

\footnotetext{
${ }^{52}$ Atendiendo a los diferentes rangos de amplitudes cubiertos en la Fig. 71(a) y la Fig. 71(b)
} 
mayor superficie. Ello conlleva una reducción de la resistencia por formación de olas, llevando a una condición de interferencia favorable.

En el caso de los resultados con $F r=0.430$, los resultados muestran como es esperable un aumento significativo de las elevaciones y presiones. La mayor energía transmitida al agua aumenta la intensidad de los fenómenos de interferencia, no alcanzándose en ninguna de las condiciones estudiadas una cancelación del oleaje. Además, la atenuación del oleaje es menos significativa a pesar de aumentar la separación, lo que explica la homogeneidad de los valores de interferencia entre las diferentes configuraciones. Por los mismos motivos, la extensión del dominio de propagación del oleaje generado por el modelo es mayor, llegando a incidir en los límites laterales del dominio de cálculo como se puede apreciar en la Fig. 72(b). Este hecho hace prever la aparición de fenómenos de bloqueo en el CEHINAV, y justifica la elección del CEHIPAR para la realización de estos ensayos. 


\subsection{Análisis global de las simulaciones numéricas}

El amplio espectro de simulaciones realizadas y su contraste con los experimentos realizados ha puesto de manifiesto la utilidad, principalmente cualitativa por el momento, de los CFD. Su aplicación para el análisis de los fenómenos de interferencia está condicionada a su precisión a la hora de predecir la resistencia al avance. Sin embargo, experimentalmente se observó cómo la principal causa de interferencia favorable reside en la cancelación entre los trenes de olas. Los efectos viscosos no juegan un papel relevante en este aspecto, lo que posibilita la utilización de modelos simplificados. Independientemente de la resistencia, si las deformaciones de la superficie libre son estimadas con suficiente precisión permitirán detectar las condiciones de interferencia más favorable.

En este trabajo se han empleado dos tipos de códigos en orden de complejidad creciente: potencial (Tdynlin) y viscoso (Star-CCM+). Como era de esperar, cuanto más simplificados son los modelos numéricos menor es su precisión. Su ventaja reside en la reducción de los requisitos computacionales para poder aumentar el número de geometrías simuladas.

En relación al $I F$, la sec. 6.2.6 analizó las predicciones obtenidas por Yeung et al. (2004) utilizando la teoría de cuerpos esbeltos, que computacionalmente presenta los requisitos más bajos. Los resultados muestran desviaciones apreciables a la hora de estimar la extensión de las zonas de interferencia favorable. Por otro lado, la utilización de Star-CCM+ a pesar de emplear un modelo de cálculo mucho más complejo no aporta una mejora significativa, como se ha visto en la sec. 7.2.3 con la misma geometría. Cabe mencionar en este apartado que las simulaciones se han realizado con un ordenador personal, SO Ubuntu 14.04 LTS (Linux), 8GB de memoria RAM y procesador Intel i5 @ $2.70 \mathrm{GHz} \times 4$. Cada una de las simulaciones del cat. S60 requirió aproximadamente $25 \mathrm{~h}$ con el modelo fijo y $20 \mathrm{~h}$ con el modelo libre. La utilización de clústeres de cálculo que aumenten la capacidad computacional permitirá aumentar la densidad de la malla lo que se espera que mejore la precisión de los resultados. En cualquier caso, esto pone de manifiesto una vez más la dudosa aplicación de estos modelos complejos como herramientas de optimización, salvo en casos muy especiales. En base a los resultados anteriores, se concluye que las herramientas numéricas predicen las tendencias del $I F$ 
observadas experimentalmente, pero no permiten determinar los rangos de interferencia favorable con precisión suficiente.

A nivel cualitativo, la predicción de las deformaciones de la superficie libre ofrece una información muy útil de cara a la comparación entre diferentes configuraciones. La utilización de los cortes de olas supone una de las principales herramientas de validación de los diferentes modelos numéricos empleados en este aspecto. Los resultados obtenidos con el cat. PESBO muestran como las predicciones más precisas corresponden a las simulaciones con modelo libre en Star-CCM+. Como era esperable, al aumentar la complejidad del modelo empleado se mejora en la precisión de los resultados a cambio de un mayor coste computacional.

Las simulaciones realizadas muestran cómo el tren de olas exterior es relativamente independiente de la separación, localizándose las diferencias en la zona entre cascos. Al reducir la separación se reduce dicha área, lo que tiende a atenuar los fenómenos de interferencia. Sin embargo, la energía de los trenes de olas al interaccionar es mayor cuanto más cerca del casco se encuentren y por ende con menor tiempo para su disipación. Consecuentemente, la intensidad de las posible cancelaciones o amplificaciones del oleaje es mayor al reducir la separación aumentando los fenómenos de interferencia, tanto favorable como desfavorable. La dependencia con ambos efectos, junto con el carácter no lineal de la atenuación del oleaje, explica la dificultad de correlacionar directamente los fenómenos de interferencia con la separación. Este hecho lleva una vez más a concluir cómo durante la fase de diseño será necesario buscar una configuración equilibrada que evite amplificar o atenuar excesivamente los fenómenos de interferencia.

Una de las mayores utilidades de los CFD es su facilidad para visualizar el flujo sobre el casco permitiendo detectar zonas problemáticas a modificar en el proceso de optimización de formas. Sin embargo, a nivel de interferencia se ha comprobado como las variaciones de los campos de presiones entre las diferentes configuraciones son un orden de magnitud inferior. 
A semejanza del $I F$ en base a la resistencia, sería útil obtener una distribución superficial sobre el casco de un factor de interferencia en base al campo de presiones, $I F_{p}$.

$$
I F_{p}=\frac{p_{M H}-p_{D}}{\frac{1}{2} \rho V^{2}}
$$

Esta opción no esta disponible en Star-CCM+ a conocimiento del autor. El desarrollo de un módulo que lo permita es un claro ejemplo de la utilidad del desarrollo de herramientas específicas para multicascos para sacar todo el partido de los CFD. Permitiría identificar las zonas con mayores variaciones frente al monocasco y ser añadido como herramienta de diseño de formas para amplificar las interferencias favorables.

Por último, la comparativa entre los resultados de los dos catamaranes considerados permite evaluar la sensibilidad de Star-CCM+ ante las diferentes características geométricas. En base a los datos de resistencia, los valores calculados de resistencia en el caso del cat. S60 muestran menores desviaciones con las curvas experimentales que los del cat. PESBO. La inclusión de una popa de espejo junto con codillos en las formas del PESBO supone una dificultad añadida para los modelos numéricos. Las inestabilidades observadas en los cortes de olas calculados con esta geometría ponen de manifiesto la complejidad del flujo a popa del espejo, consistente con las observaciones durante los ensayos. Las formas del S60 por el contrario son mucho más suaves, con una popa de crucero que evita el desprendimiento, simplificando el flujo en la realidad y facilitando las simulaciones. En defensa de los resultados numéricos merece la pena añadir que el modelo ensayado del S60 se construyó utilizando la misma definición CAD empleada en las simulaciones. En el PESBO por el contrario se utilizó un modelo existente y se creó la definición CAD en base a este. La correlación por tanto entre el modelo físico y CAD es mejor en el S60, lo que sin duda ayuda a mejorar la precisión de los resultados.

En cuanto a la interferencia en sí, las formas más estilizadas del S60 llevan a variaciones menos bruscas en las curvas de resistencia frente al cat. PESBO. Esto es beneficioso en el 
sentido que aporta una mayor flexibilidad al buque para ajustar sus condiciones de navegación, así como reduce el impacto los errores numéricos cometidos en las predicciones del $I F$. Sin embargo, se aprecia también cómo lleva a jorobas menos acusadas lo que reduce la capacidad de aprovecharse de las posibles interferencias favorables. No obstante, con sólo dos geometrías analizadas es difícil extraer conclusiones detalladas extrapolables a otras configuraciones. En el futuro se espera ampliar el espectro de catamaranes considerado para comprender mejor el impacto de la geometría en la resistencia de interferencia. 


\section{APLICACIONES Y TRABAJO FUTURO}

\subsection{Aplicaciones}

Como se indicó en el comienzo de este trabajo, el uso de multicascos es cada vez mayor en todas las áreas marítimas (comercial, ocio, competición, militar, etc.). Los fenómenos de interferencia forman parte implícita de este tipo de embarcación, cuya mejor comprensión es el objetivo principal de este trabajo.

Su principal aplicación es durante la fase de diseño del multicasco, y más concretamente en la selección del tipo de geometría y separación así como durante la optimización de formas. Los patrones de comportamiento mostrados en los capítulos anteriores buscan proporcionar al diseñador una serie de criterios generales que faciliten la toma de decisiones. No obstante, los resultados presentados muestran cómo al englobar múltiples fenómenos físicos es muy difícil establecer una correlación directa entre las características generales del buque y el $I F$. En cada proyecto por tanto será necesario definir el grado de profundidad con el que considerar dichas interferencias: desde una mera cuantificación de su orden de magnitud hasta un análisis detallado con ensayos, pasando por la estimación mediante modelos numéricos. A tal fin se espera que el presente trabajo sirva de aplicación, al haberse acometido su estudio desde diferentes perspectivas mostrando los beneficios e inconvenientes de cada uno.

En segundo lugar, este trabajo aporta un importante volumen de datos experimentales con catamaranes. Se han cubierto dos geometrías, ensayándose con modelo fijo y libre tanto del catamarán como el monocasco, con $0.12<F r<0.55$ y $0.21<s / L<0.47$. Su aplicación como referencia para la validación de modelos de predicción de resistencia es inmediata. Para ello se incluyen todos los resultados experimentales, junto con los modelos CAD de las geometrías y vídeos de los experimentos en el DVD adjunto. Estos se pueden encontrar online en:

- http://canal.etsin.upm.es/papers/catamaran/

- http://canal.etsin.upm.es/ftp/2012/S60/ 
Adicionalmente, la metodología seguida en este trabajo se espera que sirva para establecer un estándar de análisis de los efectos de interferencia, tanto a nivel experimental como numérico. Se pretende así crear un marco de estudio que pueda ser utilizado por la comunidad hidrodinámica para ir construyendo una base de datos detallada con múltiples geometrías. Cuanto más completa sea la información referente a valores de $R_{T}, I F$, cortes de olas, asientos y trimados dinámicos, y campos de elevaciones y presiones, más útil será de cara a su aplicación tanto en el diseño de nuevas embarcaciones como en la validación de modelos numéricos y/o analíticos.

Por otro lado, los resultados encontrados pueden utilizarse en los modelos operativos del buque, como sistemas de ahorro de combustible o posicionamiento dinámico. Las jorobas en la curva de resistencia, característica de multicascos, introducen mínimos locales cuya consideración en los algoritmos de control pueden reportar mejoras en la respuesta del buque. Otro de los riesgos operacionales de los multicascos son los impactos del oleaje sobre la superestructura entre cascos. Si bien los movimientos de arfada y cabeceo son su principal causa, los cuales no se han cubierto en este trabajo, la interacción entre los trenes de olas en la zona interior es de aplicación directa. En caso de estar en fase dan lugar a elevaciones significativamente mayores de las esperadas para a la geometría del casco, aumentando el riesgo de pantocazos.

\subsection{Trabajo futuro}

A lo largo de este trabajo se han ido mencionando las diferentes áreas en las que se espera continuar trabajando en el futuro. En general, estas están encaminadas a expandir el rango de configuraciones, separaciones, velocidades y estado de la mar estudiadas como se resume a continuación.

\section{Estudio de los fenómenos de interferencia con $\mathrm{Fr}>\mathbf{0 . 5 0}$}

Los modelos utilizados están diseñados para navegar en un rango de $\mathrm{Fr}$ de no planeo. Al tratar de ensayarse a velocidades mayores su comportamiento hidrodinámico no es realista, 
disparándose la generación de olas. De hecho, la separación menor del catamarán $\mathrm{S} 60$ ( $\mathrm{s} / \mathrm{L}=$ 0.226 ) no se pudo ensayar a más de $F r=0.45$ debdo al embarque de agua de la propia ola generada por el modelo. En cualquier caso, los multicascos son embarcaciones rápidas en la mayoría de casos, por lo que en un futuro se espera expandir el rango de $F r$ cubierto en este trabajo. Para ello será necesario utilizar nuevas geometrías de planeo y semi-planeo, diseñadas para navegar a altos Fr. Esto permitirá obtener resultados realistas, donde se espera que el asiento y trimado dinámicos cobren gradualmente una importancia mayor.

\section{Estudio de la respuesta de buques en olas}

Una de las variables que no se ha incluido en este estudio es el estado de la mar en relación a los efectos de interferencia. Si bien la navegación en olas es un escenario más realista, se mantuvo el enfoque clásico de resistencia asumiendo como indicativa la condición de aguas tranquilas. No obstante, el comportamiento oscilatorio mostrado por el $I F$ induce a esperar ciertas variaciones en las curvas obtenidas al incluir los movimientos de arfada, cabeceo y balance. Esto se basa en la nueva interacción entre los trenes de olas al tener que añadir el oleaje incidente, así como las componentes de radiación debidas a los nuevos movimientos del buque.

\section{Estudio experimental del monocasco equivalente}

En la sección 4.2 se discutió el concepto de monocasco equivalente, concluyendo cómo este depende de los requisitos operacionales del buque. Al ser mucho más abstractos que el $I F$ se escogió este último para cuantificar los efectos de interferencia. Sin embargo, la comparativa con posibles monocascos independientes permitiría apreciar de forma práctica la superioridad o no del multicasco a la hora de cumplir con la misión asignada. En el futuro se espera profundizar en este concepto, buscando monocascos potencialmente equivalentes a los multicascos estudiados y optimizando sus formas para satisfacer los diferentes criterios operacionales. De esta forma podrán ser comparados con mayor precisión, aportando luz a la hora de decidir el tipo de embarcación deseada. 


\section{Expansión del espectro de geometrías y configuraciones analizado}

Las formas del S60 difieren significativamente de las del catamarán comercial. No obstante, con sólo dos configuraciones tan diferentes es difícil establecer un patrón que permita evaluar el impacto de la geometría en la resistencia de interferencia. La ventaja de las herramientas numéricas reside en su capacidad para poder considerar múltiples configuraciones y geometrías con un coste añadido muy pequeño. En base a la validación realizada con los datos experimentales, en el futuro se espera utilizar Star-CCM+ para analizar más en detalle la influencia de la geometría de los cascos en la resistencia de interferencia. Para ello se expandirá el rango de casos considerados, considerando variaciones de sus parámetros característicos.

\section{Estudio con configuraciones asimétricas y con separación longitudinal}

Las geometrías consideradas en este trabajo presentan cascos idénticos, con simetría respecto a su plano de crujía. Como muestran diferentes estudios en la literatura, la utilización de cascos asimétricos así como separaciones longitudinales entre estos, no sólo transversales, puede ayudar a maximizar los efectos de interferencia favorable (Söding (1997), Bruzzone et al. (1999) Zaraphonitis et al. (2001), Chen et al. (2003b), Yeung \& Wan (2008) o Yu (2015)). Evidentemente, esto implicará la construcción y/o simulación con nuevos modelos, así como la construcción de una tercera generación del bastidor de unión.

\section{Implementación y ajuste de un modelo numérico basado en flujo potencial con los} resultados experimentales

La principal virtud del uso de la teoría lineal para predecir los efectos de interferencia reside en el poco tiempo computacional necesario frente a las simulaciones con Star-CCM+. Esto convierte a la primera en una herramienta ideal de cara a la optimización de formas, si bien el problema reside en la gran sensibilidad del $I F$ al $F r$ y la separación. Esto puede derivar en desviaciones críticas a la hora de estimar la posición de las interferencias favorables, como se observó al analizar los resultados presentados por Yeung et al. (2004). En el futuro se espera implementar un nuevo módulo de catamaranes con condición no lineal de superficie libre dentro de Tdynlin, y proporcionar unos parámetros de ajuste en base a los resultados 
experimentales que permitan mejorar su precisión. Combinando técnicas numéricas y resultados experimentales se conseguirá crear un entorno de optimización más eficiente.

\section{Estudio de las variaciones de estela para cuantificar las interferencias en los efectos viscosos}

En base a los resultados de resistencia total se concluyó que las principales causas de interferencia son debidas a las interacciones entre los trenes de olas. Las elevaciones medidas en los cortes de ola permiten intuir ciertos efectos de cancelación, si bien disponer del campo de velocidades en la zona sumergida permitiría apreciar dichas interacciones mucho mejor. La instrumentación requerida para ello es mucho más complicada, como se mostró en el apartado 4.3.1, por lo que no se ha incluido dentro del alcance de este trabajo. No obstante, su medición en el futuro permitiría poder cuantificar mejor la influencia de los efectos viscosos en la interferencia, así como su influencia en la propia resistencia por formación de olas (Moreno et al. (1975)). 


\section{CONCLUSIONES Y APORTACIONES}

\subsection{Conclusiones}

Como resultado principal de este trabajo se ha presentado un análisis de los fenómenos de interferencia presentes en multicascos desde diferentes puntos de vista. Se han realizado ensayos y simulaciones numéricas con modelo fijo y libre, determinando la resistencia al avance, asiento, trimado y cortes de olas para diferentes separaciones entre cascos y velocidades. Respecto a las geometrías, se han empleado un catamarán de servicio comercial PESBO y un catamarán S60, una geometría de uso muy extendido en la comunidad hidrodinámica. Mención aparte merece la reflexión sobre el concepto de monocasco equivalente presentada en la sec. 4.2. Desde el punto de vista de interferencia es un concepto demasiado ambiguo, si bien es clave en la decisión inicial de apostar por un multicasco o no.

En general, se ha apreciado cómo se debe tener en cuenta no sólo la geometría de los cascos sino también la separación entre ellos a la hora de proyectar este tipo de embarcaciones. Dependiendo del $\mathrm{Fr}$ puede existir un intervalo de separaciones en el que los efectos de interferencia reducen la resistencia total al avance. En el rango de separaciones estudiado, los intervalos del $\mathrm{Fr}$ con interferencias favorables son mayores a medida que aumenta la separación. En lo referente a los resultados numéricos, a pesar de reproducir las tendencias observadas experimentalmente, destacar cómo el comportamiento oscilatorio del IF pone en duda la aplicación directa de los valores calculados. Pequeñas desviaciones en su estimación pueden resultar en conseguir un resultado completamente opuesto al real. Su principal virtud reside en la facilidad para visualizar el las deformaciones de la superficie libre, permitiendo comparar diferentes geometrías y detectar las condiciones de interferencia favorable.

Los resultados con el catamarán PESBO incluyen una extensa colección de cortes de olas. En primer lugar se concluyó que es recomendable utilizar los cortes de los ensayos del monocasco como referencia. Al comparar estos con los correspondientes cortes laterales del catamarán se detectó una cierta interferencia del segundo casco. Quedó patente además cómo los cortes de olas centrales no muestran los efectos de interferencia al estar tomados 
sobre un plano de simetría. Se han tomado por tanto cortes de olas interiores descentrados, los cuales sí muestran la interacción favorable y desfavorable entre los trenes de olas de cada casco. Respecto a las condiciones de ensayo, el análisis con modelo fijo se efectuó únicamente con la separación mayor $(s / L=0.388)$ al ser esta la que mostraba mayores interferencias favorables. Al comparar con la condición de modelo libre quedó patente cómo las diferencias no son sustanciales. Esto es consistente con los valores moderados de asiento y trimado dinámicos, similares en ambos casos. En el caso del S60 las diferencias observadas son mayores, amplificando el modelo libre los fenómenos de interferencia, tanto favorables como desfavorables. Los datos experimentales con esta geometría se han utilizado para contrastar los presentados por Yeung et al. (2004) utilizando un modelo numérico basado en la teoría lineal. El $I F$ óptimo (-0.2) ocurre con $F r=0.33$, concordando con la predicción numérica. Sin embargo, esta condición favorable ocurre con una separación significativamente superior (0.4) que la predicha teóricamente (0.226). Las transiciones entre las regiones de interferencia favorable y desfavorable son más acusadas para la condición de modelo fijo. En general, se ha mostrado cómo para cada separación existe un desfase en los $\mathrm{Fr}$ con máxima interferencia favorable y desfavorable entre los datos experimentales y numéricos. La sección 6.5 recoge un resumen más detallado de los diferentes resultados obtenidos.

A tenor de los resultados encontrados, no existe un procedimiento idóneo para la estimación de la resistencia de interferencia. Dependiendo del grado de profundidad deseado, en cada proyecto se deberá decidir la metodología a seguir. En cualquier caso, al incluir el IF como factor de decisión en el diseño de un multicasco es recomendable combinar diferentes técnicas de predicción para conseguir resultados con suficiente resolución y precisión. Se espera que las diferencias descritas en este trabajo entre los resultados experimentales y numéricos, así como en la $R_{W}$ en las condiciones de modelo libre y fijo, sean relevantes en la toma de decisiones durante las etapas iniciales de diseño.

Por último, la extensa caracterización experimental de las geometrías estudiadas, y en especial del S60, se presenta con la intención de servir como caso de referencia para futuros estudios con catamaranes. 


\subsection{Aportaciones}

Resumen de las aportaciones de este trabajo:

- Análisis conceptual de los fenómenos de interferencia en multicascos (capítulo 4)

- Extensa documentación experimental con datos de resistencia (Tabla 11), cortes de olas a múltiples separaciones (Tabla 13), asientos, trimados, etc. El conjunto completo de geometrías y resultados se muestra en los apéndices $A-C$, pudiéndose encontrar su versión electrónica en el DVD adjunto u online en

- http://canal.etsin.upm.es/papers/catamaran/

○ http://canal.etsin.upm.es/ftp/2012/S60

- Fabricación de un sistema de trimado fijo para la realización de ensayos, así como de una estructura de soporte para la toma de cortes de ola centrales (5.1)

- Implementación y validación del módulo de catamaranes del código potencial Tdynlin, así como verificación de la precisión de los resultados de CD-Adapco Star-CCM+ con modelo fijo y libre (capítulo 7)

- Comparación de los resultados experimentales con predicciones teóricas simplificadas utilizando la teoría de cuerpos esbeltos (apartado 6.2.6) 


\section{PUBLICACIONES REALIZADAS DURANTE ESTA TESIS}

\section{Publicaciones indexadas}

- Souto Iglesias, A., Fernández Gutiérrez, D. \& Pérez Rojas, L. (2012). Experimental assessment of interference resistance for a Series 60 catamaran in free and fixed trimsinkage conditions. Ocean Engineering, Vol. 53, 38-47.

Factor de impacto 2011: 1.178. Cuartil en categoría ENGINEERING, OCEAN: Q1 (3/15).

- Souto Iglesias, A., Zamora, R., Fernández Gutiérrez, D. \& Pérez Rojas, L. (2007). Analysis of the wave system of a catamaran for CFD validation. Experiments in Fluids, Vol. 42/2, 321-332. DOI: http://dx.doi.org/10.1007/s00348-006-0244-4

Factor de impacto 2006: 1.112. 2014: 1.670, Cuartil en categoría ENGINEERING, MECHANICAL: Q1 (30/130), MECHANICS: Q2 (44/137).

\section{Publicaciones en congresos}

- Souto Iglesias, A., Zamora, R., Fernández Gutiérrez, D. \& López, C. (2005). Catamaran wave resistance and central wave cuts for CFD validation. 12th International Congress of the International Maritime Association of the Mediterranean (IMAM 2005), Vol. 1, 157-165.

- Fernández-Gutiérrez, D., Le Garrec, M., García-Espinosa, J., Menec, M., Souto-Iglesias, A. (2006), A GiD-based implementation of a panel method for sailing yachts flow computation. 2ㅇ Simposio Internacional de diseño y producción de yates de motor y vela. Madrid (España) 


\section{BIBLIOGRAFÍA}

[ACRM (2013)] ACRM (2013). AC72 Class Rule. Version 1.1. America's Cup Race Management.

[ACRM (2014a)] ACRM (2014). AC45 Class Rule. Version 2.0. America's Cup Race Management.

[ACRM (2014b)] ACRM (2014). AC62 Class Rule. Version 1.0. America's Cup Race Management.

[Aláez (1980)] Aláez Zazurca, J.A. (1980) Teoría del Buque (1): Olas. Publicaciones E.T.S.I.N.

[Aláez (1991)] Aláez Zazurca, J.A. (1991). Catamaranes de alta velocidad. Ingeniería Naval, №59, 151-161.

[Alvariño et al. (1991)] Alvariño Castro, R., Azpíroz Azpíroz, J.J. \& Meizoso Fernández, M. (1997). El proyecto básico del buque mercante. FEIN.

[Atreyapurapu et al. (2014)] Atreyapurapu, K., Tallapragada, B. \& Voonna, K. (2014). Simulation of a Free Surface Flow over a Container Vessel Using CFD. Intl. Journal of Engineering Trends and Technology (IJETT), Vol. 18/7, 334-339

[Aubault \& Yeung (2012)] Aubault, A. \& Yeung, R.W. (2012). Interference resistance of multihull vessels in finite depth waters. OMAE 2012, Rio de Janeiro. ASME.

[Bailey (1976)] Bailey, D. (1976). The NPL High Speed Round Bilge Displacement Hull Series: Resistance, Propulsion, Manoevring and Seakeeping Data. Maritime Technology Monograph Vol. 4, RINA.

[Baquero (2002)] Baquero, A. (2002). Teoría del buque: Lecciones de Resistencia al avance. Publicaciones de la ETSIN

[Berenguer et al. (2002)] Berenguer, I., Iribarren, J.R., López Pavón, C., Herreros, M.A. \& Souto Iglesias, A. (2002) Estudio de Generación de oleaje por el buque en la ría de Sevilla. Ingeniería Naval, Vol 71 (803), 89-95. 
[Broglia et al. (2011)] Broglia, R., Zaghi, S., \& Di Mascio, A. (2011). Numerical simulation of interference effects for a high-speed catamaran. Journal of marine science and technology, Vol. 16(3), 254-269.

[Bruzzone (1994)] Bruzzone, D. (1994). Numerical Evaluation of the Steady Free Surface Waves. Proceedings of CFD Workshop Tokyo 94. Vol 1, 126-134.

[Bruzzone et al. (1999)] Bruzzone, D., Ferrando, M. \& Gualeni, P. (1999). Numerical and experimental investigation into resistance characteristics of symmetrical and unsymmetrical catamaran hull forms. $5^{\text {th }}$ International Symposium HSMV99.

[Centeno et al. (2001)] Centeno, R., Varyani, K.S. \& Guedes Soares, C. (2001). Experimental study on the influence of hull spacing on hard-chine catamaran motions. Journal of Ship Research, Vol. 45/3, 216-227.

[Chen \& Sharma (1997)] Chen, X.N. \& Sharma, S.D. (1997). Zero wave resistance for ships moving in shallow channels at supercritical speeds. Journal of Fluid Mechanics, Vol. $335,305-312$.

[Chen et al. (2003a)] Chen, X.N., Sharma, S.D. \& Stuntz, N. (2003). Zero wave resistance for ships moving in shallow channels at supercritical speeds. Part 2. Improved theory and model experiment. Journal of Fluid Mechanics, Vol. 478, 111-124.

[Chen et al. (2003b)] Chen, X.N., Sharma, S.D. \& Stuntz, N. (2003). Wave reduction by SCatamaran at supercritical speeds. Journal of Ship Research, Vol. 47/2, 145-154.

[Chen \& Huang (2004)] Chen, P.F. \& Huang, C.H. (2004). An inverse hull design approach in minimizing the ship wave. Ocean Engineering, Vol. 31, 1683-1712.

[Chen \& Guedes-Soares (2008)] Chen, N.Z. \& Guedes Soares, C. (2008). Ultimate longitudinal strength of ship huls of composite materials. Journal of Ship Research, Vol. 52/3, 184193. 
[Couser et al. (1998)] Couser, P.R., Wellicome, J.F., \& Molland, A.F. (1998). Experimental measurement of sideforce and induced drag on catamaran demihulls. International shipbuilding progress, Vol. 45(443), 224-235.

[Davis \& Holloway (2003)] Davis, M.R. \& Holloway, D.S. (2003). The influence of hull form on the motions of high speed vessels in head seas. Ocean Engineering, Vol. 30, 2091-2115.

[Davis et al (2005)] Davis, M.R., Watson, N.L. \& Holloway, D.S. (2005). Measurement of response amplitude operators for an $86 \mathrm{~m}$ high-speed catamaran. Journal of Ship Research, Vol. 49/2, 121-143.

[Dawson (1977)] Dawson C.W. (1977). A Practical Computer Method for Solving Ship-Wave Problems. Proceedings of Second International Conference on Numerical Ship Hydrodynamics, University of California at Berkeley. Vol 1, 30-38

[Day \& Doctors (2001)] Day, A.H. \& Doctors, L.J. (2001). Rapid Estimation of Near- and FarField Wave Wake from Ships and Application to Hull Form Design and Optimization. Journal of Ship Research, Vol. 45/1, 73-84.

[Doctors (2006)] Doctors, L.J. (2006). A numerical study of the resistance of transom-stern monohulls. Proc. 5th International Conference on High performance marine vehicles, Australia.

[Dumez \& Cordier (1997)] Dumez, F.X. \& Cordier, S. (1997). Accuracy of Wave Pattern Analysis Methods in Towing Tanks. Proc. 21st Symposium on Naval Hydrodynamics, Noruega. Vol 1, 147-160

[Fang et al. (2009)] Fang, M.C., Wu, Y.C., Hu, D.K., and Lee, Z.Y. (2009). The Prediction of the Added Resistance for the Trimaran Ship With Different Side Hull Arrangements in Waves. Journal of Ship Research, Vol. 53/4, 227-235.

[Fernández-Gutiérrez (2010)] Fernández-Gutiérrez, D. (2010). Análisis de la influencia del trimado en la resistencia mediante técnicas numéricas y experimentales. Diploma de Estudios Avanzados (DEA), ETSIN (UPM). 
[Froude (1868)] Froude, W. (1868) Observations and suggestions of determining by experiment the resistance of ships. Correspondence with Admiralty. Reprinted in "The Papers of William Froude", INA, 1955.

[Froude (1874)] Froude, W. (1874). Experiments for the determination of the frictional resistance of water on a surface under various conditions. Report to admiralty and British association for the advancement of Science. Reprinted in "The Papers of William Froude", INA, 1955.

[García Espinosa et al. (2002)] García-Espinosa, J., Luco-Salman, R., Salas, M., LópezRodríguez, M., \& Oñate, E. (2002). An Advanced finite element method for fluid dynamic analysis of America's Cup boats. Proceedings of HPYD Conference (RINA), Auckland (New Zealand).

[Ge et al. (2005)] Ge, C., Faltinsen. O.M. \& Moan, T. (2005). Global hydroelastic response of catamarans due to wetdeck slamming. Journal of Ship Research, Vol. 49/1, 24-42.

[González Álvarez-Campana (1998)] González Álvarez-Campana, J.M. (1998). Teoría del Buque. Principales aspectos hidrodinámicos de algunas embarcaciones rápidas y no convencionales. Publicaciones E.T.S.I.N.

[González Gutiérrez \& Bermejo (2005)] González Gutiérrez, L. M., \& Bermejo, R. (2005). A semi-Lagrangian level set method for incompressible Navier-Stokes equations with free surface. International journal for numerical methods in fluids, Vol. 49(10), 1111-1146.

[Gourlay (2008)] Gourlay, T. (2008). Sinkage and Trim of a Fast Displacement Catamaran in Shallow Water. Journal of Ship Research, Vol. 52(3), 175-183.

[He et al. (2015)] He, W., Castiglione, T., Kandasamy, M., \& Stern, F. (2015). Numerical analysis of the interference effects on resistance, sinkage and trim of a fast catamaran. Journal of Marine Science and Technology, Vol. 20(2), 292-308.

[Heggelund \& Moan (2002)] Heggelund, S.E. \& Moan, T. (2002). Analysis of Global Load Effects in Catamarans. Journal of Ship Research, Vol. 46/2, 81-91. 
[Hughes (1954)] Hughes, G. (1954). Friction and form resistance in turbulent flow, and a proposed formulation for use in model and ship correlation. Transactions of RINA, Vol. 96.

[Hughes (1966)] Hughes, G. (1966). An analysis of ship model resistance into viscous and wave components. Transactions of RINA, Vol. 108.

[Inoue \& Kamruzzaman (2008)] Inoue, Y. \& Kamruzzaman, M.D. (2008). Analysis of hydrodynamic characteristics for arbitrary multihull ships advancing in waves. Journal of Marine Scientific \& Technology, Vol 13, 231-243.

[Insel (1990)] Insel, M. (1990). An investigation into the resistance components of high speed displacement catamarans. Tesis Doctoral, University of Southampton (Reino Unido).

[Insel \& Molland (1991)] Insel, M. \& Molland, A.F. (1991). An investigation into the resistance components of high speed displacement catamarans. RINA Vol 134, 1-20.

[Insel et al. (1994)] Insel, M., Molland, A.F. \& Wellicome, J.F. (1994). Wave resistance prediction of a catamaran by Linearised Theory. CAMO'94: the built environment, Vol 5, 59-67.

[Insel \& Doctors (1995)] Insel, M. \& Doctors, L.J. (1995). Wave pattern prediction on monohulls and catamarans in shallow water canal by Linearised Theory. $12^{\text {th }}$ AFMC, 259-262.

[ITTC (1957)] ITTC (1957). Proceedings of $8^{\text {th }}$ International Towing Tank (ITTC), Madrid, Spain.

[ITTC (1978)] ITTC (1978). Proceedings of $8^{\text {th }}$ International Towing Tank (ITTC), Madrid, Spain.

[ITTC (2011)] ITTC Recommeded Procedure 7.5.02-02-01.3 (2011). International Towing Tank Conference (ITTC) Recommended Procedures and Guidelines, Testing and Data Analysis, Resistance Test. 
[Izaguirre Alza et al. (2010)] Izaguirre Alza, P., Pérez Rojas, L., \& Núñez Basáñez, J. F. (2010). Drag Reduction trough Special paints Coated on the hull. International Conference on Ship Drag Reduction SMOOTH-SHIPS, Istanbul, (Turkey)

[Izaguirre Alza (2012)] Izaguirre Alza, P. (2012). Numerical and Experimental Studies of Sail Aerodynamics. Tesis Doctoral, Universidad Politécnica de Madrid (España)

[Janson et al. (2003)] Janson, C.E., Leer-Andersen, M. \& Larsson, L. (2003). Calculation of Deep-Water Wash Waves using a combined Rankine/Kelvin source method. Journal of Ship Research, Vol. 47/4, 313-326.

[Jiankang et al. (2001)] Jiankang, W., Lee, T.S., \& Shu, C. (2001). Numerical study of wave interaction generated by two ships moving parallely in shallow water. Computer methods in applied mechanics and engineering, Vol. 190(15), 2099-2110.

[Kim \& Jenkins (1981)] Kim, Y. H., \& Jenkins, D. (1981). Trim and Sinkage Effects on Wave Resistance with Series 60, $C_{B}=0.60$. Informe ténico No. DTNSRDC/SPD-1013-01. David W Taylor Naval Ship Research and Development Center, Ship Performance Dept., Bethesda (MD), USA.

[Kumar et al. (2005)] Kumar Saha, G., Suzuki, K. \& Kai, H. (2005). Hydrodynamic optimization of a catamaran hull with large bow and stern bulbs. Journal of Marine Scientific \& Technology, Vol 10, 32-40.

[Kundu et al. (2012)] Kundu, P.J., Cohen I.M. \& Dowling, D.R. (2012). Fluid Mechanics. Academic Press (Elsevier). 5a ed.

[Laverón-Simavilla et al. (2008)] Laverón-Simavilla, A., Lapuerta, V., Franchini, S. \& Sanz, A. (2008). Sail optimization for upwind sailing: application in a Tornado, the Olympic class catamaran. Journal of Marine Scientific \& Technology, Vol 13, 190-206.

[Lee et al. (2007)] Lee, S.H., Lee, Y.G., Kim, S.H. (2007). On the development of a small catamaran boat. Ocean Engineering, Vol 34, 2061-2073. 
[Lin (1974)] Lin, W.C. (1974). Force and moment on a twin-hull ship in a steady potential flow. In Proc. 10th Symposium on Naval Hydrodynamics, Washington D.C., Jun 24-28, 1974.

[Liu \& Yue (1996)] Liu, Y., \& Yue, D.K. (1996). On the time dependence of the wave resistance of a body accelerating from rest. Journal of Fluid Mechanics, Vol. 310, 337-364.

[Librescu et al. (2007)] Librescu, L., Oh, S.Y. \& Hohe, J. (2007). Implication of Nonclassical effects on dynamic response of sandwich structures exposed to underwater and in-air explosions. Journal of Ship Research, Vol. 51/2, 83-93.

[Longo et al. (1993)] Longo, J., Stern, F., \& Toda, Y. (1993). Mean-flow measurements in the boundary layer and wake and wave field of a Series $60 C_{B}=0.6$ ship model-part 2: scale effects on near-field wave patterns and comparisons with inviscid theory. Journal of Ship Resistance, Vol. 37(1), 16-25.

[Lugni et al. (2004)] Lugni, C., Colagrossi, A., Landrini, M., \& Faltinsen, O.M. (2004). Experimental and numerical study of semi-displacement mono-hull and catamaran in calm water and incident waves. En Proc. of 25th symposium on naval hydrodynamics, St. John's, Canada.

[Lunde (1951)] Lunde, J.K. (1951). On the linearized theory of wave resistance for displacement ships in steady and accelerated motion. Society of Naval Architects ans Marine Engineers.

[Martínez \& Castells (2005)] Martínez de Osés, F.X. \& Castells, M. (2009). High speed craft viability analysis. Journal of Maritime Research, Vol 2 (3), 59-76.

[Martínez Legazpi et al. (2012)] Martínez Legazpi, P., Rodríguez Rodríguez, J., Marugán Cruz, C., \& Lasheras, J. C. (2012). Plunging to spilling transition in corner surface waves in the wake of a partially submerged vertical plate. Experiments in Fluids, Vol. 54(1).

[Martínez Legazpi et al. (2015)] Martínez Legazpi, P., Rodríguez Rodríguez, J., Korobkin, A. \& Lasheras, J.C. (2015). Formation of corner waves in the wake of a partially submerged bluff body. Journal of Fluid Mechanics, Vol 771, 547-563. doi:10.1017/jfm.2015.192 
[Macfarlane (2009)] Macfarlane, G.J. (Enero 2009). Correlation of Prototype and Model-Scale Wave Wake Characteristics of a Catamaran. Marine Technology, Vol 46/1, 1-15.

[McDonald et al. (2011)] McDonald, T.P., Bucknall, R.W., \& Greig, A.R. (2013). Comparing trimaran small waterplane area center hull (TriSWACH), monohull, and trimaran hullforms: some initial results. Journal of Ship Production and Design, Vol. 29(4), 211220.

[Michell (1898)] Michell, J.H. (1898). XI. The wave-resistance of a ship. The London, Edinburgh, and Dublin Philosophical Magazine and Journal of Science, 45(272), 106-123.

[Migali et al. (2001)] Migali, A., Miranda, S. \& Pensa, C. (2001). Experimental study on the efficiency of trimaran configuration for high-speed very large ships. FAST, RINA, 4-6.

[Miller et al. (2006)] Miller, R., Gorski, J., Xing, T., Carrica, P. \& Stern, F. (2006). Resistance predictions of high speed Mono and Multihull ships with and without water jet propulsors using URANS. $26^{\text {th }}$ symposium on naval hidrodinamics, Vol. 3.

[Min \& Kang (2010)] Min, K.S. \& Kang, S.H. (2010). Study on the form factor and full-scale ship resistance prediction method. Journal of Marine Science and Technology, Vol 15 (2), 108-118.

[Molland et al. (1996)] Molland, A.F., Wellicome, J.F. \& Couser, P.R. (1996). Resistance experiments on a systematic series of high speed displacement catamaran forms: variation of length-displacement ratio and breadth-draught ratio. RINA, Vol. 138, 5571.

[Molland et al. (2004)] Molland, A.F., Wilson, P.A., Taunton, D.J., Chandrapabha, S. \& Ghani, P.A. (2004). Resistance and wash wave measurements on a series of high speed displacement monohull and catamaran forms in shallow water. RINA, Vol. 146/A2, 1938.

[Moraes et al. (2004)] Moraes, H.B., Vasconcellos, J.M. \& Latorre, R.G. (2004). Wave resistance for high speed catamarans. Ocean Engineering, Vol 31, 2253-2282. 
[Moraes et al. (2007)] Moraes, H.B., Vasconcellos, J.M. \& Almeida, P.M. (2007). Multiple criteria optimization applied to high speed catamaran preliminary design. Ocean Engineering, Vol 34, 133-147.

[Moreno et al. (1975)] Moreno, M., Pérez Rojas, L. \& Landweber, L. (1975) Effect of wake on wave resistance of a ship model. IIHR Rep 180:1-32

[Muscat-Fenech \& Rosa (2014)] Muscat-Fenech, C. \& Rosa, A. (2014). Resistance analysis for a trimaran. International Journal of Mechanical, Aerospace, Industrial and Mechatronics Engineering, Vol 8(1), 7-15. World Academy of Science, Engineering and Technology.

[Nakatake \& Takeshi (1994)] Nakatake, K., Takeshi, H. (1994). Review of program 2, inviscid flow around series 60 with free surface. CFD Workshop, Vol.2, 130-159.

[Pavkov \& Morabito (2014)] Pavkov, M., \& Morabito, M. (2014). Experimental investigation of trimaran models in shallow water. Journal of Ship Production and Design, Vol. 30(2), 66-78.

[Peng (2001)] Peng, H. (2001). Numerical computation of multi-hull ship resistance and motion. Tesis Doctoral, Dalhouse University (Canada).

[Pérez Arribas (2000)] Pérez Arribas, F.L. (2000). Estudio hidrodinámico de un catamaran tipo SWATH. Tesis Doctoral, Universidad Politécnica de Madrid (España).

[Pérez Arribas et al. (2004)] Pérez Arribas, F., López Pavón, C. \& Pérez Rojas, L. (2004). Optimización de las formas de un buque atunero mediante el cfd TDYNLIN y tecnicas experimentales. Métodos Computacionais em Engenharia. Lisboa, (Portugal).

[Pérez Rojas (1994)] Pérez Rojas, L. (1994). Métodos matemáticos en hidrodinámica : (notas bibliográficas). Publicaciones ETSIN (UPM)

[Pérez Rojas (2009)] Pérez Rojas, L. (2009). Los canales de ensayo frente a los cálculos numéricos. Actas del XXI Congreso Panamericano de Ingeniería Naval (COPINAVAL), Montevideo (Uruguay). 
[Perić (2013)] M. Perić, F. Schäfer, E. Schreck \& J. Singh (2013). Application of Star-CCM+ in marine and offshore engineering: state-of-the-art and new developments. STAR South Asian Conference, Malasia.

[Prandtl (1904)] Prandtl, L. (1904) Über Flüssigkeitsbewegung bei sehr kleiner Reibung. Verh. III. Intern. Math. Kongr., Heidelberg, 1904, S. 484-491, Teubner, Leipzig, 1905.

[Prohaska (1966)] Prohaska, C.W. (1966). Discussion of Hughes' “An analysis of Ship Model Resistance into Viscous and Wave Components". Transactions of RINA, Londres.

[Rigterink (2014)] Rigterink, D.T. (2014). Methods for Analyzing Early Stage Naval Distributed Systems Designs, Employing Simplex, Multislice, and Multiplex Networks. Tesis doctoral, Naval Architecture and Marine Engineering (Univ. of Michigan).

[Sahoo et al. (2004)] Sahoo, P.K., Browne, N.A., \& Salas, M. (2004). Experimental and CFD study of wave resistance of high-speed round bilge catamaran hull forms. En Proc. of $4^{\text {th }}$ International Conference on High Performance Marine Vehicles, Rome, Italy.

[Sarles et al. (2011)] Sarles, C., Gelles, B., \& Malarkey, A. (2011). An Investigation into the effect of section shape on the interference resistance of catamarans. En Proc. $11^{\text {th }}$ International Conference on Fast Sea Transportation (FAST2011), pp. 335-362.

[Savaş (2014)] Savaş, Ö. (2014). ME260A/B Advanced Fluid Mechanics. Class notes. Dept. of Mechanical Engineering, University of California at Berkeley.

[Schlichting et al. (2000)] Schlichting, H., Gersten, K., \& Gersten, K. (2000). Boundary-layer theory. Springer Science \& Business Media.

[Schoenherr (1932)] Schoenherr, K.E. (1932). Resistance of flat surfaces moving through a fluid. SNAME Transactions, Vol 40, 279-313.

[SNAME (1941)] SNAME (1941). Principles of Naval Architecture, Vol II: Resistance. Society of naval architects and marine engineers. Editors Henry Eastin Rossell, L. B. Chapman. 
[Söding (1997)] Söding, H. (1997). Drastic resistance reductions in catamarans by staggered hulls. En Proc. $4^{\text {rd }}$ International Conference on Fast Sea Transportation (FAST'97), pp. 225-230.

[Souto-Iglesias (2001)] Souto Iglesias, A. (2001). Nuevas herramientas de diseño de formas de buques basadas en códigos de flujo potencial. Tesis doctoral, ETSIN (UPM).

[Souto-Iglesias et al. (2005)] Souto Iglesias, A., Zamora, R., Fernández, D. \& López, C. (2005). Catamaran wave resistance and central wave cuts for CFD validation. $12^{\text {th }}$ International Congress of the International Maritime Association of the Mediterranean (IMAM 2005), Vol. 1, 157-165.

[Souto-Iglesias et al. (2007)] Souto Iglesias, A., Zamora, R., Fernández, D. \& Pérez Rojas, L. (2007). Analysis of the wave system of a catamaran for CFD validation. Experiments in Fluids, Vol. 42/2, 321-332.

[Souto-Iglesias et al. (2012)] Souto-Iglesias, A., Fernández-Gutiérrez, D., \& Pérez-Rojas, L. (2012). Experimental assessment of interference resistance for a Series 60 catamaran in free and fixed trim-sinkage conditions. Ocean Engineering, Vol. 53, 38-47.

[Subramani et al. (2000)] Subramani, A.K., Paterson, E.G. \& Stern, F. (2000). CFD Calculation of Sinkage and Trim. Journal of Ship Research, Vol 44/1, 59-82.

[Tarafder \& Suzuki (2007)] Tarafder, S. \& Suzuki, K. (2007). Computation of a wave-making resistance of a catamaran in deep water using a potential-based panel method. Ocean Engineering, Vol 34, 1892-1900.

[Tarafder \& Suzuki (2008a)] Tarafder, S. \& Suzuki, K. (2008). Wave-Making Resistance of a Catamaran Hull in Shallow Water Using a Potential-Based Panel Method. Journal of Ship Research, Vol. 52/1, 16-29.

[Tarafder \& Suzuki (2008b)] Tarafder, S., \& Suzuki, K. (2008). Numerical calculation of freesurface potential flow around a ship using the modified Rankine source panel method. Ocean Engineering, Vol. 35(5), 536-544. 
[Tarafder et al. (2010)] Tarafder, M.S., Khalil, G.M., \& Nizam, M.S. (2010) Computation of wave-making resistance of tetramaran hull in unbounded water. Proceedings of the International Conference on Marine Technology (MARTEC), Bangladesh

[Toda et al. (1992)] Toda, Y., Stern, F., \& Longo, J. (1992). Mean-flow measurements in the boundary layer and wake and wave field of a series $60 C_{B}=0.6$ ship model-Part 1: Froude numbers 0.16 and 0.316. Journal of Ship Resistance, Vol. 36(4), 360-377.

[Todd (1963)] Todd, F.H. (1963). Series 60 methodical experiments with models of single-screw merchant ships. Informe técnico No. DTMB-1712. David W.Taylor Model Basin, Washington DC, USA.

[Tuck (1987)] Tuck, E.O. (1987). Wave resistance of Thin Ships and Catamaran. Internal report T8701. Adelaide: Applied Mathematics Department of the University of Adelaide, Adelaide.

[Tuck \& Lazauskas (1998)] Tuck, E.O., \& Lazauskas, L. (1998). Optimum hull spacing of a family of multihulls. Ship Technology Research-Schiffstechnik, Vol. 45(4), 180.

[Tuck et al. (2002)] Tuck, E.O., Scullen, D.C., \& Lazauskas, L. (2002). Wave patterns and minimum wave resistance for high-speed vessels. Proc. 24th Symposium on Naval Hydrodynamics, Fukuoka, Japan (pp. 8-13).

[Tuck (2005)] Tuck, E.O. (2005). Can lateral asymmetry of the hulls reduce catamaran wave resistance? En $20^{\text {th }}$ International workshop on water waves and floating bodies, Spitzbergen, Norway.

[Turner \& Taplin (1968)] Turner, H. \& Taplin, A. (1968). The resistance of large powered catamarans. SNAME, Vol 76, 180-213.

[Wan \& Yeung (2012)] Wan, H., \& Yeung, R.W. (2012). Interaction of bodies in free surface with consideration of cross-flow. Computers \& Fluids, 54, 127-142. 
[Wu \& Moan (2006)] Wu, M.K. \& Moan, T. (2006). Numerical prediction of wave-induced long-term extreme load effects in a flexible high-speed pentamaran. Journal of Marine Science and Technology, Vol 11 (1), 39-51.

[Yeung (1978)] Yeung, R. W. (1978). On the interactions of slender ships in shallow water. Journal of Fluid Mechanics, Vol. 85(01), 143-159.

[Yeung et al. (2004)] Yeung, R.W., Poupard, G. \& Toilliez, J.O (2004). Interference-resistance prediction and its applications to optimal multi-hull configuration design. Transactions of the Society of Naval Architects and Marine Engineers (SNAME), Vol. 112, 142-168.

[Yeung (2005)] Yeung, R.W. (2005). Interference Resistance of Multi-hulls per thin-ship theory. In 20th International Workshop on Water Waves and Floating Bodies (IWWWFB), Longyearbyen, Spitsbergen.

[Yeung \& Wan (2008)] Yeung, R.W., Wan, H. (2008). Multihull and surface-effect ship configuration design: a framework for powering minimization. J. Offshore Mech. Arct. Eng. Vol 130(3), 031005, 1-9. doi:10.1115/1.2904590

[Yu (2015)] Yu, D. (2015). Interference Resistance Optimization for Multi-hull Vessels, Model Test and Simulation. Tesis Máster de Ciencia (University of California at Berkeley)

[Zaghi et al (2011)] Zaghi, S., Broglia, R., \& Di Mascio, A. (2011). Analysis of the interference effects for high-speed catamarans by model tests and numerical simulations. Ocean Engineering, Vol. 38(17), 2110-2122.

[Zaraphonitis et al. (2001)] Zaraphonitis, G., Spanos, D. \& Papanikolau, A. (2001). Numerical and experimental study on the wave resistance of fast displacement asymmetric catamarans. $2^{\text {nd }}$ International Euroconference HIPER'01. 


\section{APÉNDICES}




\section{A PLANOS DE FORMAS}

- Catamarán de servicio comercial PESBO

- Catamarán $\mathbf{5 6 0}$ 


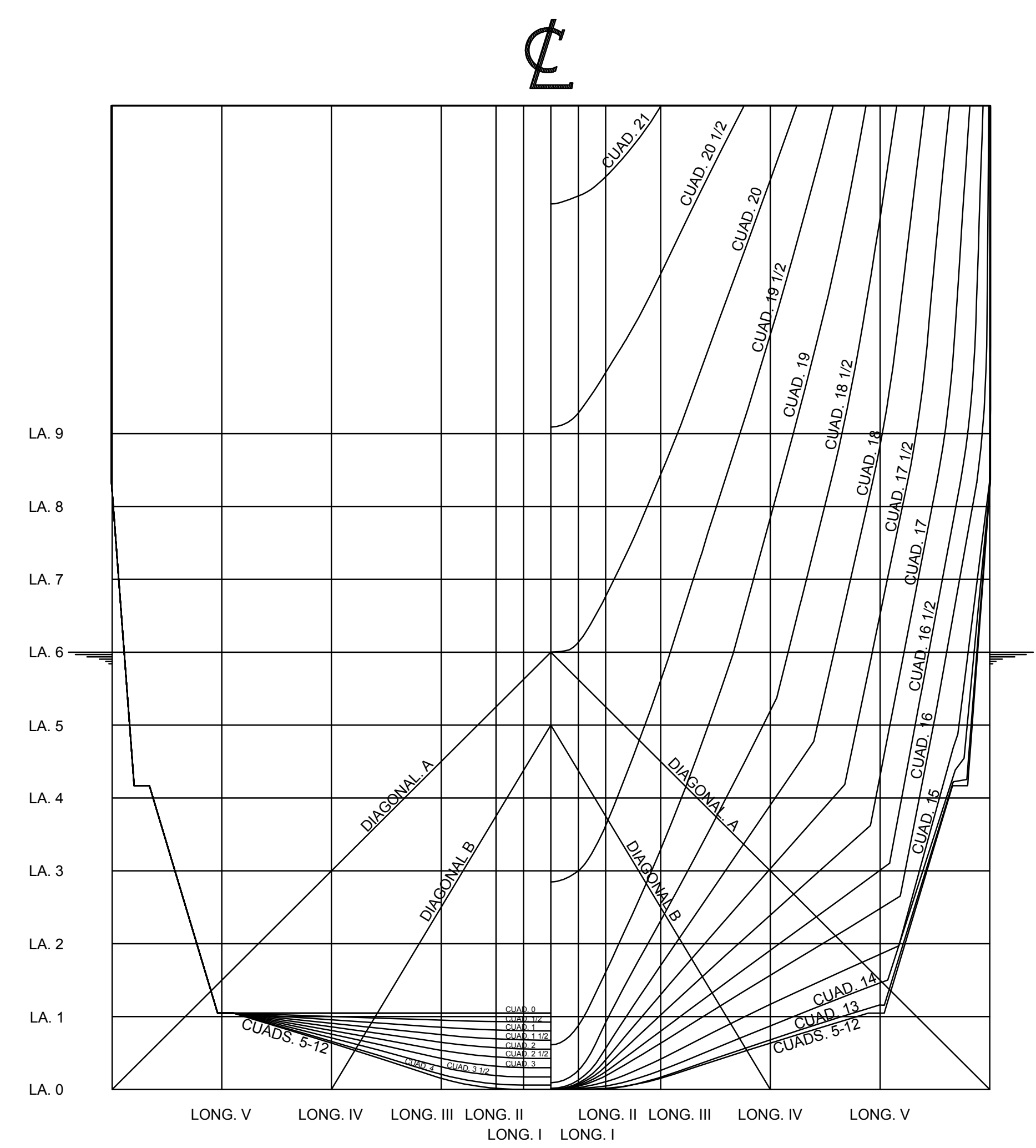

\begin{tabular}{|c|c|c|c|c|c|}
\hline 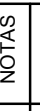 & & & & \multicolumn{2}{|c|}{$\begin{array}{l}\text { Apartado: } \\
\text { APÉNDICE A }\end{array}$} \\
\hline & \multirow{2}{*}{\multicolumn{2}{|c|}{$\begin{array}{l}\text { CAJA DE CUADERNAS } \\
\text { PESBO (Modelo a escala 1:12.5) }\end{array}$}} & \begin{tabular}{|l|} 
Escala: \\
$1 / 1.5$
\end{tabular} & \multicolumn{2}{|c|}{$\begin{array}{l}\text { Formato: } \\
\text { A3 HORIZONTAL }\end{array}$} \\
\hline & & & \begin{tabular}{|c|} 
Dibujado: \\
DFG
\end{tabular} & \multicolumn{2}{|c|}{\begin{tabular}{|l|} 
Fecha: \\
$11 /$ DIC / 2015
\end{tabular}} \\
\hline \multirow{2}{*}{$\mid$} & \multirow{2}{*}{\multicolumn{2}{|c|}{$\begin{array}{l}\text { TESIS DOCTORAL: } \\
\text { Análisis y optimización de la resistencia de } \\
\text { interferencia en multicascos }\end{array}$}} & Proyecto $\mathrm{n}^{0}:$ & \multicolumn{2}{|l|}{-- } \\
\hline & & & Plano no: & \multicolumn{2}{|c|}{ AA / 001B } \\
\hline \multirow{2}{*}{ 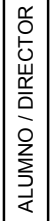 } & $\begin{array}{l}\text { ALUMNO: } \\
\text { DAVID FENÁ́NDEZ GUTIÉRREZ } \\
\text { e-maili: davididgaluamnos.upmes }\end{array}$ & \multirow{2}{*}{ UNIVERSIDA } & $\begin{array}{l}\text { UELA TÉCNI } \\
\text { JPERIOR DE } \\
\text { IEROS NAV }\end{array}$ & & ${ }^{\text {Rev. }} 0$ \\
\hline & $\begin{array}{l}\text { DIRECTOR: } \\
\text { ANTONIO SOUTO IGLESIAS }\end{array}$ & & $\begin{array}{l}913367140 \\
: 91542149 \\
\text { LITECNICAD }\end{array}$ & ADRID (UPM) & 2 of 2 \\
\hline
\end{tabular}




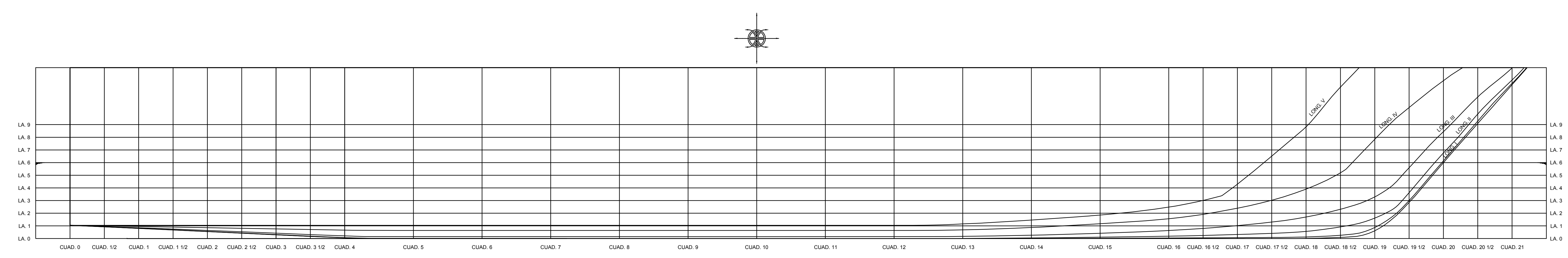

SISTEMA DE LONGITUDINALES

幽

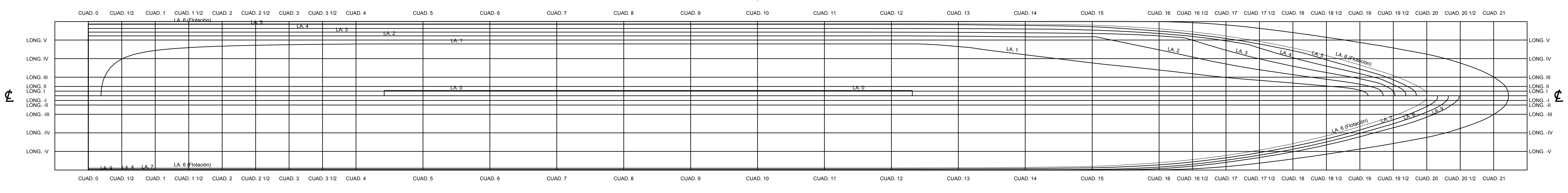

LÍNEAS DE AGUA

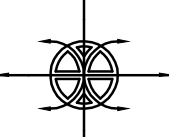

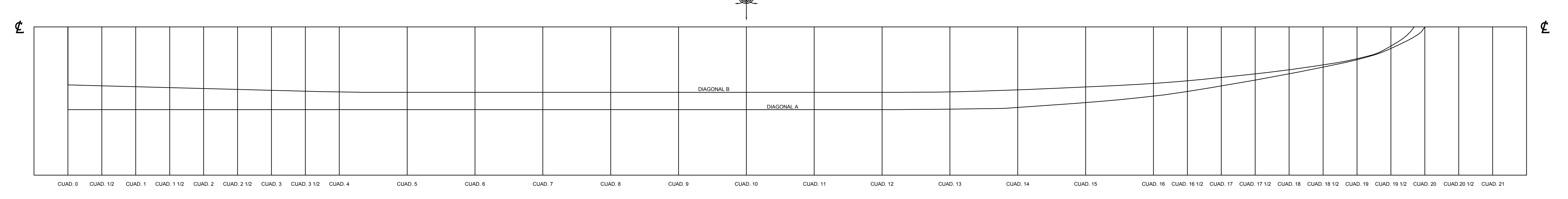

DIAGONALES DESARROLLADAS

\begin{tabular}{|c|c|c|c|}
\hline & CARA & STICAA & \\
\hline 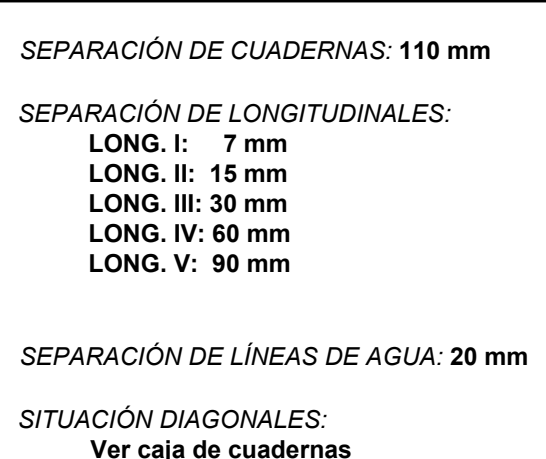 & 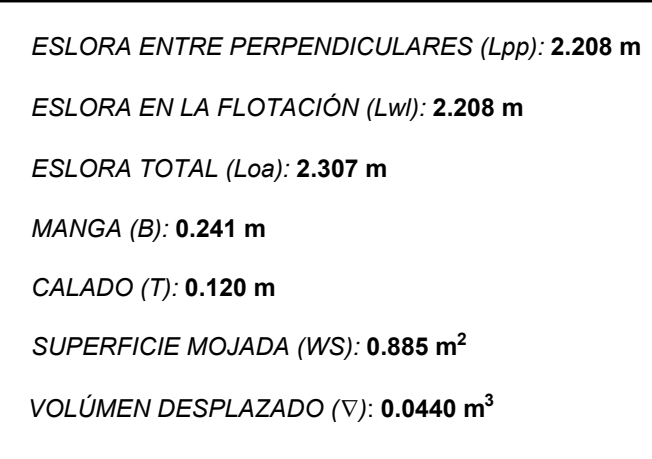 & 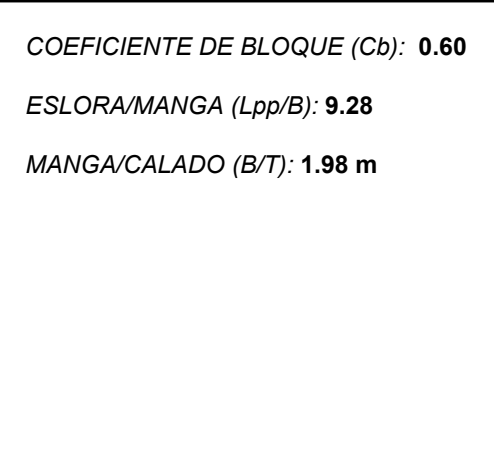 & \\
\hline
\end{tabular}

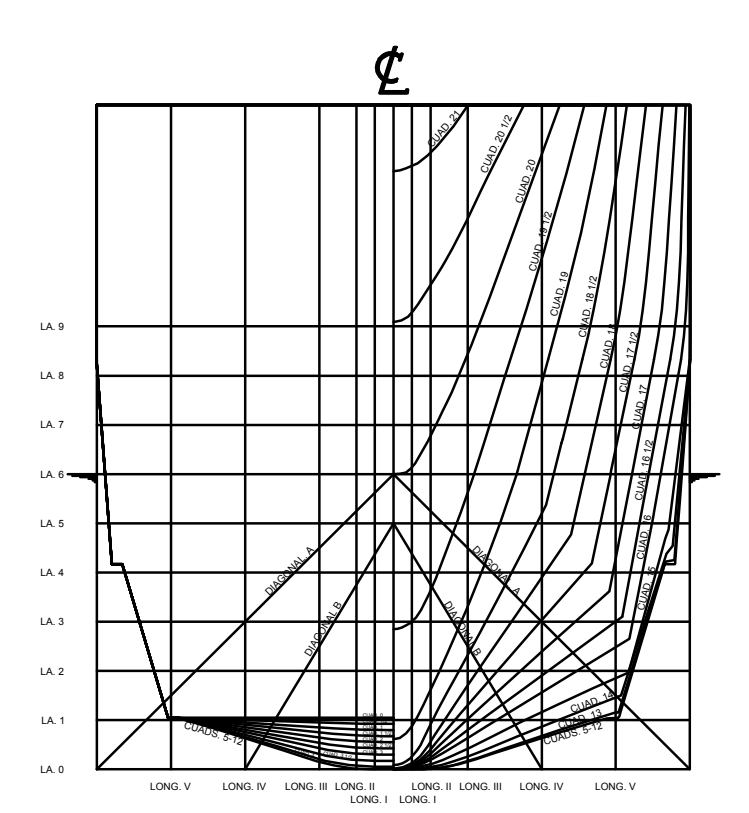

CAJA DE CUADERNAS 


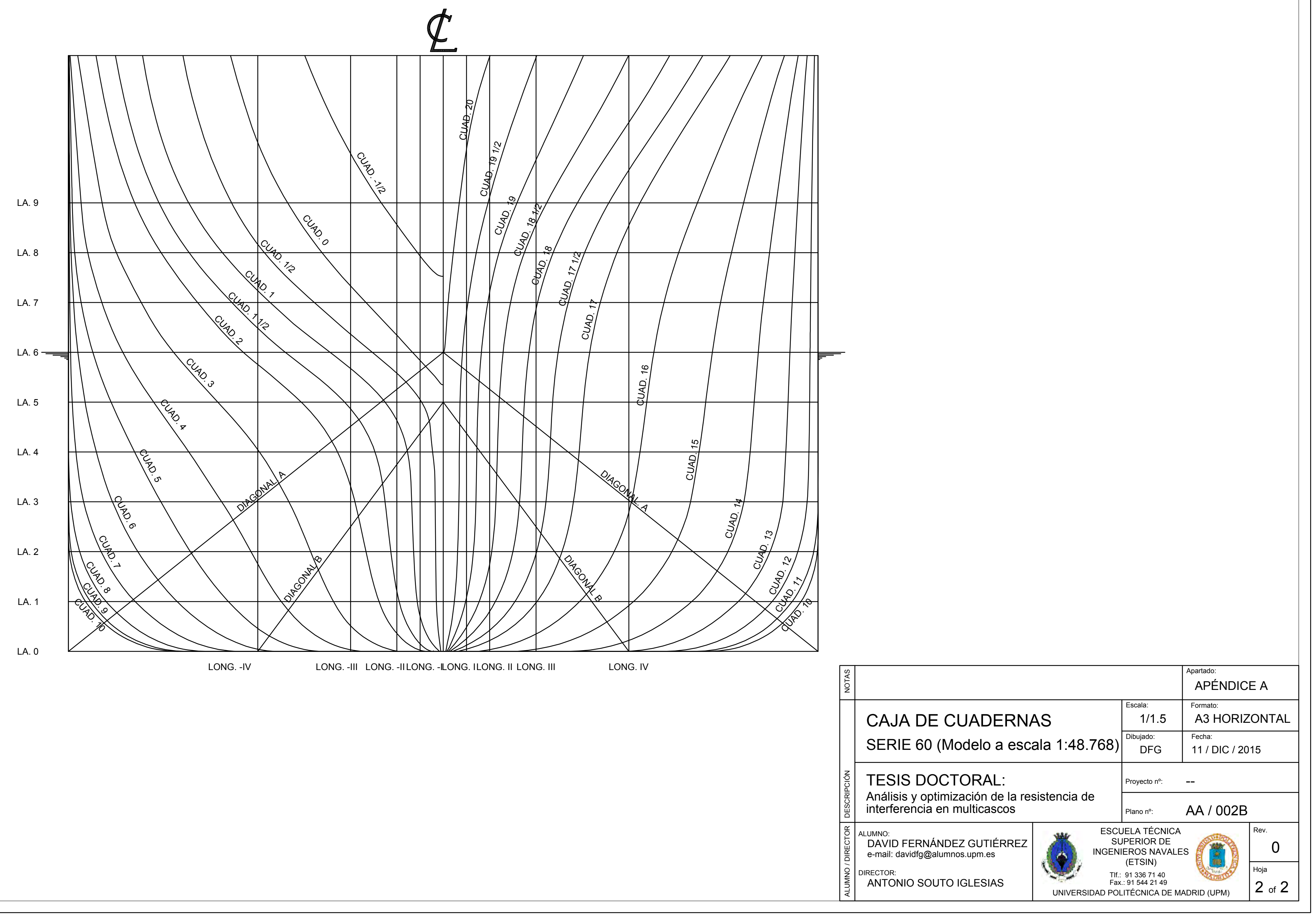




\section{B RESULTADOS EXPERIMENTALES}

\section{B.1. Resistencia al avance}

- $\quad$ Fig. 73. $\boldsymbol{R}_{\boldsymbol{T}}$ cat. PESBO, modelo libre

- $\quad$ Fig. 74. $\boldsymbol{R}_{\boldsymbol{T}}$ cat. PESBO, modelo fijo

- $\quad$ Fig. 75. $\boldsymbol{R}_{T}$ cat. S60, modelo libre

- $\quad$ Fig. 76. $\boldsymbol{R}_{\boldsymbol{T}}$ cat. S60, modelo fijo 


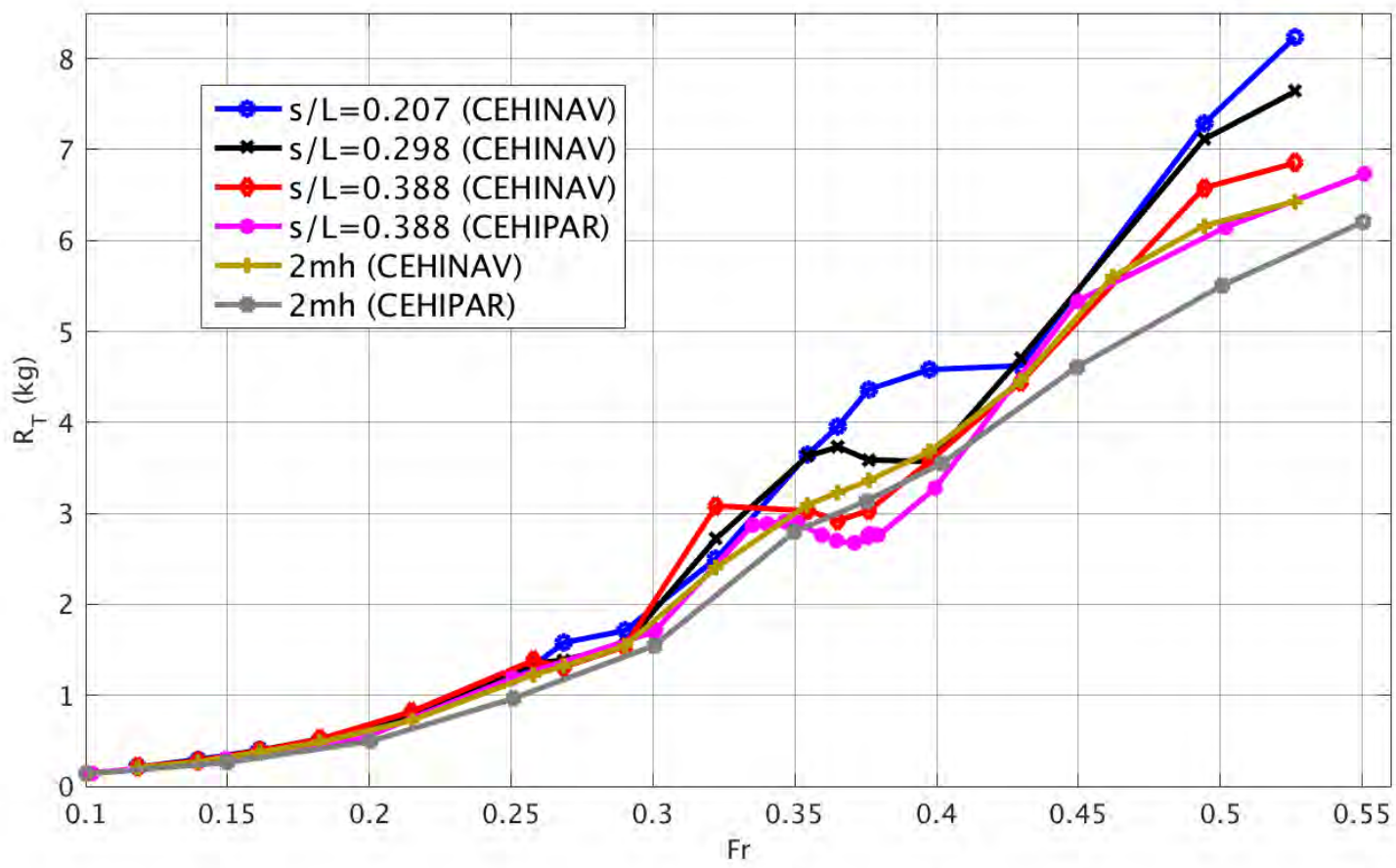

Fig. 73. $R_{T}$ cat. PESBO, modelo libre

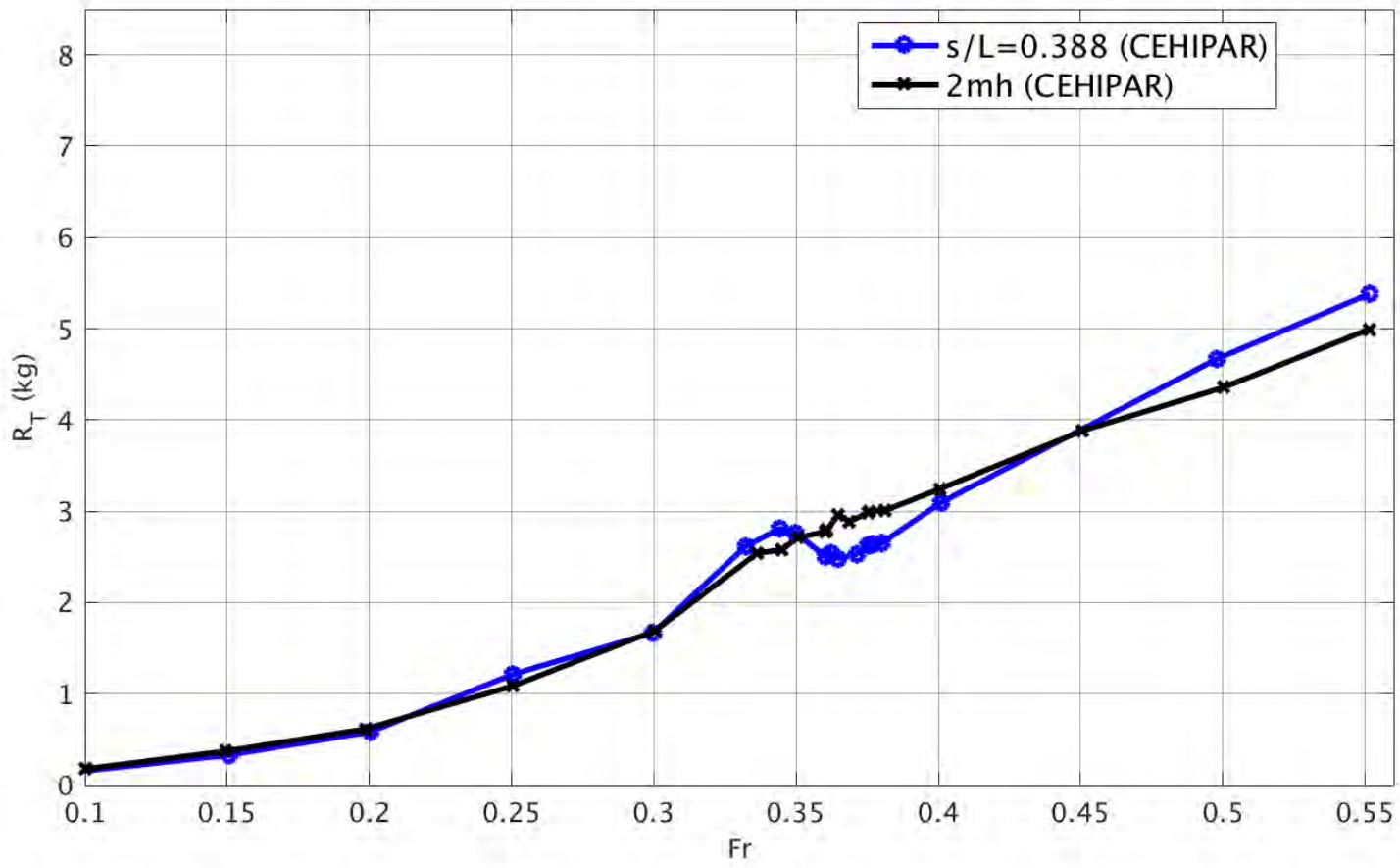

Fig. 74. $R_{T}$ cat. PESBO, modelo fijo 


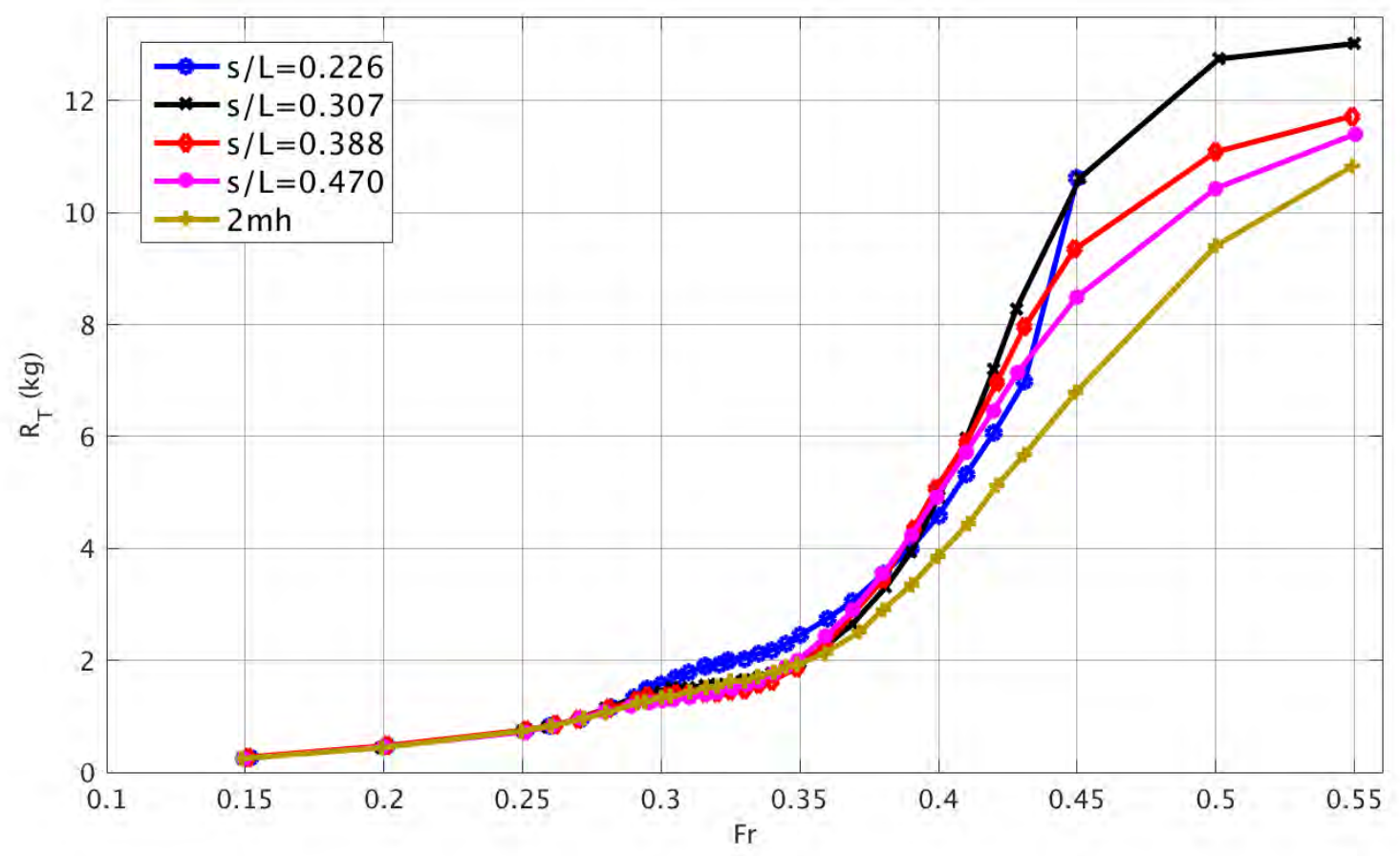

Fig. 75. $\boldsymbol{R}_{T}$ cat. 560 , modelo libre

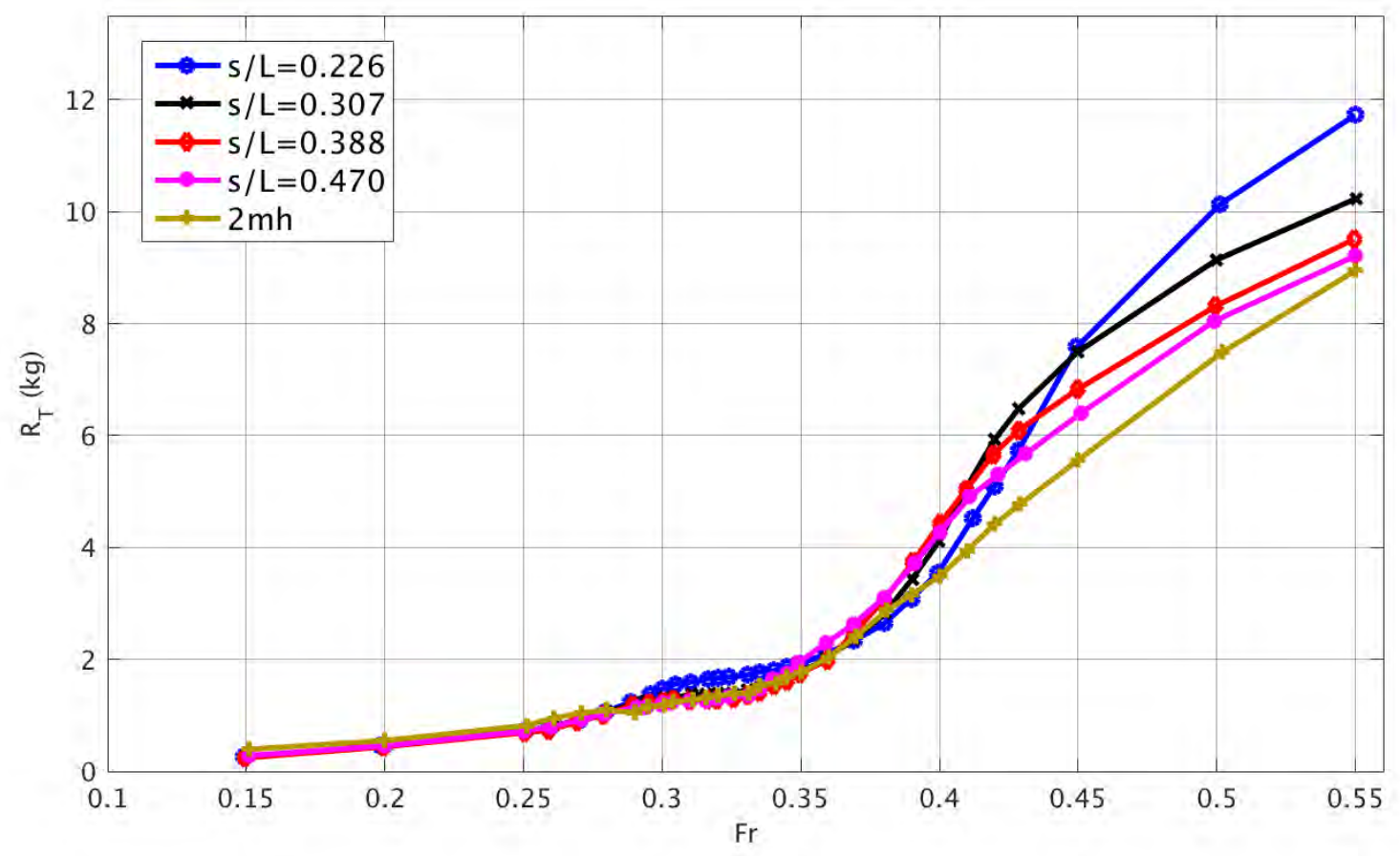

Fig. 76. $R_{T}$ cat. S60, modelo fijo 


\section{B.2. Factores de interferencia}

- $\quad$ Fig. 77. IF cat. PESBO, modelo libre

- $\quad$ Fig. 78. IF cat. PESBO, modelo fijo

- $\quad$ Fig. 79. IF cat. S60, modelo libre

- Fig. 80. IF cat. S60, modelo fijo 


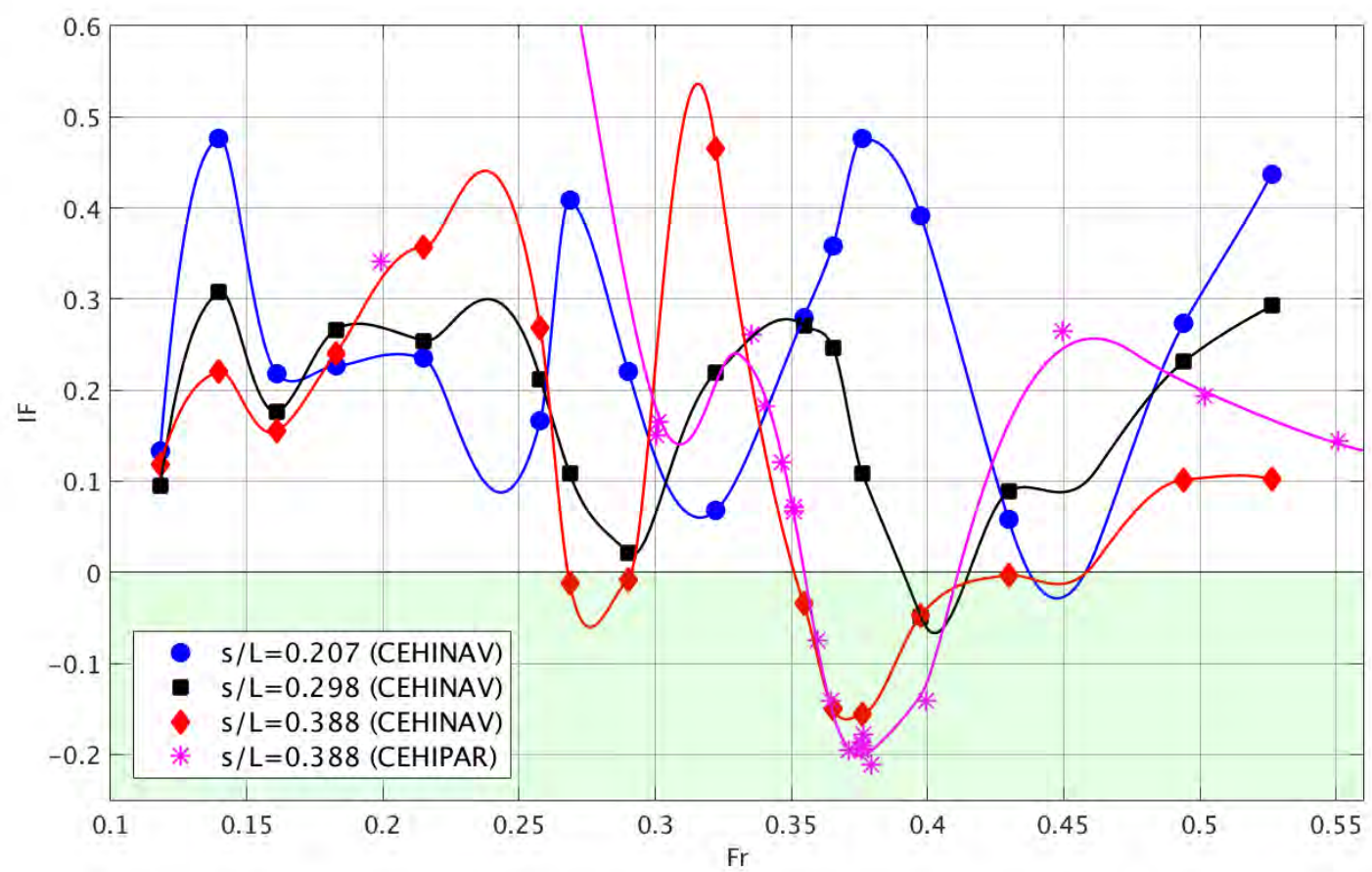

Fig. 77. IF cat. PESBO, modelo libre

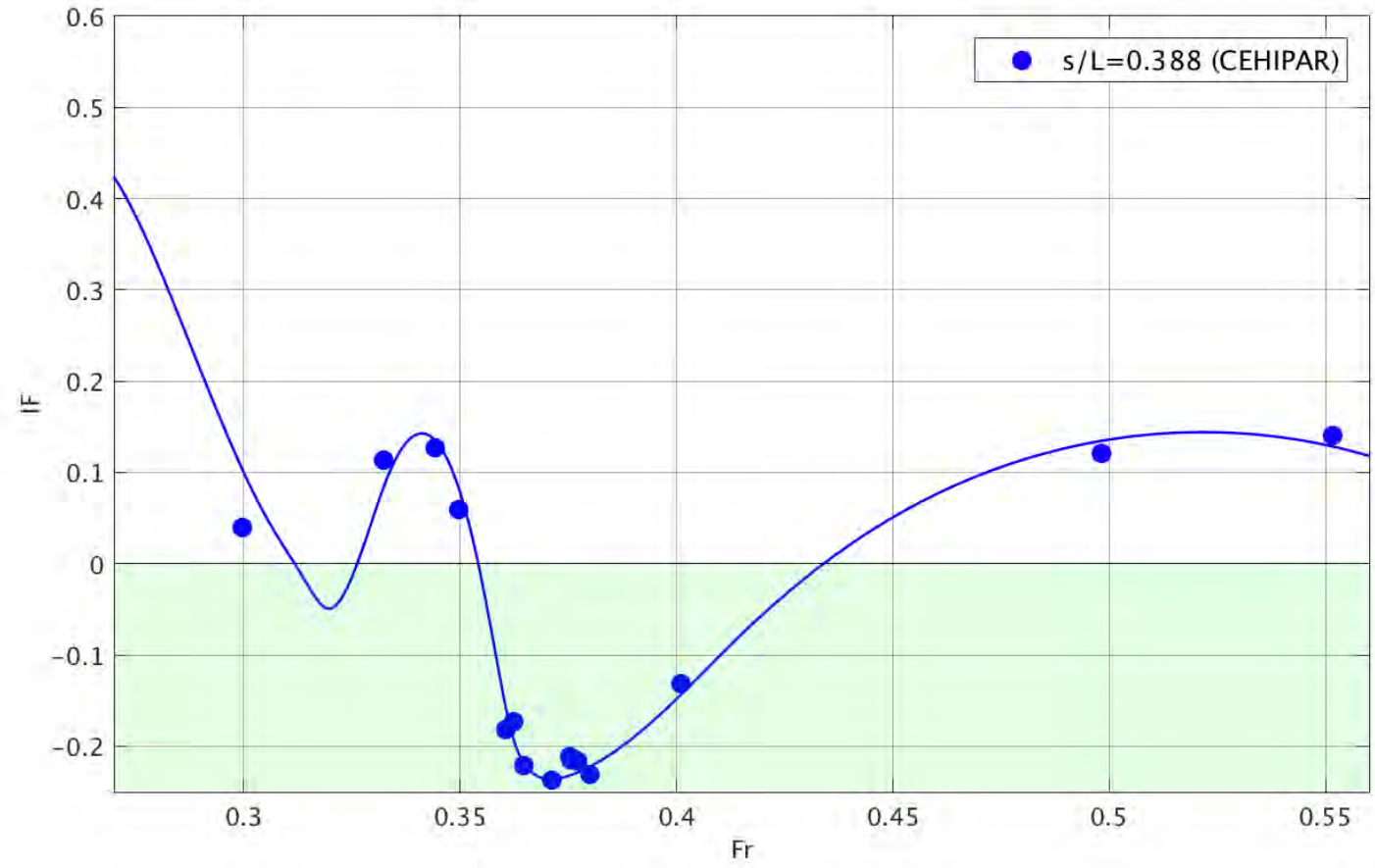

Fig. 78. IF cat. PESBO, modelo fijo 


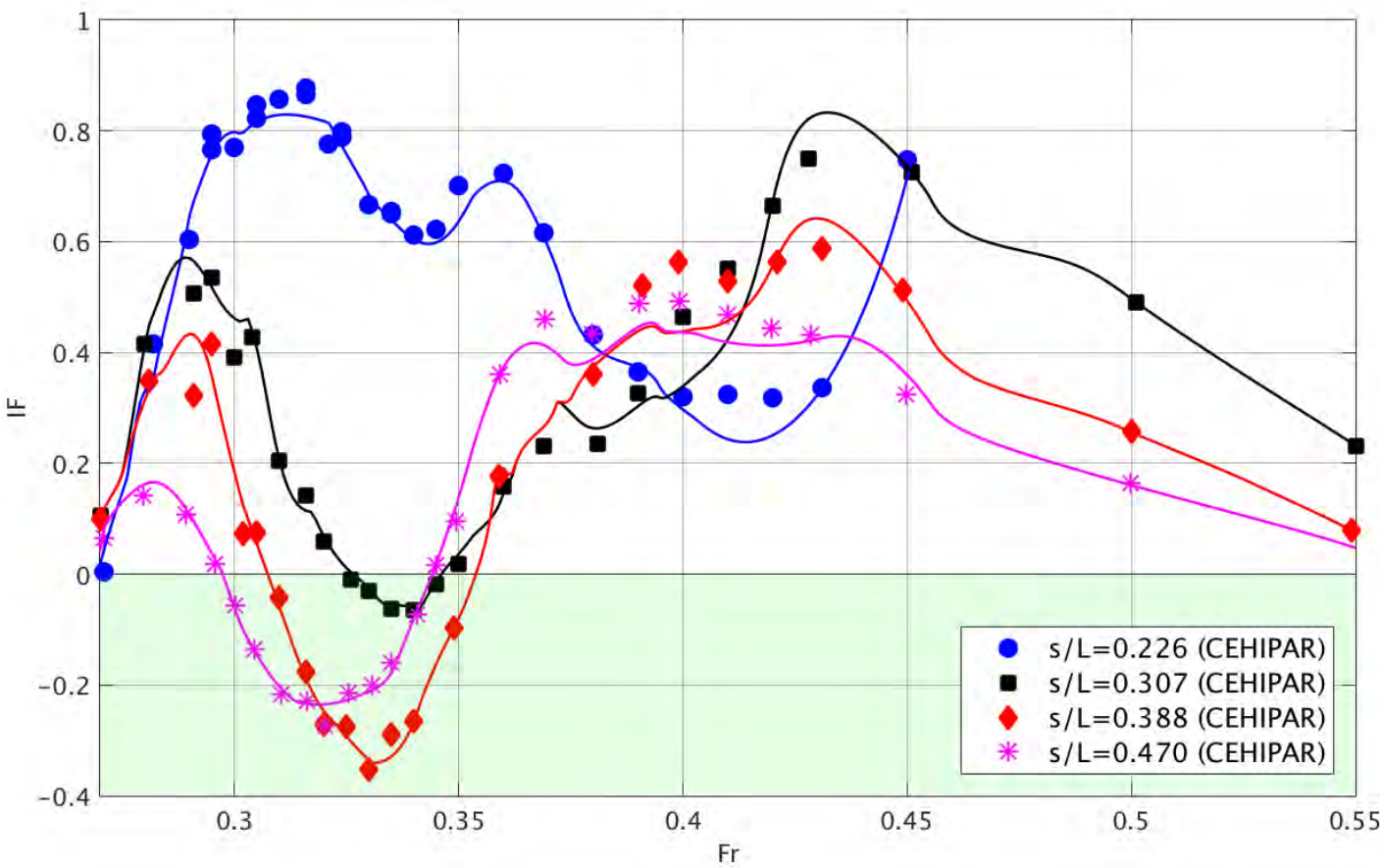

Fig. 79. IF cat. S60, modelo libre

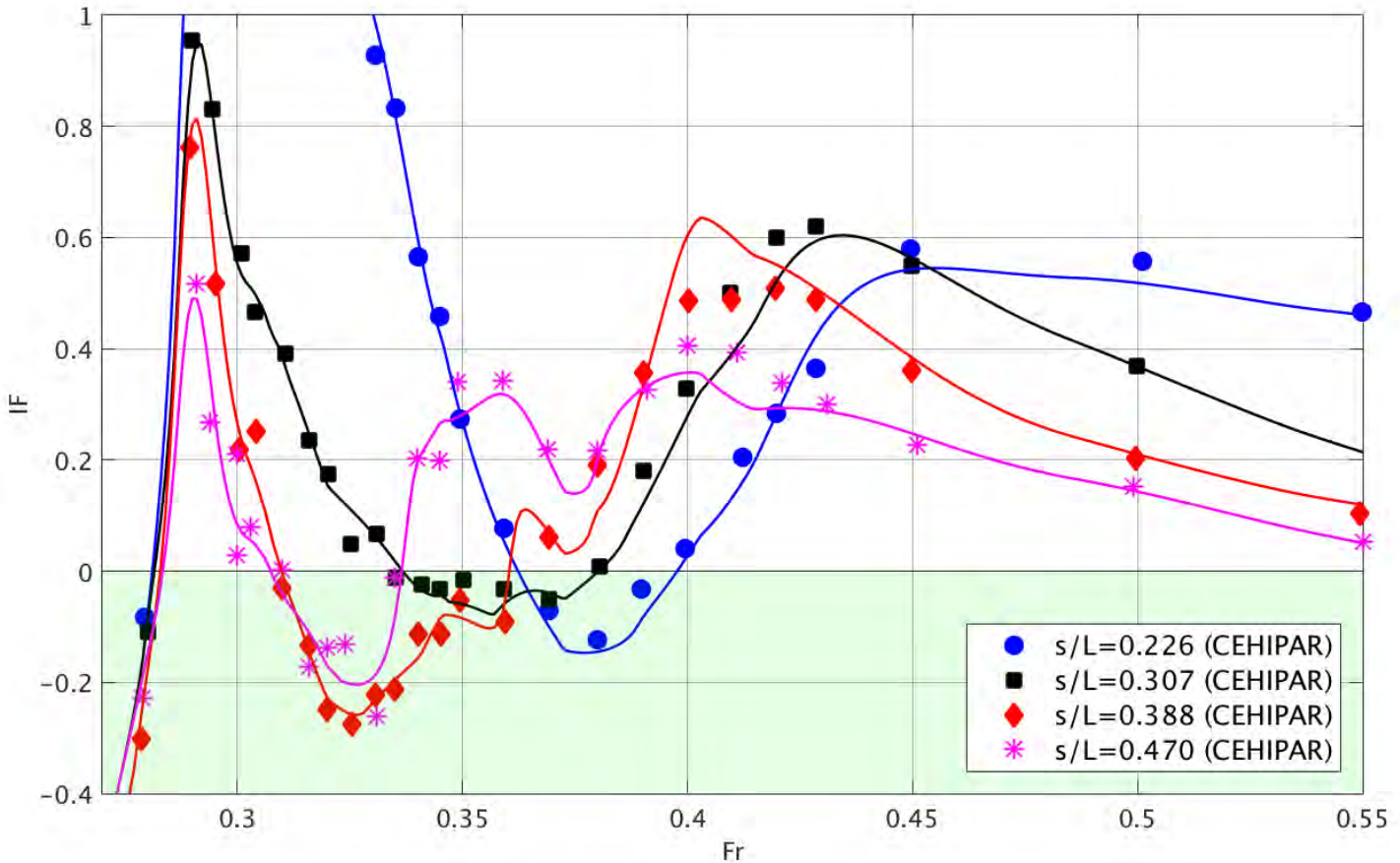

Fig. 80. IF cat. S60, modelo fijo 


\section{B.3. Asiento y trimado dinámicos}

- Fig. 81. Asiento dinámico, cat. PESBO

- Fig. 82 Trimado dinámico, cat. PESBO

- Fig. 83. Asiento dinámico, cat. S60

- Fig. 84. Trimado dinámico, cat. S60

- Fig. 85. $\boldsymbol{\delta} \boldsymbol{R}_{\boldsymbol{T}}$ cat. PESBO

- Fig. $86 . \boldsymbol{\delta} \boldsymbol{R}_{T}$ cat. $\mathrm{S} 60$

- Fig. 87. IF $\boldsymbol{F}_{\boldsymbol{S} \& \boldsymbol{T}}$ cat. PESBO

- Fig. 88. $\boldsymbol{I} \boldsymbol{F}_{\boldsymbol{S} \& \boldsymbol{T}}$ cat. $\mathrm{S} 60$ 


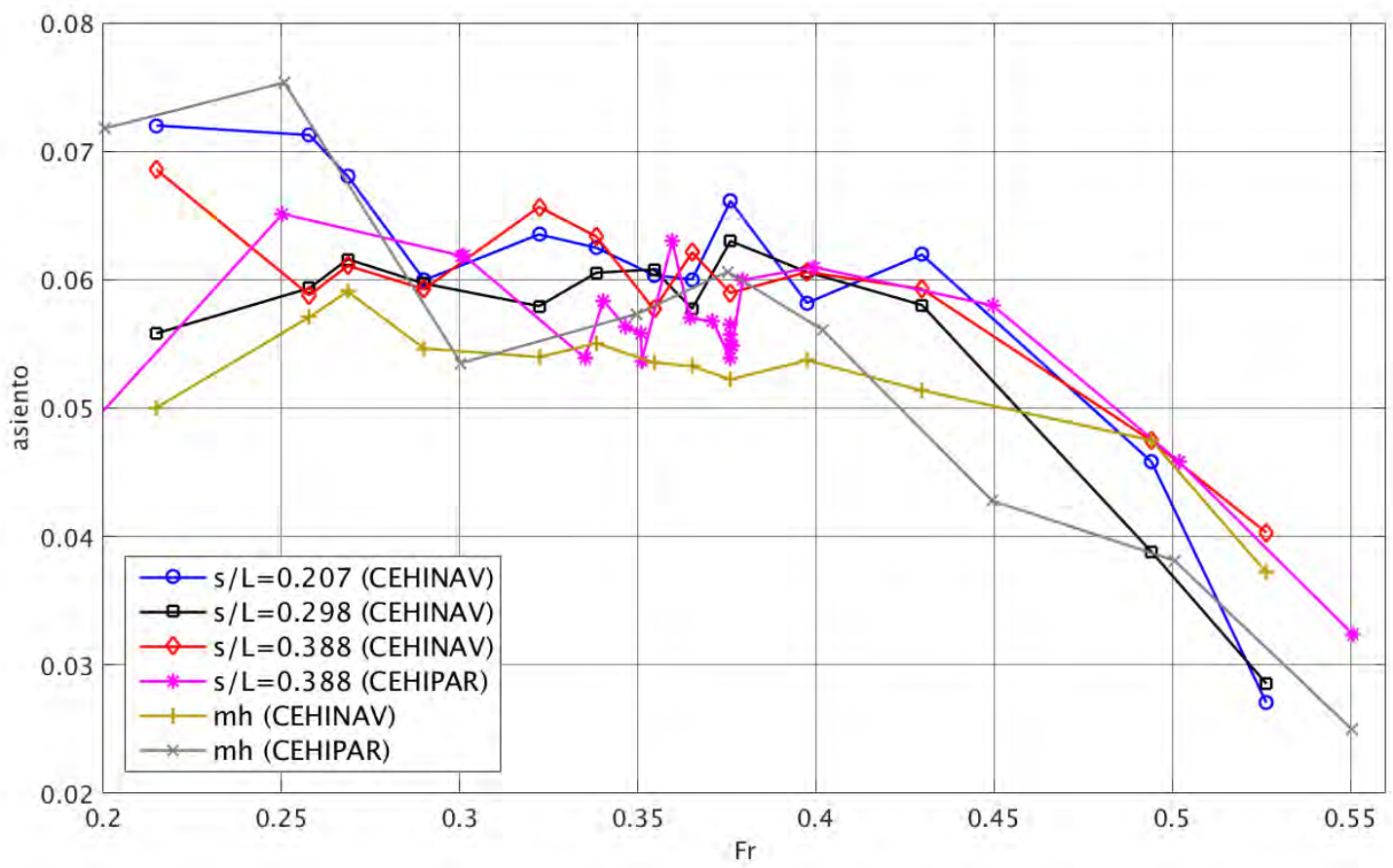

Fig. 81. Asiento dinámico, cat. PESBO

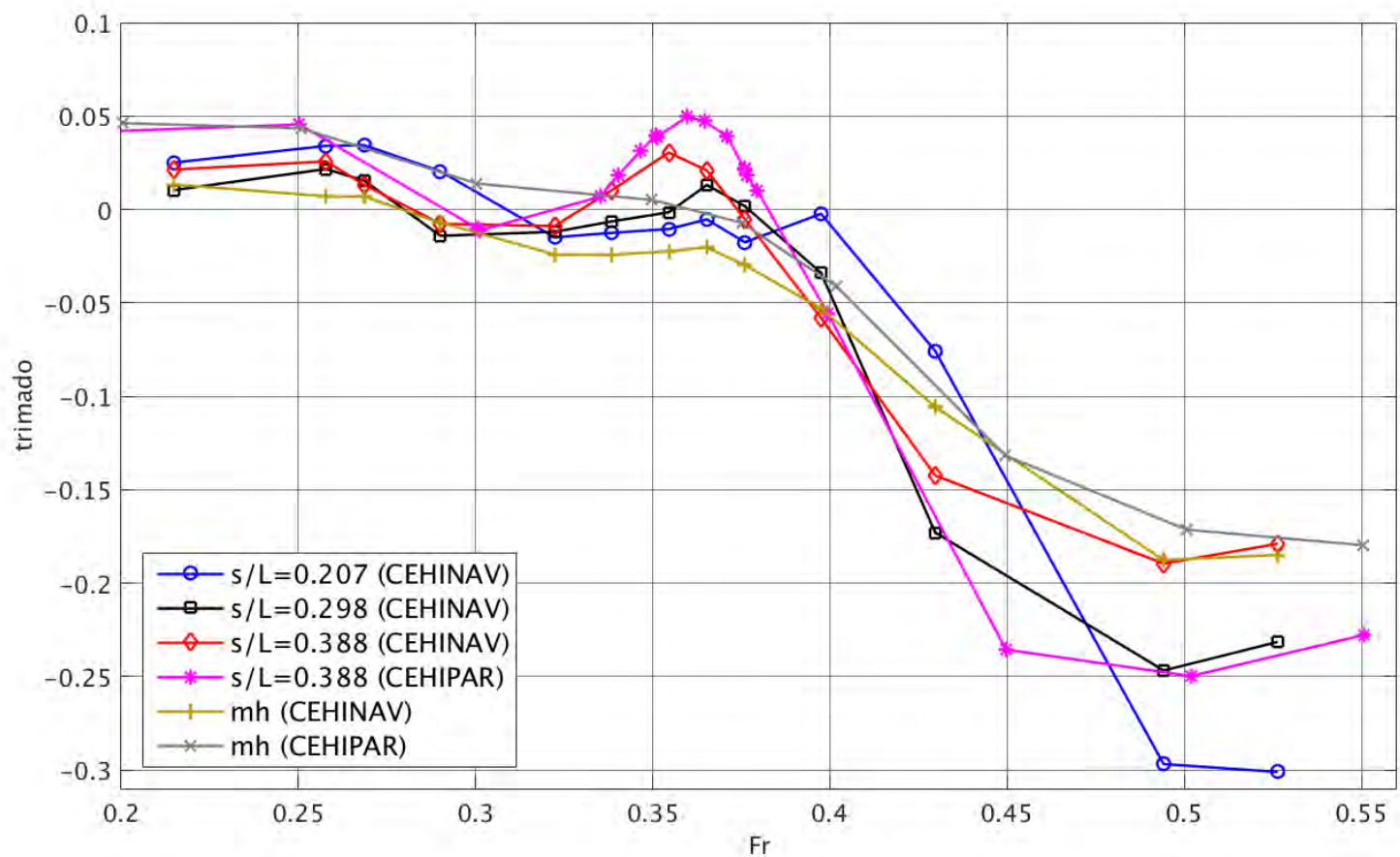

Fig. 82 Trimado dinámico, cat. PESBO 


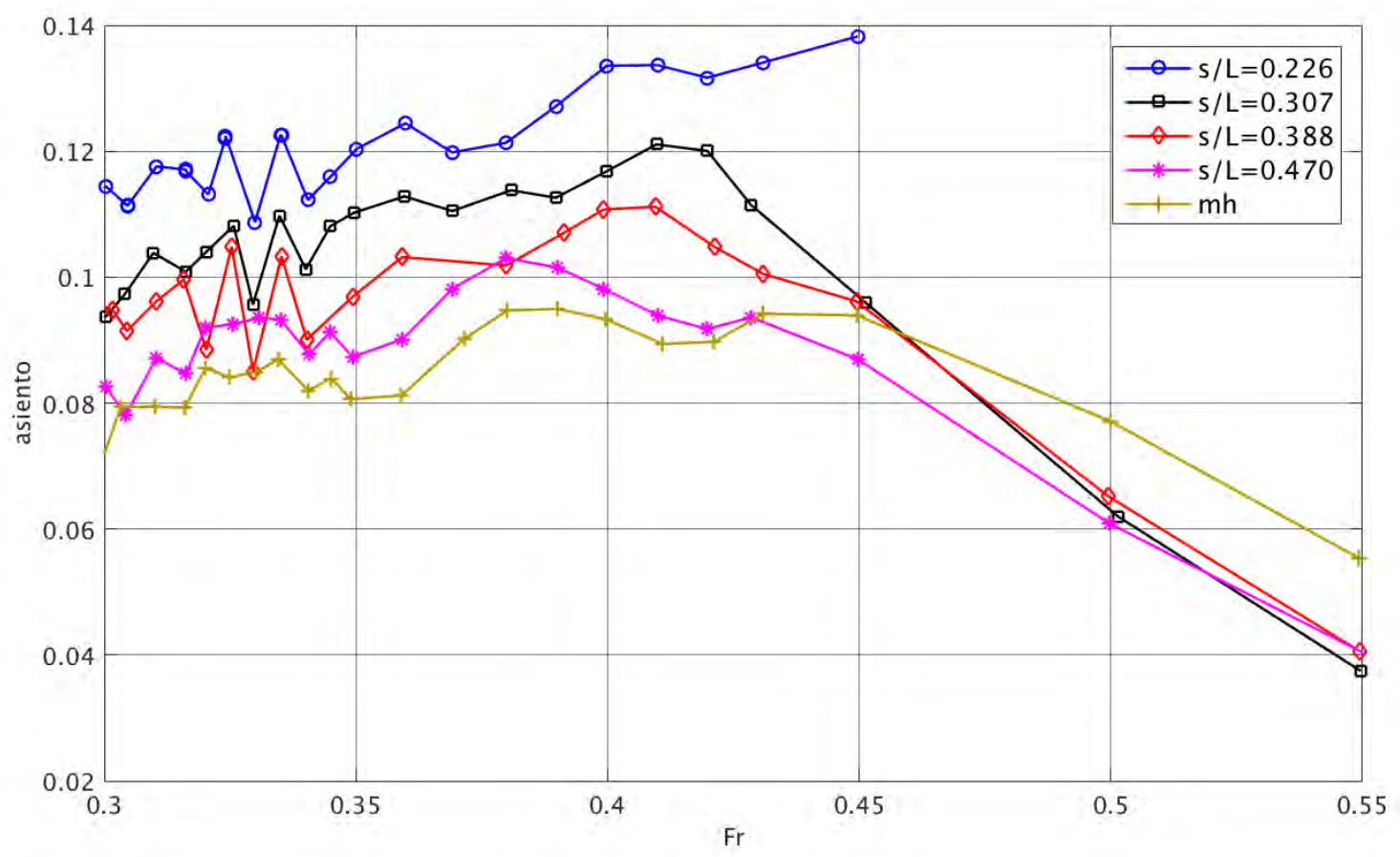

Fig. 83. Asiento dinámico, cat. $S 60$

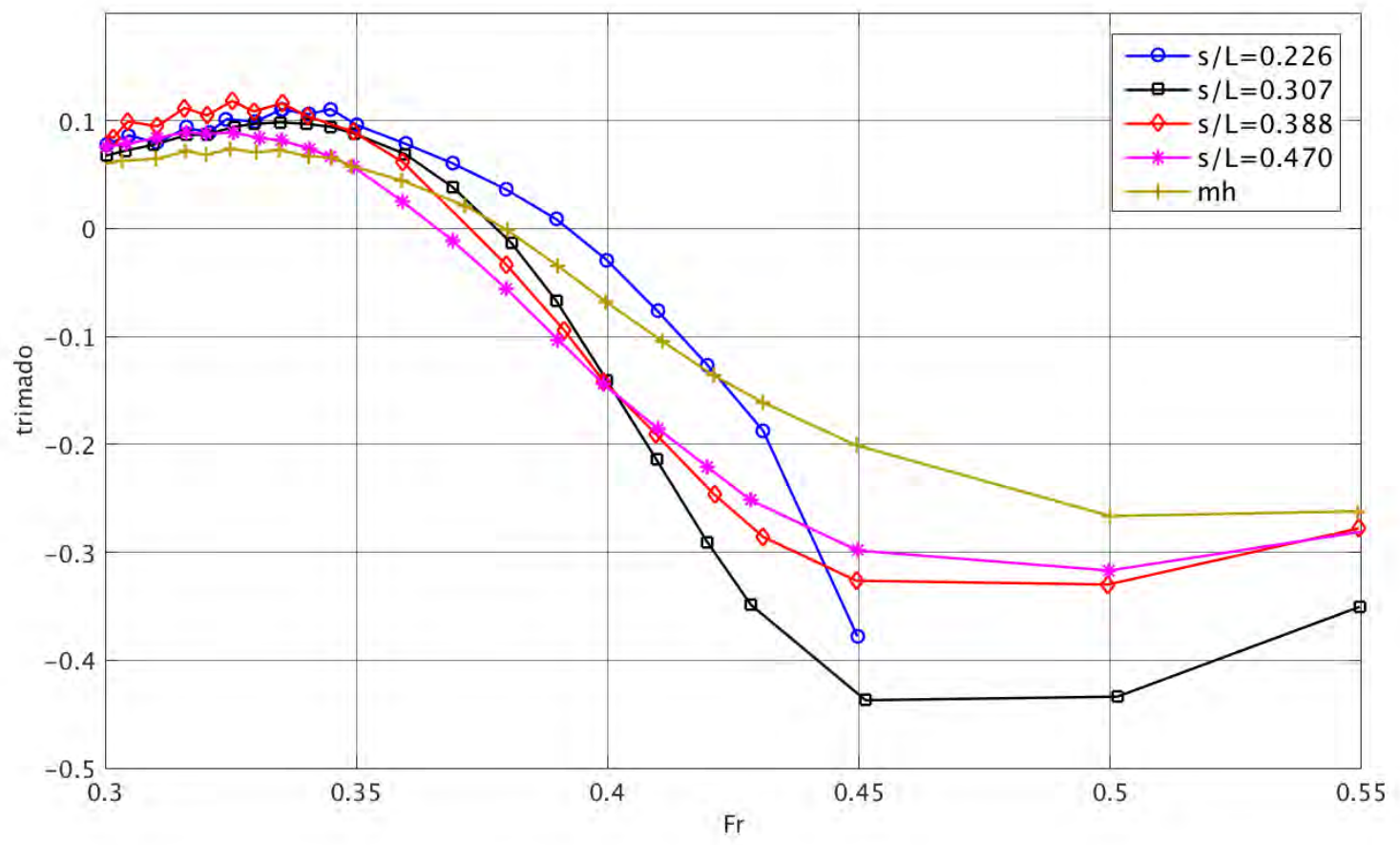

Fig. 84. Trimado dinámico, cat. 560 


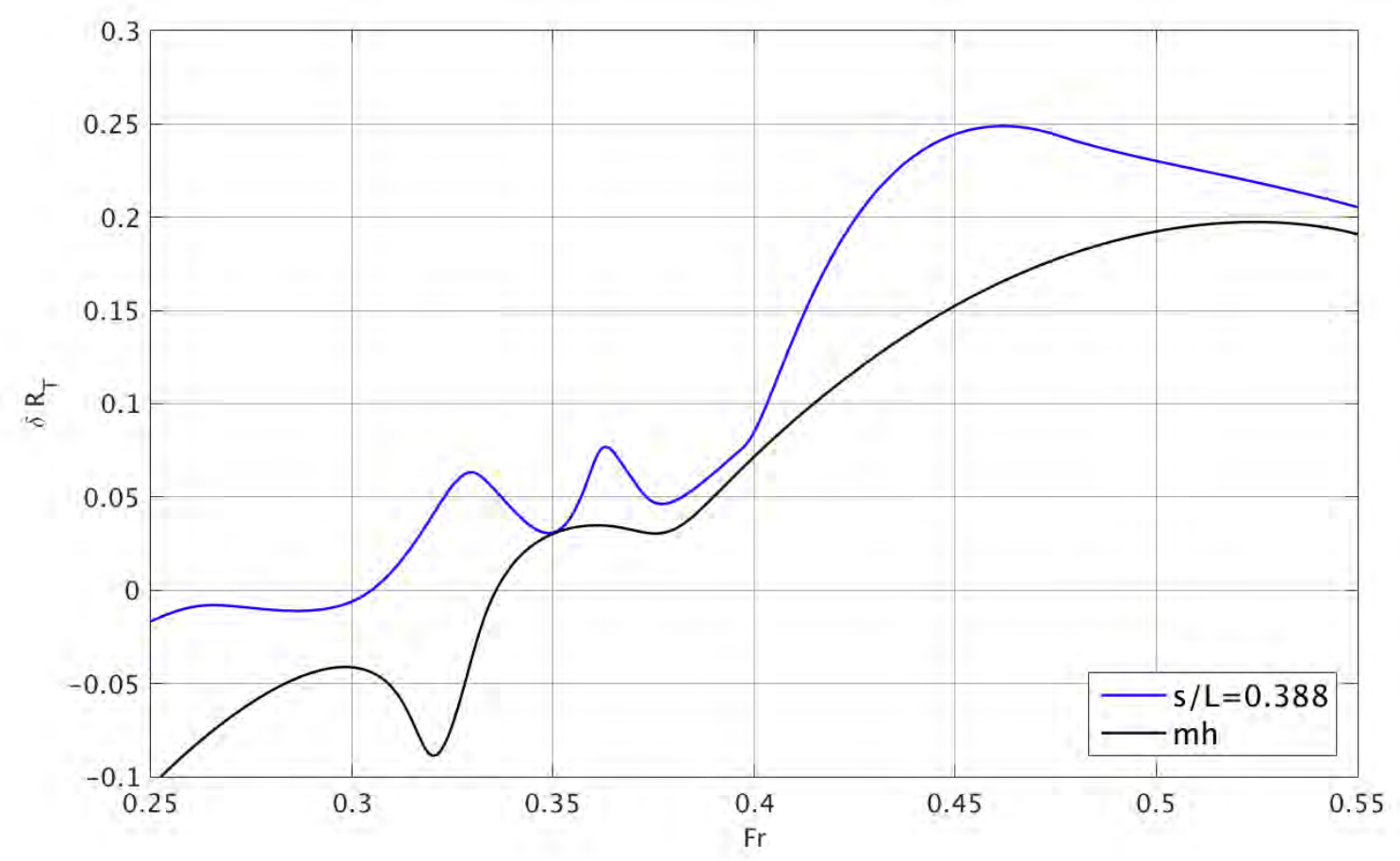

Fig. 85. $\delta R_{T}$ cat. PESBO

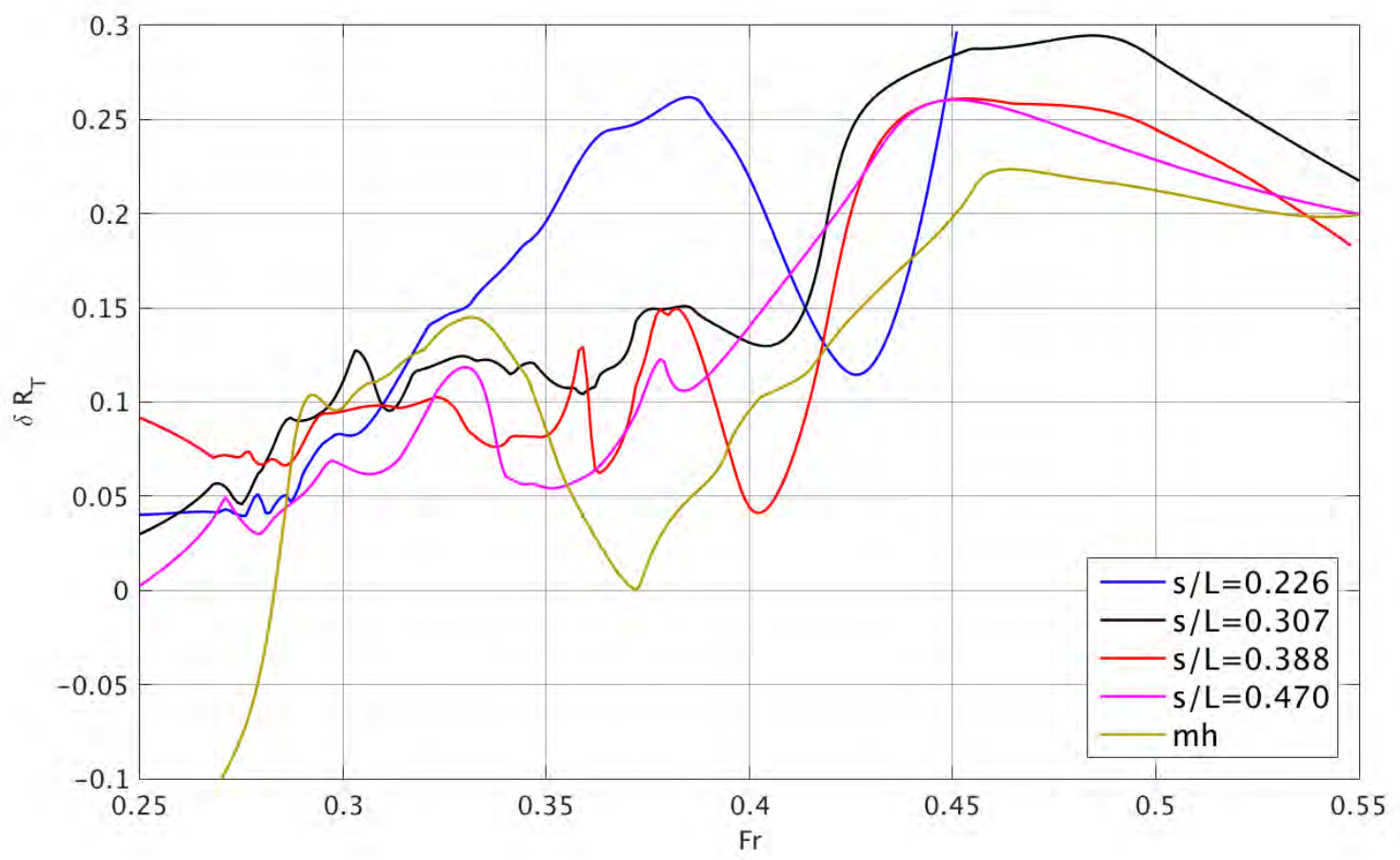

Fig. 86. $\delta R_{T}$ cat. $S 60$ 


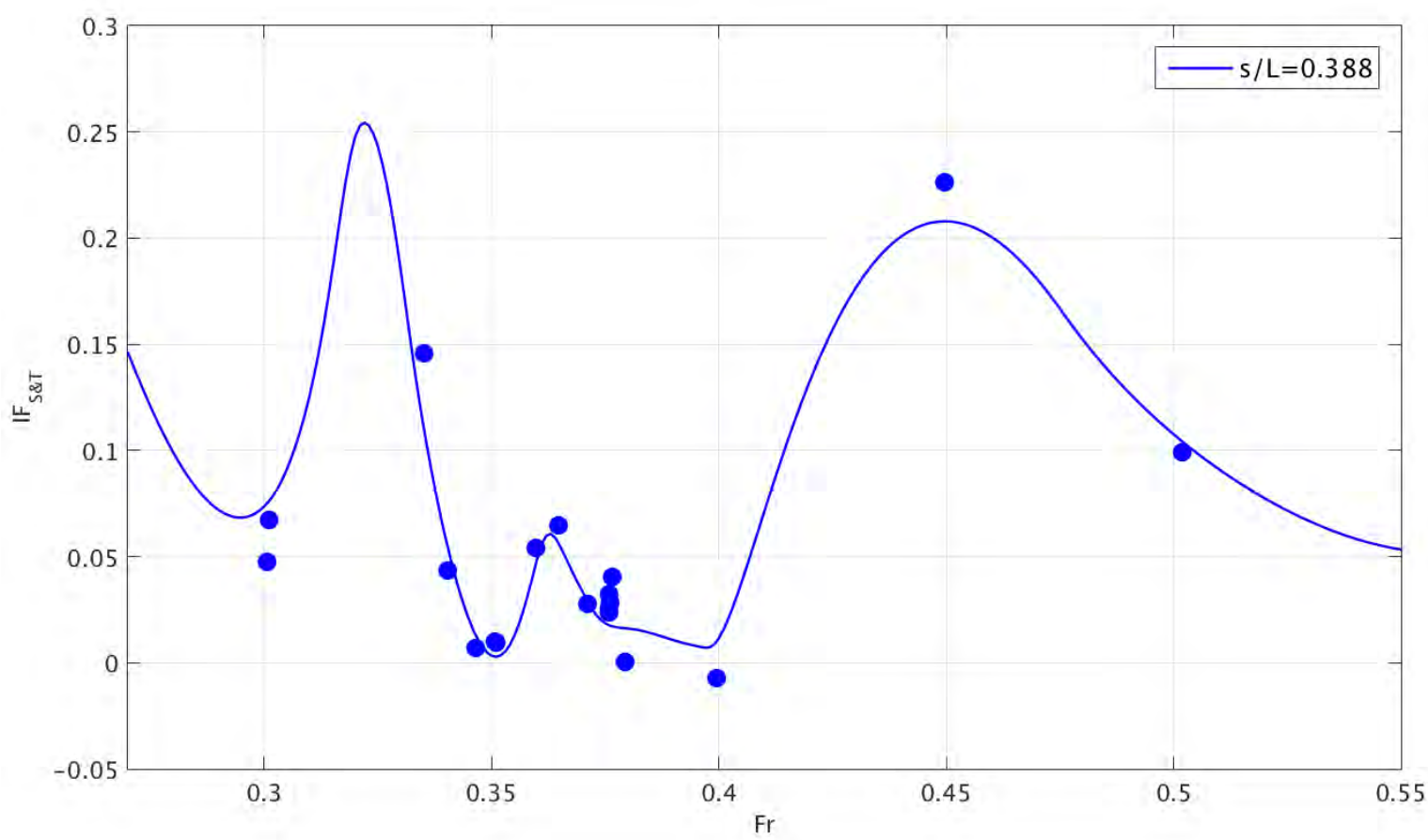

Fig. 87. IF ${ }_{S \& T}$ cat. PESBO

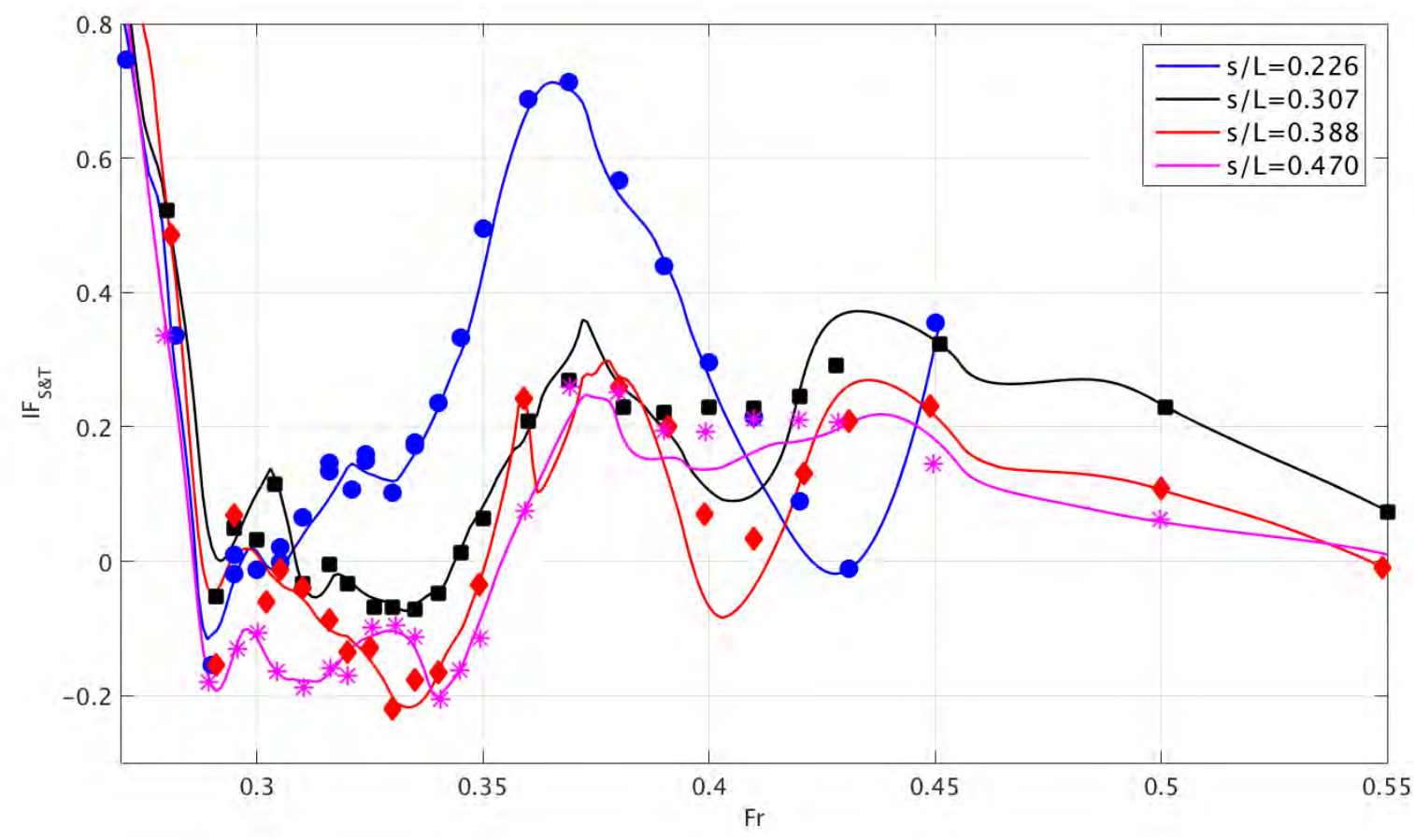

Fig. 88. IF $F_{S \& T}$ cat. $S 60$ 


\section{B.4. Cortes de olas centrales}

- Fig. 89. $s / L=0.207 F r=0.022$

- Fig. 90. $s / L=0.207 \quad F r=0.375$

- Fig. 91. $s / L=0.207 F r=0.398$

- Fig. 92. $s / L=0.207 F r=0.430$

- Fig. 93. $s / L=0.298 F r=0.322$

- Fig. 94. $s / L=0.298 F r=0.375$

- Fig. 95. $s / L=0.298 F r=0.398$

- Fig. 96. $s / L=0.298 F r=0.430$

- Fig. 97. $s / L=0.388 F r=0.322$

- Fig. 98. $s / L=0.388 F r=0.375$

- Fig. 99. $s / L=0.388 F r=0.398$

- Fig. 100. $s / L=0.388 F r=0.430$ 


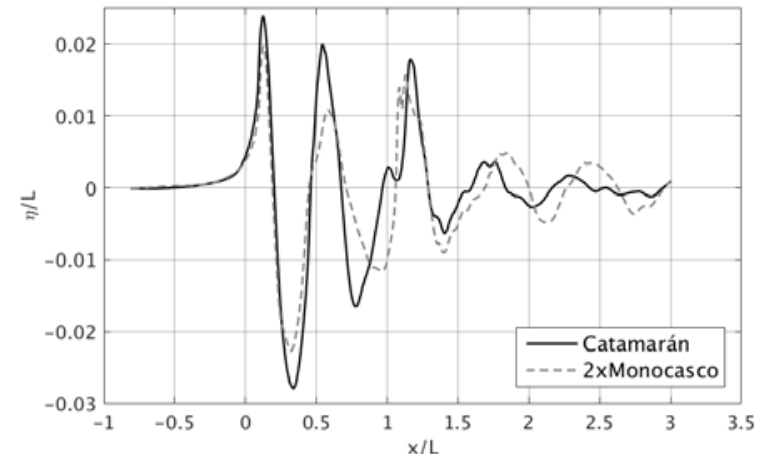

Fig. 89. $s / L=0.207 \quad F r=0.022$

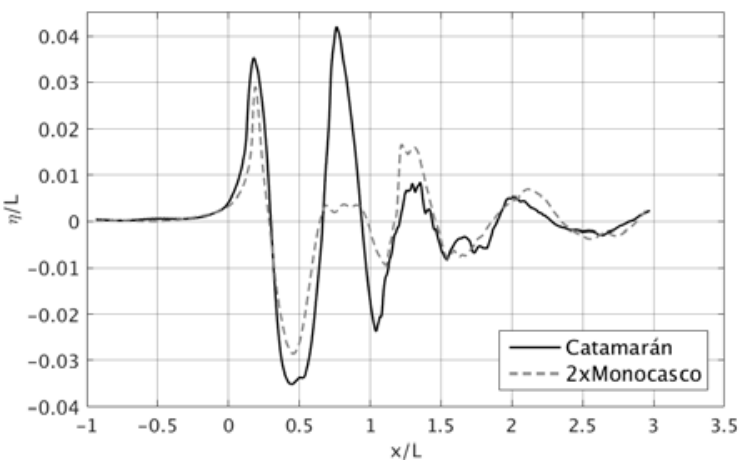

Fig. 91. $s / L=0.207 \quad F r=0.398$

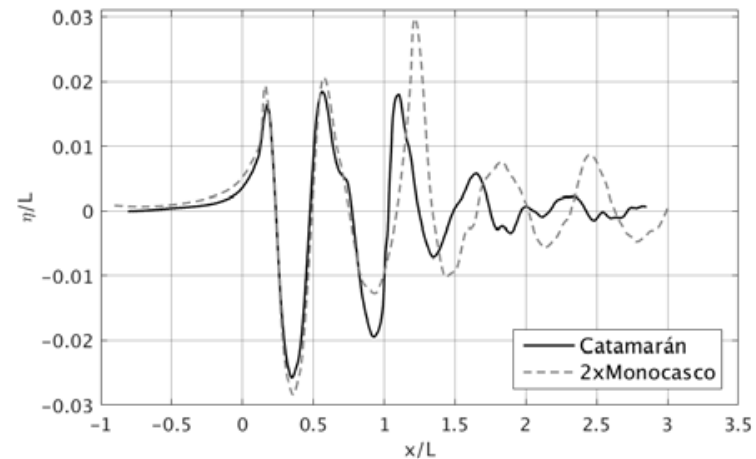

Fig. 93. $s / L=0.298 \quad F r=0.322$

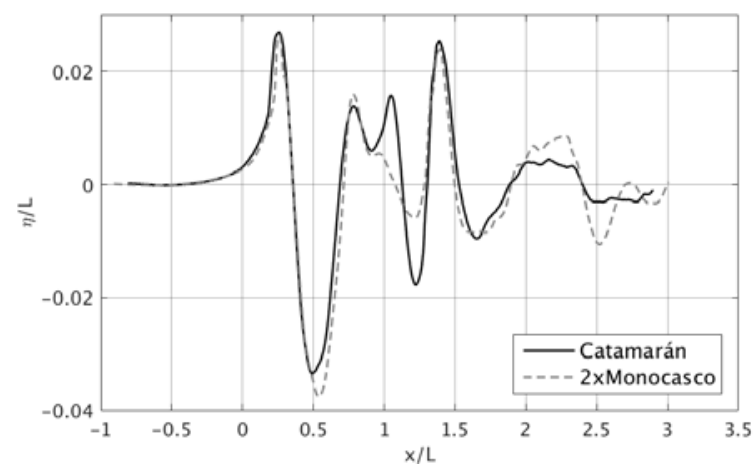

Fig. 95. $s / L=0.298 F r=0.398$

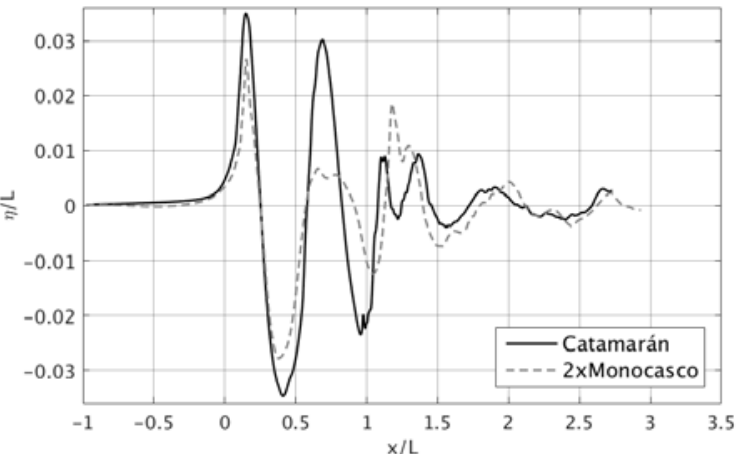

Fig. 90. $s / L=0.207 \quad F r=0.375$

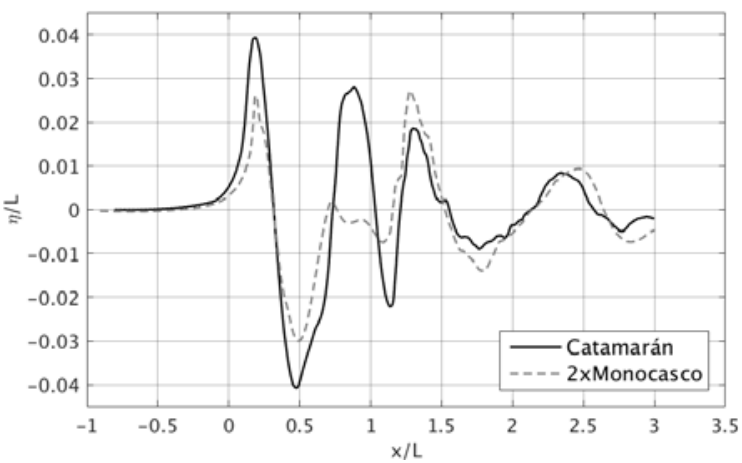

Fig. 92. $s / L=0.207 \quad F r=0.430$

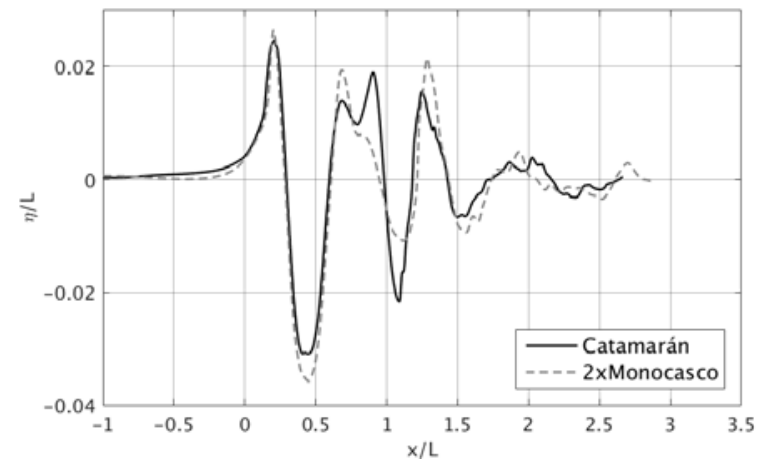

Fig. 94. $s / L=0.298 \quad F r=0.375$

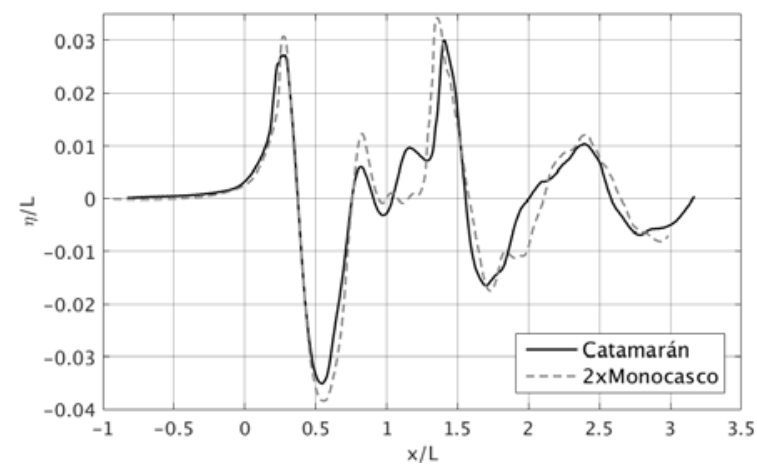

Fig. 96. $s / L=0.298 \quad F r=0.430$ 


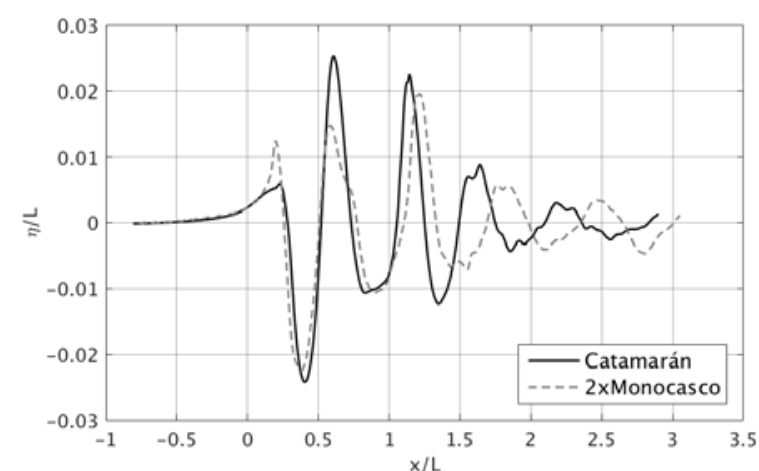

Fig. 97. $s / L=0.388 \quad F r=0.322$

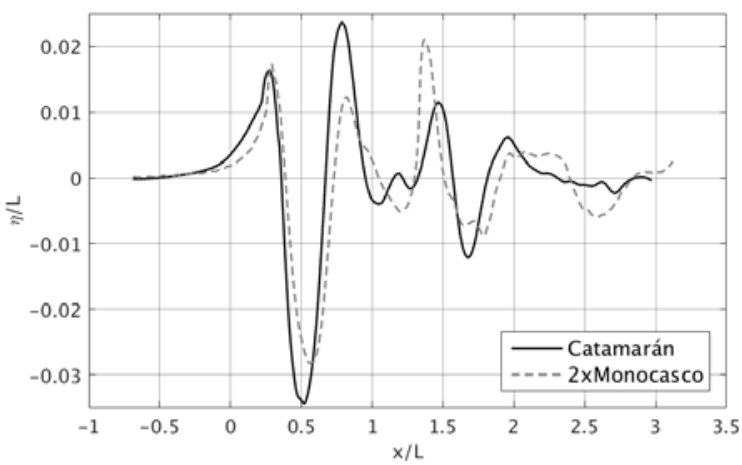

Fig. 99. $s / L=0.388 \quad F r=0.398$

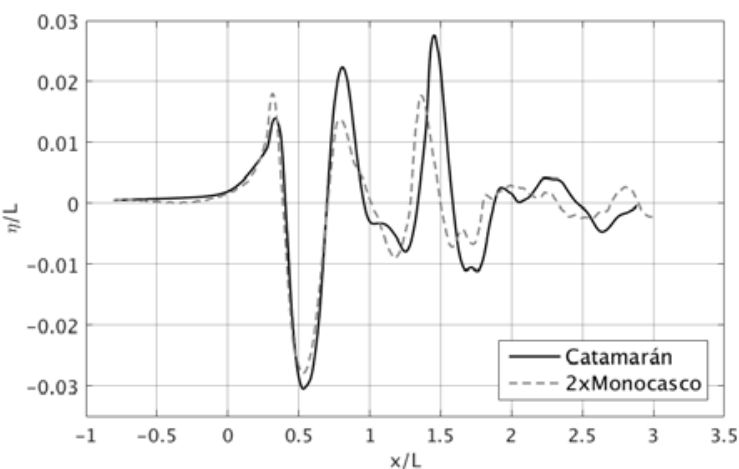

Fig. 98. $s / L=0.388 \quad F r=0.375$

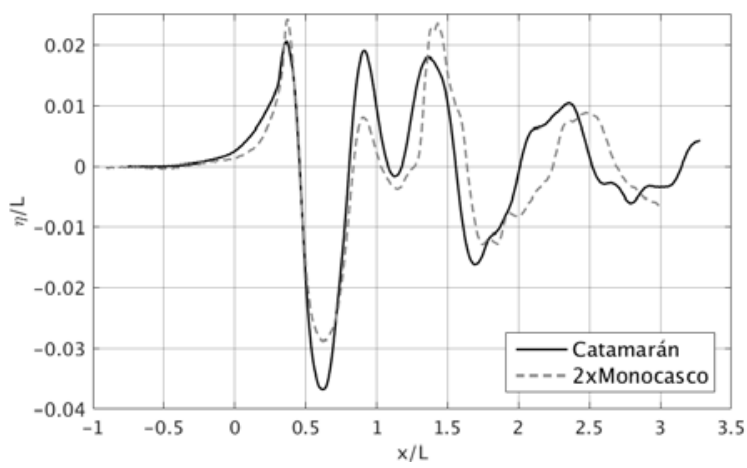

Fig. 100. $s / L=0.388 \quad F r=0.430$ 


\section{B.5. Cortes de olas interiores}

- Fig. 101. $s / L=0.207 \quad F r=0.322 \Delta y / s_{D}=1 / 3$

- Fig. 102. $s / L=0.207 \quad F r=0.322 \Delta y / s_{D}=1 / 4$

- Fig. 103. $s / L=0.207 \quad F r=0.375 \Delta y / s_{D}=1 / 3$

- Fig. 104. $s / L=0.207 F r=0.375 \Delta y / s_{D}=1 / 4$

- Fig. 105. $s / L=0.207 F r=0.398 \Delta y / s_{D}=1 / 3$

- Fig. 106. $s / L=0.207 F r=0.398 \Delta y / s_{D}=1 / 4$

- Fig. 107. $s / L=0.207 \quad F r=0.430 \Delta y / s_{D}=1 / 3$

- Fig. 108. $s / L=0.207 \quad F r=0.430 \Delta y / s_{D}=1 / 4$

- Fig. 109. $s / L=0.298 F r=0.322 \Delta y / s_{D}=1 / 3$

- Fig. 110. $s / L=0.298 F r=0.322 \Delta y / s_{D}=1 / 4$

- Fig. 111. $s / L=0.298 F r=0.322 \Delta y / s_{D}=1 / 6$

- Fig. 112. $s / L=0.298 F r=0.375 \Delta y / s_{D}=1 / 3$

- Fig. 113. $s / L=0.298 F r=0.375 \Delta y / s_{D}=1 / 4$

- Fig. 114. $s / L=0.298 F r=0.375 \Delta y / s_{D}=1 / 6$

- Fig. 115. $s / L=0.298 F r=0.398 \Delta y / s_{D}=1 / 3$

- Fig. 116. $s / L=0.298 F r=0.398 \Delta y / s_{D}=1 / 4$

- Fig. 117. $s / L=0.298 F r=0.398 \Delta y / s_{D}=1 / 6$

- Fig. 118. $s / L=0.298 F r=0.430 \Delta y / s_{D}=1 / 3$

- Fig. 119. $s / L=0.298 F r=0.430 \quad \Delta y / s_{D}=1 / 4$

- Fig. 120. $s / L=0.298 F r=0.430 \Delta y / s_{D}=1 / 6$

- Fig. 121. $s / L=0.388 F r=0.322 \Delta y / s_{D}=1 / 3$

- Fig. 122. $s / L=0.388 F r=0.322 \Delta y / s_{D}=1 / 4$

- Fig. 123. $s / L=0.388 F r=0.322 \Delta y / s_{D}=1 / 6$

- Fig. 124. $s / L=0.388 F r=0.375 \Delta y / s_{D}=1 / 3$

- Fig. 125. $s / L=0.388 F r=0.375 \Delta y / s_{D}=1 / 4$

- Fig. 126. $s / L=0.388 F r=0.375 \Delta y / s_{D}=1 / 6$

- Fig. 127. $s / L=0.388 F r=0.398 \Delta y / s_{D}=1 / 3$

- Fig. 128. $s / L=0.388 F r=0.398 \Delta y / s_{D}=1 / 4$

- Fig. 129. $s / L=0.388 F r=0.398 \Delta y / s_{D}=1 / 6$

- Fig. 130. $s / L=0.388 F r=0.430 \quad \Delta y / s_{D}=1 / 3$

- Fig. 131. $s / L=0.388 F r=0.430 \Delta y / s_{D}=1 / 4$

- Fig. 132. $s / L=0.388 F r=0.430 \Delta y / s_{D}=1 / 6$ 


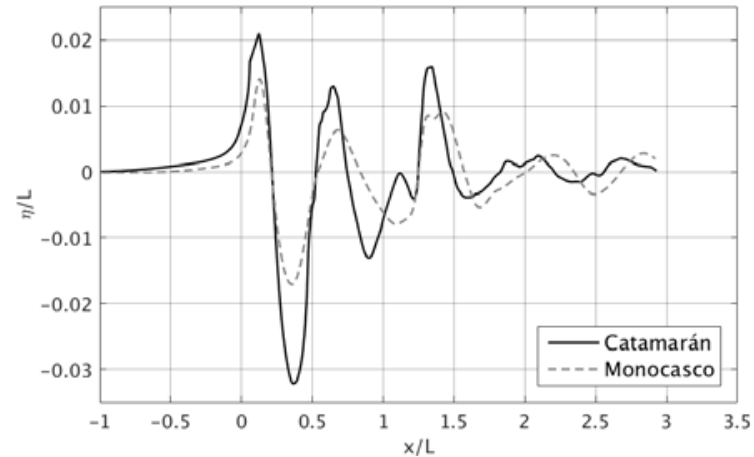

Fig. 101. $s / L=0.207 \quad F r=0.322 \Delta y / s_{D}=1 / 3$

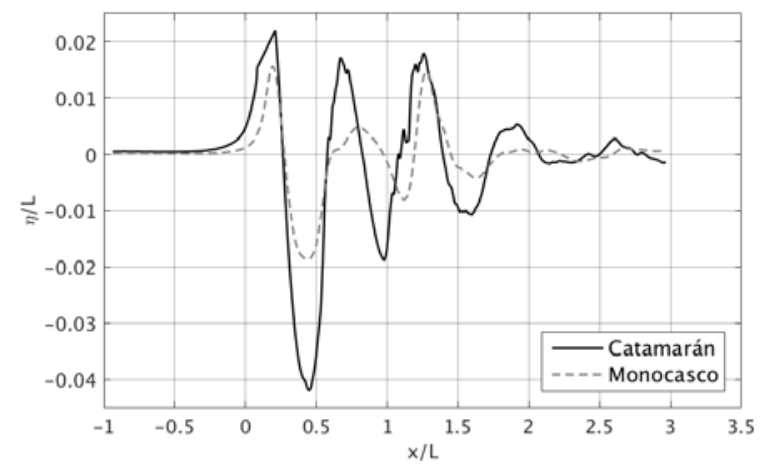

Fig. 103. $s / L=0.207 \quad F r=0.375 \Delta y / s_{D}=1 / 3$

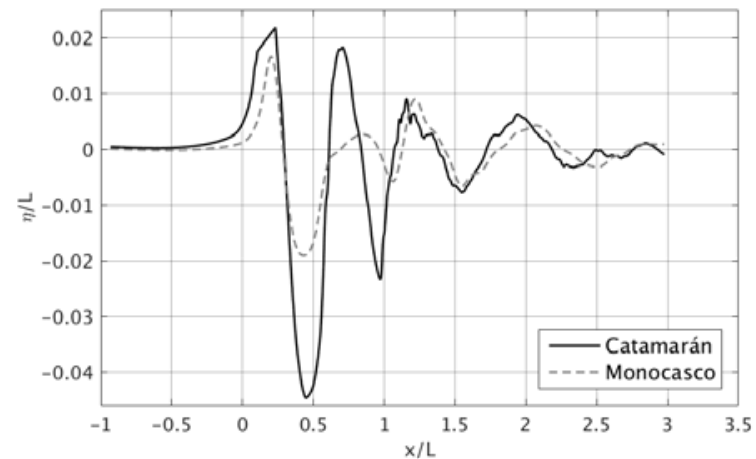

Fig. 105. $s / L=0.207 \quad F r=0.398 \Delta y / s_{D}=1 / 3$

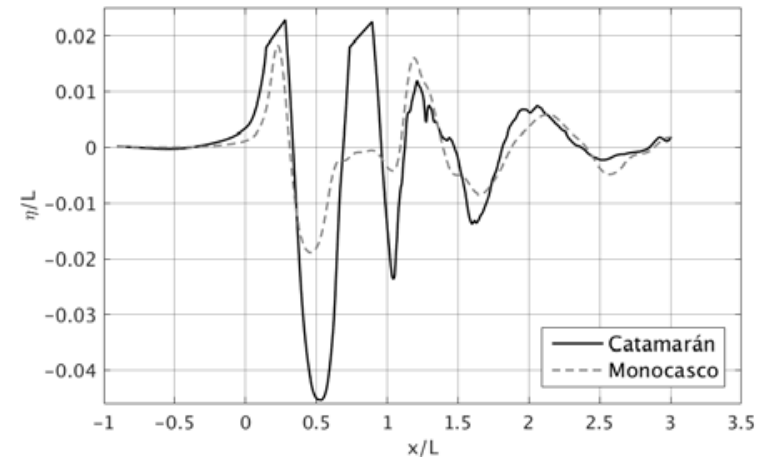

Fig. 107. $s / L=0.207 \quad F r=0.430 \quad \Delta y / s_{D}=1 / 3$

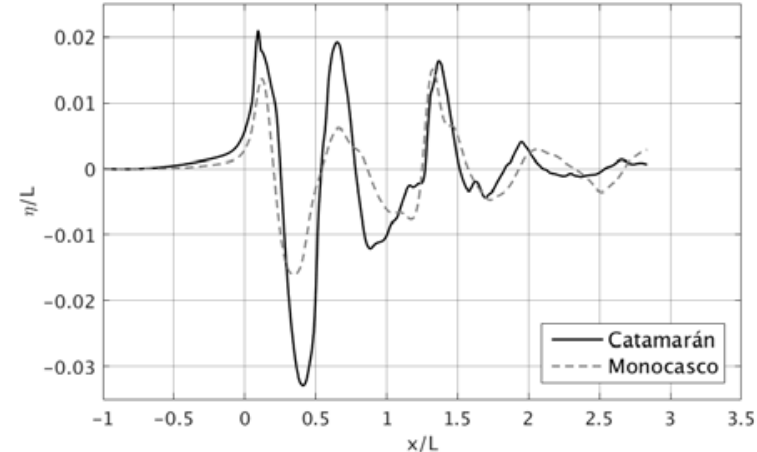

Fig. 102. $s / L=0.207 \quad F r=0.322 \Delta y / s_{D}=1 / 4$

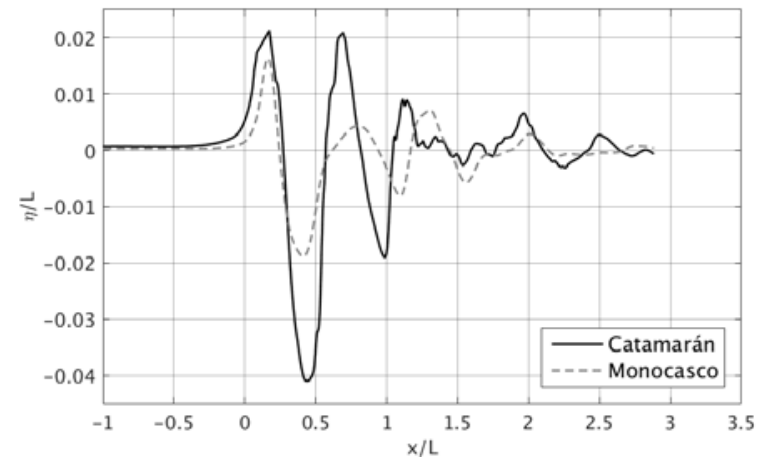

Fig. 104. $s / L=0.207 \quad F r=0.375 \Delta y / s_{D}=1 / 4$

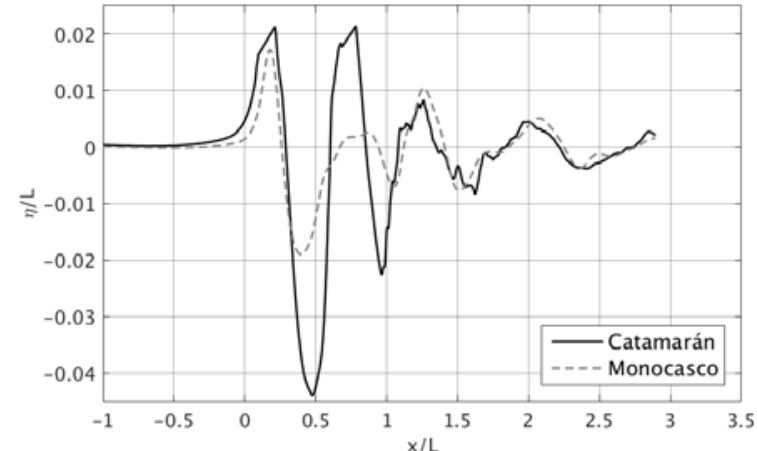

Fig. 106. $s / L=0.207 \quad F r=0.398 \Delta y / s_{D}=1 / 4$

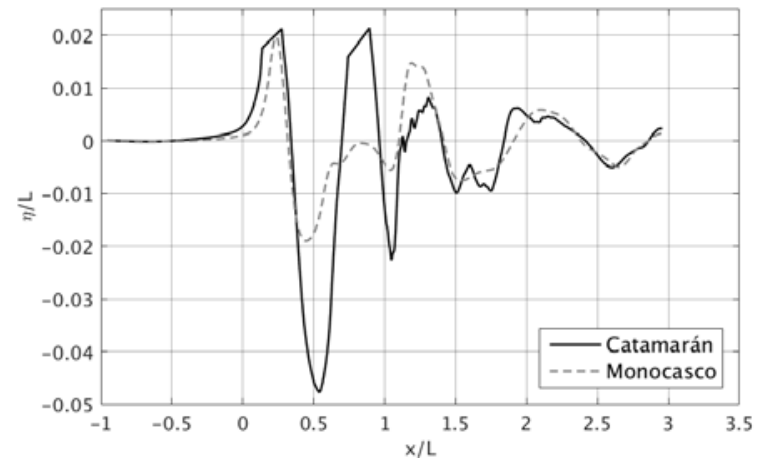

Fig. 108. $s / L=0.207 \quad F r=0.430 \Delta y / s_{D}=1 / 4$ 


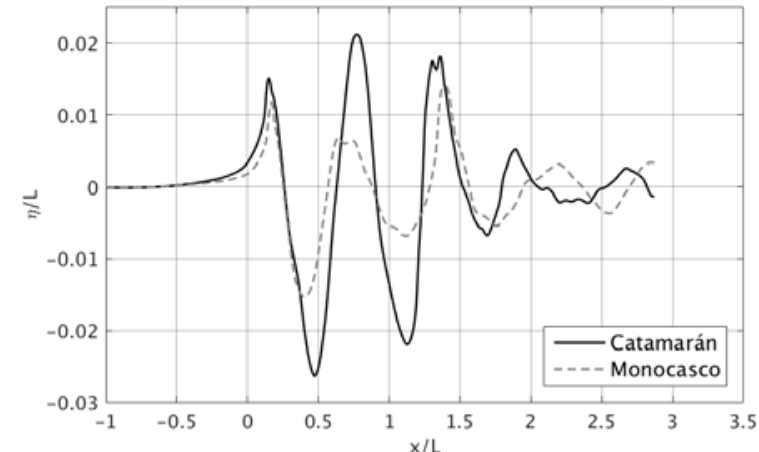

Fig. 109. $s / L=0.298 \quad F r=0.322 \quad \Delta y / s_{D}=1 / 3$

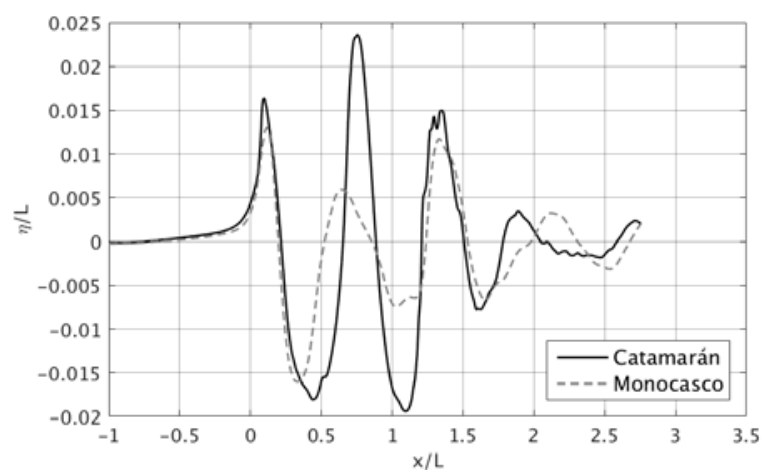

Fig. 111. $s / L=0.298 \quad F r=0.322 \quad \Delta y / s_{D}=1 / 6$

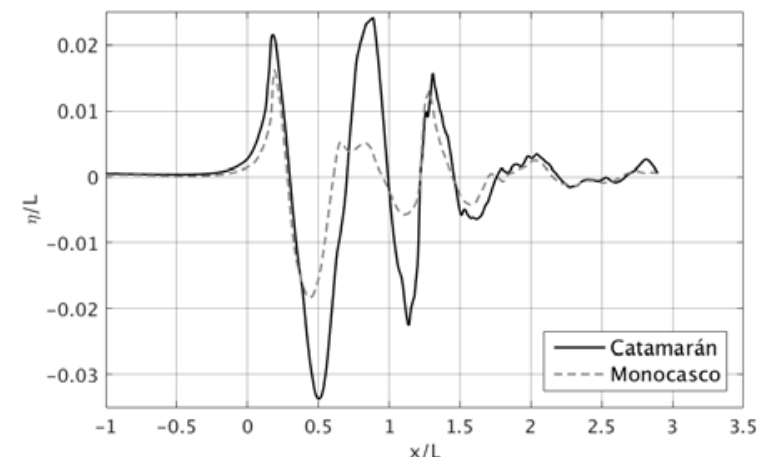

Fig. 112. $s / L=0.298 \quad F r=0.375 \quad \Delta y / s_{D}=1 / 3$

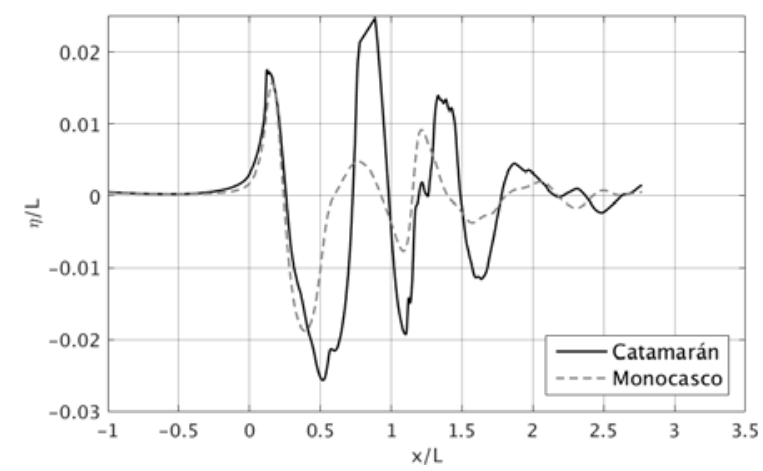

Fig. 114. $s / L=0.298 \quad F r=0.375 \quad \Delta y / s_{D}=1 / 6$

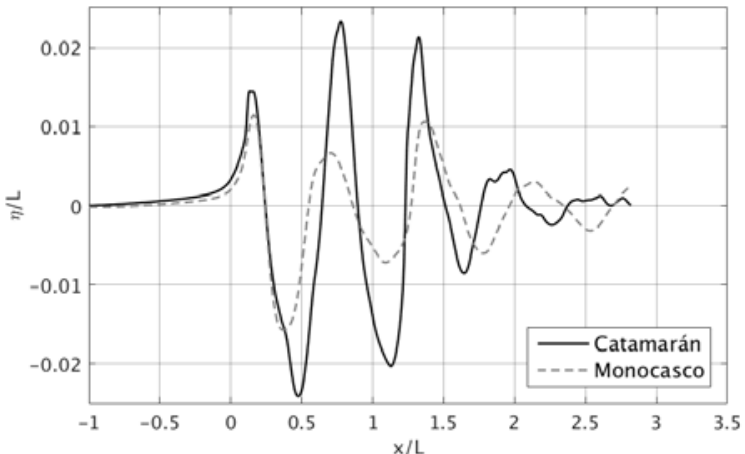

Fig. 110. $s / L=0.298 \quad F r=0.322 \quad \Delta y / s_{D}=1 / 4$

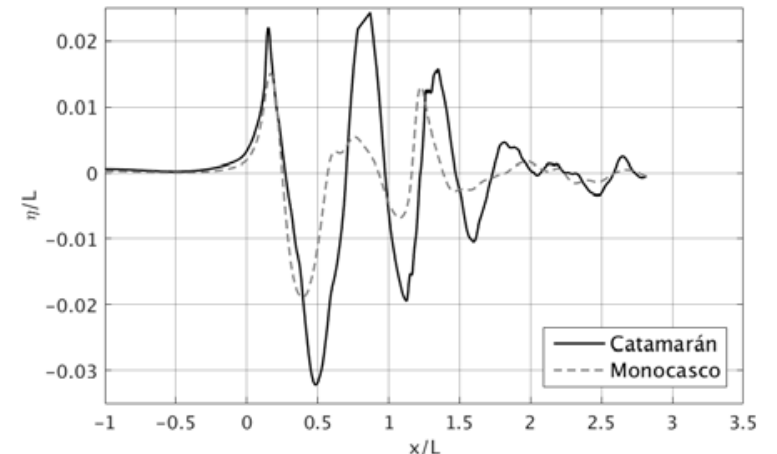

Fig. 113. $s / L=0.298 \quad F r=0.375 \quad \Delta y / s_{D}=1 / 4$ 


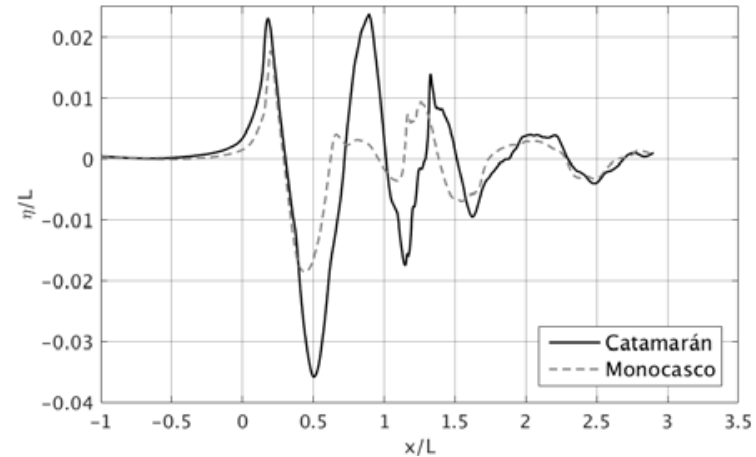

Fig. 115. $s / L=0.298 \quad F r=0.398 \Delta y / s_{D}=1 / 3$

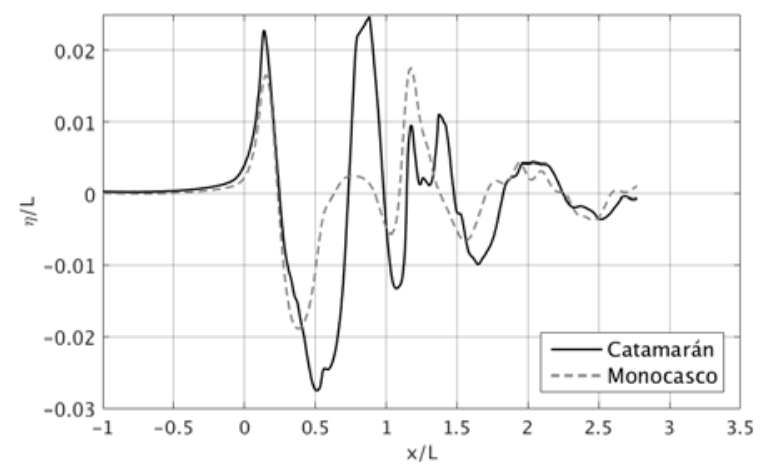

Fig. 117. $s / L=0.298 \quad F r=0.398 \Delta y / s_{D}=1 / 6$

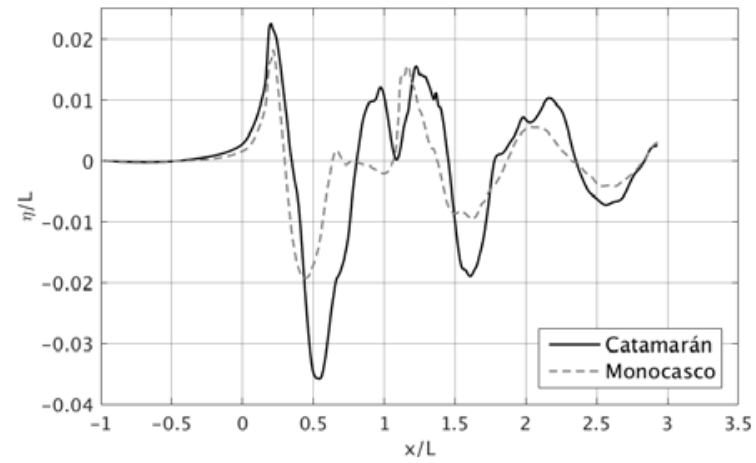

Fig. 118. $s / L=0.298 \quad F r=0.430 \Delta y / s_{D}=1 / 3$

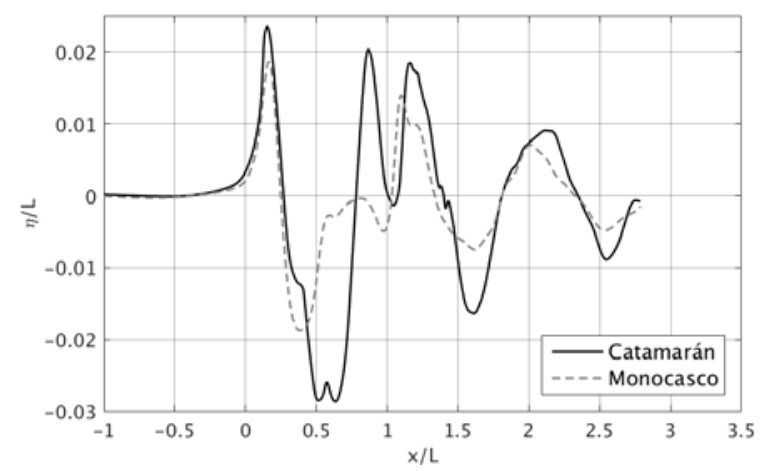

Fig. 120. $s / L=0.298 \quad F r=0.430 \Delta y / s_{D}=1 / 6$

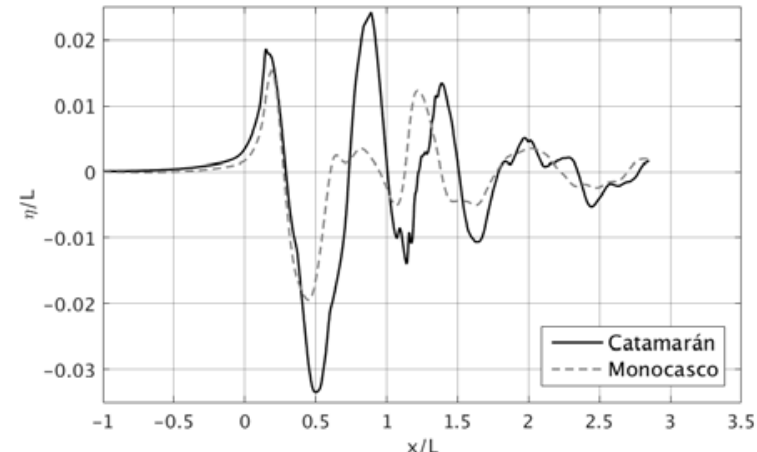

Fig. 116. $s / L=0.298 F r=0.398 \Delta y / s_{D}=1 / 4$

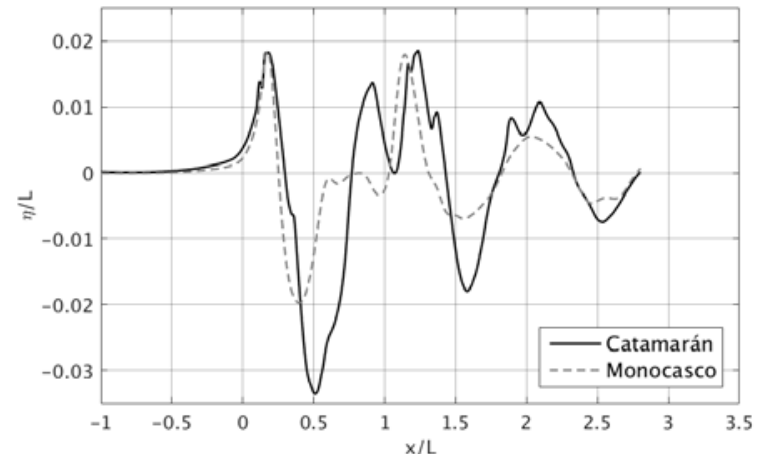

Fig. 119. $s / L=0.298 \quad F r=0.430 \Delta y / s_{D}=1 / 4$ 
DAVID FERNÁNDEZ GUTIÉRREZ

ESCUELA TÉCNICA SUPERIOR DE INGENIEROS NAVALES (UPM)

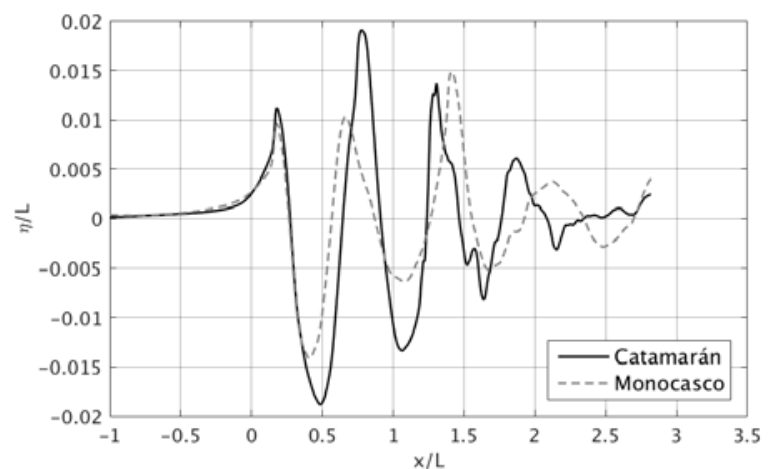

Fig. 121. $s / L=0.388 \quad F r=0.322 \quad \Delta y / s_{D}=1 / 3$

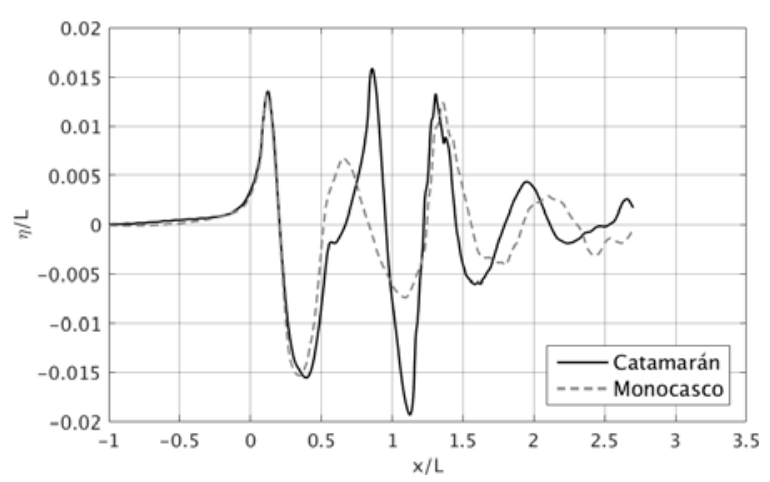

Fig. 123. $s / L=0.388 F r=0.322 \Delta y / s_{D}=1 / 6$

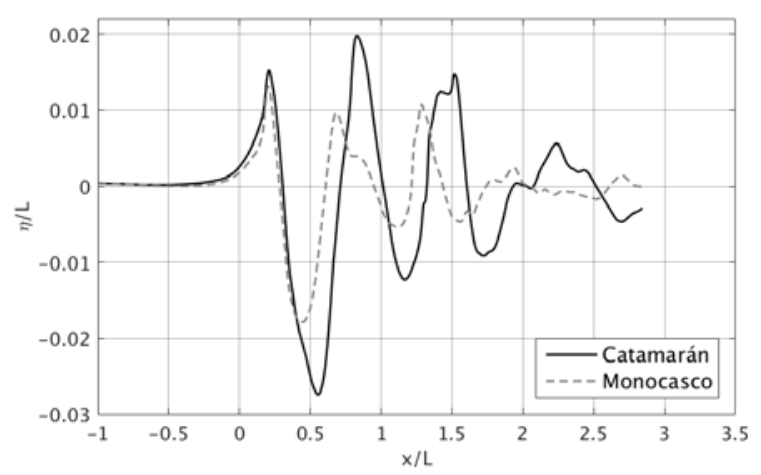

Fig. 124. $s / L=0.388 \quad F r=0.375 \Delta y / s_{D}=1 / 3$

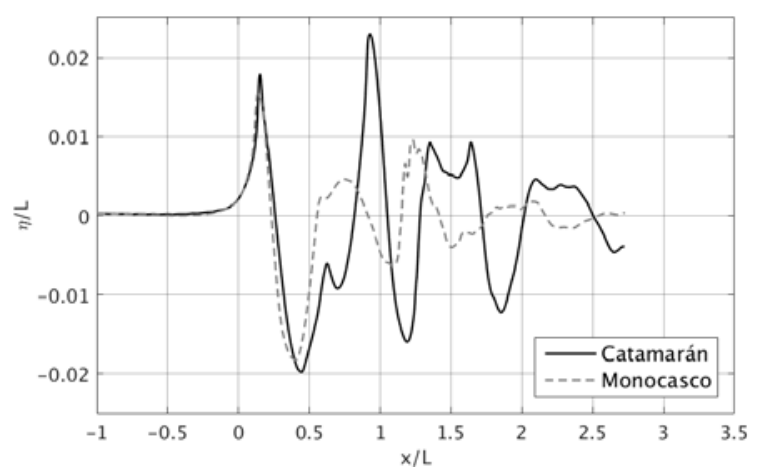

Fig. 126. $s / L=0.388 \quad F r=0.375 \Delta y / s_{D}=1 / 6$

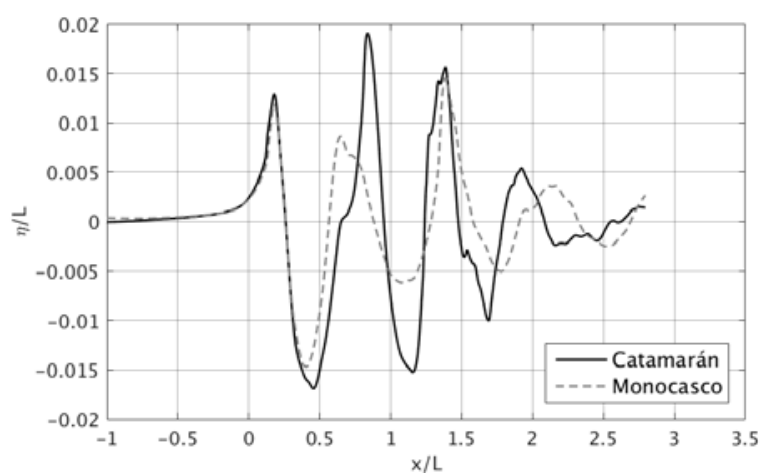

Fig. 122. $s / L=0.388 \quad F r=0.322 \quad \Delta y / s_{D}=1 / 4$

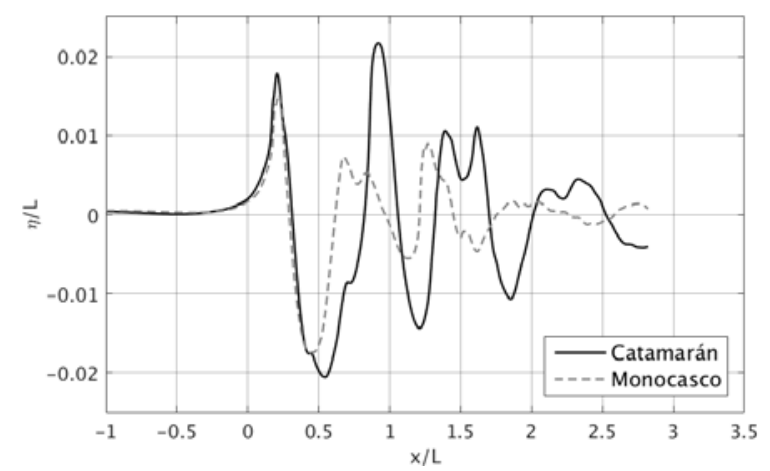

Fig. 125. $s / L=0.388 F r=0.375 \Delta y / s_{D}=1 / 4$

ANÁLISIS Y OPTIMIZACIÓN DE LA RESISTENCIA DE INTERFERENCIA EN MULTICASCOS

Página 216 de 258 


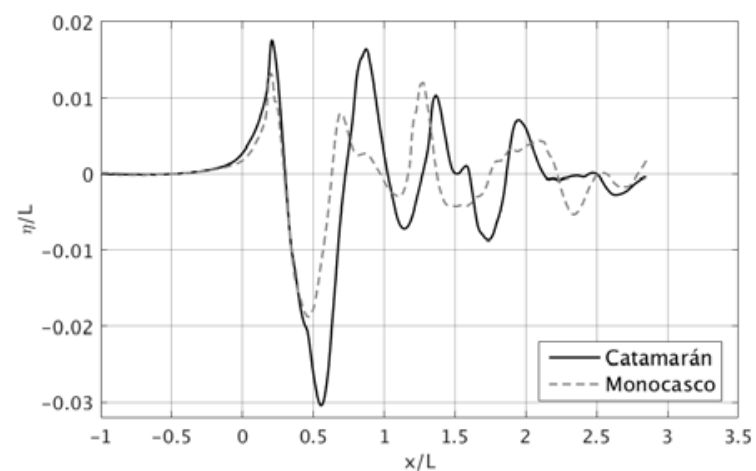

Fig. 127. $s / L=0.388 \quad F r=0.398 \Delta y / s_{D}=1 / 3$

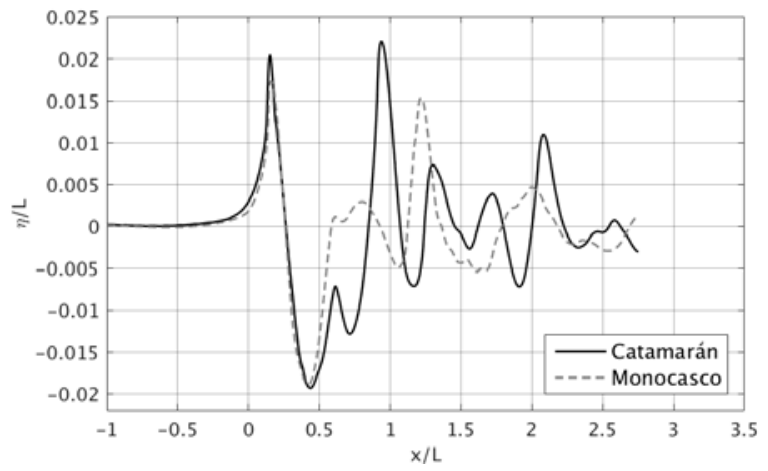

Fig. 129. $s / L=0.388 \quad F r=0.398 \Delta y / s_{D}=1 / 6$

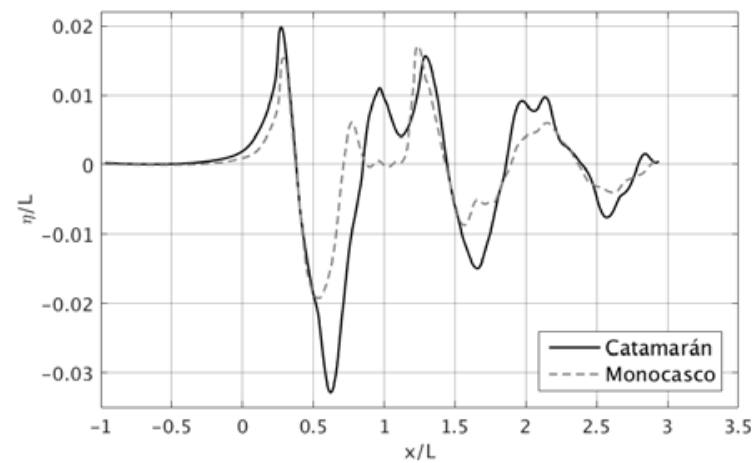

Fig. 130. $s / L=0.388 \quad F r=0.430 \quad \Delta y / s_{D}=1 / 3$

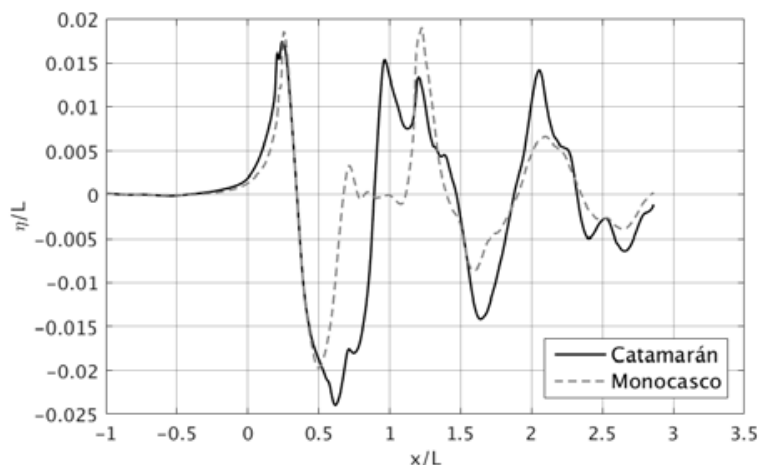

Fig. 131. $s / L=0.388 \quad F r=0.430 \quad \Delta y / s_{D}=1 / 4$

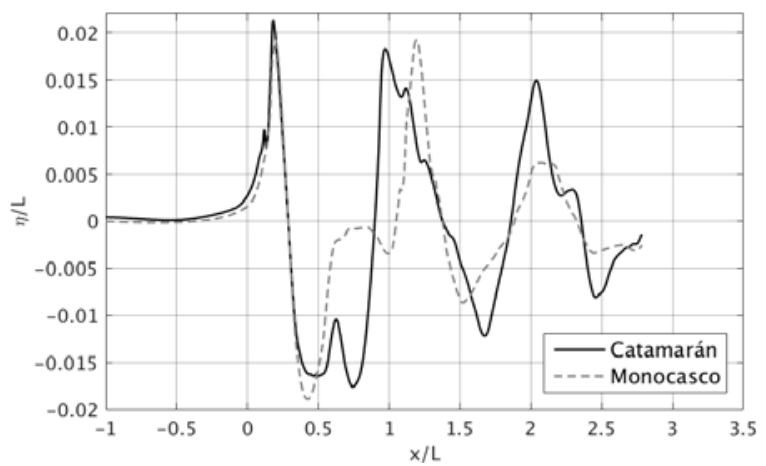

Fig. 132. $s / L=0.388 \quad F r=0.430 \Delta y / s_{D}=1 / 6$ 


\section{B.6. Cortes de olas exteriores}

- Fig. 133. $s / L=0.207 \quad F r=0.322 \Delta y / s_{D}=1 / 2$

- Fig. 134. $s / L=0.207 \quad F r=0.430 \Delta y / s_{D}=1 / 2$

- Fig. 135. $s / L=0.388 F r=0.322 \Delta y / s_{D}=1 / 2$

- Fig. 136. $s / L=0.388 F r=0.430 \Delta y / s_{D}=1 / 2$ 

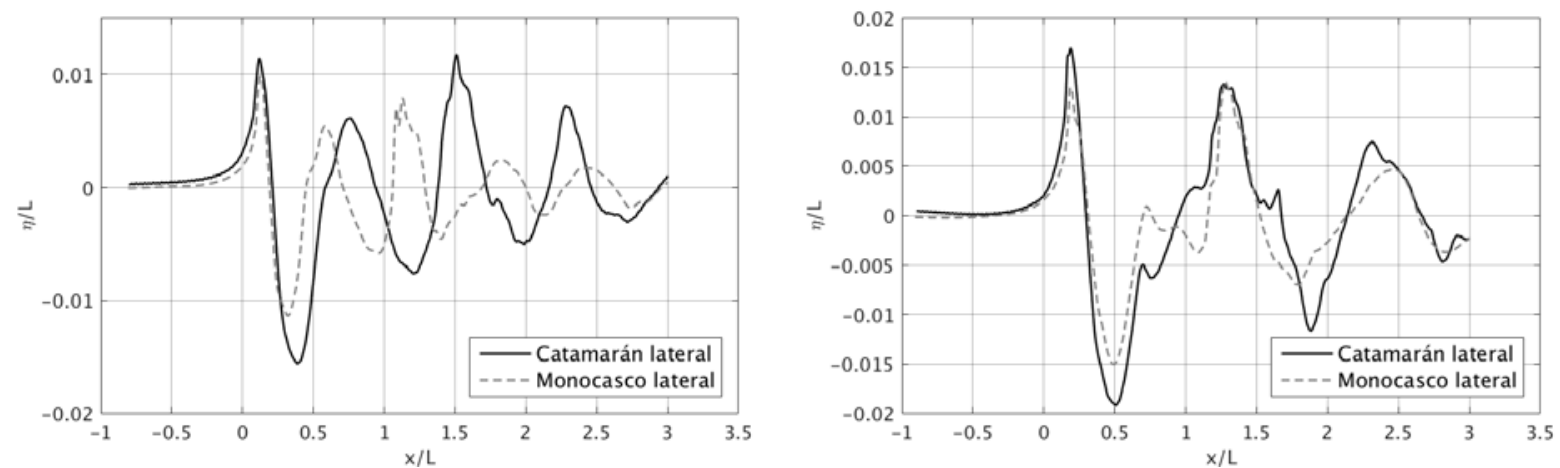

Fig. 133. $s / L=0.207 \quad F r=0.322 \Delta y / s_{D}=1 / 2$

Fig. 134. $s / L=0.207 \quad F r=0.430 \quad \Delta y / s_{D}=1 / 2$
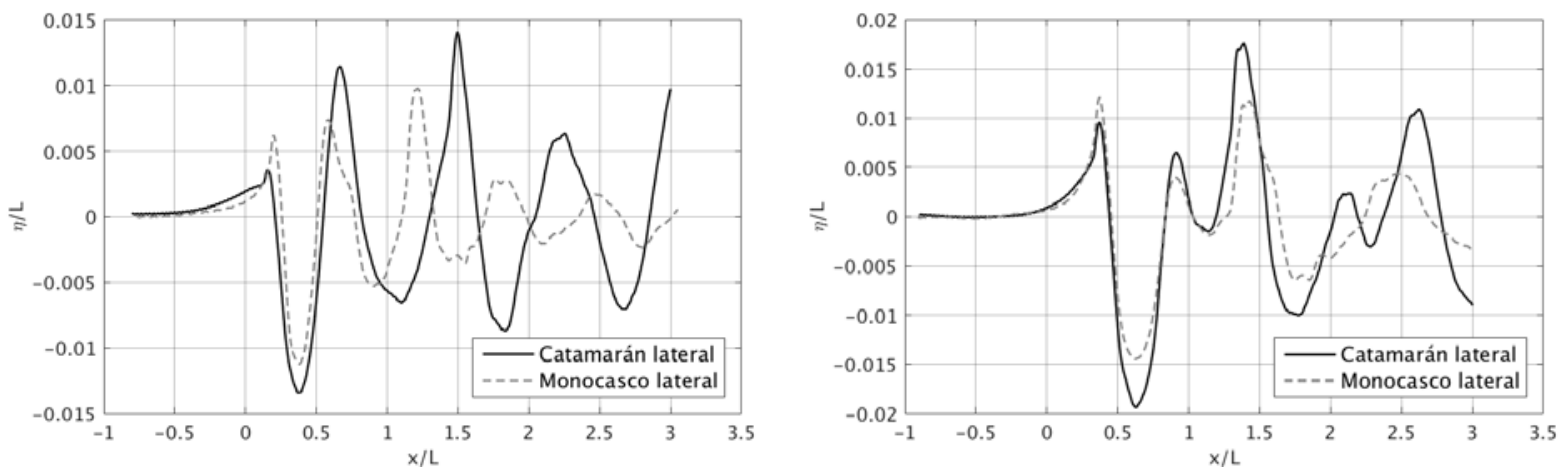

Fig. 135. $s / L=0.388 \quad F r=0.322 \quad \Delta y / s_{D}=1 / 2 \quad$ Fig. 136. $s / L=0.388 \quad F r=0.430 \quad \Delta y / s_{D}=1 / 2$ 


\section{RESULTADOS NUMÉRICOS}

\section{C.1. Catamarán PESBO (Tdynlin)}

- Fig. 137. $s / L=0.207 \quad F r=0.022$

- Fig. 138. $s / L=0.207 F r=0.375$

- Fig. 139. $s / L=0.207 \quad F r=0.398$

- Fig. 140. $s / L=0.207 \quad F r=0.430$

- Fig. 141. $s / L=0.298 F r=0.322$

- Fig. 142. $s / L=0.298 F r=0.375$

- Fig. 143. $s / L=0.298 F r=0.398$

- Fig. 144. $s / L=0.298 F r=0.430$

- Fig. 145. $\boldsymbol{s} / \boldsymbol{L}=\mathbf{0 . 3 8 8} \boldsymbol{F r}=\mathbf{0 . 3 2 2}$

- Fig. 146. $s / L=0.388 F r=0.375$

- Fig. 147. $s / L=0.388 F r=0.398$

- Fig. 148. $\boldsymbol{s} / \boldsymbol{L}=\mathbf{0 . 3 8 8} \boldsymbol{F r}=\mathbf{0 . 4 3 0}$ 


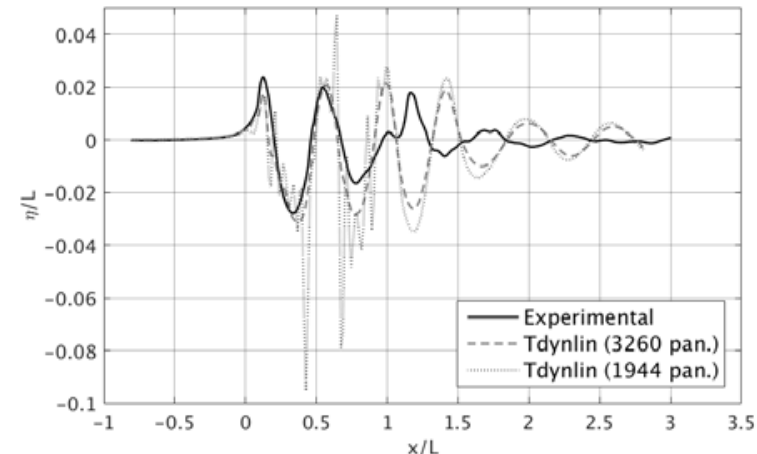

Fig. 137. $s / L=0.207 \quad F r=0.022$

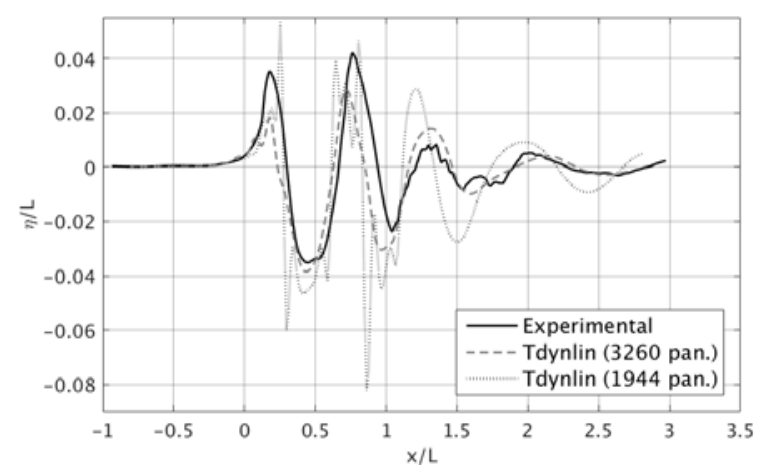

Fig. 139. $s / L=0.207 \quad F r=0.398$

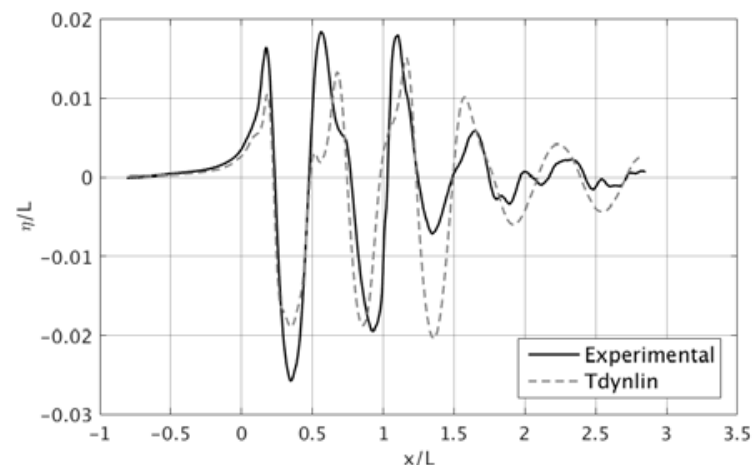

Fig. 141. $s / L=0.298 \quad F r=0.322$

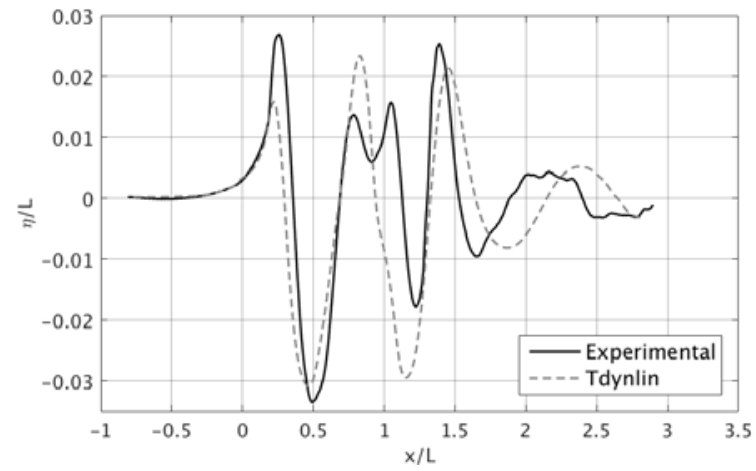

Fig. 143. $s / L=0.298 \quad F r=0.398$

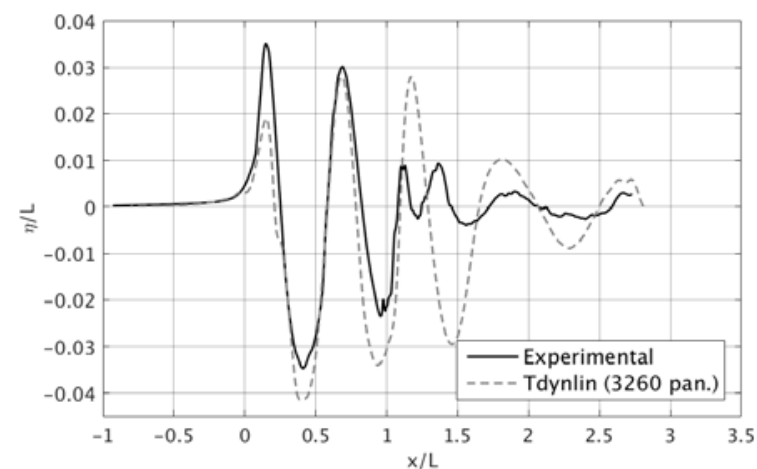

Fig. 138. $s / L=0.207 \quad F r=0.375$

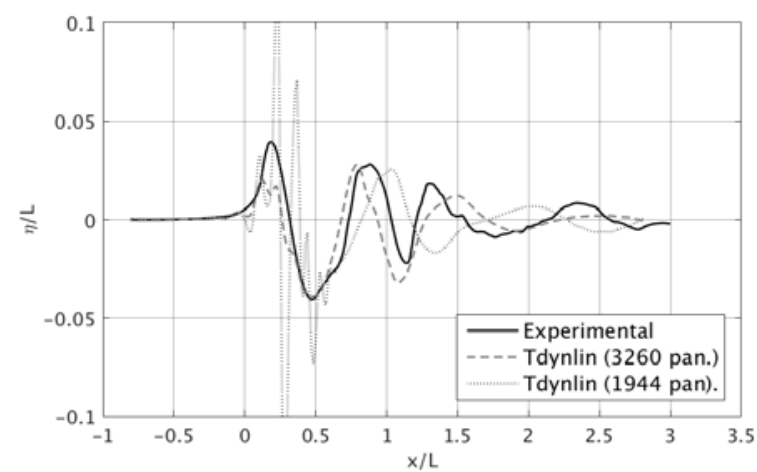

Fig. 140. $s / L=0.207 \quad F r=0.430$

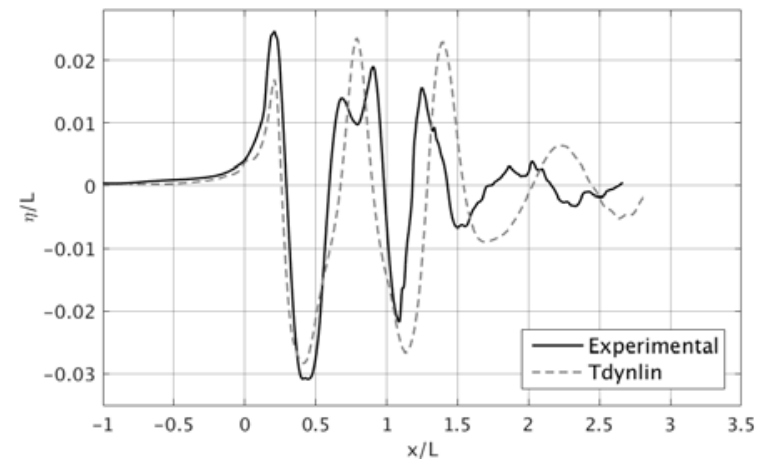

Fig. 142. $s / L=0.298 \quad F r=0.375$

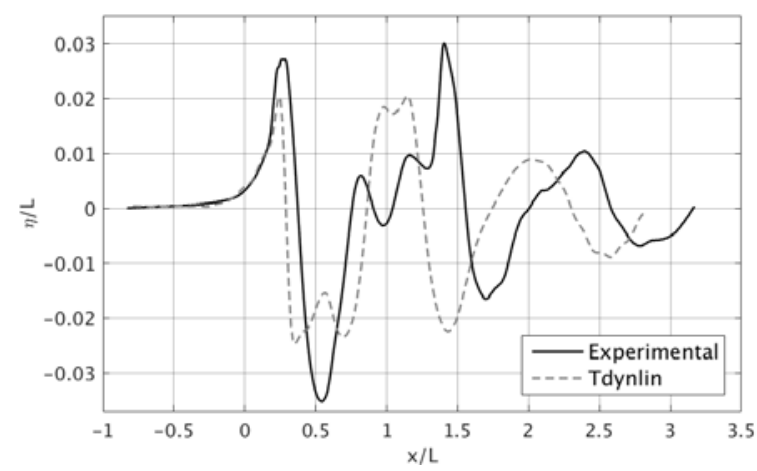

Fig. 144. $s / L=0.298 F r=0.430$ 


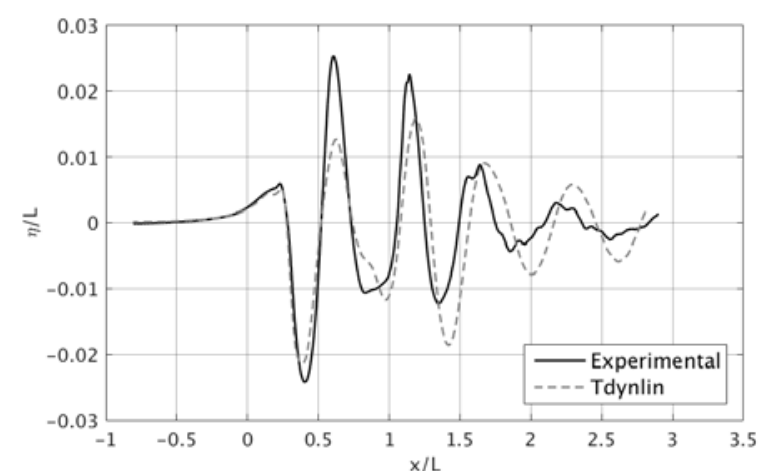

Fig. 145. $s / L=0.388 \quad F r=0.322$

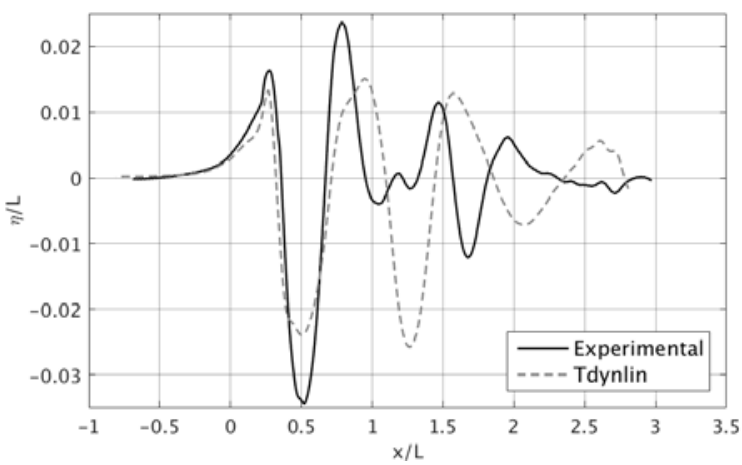

Fig. 147. $s / L=0.388 \quad F r=0.398$

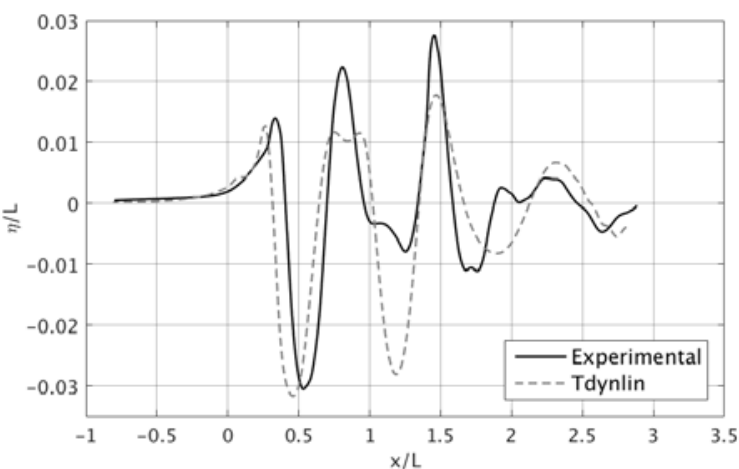

Fig. 146. $s / L=0.388 \quad F r=0.375$

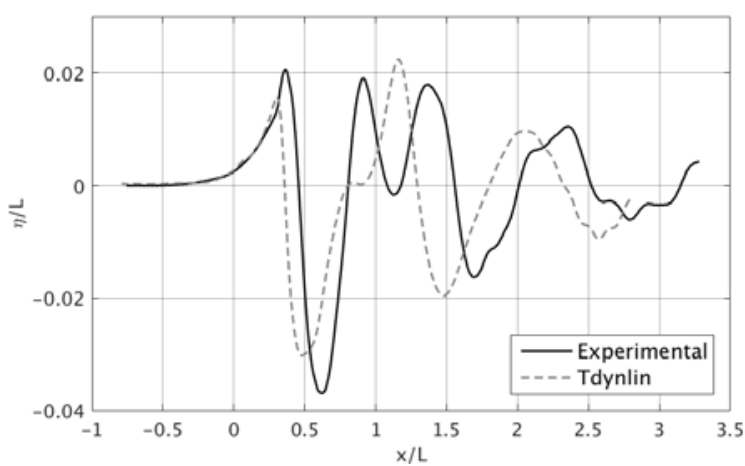

Fig. 148. $s / L=0.388 \quad F r=0.430$ 


\section{C.2. Catamáran PESBO (CD-Adapco Star-CCM+)}

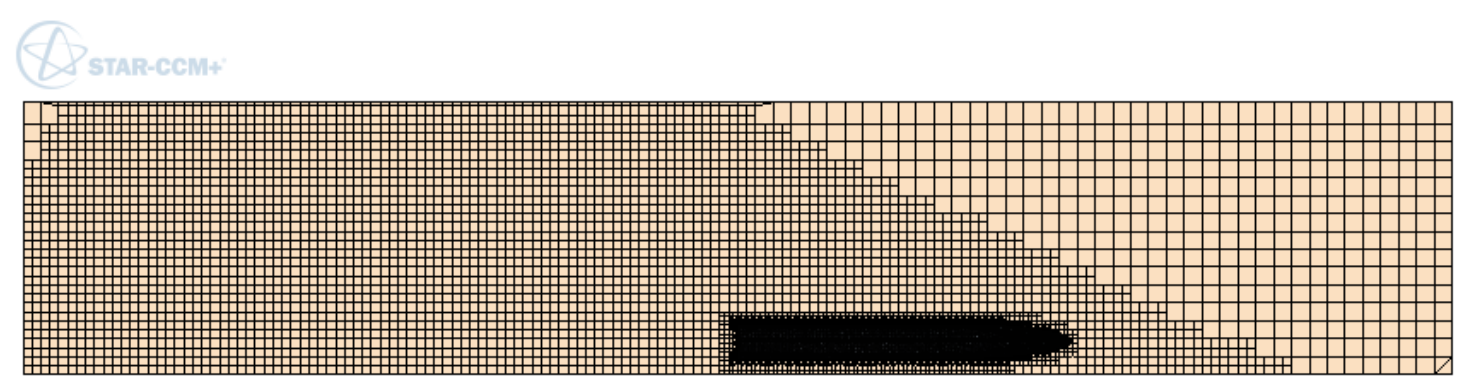

a) $s / L=0.207$

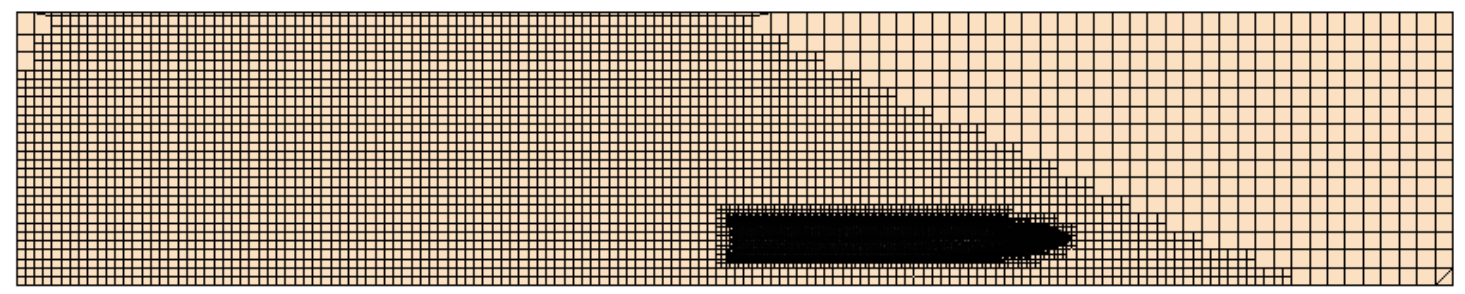

b) $s / L=0.298$

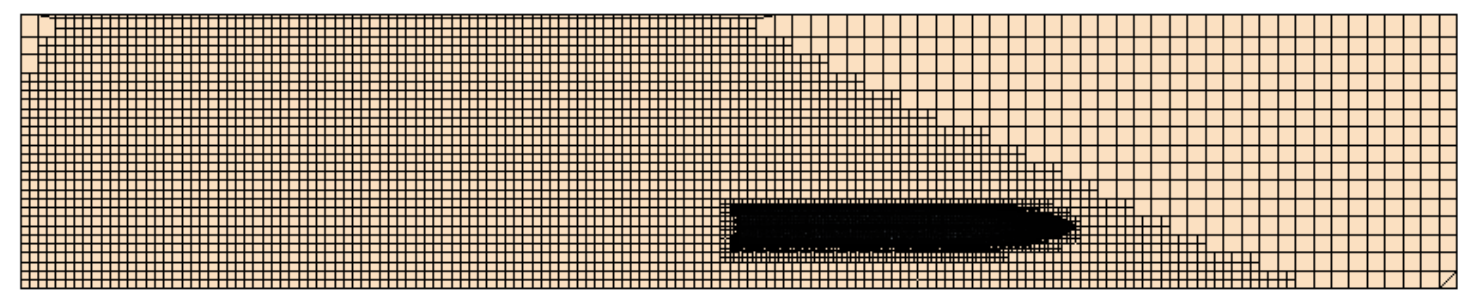

c) $s / L=0.388$

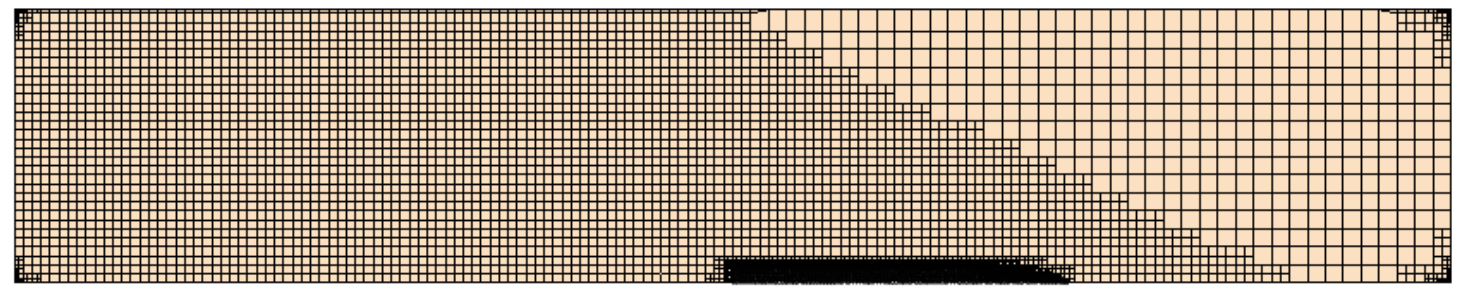

a) Monocasco

Fig. 149: Variaciones del mallado para cada separación en la superficie libre del cat. PESBO en Star-CCM+ 

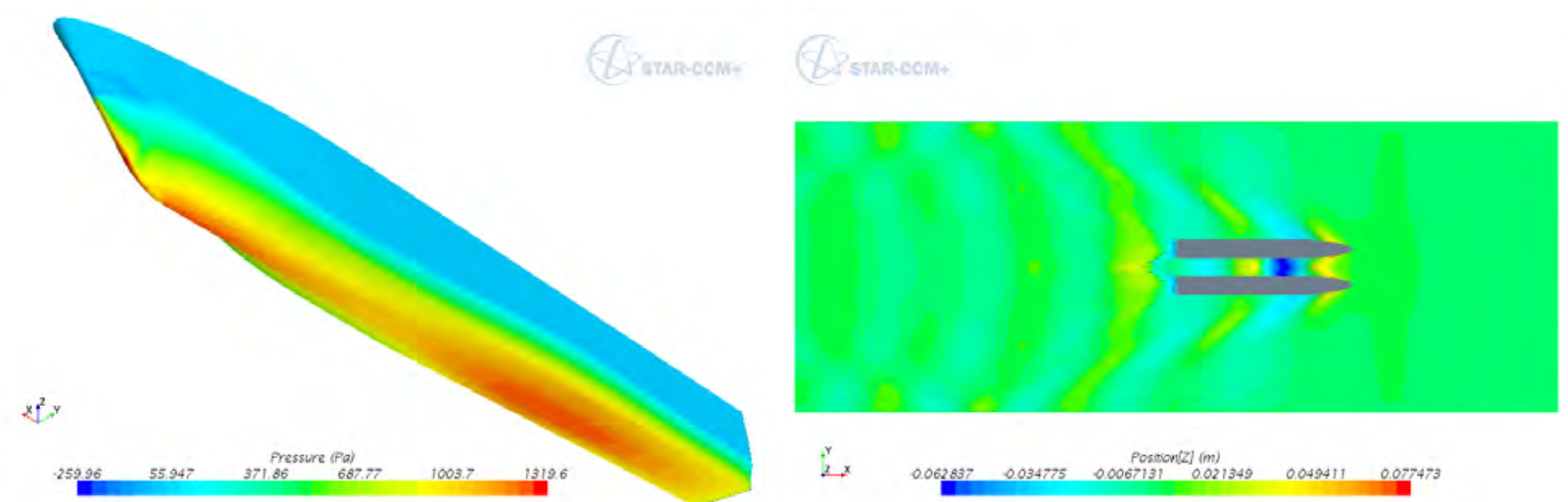

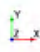

Posecon $[2](m)$

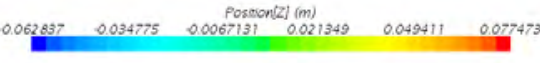

Modelo libre

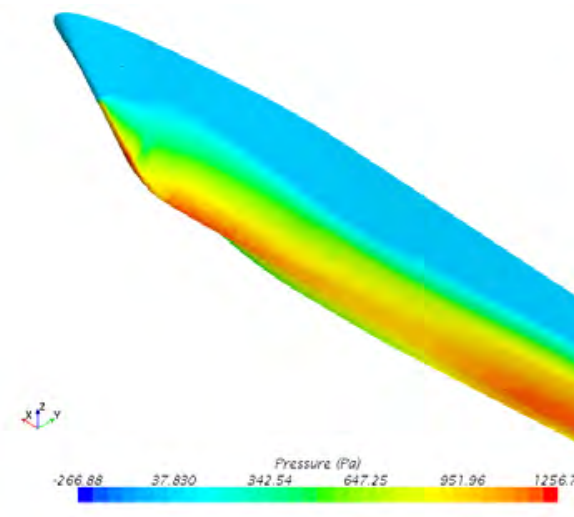

Gestar-com

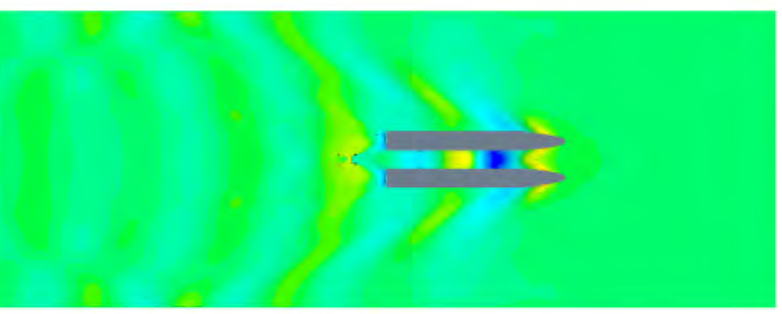

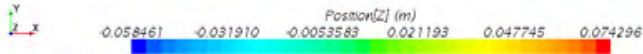

Modelo fijo

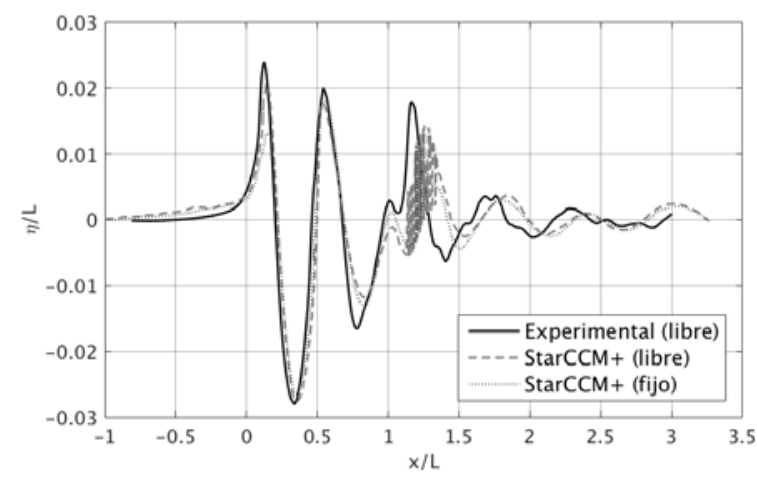

$\Delta y / s_{D}=1 / 2$ (corte de olas central)

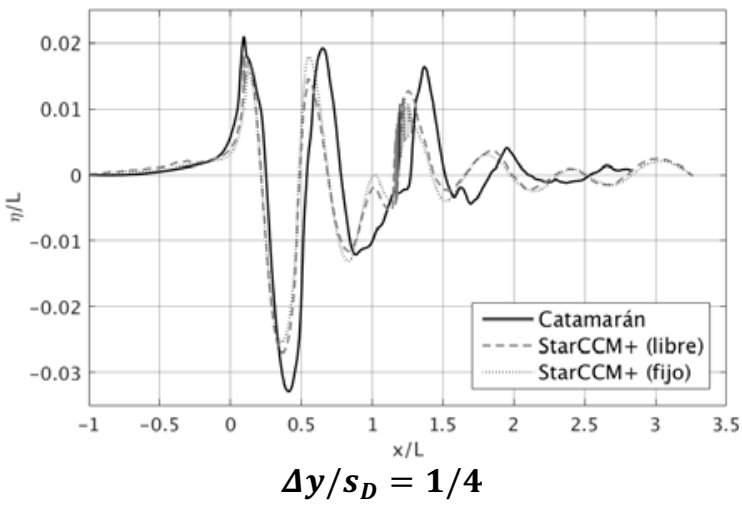

Fig. 150. $s / L=0.207 \quad F r=0.322$ 

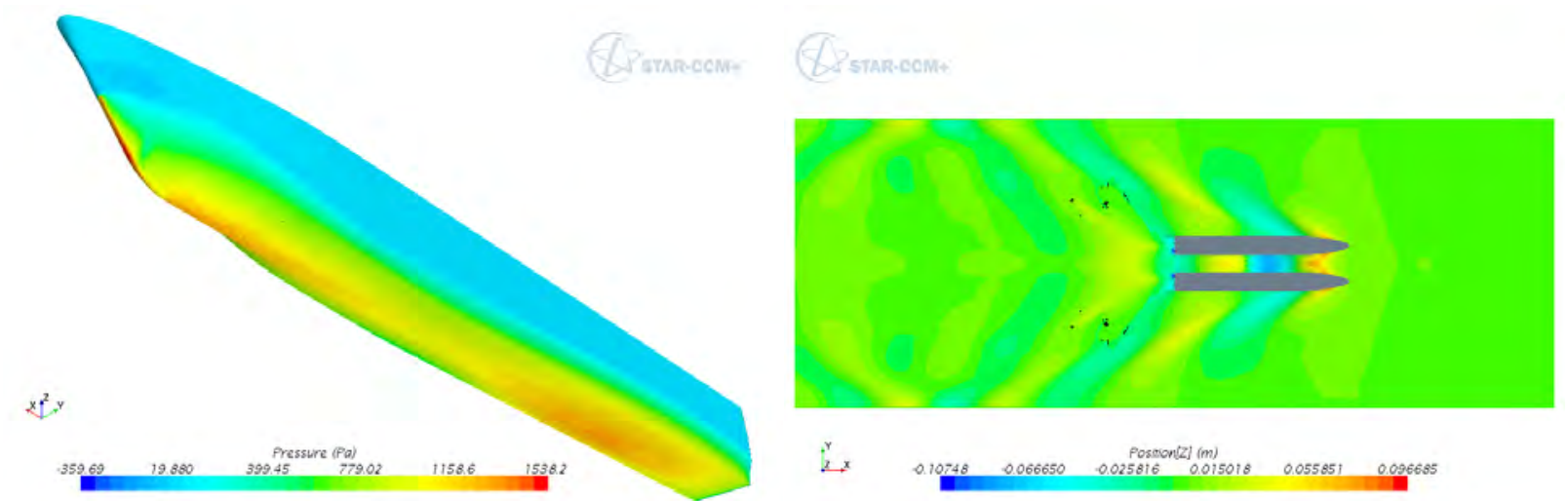

Modelo libre
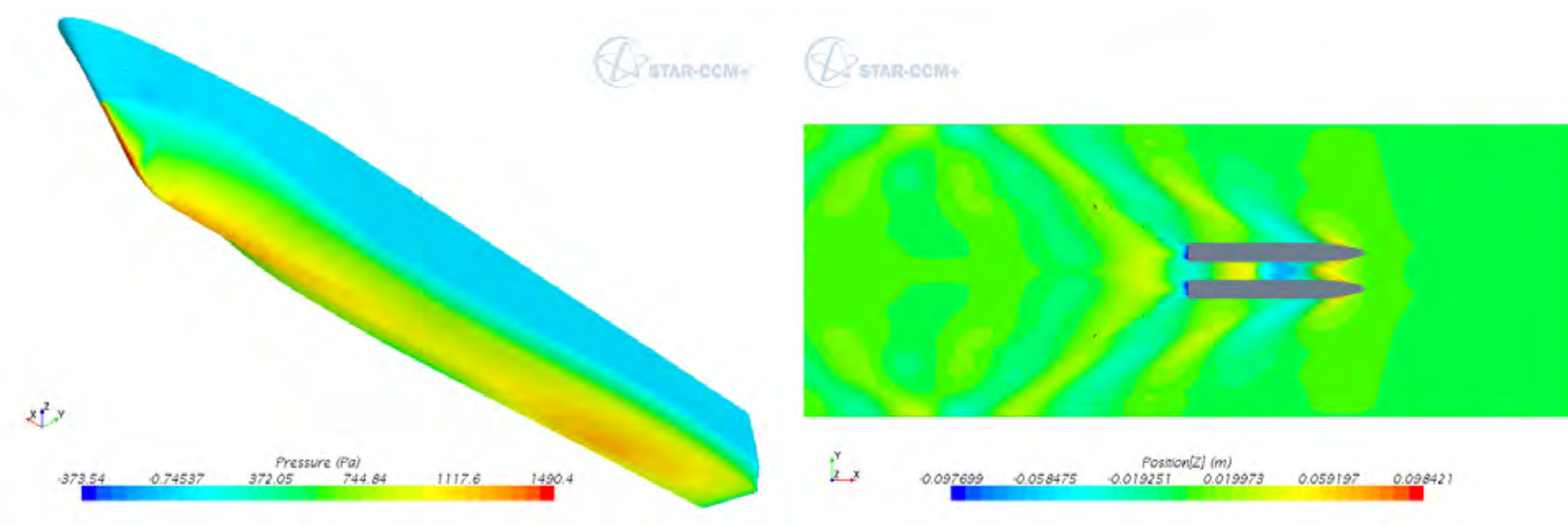

Modelo fijo

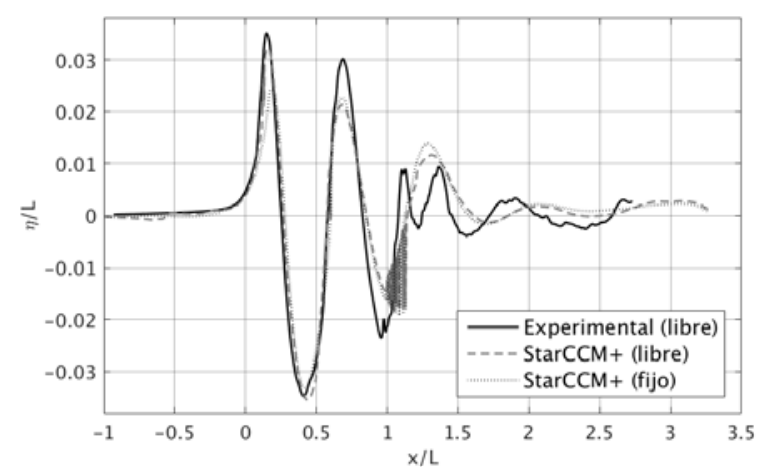

$\Delta y / s_{D}=1 / 2$ (corte de olas central)

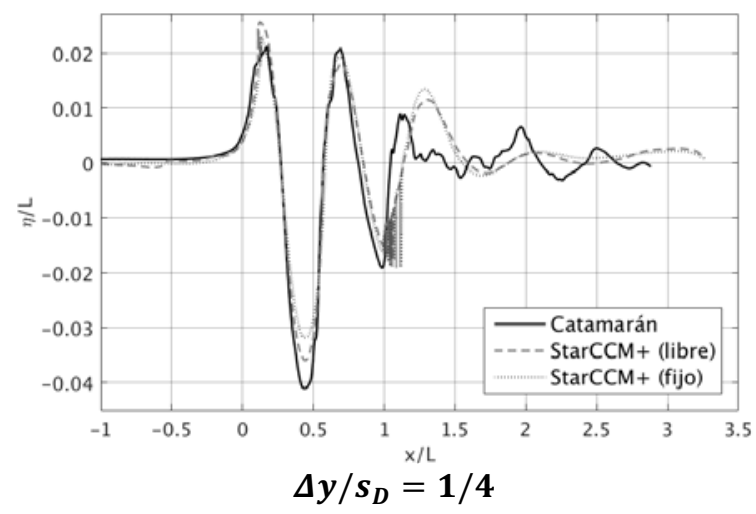

Fig. 151. $s / L=0.207 \quad F r=0.375$ 

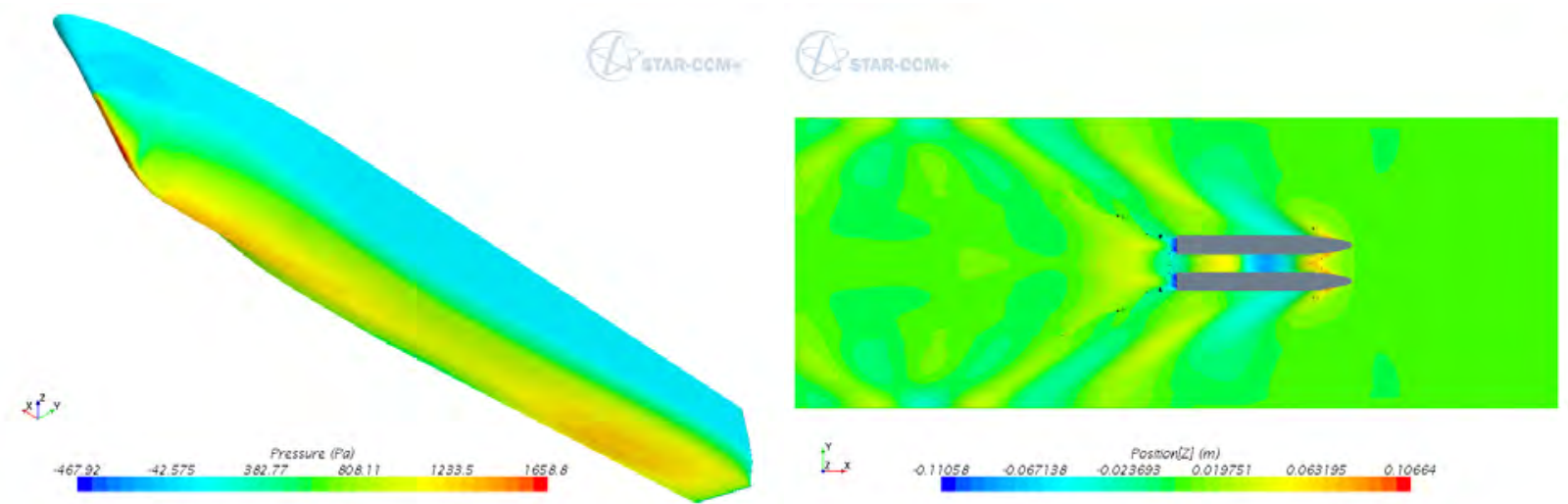

Modelo libre
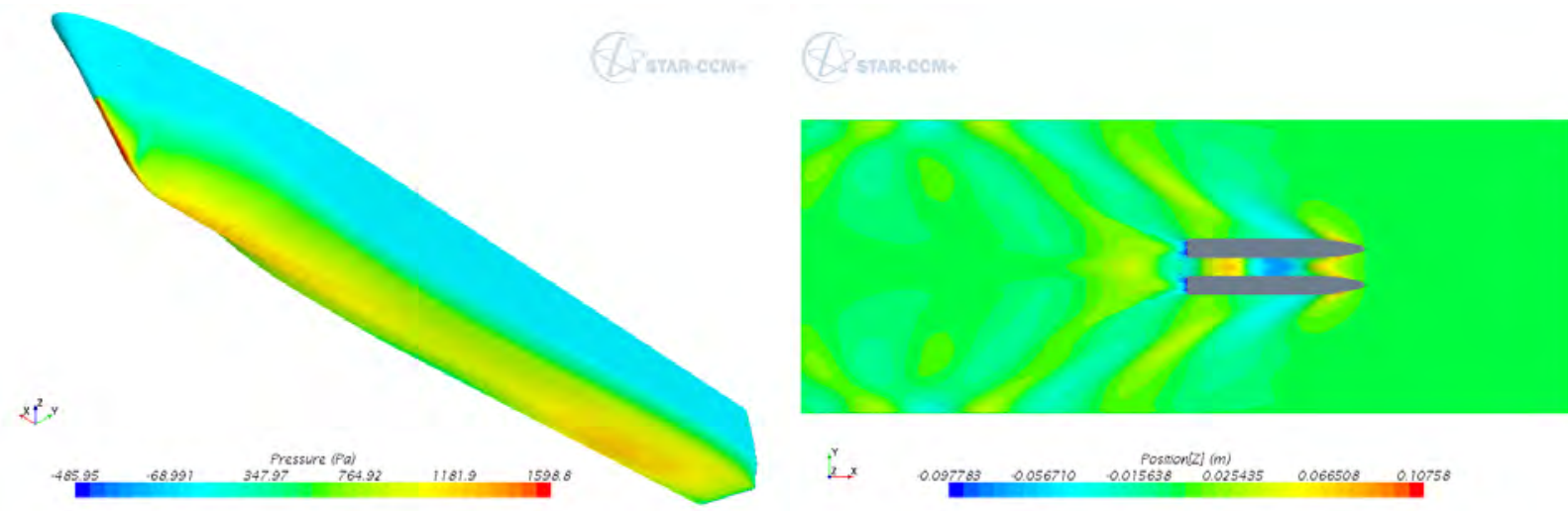

Modelo fijo

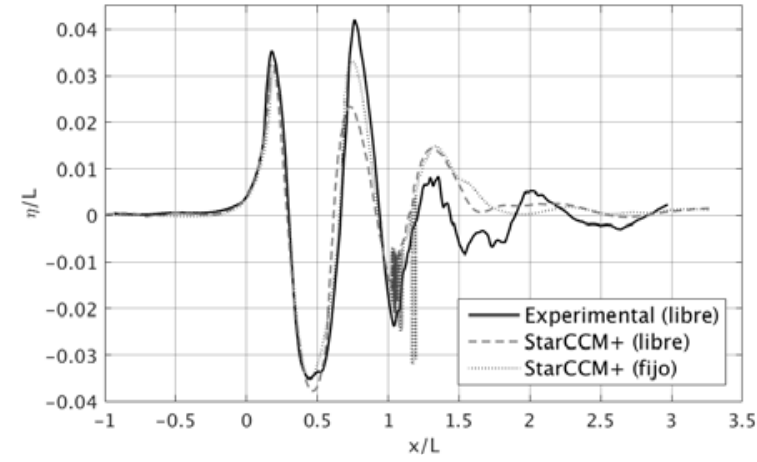

$\Delta y / s_{D}=1 / 2$ (corte de olas central)

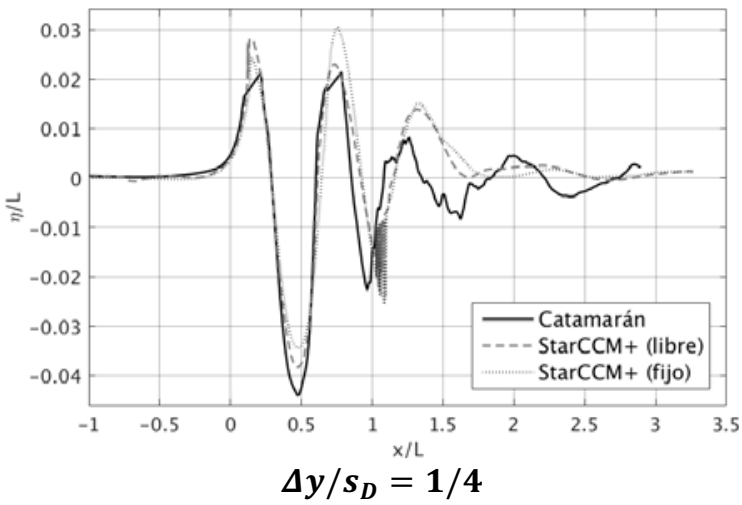

Fig. 152. $s / L=0.207 \quad F r=0.398$ 

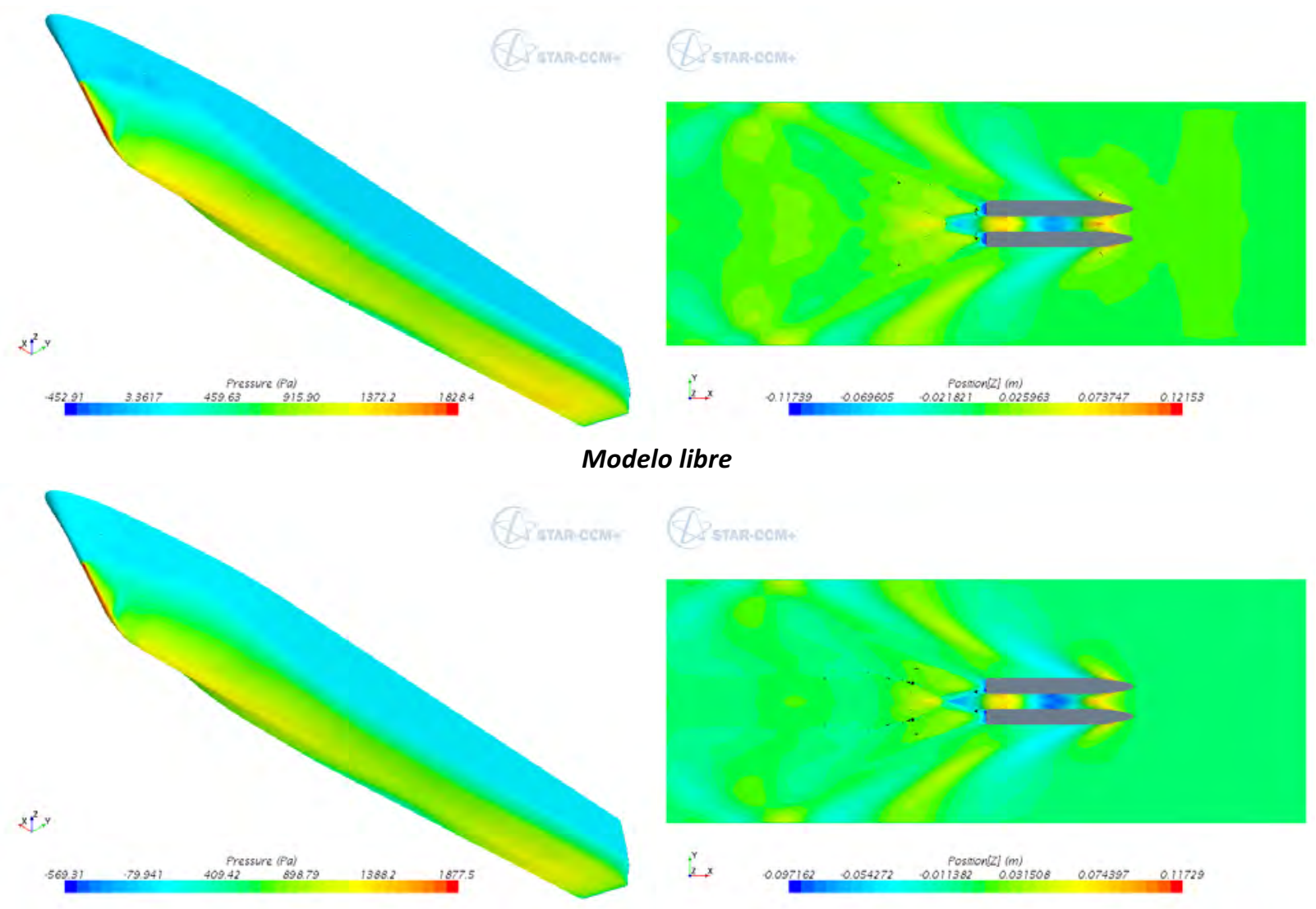

Modelo fijo

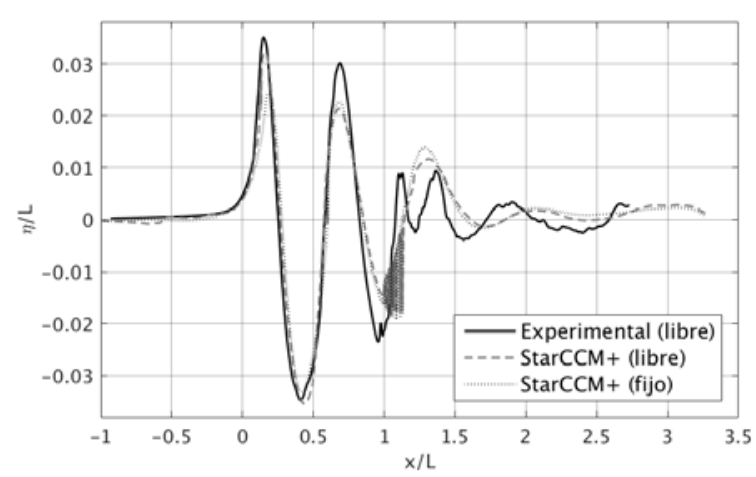

$\Delta y / s_{D}=1 / 2$ (corte de olas central)

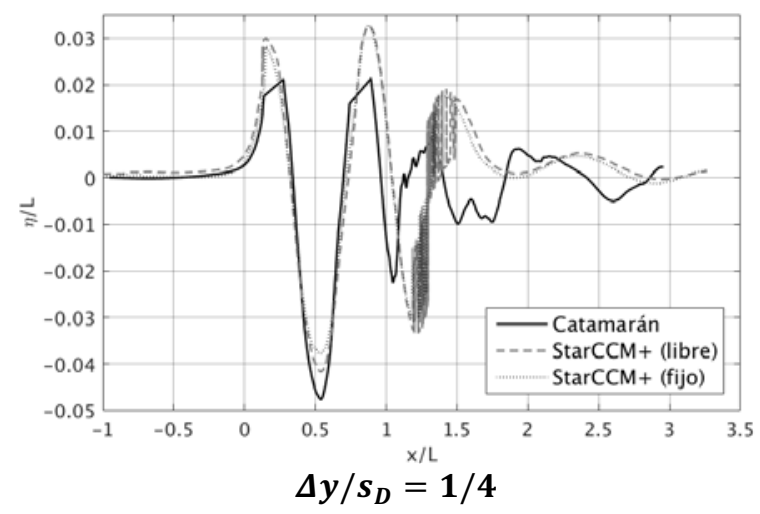

Fig. 153. $s / L=0.207 \quad F r=0.430$ 

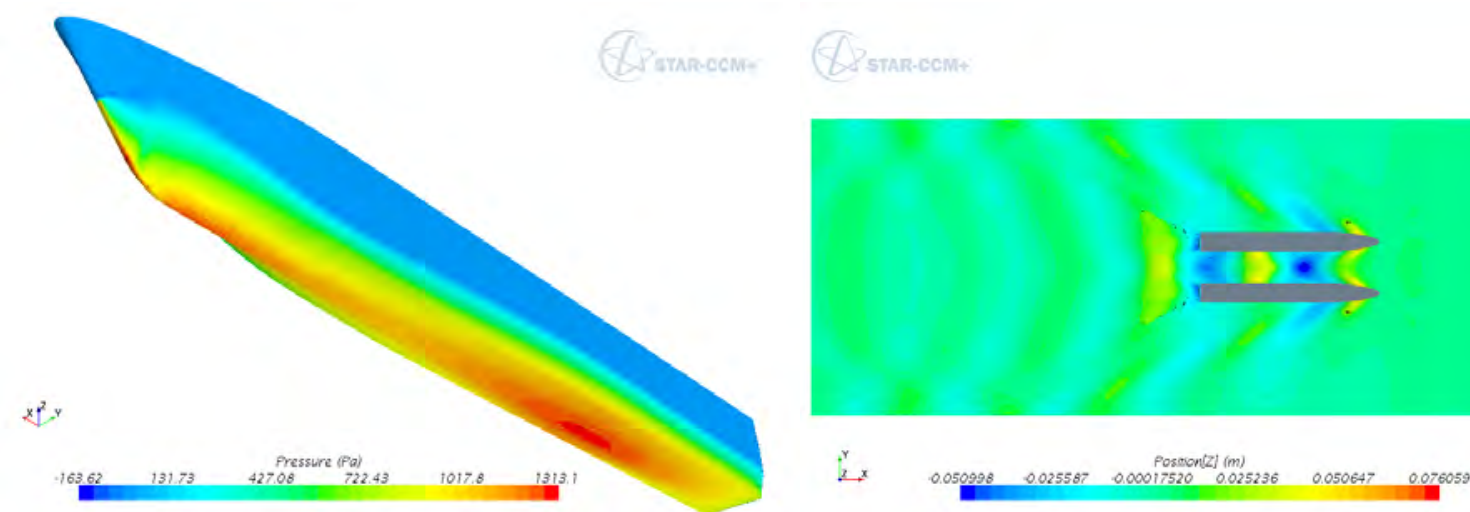

Modelo libre
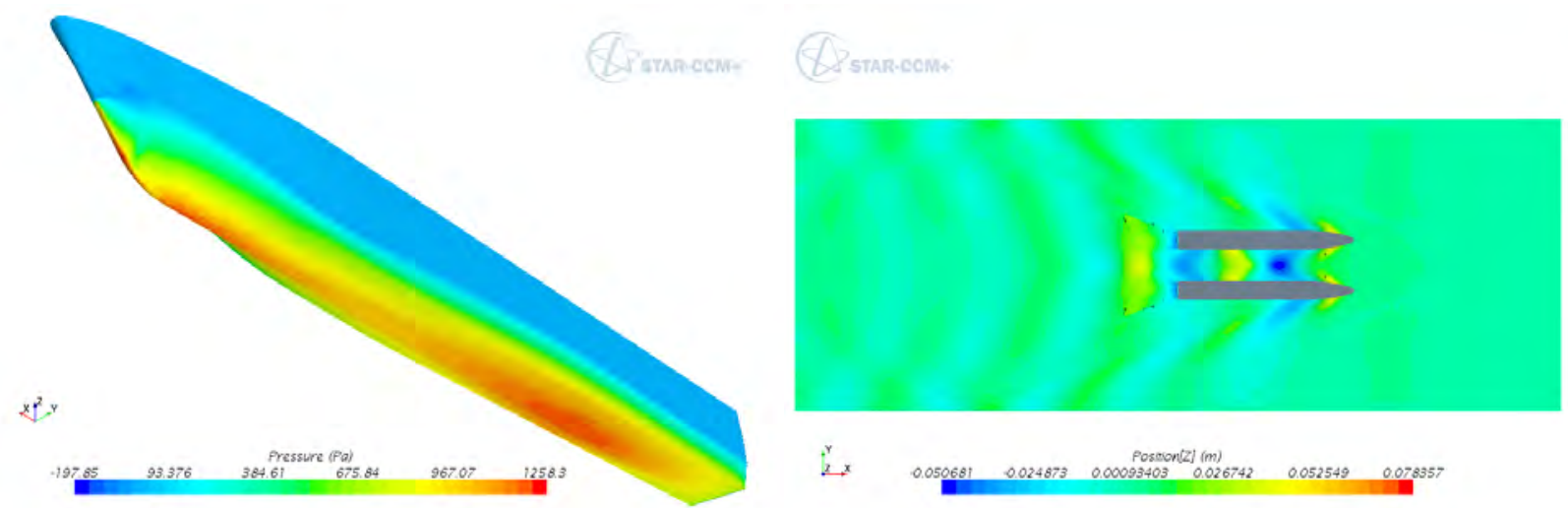

Modelo fijo
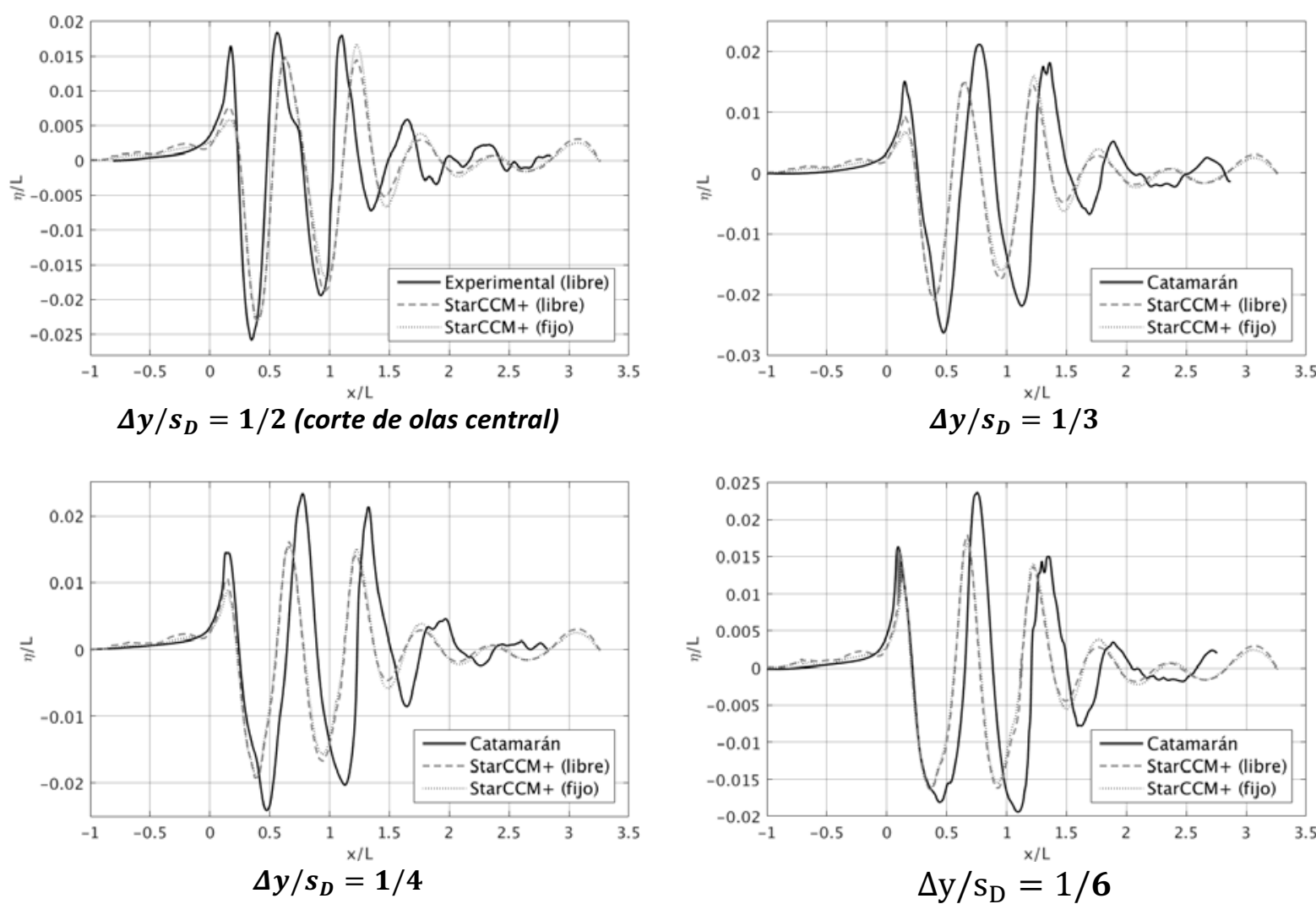

Fig. 154. $s / L=0.298 F r=0.322$

ANÁLISIS Y OPTIMIZACIÓN DE LA RESISTENCIA DE INTERFERENCIA EN MULTICASCOS 

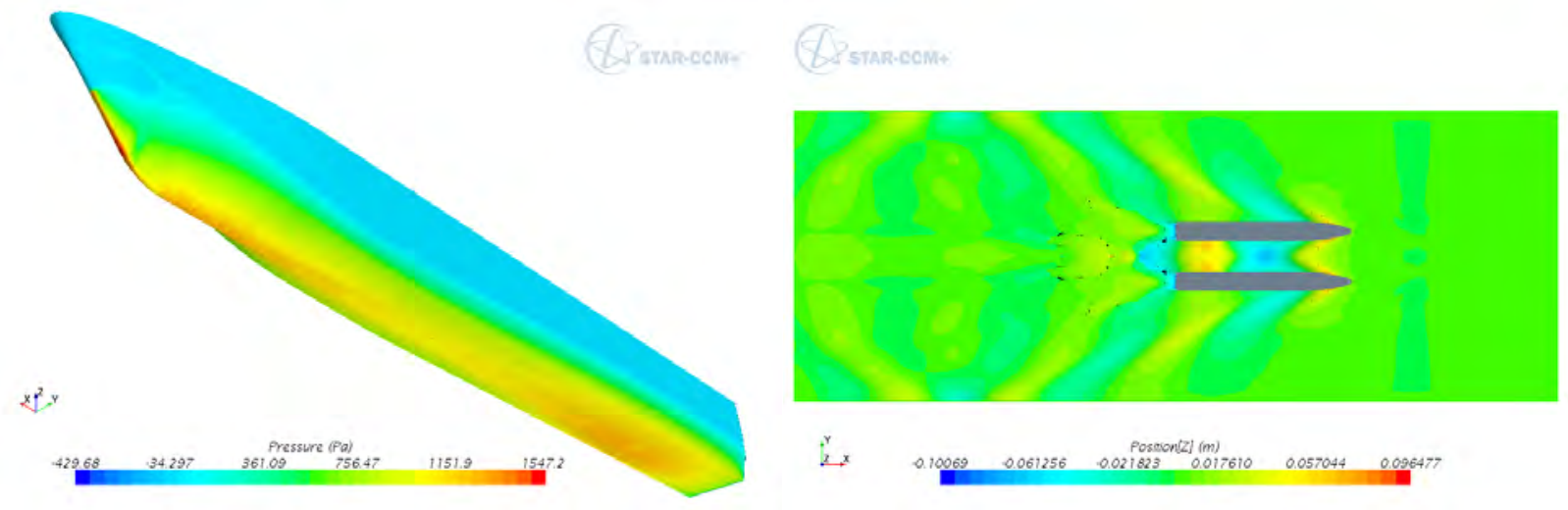

Modelo libre
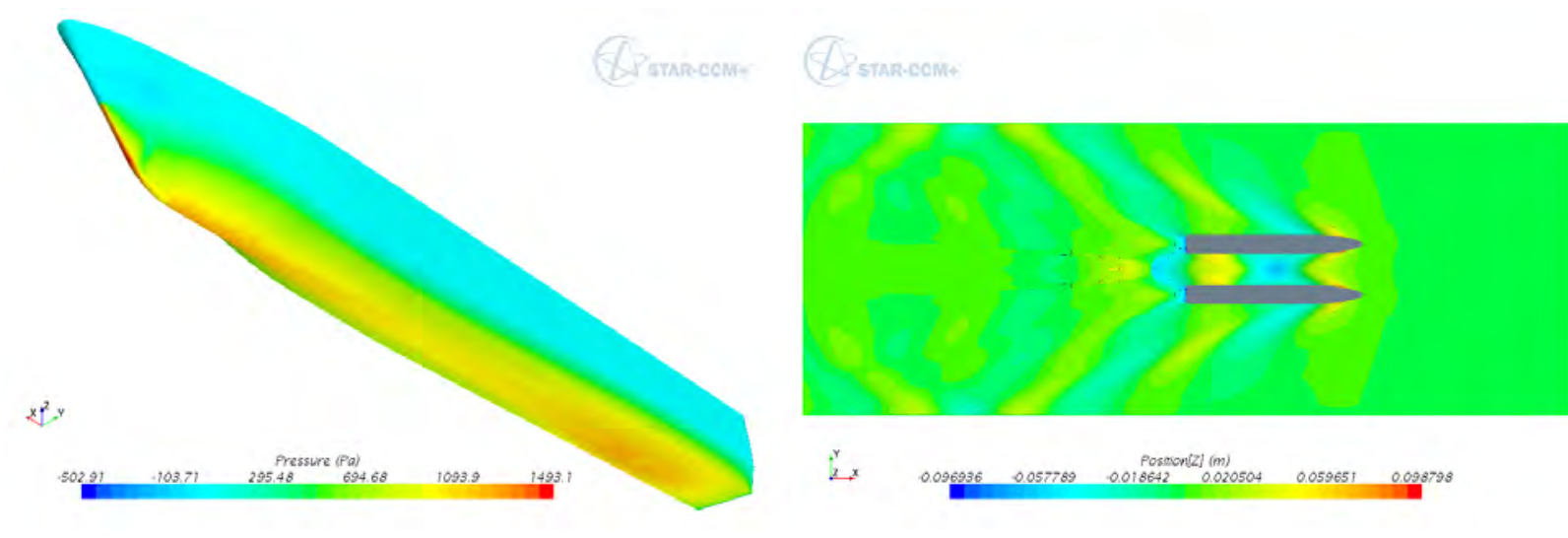

Modelo fijo
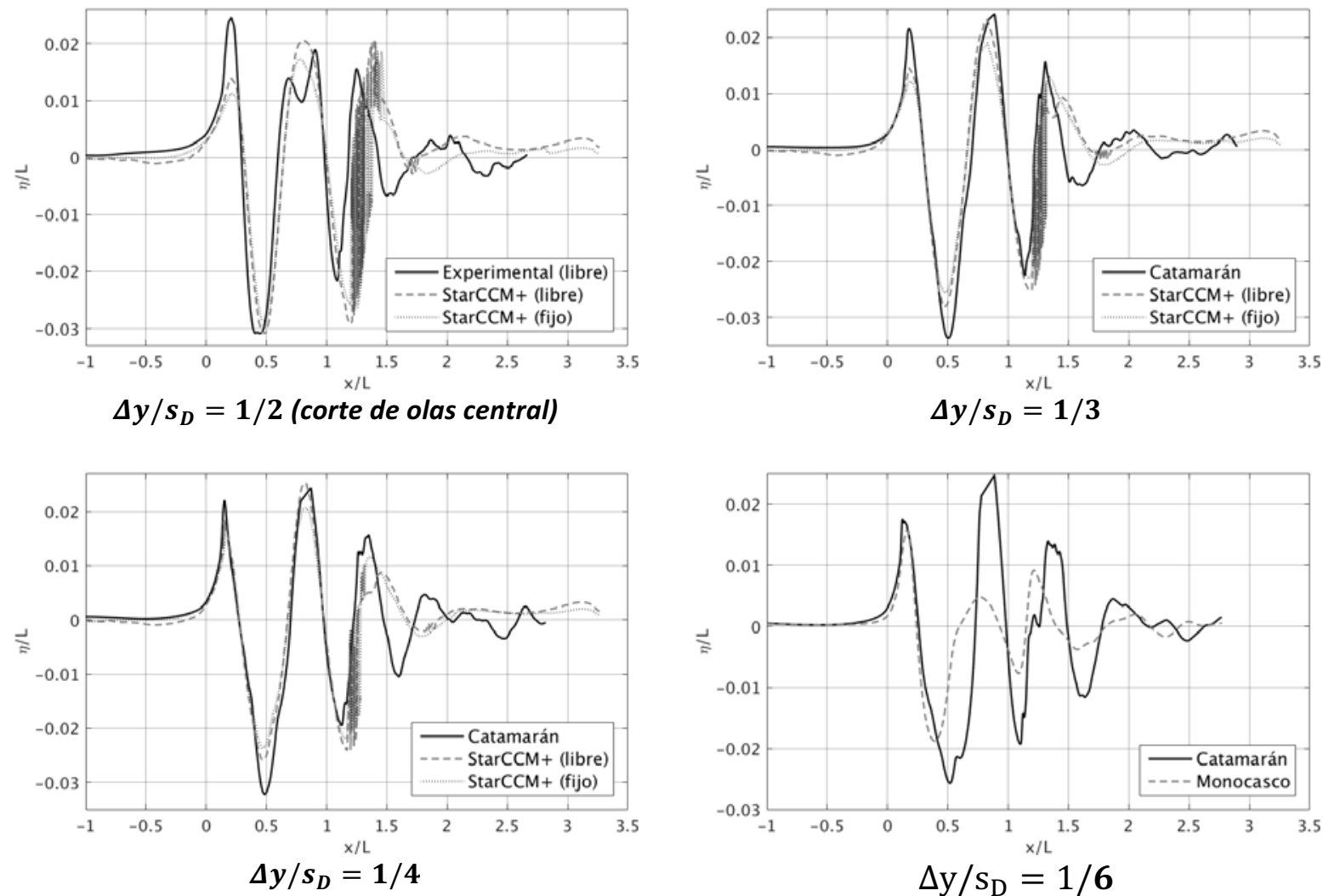

Fig. 155. $s / L=0.298 \quad F r=0.375$

ANÁLISIS Y OPTIMIZACIÓN DE LA RESISTENCIA DE INTERFERENCIA EN MULTICASCOS

Página 233 de 258 

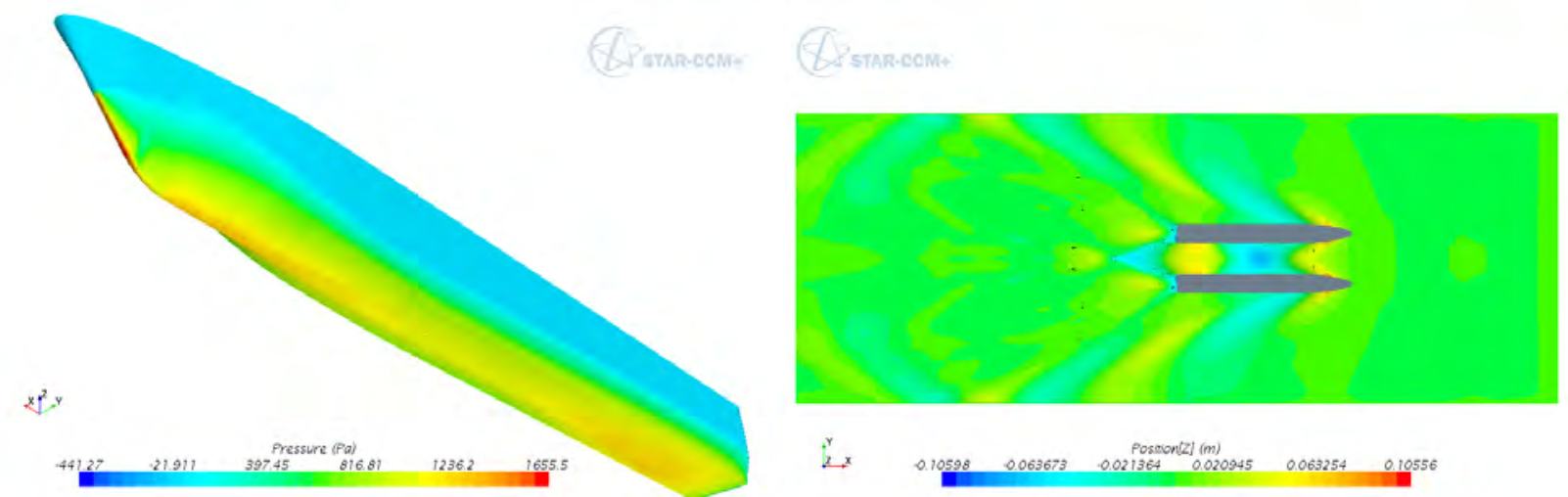

Modelo libre
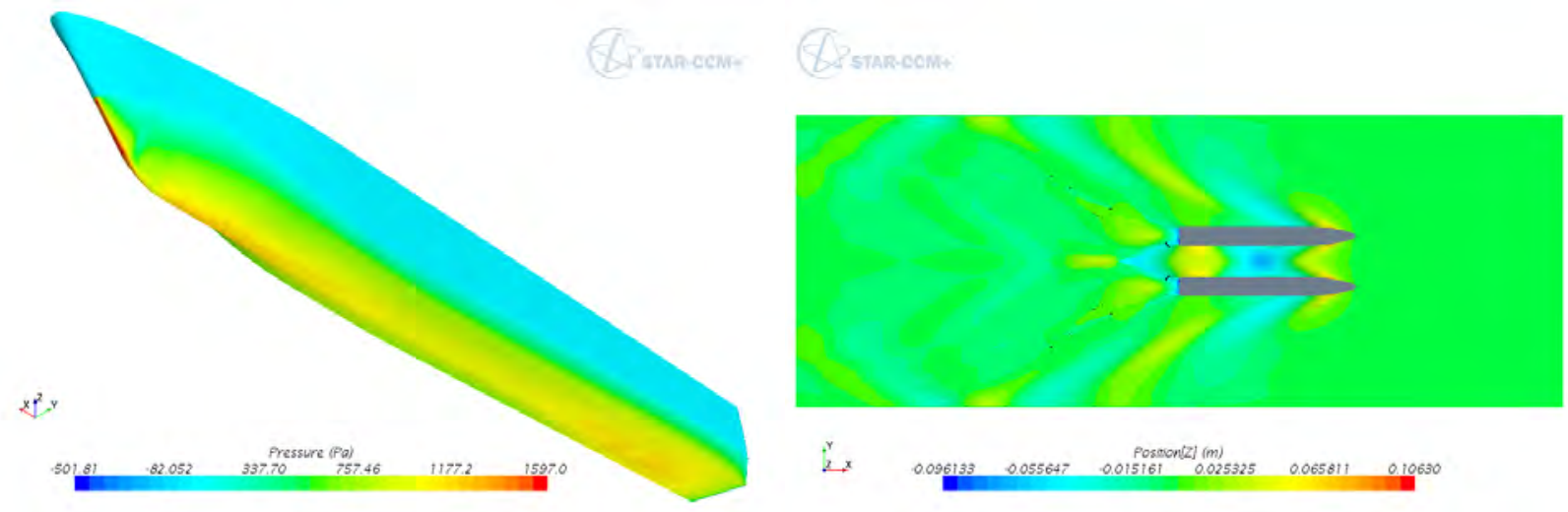

Modelo fijo
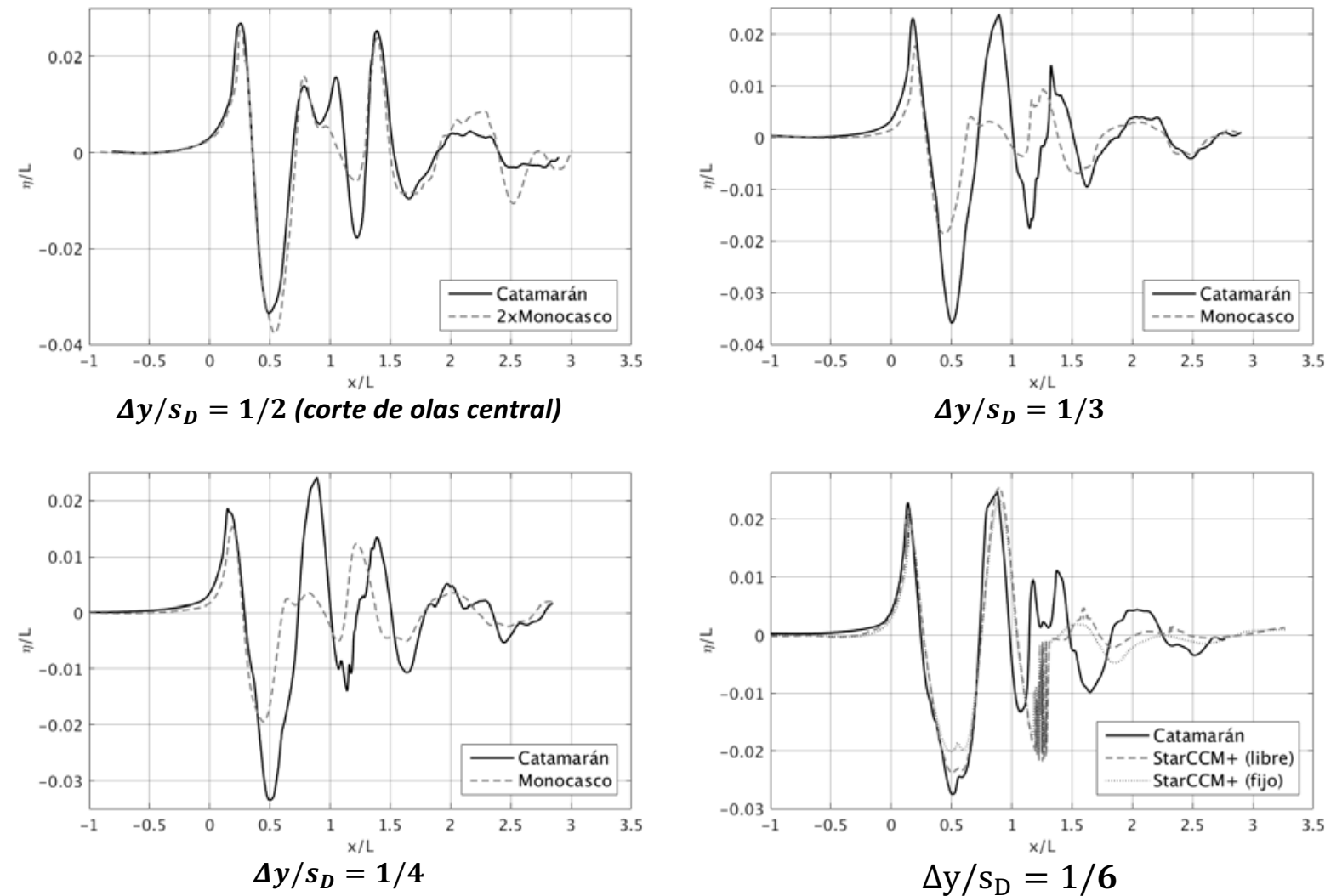

Fig. 156. $s / L=0.298 \quad F r=0.398$

ANÁLISIS Y OPTIMIZACIÓN DE LA RESISTENCIA DE INTERFERENCIA EN MULTICASCOS 

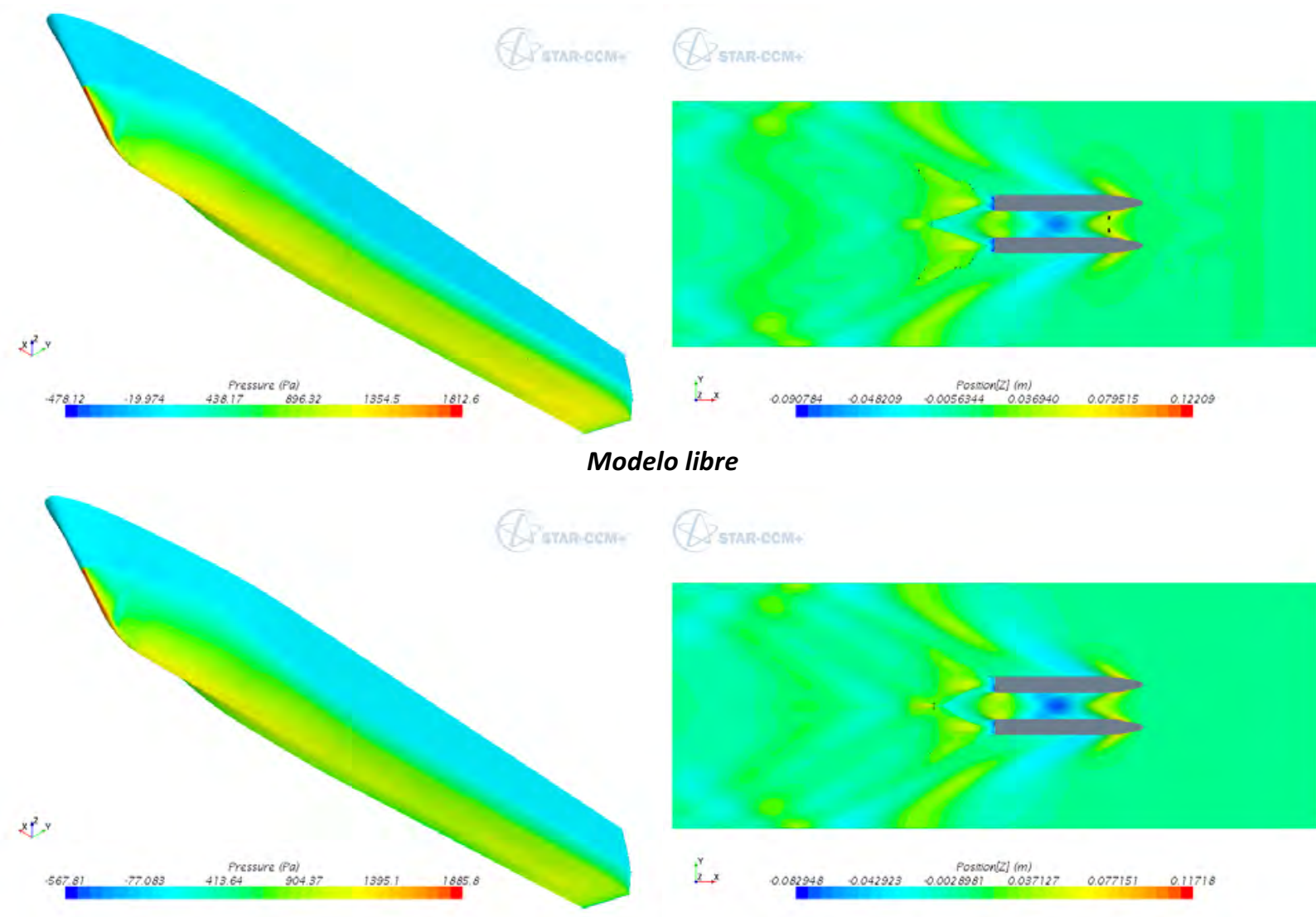

\section{Modelo fijo}

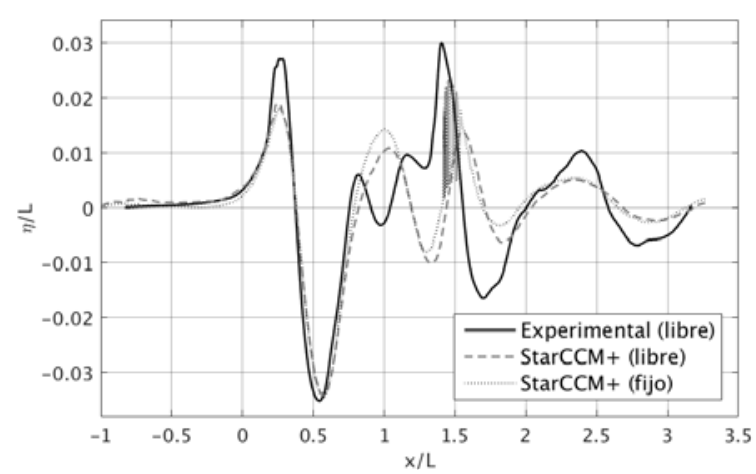

$\Delta y / s_{D}=1 / 2$ (corte de olas central)

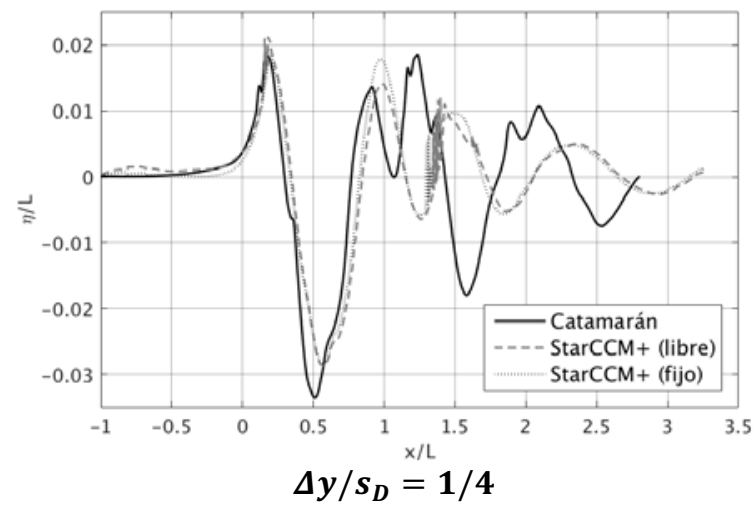

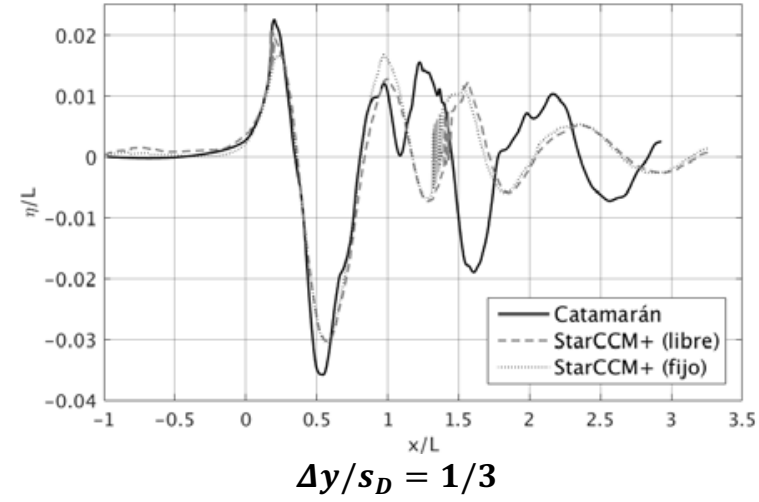

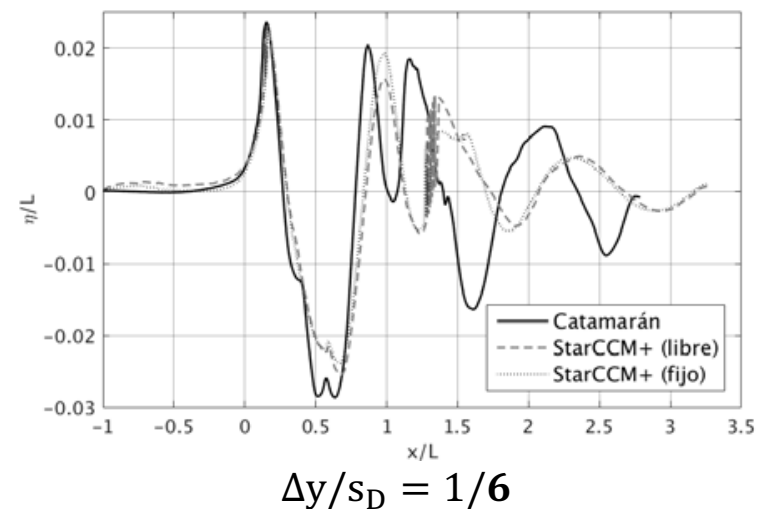

Fig. 157. $s / L=0.298 \quad F r=0.430$

ANÁLISIS Y OPTIMIZACIÓN DE LA RESISTENCIA DE INTERFERENCIA EN MULTICASCOS 

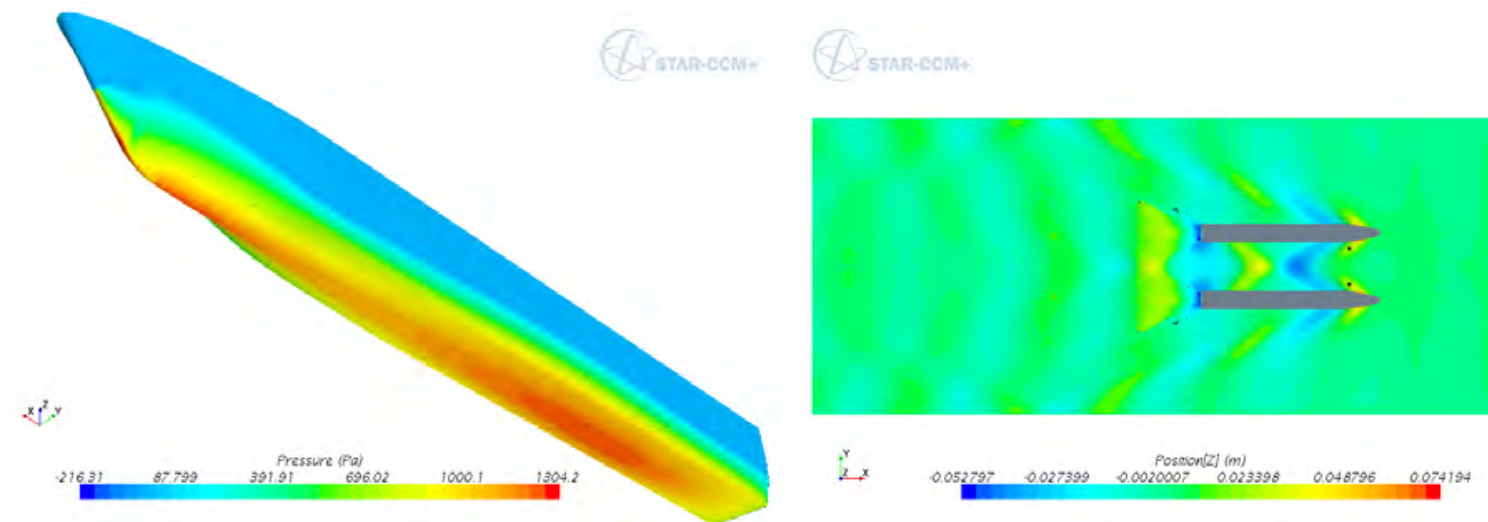

Modelo libre
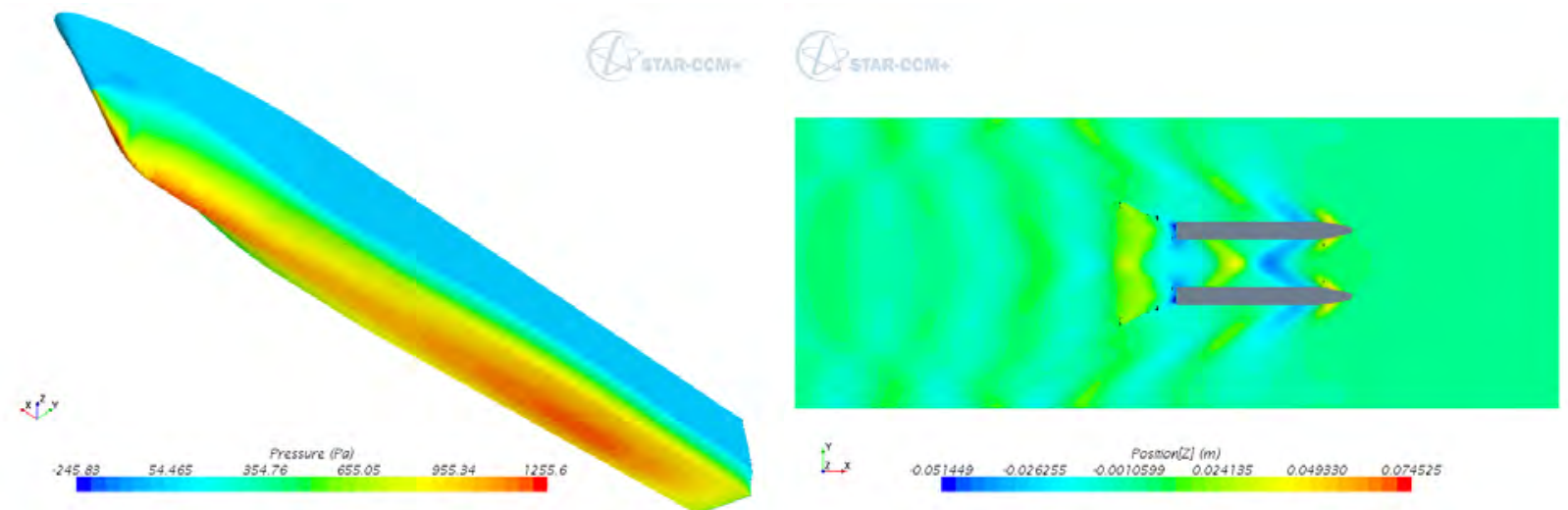

Modelo fijo
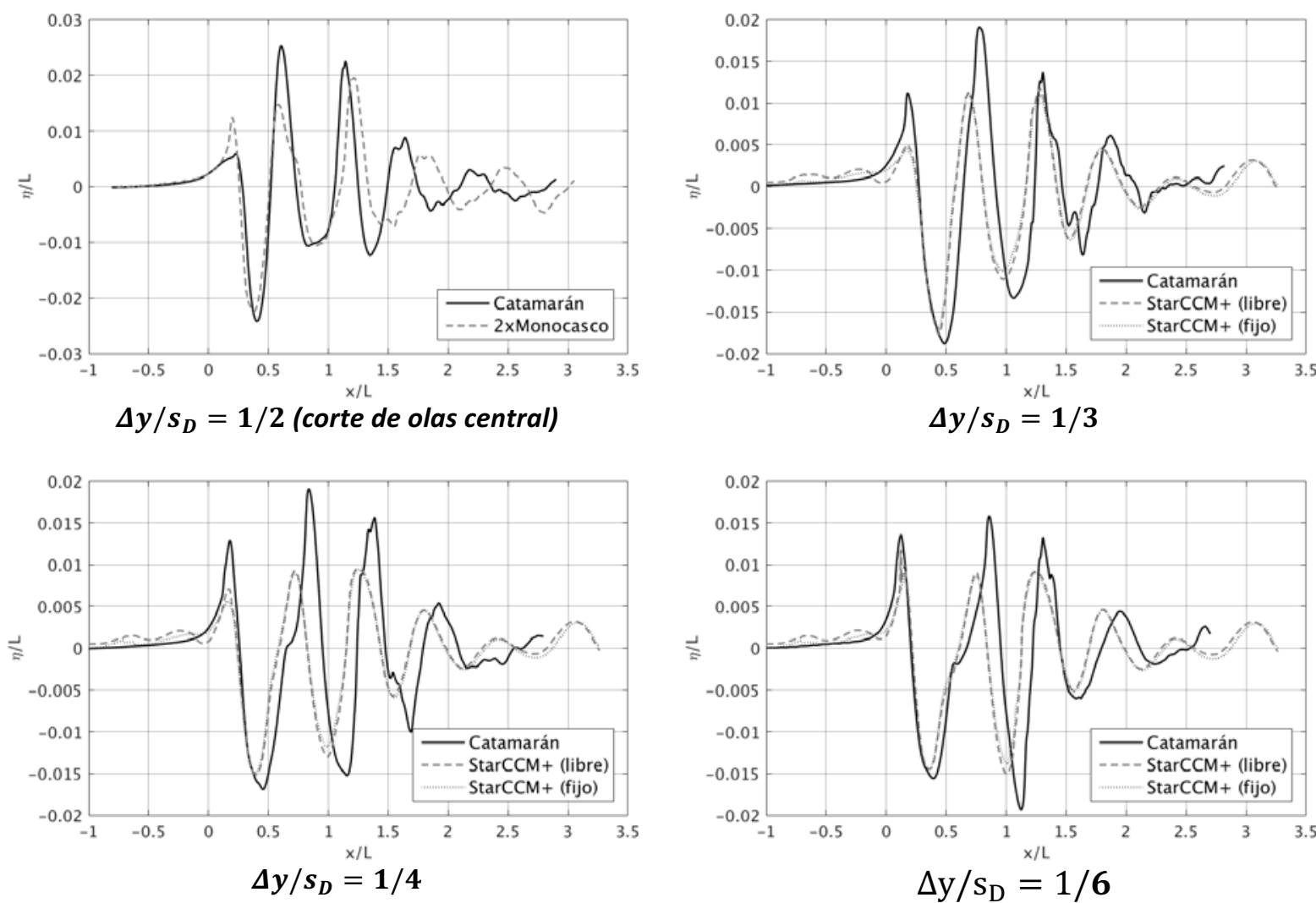

Fig. 158. $s / L=0.388 \quad F r=0.322$

ANÁLISIS Y OPTIMIZACIÓN DE LA RESISTENCIA DE INTERFERENCIA EN MULTICASCOS 


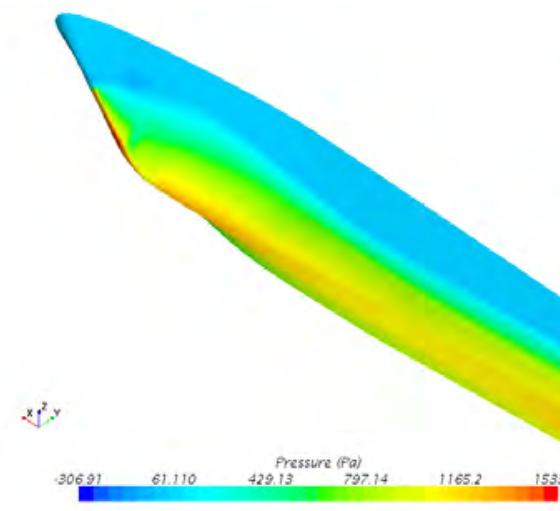

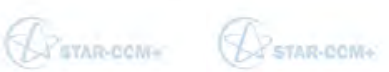
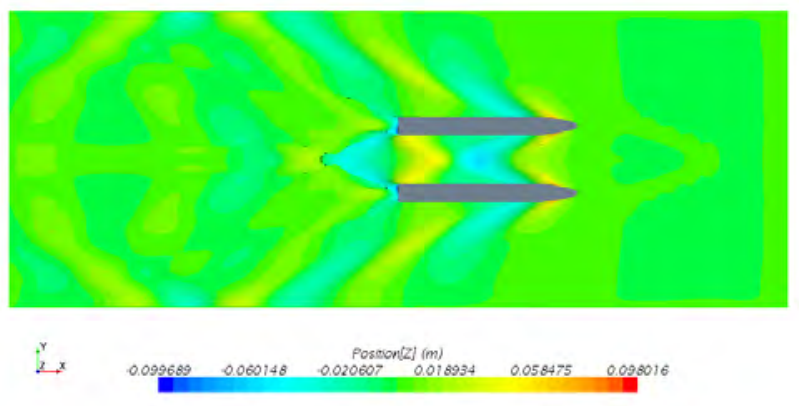

Modelo libre
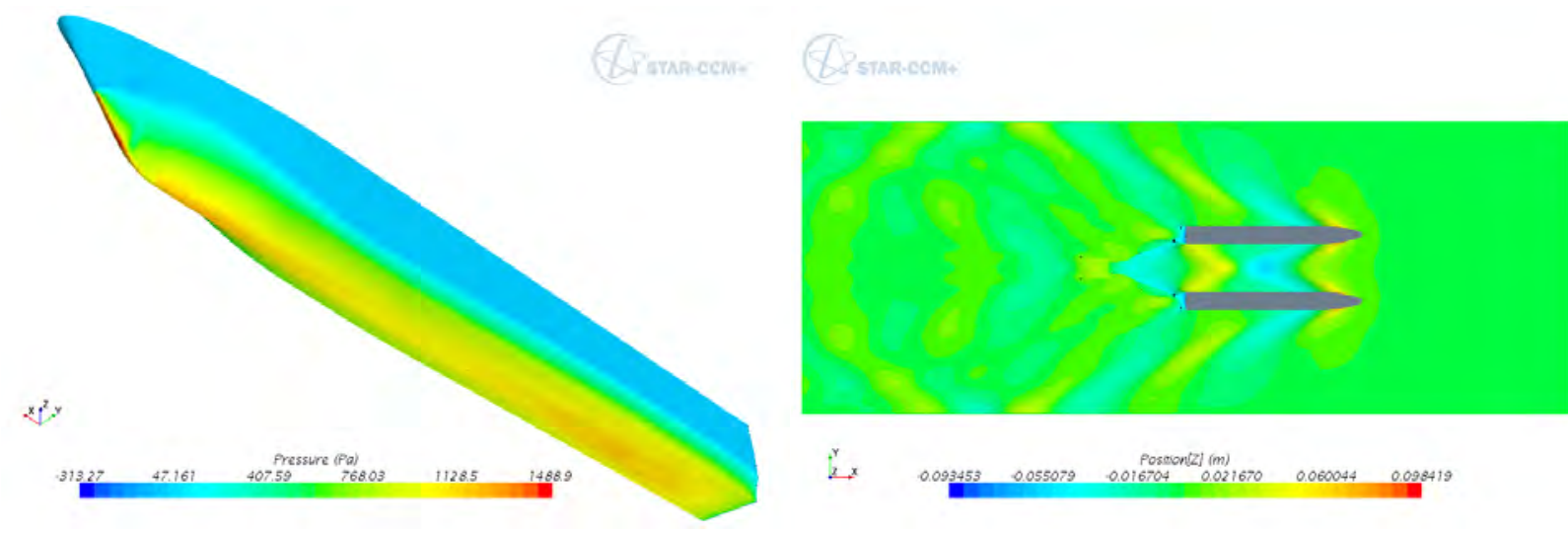

Modelo fijo

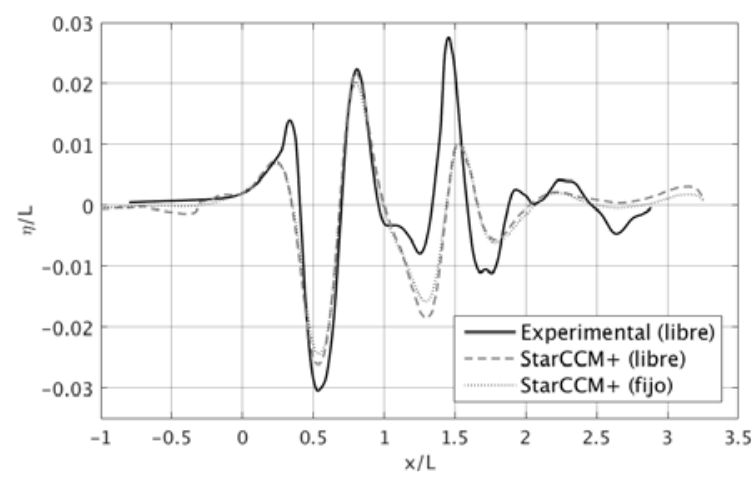

$\Delta y / s_{D}=1 / 2$ (corte de olas central)
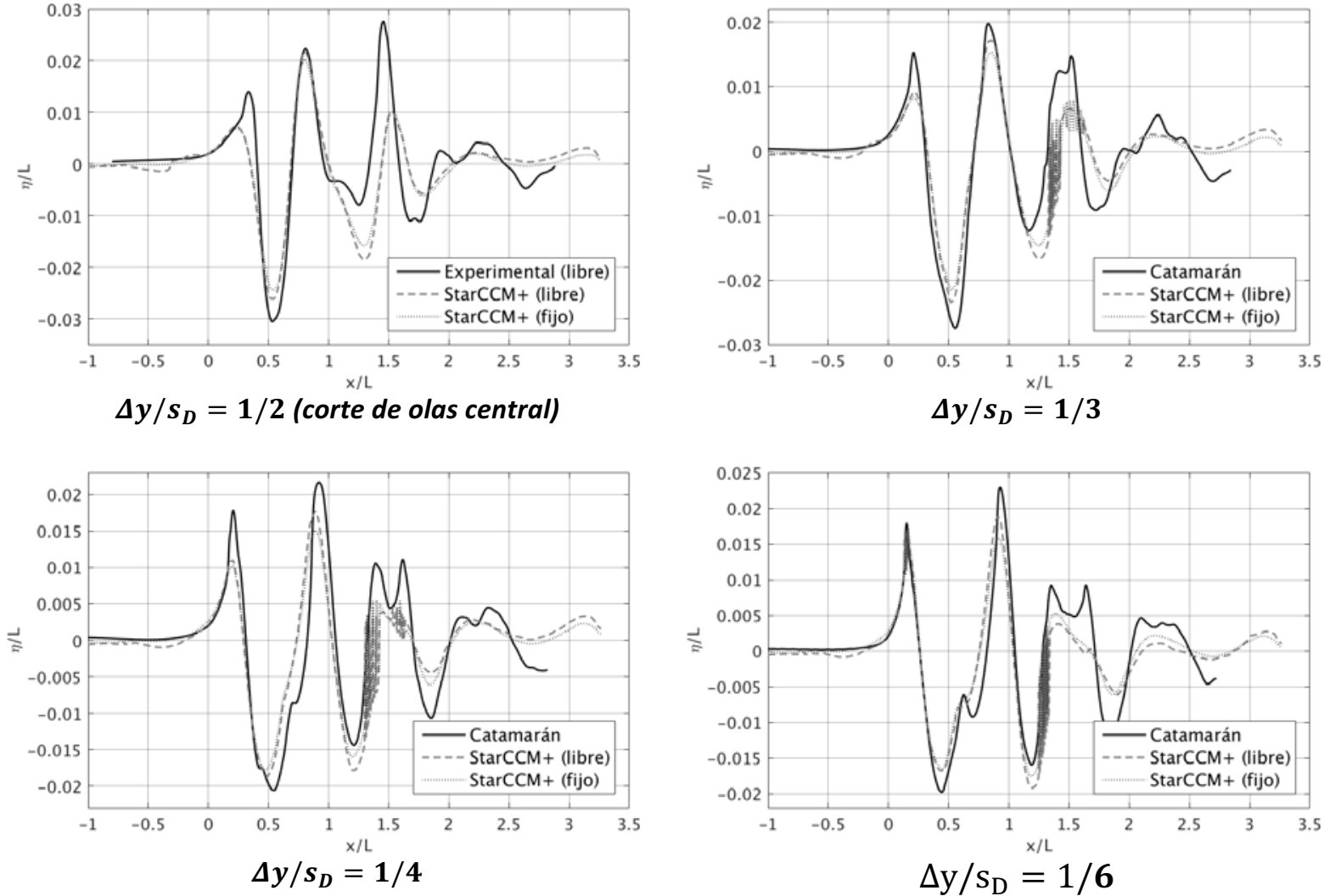

Fig. 159. $s / L=0.388 \quad F r=0.375$

ANÁLISIS Y OPTIMIZACIÓN DE LA RESISTENCIA DE INTERFERENCIA EN MULTICASCOS 

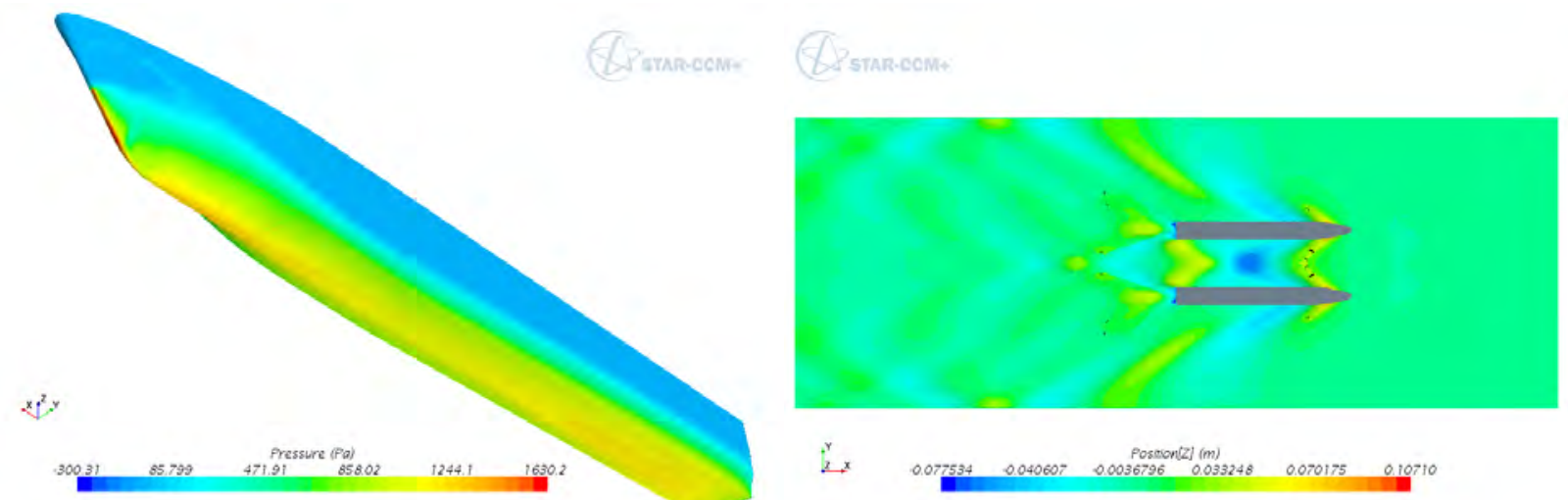

Modelo libre
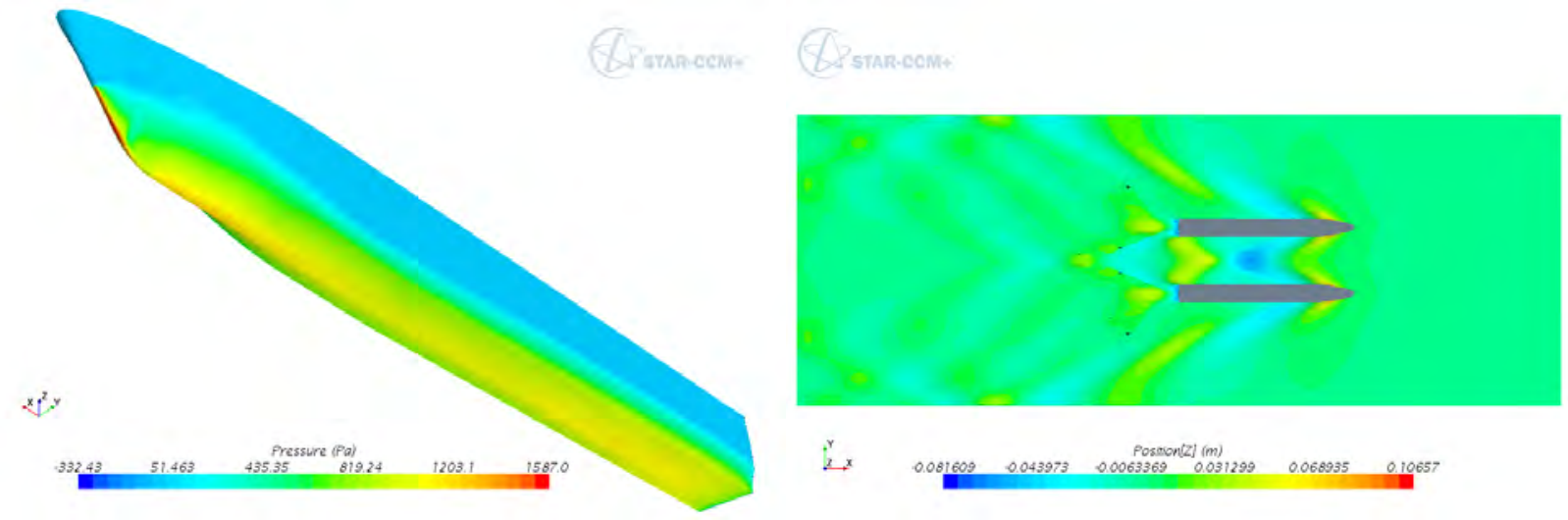

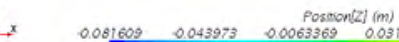

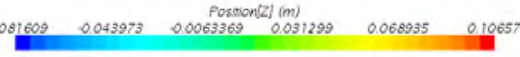

Modelo fijo
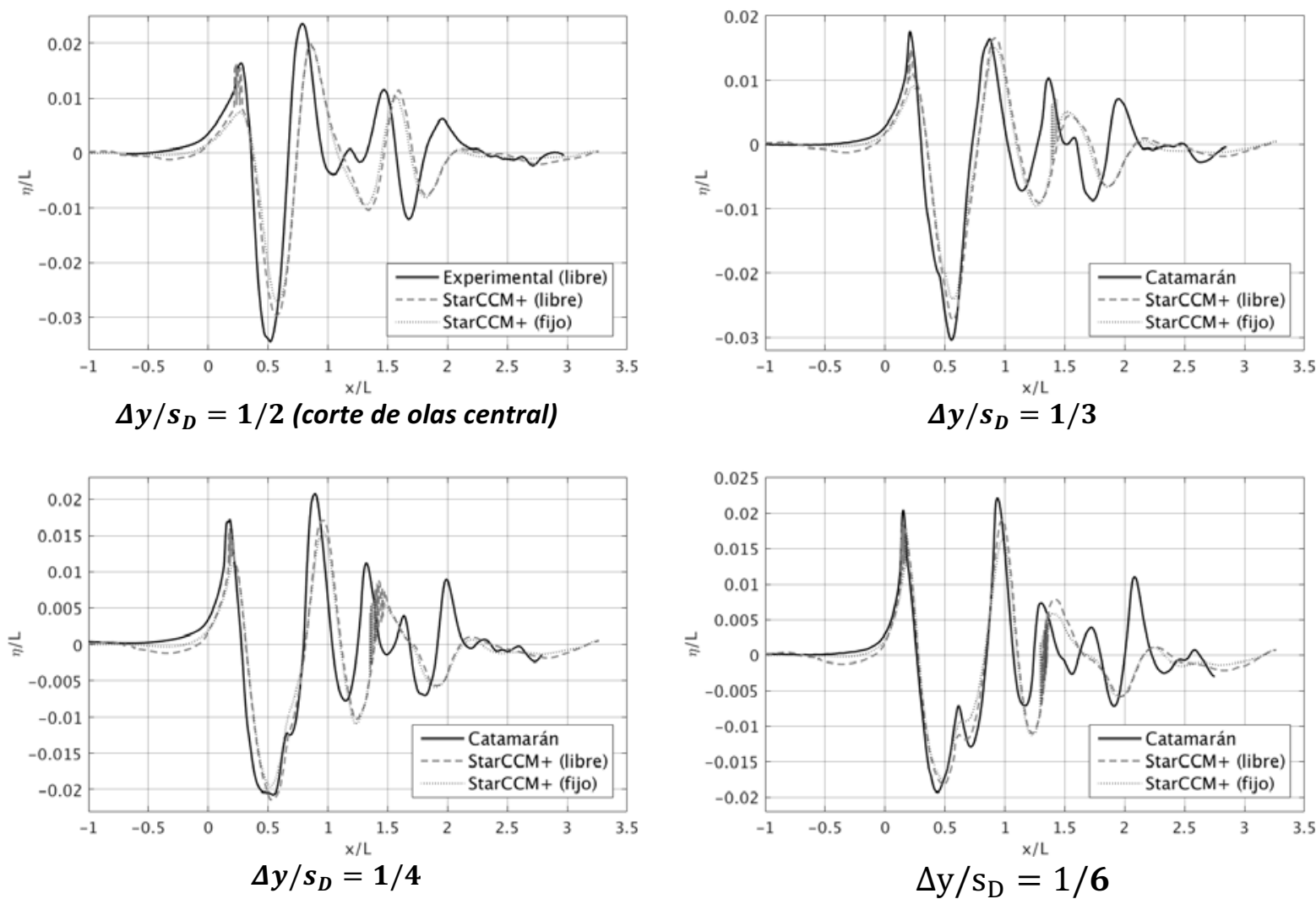

Fig. 160. $s / L=0.388 \quad F r=0.398$

ANÁLISIS Y OPTIMIZACIÓN DE LA RESISTENCIA DE INTERFERENCIA EN MULTICASCOS 

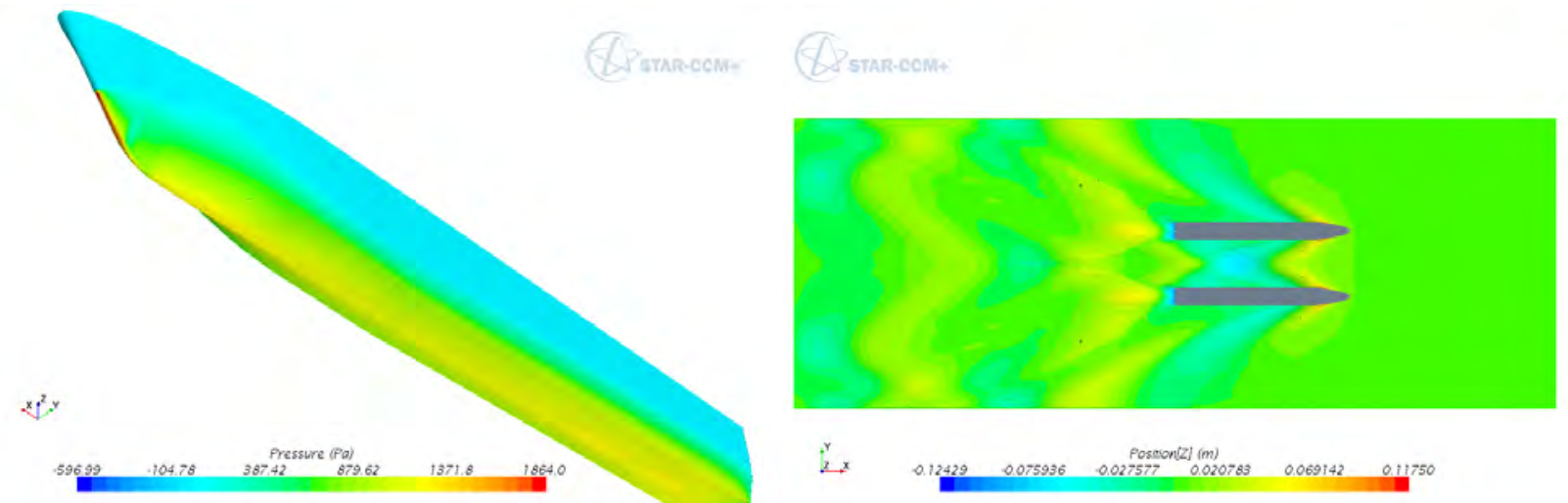

Modelo libre
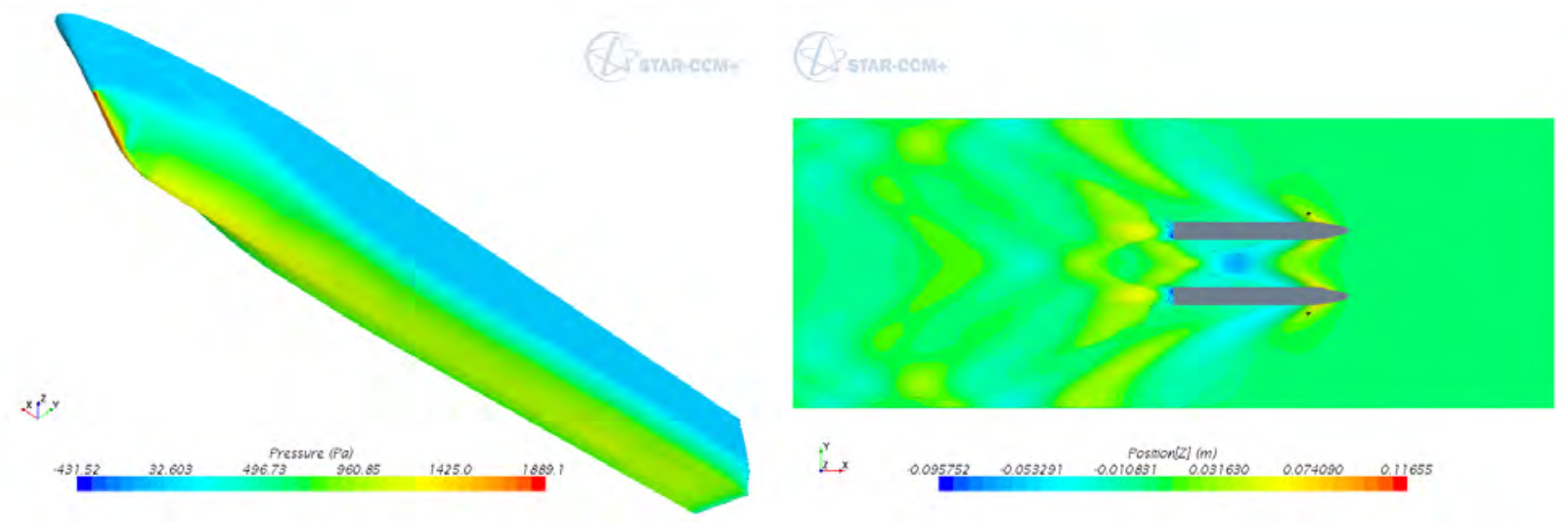

Modelo fijo

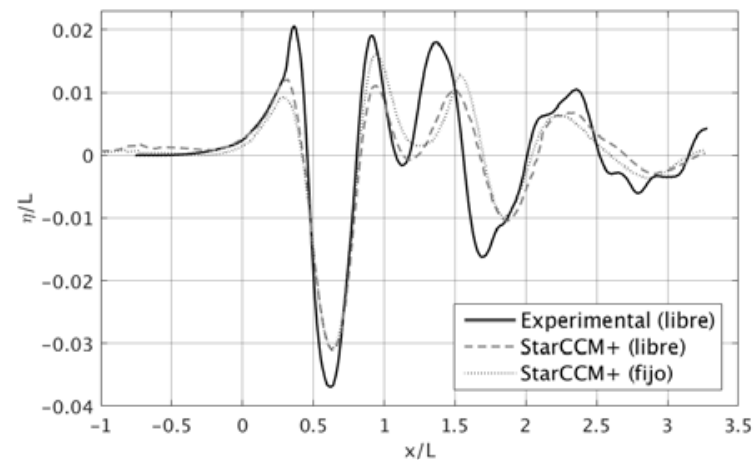

$\Delta y / s_{D}=1 / 2$ (corte de olas central)
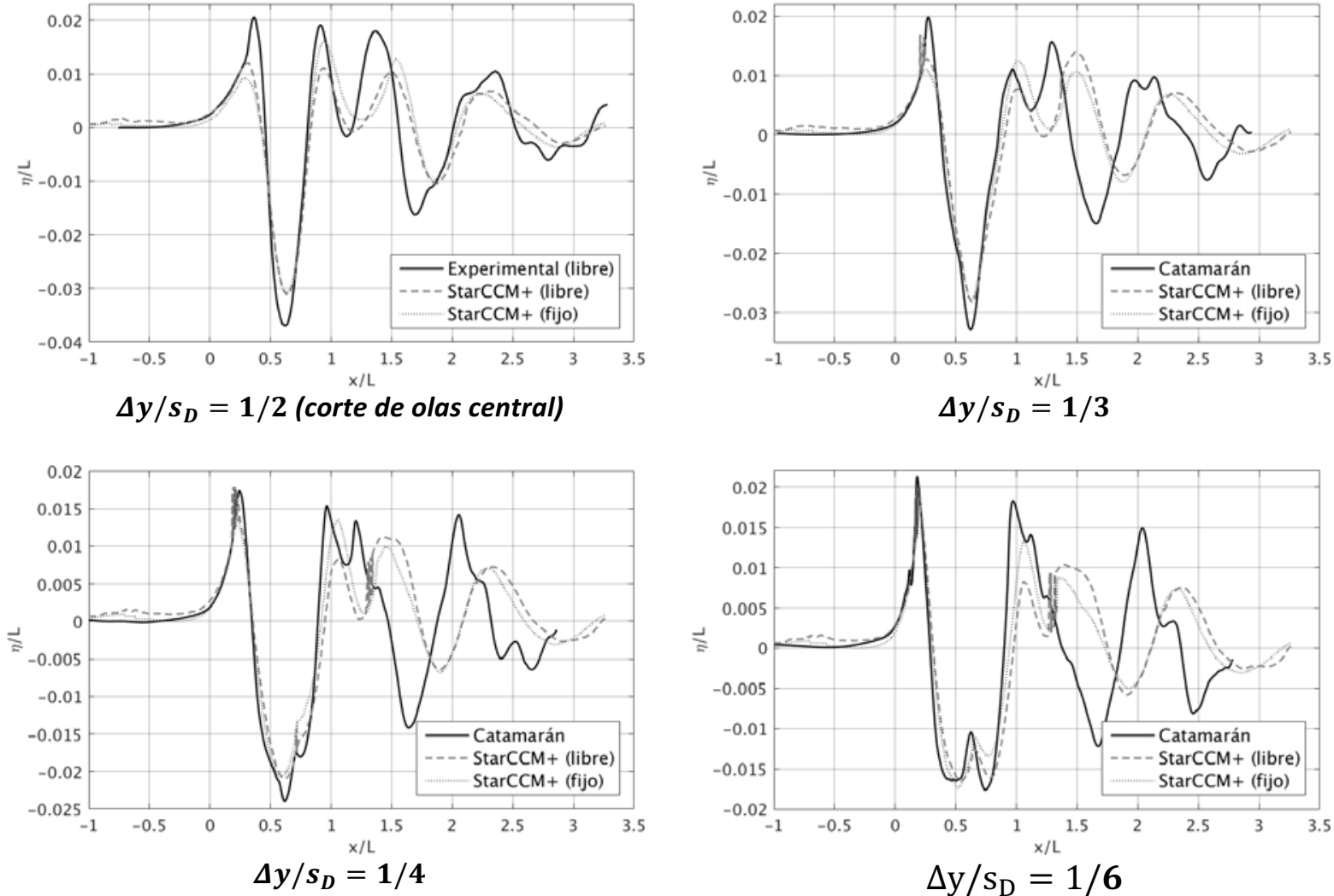

Fig. 161. $s / L=0.398 \quad F r=0.430$

ANÁLISIS Y OPTIMIZACIÓN DE LA RESISTENCIA DE INTERFERENCIA EN MULTICASCOS 

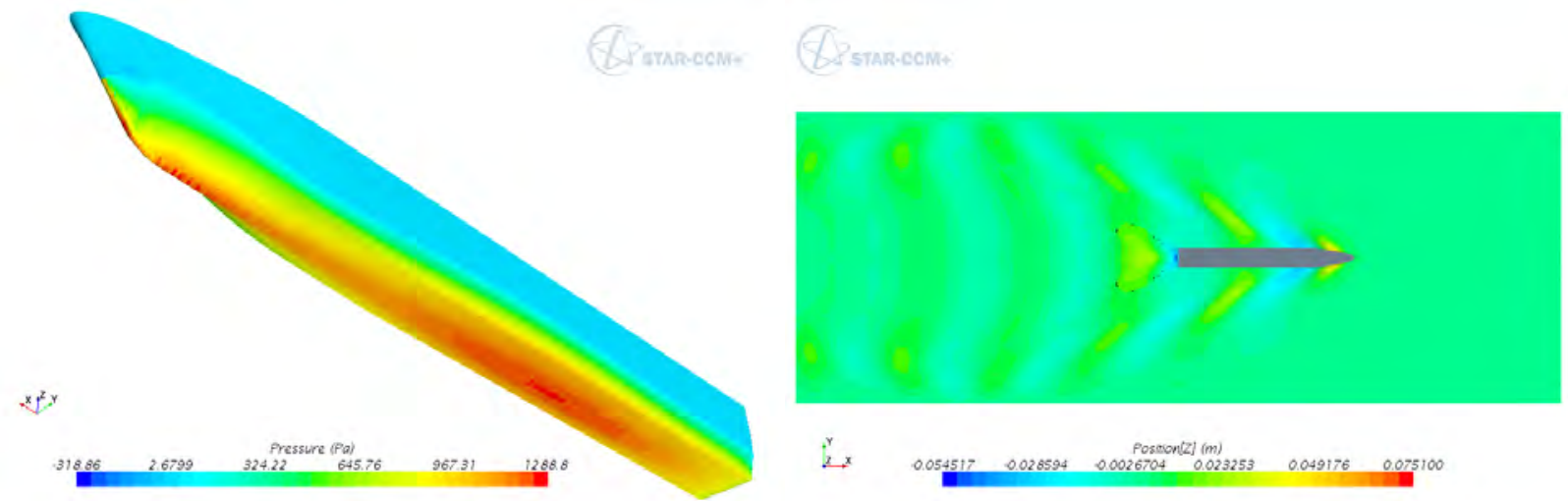

Fig. 162. Monocasco, $F r=0.322$, modelo libre
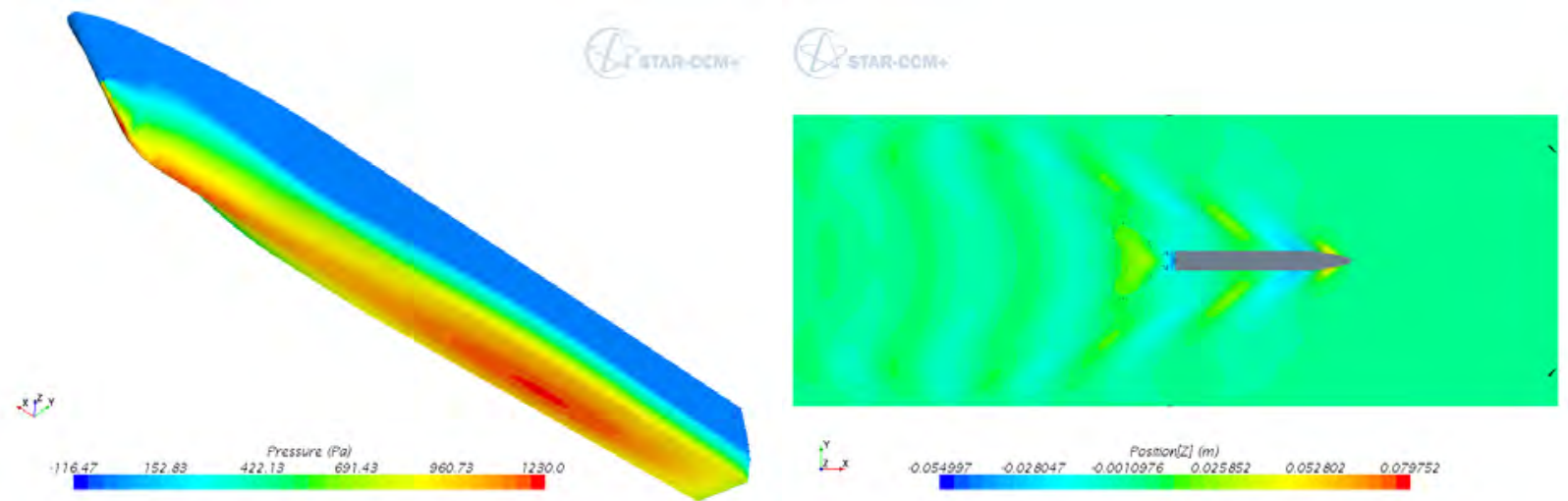

Fig. 163. Monocasco, $\mathrm{Fr}=0.322$, modelo fijo
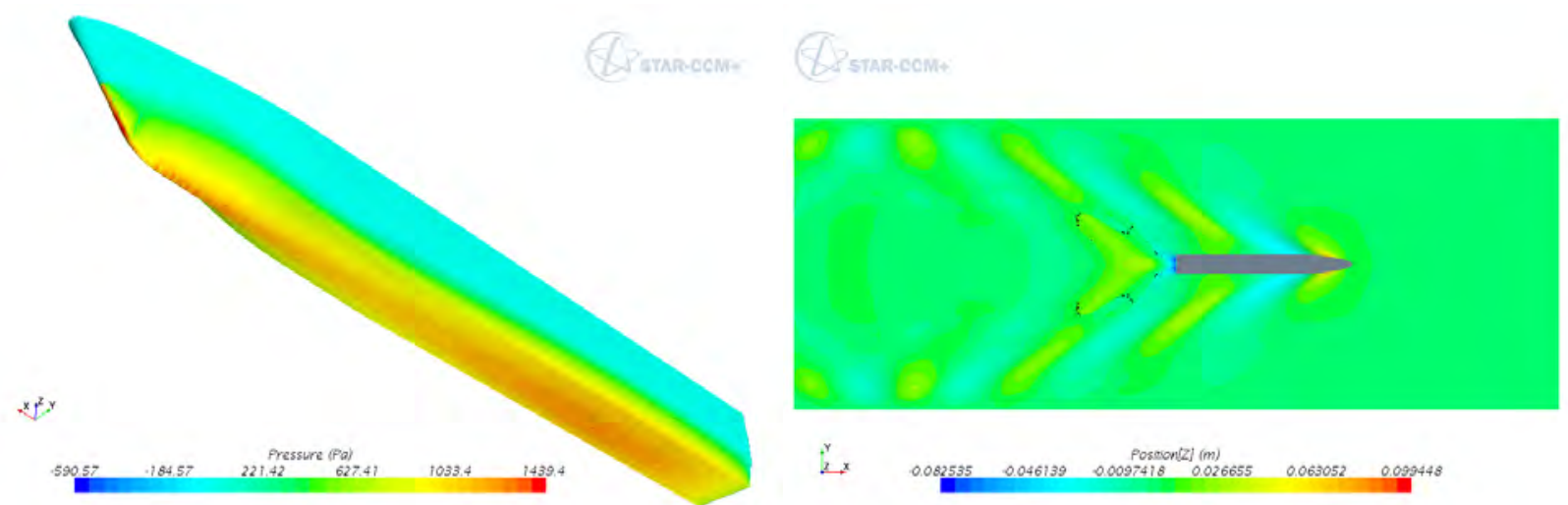

Fig. 164. Monocasco, $F r=0.375$, modelo libre
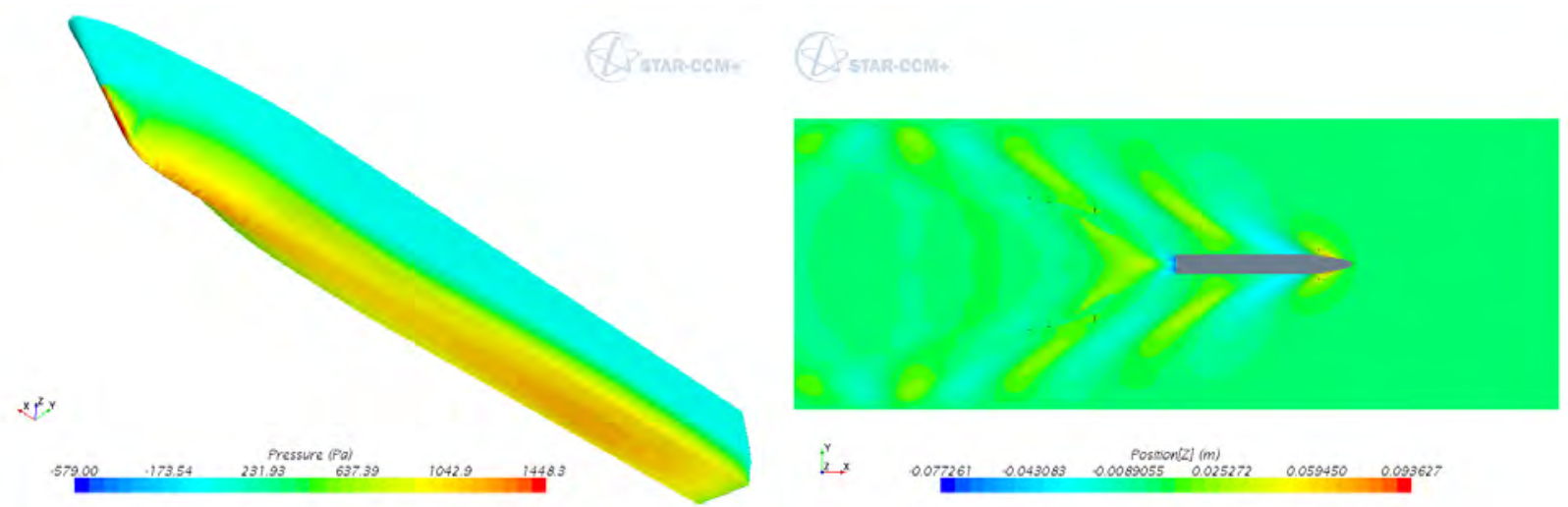

Fig. 165. Monocasco, $\mathrm{Fr}=\mathbf{0} .375$, modelo fijo

ANÁLISIS Y OPTIMIZACIÓN DE LA RESISTENCIA DE INTERFERENCIA EN MULTICASCOS 

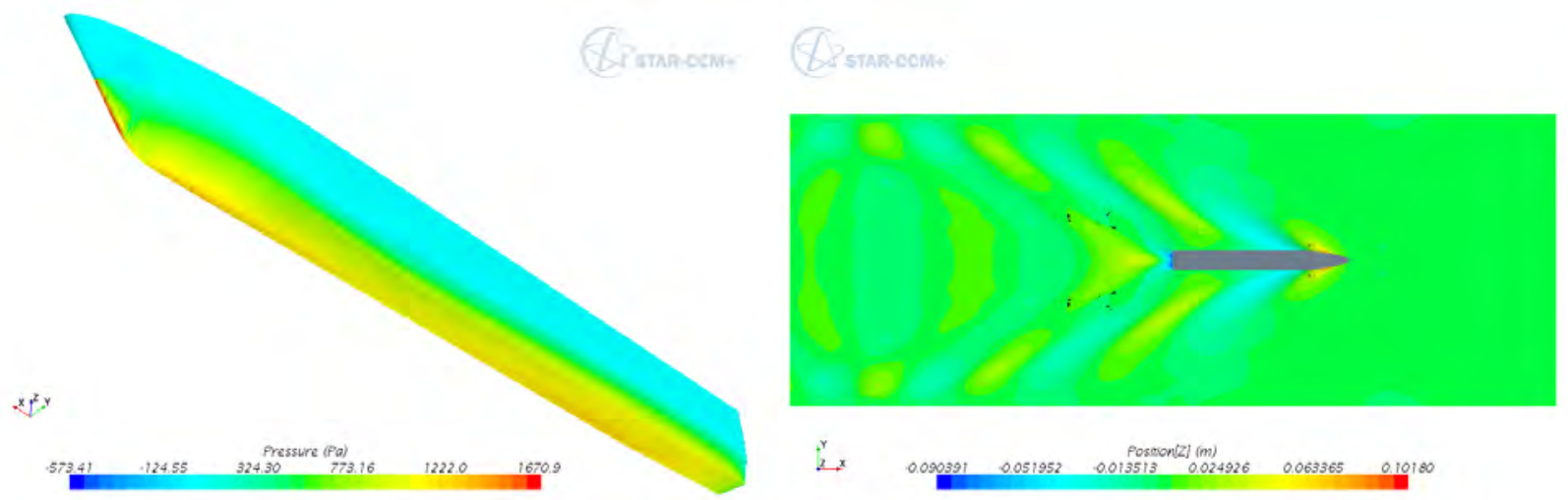

Fig. 166. Monocasco, $F r=0.398$, modelo libre
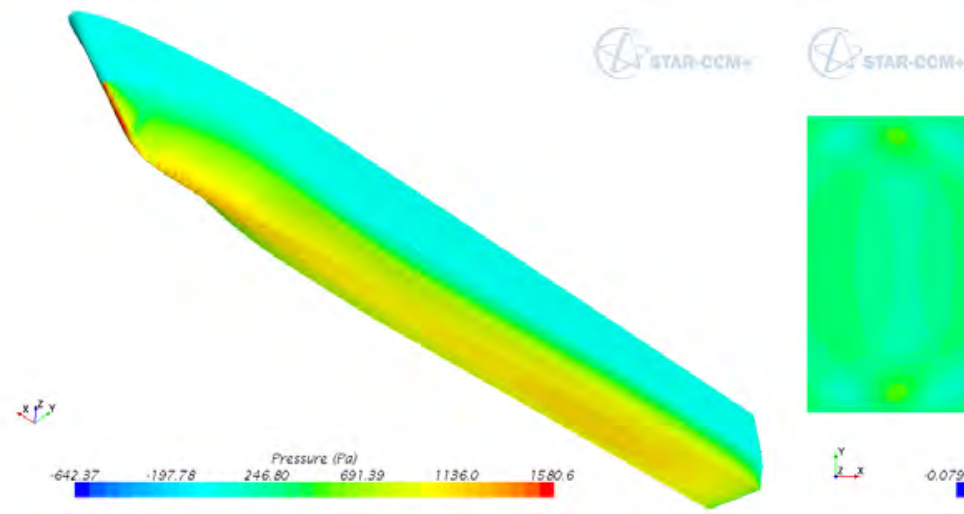

Fig. 167. Monocasco, $\mathrm{Fr}=0.398$, modelo fijo
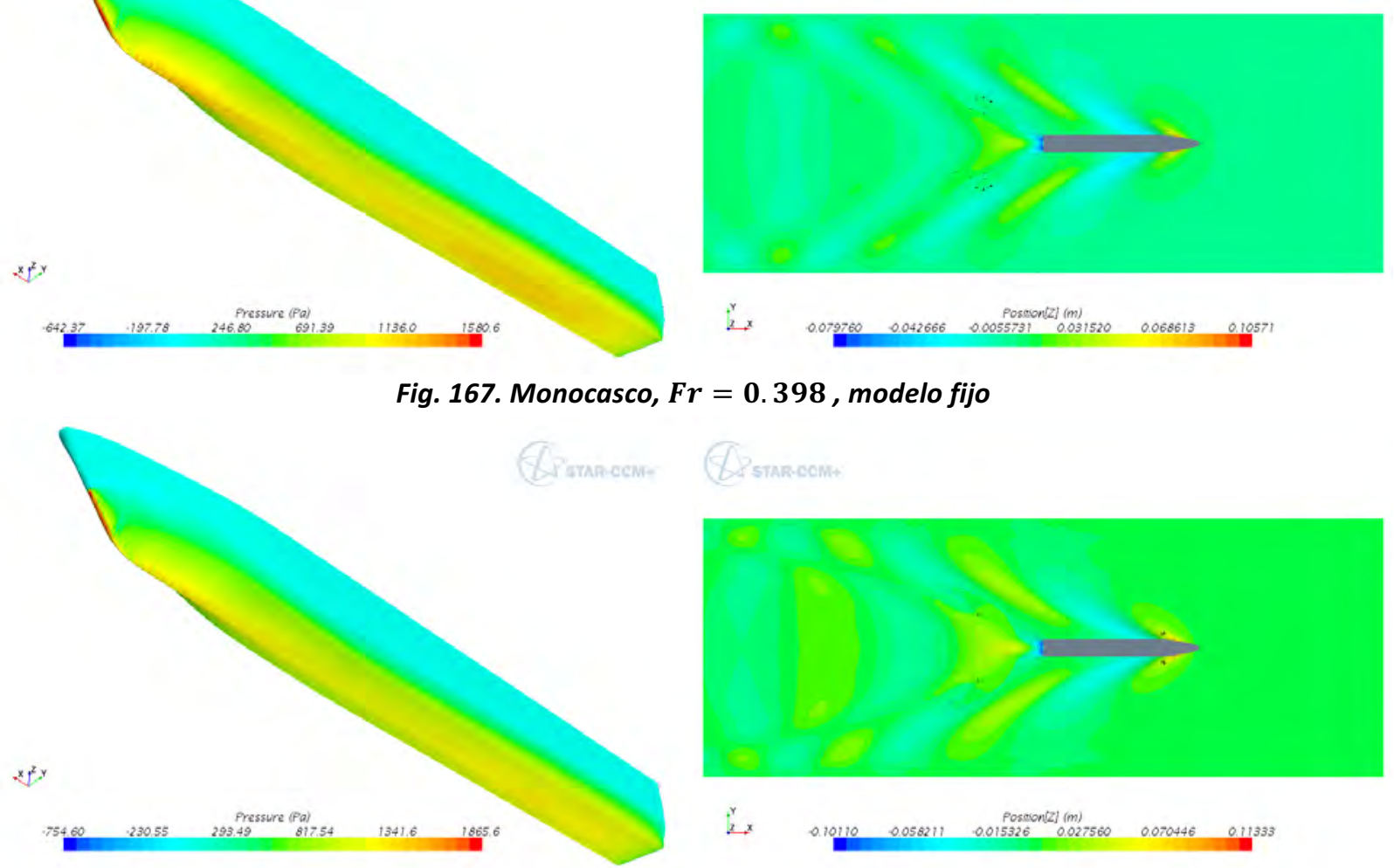

Fig. 168. Monocasco, $\mathrm{Fr}=0.430$, modelo libre
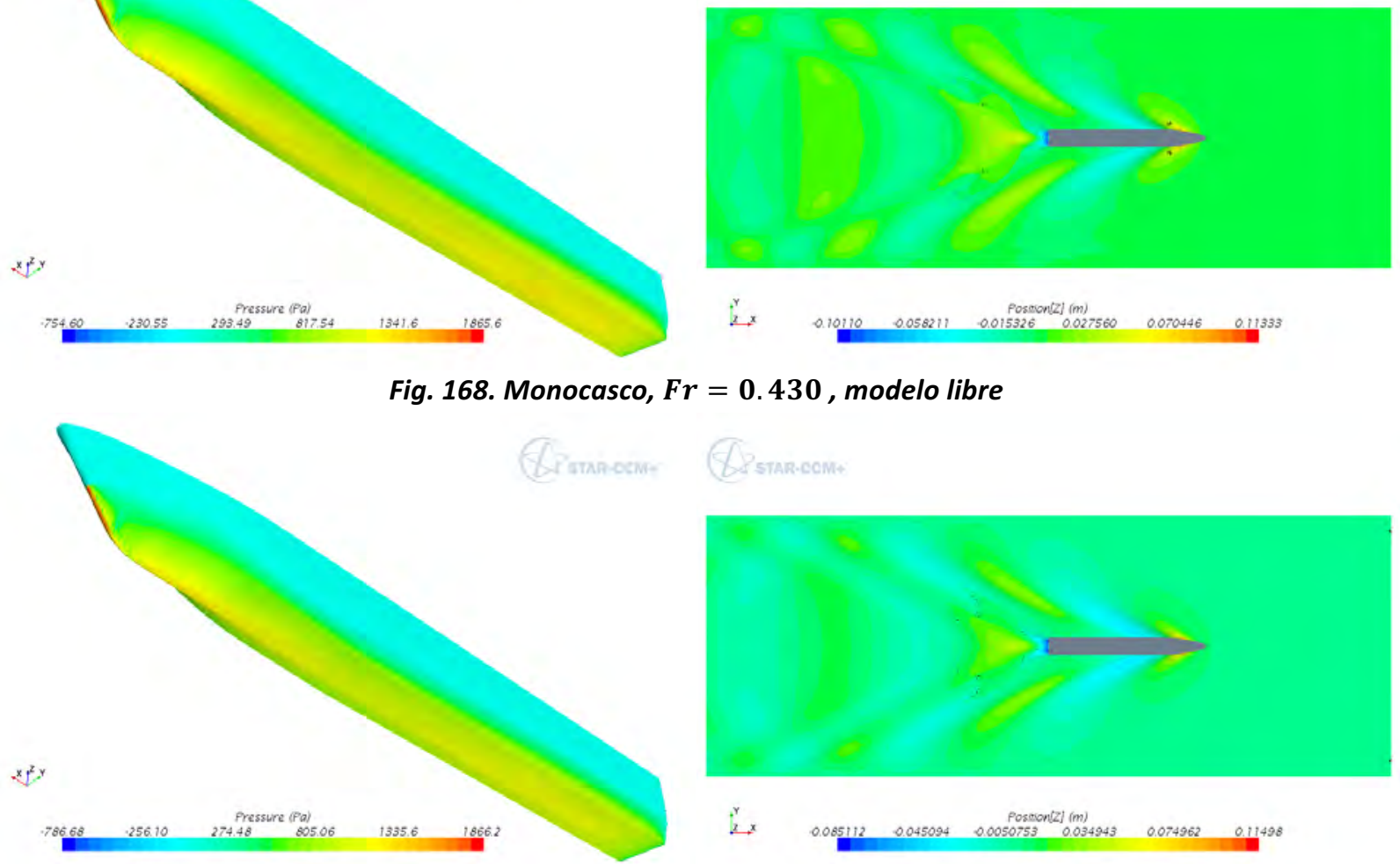

Fig. 169. Monocasco $\mathrm{Fr}=0.430$, modelo fijo ANÁLISIS Y OPTIMIZACIÓN DE LA RESISTENCIA DE INTERFERENCIA EN MULTICASCOS 


\section{C.3. Catamarán S60}

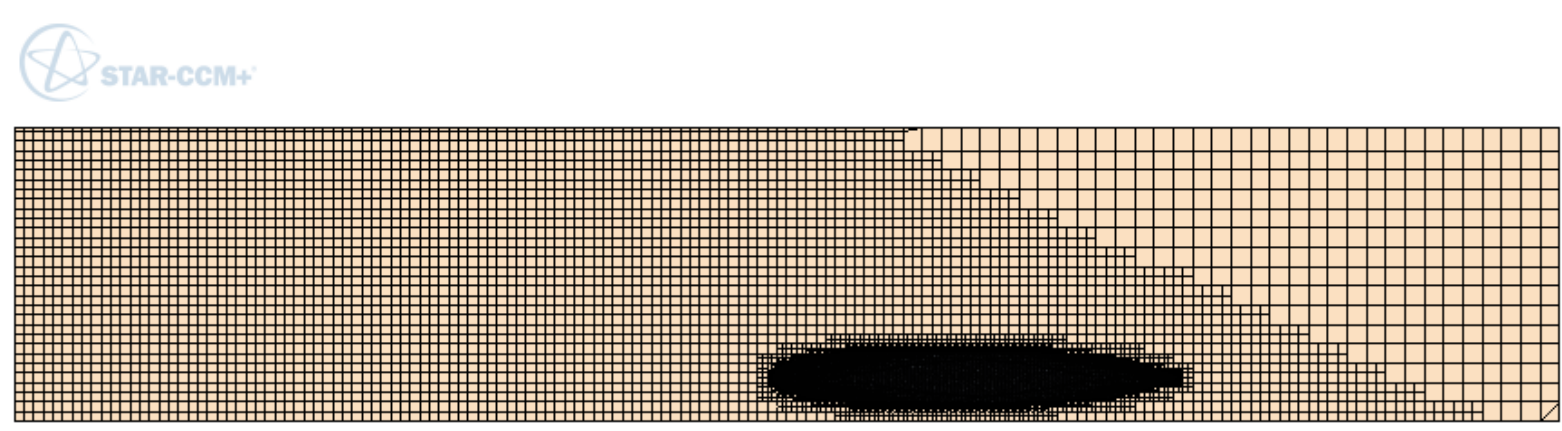

a) $s / L=0.226$

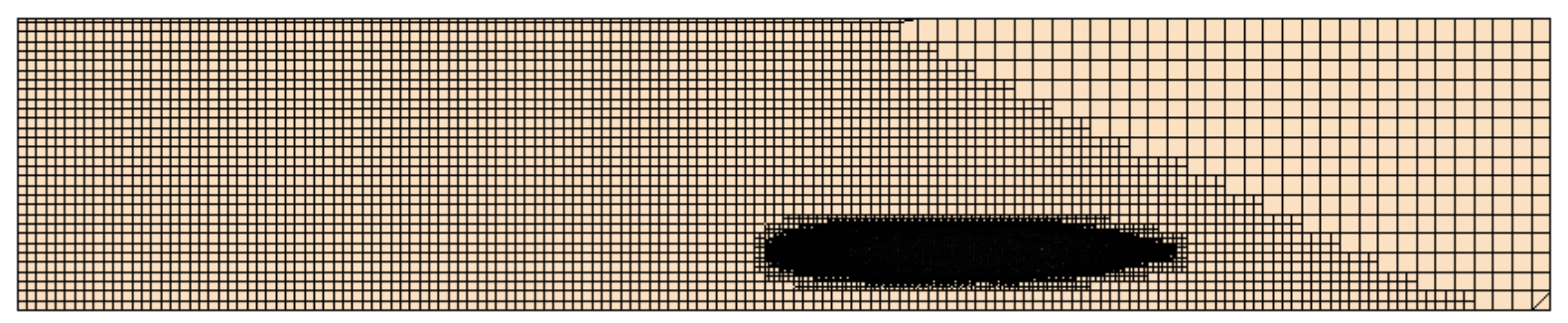

b) $s / L=0.307$

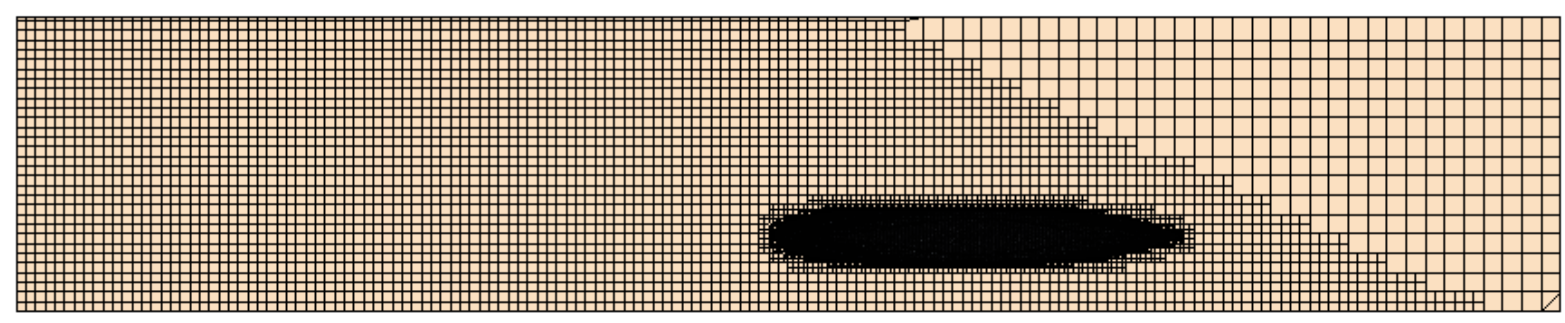

c) $s / L=0.388$

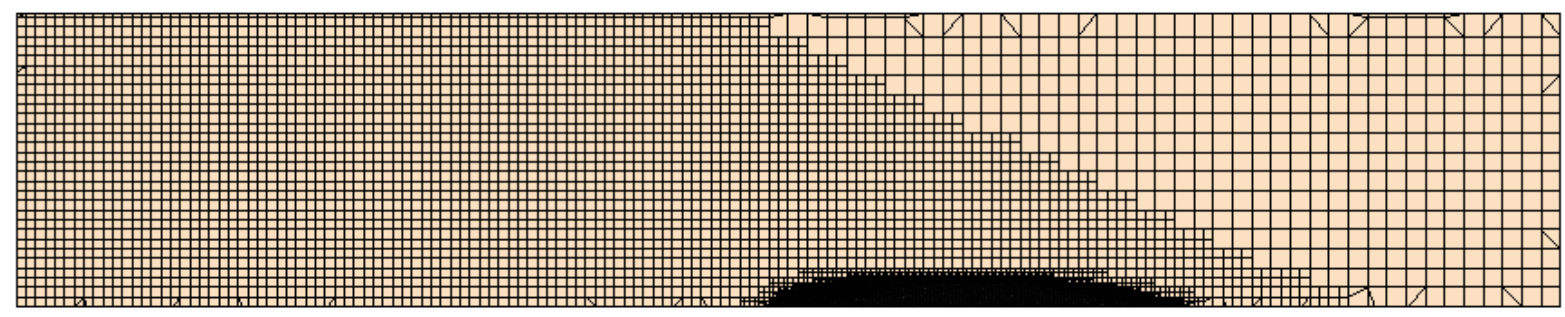

d) Monocasco

Fig. 170: Variaciones del mallado para cada separación en la superficie libre 

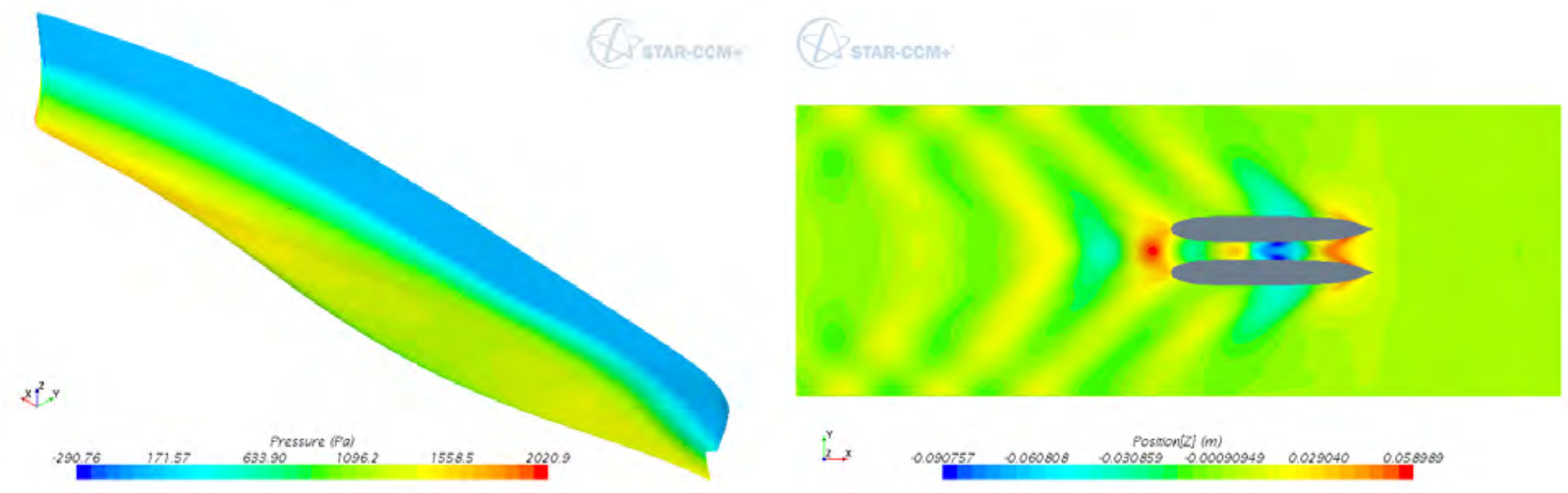

Fig. 171. $s / L=0.226 \quad F r=0.335$ modelo libre
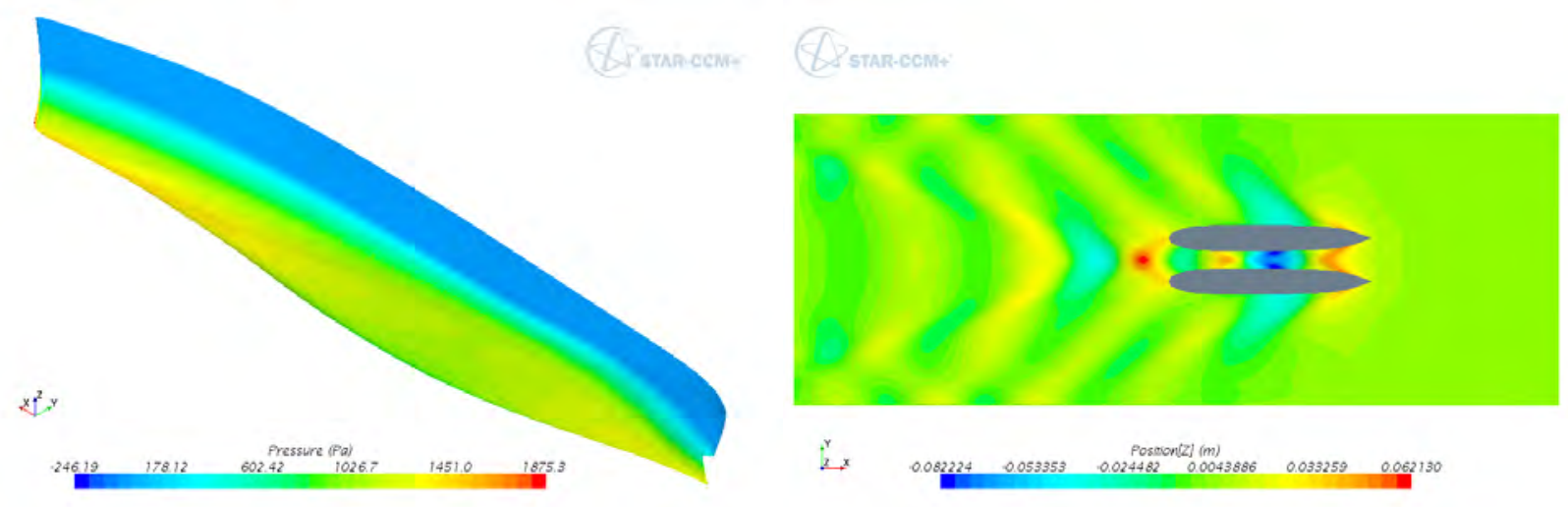

Fig. 172. $s / L=0.226 \quad F r=0.335$ modelo fijo
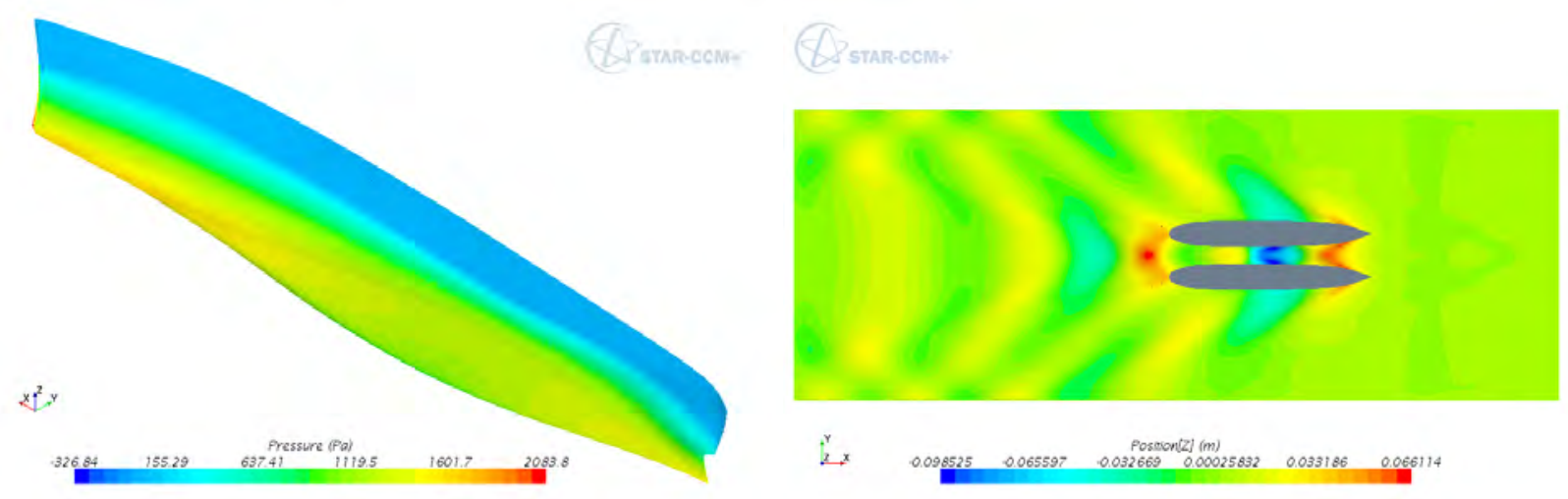

Fig. 173. $s / L=0.226 \quad F r=0.350$ modelo libre
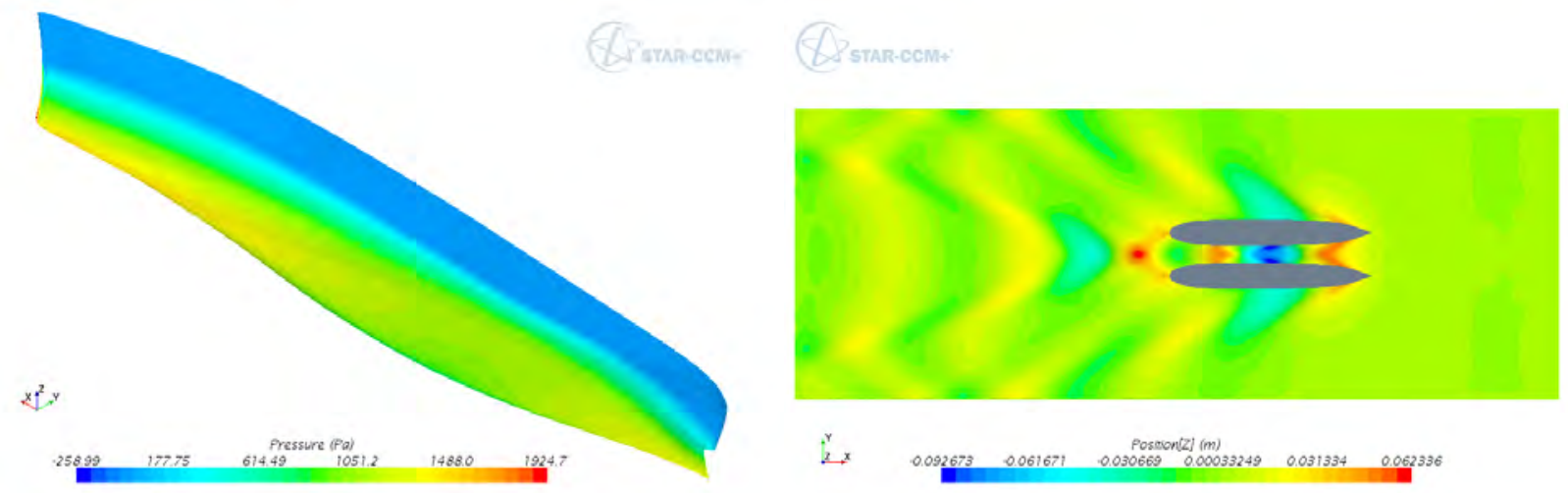

Fig. 174. $s / L=0.226 \quad F r=0.350$ modelo fijo ANÁLISIS Y OPTIMIZACIÓN DE LA RESISTENCIA DE INTERFERENCIA EN MULTICASCOS 

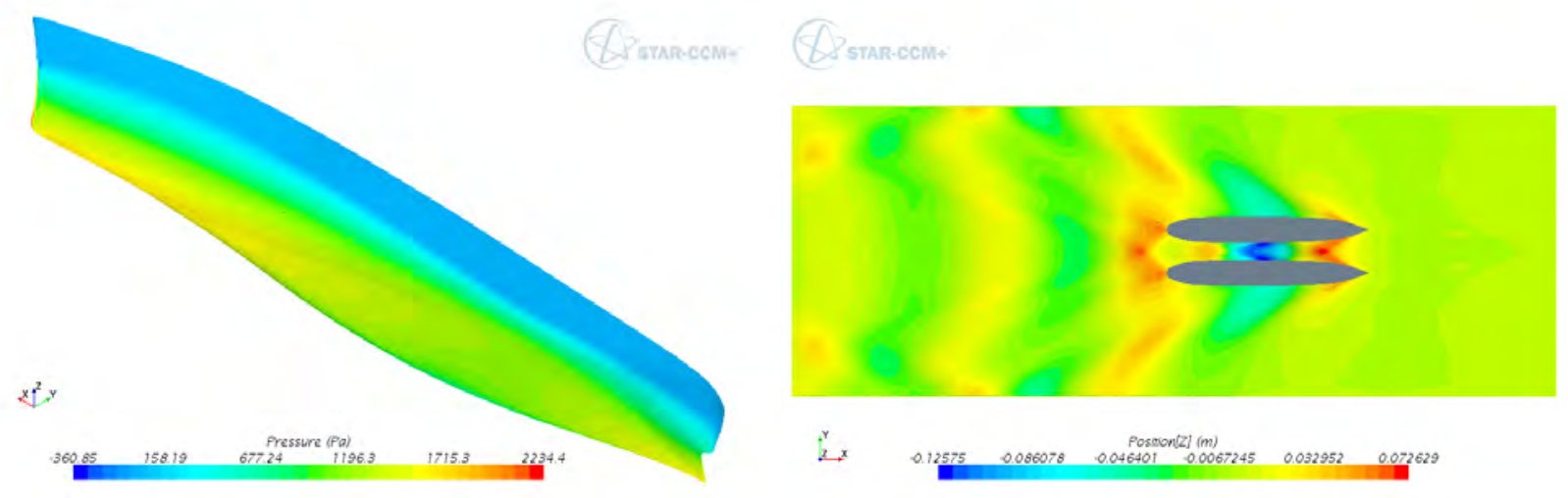

Fig. 175. $S / L=0.226 \quad F r=0.376$ modelo libre
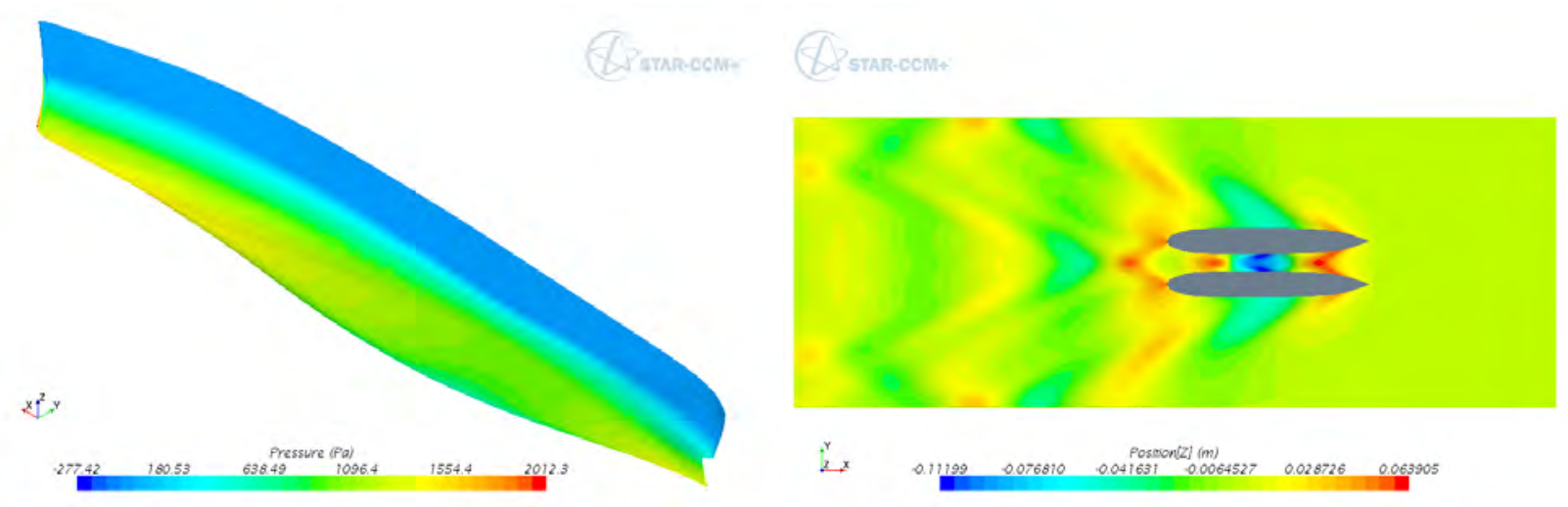

Fig. 176. $s / L=0.226$

$F r=0.376$ modelo fijo
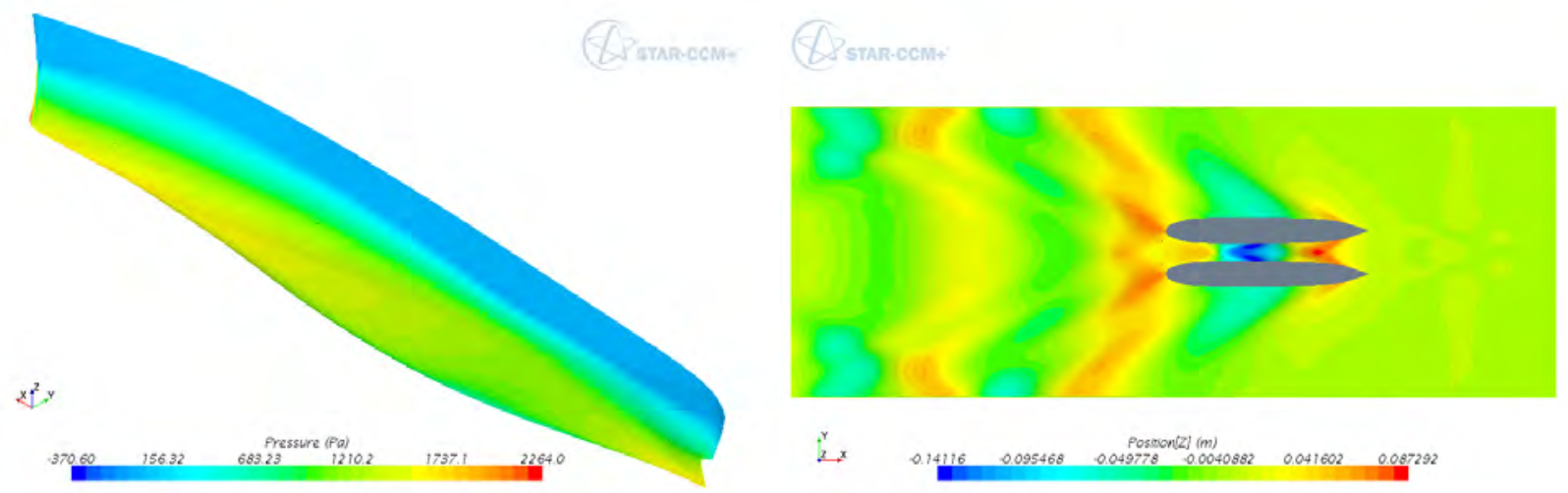

Fig. 177. $s / L=0.226 \quad F r=0.400$ modelo libre
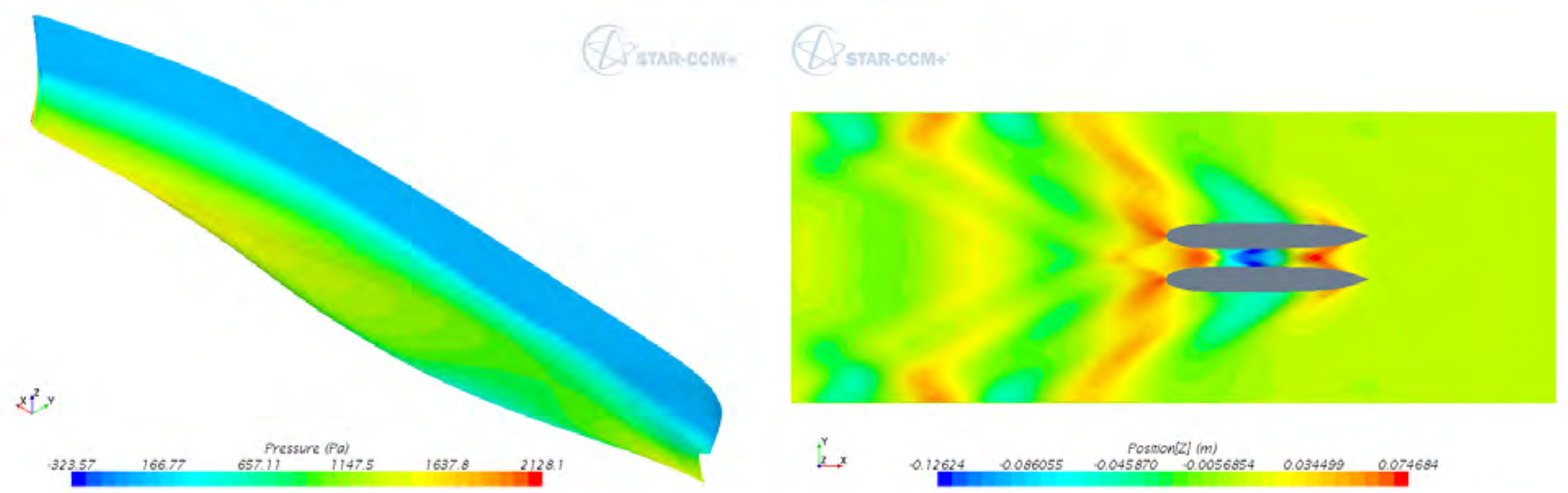

Fig. 178. $s / L=0.226 \quad F r=0.400$ modelo fijo ANÁLISIS Y OPTIMIZACIÓN DE LA RESISTENCIA DE INTERFERENCIA EN MULTICASCOS 

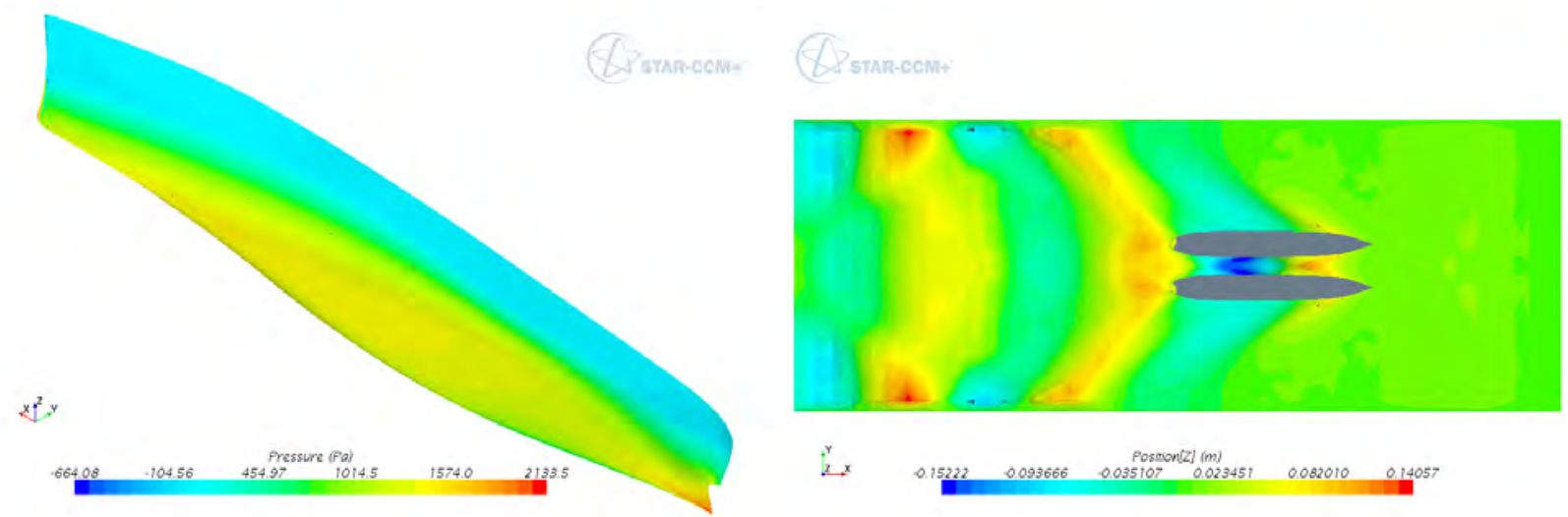

Fig. 179. $s / L=0.226 \quad F r=0.430$ modelo libre
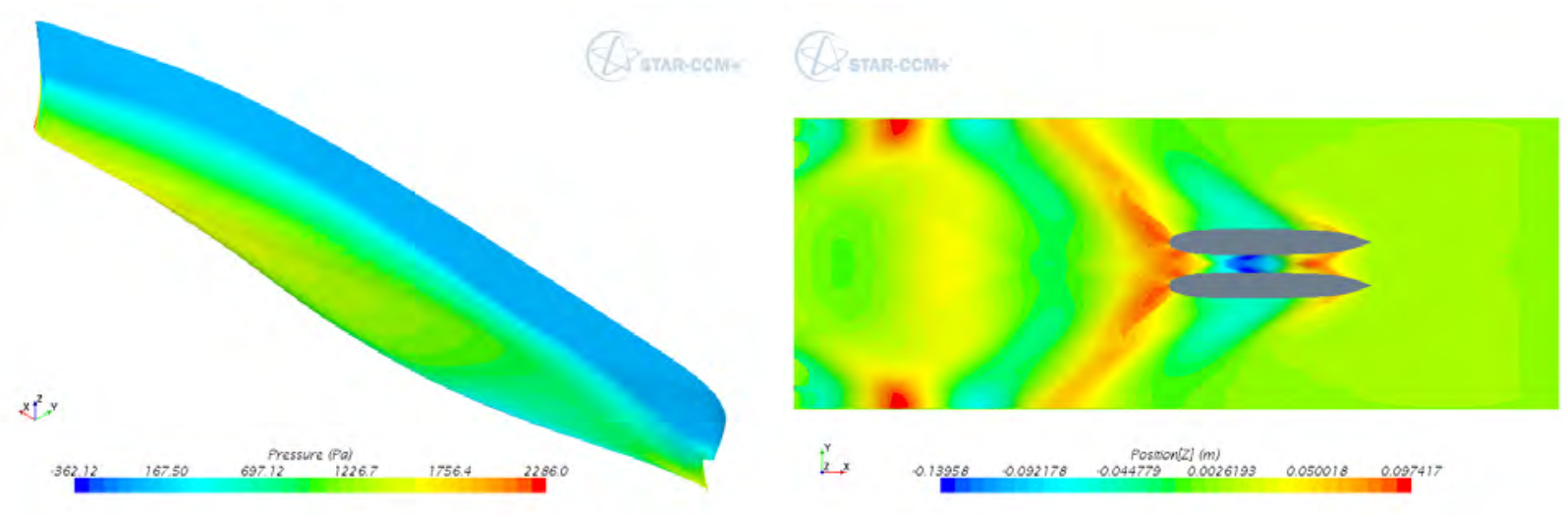

Fig. 180. $s / L=0.226 \quad F r=0.430$ modelo fijo 

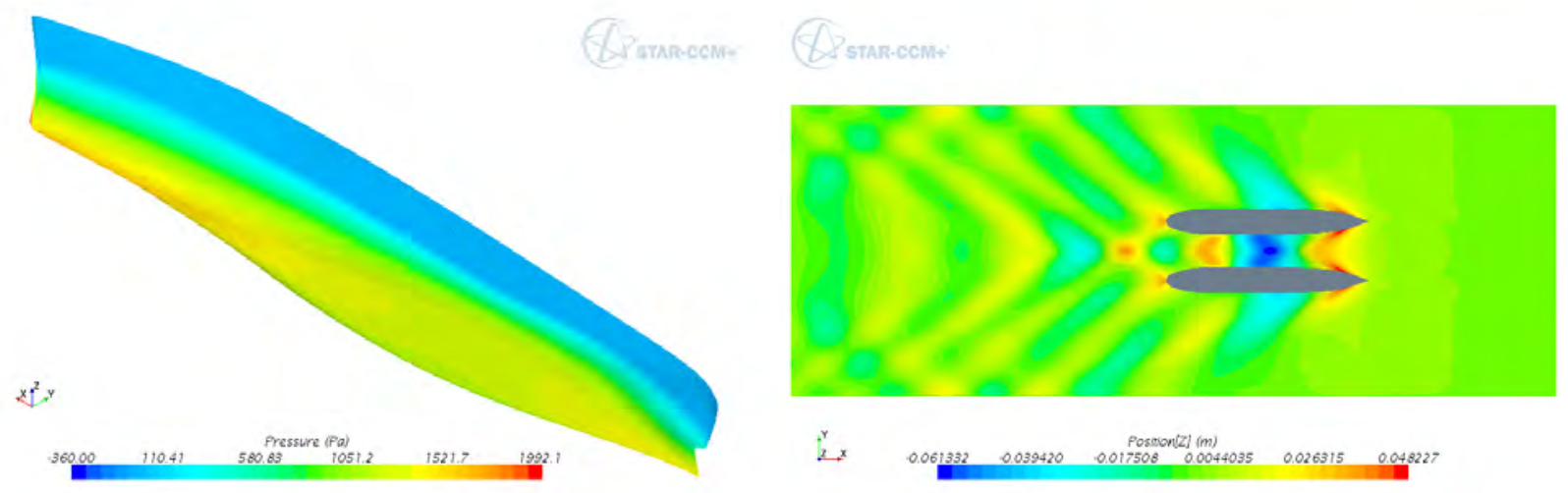

Fig. 181. $s / L=0.307 \quad F r=0.335$ modelo libre
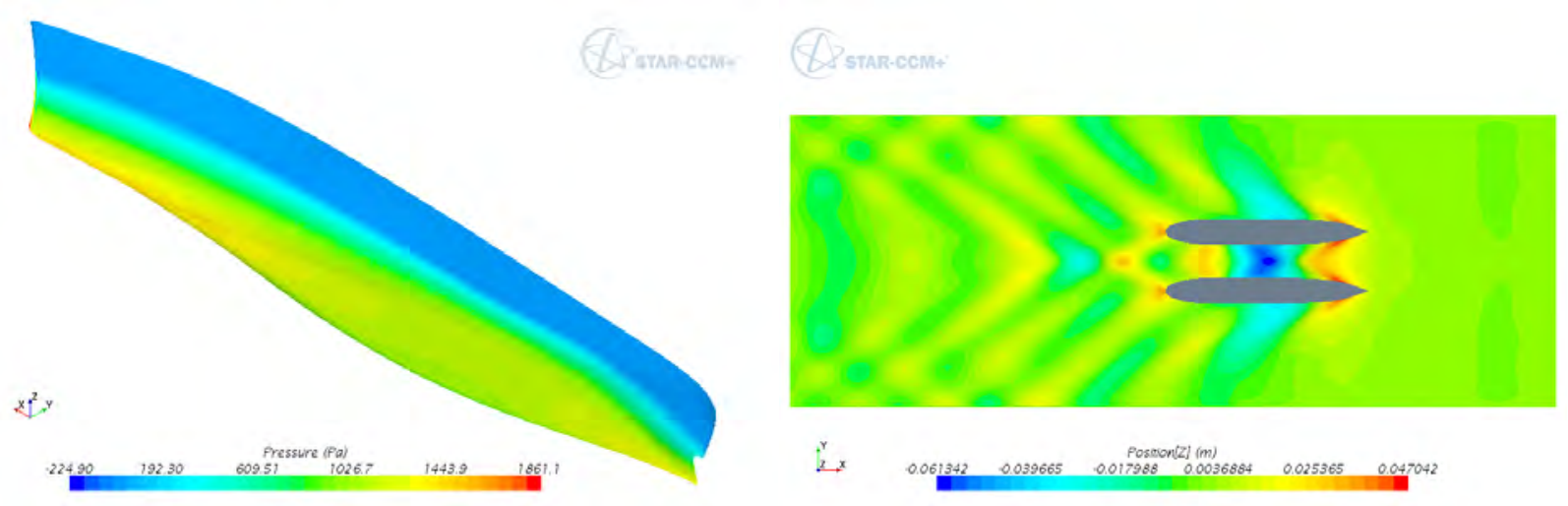

Fig. 182. $s / L=0.307 \quad F r=0.335$ modelo fijo
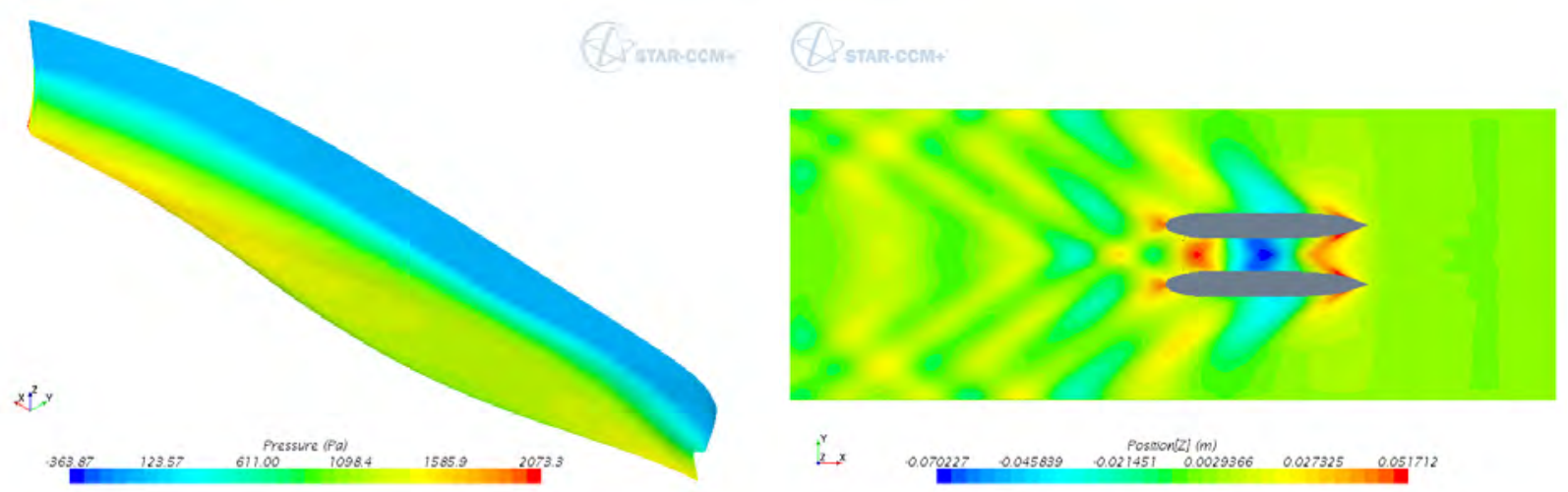

Fig. 183. $s / L=0.307 \quad F r=0.350$ modelo libre
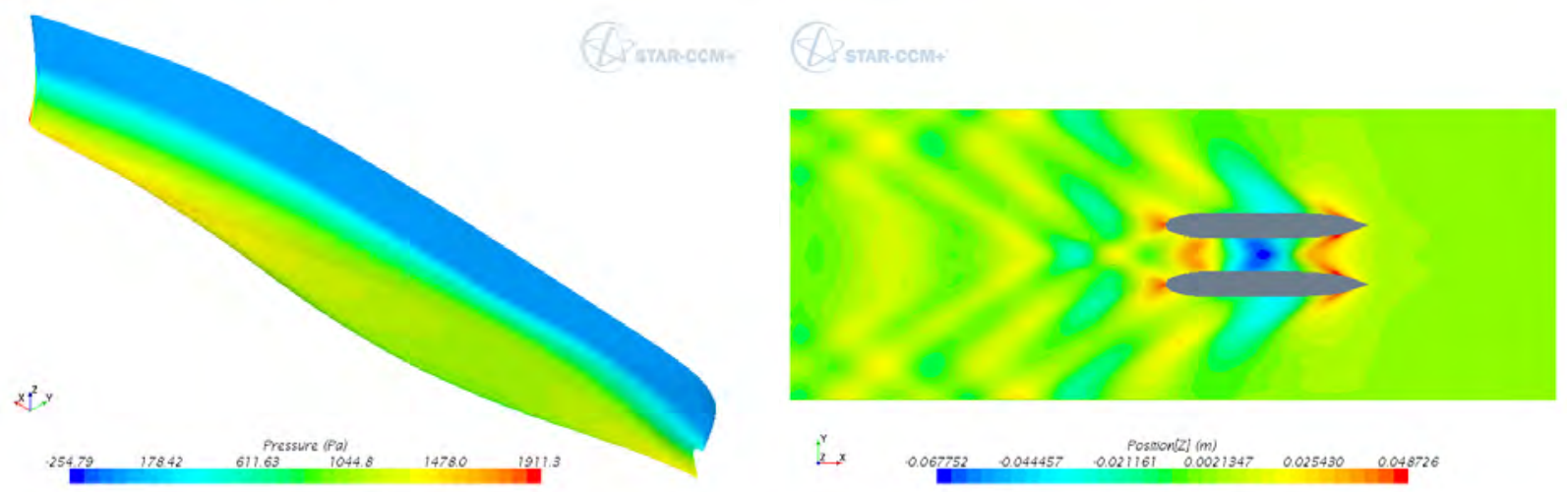

Fig. 184. $s / L=0.307 \quad F r=0.350$ modelo fijo ANÁLISIS Y OPTIMIZACIÓN DE LA RESISTENCIA DE INTERFERENCIA EN MULTICASCOS 

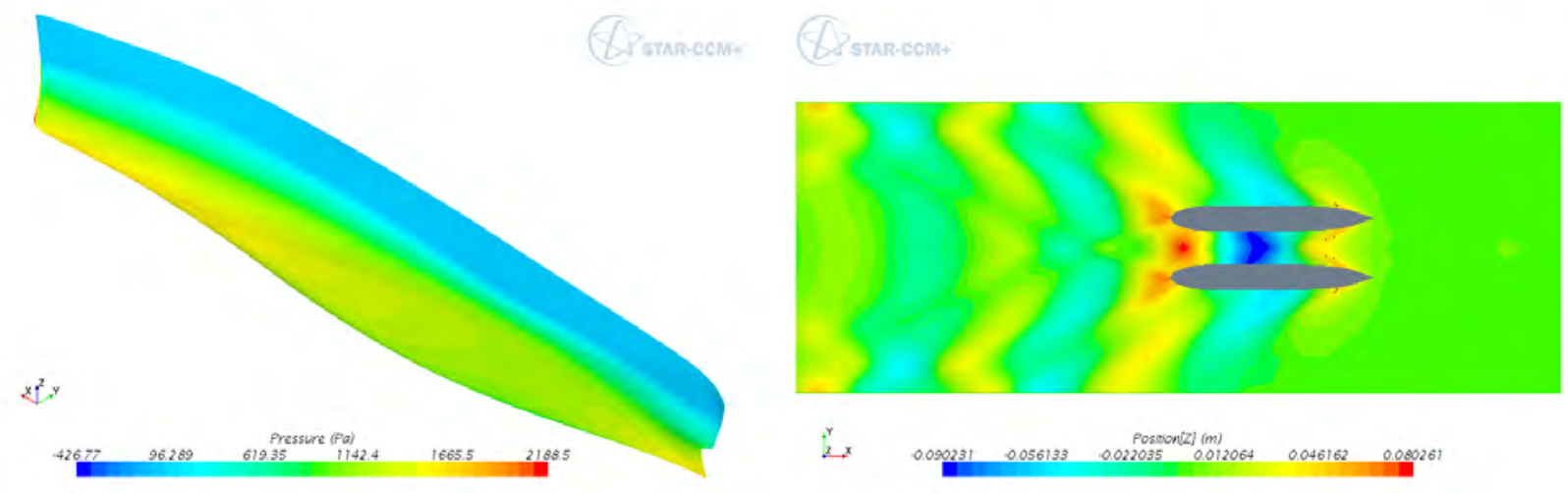

Fig. 185. $S / L=0.307 \quad F r=0.376$ modelo libre
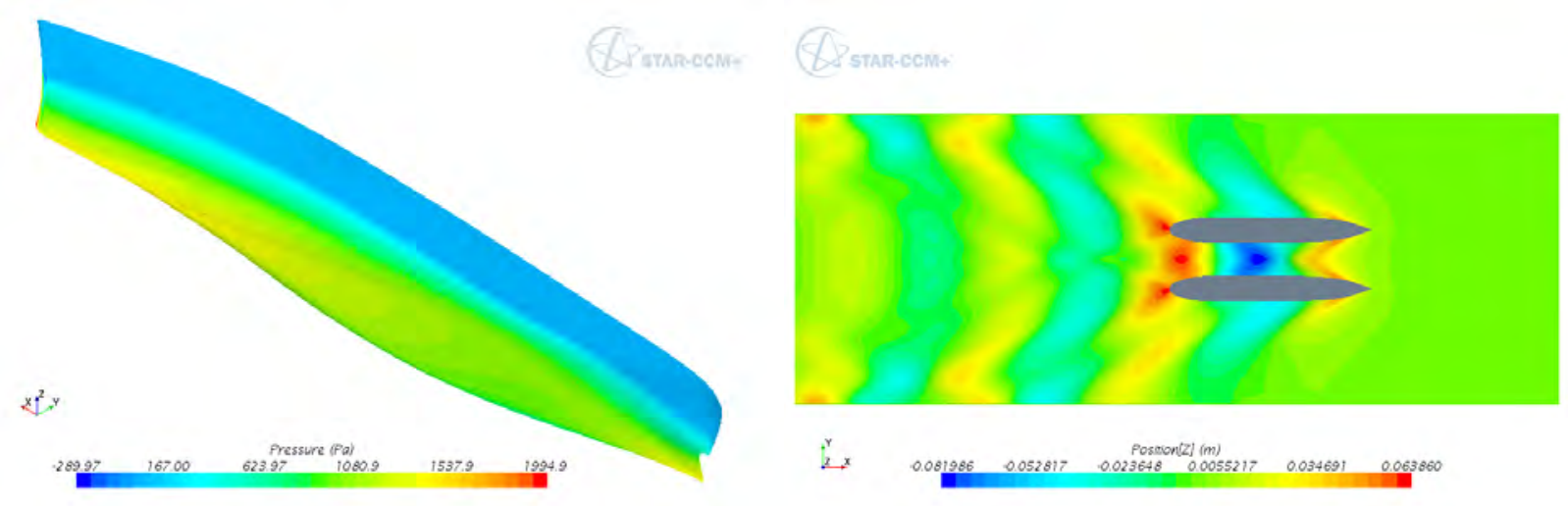

Fig. 186. $s / L=0.307 \quad F r=0.376$ modelo fijo
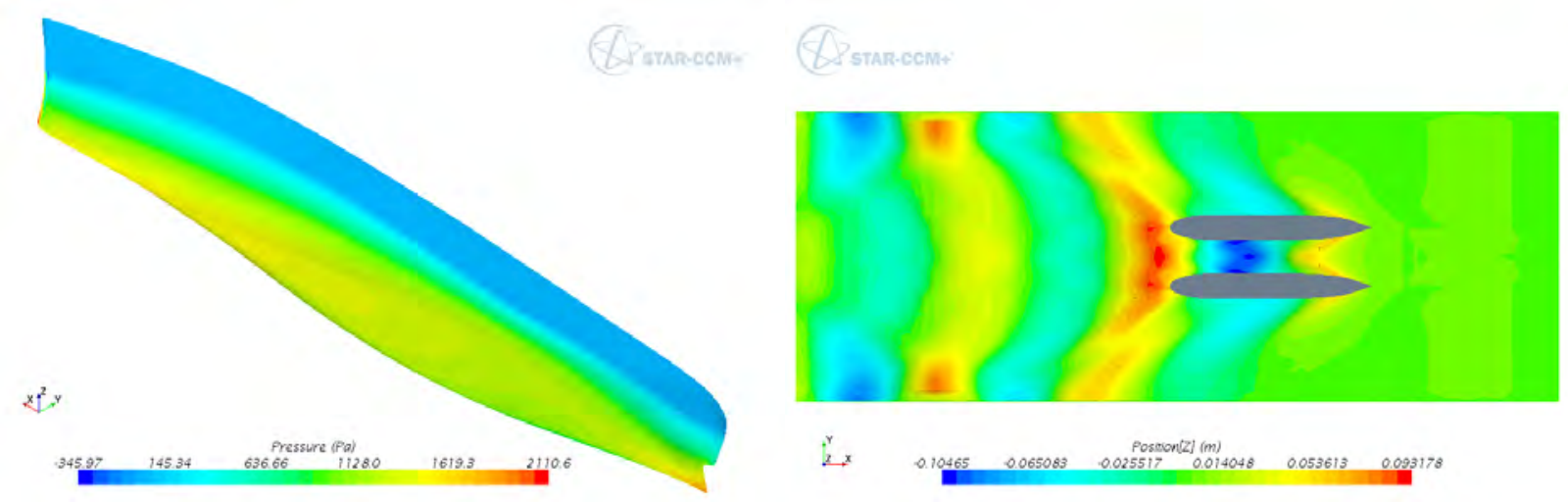

Fig. 187. $s / L=0.307 \quad F r=0.400$ modelo libre
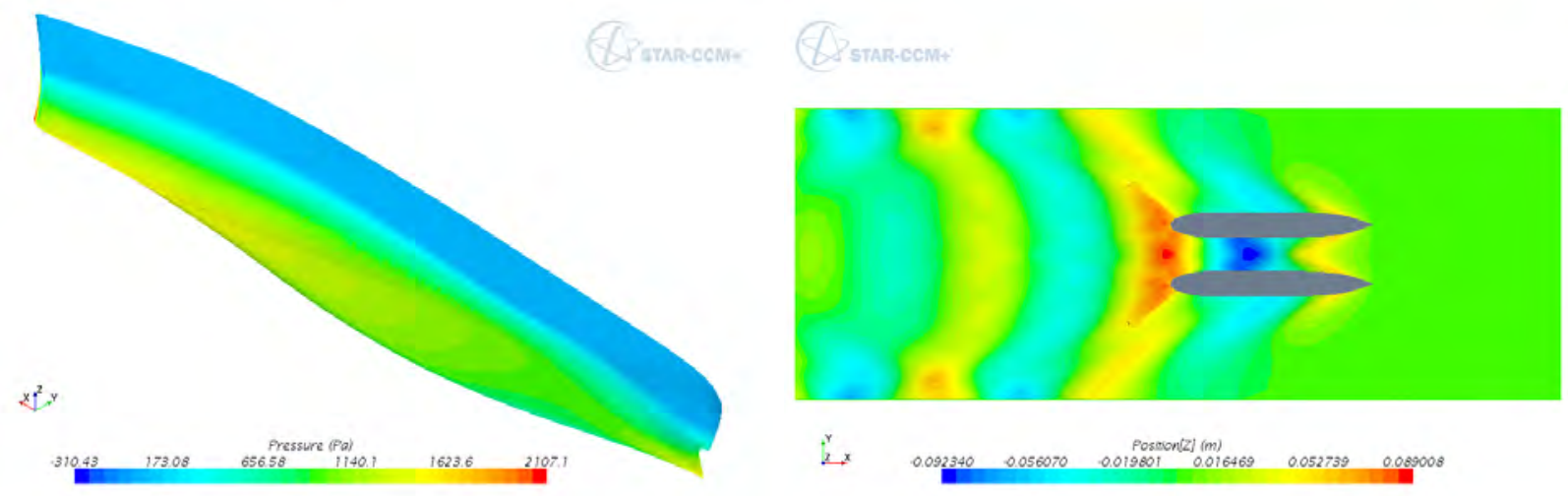

Fig. 188. $s / L=0.307 \quad F r=0.400$ modelo fijo ANÁLISIS Y OPTIMIZACIÓN DE LA RESISTENCIA DE INTERFERENCIA EN MULTICASCOS 

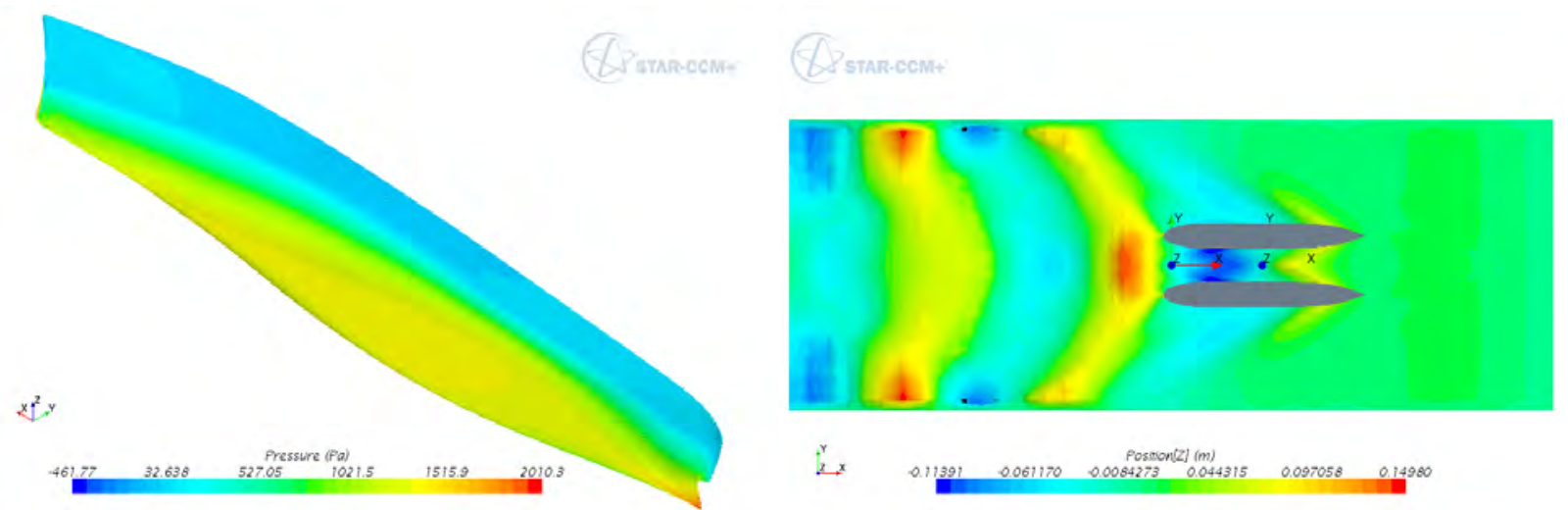

Fig. 189. $s / L=0.307 \quad F r=0.430$ modelo libre
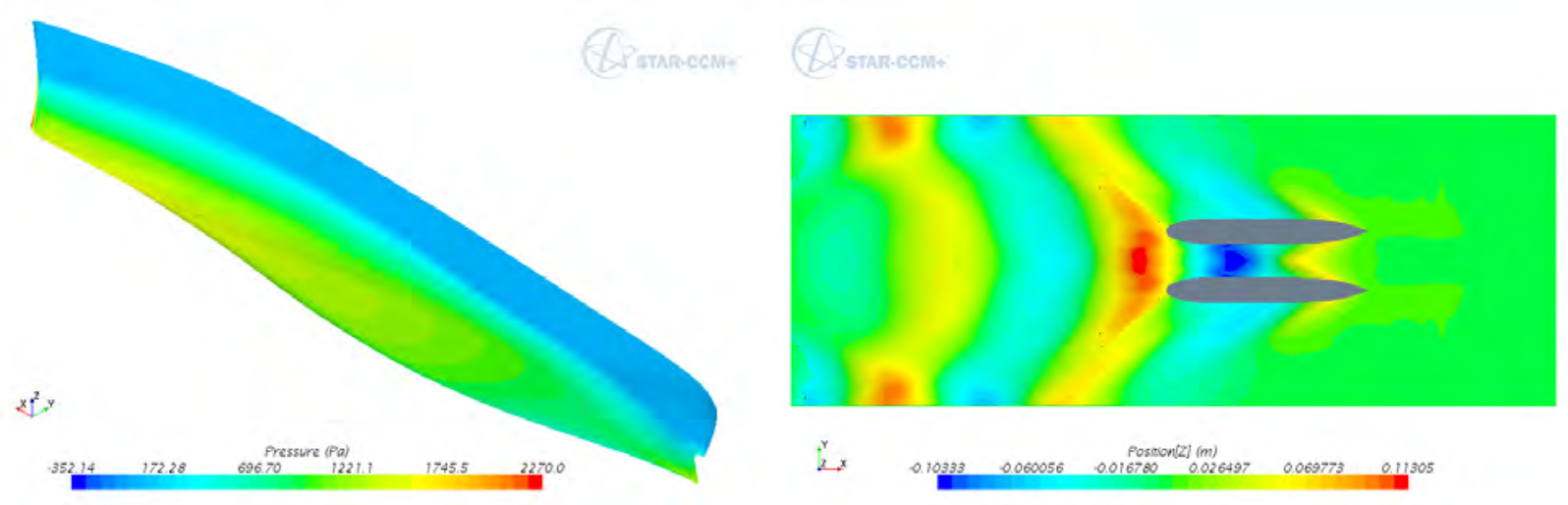

Fig. 190. $s / L=0.307 \quad F r=0.430$ modelo fijo 

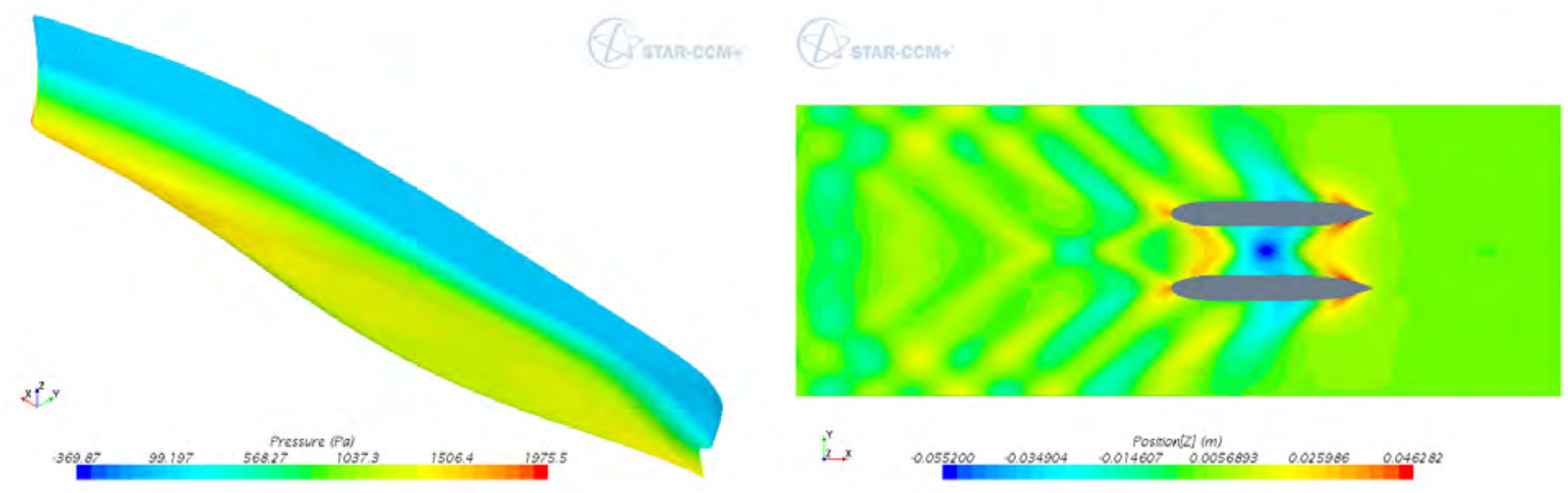

Fig. 191. $s / L=0.388 \quad F r=0.335$ modelo libre
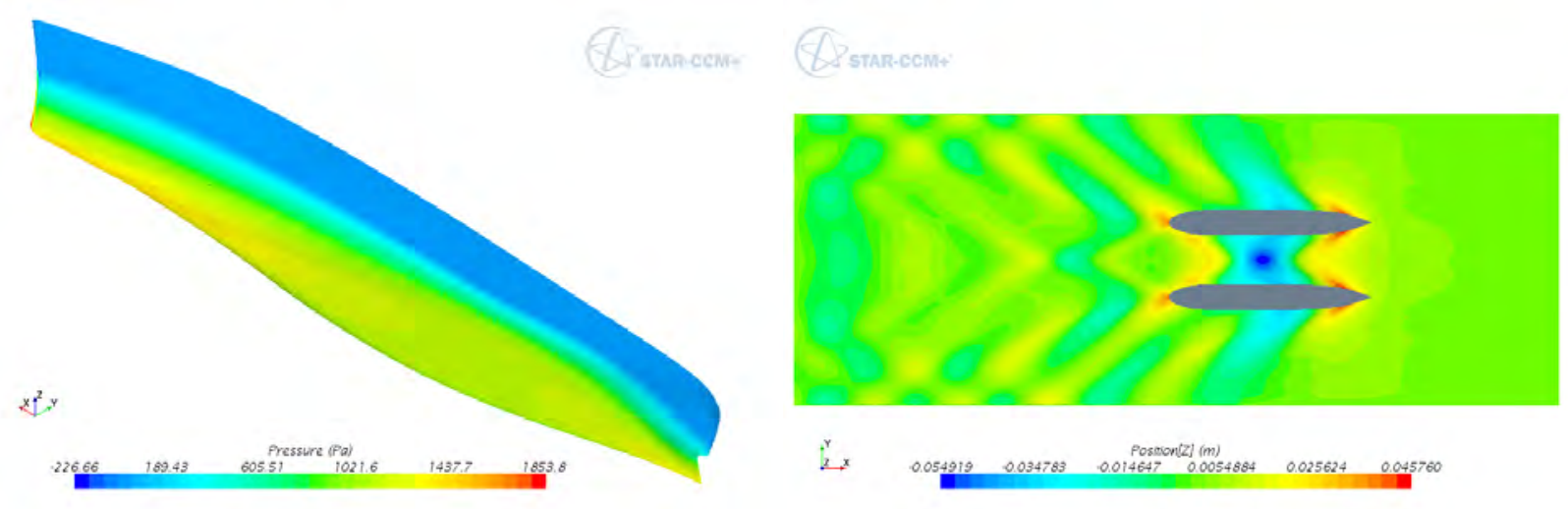

Fig. 192. $s / L=0.388 \quad F r=0.335$ modelo fijo
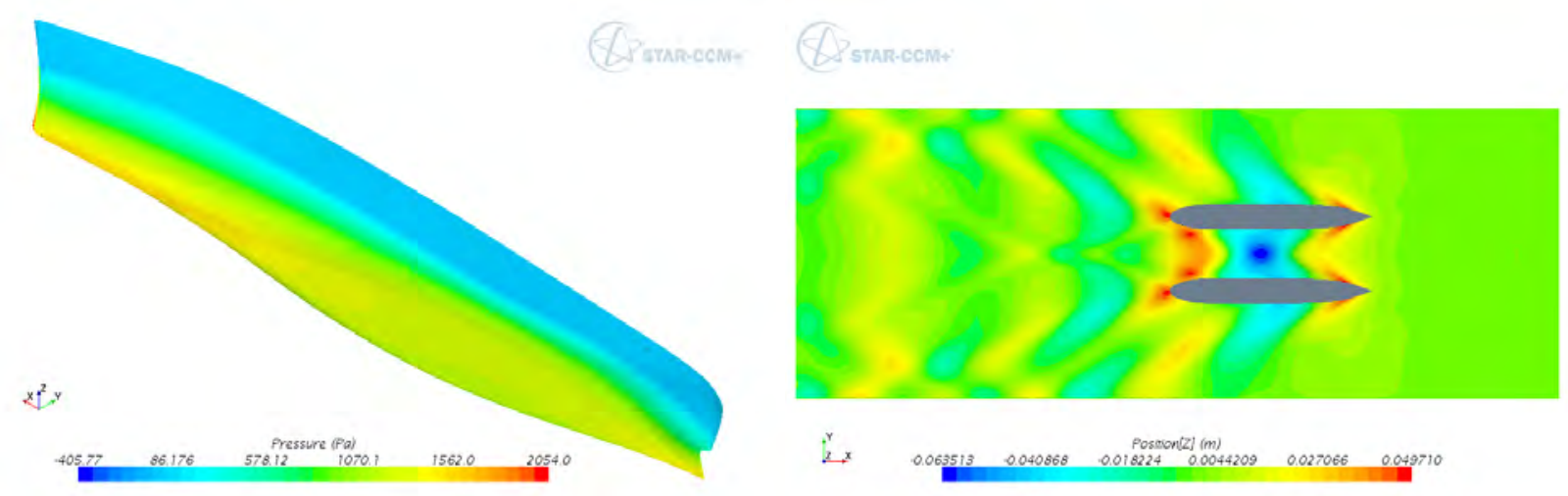

Fig. 193. $s / L=0.388 \quad F r=0.350$ modelo libre
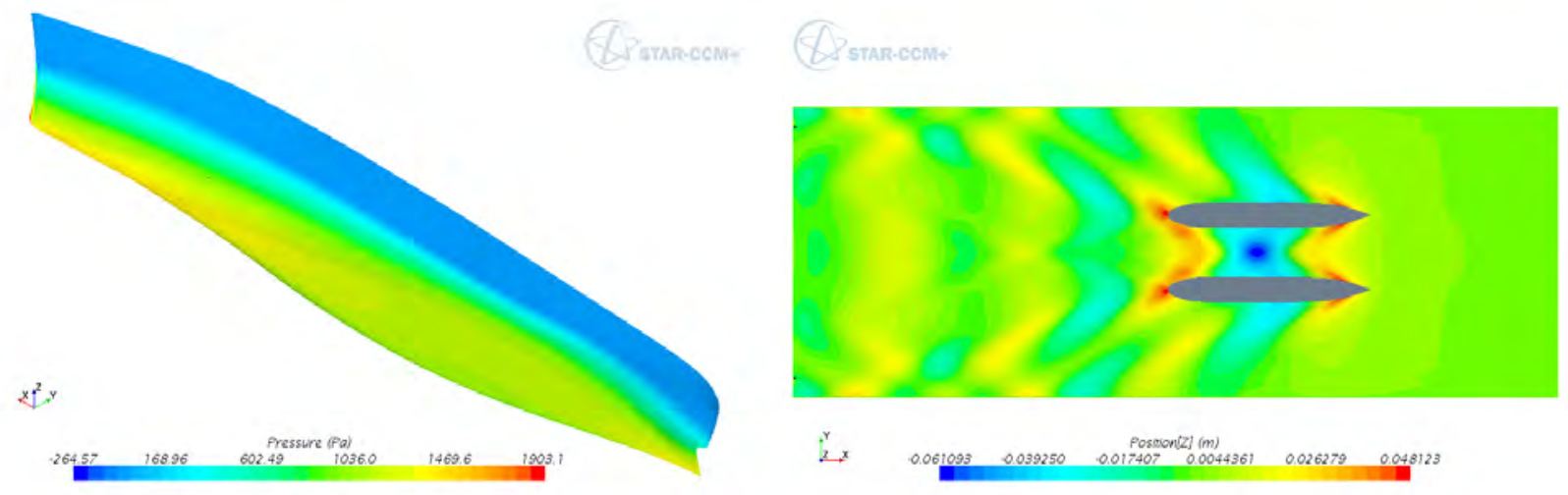

Fig. 194. $s / L=0.388 F r=0.350$ modelo fijo ANÁLISIS Y OPTIMIZACIÓN DE LA RESISTENCIA DE INTERFERENCIA EN MULTICASCOS 

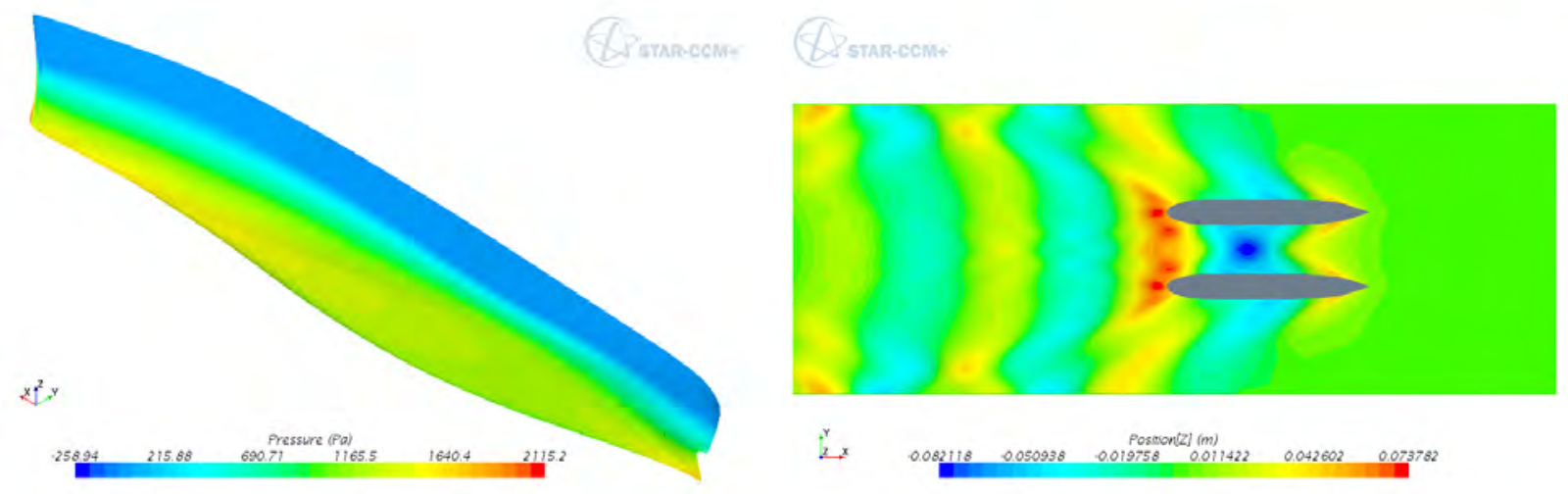

Fig. 195. $S / L=0.388 \quad F r=0.376$ modelo libre
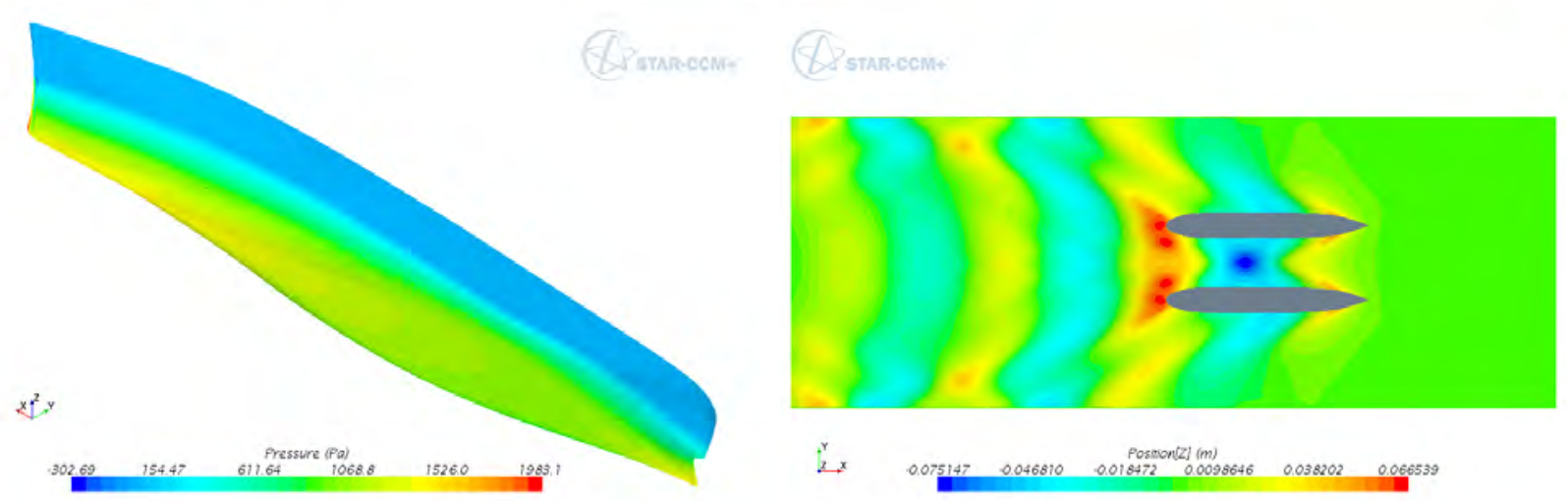

Fig. 196. $s / L=0.388$

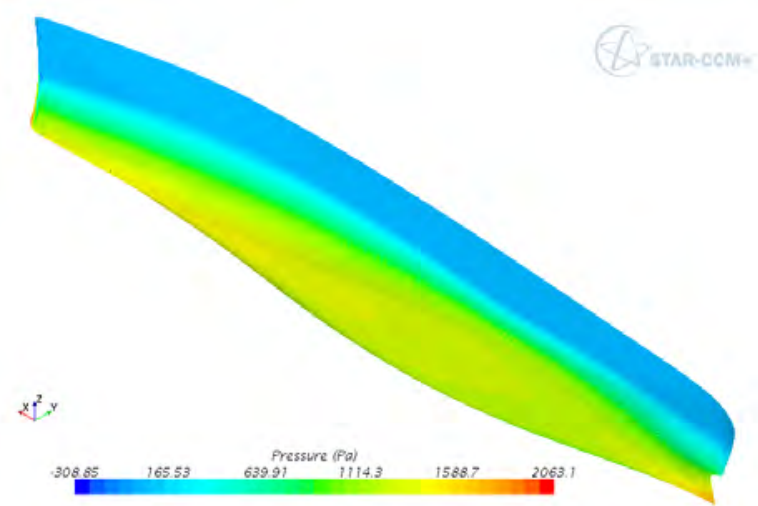

$F r=0.376$ modelo fijo

Fig. 197. $s / L=0.388$

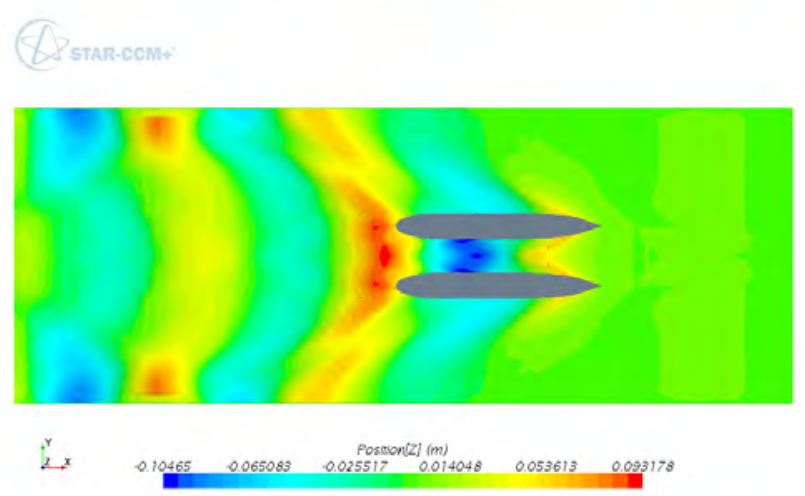

Fr $=0.400$ modelo libre
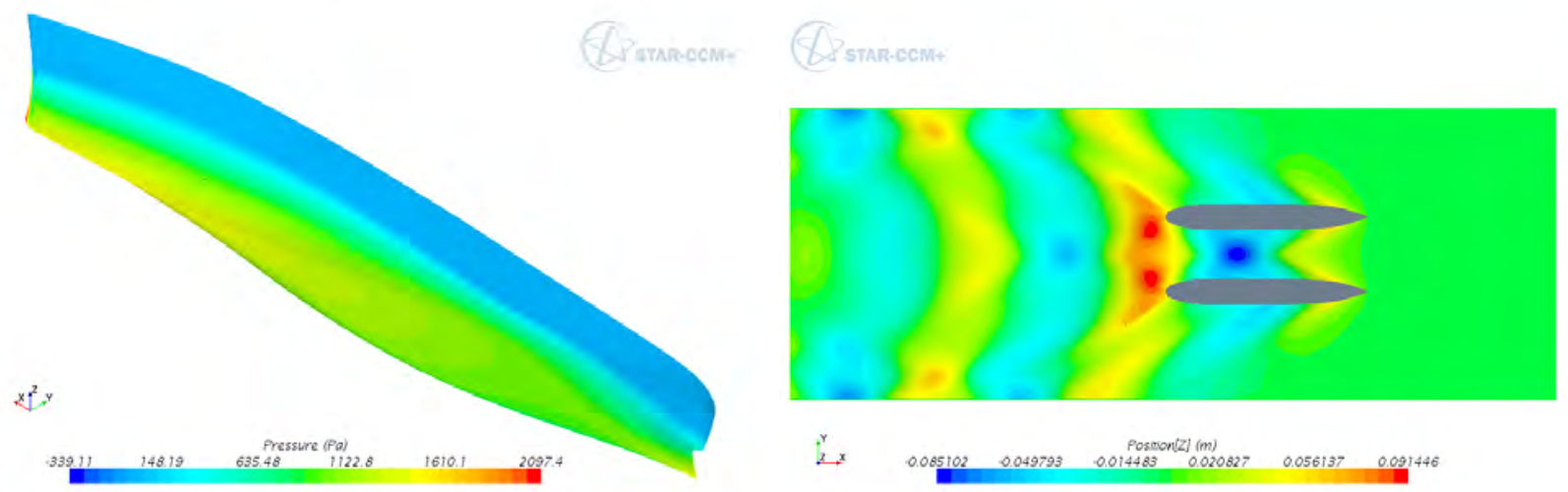

Fig. 198. $s / L=0.388 F r=0.400$ modelo fijo ANÁLISIS Y OPTIMIZACIÓN DE LA RESISTENCIA DE INTERFERENCIA EN MULTICASCOS 

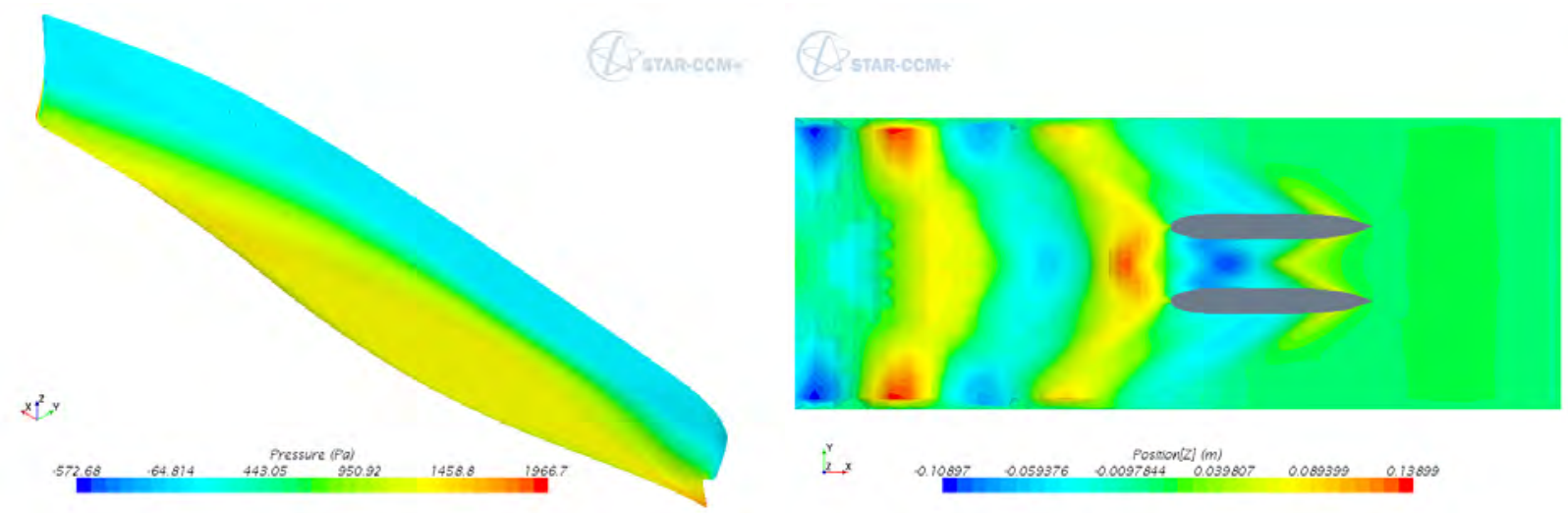

Fig. 199. $s / L=0.388 F r=0.430$ modelo libre
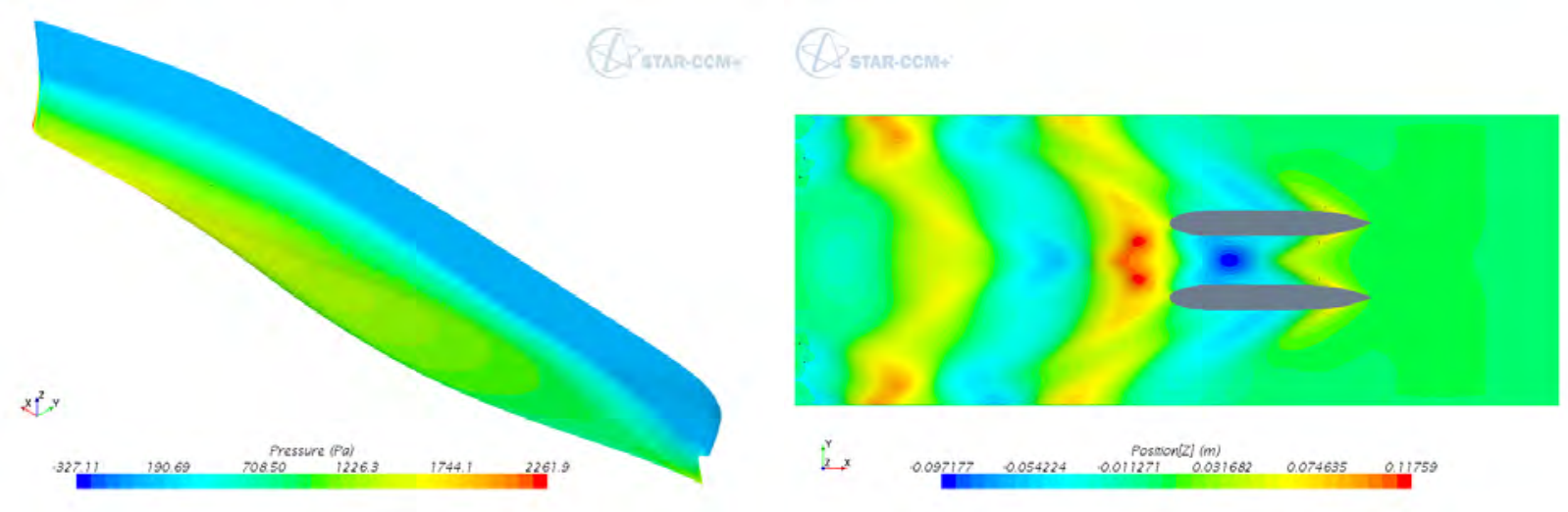

Fig. 200. $s / L=0.388 F r=0.430$ modelo fijo 

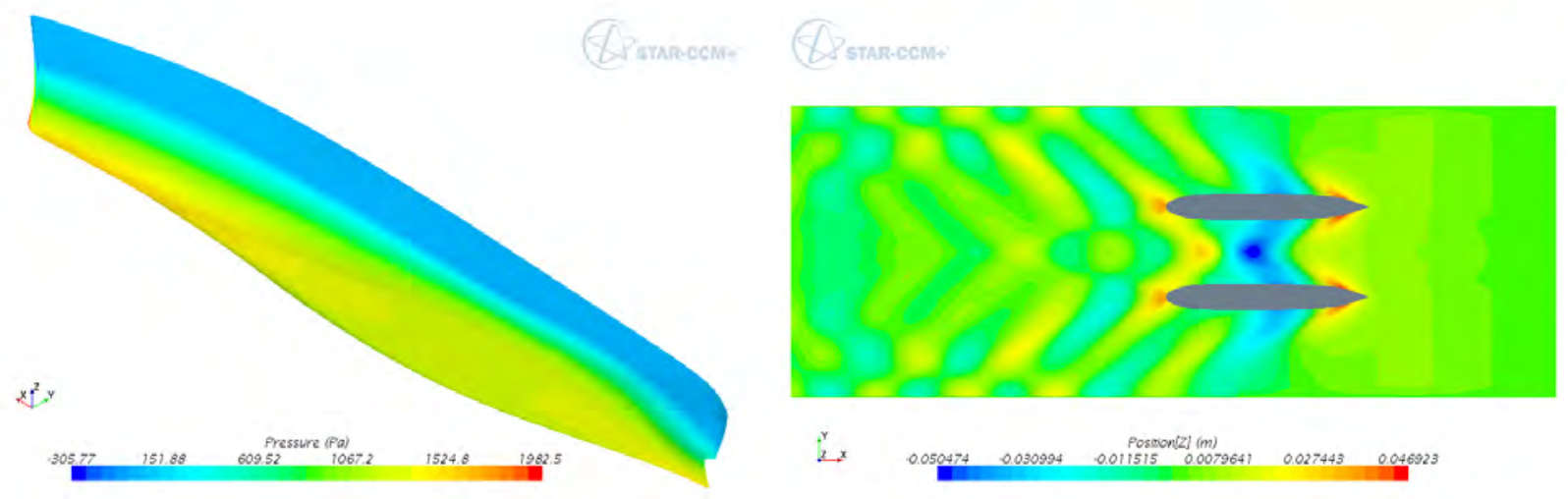

Fig. 201. $s / L=0.470 \quad F r=0.335$ modelo libre
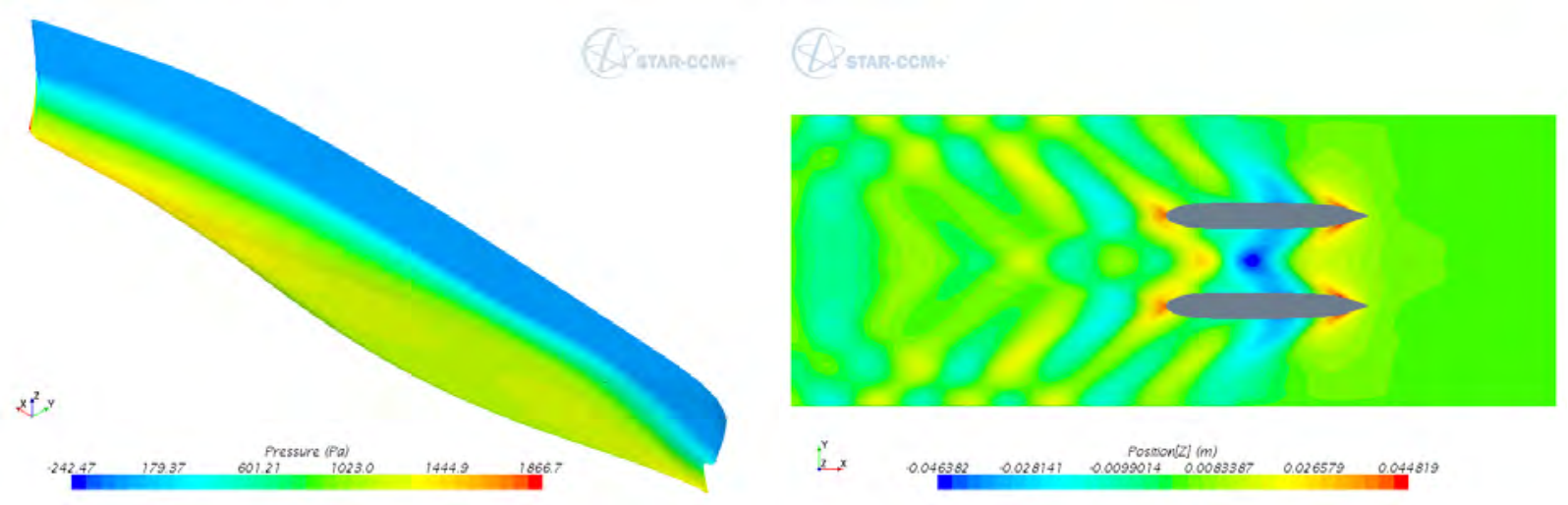

Fig. 202. $s / L=0.470 \quad F r=0.335$ modelo fijo
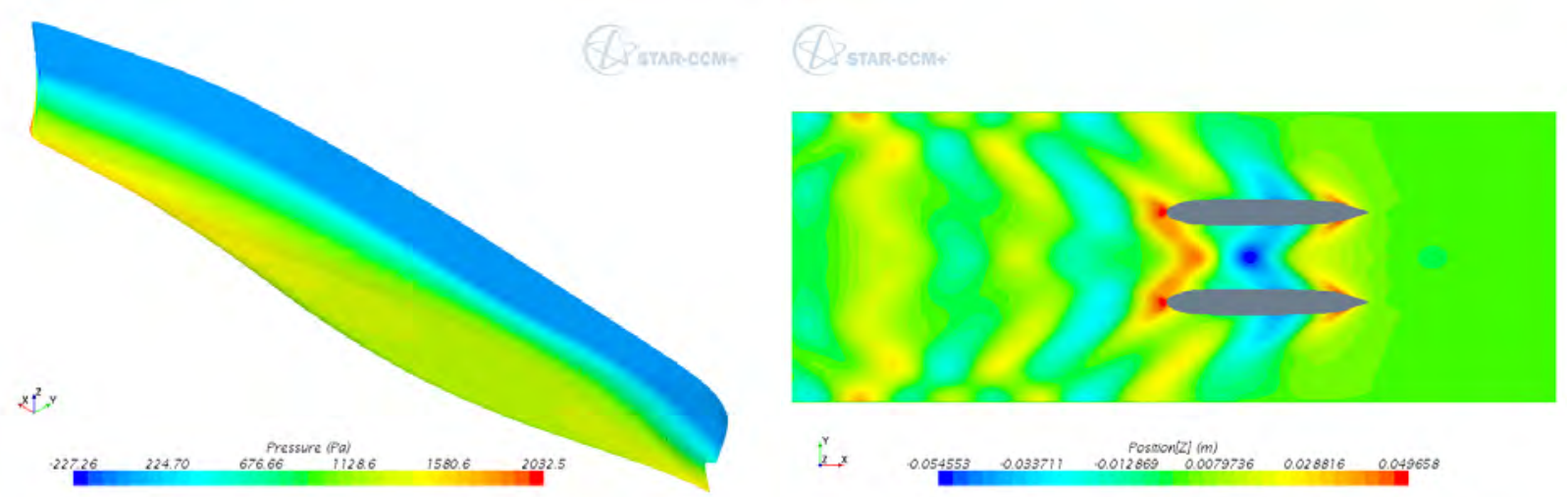

Fig. 203. $s / L=0.470 \quad F r=0.350$ modelo libre
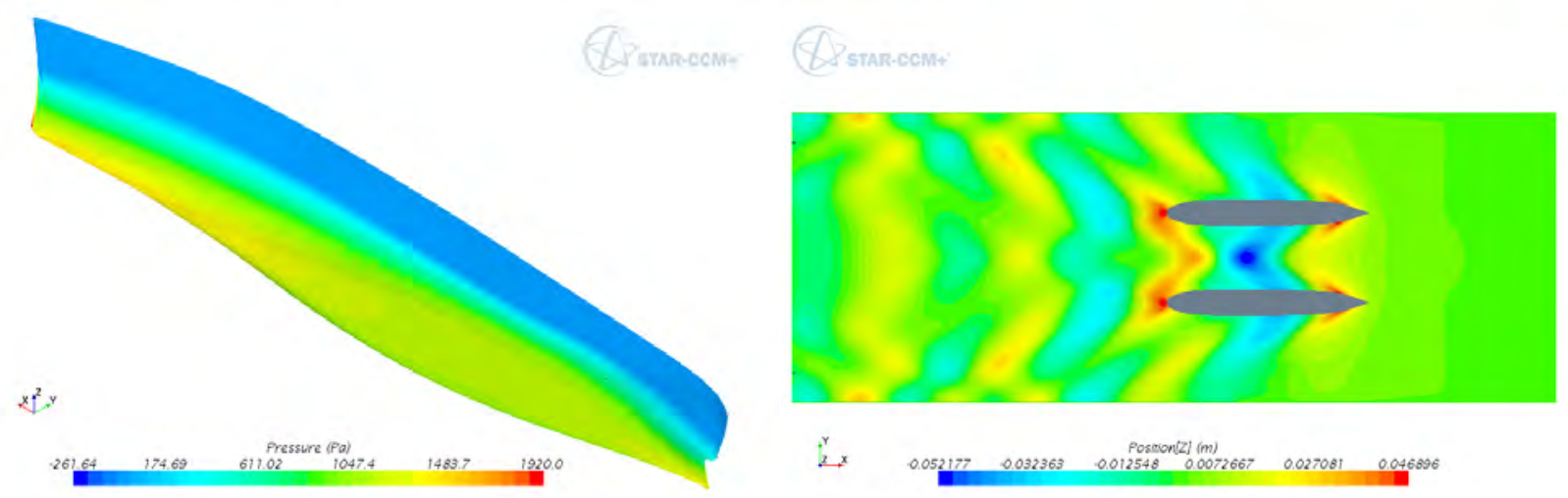

Fig. 204. $s / L=0.470 \quad F r=0.350$ modelo fijo ANÁLISIS Y OPTIMIZACIÓN DE LA RESISTENCIA DE INTERFERENCIA EN MULTICASCOS 

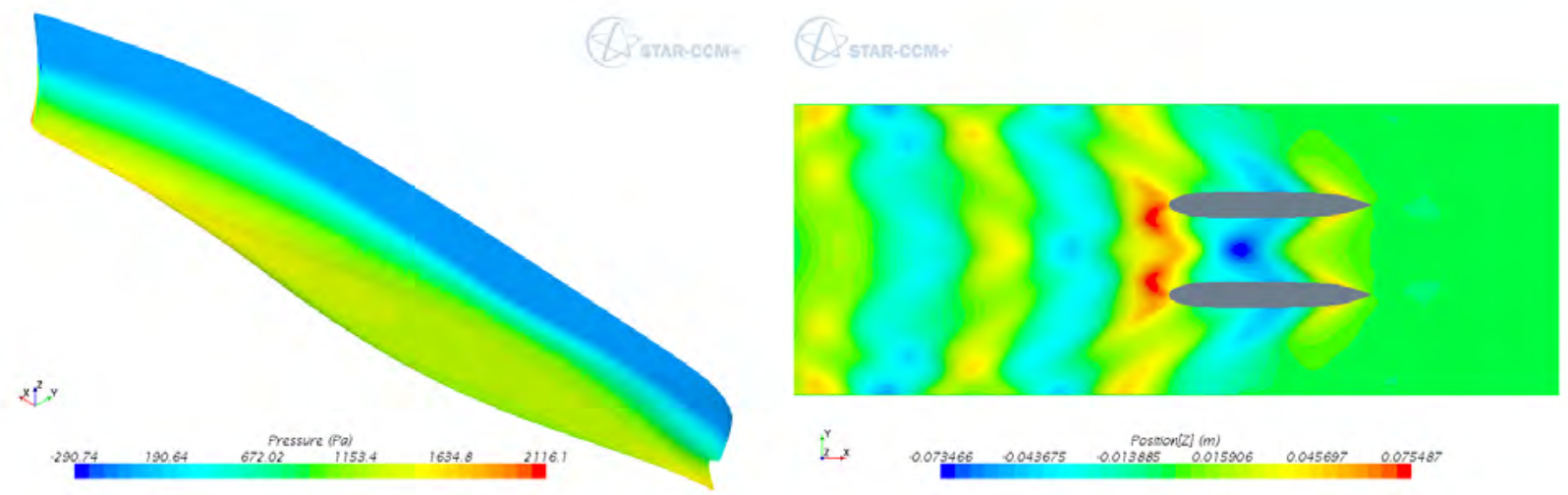

Fig. 205. $s / L=0.470 \quad F r=0.376$ modelo libre
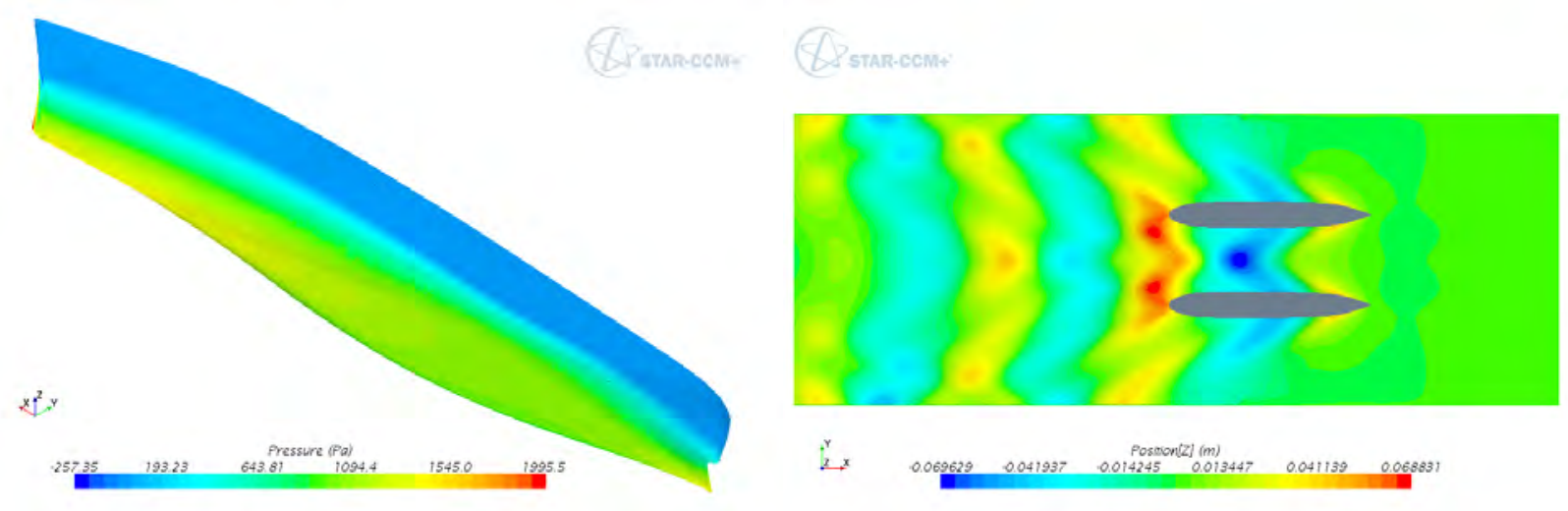

Fig. 206. $s / L=0.470 \quad F r=0.376$ modelo fijo
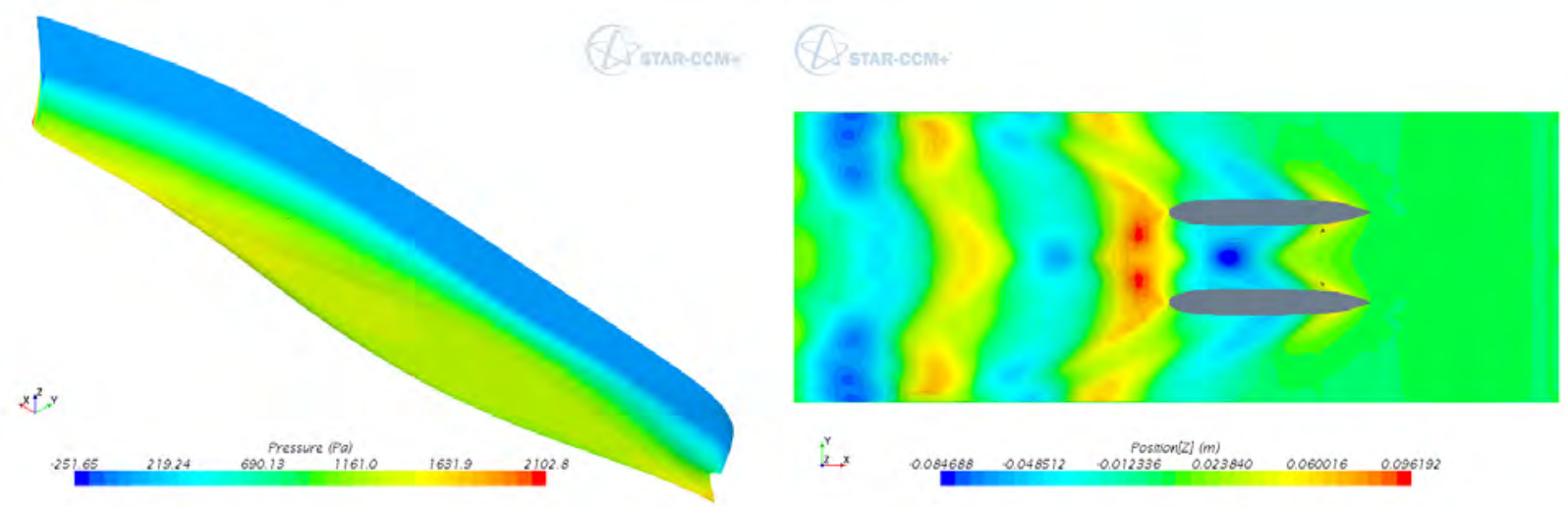

Fig. 207. $s / L=0.470 \quad F r=0.400$ modelo libre
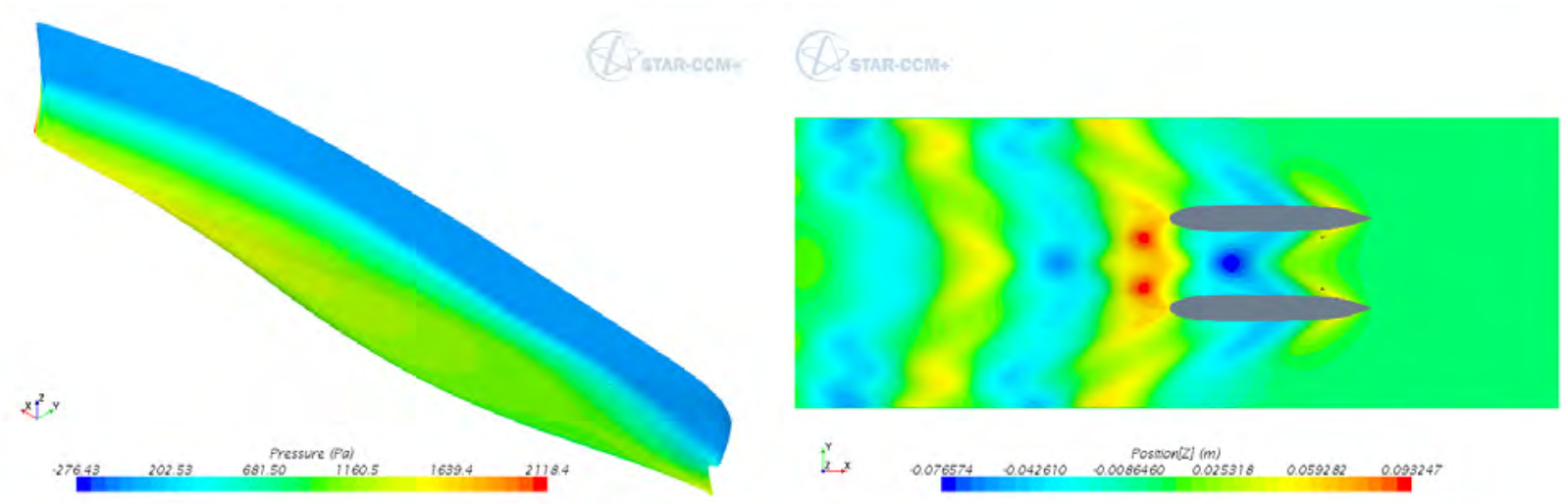

Fig. 208. $s / L=0.470 \quad F r=0.400$ modelo fijo ANÁLISIS Y OPTIMIZACIÓN DE LA RESISTENCIA DE INTERFERENCIA EN MULTICASCOS 

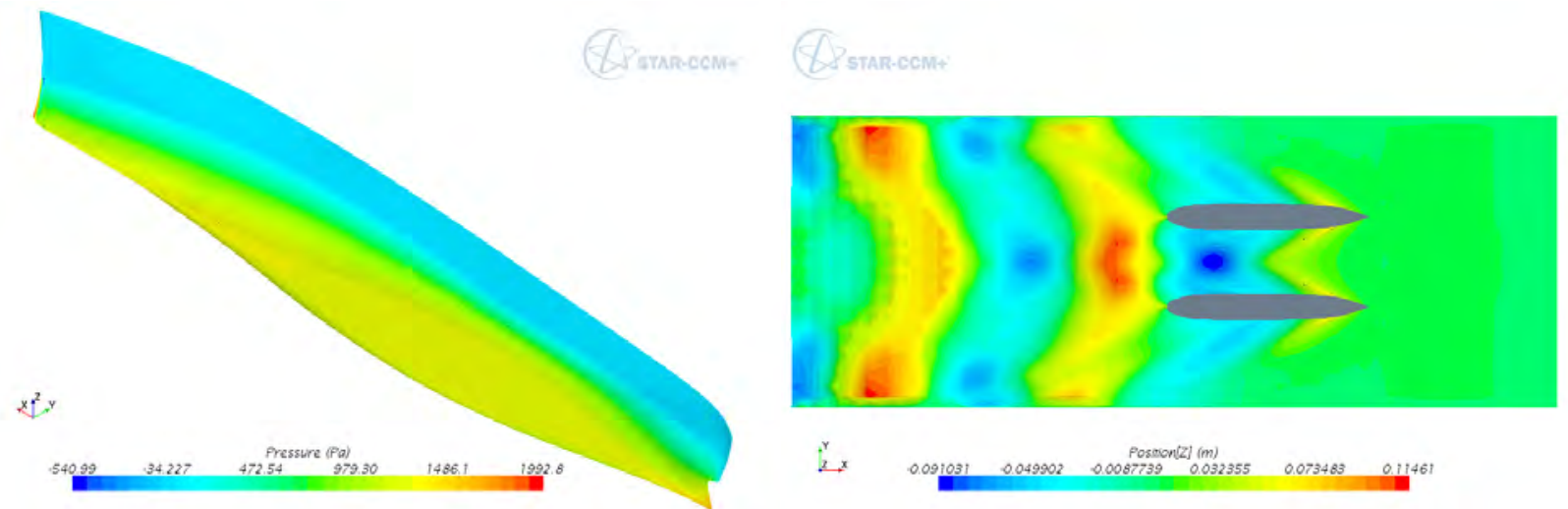

Fig. 209. $s / L=0.470 \mathrm{Fr}=0.430$ modelo libre
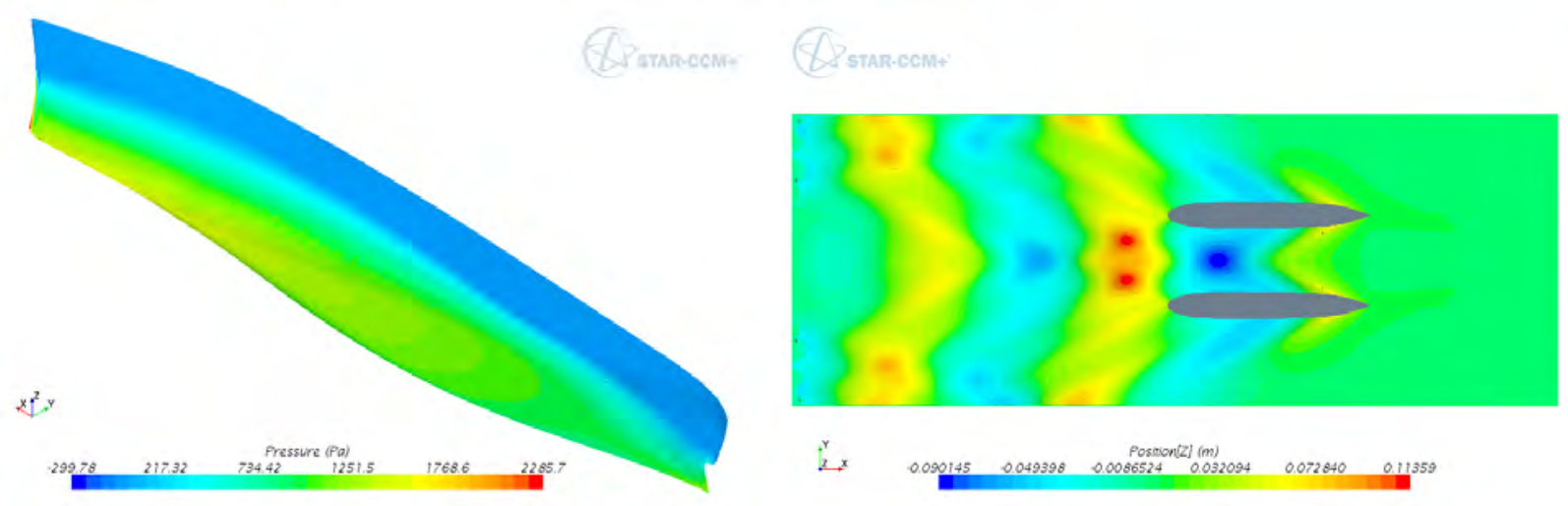

Fig. 210. $s / L=0.470 \quad F r=0.430$ modelo fijo 

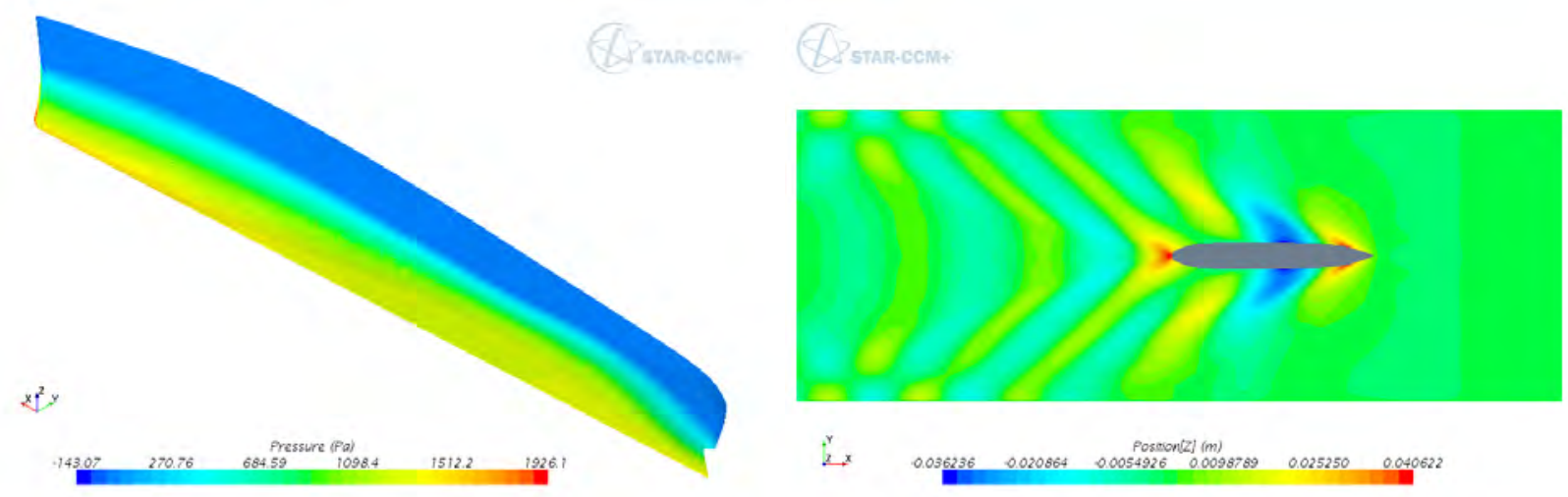

Fig. 211. Monocasco $\mathrm{Fr}=0.335$ modelo libre
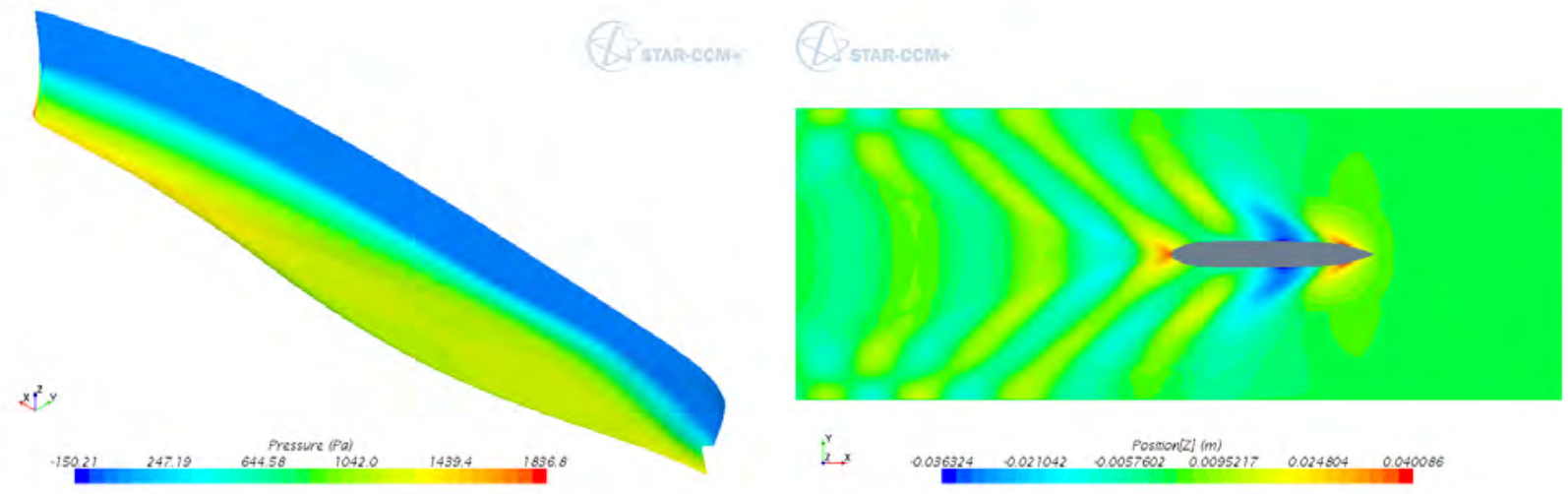

Fig. 212. Monocasco $\mathrm{Fr}=\mathbf{0 . 3 3 5}$ modelo fijo
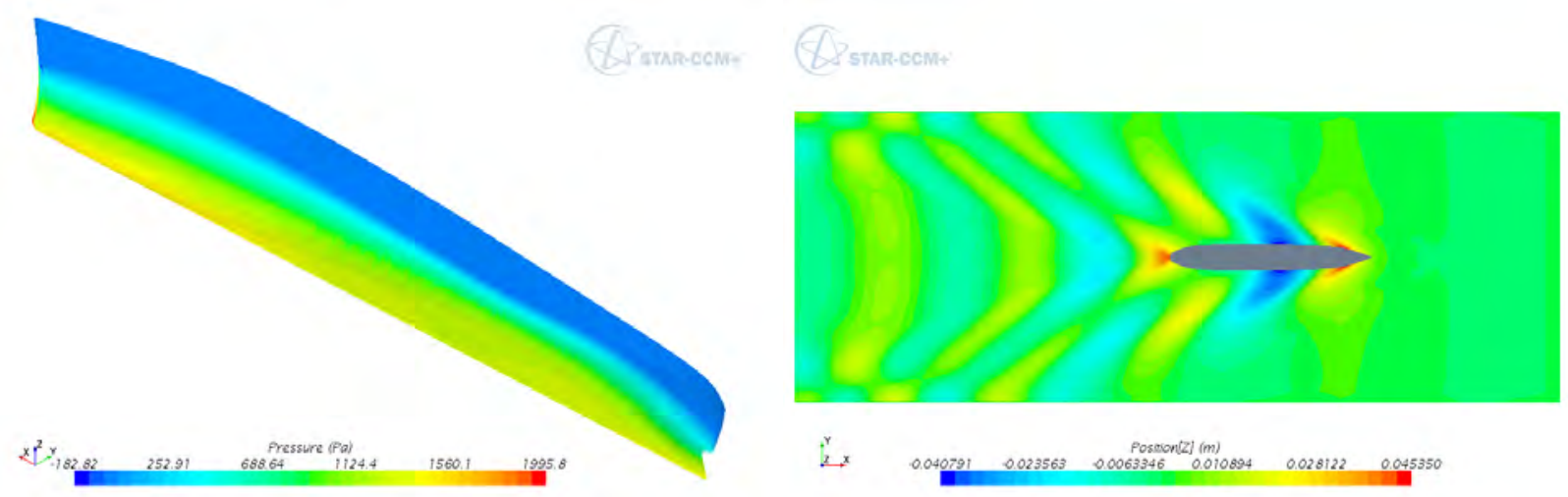

Fig. 213. Monocasco $\mathrm{Fr}=0.350$ modelo libre
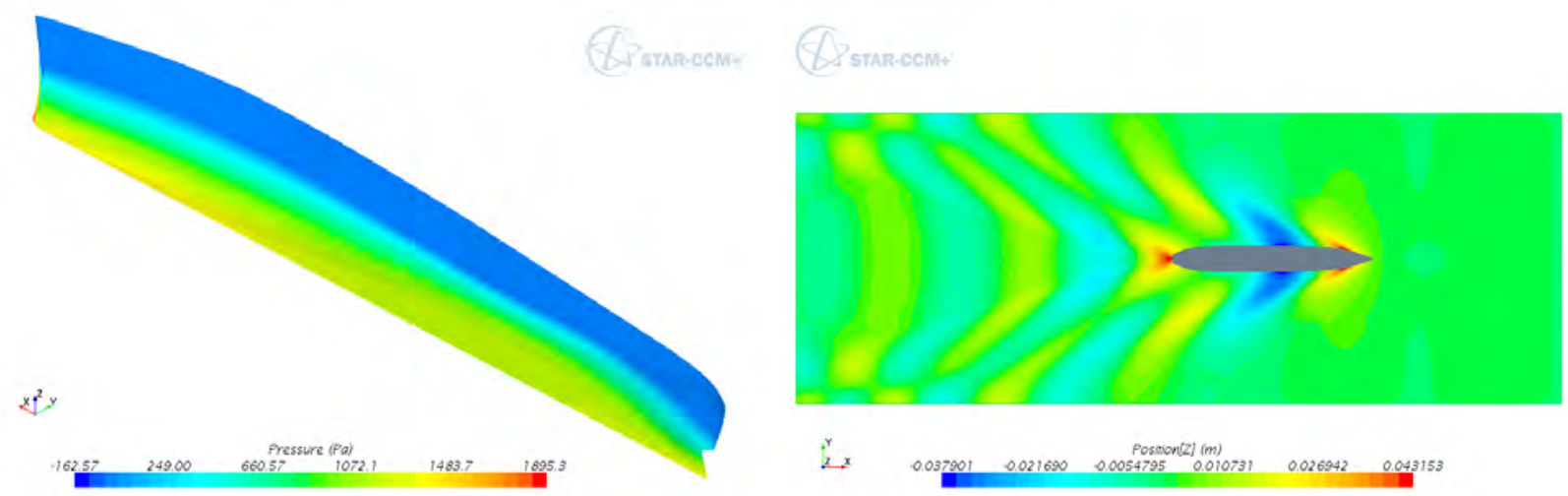

Fig. 214. Monocasco $\mathrm{Fr}=\mathbf{0} .350$ modelo fijo

ANÁLISIS Y OPTIMIZACIÓN DE LA RESISTENCIA DE INTERFERENCIA EN MULTICASCOS

Página 256 de 258 

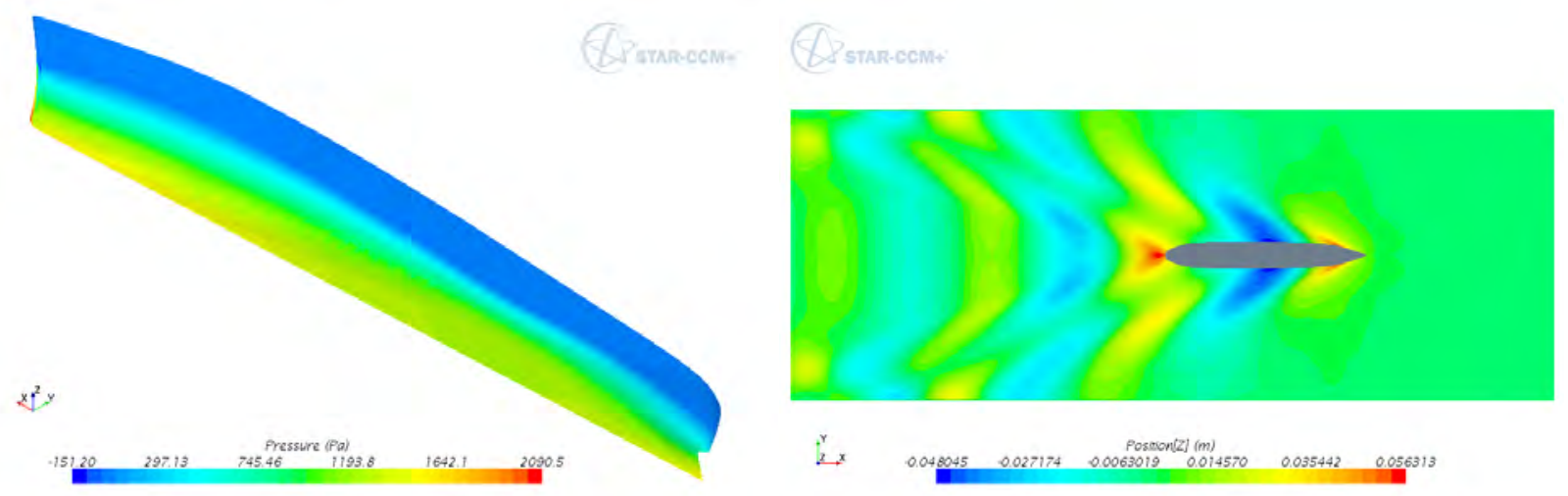

Fig. 215. Monocasco $\mathrm{Fr}=\mathbf{0} .376$ modelo libre
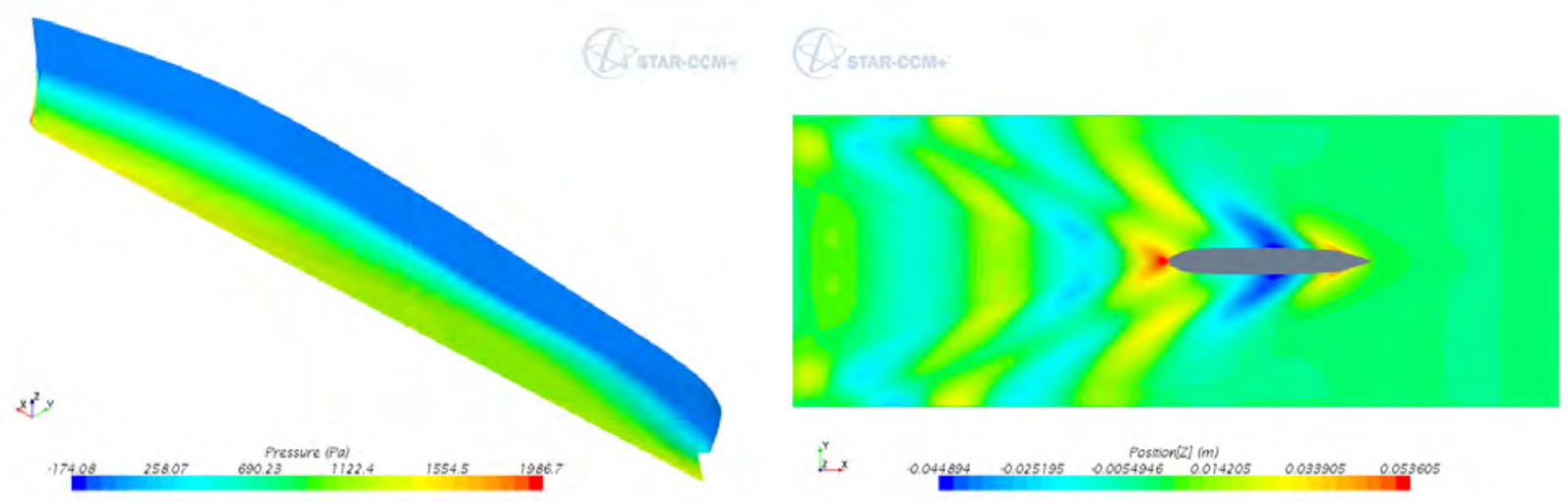

Fig. 216. Monocasco $\mathrm{Fr}=0.376$ modelo fijo
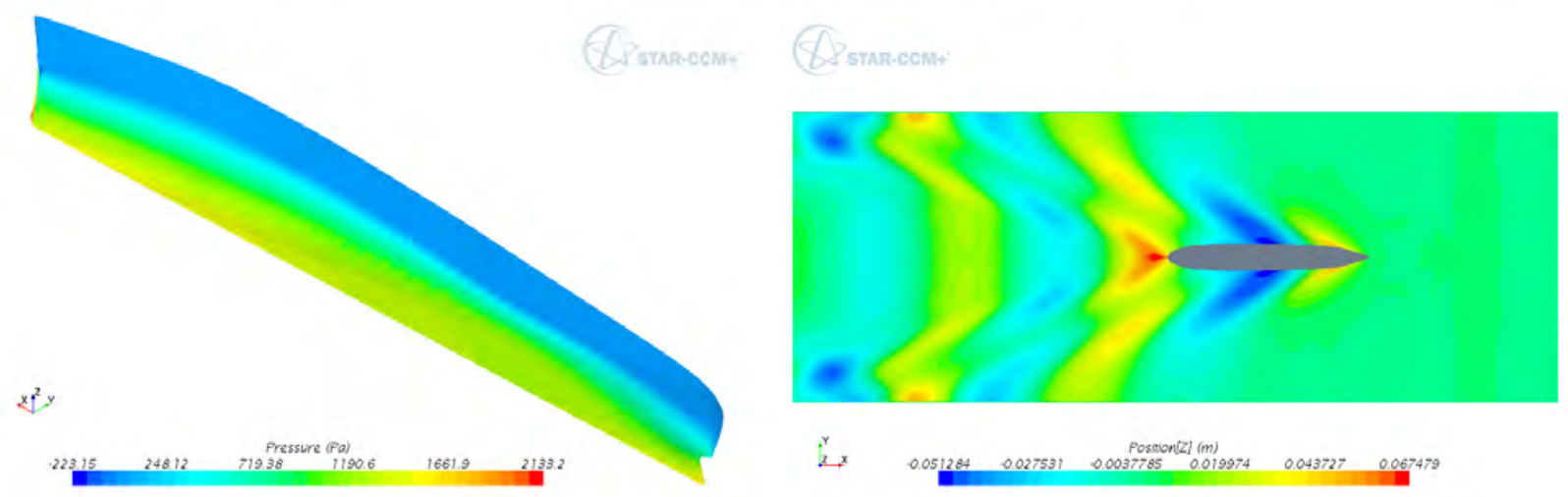

Fig. 217. Monocasco $F r=0.400$ modelo libre
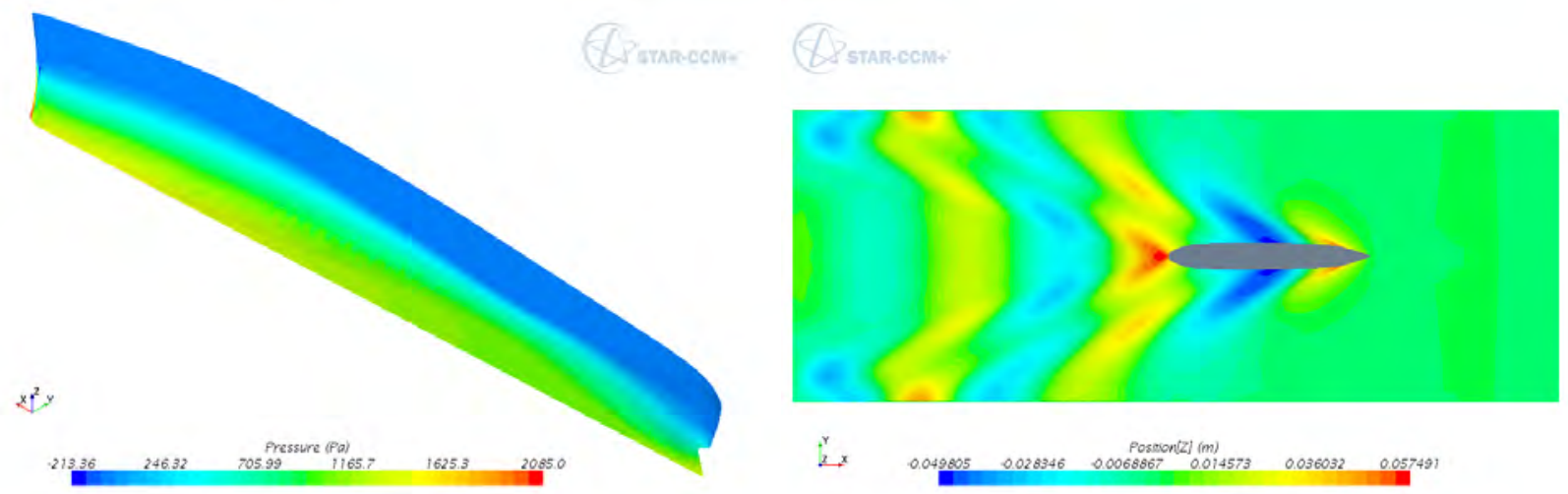

Fig. 218. Monocasco $\mathrm{Fr}=\mathbf{0 . 4 0 0}$ modelo fijo ANÁLISIS Y OPTIMIZACIÓN DE LA RESISTENCIA DE INTERFERENCIA EN MULTICASCOS 

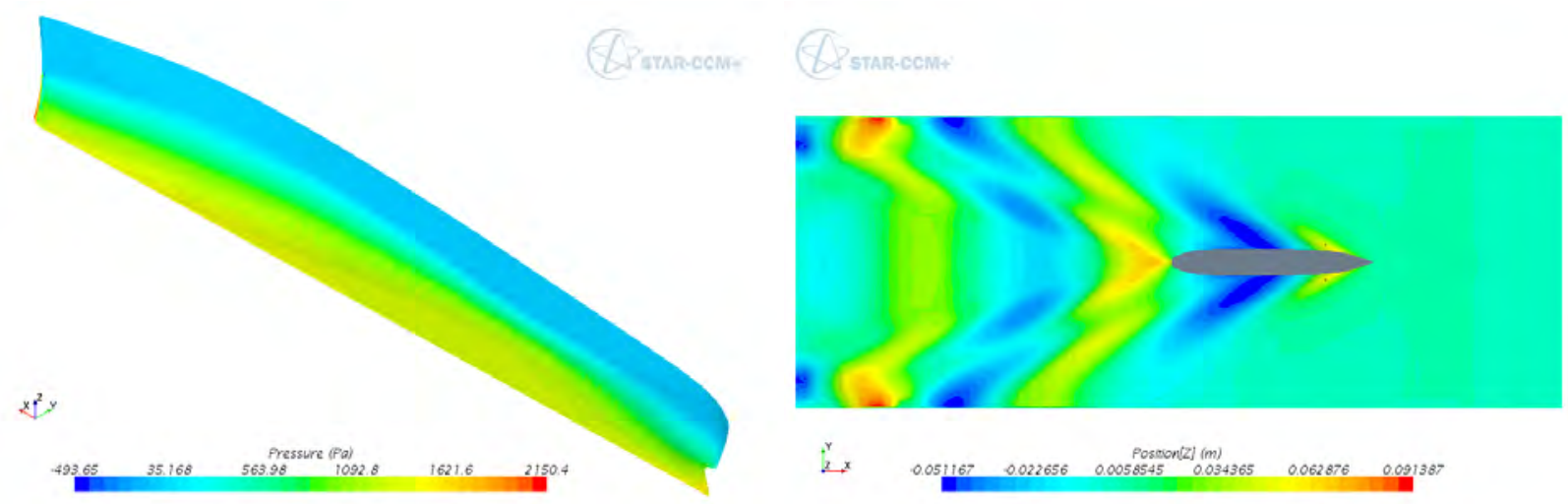

Fig. 219. Monocasco $\mathrm{Fr}=\mathbf{0 . 4 3 0}$ modelo libre
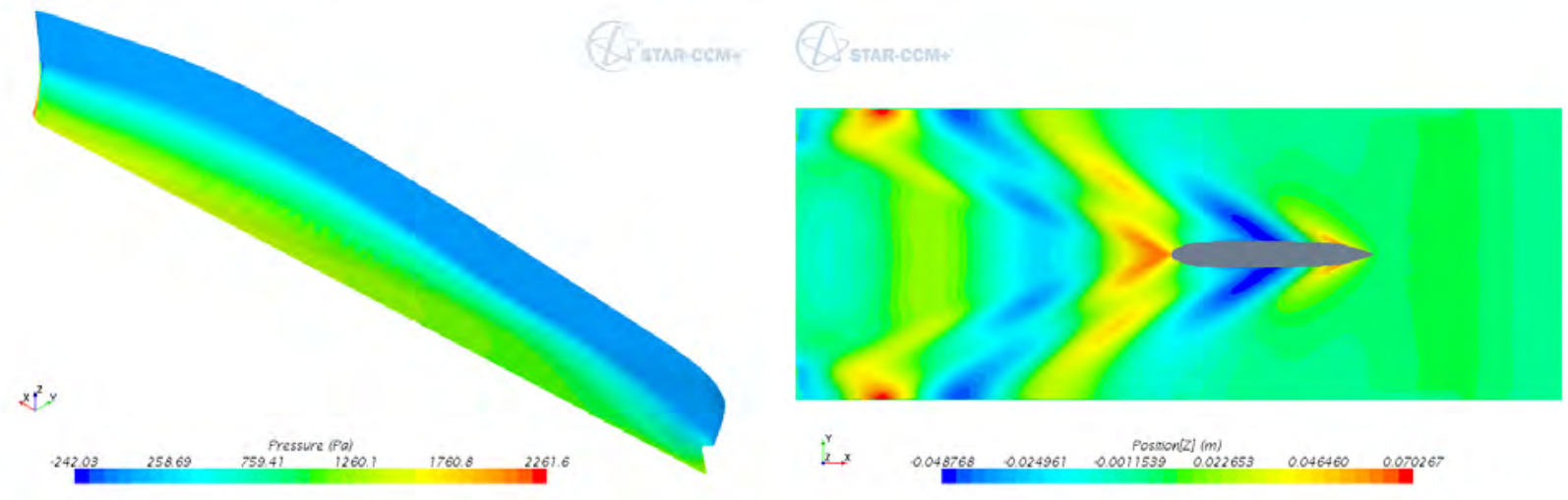

Fig. 220. Monocasco $\mathrm{Fr}=0.430$ modelo fijo

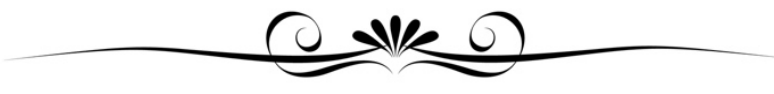

